\title{
Public Water Supplies In Western Texas
}

By W. L. Broadhurst, R. W. Sundstrom, and D. E. Weaver

GEOLOGICAL SURVEY WATER-SUPPLY PAPER 1106

Prepared by Water Resources

Division, in cooperation with the Texas State Board of Water Engineers

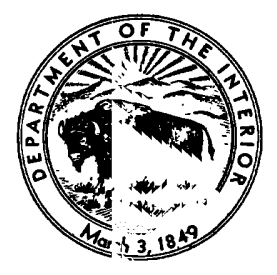

This copy is PUBLIC PROPERTY sad is not to bo revoved from the of piolal plles. PRIVATE POSSESSION IS UNLAWFUL (R. S. Sap. VOI. 2, pp. 380. Sec. 749) 


\section{UNITED STATES DEPARTMENT OF THE INTERIOR}

Oscar L. Chapman, Secretary

\section{GEOLOGICAL SURVEY}

W. E. Wrather, Director

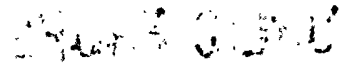

$$
\begin{aligned}
& \therefore: \text { A : : " } \\
& -40 \quad 1,26,1,
\end{aligned}
$$

For sale by the Superintendent of Documents, U. S. Government Printing Office Washington, D. C.-Price 50 cents (paper cover) 


\section{CONTENTS}

Abstract
Introduction
Extent of area and scope of report
Acknowledgments
Ground water
Area A
Area B
Area C
Area D
Area E

Municipalities and probable source of supply

Surface water

Chemical character of water

Analyses of water._._. 9

Mineral constituents in solution 10

Hardness _- 12

Alkalinity _._. 13

Hydrogen-ion concentration _._. 13

Selected bibliography

Published reports _._. 14

Unpublished reports _._. 15

Public water supplies

Andrews County _. 15

Andrews _- 15

Armstrong County _-_. 17

Claude _-_. 17

Bailey County _- 17

Muleshoe _- 17

Brewster County _._. 18

Alpine _-_ 18

Briscoe County _- 19

Quitaque

Silverton _- 20

Carson County - 21

Groom - _- 21

Panhandle _.._- 21

Skellytown _._._. 22

White Deer _... 23

Castro County _.

Dimmitt _._. 24

Childress County - 24

Childress _...

Cochran County _-_._- 28

Morton _._. 28 
Public water supplies-continued

Coke County __a 28

Bronte -._.

Robert Lee _._. 29

Collingsworth County _-_._. 30

Dodsonville _-_ 30

Wellington _._. 30

Cottle County _- 31

Paducah _. 31

Crane County _- 32

Crane -

Crockett County _.. 34

Ozona -

Crosby County _- 35

Crosbyton _..._. 35

Lorenzo _- 36

Ralls _-_-_- 36

Culberson County

Van Horn

Dallam County _- 37

Dalhart __- 37

Texline _. 39

Dawson County _. 40

Lamesa _-

Deaf Smith County_______ 44

Hereford _- 44

Dickens County _._. 44

Dickens _- 44

Spur _- 46

Donley County

Clarendon _._. 47

Hedley -

Ector County _. 49

Odessa - _. 49

Edwards County _._. 61

Rocksprings - 61

El Paso County

El Paso _. 61

Fabens _- 65

Tornillo _-_- 66

Ysleta

Fisher County _- 67

Roby _- 67

Rotan _- 67

Floyd County _. 69

Dougherty _. 69

Floydada _-_. 69

Lockney _-_ _..._- 70

South Plains _. 71 
Public water supplies-continued

Gaines County - 71

Seagraves _-__- 71

Seminole _._- 73

Garza County - 74

Post _-

Gray County _- 77

Lefors

MeLean - 78

Pampa _- 79

Hale County - 80

Abernathy _....... 80

Hale Center _-

Petersburg _-

Plainview _. 82

Hall County

Estelline _- 82

Memphis _- 83

Turkey _. 84

Hansford County - 85

Gruver -

Hitchland _. 85

Morse _. 86

Spearman _.

Hartley County

Channing - 87

Hartley _..- 88

Hemphill County __. 88

Canadian - 88

Hockley County

Anton - 89

Levelland _... 90

Howard County _._. 91

Big Spring -

Coahoma _.

Forsan - 95

Hudspeth County

Fort Hancock

Sierra Blanca _. 96

Hutchinson County _- 97

Borger _._. 97

Pringle _... 98

Stinnett _- 99

Irion County _. 99

Barnhart _... 99

Mertzon -

Jeff Davis County

Valentine -

Kent County _. 101

Jayton _ 
Public water supplies-continued

Lamb County _...... 101

Amherst _._. 101

Littlefield _..._. 102

Olton

Sudan _- 103

Lipscomb County _-_ 104

Booker … 104

Follett

Higgins _-_._- 105

Lubbock County _. 105

Idalou _._. 105

Lubbock - _. 106

Slaton - 109

Lynn County _- 110

O'Donnell _- 110

Tahoka _. 111

Martin County _. 112

Stanton _-_._. 112

Midland County _._. 113

Midland _-_._- 113

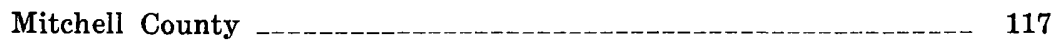

Colorado City _. 117

Moore County _- 119

Dumas _._. 119

Motley County _- 120

Matador

Roaring Springs _.. 121

Nolan County _- 122

Roscoe -

Sweetwater _... 122

Ochiltree County _. 123

Perryton -

Oldham County _. 124

Adrian - 124

Vega _- 125

Wildorado _..._. 125

Parmer County _._. 126

Farwell _. 126

Friona

Pecos County _._. 127

Fort Stockton _._. 127

Imperial _. 128

Iraan

Potter County _. 129

Amarillo _._. 129

Presidio County _-__ 135

Marfa _. 135

Presidio _ _ 136 
Public water supplies-continued

Randall County _._- 136

Canyon _-_. 136

Reagan County _- 138

Big Lake _._. 138

Santa Rita _- 139

Texon _- 139

Reeves County _- 141

Balmorhea __. 141

Pecos _. 141

Toyah _.

Roberts County _._. 144

Miami _

Schleicher County _-

Eldorado _. 144

Scurry County _. 145

Hermleigh -___-_ 145

Snyder _.

Sherman County _._. 148

Stratford _... 148

Stonewall County _._. 148

Aspermont _... 148

Sutton County _. 149

Sonora -

Swisher County

Happy _._.

Tulia _- 151

Terrell County ___ 151

Sanderson _._.

Terry County _- 154

Brownfield _. 154

Tom Green County - 155

Christoval _- 155

San Angelo

Sanatorium _- 156

Upton County _. 157

McCamey _. 157

Rankin _._._. 158

Val Verde County _._. 159

Del Rio _. 159

Ward County _- 160

Barstow _._. 160

Grandfalls _._._. 160

Monahans _._. 161

Wheeler County _- 162

Shamrock _.

Wheeler _... 163

vii 
Public water supplies-continued

Winkler County

Kermit

Yoakum County

Plains

Denver City

Literature cited 166

Index 167

\section{ILLUSTRATION}

Plate 1. Index map showing location and type of public water supplies and ground-water subdivisions in western Texas. 


\title{
PUBLIC WATER SUPPLIES IN WESTERN TEXAS
}

\author{
By W. L. BROAdHURST, R. W. SUNDSTROM, and D. E. V'EAVER
}

\begin{abstract}
This report gives a summarized description of the public water supplies in a region comprising 81 counties of western Texas and lying genorally west of the hundredth meridian. It is the fourth and last of this series of reports concerning the public water supplies of the State. It gives the avc.ilable data for each of 142 communities, as follows: The population of the community; the name of the official from whom the information was obtained; the ownership of the waterworks, whether private or municipal; the source of supply, whether ground water or surface water; the amount of water con rumed; the facilities for storage; the number of customers served; the charanter of the chemical and sanitary treatment of the water, if any; and the chemical analyses of the water. Where ground water is used the following also are given: Records of wells, including drillers' logs; character of the pumping equipment; and yield of the wells and water-level records where they ar? available.
\end{abstract}

Of the 142 public supplies, 133 are obtained from ground water, 5 from surface water, and 4 from a combination of both. The total amount of water used for public supply in the region averages about $78,000,000$ gallons a day. Of this about $61,000,000$ gallons a day is ground water and about $17,000,000$ gallons a day is surface water.

The ground-water resources of the region from which public water supplies are drawn are in rocks that range in age from Permian to Quaternary. The Ogallala formation of Tertiary age (Pliocene), which covers about 35,000 square miles of the High Plains in Texas, is the most important ground-water reservoir in the region. The formation furnishes water for 78 public supplies and for irrigating about $1,000,000$ acres of land. The amount of water used for irrigating amounted to about 1,000,000 acre-feet in 1948. The Trinity and Fredericksburg groups of Lower Cretaceous age supply ground water in the western part of the Edwards Plateau, which constitutes an area of more than 22,000 square miles. These formations furnish small to large supplies to 20 municipalities. Sands of the Dockum group of Triassic a que furnish neager to moderate supplies of water for 10 municipalities in areas east of the southern part of the High Plains and in the northern Pecos Valley in Texas. Local alluvial, bolson, or volcanic deposits furnish ground water in small to large amounts in scattered localities in the remairder of the region. The Permian rocks are of little importance as a source of ground water for public supply, owing to the highly mineralized water in them.

The results of the chemical analyses of 206 samples of water obtained from the public supplies of the region are given in this report. T'e analyses are reported in parts per million and in equivalents per million for those ions entering into ionic balance. Of the samples analyzed 57 percent contained silica in excess of 20 parts per million; about 9 percent contained iron in excess of 0.3 part per million; 78 percent had hardness in excess of 200 
parts per million; about 18 percent contained sulfate in excess of 250 parts per million; 10 percent contained chloride in excess of 250 parts per million; 3 percent contained nitrate in excess of 20 parts per million; 37 percent contained fluoride in excess of 2 parts per million; and 12 percent contained dissolved solids in excess of 1,000 parts per million.

\section{INTRODUCTION}

\section{EXTENT OF AREA AND SCOPE OF REPORT}

This report gives a summarized description of the public water supplies in a region comprising 81 counties of western Texas and lying generally west of the hundredth meridian. It is the fourth and last of a series of reports on the public water supplies of Texas (Sundstrom, Hastings, and Broadhurst, 1948; Sundstrom, Broadhurst, and Dwyer, 1949; and Broadhurst, Sundstrom, and Rowley, 1950) ${ }^{1}$ prepared by the United States Geological Survey in cooperation with the Texas State Board of Water Encineers.

The need for certain basic data in the study of quantitative and qualitative problems of public water supply has long been apparent. This has been brought into sharper focus in Texas in recent years by the great increase in the demands for water for public and industrial supply. The phenomenal growth of many Texas cities has resulted in the need from time to time for expanding or rebuilding the waterworks systems. Some of the municipalities still use the original source of suppl $r$, some have developed additional sources of ground water, and others have replaced inadequate supplies of ground water with surface water.

This report gives in condensed form the available data for each municipality, as follows: Population of the community; name of the official from whom the information was obtainer; ownership of the waterworks, whether private or municipal ; source of supply, whether ground water or surface water; amount of water consumed; facilities for storage; number of service connections; character of the chemical and sanitary treatment of the water, if any; and the chemical analyses of the water. Where ground water is used the following also are given: Records of wells, including drillers' logs; character of the pumping equipment; and yield of the wells and water-level records where they are available. Unfortunately, many of the municipalities have kept poor records and some no records at all, and the information given for such municipalities necessarily is incomplete. The lack of data regarding the amount of water pumped and the resulting changes in

1 Names of authors, with dates, in parentheses refer to publications listed in the Literature Cited, p. 166. 
water level or artesian pressure in the wells since they were drilled is unfortunate. Such information is of great importance, particularly in areas where the draft on the underground supplies approaches the practical limits of development.

Five of the 81 counties in the region have no public water supplies. The region has an area of 107,272 square miles, and in 1940 it had a population of 892,418 . About half the people live in communities served by the public water supplies described in this report. The total amount of water pumped by these communities averages about $78,000,000$ gallons a day. Of this amount about $61,000,000$ gallons is ground water and about $17,000,000$ gallons is surface water. Ground water is used at 133 localities, surface water at 5 , and a combination of ground and surface water at 4.

\section{ACKNOWLEDGMENTS}

The data presented in this report were collected by engineers and geologists of the Federal Geological Survey and th? Texas Board of Water Engineers who are engaged in ground-water studies in Texas. The field work was done by J. R. Barnes, R. L. Cushman, J. H. Dante, W. C. Ellis, D. B. Knowles, J. W. Lang, E. R. Leggatt, R. A. Scalapino, and the writers. This report was prepared and written jointly by W. L. Broadhurst and R. W. Sundstrom, except for the section on the analyses of water which was written by D. E. Weaver. The analyses of water were made in the laboratory of the Geological Survey at Austin. Assistance given by city officials and well drillers, who furnished most of the information, is gratefully acknowledged.

\section{GROUND WATER}

The ground-water reservoirs of the region from which the public-water supplies are drawn occur in rocks that range in age from Permian to Quaternary. The Permian rocks are of minor importance as a source of public water supply in Texas, owing to the highly mineralized water in them. The city of Paducah in Cottle County is believed to be the only city in the region using ground water from the Permian rocks, and the water it uses is highly mineralized. The most important sources of ground water are the Dockum group of Triassic age, which furnisher ground water for 10 localities; the Trinity and Fredericksburg groups of Lower Cretaceous age, which supply 21 localities; the lavas of Tertiary age, which are believed to supply 3 localities; the Ogallala formation of Tertiary age, which supplies 78 localities; and al- 
luvial and bolson deposits of Quaternary age, which supply 27 localities.

For convenience in summarizing the sources of ground water, the region has been divided into five areas as shown in plate 1.

Area A.-In Area A the ground-water supplies are obtained principally from the Ogallala formation of Tertiary age, and in a few localities in the southern part of the area some ground water for public supply is obtained from the underlying sands of Cretaceous age. The Ogallala formation, which cover about 35,000 square miles of the High Plains in Texas, is by far the most important ground-water reservoir in the region. It not only furnishes water for 78 public supplies in Area A, but approximately $1,000,000$ acre-feet of water was pumped from about 8,500 irrigation wells to irrigate about $1,000,000$ acres of land in 1948. The sediments were deposited by wind action and by str?ams, some of which long ago in geologic time had their headwaters in the Rocky Mountains. The Ogallala rests on an uneven floor of older rocks which were eroded into valleys and ridges before the Ogallala was deposited. However, owing to subsequent erosion, it has been completely removed from the valleys of the Pecos and Canadian Rivers. The formation ranges from a $f \in$ ather edge to nearly 600 feet in thickness, but in most places it is between 200 and 300 feet thick. It is composed chiefly of silt and fine-grained sand but contains some coarse sand and gravel. The coarser sediments, which usually yield water freely to wells, are present at all horizons but are most prominent in the lower part of the formation. The cities that probably draw some water from the underlying sands of the Cretaceous in the southern part of Area A are Lubbock, Lamesa, Seagraves, and Seminole.

Area B.-Area B lies eastward from the High Plains escarpment. Along the border of the escarpment and in the southwestern part of the area, ground-water supplies are obtained from sandstones in the Dockum group of Triassic age in six localities, but these sandstones generally yield only meager supplies to wells. In the remainder of the area all the ground-water supplies are obtained from shallow alluvial deposits, except at Paducah where highly mineralized water is drawn from Permian rocks. Alluvial deposits furnish ground water for public supply ir 15 localities, but in general these deposits are thin, and ground water suitable for public supplies and in large quantities is difficult to obtain in most of the area.

Area C.-Area C extends southward from Areas A and B. It covers about 22,000 square miles and includes the western part 
of the Edwards Plateau. Sands and limestones of the Trinity and Fredericksburg groups, of Lower Cretaceous age, furnirh all the public water supplies in the area. Del Rio uses water from San Felipe Springs, which issue from these rocks. The amount of ground water that can be obtained from the Lower Cretaceous rocks in the area varies greatly from place to place. In parts of the area erosion has formed deep valleys, and much of the groundwater reservoir has been drained; in some places the sands of the Trinity group are thin or have a low permeability, or both; but in other places large supplies are furnished from both the sands of the Trinity group and limestones of the Fredericksburg group.

Area D.-Area D constitutes a large part of the region lying west of Area C. The ground-water reservoirs that furnish water for public supply in the area are found in the alluvial and bolson outwash deposits of Quaternary age, except in the northeastern part of the area where the public supplies of Pecos, Barstow, Kermit, Monahans, and Imperial are obtained from ground-water reservoirs in the Dockum group of Triassic age. The amount of ground water that can be obtained from the alluvial ard bolson deposits varies greatly from place to place, and in many places the ground water in these deposits is highly mineralized. The Triassic rocks yield water acceptable for domestic purposes but the reservoirs generally do not yield large quantities of water to wells.

Area E.-In Area E, which lies within Area D, ground water for public supply is obtained in part from volcanic lavas of Tertiary age, and perhaps in part from Cretaceous rocks that underlie the lava and from alluvium.

\section{MUNICIPALITIES AND PROBABLE SOURCE OF SUPPLY}

The following table lists 127 of the municipalities in the region covered by this report that obtain their public water supplies in whole or in part from ground water and gives the probable waterbearing formation or groups of formations from which the water is drawn. For 10 of the 137 communities using ground water in whole or in part the source of the water is not known.

Municipalities and probable water-bearing formation from which water is drawn

Adrian

Alpine

Amarillo

Amherst
Ogallala formation.

Tertiary volcanic lava.

Ogallala formation.

Do. 
Andrews

Anton

Barnhart

Barstow

Big Lake

Big Springs

Booker

Borger

Bronte

Brownfield

Canadian

Canyon

Channing

Childress

Clarendon

Claud

Coahoma

Colorado City

Crane

Cristoval

Crosbyton

Denver City

Dickens

Dimmit

Dodson

Dougherty

Dumas

Eldorado

El Paso

Estelline

Fabens

Farwell

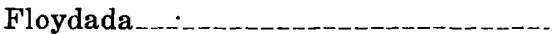

Follett

Forsan

Fort Hancock

Fort Stockton

Friona

Grandfalls

Groom

Gruver

Hale Center

Happy

Hartley

Hedley

Hereford

Hermleigh

Higgins

Hitchland

Idalou
Ogallala formation.

Do.

Trinity group.

Dockum group.

Trinity group.

Alluvium.

Ogallala formation.

Do.

Alluvium.

Ogallala formation.

Alluvium.

Dockum group.

Ogallala formation.

Alluvium.

Ogallala formation.

Do.

Do.

Alluvium.

Do.

Trinity group

Ogallala formation.

Do.

Dockum group.

Ogallala formation.

Alluvium.

Ogallala formation.

Do.

Fredericksburg group.

Alluvium and bolson deposits.

Alluvium.

Bolson deposits.

Ogallala formation.

Do.

Do.

Trinity group.

Bolson deposits.

Trinity group.

Ogallala formation.

Alluvium.

Ogallala formation.

Do.

Do.

Do.

Do.

Do.

Do.

Dockum group.

Ogallala formation.

Do.

Do. 
Imperial

Iraan

Jayton

Kermit

Lamesa

Lefors

Levelland

Littlefield

Lockney

Lorenzo

Lubbock

McCamey

\section{McLean}

Memphis

Mertzon

Miami

Midland

Monahans

Morse

Morton

Muleshoe

Odessa

O'Donnell

Olton

Ozona

Paducah

Pampa

Panhandle

Perryton

Petersburg

Plainview

Plains

Post

Pringle

Quitaque

Ralls

Rankin

Roaring Springs

Robert Lee

Rock Springs

Roscoe

Rotan

Sanatorium

Sanderson

Santa Rita

Seagraves.

Seminole
Dockum group.

Trinity group.

Alluvium.

Dockum group.

Ogallala formation ard

Trinity group.

Ogallala formation.

Do.

Do.

Do.

Do.

Ogallala formation and

Trinity group.

Trinity group.

Ogallala formation.

Do.

Trinity group.

Ogallala formation.

Do.

Dockum group.

Ogallala formation.

Do.

Do.

Trinity group.

Ogallala formation.

Do.

Trinity group.

Permian rocks.

Ogallala formation.

Do.

Do.

Do.

Do.

Do.

Do.

Do.

Alluvium.

Ogallala formation.

Trinity group.

Alluvium.

Do.

Trinity group.

Ogallala formation.

Dockum group.

Alluvium.

Trinity group.

Do.

Ogallala formation and Trinity group.

Do. 


Shamrock
Silverton
Skellytown
Slaton
Snyder
Sonora
South Plains
Spearman
Spur
Stanton
Stinnett
Stratford
Sudan
Tahoka
Texline
Texon
Tornilla
Tulia
Turkey
Van Horn
Vega
Wellington
Wheeler
White Deer
Wilderado
Ysleta

Ogallala formation.

Do.

Do.

Do.

Alluvium and Dockum group.

Fredericksburg group.

Ogallala formation.

Do.

Alluvium.

Ogallala formation.

Do.

Do.

Do.

Do.

Do.

Trinity group.

Bolson deposits.

Ogallala formation.

Alluvium.

Bolson deposits.

Ogallala formation.

Alluvium.

Ogallala formation.

Do.

Do.

Bolson deposits.

\section{SURFACE WATER}

In the region covered by this report only five municipalities use surface water exclusively, and four use a combination of ground and surface water. The total amount of surface water used by the nine municipalities amounts to an average of about $17,000,000$ gallons a day. Of this amount San Angelo uses an average of about $6,000,000$ gallons a day from reservoirs on the North, South, and Middle forks of the Concho River; El Paso uses an average of about 5,000,000 gallons a day from a diversion canal on the Rio Grande, and also uses about 11,010,000 gallons a day of ground water; Big Spring uses an average of about $1,300,000$ gallons a day from reservoirs on Powell and Moss Creeks, and also uses about 1,000,000 gallons a c'ay of ground water; and Sweetwater uses an average of about 2,300,000 gallons a day from reservoirs on Sweetwater and Bitter Creeks. Small amounts of surface water are used by Aspermont, Roby, Robert Lee, and Bronte, at the last two in combination with ground water. 


\section{CHEMICAL CHARACTER OF WATER}

\section{ANALYSES OF WATER}

The analyses given in this report show the chemical quality but not the sanitary fitness of the waters. However, with tro exception of the water supply at Roby, all the surface supplies and many of the ground-water supplies are reported to be chlorinated.

A single sample is ordinarily representative of a well water, as the chemical character of ground waters usually shows no material change in quality over long periods of time. Surface waters vary in chemical quality with rainfall and runoff. For this reason a single sample of a surface source is only an indication of the general nature of the water furnished to the public. Variation in quality of water from lakes is usually less than that from streams.

Water used for public supplies must be potable and should be low in mineral content so as to be free from tastes and any physiological reactions. All natural waters contain some dissolved mineral matter, because water is a very good solvent and soluble material is widely distributed in the atmosphere and in the ground. In addition to the exact limits set for concentrations of toxic salts, the United States Public Health Service drinking-water standards give the following limits for concentrations of other minerals quite commonly found in natural water:

Iron $(\mathrm{Fe})$ and manganese $(\mathrm{Mn})$ together should not exceed $0 . \AA$ part per million.

Magnesium ( $\mathrm{Mg}$ ) should not exceed 125 parts per million.

Chloride $(\mathrm{Cl})$ should not exceed 250 parts per million.

Sulfate $\left(\mathrm{SO}_{4}\right)$ should not exceed 250 parts per million.

Total solids should not exceed 500 parts per million for water of good chemical quality. However, if such water is not available, total solids of 1,000 parts per million may be permitted.

Sometimes water that exceeds the recommended limits is used when no other water supply is available.

Water passes through a natural cycle beginning with precipitation from clouds, followed by percolation into the ground or runoff into surface streams and thence into the sea, and, finally liy evaporation into the atmosphere. The chemical quality of surface water is determined by the solubility of the material of the vatershed over which it passes. Therefore, the amount of dissolved solids in surface waters differs greatly. For example, the water from Cottonwood Creek at Roby in Fisher County has more tr an 4,000 parts per million of dissolved solids, whereas the other surface waters used for public supplies in western Texas are relatively low in dissolved solids. 
In general, ground waters are somewhat more highly mineralized than surface waters. Some minerals, such as iron, are found in much greater amounts in ground waters than in scrface waters. Ground waters that derive their chemical content from igneous rocks usually have a relatively high alkalinity, low hardness, and considerable silica, and sometimes they contain iron and manganese. Waters derived from sedimentary rocks are more variable in composition and usually contain bicarbonates of calcium and magnesium and some chloride. Chloride and sulfate are often the predominant anions in waters from formations containing deposits of salt and gypsum. Most of the ground-rater supplies of cities in western Texas come from sedimentary jocks.

The most important considerations in evaluating the quality of the water supplies are hardness, alkalinity, and total mineral content. The hardness of natural waters is caused largely by compounds of calcium and magnesium and sometimes iron and aluminum. The alkalinity of natural waters is caused by carbonates, bicarbonates, hydroxides, and, occasionally, silicates, borates, and phosphates.

The analyses are reported in parts per million, $\epsilon x c e p t$ for $\mathrm{pH}$, and also in equivalents per million for those ions entering into ionic balance. Methods of analysis are those in general use by the United States Geological Survey (Collins, 192\&; Amer. Pub. Health Assoc. 1946).

\section{MINERAL CONSTITUENTS IN SOLUTION}

Silica $\left(\mathrm{SiO}_{2}\right)$ is found in most natural waters. It probably occurs in the form of very finely divided particles in colloidal suspension. In the more alkaline waters some of the silica may be present in ionic form. Silica has no effect on the use of water for irrigation or domestic purposes, but it causes a hard boiler scale if present in appreciable quantities in boiler-feed water. In lowpressure boilers the scale formation results in inefficient heat transfer, and in high-pressure boilers the scale may cause overheating and boiler-tube failure. Fifty-seven percent of the waters analyzed had more than 20 parts per million of silica; the greatest quantity was 80 parts per million, the lowest 5 parts per million.

Iron $(\mathrm{Fe})$ found in natural waters is usually in the form of ferrous bicarbonate or ferrous or ferric sulfate. In the presence of oxygen these iron compounds are converted to ferric oxide and precipitated; hence, in surface water the iron content seldom is high. Iron is commonly found in ground water because of the wide distribution of iron in nature as hematite and its ready 
INDEX MAP SHOWING LOCATION AND TYPE OF PUBLIC WATER SUPPLIES AND GROUND-WATER SUBDIVISIONS IN WESTERN TEXAS

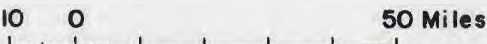

EXPLANATION

- Supply from ground water

- Supply from surfoce water

- Supply from ground and surface water

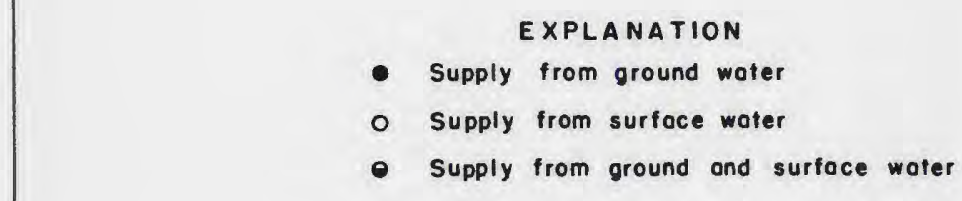

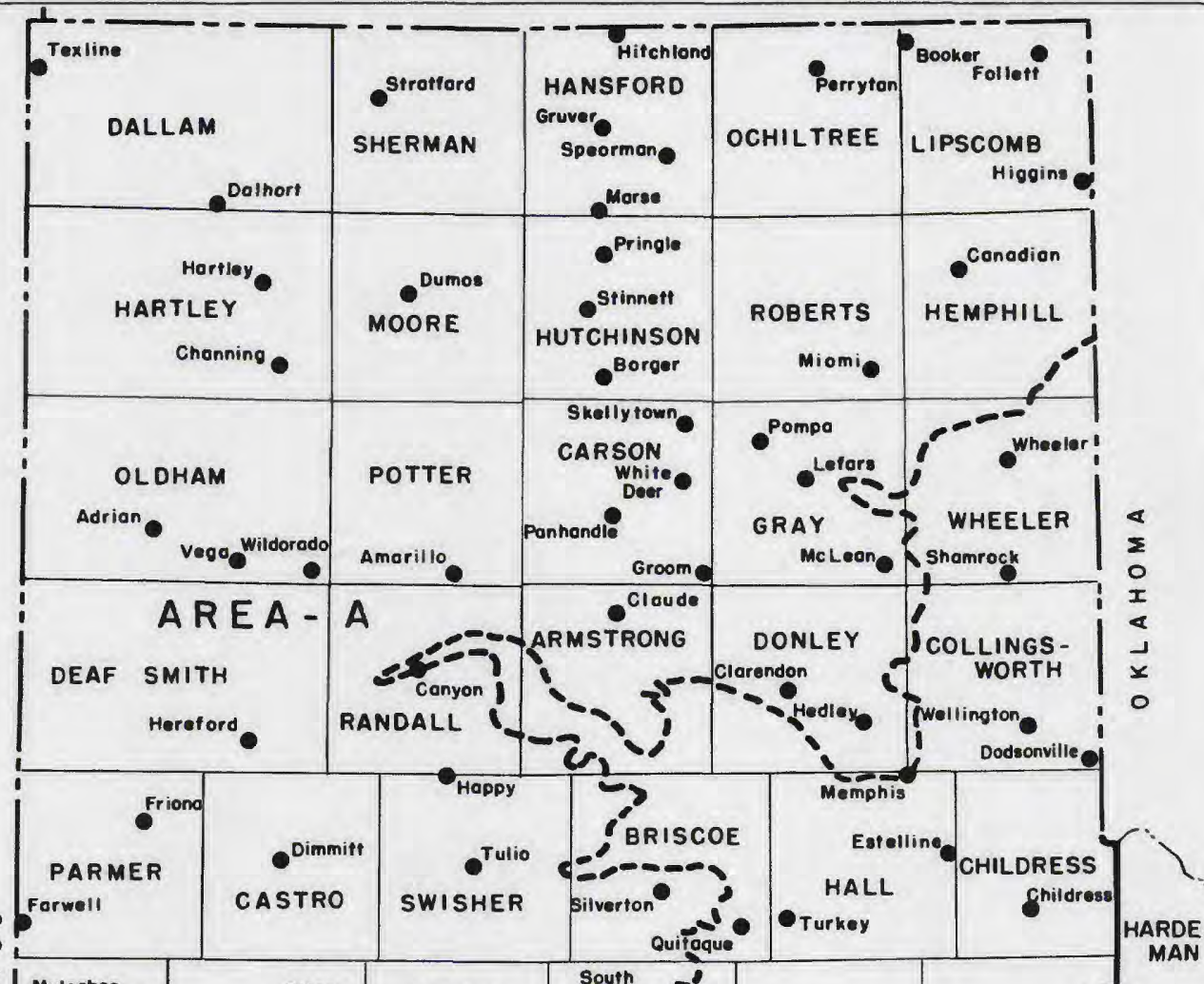

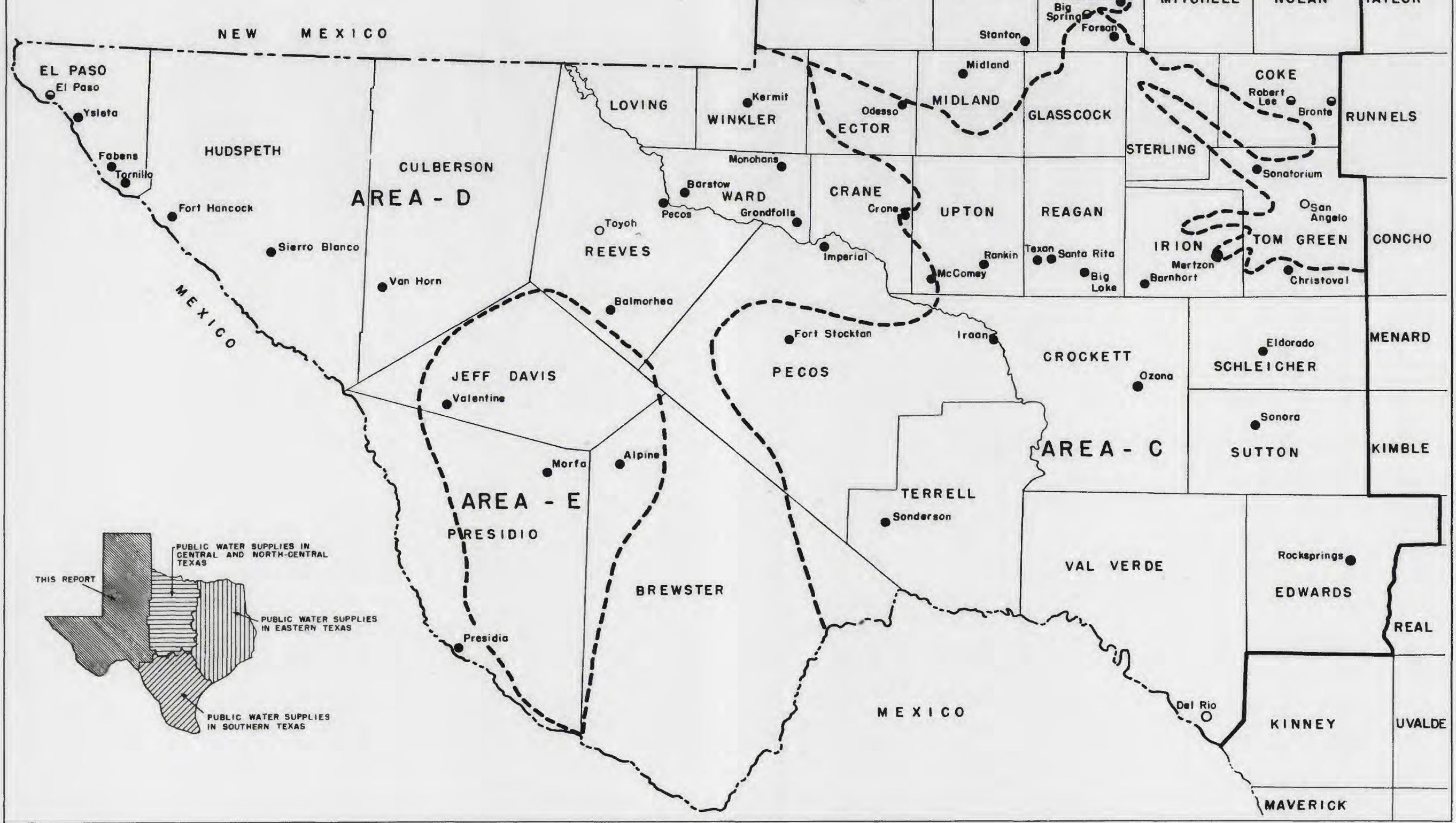

$9475350-51$ (Face p. 10) 
folution in those waters containing carbonic acid. In Frblic water supplies iron is objectionable, because it causes stains c $\eta$ plumbing fixtures and on clothing washed in the iron-bearing water. Iron is particularly troublesome in water used for many industrial purposes. In the water supplies from wells examined in western Texas, the average iron content was 0.23 part per million, and in about 9 percent of the supplies it was above the limit of 0.3 part set by the United States Public Health Service standards. Concentrations of iron above 2 parts per million were noted in Dallam, Dickens, and Reagan Counties. The surface-water samples averaged 0.35 part per million.

Calcium $(\mathrm{Ca})$ and magnesium $(\mathrm{Mg})$ in natural waters are usually derived from limestone, dolomite, gypsum, ant, in places such as the Permian basin in western Texas, from bedded salt deposits containing chlorides and other salts of calcium and magnesium. Calcium and magnesium are objectionable bacause they are the principal causes of hardness.

Sodium ( $\mathrm{Na}$ ) and Potassium (K) salts are highly soluble and are present in many rocks. The more saline waters usually contain high proportions of sodium and a relatively small amount of potassium. Large amounts of these elements will cause "foaming" in boiler operations. Low sodium content is irnportant in irrigation water, owing to the formation of "black alkali" in the soil by high-sodium waters. Sodium in water for irrigation use should not exceed 60 percent of the equivalents per million of the positive ions (Wilcox, 1948).

In public supplies of western Texas the content of sodium and potassium ranged from 12 to 400 parts per million. Ir 87 percent of the supplies the sodium percentage was less than 50 .

Sulfate $\left(\mathrm{SO}_{4}\right)$ is dissolved in large quantities from gypsum beds and occasionally from alkali deposits of sodium sulfate and from iron pyrite ores. Sulfate in waters that contain much calcium and magnesium contributes to the formation of hard scale in steam boilers and may increase the cost of softening. In the absence of air certain bacteria reduce sulfates to hydrogen sulfide, which is objectionable because of its offensive odor and corrosiveness to iron pipes. In 18 percent of the water supplies analyzed the sulfate content exceeded 250 parts per million.

Chloride ( $\mathrm{Cl}$ ) has little effect on the utility of water except when present in large amounts. However, when chloride is in equilibrium with calcium and magnesium, it may incresse the corrosiveness of water. Water having a chloride content above about 
500 parts per million will taste salty to most people. Chloride exceeded 250 parts per million in 10 percent of the public water supplies of western Texas, and 100 parts per millior in more than 21 percent.

Nitrate $\left(\mathrm{NO}_{3}\right)$ is the final oxidation product of organic nitrogen compounds. Generally, in mineral analyses of ground waters, nitrate has no sanitary significance, but at times nitrate in shallow wells is due to human or animal contamination.

Recent studies made in Illinois indicate that nit:ate in excess of 70 parts per million may contribute to the ailment methemoglobinemia, "blue babies" (Faucett and Miller, I.946 p. 593). Further investigations are being made in Texas by the Texas State Department of Health in areas where nitrate is high. Examination of the public supplies listed showed the righest nitrate to be 35 parts per million, and only 3 percent of the supplies have nitrates exceeding 20 parts per million.

Fluoride $(F)$ is found in natural waters obtained from regions where fluorspar and cryolite occur. It has been identified as the causative agent of mottled tooth enamel, and the evidence indicates that its action on the teeth occurs during their period of formation (Smith, Smith, and Foster, 1936). The United States Public Health Service has established a fluoride limit of 1.5 parts per million when the water is to be used on interstate carriers. Approximately 1 part per million of fluoride in water is thought to be desirable as a preventative of tooth decay (Jean, 1938, $p$. 1443). Some of the western Texas ground-water supplies contain undesirable amounts of fluoride; analyses showed th at 37 percent of the ground-water supplies contained more than 2 parts per million and 11 percent contained more than $4 \mathrm{par}^{+} \mathrm{s}$ per million of fluoride.

The dissolved solids represent the residue on evaroration of the total mineral content and organic matter present an 7 may include some water of crystallization. More than 1,000 parts per million of dissolved solids is likely to produce a noticeable taste or in other respects to make the water undesirable for public supply. The average dissolved solids found in west Texas water supplies examined was 638 parts per million. The dissolved solids was in excess of 1,000 parts per million in only 12 percent of the supplies.

\section{HARDNESS}

A hard water requires a relatively large quantity of soap to form lather. Hardness is caused for the most part by the bicarbonates and sulfates of calcium and magnesium. Temporary or 
carbonate hardness is that part of the hardness which can be removed by boiling. It is caused principally by the presence of bicarbonates of calcium and magnesium. Permanent or noncarbonate hardness is caused by the combination of calcium and magnesium with sulfate, chloride, and nitrate.

Water having less than 50 to 75 parts per million of hardness is generally considered as sufficiently soft for ordinary use in a public water supply. Water having 75 to 150 parts per million of hardness is considered moderately hard, but still not sufficiently hard to interfere seriously in its uses or to cause much public demand for water softening. Hardness above 150 part per million is noticed by most persons, and where the hardness is above 200 parts per million many users employ household softeners. The average hardness of public supplies in western Texas is about 350 parts per million. Only 22 percent of the ground-vrater supplies showed less than 200 parts per million of hardness. In the surface supplies the average hardness found, with one exception, was 115 parts per million. At Roby, not included in the average, the hardness was 2,310 parts per million.

\section{ALKALINITY}

Carbonate $\left(\mathrm{CO}_{3}\right)$, bicarbonate $\left(\mathrm{HCO}_{3}\right)$, and occasionally borate, silicate, and phosphate are responsible for the alkalinity of natural waters. Bicarbonate in natural waters results from the action on carbonate rocks of the carbon dioxide dissolved in water. Aside from its effect on the palatability of the water when present in excessive amounts, bicarbonate is of little significance in public water supplies. Carbonate is found in some samples that have been in contact with glass sampling bottles for some time, owing to the solvent action of water on soft glass.

\section{HYDROGEN-ION CONCENTRATION (pH)}

The $\mathrm{pH}$ of a solution is a direct measure of its chemical activity and may also have some bearing on the utility of the supply for domestic or industrial purposes. Knowledge of hydrogen-ion concentration is used in waterworks, principally in chemical control of purification. The degree of acidity or alkalinity of the water, as indicated by the hydrogen-ion concentration, or $\mathrm{pH}$, has an important bearing on the corrosiveness of water. Values lower than 7.0 denote increasing acidity and values higher than 7.0 denote increasing alkalinity. Acid waters are generally more corrosive than alkaline waters. Raw water in most west Texas wells and streams has a pH greater than 7.0. 


\section{SELECTED BIBLIOGRAPHY}

\section{PUBLISHED REPORTS}

Alexander, W. H., 1946, Ground-water resources of the areas southwest of Amarillo, Texas: Texas State Board of Water Engineers (mimeographed).

Baker, C. L., 1915, Geology and underground waters of the northern Llano Estacado: Texas Univ. Bull. 57.

Barnes, J. R., and others, 1949, Geology and ground water in the irrigated region of the southern High Plains in Texas: Texas State Board of Water Engineers, Progress Rept. 7 (mimeographed).

Broadhurst, W. L., 1946, Ground water in the High Plains of Texas: Texas State Board of Water Engineers, Progress Rept. 6 (mimeographed).

Gould, C. N., 1906, The geology and water resources of the eastern portion of the Panhandle of Texas: U. S. Geol. Survey Water-Supply Paper 154.

1907, The geology and water resources of the weste"n portion of the Panhandle of Texas: U. S. Geol. Survey Water-Supply Paper 191.

Johnson, W. D., 1901, The High Plains and their utilization: U. S. Geol. Survey 21st Ann. Rept., pt. 4, pp. 609-741.

1902. The High Plains and their utilization: U. S. Geol. Survey 22d Ann. Rept., pt. 4, pp. 637-669.

Knowles, D. B., 1947, Preliminary report on the geology and ground-water resources of Reeves County, Texas: Texas State Board of Water Engineers (mimeographed).

Lang, J. W., 1945, Ground water in the High Plains of Texas: Texas State Board of Water Engineers, Progress Rept. 5 (mimeographed).

Livingston, Penn, and Bennett, R. R., 1944, Geology and cround-water resources of the Big Spring area, Texas: U. S. Geol. Survey Water-Supply Paper 913.

National Resources Planning Board, 1942, The Pecos River Basin joint investigation.

Sayre, A. N., and Livingston, Penn, 1945, Ground-water resources of the El Paso area, Texas: U. S. Geol. Survey. Water-Suppl": Paper 919.

White, W. N., Broadhurst, W. L., and Lang, J. W., 1946, Ground water in the High Plains of Texas: U. S. Geol. Survey Water-Supply Paper 889-F.

White, W. N., Gale, H. S., and Nye, S. S., 1941, Geology and ground-water resources of the Balmorhea area, western Texas: U. S. Geol. Survey Water-Supply Paper 849-C.

In addition to the above-listed reports, mimeogre.phed publications containing records of wells and springs, driller;' logs, partial chemical analyses of water from wells and springs, and a map showing the location of wells have been published by the Texas State Board of Water Engineers for the following counties in the region: 


$\begin{array}{lll}\text { Andrews } & \text { Edwards } & \text { Ochiltree } \\ \text { Armstrong } & \text { Floyd } & \text { Oldhar } \\ \text { Bailey } & \text { Gaines } & \text { Parmer } \\ \text { Briscoe } & \text { Hale } & \text { Pecos } \\ \text { Carson } & \text { Hansford } & \text { Potter } \\ \text { Castro } & \text { Hartley } & \text { Randall } \\ \text { Childress } & \text { Hockley } & \text { Robert } \\ \text { Collingsworth } & \text { Howard } & \text { Scurry } \\ \text { Crosby } & \text { Irion } & \text { Swisher } \\ \text { Dallam } & \text { Lamb } & \text { Terry } \\ \text { Dawson } & \text { Lubbock } & \text { Tom Green } \\ \text { Deaf Smith } & \text { Martin } & \text { Val Verde } \\ \text { Donley } & \text { Midland } & \text { Winkler } \\ \text { Ector } & \text { Nolan } & \text { Yoakum }\end{array}$

\section{UNPUBLISHED REPORTS}

The following typewritten reports giving results of groundwater investigations are available for reference in the offices of the Geological Survey and the Texas Board of Water Engineers at Austin :

1942, Ground-water resources of the Toyah area, Reeves County.

1944, Midland city water supply.

1944, Ground-water conditions in the Roby-Camp Springs area.

1944, Progress report on the ground-water supply of the El Paso area.

1945, Exploration for ground water for city of Childress.

1945, Water resqurces of the Lubbock district.

1945, Progress report on ground-water resources of the El Paso ares.

1946, Ground water in the vicinity of Paducah, Cottle County.

1948, Ground water in the area northwest of Lubbock.

\section{PUBLIC WATER SUPPLIES}

\section{ANDREWS COUNTY}

\section{ANDREWS}

Population in 1940: 611.

Source of information: H. T. Wilson, president, Andrews County Irdependent School District, Nov. 27, 1946.

Source of supply: Four wells.

Well 1. Nine miles north of Andrews; drilled in 1946 by G. L. Taylor; depth, 190 feet; diameter, 8 inches; deep-well turbine pump and 10-horsepower electric motor; pump set at 120 feet; st?.tic water level, 73 feet below land surface April 1946; yield reported, 200 gallons a minute.

Well 2. A quarter of a mile east of well 1; drilled in March 1946 by G. L. Taylor; depth, 200 feet; diameter, 8 inches; deep-w 911 turbine pump and 10-horsepower electric motor; pump set at 120 feet; static water level, 86 feet below land surface April 1946; yield reported, 300 gallons a minute. 
Well 3. A quarter of a mile east of well 2; drilled in March 1946 by G.

L. Taylor; depth, 200 feet; diameter, $131 \frac{1}{2}$ inches; deep-well turbine pump and 15-horsepower electric motor; pump set at 120 feet; static water level, 72 feet below land surface Apri 1946; yield reported, 300 gallons a minute.

Well 4. A quarter of a mile east of well 3; drilled in March 1946 by G. L. Taylor; depth, 200 feet; diameter, $13 \frac{1}{2}$ inches; deep-well turbine pump and 20-horsepower electric motor; pump set at 120 feet; static water level, 72 feet below land surface April 1946; yiald reported, 300 gallons a minute.

Pumpage (estimated): Average, 115,000 gallons a day.

Storage: Elevated tank, 50,000 gallons; elevated tank, 100,000 gallons; two concrete ground reservoirs, 100,000 gallons each.

Number of customers: 350 .

Treatment: Chlorination.

\section{Analysis}

[Composite sample of all wells. Collected Nov. 27, 1946. pH is 8.0. Analyzed by B. C. Dwyer]

\begin{tabular}{|c|c|c|c|c|c|}
\hline & $\begin{array}{c}\text { Parts per } \\
\text { million }\end{array}$ & $\begin{array}{l}\text { Equivalents } \\
\text { per million }\end{array}$ & & $\begin{array}{l}\text { Parts per } \\
\text { million }\end{array}$ & $\begin{array}{l}\text { Equivalents } \\
\text { per million }\end{array}$ \\
\hline $\begin{array}{l}\text { Silica }\left(\mathrm{SiO}_{2}\right) \\
\text { Iron }(\mathrm{Fe}) \\
\text { Calcium }(\mathrm{Ca}) \\
\text { Magnesium }(\mathrm{Mg}) \\
\text { Sodium }(\mathrm{Na}) \\
\text { Potassium }(\mathrm{K}) \\
\left.\text { Bicarbonate }(\mathrm{HCO})_{3}\right)^{-}\end{array}$ & $\begin{array}{l}21 \\
41 \\
42 \\
67 \\
8.0 \\
298\end{array}$ & $\begin{array}{r}-2.05 \\
3.45 \\
2.91 \\
.20 \\
4.88\end{array}$ & $\begin{array}{l}\text { Sulfate }\left(\mathrm{SO}_{4}\right) \\
\text { Chloride }(\mathrm{Cl}) \\
\text { Fluoride (F) } \\
\left.\text { Nitrate ( } \mathrm{NO}_{3}\right) \\
\text { Dissolved solids } \\
\text { Total hardness as } \mathrm{CaCO}_{3}\end{array}$ & \begin{tabular}{|c|}
75 \\
66 \\
5.2 \\
1.2 \\
494 \\
275
\end{tabular} & \begin{tabular}{r}
1.56 \\
1.86 \\
.27 \\
.02 \\
\hdashline \\
\\
\end{tabular} \\
\hline
\end{tabular}

\section{Driller's logs}

\begin{tabular}{|c|c|c|c|c|}
\hline & $\begin{array}{c}\text { Thickness } \\
\text { (feet) }\end{array}$ & $\begin{array}{c}\text { Depth } \\
\text { (feet) }\end{array}$ & $\begin{array}{c}\text { Thickness } \\
\text { (feet) }\end{array}$ & $\begin{array}{c}\text { Depth } \\
\text { (feet) }\end{array}$ \\
\hline
\end{tabular}

Well 1

\begin{tabular}{|c|c|c|c|c|c|}
\hline $\begin{array}{l}\text { Surface soil } \\
\text { Red sand } \\
\text { Pack sand }\end{array}$ & $\begin{array}{r}3 \\
9 \\
63\end{array}$ & $\begin{array}{r}3 \\
12 \\
75\end{array}$ & $\begin{array}{l}\text { Hard rock. } \\
\text { Medium-grained water-bear- } \\
\text { ing sand. }\end{array}$ & $\begin{array}{l}20 \\
95\end{array}$ & $\begin{array}{r}95 \\
190\end{array}$ \\
\hline
\end{tabular}

Well 2

\begin{tabular}{|c|c|c|c|c|c|}
\hline $\begin{array}{l}\text { Surface soil } \\
\text { Red sand. } \\
\text { Pack sand. }\end{array}$ & $\begin{array}{r}3 \\
\mathbf{9} \\
63\end{array}$ & $\begin{array}{r}3 \\
12 \\
75\end{array}$ & $\begin{array}{l}\text { Hard rock. } \\
\text { Medium-grained water-bear- } \\
\text { ing sand. }\end{array}$ & $\begin{array}{r}20 \\
105\end{array}$ & $\begin{array}{r}95 \\
200\end{array}$ \\
\hline
\end{tabular}

Well 4

\begin{tabular}{|c|c|c|c|c|c|}
\hline $\begin{array}{l}\text { Surface soil. } \\
\text { Red sand. } \\
\text { Pack sand } \\
\text { Sand } \\
\text { Hard rock. }\end{array}$ & $\begin{array}{r}2 \\
6 \\
22 \\
47 \\
23\end{array}$ & $\begin{array}{r}2 \\
8 \\
30 \\
77 \\
100\end{array}$ & $\begin{array}{l}\text { Fine-grained water-bearing } \\
\text { sand. } \\
\text { Medium-grained water-bear- } \\
\text { ing sand. }\end{array}$ & $\begin{array}{r}55 \\
45\end{array}$ & $\begin{array}{l}155 \\
200\end{array}$ \\
\hline
\end{tabular}




\section{ARMSTRONG COUNTY}

\section{CLAUDE}

Population in 1940: 761 .

Source of information: H. G. Nave, water superintendent, Dec. 4, 1947.

Ownership: Municipal.

Source of supply: Two wells.

Well 1 Drilled in 1930 by D. L. McDonald; depth, 250 feet; diameter, 18 inches; deep-well turbine pump and electric motor; yield, 60 gallons a minute.

Well 2. Drilled in 1930 by D. L. McDonald; depth, 250 feet; diameter, 18 inches; deep-well turbine pump and electric motor; yield, 40 gallons a minute.

Pumpage: Average, 68,000 gallons a day.

Storage: Two elevated storage reservoirs, 60,000 gallons each; elevated tank, 175,000 gallons.

Number of customers: 247.

Treatment: None.

\section{Analysis}

[Composite sample of wells 1 and 2. Collected Dec. 4, 1947. pH is 7.9. Analyzed by B. C. Dwyer]

\begin{tabular}{|c|c|c|c|c|c|}
\hline & $\begin{array}{c}\text { Parts per } \\
\text { million }\end{array}$ & $\begin{array}{c}\text { Equivalents } \\
\text { per million }\end{array}$ & & $\begin{array}{l}\text { Parts per } \\
\text { million }\end{array}$ & $\begin{array}{l}\text { Equivalents } \\
\text { per million }\end{array}$ \\
\hline $\begin{array}{l}\text { Silica }\left(\mathrm{SiO}_{2}\right) \\
\text { Iron }(\mathrm{Fe}) \\
\text { Calcium }(\mathrm{Ca}) \\
\text { Magnesium }(\mathrm{Mg}) \\
\text { Sodium }(\mathrm{Na}) \\
\text { Potassium }(\mathrm{K}) \\
\text { Bicarbonate }\left(\mathrm{H} \mathrm{CO}_{3}\right)\end{array}$ & $\begin{array}{l}50 \\
31.02 \\
36 \\
40 \\
3.2 \\
290\end{array}$ & $\begin{array}{r}1.55 \\
2.96 \\
1.76 \\
.08 \\
4.75\end{array}$ & $\begin{array}{l}\text { Sulfate }\left(\mathrm{SO}_{4}\right) \\
\text { Chloride }(\mathrm{Cl}) \\
\text { Fluoride (F) } \\
\text { Nitrate (NO} \\
\text { Dissolved solids } \\
\text { Total hardness as } \mathrm{CaCO}_{3}\end{array}$ & $\begin{array}{c}41 \\
16 \\
3.2 \\
7.9 \\
374 \\
226\end{array}$ & \begin{tabular}{r}
0.85 \\
.45 \\
.17 \\
.13 \\
- \\
\hdashline \\
\end{tabular} \\
\hline
\end{tabular}

\section{BAILEY COUNTY}

\section{MULESHOE}

Population in 1940: 1,327 (estimated 2,000 in 1944).

Source of information: W. E. Young, water superintendent, Mar. 2, 1945.

Ownership: Municipal.

Source of supply: Two wells.

Well 1. Drilled in 1936; depth, 100 feet; diameter, 16 inches; deep-well turbine pump and electric motor; pumping level, 23.15 feet below land surface while pumping 250 gallons a minute; yield, $25 \mathrm{C}$ gallons a minute; temperature, $64^{\circ} \mathrm{F}$.

Well 2. Drilled in 1940; depth, 100 feet; diameter, 16 inches; deep-well turbine pump and electric motor; static water level, 19.10 feet below land surface on March 2, 1945; yield, 800 gallons a minute.

Pumpage (estimated) : 200,000 gallons a day.

Storage: Elevated tank, 50,000 gallons.

Number of customers: 380 .

Treatment: None. 
Analysis, well 1

[Collected Mar. 7, 1947. pH is 7.7. Analyzed by M. L. Begley]

\begin{tabular}{|c|c|c|c|c|c|}
\hline & $\begin{array}{l}\text { Parts per } \\
\text { million }\end{array}$ & $\begin{array}{l}\text { Equivalents } \\
\text { per million }\end{array}$ & & $\begin{array}{c}\text { Farts per } \\
\text { million }\end{array}$ & $\begin{array}{l}\text { Equivalents } \\
\text { per million }\end{array}$ \\
\hline $\begin{array}{l}\text { Silica }\left(\mathrm{SiO}_{2}\right) \\
\text { Iron }(\mathrm{Fe}) \\
\text { Calcium }(\mathrm{Ca}) \\
\text { Magnesium }(\mathrm{Mg}) \\
\text { Sodium }(\mathrm{Na}) \\
\text { Potassium }(\mathrm{K}) \\
\text { Bicarbonate }\left(\mathrm{H} \mathrm{CO}_{3}\right)\end{array}$ & $\begin{array}{l}{ }_{81}^{46} \\
83 \\
60 \\
11 \\
283\end{array}$ & $\begin{array}{l}4.04 \\
6.83 \\
2.61 \\
.28 \\
4.64\end{array}$ & $\begin{array}{l}\text { Sulfate }\left(\mathrm{SO}_{4}\right) \\
\text { Chloride }(\mathrm{Cl}) \\
\text { Fluoride (F) } \\
\text { Nitrate (NO} \\
\text { Dissolved solids. } \\
\text { Total hardness as } \mathrm{CaCO}_{3-}\end{array}$ & $\begin{array}{l}251 \\
129 \\
3.0 \\
5.4 \\
845 \\
544\end{array}$ & $\begin{array}{r}5.23 \\
3.64 \\
.16 \\
.09 \\
\\
\\
-2 .-\end{array}$ \\
\hline
\end{tabular}

Driller's log, well 1

\begin{tabular}{|c|c|c|c|c|c|}
\hline & $\begin{array}{c}\text { Thickness } \\
\text { (feet) }\end{array}$ & $\begin{array}{l}\text { Depth } \\
\text { (feet) }\end{array}$ & & $\begin{array}{c}\text { Thickness } \\
\text { (feet) }\end{array}$ & $\begin{array}{l}\text { Depth } \\
\text { (feet) }\end{array}$ \\
\hline $\begin{array}{l}\text { Surface soil } \\
\text { Chalk and clay } \\
\text { Water sand } \\
\text { Gray clay } \\
\text { White water sand } \\
\text { Gray shale } \\
\text { Lime rock. }\end{array}$ & $\begin{array}{r}2 \\
15 \\
1 \\
6 \\
4 \\
4 \\
2\end{array}$ & $\begin{array}{r}2 \\
17 \\
18 \\
24 \\
28 \\
32 \\
34\end{array}$ & $\begin{array}{l}\text { Brown clay } \\
\text { Gray packed sand } \\
\text { Red water sand. } \\
\text { Packed sand } \\
\text { Gray water sand } \\
\text { Red clay }\end{array}$ & $\begin{array}{r}6 \\
5 \\
30 \\
10 \\
9 \\
6\end{array}$ & $\begin{array}{r}40 \\
45 \\
75 \\
85 \\
94 \\
100\end{array}$ \\
\hline
\end{tabular}

\section{BREWSTER COUNTY}

Population in 1940: 3,866 .

\section{ALPINE}

Source of information: John Stovell, city engineer, Aug. 9, 1948.

Ownership: Municipal.

Source of supply: Four wells.

East Well. On north side of Southern Pacific Railroad, 1 mile east of depot; drilled in 1927; depth, 580 feet; (no water reforted below 170 feet); deep-well turbine pump and 30-horsepower electric motor; static water lêvel, 65.1 feet below land surface June 28, 1948; yield, 235 gallons a minute.

College well. On campus of Sul Ross State College; drilled in 1940; depth, 300 feet; deep-well turbine pump and 15-horsepower electric motor; yield, 70 gallons a minute.

Well 3. On Alpine Hill southwest of Southern Pacific Railroad depot; drilled in 1924 by Tony Hess; depth, 385 feet; diameter, 10 inches; deep-well turbine pump and 75-horsepower electric motor; static water level, 165 feet below land surface Aug. 1948; yield, 200 gallons a minute.

Well 4. On Alpine Hill southwest of Southern Pacific Railroad depot; drilled in 1929; depth, 700 feet; diameter, 10 inches; ceep-well turbine pump and 50-horsepower electric motor; static water level, 210 feet below land surface June 1948; yield, 200 gallons a minute.

Pumpage (estimated) : 300,000 gallons a day.

Storage: Concrete ground reservoir on Alpine Hill, 1,250,000 callons.

Treatment: None. 
Analysis, well s

[Collected Nov. 10, 1947. $\mathrm{pH}$ is 7.2. Analyzed by B. C. Dwyer]

\begin{tabular}{|c|c|c|c|c|c|}
\hline & $\begin{array}{l}\text { Parts per } \\
\text { million }\end{array}$ & $\begin{array}{l}\text { Equivalents } \\
\text { per million }\end{array}$ & & $\begin{array}{l}\text { Parts per } \\
\text { milli on }\end{array}$ & $\begin{array}{l}\text { Equivalents } \\
\text { per million }\end{array}$ \\
\hline $\begin{array}{l}\text { Silica }\left(\mathrm{SiO}_{2}\right) \\
\text { Iron }(\mathrm{Fe}) \\
\text { Calcium }(\mathrm{Ca}) \\
\text { Magnesium }(\mathrm{Mg}) \\
\text { Sodium }(\mathrm{Na}) \\
\text { Potassium }(\mathrm{K}) \\
\text { Bicarbonate }\left(\mathrm{HCO}_{3}\right)^{-}\end{array}$ & $\begin{array}{l}42 \\
47^{.00} \\
12 \\
37 \\
224.7\end{array}$ & $\begin{array}{r}2.35 \\
.99 \\
1.60 \\
.09 \\
3.67\end{array}$ & $\begin{array}{l}\text { Sulfate }\left(\mathrm{SO}_{4}\right) \\
\text { Chloride }(\mathrm{Cl}) \\
\text { Fluoride (F) } \\
\left.\text { Nitrate }(\mathrm{NO})_{3}\right) \\
\text { Dissolved solids.-. } \\
\text { Total hardness as } \mathrm{CaCO}_{3}\end{array}$ & $\begin{array}{l}18 \\
25 \\
16 \\
312 \\
167\end{array}$ & \begin{tabular}{r}
0.37 \\
.71 \\
.02 \\
.26 \\
\hdashline..- \\
\end{tabular} \\
\hline
\end{tabular}

Driller's log of abandoned city well

\begin{tabular}{|c|c|c|c|c|c|}
\hline & $\begin{array}{c}\text { Thickness } \\
\text { (feet) }\end{array}$ & $\begin{array}{l}\text { Depth } \\
\text { (feet) }\end{array}$ & & $\begin{array}{c}\text { Tickness } \\
\text { (feet) }\end{array}$ & $\begin{array}{l}\text { Depth } \\
\text { (feet) }\end{array}$ \\
\hline $\begin{array}{l}\text { Soft brown soil and subsoil } \\
\text { Brown clay and gravel } \\
\text { Gravel and clay. } \\
\text { Gravel, water } \\
\text { Gravel and boulders, water. } \\
\text { Hard brown rock. } \\
\text { Brown and yellow clay and } \\
\text { soapstone. } \\
\text { Pink shale. } \\
\text { Hard red, brown, blue and } \\
\text { black rock, water bearing. } \\
\text { Soft brown shale. } \\
\text { Hard black rock } \\
\text { Chocolate-colored shale. }\end{array}$ & $\begin{array}{r}\mathbf{5} \\
15 \\
13 \\
3 \\
4 \\
20 \\
10 \\
\\
5 \\
40 \\
5 \\
5 \\
5\end{array}$ & $\begin{array}{r}5 \\
20 \\
33 \\
36 \\
40 \\
60 \\
70 \\
75 \\
115 \\
120 \\
125 \\
130\end{array}$ & $\begin{array}{l}\text { Hard blue and black rock, } \\
\text { water bearing. } \\
\text { Red and brown clay and } \\
\text { gravel. } \\
\text { Blue and black conglomerate. } \\
\text { Hard black and blue rock } \\
\text { Soft red clay } \\
\text { Soft pink, red, and blue soap- } \\
\text { stone. } \\
\text { Blue and green sandy shale.- } \\
\text { Blue sandy rock. } \\
\text { Hard red rock } \\
\text { Green shale and conglomerate } \\
\text { Green shale and hard rock } \\
\text { Green sandy shale... }\end{array}$ & $\begin{array}{r}30 \\
5 \\
10 \\
10 \\
35 \\
8 \\
7 \\
5 \\
10 \\
18 \\
12 \\
10 \\
20\end{array}$ & $\begin{array}{l}160 \\
165 \\
175 \\
210 \\
218 \\
225\end{array}$ \\
\hline
\end{tabular}

\section{BRISCOE COUNTY}

\section{QUITAQUE}

Population in 1940: 763.

Source of information: W. Middleton, water superintendent, Sept. 2, 1946.

Ownership: Municipal.

Source of supply: Two wells.

Well 1. About 2 miles northeast of Quitaque; drilled in 1927; depth, 100 feet; diameter, 14 inches; deep-well turbine pump and 15-horsepower electric motor; pump set at $\mathbf{9 0}$ feet below land surface; static water level, 51 feet below land surface on Sept. 2, 1946; yield reported, 200 gallons a minute with a drawdown of 20 feet.

Well 2. Five hundred feet north of well 1; drilled in 192؟; depth, 100 feet; diameter, 14 inches; deep-well turbine pump and 15-horsepower electric motor; pump set at 90 feet below land surface; y:eld reported, 200 gallons a minute.

Average pumpage, in gallons a day

\begin{tabular}{|c|c|c|c|c|c|}
\hline & 1945 & 1946 & & 1945 & 1946 \\
\hline $\begin{array}{l}\text { January } \\
\text { February } \\
\text { March } \\
\text { April } \\
\text { May } \\
\text { June... }\end{array}$ & $\begin{array}{l}24,000 \\
34,500 \\
33,000 \\
42,300 \\
39,000 \\
60,100\end{array}$ & $\begin{array}{r}50,000 \\
41,500 \\
36,500 \\
66,500 \\
80,500 \\
116,000\end{array}$ & $\begin{array}{l}\text { July } \\
\text { August } \\
\text { September } \\
\text { October } \\
\text { November } \\
\text { December }\end{array}$ & $\begin{array}{r}78,400 \\
100,000 \\
\mathbf{7 5}, \mathbf{1 0 0} \\
\mathbf{5 3}, \mathbf{5 0 0} \\
\mathbf{3 9}, 000 \\
\mathbf{3 6}, \mathbf{1 0 0}\end{array}$ & \begin{tabular}{r}
113,600 \\
127,500 \\
\hdashline-10 \\
-10
\end{tabular} \\
\hline
\end{tabular}


Storage: Ground reservoir, 54,000 gallons; elevated tank, 50,000 gallons. Number of customers: 206.

Treatment: Chlorination.

Analysis of finished water, wells 1 and 2

[Collected Mar. 28, 1947. $\mathrm{pH}$ is 7.4. Analyzed by B. C. Dwyer]

\begin{tabular}{|c|c|c|c|c|c|}
\hline & $\begin{array}{c}\text { Parts per } \\
\text { million }\end{array}$ & $\begin{array}{l}\text { Equivalents } \\
\text { per million }\end{array}$ & & $\begin{array}{l}\text { Parts per } \\
\text { million }\end{array}$ & $\begin{array}{l}\text { Equivalents } \\
\text { per million }\end{array}$ \\
\hline $\begin{array}{l}\text { Silica }\left(\mathrm{SiO}_{2}\right) \\
\text { Iron }(\mathrm{Fe}) \\
\text { Calcium }(\mathrm{Ca}) \\
\text { Magnesium }(\mathrm{Mg}) \\
\text { Sodium }(\mathrm{Na}) \\
\text { Potassium }(\mathrm{K}) \\
\text { Bicarbonate }\left(\mathrm{HCO}_{3}\right)^{-}\end{array}$ & $\begin{array}{l}24 \\
66^{.26} \\
44 \\
170 \\
6.9 \\
418\end{array}$ & $\begin{array}{r}3.29 \\
3.62 \\
7.40 \\
.18 \\
6.85\end{array}$ & $\begin{array}{l}\text { Sulfate }\left(\mathrm{SO}_{4}\right) \\
\text { Chloride (Cl) } \\
\text { Fluoride (F) } \\
\text { Nitrate (NO } \\
\text { Dissolved solids } \\
\text { Total hardness as } \mathrm{CaCO}_{3}\end{array}$ & $\begin{array}{l}184 \\
122 \\
3.6 \\
11 \\
826 \\
346\end{array}$ & \begin{tabular}{r}
3.83 \\
3.44 \\
.19 \\
.18 \\
\hdashline$-1 .-$ \\
\end{tabular} \\
\hline
\end{tabular}

\section{SILVERTON}

Population in 1940 : 684.

Source of information: Mr. Summers, city secretary, Aug. 27, 1946.

Ownership: Municipal.

Source of supply: Three wells.

Well 1. Fifty feet north of City Hall; drilled in 1929; depth, 200 feet; diameter, 12 inches; cased to 142 feet below land surface; deep-well cylinder and 5-horsepower electric motor; cylinder $\mathrm{s}$ st at 165 feet; static water level reported, 120 feet below land surface; yield reported, 90 gallons a minute.

Well 2. Three-fourths mile southeast of City Hall; drilled in 1933 by Leo McDade; depth, 202 feet; diameter, 9 inches; not cased; deep-well turbine pump and 15-horsepower electric motor; pump set at 160 feet below land surface; yield reported, 70 gallons a minute.

Well 3. Fifty feet east of City Hall; drilled in 1939 by J. F. Davis; depth, 200 feet; diameter, 16 to 8 inches; deep-well turbine pump and 3-horsepower electric motor; static water level, $\mathbf{1 1 6 . 9}$ feet below land surface in August 1946; yield reported, 50 gallons a minute.

Pumpage: Average, 100,000 gallons a day.

Storage: Elevated tank, 50,000 gallons; ground reservoir, 50,000 gallons. Number of customers: 200 .

Treatment: None.

\section{Analysis}

[Composite sample of all wells. Collected Aug. 27, 1946. pH is 7.5. Analyzed by B. C. Dwyer]

\begin{tabular}{|c|c|c|c|c|c|}
\hline & $\begin{array}{l}\text { Parts per } \\
\text { million }\end{array}$ & $\begin{array}{l}\text { Equivalents } \\
\text { per million }\end{array}$ & & $\begin{array}{c}\text { Pcrts per } \\
\text { million }\end{array}$ & $\begin{array}{l}\text { Equivalents } \\
\text { per million }\end{array}$ \\
\hline $\begin{array}{l}\text { Silica }\left(\mathrm{SiO}_{2}\right) \\
\text { Iron }(\mathrm{Fe}) \\
\text { Calcium }(\mathrm{Ca}) \\
\text { Magnesium }(\mathrm{Mg}) \\
\text { Sodium }(\mathrm{Na}) \\
\text { Potassium }(\mathrm{K}) \\
\text { Bicarbonate }\left(\mathrm{HCO}_{3}\right)\end{array}$ & $\begin{array}{c}60 \\
40^{6} .01 \\
34 \\
35 \\
6.0 \\
288\end{array}$ & $\begin{array}{r}2.00 \\
2.80 \\
1.51 \\
.15 \\
4.72\end{array}$ & $\begin{array}{l}\text { Sulfate }\left(\mathrm{SO}_{4}\right) \\
\text { Chloride }(\mathrm{Cl}) \\
\text { Fluoride (F) } \\
\text { Nitrate }\left(\mathrm{NO}_{3}\right) \\
\text { Dissolved solids } \\
\text { Total hardness as } \mathrm{CaCO}_{3}\end{array}$ & $\begin{array}{c}40 \\
24 \\
3.6 \\
2.5 \\
387 \\
240\end{array}$ & $\begin{array}{r}0.83 \\
.68 \\
.19 \\
.04 \\
-0.0 \\
\end{array}$ \\
\hline
\end{tabular}




\section{CARSON COUNTY}

\section{GROOM}

Population in 1940: 475.

Source of information: V. L. McCoy, water superintendent, June 25, 1948.

Ownership: Municipal.

Source of supply: Three wells.

Well 1. Drilled in 1946 by H. H. Heiskell; depth, 518 feet; diameter, 9 inches; deep-well turbine pump and 15-horsepower electric motor; static water level, 280 feet below land surface in 1946; yiold, 105 gallons a minute.

Well 2. Drilled in 1929 by C. Meeker; depth, 450 feet; diameter, 65/8 inches; deep-well turbine pump and 10-horsepower electric motor; static water level, 270 feet below land surface in 1945; yield, 35 gallons a minute.

Well 3. Drilled in 1923; depth, 302 feet; diameter, $5 \%$ inches; deep-well turbine pump and $71 / 2$-horsepower electric motor; static water level, 285 feet below land surface in 1945; yield, 20 gallons a minute.

Pumpage: 65,000 gallons a day.

Storage: Ground storage reservoir, 58,000 gallons; elevated tank, 55,000 gallons.

Number of customers: 204.

Treatment: None.

Analysis, well 1

[Collected June 25, 1948. $\mathrm{pH}$ is 7.7. Analyzed by D. E. Weaver]

\begin{tabular}{|c|c|c|c|c|c|}
\hline & $\begin{array}{l}\text { Parts per } \\
\text { million }\end{array}$ & $\begin{array}{c}\text { Equivalents } \\
\text { per million }\end{array}$ & & $\begin{array}{l}\text { Parts per } \\
\text { million }\end{array}$ & $\begin{array}{l}\text { Equivalents } \\
\text { per million }\end{array}$ \\
\hline $\begin{array}{l}\text { Silica }\left(\mathrm{SiO}_{2}\right) \\
\text { Iron }(\mathrm{Fe}) \\
\text { Calcium }(\mathrm{Ca}) \\
\text { Magnesium }(\mathrm{Mg}) \\
\text { Sodium }(\mathrm{Na}) \\
\text { Potassium }(\mathrm{K}) \\
\text { Bicarbonate }\left(\mathrm{H}^{2} \mathrm{CO}_{3}\right)^{-}\end{array}$ & $\begin{array}{l}34 \\
42 \\
28 \\
21 \\
3.00 \\
290\end{array}$ & $\begin{array}{r}2.10 \\
2.30 \\
.91 \\
.09 \\
4.75\end{array}$ & $\begin{array}{l}\text { Silfate (SO) } \\
\text { Chlo ide (Cl) } \\
\text { Flio ide (F) } \\
\text { Nit.ate (NO})_{3} \text { ) } \\
\text { Dissol red solids } \\
\text { Total hardness as } \mathrm{CaCO}_{3}\end{array}$ & $\begin{array}{r}16 \\
5.5 \\
.4 \\
3.8 \\
295 \\
220\end{array}$ & $\begin{array}{r}0.33 \\
.16 \\
.02 \\
.06 \\
-.0 .-1.0\end{array}$ \\
\hline
\end{tabular}

\section{PANHANDLE}

Population in 1940: 978.

Source of information: Kelly M. Bender, water superintendent, N'vv. 20, 1947. Ownership: Municipal.

Source of supply: Two wells near waterworks plant in city. System connected to Santa Fe Railroad.

Well 1. Drilled in 1924; depth, 524 feet; diameter, 24 inches; air pump; static water level, 300 feet below land surface in 1937; yield, 350 gallons a minute; not used at present.

Well 2. Drilled in 1926; depth, 580 feet; diameter 24 inches; pumped with same compressor as well 1; static water level, 300 feet bel $2 w$ land surface in 1937; yield, 360 gallons a minute.

Pumpage: Average, 150,000 gallons a day.

Storage: Ground storage reservoir, 65,000 gallons; elevated tank, 75,000 gallons.

Number of customers: 380.

Treatment: Chlorination. 


\section{Analysis, well 2}

[Collected Dec. 1, 1938. Analyzed by E. W. Lohr]

\begin{tabular}{|c|c|c|c|c|c|}
\hline & $\begin{array}{c}\text { Parts per } \\
\text { million }\end{array}$ & $\begin{array}{l}\text { Equivalents } \\
\text { per million }\end{array}$ & - & $\begin{array}{c}\text { Parts per } \\
\text { million }\end{array}$ & $\begin{array}{l}\text { Equivalents } \\
\text { per million }\end{array}$ \\
\hline $\begin{array}{l}\text { Calcium }(\mathrm{Ca}) \\
\text { Magnesium }(\mathrm{Mg}) \\
\text { Sodium and potassium } \\
\text { (Na +K). } \\
\text { Bicarbonate }\left(\mathrm{HCO}_{3}\right) \\
\left.\text { Sulfate ( } \mathrm{SO}_{4}\right)\end{array}$ & $\begin{array}{r}52 \\
28 \\
12 \\
293 \\
20\end{array}$ & $\begin{array}{r}2.60 \\
2.30 \\
.62 \\
4.80 \\
.42\end{array}$ & $\begin{array}{l}\text { Chloride }(\mathrm{Cl}) \\
\text { Fluoride }(\mathrm{F}) \\
\text { Nitrate }\left(\mathrm{NO}_{3}\right) \\
\text { Dissolved solids } \\
\text { Total hardness as } \mathrm{CaCO}\end{array}$ & $\begin{array}{l}10 \\
1.1 \\
266^{.0} \\
248\end{array}$ & $\begin{array}{r}0.28 \\
.06 \\
.00 \\
\\
-0.0 \\
\end{array}$ \\
\hline
\end{tabular}

Driller's log, Panhandle and Santa Fe R.R. well in Panhandle

\begin{tabular}{|c|c|c|c|c|c|}
\hline & $\begin{array}{c}\text { Thickness } \\
\text { (feet) }\end{array}$ & $\begin{array}{l}\text { Depth } \\
\text { (feet) }\end{array}$ & & $\begin{array}{c}\text { Thickness } \\
\text { (feet) }\end{array}$ & $\begin{array}{l}\text { Depth } \\
\text { (feet) }\end{array}$ \\
\hline $\begin{array}{l}\text { Sandy soil } \\
\text { Yellow clay and sand } \\
\text { Fine-grained dry sand } \\
\text { Clay- } \\
\text { Clay and gravel } \\
\text { Sand and soapstone } \\
\text { Fine-grained sand } \\
\text { Sandrock } \\
\text { Hard-packed clay and sand } \\
\text { Dry sand } \\
\text { Clay } \\
\text { Clay and gravein } \\
\text { Sandrock }\end{array}$ & $\begin{array}{r}5 \\
111 \\
29 \\
30 \\
3 \\
4 \\
18 \\
4 \\
19 \\
47 \\
5 \\
30 \\
40\end{array}$ & $\begin{array}{r}5 \\
116 \\
145 \\
175 \\
178 \\
182 \\
200 \\
204 \\
223 \\
270 \\
275 \\
305 \\
345\end{array}$ & $\begin{array}{l}\text { Water sand } \\
\text { Sandrock } \\
\text { Sand } \\
\text { Sand and clay } \\
\text { Sandrock } \\
\text { Yellow clay } \\
\text { Sand and clay } \\
\text { Sandrock } \\
\text { Sand and clay } \\
\text { Sandrock } \\
\text { Clay and rock } \\
\text { Sandrock } \\
\text { Sand }\end{array}$ & $\begin{array}{r}7 \\
28 \\
5 \\
15 \\
20 \\
20 \\
20 \\
5 \\
15 \\
20 \\
15 \\
29 \\
6\end{array}$ & $\begin{array}{l}352 \\
380 \\
385 \\
400 \\
420 \\
440 \\
460 \\
465 \\
480 \\
500 \\
515 \\
544 \\
550\end{array}$ \\
\hline
\end{tabular}

\section{SKEILYTOWN}

Population in 1940: 650 .

Source of information: T. E. Morgan, operator, Oct. 5, 1948.

Owner: Southwestern Public Service Co.

Source of supply: Well owned by the Santa Fe Railroad; drilled in 1927 by Tye Brothers; depth, 418 feet; diameter, 14 inches; deep-well turbine pump and 40-horsepower electric motor; static water level, 300 feet below land surface in 1947; yield, 260 gallons a minute.

Pumpage (estimated) : 50,000 gallons a day.

Storage: Elevated tank, 100,000 gallons.

Number of customers: 250.

Treatment: None.

\section{Analysis}

[Collected Oct. 5, 1948. pH is 7.5. Analyzed by J. R. Avrett]

\begin{tabular}{|c|c|c|c|c|c|}
\hline & $\begin{array}{c}\text { Parts per } \\
\text { million }\end{array}$ & $\begin{array}{c}\text { Equivalents } \\
\text { per million }\end{array}$ & & $\begin{array}{c}\text { Parts per } \\
\text { million }\end{array}$ & $\begin{array}{l}\text { Equivalen ts } \\
\text { per million }\end{array}$ \\
\hline $\begin{array}{l}\text { Silica }\left(\mathrm{SiO}_{2}\right) \\
\text { Iron }(\mathrm{Fe}) \\
\text { Calcium }(\mathrm{Ca}) \\
\text { Magnesium }(\mathrm{Mg}) \\
\text { Sodium }(\mathrm{Na}) \\
\text { Potassium }(\mathrm{K}){ }^{-} \\
\text {Bicarbonate }\left(\mathrm{H} \mathrm{CO}_{3}\right)\end{array}$ & $\begin{array}{l}21 \\
43^{.05} \\
23 \\
13 \\
4.8 \\
220^{4}\end{array}$ & $\begin{array}{r}2.146 \\
1.891 \\
.565 \\
.123 \\
3.606\end{array}$ & 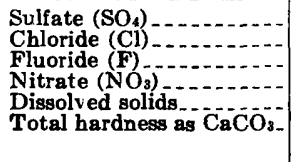 & $\begin{array}{c}27 \\
13 \\
10 \\
262 \\
202\end{array}$ & $\begin{array}{r}0.562 \\
.367 \\
.032 \\
.161 \\
-0 .- \\
\end{array}$ \\
\hline
\end{tabular}


Driller's log

\begin{tabular}{|c|c|c|c|c|c|}
\hline & $\underset{\text { (feet) }}{\text { Thickness }}$ & $\begin{array}{l}\text { Depth } \\
\text { (feet) }\end{array}$ & & $\begin{array}{c}\text { Thickness } \\
\text { (fe?t) }\end{array}$ & $\begin{array}{l}\text { Depth } \\
\text { (feet) }\end{array}$ \\
\hline $\begin{array}{l}\text { Surface soil } \\
\text { Red bed } \\
\text { Brown clay, some sand } \\
\text { Yellow clay and sand, } \\
\text { Yellow clay, sand and gravel.- } \\
\text { Soft yellow sand, some gravel } \\
\text { and clay. } \\
\text { Yellow packed sand, soft and } \\
\text { dry. } \\
\text { Red packed sand and lime } \\
\text { gravel. } \\
\text { Red packed sand } \\
\text { Brown sand, soft and dry } \\
\text { Brown sand and clay } \\
\text { Light fluffy clay, soft. } \\
\text { Packed sand, dry }\end{array}$ & $\begin{array}{r}3 \\
4 \\
21 \\
2 \\
30 \\
15 \\
20 \\
28 \\
37 \\
15 \\
15 \\
8 \\
27\end{array}$ & $\begin{array}{r}3 \\
7 \\
28 \\
30 \\
60 \\
75 \\
95 \\
\\
123 \\
160 \\
175 \\
190 \\
198 \\
225\end{array}$ & $\begin{array}{l}\text { White packed sand } \\
\text { White sandrock } \\
\text { Light-brown sand } \\
\text { Coarse gravel and sand } \\
\text { (water). } \\
\text { Fine gravel and coarse sand } \\
\text { Fine gravel and coarse brown } \\
\text { sand. } \\
\text { Coarse white sand and very } \\
\text { fine gravel. } \\
\text { Yellow clay, some gravel } \\
\text { Brown clay- } \\
\text { Red clay and sand. } \\
\text { Brown quicksand (second } \\
\text { water). }\end{array}$ & $\begin{array}{r}35 \\
27 \\
33 \\
6 \\
14 \\
10 \\
\\
21 \\
9 \\
15 \\
18 \\
5\end{array}$ & $\begin{array}{l}260 \\
287 \\
320 \\
326 \\
\\
340 \\
350 \\
\\
371 \\
\\
380 \\
395 \\
413 \\
418\end{array}$ \\
\hline
\end{tabular}

Population in 1940: 733.

\section{WHITE DEER}

Source of information: R. M. Smith, water superintendent, June 24, 1948.

Ownership: Municipal.

Source of supply: Two wells at elevated tank.

Well 1. Drilled about 1925; depth, 382 feet; diameter, 10 inches; deepwell cylinder and pump jack and 15-horsepower electric motor; yield, 50 gallons a minute.

Well 2. Drilled about 1925; depth, 400 feet; diameter, 10 inches; deepwell turbine pump and 30-horsepower electric motor; yield, 150 gallons a minute.

Pumpage (estimated) : 150,000 gallons a day.

Storage: Elevated tank, 55,000 gallons.

Number of customers: 204.

Treatment: None.

\section{Analysis, well 2}

[Collected June 24, 1948. pH is 7.4. Analyzed by D. E. Weaver]

\begin{tabular}{|c|c|c|c|c|c|}
\hline & $\begin{array}{l}\text { Parts per } \\
\text { million }\end{array}$ & $\begin{array}{l}\text { Equivalents } \\
\text { per million }\end{array}$ & & $\begin{array}{l}\text { Parts per } \\
\text { million }\end{array}$ & $\begin{array}{l}\text { Equivalents } \\
\text { per million }\end{array}$ \\
\hline $\begin{array}{l}\text { Silica }\left(\mathrm{SiO}_{2}\right) \\
\text { Iron ( } \mathrm{Fe}) \\
\text { Calcium }(\mathrm{Ca}) \\
\text { Magnesium }(\overline{\mathrm{M}} \mathrm{g}) \\
\text { Sorlium (Na) } \\
\text { Potassium }(\mathrm{K}) \\
\text { Birqrbonate }\left(\mathrm{H} \mathrm{CO}_{3}\right)\end{array}$ & $\begin{array}{l}30 \\
41 \\
20 \\
26 \\
258 \\
3.2\end{array}$ & $\begin{array}{r}1.064 \\
1.645 \\
1.131 \\
.082 \\
4.229\end{array}$ & 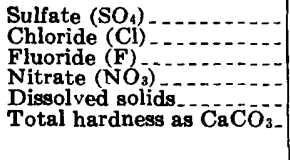 & $\begin{array}{r}18 \\
9.0 \\
5.2 \\
285 \\
185\end{array}$ & $\begin{array}{r}0.375 \\
.253 \\
.011 \\
.082 \\
0.0 \\
\end{array}$ \\
\hline
\end{tabular}

Driller's log, Panhandle and Santa Fe R.R. well in White D:er

\begin{tabular}{|c|c|c|c|c|c|}
\hline & $\begin{array}{c}\text { Thickness } \\
\text { (feet) }\end{array}$ & $\underset{\text { (feet) }}{\text { Depth }}$ & & $\underset{\text { (f :et) }}{\text { Thickness }}$ & $\begin{array}{l}\text { Depth } \\
\text { (feet) }\end{array}$ \\
\hline $\begin{array}{l}\text { Surface soil } \\
\text { Yellow clay } \\
\text { Brown caving sand } \\
\text { Sandy yellow clay } \\
\text { Yellow clay } \\
\text { Sandy yellow clay } \\
\text { Lime roek. } \\
\text { Red clay } \\
\text { Red sandrock, water } \\
\text { Sandy red clay }\end{array}$ & $\begin{array}{r}10 \\
86 \\
9 \\
95 \\
40 \\
56 \\
10 \\
26 \\
3 \\
10\end{array}$ & $\begin{array}{r}10 \\
\mathbf{9 6} \\
105 \\
200 \\
240 \\
296 \\
\mathbf{3 0 6} \\
\mathbf{3 3 2} \\
\mathbf{3 3 5} \\
\mathbf{3 4 5}\end{array}$ & $\begin{array}{l}\text { Water, gravel } \\
\text { Sandy red clay } \\
\text { Red clay } \\
\text { Sandy red clay } \\
\text { Red clay } \\
\text { Red water sand } \\
\text { Red clay } \\
\text { Red sand.- "bad" water } \\
\text { Red sand, clay } \\
\text { Red }\end{array}$ & $\begin{array}{r}3 \\
2 \\
12 \\
38 \\
95 \\
20 \\
5 \\
13 \\
24 \\
5\end{array}$ & $\begin{array}{l}348 \\
350 \\
362 \\
400 \\
495 \\
515 \\
520 \\
533 \\
557 \\
562\end{array}$ \\
\hline
\end{tabular}




\section{CASTRO COUNTY}

\section{DIMMITT}

Population in 1940: 943.

Source of information: Glen Smith, water superintendent, Mar. 7, 1941.

Ownership: Municipal.

Source of supply: Well one block east of courthouse; drilled ir 1929 by D. L.

McDonald; depth, 206 feet; diameter, 16 inches; deep-well turbine pump and 25-horsepower electric motor; yield, 600 gallons a minute; temperature, $63^{\circ} \mathrm{F}$.

Pumpage (estimated): Summer, 400,000 gallons a day; winter, 100,000 gallons a day.

Storage: Concrete reservoir, 250,000 gallons; elevated tank, 50,000 gallons. Number of customers: 250.

Treatment: None.

\section{Analysis}

[Collected Mar. 7, 1941. pH is 7.6. Analyzed by J. H. Rowley]

\begin{tabular}{|c|c|c|c|c|c|}
\hline & $\begin{array}{l}\text { Parts per } \\
\text { million }\end{array}$ & $\begin{array}{l}\text { Equivalents } \\
\text { per million }\end{array}$ & & $\begin{array}{l}\text { Parts per } \\
\text { million }\end{array}$ & $\begin{array}{l}\text { Equivalents } \\
\text { per million }\end{array}$ \\
\hline $\begin{array}{l}\text { Silica }\left(\mathrm{SiO}_{2}\right) \\
\text { Iron }(\mathrm{Fe}) \\
\text { Calcium }(\mathrm{Ca}) \\
\text { Magnesium }(\mathrm{Mg}) \\
\text { Sodium }(\mathrm{Na}) \\
\text { Potassium }(\mathrm{K}) \\
\text { Bicarbonate }\left(\mathrm{HCO}_{3}\right)^{-}\end{array}$ & $\begin{array}{l}44 \\
55 \\
33 \\
13 \\
6.2 \\
288\end{array}$ & $\begin{array}{r}2.75 \\
2.71 \\
.55 \\
.16 \\
4.72\end{array}$ & $\begin{array}{l}\text { Sulfate }\left(\mathrm{SO}_{4}\right) \\
\text { Chloride }(\mathrm{Cl}) \\
\text { Fluoride (F) } \\
\left.\text { Nitrate (NO }{ }_{3}\right) \\
\text { Dissolved solids. } \\
\text { Total hardness as } \mathrm{CaCO}_{3}\end{array}$ & $\begin{array}{c}38 \\
19 \\
2.2 \\
353 \\
273\end{array}$ & 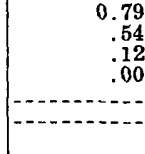 \\
\hline
\end{tabular}

Driller's log of abandoned well 160 feet east of city well

\begin{tabular}{|c|c|c|c|c|c|}
\hline & $\underset{\text { (feet) }}{\text { Thickness }}$ & $\begin{array}{l}\text { Depth } \\
\text { (feet) }\end{array}$ & & $\begin{array}{c}\text { Thickness } \\
\text { (feet) }\end{array}$ & $\begin{array}{l}\text { Depth } \\
\text { (feet) }\end{array}$ \\
\hline $\begin{array}{l}\text { Top soil } \\
\text { Caliche } \\
\text { Red sand } \\
\text { Sandrock } \\
\text { Sand and sandrock } \\
\text { Hard sand } \\
\text { White lime } \\
\text { Water sand } \\
\text { Sandrock } \\
\text { Water sand and sandrock }\end{array}$ & $\begin{array}{r}4 \\
11 \\
5 \\
15 \\
63 \\
52 \\
10 \\
4 \\
4 \\
44\end{array}$ & $\begin{array}{r}4 \\
15 \\
20 \\
35 \\
98 \\
150 \\
160 \\
164 \\
168 \\
212\end{array}$ & $\begin{array}{l}\text { Lime rock } \\
\text { Water sand and sandrock } \\
\text { Lime rock } \\
\text { Water sand and sandrock } \\
\text { Hard sandrock. } \\
\text { Water sand and sandrock } \\
\text { Yellow clay } \\
\text { Lime rock } \\
\text { "Red Beds" }\end{array}$ & $\begin{array}{r}6 \\
60 \\
12 \\
38 \\
17 \\
49 \\
8 \\
13 \\
2\end{array}$ & $\begin{array}{l}218 \\
278 \\
290 \\
328 \\
345 \\
394 \\
402 \\
415 \\
417\end{array}$ \\
\hline
\end{tabular}

\section{CHILDRESS COUNTY}

\section{CHILDRESS}

Population in $1940: 6,464$.

Source of information: Witt Johnson, city manager, Sept. 16, 1947.

Ownership: Municipal.

Source of supply: Eleven wells and Lake Childress.

Well 1. In Michie Sand Hill Well Field about 8 miles southwest of Childress; drilled by Layne-Texas Co. in 1947; depth, 80 feet; diameter $85 / 8$ inches; deep-well turbine pump and 5-horsepower electric motor; static water level, 31 feet below land surface Feb. 15, 1947; measured drawdown, 19 feet while pumping 64 gallons a minute on Aug. 30. 1947. 
Well 2. In Michie Sand Hill Well Field; drilled by Layne-Texas Co. in 1947; depth, 140 feet; diameter, 85 inches; deep-well turbine pump and 5-horsepower electric motor; static water level, 63.5 feet below land surface on Mar. 13, 1947; measured drawdown, 7.5 feet while pumping 166 gallons a minute on Aug. 30, 1947.

Well 3. In Michie Sand Hill Well Field; drilled by Layne-Texas Co. in 1947; depth, 201 feet; diameter, 16 to $5 \%$ inches; deep-woll turbine pump and 5-horsepower electric motor; static water level, 90 feet below land surface on Mar. 11, 1947; measured drawdown, 20 feet while pumping 190 gallons a minute on Aug. 30, 1947; temperature, 67 ${ }^{\circ} \mathrm{F}$.

Well 4. In Michie Sand Hill Well Field; drilled by Layne-Texas Co. in 1947; depth, 120 feet; diameter, 16 to $8 \%$ inches; deep-well turbine pump and 5-horsepower electric motor; static water level, 42.5 feet on Aug. 30, 1947; measured drawdown, 7.5 feet while pumping 272 gallons a minute on Aug. 30, 1947.

Well 5. In Michie Sand Hill Well Field; drilled by Layne-Texas Co. in 1947; depth, 133 feet; diameter, 16 to $8 \%$ inches; deep-well turbine pump and 5-horsepower electric motor; static water level, 53 feet below land surface on Aug. 30, 1947; measured drawdown, 8 feet while pumping 120 gallons a minute on Aug. 30, 1947.

Well 6. In Michie Sand Hill Well Field; drilled by Layne-Texas Co. in 1947; depth, 107 feet; diameter, 16 to $8 \%$ inches; deep-well turbine pump and 5-horsepower electric motor; static water level, 32 feet below land surface on Aug. 30, 1947; measured drawdown, 14 feet while pumping 160 gallons a minute on Aug. 30, 1947; temperature, $66^{\circ} \mathrm{F}$.

Well 7. In Michie Sand Hill Well Field; drilled by Layne-Texas Co. in 1947 ; depth, 92 feet; diameter, 16 to 85 inches; deep-well turbine pump and 5-horsepower electric motor; static water level, 34 feet below land surface on Aug. 30, 1947; measured drawdown, 6 feet whil? pumping 214 gallons a minute on Aug. 30, 1947.

Well 8. In Michie Sand Hill Well Field; drilled by Layne-Texas Co. in 1947; depth, 111 feet; diameter, 16 to $8 \%$ inches; deep-well turbine pump and 5-horsepower electric motor; static water level, 36 feet below land surface on Mar. 11, 1947; measured drawdown, 9 feet while pumping 226 gallons a minute on Aug. 30, 1947.

Well 9. In Michie Sand Hill Well Field; drilled by Layne-Texas Co. in 1947; depth, 87 feet; diameter, 16 to $8 \%$ inches; deep-well turbine pump and 5-horsepower electric motor; static water level, 36 feet below land surface on Mar. 11, 1947; measured drawdown, 4.5 feet while pumping 350 gallons a minute on Aug. 30, 1947.

Well 10. In Michie Sand Hill Well Field; drilled by Layne-Texas Co. in 1947; depth, 132 feet; diameter, 16 to $8 \%$ inches; deep-well turbine pump and 5-horsepower electric motor; static water level, 62 feet below land surface on Aug. 30, 1947; measured drawdown, 13 feet while pumping 180 gallons a minute on Aug. 30, 1947.

62 Ranch Well. About 14 miles west of Childress on 62 Ranch; drilled in 1942; depth, 443 feet; diameter, 10 inches; deep-well turbine pump and 25-horsepower electric motor; pumping level, 346 feet below land surface on Sept. 16, 1947; static water level, 296 feet below land surface in January 1943 and 334 feet below land surface on Sept. 20, 1945; yield, -480 gallons a minute. 
Lake Childress. Dam constructed in 1943; drainage area, ab iut 12 square miles; lake floods 322 acres; average depth, 15 feet; ce vacity, 4,830 acre-feet.

\section{Average pumpage in gallons a day}

\begin{tabular}{|c|c|c|c|c|c|}
\hline & 1946 & 1947 & & 1946 & 1947 \\
\hline $\begin{array}{l}\text { January } \\
\text { February } \\
\text { March } \\
\text { April } \\
\text { May } \\
\text { June. }\end{array}$ & $\begin{array}{l}603,000 \\
606,000 \\
691,000 \\
821,000 \\
819,000 \\
699,000\end{array}$ & $\begin{array}{l}\mathbf{5 0 2}, 000 \\
\mathbf{4 8 9}, 000 \\
\mathbf{5 1 9}, 000 \\
\mathbf{5 3 8}, 000 \\
\mathbf{6 0 1}, 000 \\
\mathbf{7 3 4}, 000\end{array}$ & $\begin{array}{l}\text { July } \\
\text { August } \\
\text { September } \\
\text { October } \\
\text { November } \\
\text { December }\end{array}$ & $\begin{array}{l}886,000 \\
852,000 \\
561,0>0 \\
510,000 \\
507,000 \\
498,000\end{array}$ & 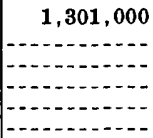 \\
\hline
\end{tabular}

Storage: Ground reservoir, 1,000,000 gallons; ground reservir, 1,500,000 gallons; elevated tank, 75,000 gallons.

Number of customers: 2,146.

Treatment: Chlorination.

\section{Analysis}

[Collected Sept. 17, 1947. pH: well 3, 7.2; nell 6, 7.7. Analyzed by B. C. Dwyer]

\begin{tabular}{|c|c|c|c|c|}
\hline & \multicolumn{2}{|c|}{ Well 3} & \multicolumn{2}{|c|}{ Well 6} \\
\hline & $\begin{array}{l}\text { Parts per } \\
\text { million }\end{array}$ & $\begin{array}{l}\text { Equivalents } \\
\text { per million }\end{array}$ & $\begin{array}{l}\text { Parts per } \\
\text { million }\end{array}$ & $\begin{array}{l}\text { Equivalents } \\
\text { per million }\end{array}$ \\
\hline 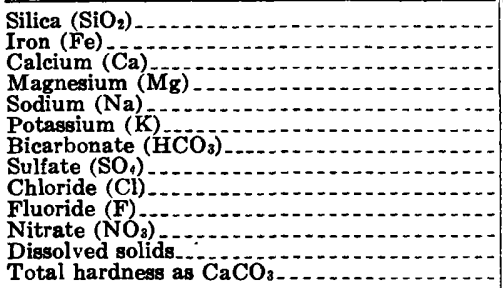 & $\begin{array}{l}20 \\
97 \\
90 \\
20 \\
33 \\
7.04 \\
226 \\
85 \\
54 \\
{ }^{67} .0 \\
604 \\
324\end{array}$ & $\begin{array}{r}4.84 \\
1.64 \\
1.42 \\
3.18 \\
1.70 \\
1.52 \\
1.01 \\
1.08\end{array}$ & $\begin{array}{l}16 \\
74 \\
22 \\
54 \\
5.8 \\
266 \\
84 \\
44 \\
38^{-25} \\
470 \\
275\end{array}$ & $\begin{array}{r}\mathbf{3} .69 \\
1.81 \\
\mathbf{2 . 3 5} \\
.15 \\
4.36 \\
1.75 \\
1.24 \\
.04 \\
.61 \\
\hdashline-. .\end{array}$ \\
\hline
\end{tabular}

[Collected Sept. 17, 1947. pH: raw water, 7.2; finished water, 7.8. Analyzed by B. C. Dwyer]

\begin{tabular}{|c|c|c|c|c|}
\hline & \multicolumn{4}{|c|}{ Lake Childress } \\
\hline & \multicolumn{2}{|c|}{ Raw water } & \multicolumn{2}{|c|}{ Fin:shed water } \\
\hline & $\begin{array}{c}\text { Parts per } \\
\text { million }\end{array}$ & $\begin{array}{l}\text { Equivalents } \\
\text { per million }\end{array}$ & $\begin{array}{l}\text { Parts per } \\
\text { million }\end{array}$ & $\begin{array}{l}\text { Equivalents } \\
\text { per million }\end{array}$ \\
\hline 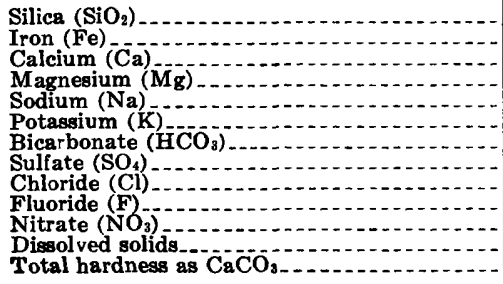 & $\begin{array}{l}8.0 \\
184.18 \\
23 \\
2.5 \\
3.2 \\
84 \\
456 \\
12 \\
2.2 \\
202 \\
558\end{array}$ & \begin{tabular}{r}
-1.89 \\
.11 \\
.08 \\
1.38 \\
9.49 \\
.34 \\
.01 \\
.04 \\
\hdashline$-0 .-18$ \\
\end{tabular} & $\begin{array}{l}17 \\
93 \\
23 \\
70 \\
6.0 \\
230 \\
161 \\
70 \\
38 \\
592 \\
326\end{array}$ & $\begin{array}{r}4.64 \\
1.89 \\
3.04 \\
.15 \\
3.77 \\
3.35 \\
1.97 \\
.02 \\
.61 \\
\\
\hdashline\end{array}$ \\
\hline
\end{tabular}




\section{Drillers' Logs}

\begin{tabular}{|c|c|c|c|c|c|}
\hline & $\begin{array}{l}\text { Thickness } \\
\text { (feet) }\end{array}$ & $\begin{array}{l}\text { Depth } \\
\text { (feet) }\end{array}$ & & $\begin{array}{l}\text { Thickness } \\
\text { (feet) }\end{array}$ & $\begin{array}{l}\text { Depth } \\
\text { (feet) }\end{array}$ \\
\hline \multicolumn{6}{|c|}{ Well 1} \\
\hline $\begin{array}{l}\text { Sandy soil } \\
\text { Sand and boulders }\end{array}$ & $\begin{array}{r}4 \\
46\end{array}$ & $\begin{array}{r}4 \\
50\end{array}$ & Fine-grained cemented sand & 30 & 80 \\
\hline \multicolumn{6}{|c|}{ Well 2} \\
\hline $\begin{array}{l}\text { Sandy soil } \\
\text { Sandy clay } \\
\text { Fine-grained cemented sand } \\
\text { Clay } \\
\text { Fine-grained sand }\end{array}$ & $\begin{array}{r}3 \\
12 \\
65 \\
3 \\
10\end{array}$ & $\begin{array}{r}3 \\
15 \\
80 \\
83 \\
93\end{array}$ & $\begin{array}{l}\text { Clay } \\
\text { Fine-grained cemented sand } \\
\text { Flaye-grained cemented sand } \\
\text { Clay_. }\end{array}$ & $\begin{array}{r}5 \\
25 \\
5 \\
10 \\
2\end{array}$ & $\begin{array}{r}98 \\
123 \\
128 \\
138 \\
140\end{array}$ \\
\hline
\end{tabular}

\section{Well 3}

\begin{tabular}{|c|c|c|c|c|c|}
\hline $\begin{array}{l}\text { Top soil } \\
\text { Red sand } \\
\text { Gray sand. }\end{array}$ & $\begin{array}{r}3 \\
52 \\
45\end{array}$ & $\begin{array}{r}\mathbf{3} \\
\mathbf{5 5} \\
\mathbf{1 0 0}\end{array}$ & $\begin{array}{l}\text { White sand and fine gravel. } \\
\text { Red beds.. }\end{array}$ & $\begin{array}{r}100 \\
1\end{array}$ & $\begin{array}{l}200 \\
201\end{array}$ \\
\hline
\end{tabular}

\section{Well 5}

\begin{tabular}{|c|c|c|c|c|c|}
\hline $\begin{array}{l}\text { Surface soil. } \\
\text { Red sand.... } \\
\text { Clay.2. }\end{array}$ & $\begin{array}{r}6 \\
32 \\
3\end{array}$ & $\begin{array}{r}6 \\
38 \\
41\end{array}$ & $\begin{array}{l}\text { Fine to coarse-grained sand.- } \\
\text { Red beds. }\end{array}$ & $\begin{array}{r}91 \\
1\end{array}$ & $\begin{array}{l}132 \\
133\end{array}$ \\
\hline
\end{tabular}

Well 6

\begin{tabular}{|c|c|c|c|c|c|}
\hline $\begin{array}{l}\text { Surface soil } \\
\text { Red sand } \\
\text { Brown sand } \\
\text { Coarse-grained sand }\end{array}$ & $\begin{array}{r}5 \\
40 \\
18 \\
12\end{array}$ & $\begin{array}{r}\mathbf{5} \\
4 \mathbf{5} \\
63 \\
\mathbf{7 5}\end{array}$ & $\begin{array}{l}\text { Sandy clay } \\
\text { Coarse-grained brown sand.- } \\
\text { Red beds. }\end{array}$ & $\begin{array}{r}20 \\
10 \\
2\end{array}$ & $\begin{array}{r}95 \\
105 \\
107\end{array}$ \\
\hline
\end{tabular}

Well 7

\begin{tabular}{|c|c|c|c|c|c|}
\hline $\begin{array}{l}\text { Surface soil } \\
\text { Red sand }\end{array}$ & $\begin{array}{r}4 \\
42\end{array}$ & $\begin{array}{r}4 \\
46\end{array}$ & $\begin{array}{l}\text { Coarse sand and gravel } \\
\text { Red beds. }\end{array}$ & $\begin{array}{r}45 \\
1\end{array}$ & $\begin{array}{l}91 \\
92\end{array}$ \\
\hline
\end{tabular}

\section{Well 8}

\begin{tabular}{|c|c|c|c|c|c|}
\hline $\begin{array}{l}\text { Surface soil. } \\
\text { Red sand } \\
\text { Coarse-grained sand }\end{array}$ & $\begin{array}{r}6 \\
39 \\
12\end{array}$ & $\begin{array}{r}6 \\
45 \\
57\end{array}$ & $\begin{array}{l}\text { Gravel and coarse-grained } \\
\text { sand. } \\
\text { Sand and gravel. } \\
\text { Red beds. }\end{array}$ & $\begin{array}{r}32 \\
6 \\
16\end{array}$ & $\begin{array}{r}89 \\
95 \\
111\end{array}$ \\
\hline
\end{tabular}

\section{Well 9}

\begin{tabular}{|c|c|c|c|c|c|}
\hline $\begin{array}{l}\text { Surface } \\
\text { Red sand } \\
\text { Medium coarse-grained sand. }\end{array}$ & $\begin{array}{r}4 \\
36 \\
5\end{array}$ & $\begin{array}{r}4 \\
40 \\
45\end{array}$ & $\begin{array}{l}\text { Coarse-grained sand } \\
\text { Coarse-grained sand and } \\
\text { gravel. } \\
\text { Red beds }\end{array}$ & $\begin{array}{l}\mathbf{8} \\
1 / 2\end{array}$ & $\begin{array}{l}53 \\
86 \\
861 / 2\end{array}$ \\
\hline
\end{tabular}

\section{Well 10}

\begin{tabular}{|c|c|c|c|c|c|}
\hline $\begin{array}{l}\text { Surface soil } \\
\text { Red sand } \\
\text { Soft brown sand } \\
\text { Gravel and sand }\end{array}$ & $\begin{array}{r}2 \\
43 \\
35 \\
18\end{array}$ & $\begin{array}{r}2 \\
45 \\
80 \\
98\end{array}$ & $\begin{array}{l}\text { Sandy clay and gravel } \\
\text { Gravel } \\
\text { Red and blue clay. }\end{array}$ & $\begin{array}{r}21 \\
12 \\
1\end{array}$ & $\begin{array}{l}119 \\
131 \\
132\end{array}$ \\
\hline
\end{tabular}




\section{COCHRAN COUNTY}

\section{MORTON}

Population in 1940: 1,137.

Source of information: O. D. Vernon, water superintendent, March 5, 1947.

Ownership: Municipal.

Source of supply: Two wells.

Well 1. Drilled in 1933 and drilled deeper in 1947; derth, 152 feet in 1933, 233 feet in 1947; diameter, 12 inches; deep-well turbine pump and 50-horsepower electric motor; static water level, 132 feet below land surface in 1946; yield, 750 gallons a minute.

Well 2. Drilled in 1941 by Carl Williams; depth, 207 feet; diameter, 10 inches; deep-well turbine pump and 35-horsepower electric motor; drawdown, 108 feet while pumping 350 gallons a minute, 130 feet while pumping 600 gallons a minute; static water leve'. 95 feet below land surface on August 2, 1941; normal yield reported, 350 gallons a minute.

Pumpage (estimated) : 100,000 gallons a day.

Storage: Elevated tank, 50,000 gallons.

Number of customers: 500 .

Treatment: None.

Analysis, Well 2

[Collected Mar. 5, 1947. $\mathrm{pH}$ is 7.6. Analyzed by B. C. Dwye-]

\begin{tabular}{|c|c|c|c|c|c|}
\hline & $\begin{array}{l}\text { Parts per } \\
\text { million }\end{array}$ & $\begin{array}{l}\text { Equivalents } \\
\text { per million }\end{array}$ & & $\begin{array}{l}\text { Parts per } \\
\text { Inillion }\end{array}$ & $\begin{array}{l}\text { Equivalents } \\
\text { per million }\end{array}$ \\
\hline $\begin{array}{l}\text { Silica }\left(\mathrm{SiO}_{2}\right) \\
\text { Iron }(\mathrm{Fe}) \\
\text { Calcium }(\mathrm{Ca}) \\
\text { Magnesium }(\mathrm{Mg}) \\
\text { Sodium }(\mathrm{Na}) \\
\text { Potassium }(\mathrm{K}) \\
\text { Bicarbonate }\left(\mathrm{H}^{2} \mathrm{CO}_{3}\right)\end{array}$ & $\begin{array}{r}42 \\
37 \\
51 \\
103 \\
12 \\
286\end{array}$ & $\begin{array}{r}1.85 \\
4.19 \\
4.47 \\
.31 \\
4.69\end{array}$ & $\begin{array}{l}\text { Sulfate }\left(\mathrm{SO}_{4}\right) \\
\text { Chloride }(\mathrm{Cl}) \\
\text { Fluoride (F) } \\
\text { Nitrate (NO} \\
\text { Dissolved solids } \\
\text { Total hardness as } \mathrm{CaCO}_{3}\end{array}$ & $\begin{array}{c}200 \\
60 \\
4.0 \\
4.5 \\
654 \\
302\end{array}$ & $\begin{array}{r}4.16 \\
1.69 \\
.21 \\
.07 \\
\hdashline-.0- \\
\hdashline-0 .-0\end{array}$ \\
\hline
\end{tabular}

Driller's Log, Well 2

\begin{tabular}{|c|c|c|c|c|c|}
\hline & $\begin{array}{c}\text { Thickness } \\
\text { (feet) }\end{array}$ & $\begin{array}{l}\text { Depth } \\
\text { (feet) }\end{array}$ & & $\underset{\text { (feet) }}{\text { Thickness }}$ & $\begin{array}{l}\text { Depth } \\
\text { (feet) }\end{array}$ \\
\hline $\begin{array}{l}\text { Soil } \\
\text { Sandy caliche sand and caliche. } \\
\text { Fine packed sand } \\
\text { Hard limestone. } \\
\text { Fine packed sand } \\
\text { Hard limestone. } \\
\text { Fine packed sand }\end{array}$ & $\begin{array}{r}6 \\
8 \\
21 \\
30 \\
10 \\
5 \\
5 \\
5\end{array}$ & $\begin{array}{r}6 \\
14 \\
35 \\
65 \\
75 \\
80 \\
85 \\
90\end{array}$ & $\begin{array}{l}\text { Fine sand (little water) } \\
\text { Fine sand (some clay) } \\
\text { Gravel (some lime) } \\
\text { Yellow clay and gravel. } \\
\text { Yellow clay and sand. } \\
\text { Sand with some clay. } \\
\text { Sand and gravel } \\
\text { Sand }\end{array}$ & $\begin{array}{r}18 \\
17 \\
7 \\
23 \\
5 \\
20 \\
12 \\
15\end{array}$ & $\begin{array}{l}108 \\
125 \\
132 \\
155 \\
160 \\
180 \\
192 \\
207\end{array}$ \\
\hline
\end{tabular}

\section{COKE COUNTY}

\section{BRONTE}

Population in 1940: 754 .

Source of information: O. W. Chapman, city secretary, May $\& .1946$.

Ownership: Municipal. 
Source of supply: One well and two lakes.

Well: Two miles south of Bronte about 60 feet north of the north bank of the Colorado River; dug in 1932; depth, 20 feet; diameter, 8 feet; jet pump and 5-horsepower electric motor; pump yields 60 gallons a minute, but pump runs dry in 40 minutes; well recovers in about 15 minutes.

Lake Chapman: About 1.2 miles southeast of Bronte; catchment area, 800 acres; lake supply inadequate in dry periods.

Lake Kaierim: About .6 mile east of Bronte; constructed in 1922; lake supply inadequate in dry periods.

Pumpage: Average, 45,000 gallons a day.

Storage: Elevated tank, 30,000 gallons.

Number of customers: 225 .

Treatment: Aeration, sedimentation, and filtration.

\section{Analysis}

[Composite sample from lakes. Collected May 8, 1946. $\mathrm{pH}$ is 7.2. Analyzed by C. B. Cibulka]

\begin{tabular}{|c|c|c|c|c|c|}
\hline & $\begin{array}{c}\text { Parts per } \\
\text { million }\end{array}$ & $\begin{array}{l}\text { Equivalents } \\
\text { per million }\end{array}$ & & $\begin{array}{c}\text { Parts per } \\
\text { million }\end{array}$ & $\begin{array}{l}\text { Equivalents } \\
\text { per million }\end{array}$ \\
\hline $\begin{array}{l}\text { Silica }\left(\mathrm{SiO}_{2}\right) \\
\text { Iron }(\mathrm{Fe}) \\
\text { Calcium }(\mathrm{Ca}) \\
\text { Magnesium }(\mathrm{Mg}) \\
\text { Sodium and potassium } \\
\quad(\mathrm{Na}+\mathrm{K}) \text {. } \\
\text { Bicarbonate }\left(\mathrm{HCO}_{3}\right)\end{array}$ & $\begin{array}{r}5.6 \\
1.0 \\
30 \\
7.1 \\
5.8 \\
113\end{array}$ & $\begin{array}{r}.584 \\
.254 \\
1.852\end{array}$ & $\begin{array}{l}\text { Sulfate }\left(\mathrm{SO}_{4}\right) \\
\text { Chloride (Cl) } \\
\text { Fluoride (F) } \\
\text { Nitrate (NO})_{3} \text { ) } \\
\text { Dissolved solids } \\
\text { Total hardness as } \mathrm{CaCO}_{3}\end{array}$ & $\begin{array}{r}17 \\
4.0 \\
1.0 \\
135 \\
104\end{array}$ & $\begin{array}{r}.354 \\
.113 \\
.000 \\
.016 \\
-0 .- \\
-0.0\end{array}$ \\
\hline
\end{tabular}

\section{ROBERT LEE}

Population in 1940: 662 .

Source of information: Fred O. Green, city secretary, May 9, 1946.

Ownership: Municipal.

Source of supply: Lake and well.

Lake: About $1 \frac{1 / 2}{2}$ miles southeast of Robert Lee; catchment area, 800 acres. Does not furnish sufficient water during dry period?

Well: Dug in 1943 on G. W. Hill Estate below city lake; denth, 24 feet; diameter, 5 feet; centrifugal pump and gasoline engine; static water level, 14.9 feet below land surface, May 19, 1946; yield, 30 gallons a minute.

Pumpage: Average, 40,000 gallons a day.

Storage: Elevated tank, 5u,000 gallons.

Number of customers: 200.

Treatment: Coagulation, sedimentation, and filtration.

Analysis of water from well

[Collected May 9, 1946. $\mathrm{pH}$ is 7.4. Analyzed by C. B. Cibulka]

\begin{tabular}{|c|c|c|c|c|c|}
\hline & $\begin{array}{c}\text { Parts per } \\
\text { million }\end{array}$ & $\begin{array}{c}\text { Equivalents } \\
\text { per million }\end{array}$ & & $\underset{\text { millic } n}{\text { Parts per }}$ & $\begin{array}{l}\text { Equivalents } \\
\text { per million }\end{array}$ \\
\hline $\begin{array}{l}\text { Silica }\left(\mathrm{SiO}_{2}\right) \\
\text { Iron }(\mathrm{Fe}) \\
\text { Calcium }(\mathrm{Ca}) \\
\text { Magnesium }(\mathrm{Mg}) \\
\text { Sodium }(\mathrm{Na}) \\
\text { Potassium }(\mathrm{K}) \\
\text { Bicarbonate }\left(\mathrm{HCOO}_{3}\right)\end{array}$ & $\begin{array}{l}16 \\
132 \\
48 \\
49 \\
14 \\
328\end{array}$ & $\begin{array}{r}6.59 \\
3.95 \\
2.15 \\
.36 \\
5.38\end{array}$ & $\begin{array}{l}\text { Sulfate }\left(\mathrm{SO}_{4}\right) \\
\text { Chloride }(\mathrm{Cl}) \\
\text { Fluoride (F) } \\
\left.\text { Nitrate (NO})_{3}\right) \\
\text { Dissolved solids } \\
\text { Total hardness as } \mathrm{CaCO}_{3}\end{array}$ & $\begin{array}{c}269 \\
68 \\
.2 \\
8.8 \\
838 \\
527\end{array}$ & \begin{tabular}{r}
5.60 \\
1.92 \\
.01 \\
.14 \\
\hdashline.-- \\
\end{tabular} \\
\hline
\end{tabular}




\section{COLLINGSWORTH COUNTY}

\section{DODSONVILLE}

Population in 1940: 357.

Source of information: Arthur Weaver, pump operator, June 22, 1948.

Ownership: Municipal.

Source of supply: Well 2 miles northeast of Dodsonville on top of hill at concrete reservoir; drilled in 1948; depth, 130 feet; diameter, 8 inches; deepwell turbine pump and 5-horsepower electric motor; yield. 50 gallons a minute.

Pumpage: No record.

Storage: Concrete ground reservoir, 50,000 gallons.

Number of customers: About 200.

Treatment: None.

Analysis

[Collected June 22, 1948. pH is 7.9. Analyzed by H. D. Smith]

\begin{tabular}{|c|c|c|c|c|c|}
\hline & $\begin{array}{c}\text { Parts per } \\
\text { million }\end{array}$ & $\begin{array}{l}\text { Equivalents } \\
\text { per million }\end{array}$ & & $\begin{array}{l}\text { Parts per } \\
\text { million }\end{array}$ & $\begin{array}{l}\text { Equivalents } \\
\text { per million }\end{array}$ \\
\hline $\begin{array}{l}\text { Silica }\left(\mathrm{SiO}_{2}\right) \\
\text { Iron }(\mathrm{Fe}) \\
\text { Calcium }(\mathrm{Ca}) \\
\text { Magnesium }(\mathrm{Mg})^{-} \\
\text {Sodium }(\mathrm{Na}) \\
\text { Potassium }(\mathrm{K}) \\
\text { Bicarbonate }\left(\mathrm{HCO}_{3}\right)^{-}\end{array}$ & $\begin{array}{l}29 \\
67.00 \\
21 \\
9.2 \\
5.2 \\
\mathbf{2 9 0}\end{array}$ & $\begin{array}{r}3.34 \\
1.73 \\
.40 \\
.13 \\
4.75\end{array}$ & $\begin{array}{l}\text { Sulfate }\left(\mathrm{SO}_{4}\right) \\
\text { Chloride (Cl) } \\
\text { Fluoride (F) } \\
\text { Nitrate (NO} \\
\text { Dissolved solids.. } \\
\text { Total hardness as } \mathrm{CaCO}_{3}\end{array}$ & $\begin{array}{l}23 \\
6.1 \\
17.8 \\
320 \\
254\end{array}$ & $\begin{array}{r}.48 \\
.17 \\
.04 \\
.27 \\
-.0 .-1 \\
-0.0\end{array}$ \\
\hline
\end{tabular}

\section{WELLINGTON}

Population in $1940: 3,308$.

Source of information: B. O. Handley, water superintendent, June 22, 1948. Ownership: Municipal.

Source of supply: Eleven wells $2 \frac{1}{2}$ miles northeast of Wellington.

Well 1. Drilled in 1932; depth, 45 feet; diameter, 8 inches; gravelwalled; deep-well turbine pump and 3-horsepower electric motor; static water level, 15 feet below land surface June 1938; yield, 50 gallons a minute.

Well 2. About 450 feet from well 1; drilled in 1932; depth, 45 feet; diameter, 8 inches; gravel-walled; deep-well turbine pump and 3-horsepower electric motor; yield, 50 gallons a minute.

Well 3. About 450 feet from well 2; drilled in 1932; cepth, 45 feet; diameter, 8 inches; gravel-walled; deep-well turbine pump and 3horsepower electric motor; yield, 50 gallons a minute.

Well 4. About 450 feet from well 3; drilled in 1926; depth, 50 feet; diameter, 8 inches; concrete casing and screen; vertical certrifugal pump and 5-horsepower electric motor; yield, 50 gallons a minute.

Well 5. About 450 feet from well 4; drilled in 1926; depth, 50 feet; diameter, 8 inches; concrete casing and screen; vertical certrifugal pump and 5-horsepower electric motor; yield, 50 gallons a mirute.

Well 6. About 450 feet from well 5; drilled in 1934; depth, 40 feet; diameter, 8 inches; gravel-walled; deep-well turbine pump and 3-horsepower electric motor; yield, 50 gallons a minute. 
Well 7. About 450 feet from well 6; drilled in 1926; depth, 50 feet, diameter, 18 inches; concrete casing and screen; vertical centrifugal pump and 5-horsepower electric motor; yield, 50 gallons a minute.

Well 8. About 500 feet from well 7; drilled in 1926; depth, $5 C$ feet; diameter, 18 inches; concrete casing and screen; deep-well cylinder pump and electric motor; yield, 20 gallons a minute.

Well 9. About 500 feet from well 8; drilled in 1926; depth, 5C feet; diameter, 8 inches; gravel-walled; deep-well turbine pump ard 3-horsepower electric motor; yield, 75 gallons a minute.

Well 10. About 500 feet from well 9; drilled in 1946; depth, 45 feet; diameter, 8 inches; gravel-walled; deep-well turbine pump and 3 horsepower electric motor; yield, 100 gallons a minute.

Well 11. About 500 feet from well 10; drilled in 1946; depth, 45 feet; diameter, 8 inches; gravel-walled; deep-well turbine pump and 3horsepower electric motor; yield, 100 gallons a minute.

Pumpage: Average, 250,000 gallons a day in winter and $600,00 \mathrm{C}$ gallons a day in summer.

Storage: Ground storage reservoir, 25,000 gallons; elevated tark, 100,000 gallons; ground storage reservoir under construction, 200,000 gellons.

Number of customers: 1,100 .

Treatment: None.

\section{Analysis}

[Composite sample of all wells. Collected June 22, 1948. pH is 7.4. Analyzed by Ir. D. Smith]

\begin{tabular}{|c|c|c|c|c|c|}
\hline & $\begin{array}{l}\text { Parts per } \\
\text { million }\end{array}$ & $\begin{array}{l}\text { Equivalents } \\
\text { per million }\end{array}$ & & $\begin{array}{c}\text { Parts per } \\
\text { million }\end{array}$ & $\begin{array}{l}\text { Equivalents } \\
\text { per million }\end{array}$ \\
\hline $\begin{array}{l}\text { Silica }\left(\mathrm{SiO}_{2}\right) \\
\text { Iron }(\mathrm{Fe}) \\
\text { Calcium }(\mathrm{Ca}) \\
\text { Magnesium }(\mathrm{Mg}) \\
\text { Sodium }(\mathrm{Na}) \\
\text { Potassium }(\mathrm{K}) \\
\text { Bicarbonate }\left(\mathrm{HCOO}_{3}\right)^{-}\end{array}$ & $\begin{array}{l}32 \\
82 \\
18 \\
71 \\
4.00 \\
388\end{array}$ & $\begin{array}{l}4.09 \\
1.48 \\
3.10 \\
.10 \\
6.36\end{array}$ & $\begin{array}{l}\text { Sulfate }\left(\mathrm{SO}_{4}\right) \\
\text { Chloride }(\mathrm{Cl}) \\
\text { Fluoride (F) } \\
\text { Nitrate }\left(\mathrm{NO}_{3}\right) \\
\text { Dissolved solids } \\
\text { Total hardness as } \mathrm{CaCO}_{3}\end{array}$ & $\begin{array}{c}66 \\
30 \\
9.6 \\
996 \\
296\end{array}$ & $\begin{array}{r}1.37 \\
.85 \\
.03 \\
.16 \\
-1 .-\end{array}$ \\
\hline
\end{tabular}

\section{COTTLE COUNTY}

\section{PADUCAH}

Population in 1940: 2,677.

Source of information: J. A. Carroll, water superintendent, Sept. 16, 1947. Ownership: Municipal.

Source of supply: Four wells in field 8 miles west of Paducah near South Pease River.

Well 2. Drilled about 1931; depth, 225 feet; diameter, 7 inch $\epsilon$; and 35.6 feet below land surface on Sept. 16, 1947; deep-well turbine pump and 71/2-horsepower electric motor; static water level, 24.9 feet below land surface on October 25, 1945, yield reported, 120 gallons a minute.

Well 3. Drilled about 1931; depth, 130 feet; diameter, 7 inches; static water level, 21.1 feet below land surface on October 25, 1945, and 38.3 feet below land surface on Sept. 6, 1947; pumped with air; yield reported, 150 gallons a minute; temperature, $67^{\circ} \mathrm{F}$. 
Well 4. Drilled about 1931; depth 130 feet; diameter, 7 inches; static water level, 35.8 feet below land surface on Sept. 16, 1947; pumped with air; yield reported, 150 gallons a minute.

Well 5. Drilled about 1931; depth, 127 feet; diameter, 7 inches; deepwell turbine pump and $7 \frac{1}{2}$-horsepower electric motor; static water level, 28.2 feet below land surface on October 25, 1945, and 33 feet below land surface on Sept. 16, 1947; yield reported, 150 gallons a minute.

Average pumpage in gallons a day

\begin{tabular}{|c|c|c|c|c|}
\hline & 1944 & 1945 & 1946 & 1947 \\
\hline $\begin{array}{l}\text { January } \\
\begin{array}{l}\text { February } \\
\text { March }\end{array} \\
\text { April } \\
\text { May } \\
\begin{array}{l}\text { June } \\
\text { July }\end{array} \\
\begin{array}{l}\text { August } \\
\text { Septem }\end{array} \\
\begin{array}{l}\text { Oetober } \\
\text { Novem }\end{array} \\
\text { December }\end{array}$ & $\begin{array}{r}108,000 \\
137,000 \\
214,000 \\
244,000 \\
281,000 \\
150,000 \\
143,000 \\
124,000\end{array}$ & $\begin{array}{l}129,000 \\
114,000 \\
117,000 \\
149,000 \\
230,000 \\
263,000 \\
214,000 \\
251,000 \\
202,000 \\
271,000 \\
143,000 \\
130,000\end{array}$ & $\begin{array}{l}123,000 \\
124,000 \\
160,000 \\
2 C 7,000 \\
203,000 \\
279,000 \\
326,000 \\
260,000 \\
176,000 \\
140,000 \\
180,000 \\
124,000\end{array}$ & $\begin{array}{r}119,000 \\
117,000 \\
147,000 \\
222,000 \\
162,000 \\
256,000 \\
331,000 \\
351,000 \\
\hdashline \\
\hdashline \\
\hdashline\end{array}$ \\
\hline
\end{tabular}

Storage: Concrete reservoir at well field, 286,000 gallons; ground storage reservoir 3 miles west of Paducah, 250,000 gallons; elevated tank, 100,000 gallons.

Number of customers: 680 .

Treatment: Chlorination.

Analyses

[Collected Oct. 25, 1945. Analyzed by B. C. Dwyer and J. H. Rowlev]

\begin{tabular}{|c|c|c|c|c|}
\hline & \multicolumn{2}{|c|}{ Well 3} & \multicolumn{2}{|c|}{ Well 5} \\
\hline & $\begin{array}{l}\text { Parts per } \\
\text { million }\end{array}$ & $\begin{array}{l}\text { Equivalents } \\
\text { per million }\end{array}$ & $\begin{array}{l}\text { Parts per } \\
\text { millior }\end{array}$ & $\begin{array}{l}\text { Equivalents } \\
\text { per million }\end{array}$ \\
\hline 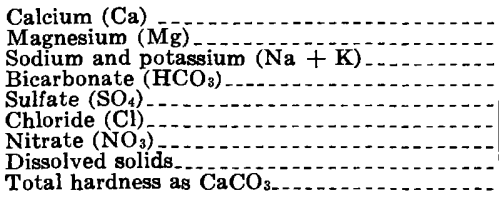 & $\begin{array}{r}354 \\
101 \\
13 \\
202 \\
1,090 \\
18 \\
2.8 \\
1,680 \\
1,300\end{array}$ & $\begin{array}{r}17.67 \\
8.31 \\
.57 \\
3.31 \\
22.69 \\
.51 \\
.05 \\
\hdashline-0 .--0\end{array}$ & $\begin{array}{r}534 \\
144 \\
337 \\
224 \\
1,37 C \\
73 C \\
1.2 \\
3,24 C \\
1,92 C\end{array}$ & $\begin{array}{r}26.65 \\
11.84 \\
14.65 \\
3.67 \\
28.52 \\
20.59 \\
.02 \\
-0--\end{array}$ \\
\hline
\end{tabular}

\section{CRANE COUNTY}

\section{CRANE}

Population in 1940: 1,420.

Source of information: Albert Wright, water superintendent, Dec. 13, 1946. Ownership: Municipal.

Source of supply: Five wells about $6 \frac{1}{4}$ miles northwest of Crane, (sec. 35, blk. 31, University of Texas.) This well field was not in operation on Dec. $13,1946$. 
Well 2. Drilled in September 1946 by W. O. Bower; depth, 79 feet; diameter, 8 inches; casing perforated from 54 to 79 feet; deep-well turbine pump and 3-horsepower electric motor, pump set at 69 feet; static water level reported, 40 feet below land surface wl on drilled; yield reported, 60 gallons a minute when drilled.

Well 3. Drilled in November 1946 by W. O. Bower; depth, 82 feet; diameter, 8 inches; casing perforated from 52 to 82 feet; deep-well . turbine pump and 3-horsepower electric motor, pump set at 72 feet; static water level reported, 42 feet below land surface wh en drilled; yield reported, 65 gallons a minute when drilled.

Well 4. Drilled in November 1946 by W. O. Bower; depth, 92 feet; diameter, 8 inches; casing perforated from 62 to 92 feet; deep-well turbine pump and 3-horsepower electric motor, pump set at 82 feet; static water level reported, 45 feet below land surface when drilled; yield reported, 70 gallons a minute when drilled.

Well 6. Drilled in November 1946 by W. O. Bower; depth, 90 feet; diameter, 14 inches; deep-well turbine pump and 3-horseporver electric motor; static water level reported, 43 feet below land surface when drilled; yield reported, 50 gallons a minute when drilled.

Well 7. Drilled in September 1946 by W. O. Bower; depth, 92 feet; diameter, 14 inches; casing perforated from 62 to 92 feet; deep-well turbine pump and 3-horsepower electric motor; pump set at 82 feet; static water level reported, 40 feet below land surface wren drilled; yield reported, 80 gallons a minute when drilled.

Average pumpage in gallons a day

\begin{tabular}{|c|c|c|c|c|c|}
\hline & 1945 & 1946 & & 1945 & 1946 \\
\hline $\begin{array}{l}\text { January } \\
\text { February } \\
\text { Mareh } \\
\text { April } \\
\text { May } \\
\text { June... }\end{array}$ & 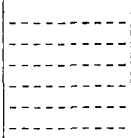 & $\begin{array}{l}52,370 \\
63,048 \\
58,410 \\
79,855 \\
75,907 \\
92,324\end{array}$ & $\begin{array}{l}\text { July } \\
\text { August } \\
\text { September } \\
\text { Oetober } \\
\text { November } \\
\text { December. }\end{array}$ & 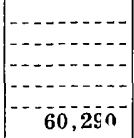 & $\begin{array}{r}91,994 \\
111,565 \\
89,542 \\
62,926 \\
58,306 \\
\end{array}$ \\
\hline
\end{tabular}

Storage: Elevated tank, 50,000 gallons; surface storage reservoir, 210,000 gallons.

Number of customers : 500 .

Treatment: Zeolite softening and chlorination.

Analysis

[Composite sample of five wells. Collected Dec. 13, 1946. pH is 7.6. Analyzed by C. B. Cibulka]

\begin{tabular}{|c|c|c|c|c|c|}
\hline & $\begin{array}{l}\text { Parts per } \\
\text { million }\end{array}$ & $\begin{array}{l}\text { Equivalent } \\
\text { per million }\end{array}$ & & $\begin{array}{l}\text { Parts per } \\
\text { million }\end{array}$ & $\begin{array}{l}\text { Equivalents } \\
\text { per million }\end{array}$ \\
\hline $\begin{array}{l}\text { Silica }\left(\mathrm{SiO}_{2}\right) \\
\text { Iron }(\mathrm{Fe}) \\
\text { Calcium }(\mathrm{Ca}) \\
\text { Magnesium }(\mathrm{Mg}) \\
\text { Sodium }(\mathrm{Na}) \\
\text { Potassium }(\mathrm{K})\end{array}$ & $\begin{array}{l}48 \\
32.50 \\
5.2 \\
8.4 \\
2.1\end{array}$ & $\begin{array}{r}.597 \\
.428 \\
.364 \\
.054\end{array}$ & $\begin{array}{l}\text { Bicarbonate }\left(\mathrm{HCO}_{3}\right) \\
\text { Sulfate }\left(\mathrm{SO}_{4}\right) \\
\text { Chloride (Cl) } \\
\text { Fluoride (F) } \\
\text { Dissol red solids. } \\
\text { Total hardness as } \mathrm{CaCO}_{3}\end{array}$ & $\begin{array}{c}95 \\
15 \\
8 \\
1.4 \\
199 \\
101\end{array}$ & \begin{tabular}{r}
1.557 \\
.312 \\
.226 \\
.074 \\
\hdashline-10 \\
\end{tabular} \\
\hline
\end{tabular}


Driller's logs

\begin{tabular}{|c|c|c|c|c|c|}
\hline & $\begin{array}{c}\text { Thickness } \\
\text { (feet) }\end{array}$ & $\begin{array}{c}\text { Depth } \\
\text { (feet) }\end{array}$ & & $\begin{array}{l}\text { Thickness } \\
\text { (feet) }\end{array}$ & $\begin{array}{l}\text { Depth } \\
\text { (feet) }\end{array}$ \\
\hline \multicolumn{6}{|c|}{ Well 2} \\
\hline $\begin{array}{l}\text { Surface sand } \\
\text { White caliche } \\
\text { Pink flint rock } \\
\text { Brown sand } \\
\text { Sand, little water. }\end{array}$ & $\begin{array}{r}13 \\
20 \\
4 \\
10 \\
6\end{array}$ & $\begin{array}{l}13 \\
33 \\
37 \\
47 \\
\mathbf{6 3}\end{array}$ & $\begin{array}{l}\text { Red sand } \\
\text { Coarse brown sand and } \\
\text { gravel, water. } \\
\text { Fine brown sand, water. }\end{array}$ & $\begin{array}{r}6 \\
11 \\
9 \\
1\end{array}$ & $\begin{array}{l}59 \\
70 \\
79 \\
80\end{array}$ \\
\hline \multicolumn{6}{|c|}{ Well 3} \\
\hline $\begin{array}{l}\text { Surface sand } \\
\text { Caliche, nhite } \\
\text { Brown sand, } \\
\text { Brown sand, water }\end{array}$ & $\begin{array}{r}16 \\
24 \\
6 \\
5\end{array}$ & $\begin{array}{l}16 \\
40 \\
46 \\
51\end{array}$ & $\begin{array}{l}\text { Brown sand, more water } \\
\text { Sand and gravel, coarse } \\
\text { Red rock }\end{array}$ & $\begin{array}{r}3 \\
24 \\
1\end{array}$ & $\begin{array}{l}54 \\
78 \\
79\end{array}$ \\
\hline \multicolumn{6}{|c|}{ Well 4} \\
\hline $\begin{array}{l}\text { Surface sand } \\
\text { Caliche, w hite } \\
\text { Sandy red rock } \\
\text { Red rock }\end{array}$ & $\begin{array}{r}14 \\
21 \\
20 \\
5\end{array}$ & $\begin{array}{l}14 \\
35 \\
55 \\
60\end{array}$ & $\begin{array}{l}\text { Sand, rock shell, brown } \\
\text { Brown sand, water } \\
\text { Coarse sand and gravel } \\
\text { Red rock. }\end{array}$ & $\begin{array}{r}1 \\
4 \\
20 \\
2\end{array}$ & $\begin{array}{l}61 \\
65 \\
85 \\
87\end{array}$ \\
\hline \multicolumn{6}{|c|}{ Well 6} \\
\hline $\begin{array}{l}\text { Surface sand } \\
\text { Caliche, white } \\
\text { Flint rock } \\
\text { Brown sand. } \\
\text { Red rock }\end{array}$ & $\begin{array}{r}12 \\
14 \\
2 \\
20 \\
3\end{array}$ & $\begin{array}{l}12 \\
26 \\
28 \\
48 \\
51\end{array}$ & $\begin{array}{l}\text { Fine brown sand } \\
\text { Coarse sand and gravel } \\
\text { Coarse sand } \\
\text { Coarse sand and gravel } \\
\text { Red rock }\end{array}$ & $\begin{array}{r}9 \\
5 \\
16 \\
6 \\
3\end{array}$ & $\begin{array}{l}60 \\
65 \\
81 \\
87 \\
90\end{array}$ \\
\hline
\end{tabular}

\section{CROCKETT COUNTY}

\section{OZONA}

Population in 1940: 2,150.

Source of information: W. D. Cooper, manager, July 22, 1947.

Owner: Crockett County Water Control and Improvement District No. 1.

Source of supply: Three wells.

Well 1. Drilled in 1941 by J. C. Crowder; depth, 450 feet; diameter, 10 inches; deep-well turbine pump and 50-horsepower el actric motor; static water level reported, 365 feet below land surface in July 1947; drawdown reported, 7 feet after pumping several hours at a rate of 450 gallons a minute.

Well 2. 125 feet southwest of well 1; drilled in 1941 by J. C. Crowder; depth, 450 feet; diameter, 10 inches; deep-well subme'sible turbine pump and 30-horsepower electric motor; yield reported, 225 gallons a minute.

Well 3. 125 feet west of well 1; well not completed July 22, 1947.

Pumpage (estimated) : Average 500,000 gallons a day.

Storage: Ground reservoir, 200,000 gallons.

Number of customers: 673.

Treatment: None. 


\section{Analyses}

[Composite sample of wells 1 and 2. Collected July 22, 1947. pH is 7.7. Analyzed by B. C. Dwyer]

\begin{tabular}{|c|c|c|c|c|c|}
\hline & $\begin{array}{l}\text { Parts per } \\
\text { million }\end{array}$ & $\begin{array}{l}\text { Equivalents } \\
\text { per million }\end{array}$ & & $\begin{array}{c}\text { Parts Fer } \\
\text { million }\end{array}$ & $\begin{array}{l}\text { Equivalents } \\
\text { per million }\end{array}$ \\
\hline $\begin{array}{l}\text { Silica }\left(\mathrm{SiO}_{2}\right) \\
\text { Iron }(\mathrm{Fe}) \\
\text { Calcium }(\mathrm{Ca}) \\
\text { Magnesium }(\mathrm{Mg}) \\
\text { Sodium }(\mathrm{Na}) \\
\text { Potassium }(\mathrm{K}) \\
\text { Bicarbonate }\left(\mathrm{H} \overline{\mathrm{C}} \mathrm{O}_{3}\right)\end{array}$ & $\begin{array}{l}15 \\
72 \\
17 \\
11 \\
272.0\end{array}$ & $\begin{array}{r}3.59 \\
1.40 \\
.46 \\
.13 \\
4.46\end{array}$ & $\begin{array}{l}\text { Sulfate (SO4) } \\
\text { Chloride (Cl) } \\
\text { Fluoride (F) } \\
\text { Nitrate (NO }{ }_{3} \text { ) } \\
\text { Dissolved golids } \\
\text { Total hardness as } \mathrm{CaCO}_{3}\end{array}$ & $\begin{array}{l}16 \\
22 \\
7.8 \\
301.8 \\
250\end{array}$ & $\begin{array}{r}.33 \\
.62 \\
.04 \\
.13 \\
\hdashline-1.0 .-2\end{array}$ \\
\hline
\end{tabular}

\section{CROSBY COUNTY}

\section{CROSBYTON}

Population in 1940: 1,615 .

Source of information: C. R. Saffel, water superintendent, Mar. 6, 1945.

Ownership: Municipal.

Source of supply: Three wells.

Well 1. Drilled in 1938 by L. A. Peeples; depth, 301 feet; diameter, 10 inches; deep-well turbine pump and 40-horsepower electric motor; yield, 190 gallons a minute.

Well 2. Drilled in 1938 by L. A. Peeples; depth, 312 feet; diameter, 10 inches; deep-well turbine pump and 15-horsepower electric motor; yield, 225 gallons a minute.

Well 3. Drilled in 1939 by Ed. Ballard; depth, 314 feet; ciameter, 10 inches; deep-well turbine pump and 25-horsepower electric motor; yield, 350 gallons a minute.

Pumpage (estimated) : 150,000 gallons a day.

Storage: Ground reservoir, 110,000 gallons; elevated tank, 55,0?0 gallons. Number of customers (estimated) : 400 .

Treatment: None.

\section{Analysis, well 3}

[Collected April 1939. pH is 7.5. Analyzed by State Health Dept.]

\begin{tabular}{|c|c|c|c|c|c|}
\hline & $\begin{array}{c}\text { Parts per } \\
\text { million }\end{array}$ & $\begin{array}{l}\text { Equivalents } \\
\text { per million }\end{array}$ & & $\begin{array}{l}\text { Parts fer } \\
\text { million }\end{array}$ & $\begin{array}{l}\text { Equivalents } \\
\text { per million }\end{array}$ \\
\hline $\begin{array}{l}\text { Silica }\left(\mathrm{SiO}_{2}\right) \\
\text { Calcium }(\mathrm{Ca}) \\
\text { Magnesium }(\mathrm{Mg}) \\
\text { Sodium }(\mathrm{Na}) \\
\left.\text { Bicarbonate }(\overline{\mathrm{HCO}})_{3}\right) \\
\text { Sulfate }\left(\mathrm{SO}_{4}\right)\end{array}$ & $\begin{array}{r}53 \\
40 \\
31 \\
69 \\
\mathbf{3 6 9} \\
\mathbf{4 1}\end{array}$ & $\begin{array}{r}2.00 \\
2.55 \\
3.00 \\
6.05 \\
.85\end{array}$ & $\begin{array}{l}\text { Chloride (Cl) } \\
\text { Fluoride (F) } \\
\left.\text { Nitrate (N }{ }_{3}\right)^{2} \\
\text { Dissol ed solids } \\
\text { Total hardness as } \mathrm{CaCO}{ }_{3}\end{array}$ & $\begin{array}{r}21 \\
2.8 \\
1.3 \\
477 \\
227\end{array}$ & \begin{tabular}{r}
0.59 \\
.15 \\
.02 \\
\hdashline-9 \\
-9
\end{tabular} \\
\hline
\end{tabular}

\section{Driller's log, well 2}

\begin{tabular}{|c|c|c|c|c|c|}
\hline & $\begin{array}{c}\text { Thickness } \\
\text { (feet) }\end{array}$ & $\begin{array}{l}\text { Depth } \\
\text { (feet) }\end{array}$ & & $\begin{array}{c}\text { Thickness } \\
\text { (feet) }\end{array}$ & $\begin{array}{l}\text { Depth } \\
\text { (feet) }\end{array}$ \\
\hline $\begin{array}{l}\text { Surface soil } \\
\text { Hard white caliche } \\
\text { Soft red caliche } \\
\text { Sticky red clay } \\
\text { Sandy clay } \\
\text { Hard sand rock }\end{array}$ & $\begin{array}{r}3 \\
42 \\
22 \\
36 \\
67 \\
7\end{array}$ & $\begin{array}{r}3 \\
45 \\
67 \\
103 \\
170 \\
177\end{array}$ & $\begin{array}{l}\text { Red clay } \\
\text { Sand and white pebbles, } \\
\text { water at } 208 \text { feet. } \\
\text { Fine-grained yellow sand } \\
\text { Coarse-grained yellow sand.- } \\
\text { Blue shale. }\end{array}$ & $\begin{array}{l}\mathbf{3 1} \\
\mathbf{3 7} \\
\mathbf{1 5} \\
\mathbf{3 5} \\
\mathbf{1 7}\end{array}$ & $\begin{array}{l}208 \\
245 \\
260 \\
295 \\
312\end{array}$ \\
\hline
\end{tabular}


Population in 1940: 616.

\section{LORENZO}

Source of information: Wm. W. Mitchell, city secretary, Mar. 21, 1947.

Ownership: Municipal.

Source of supply: Well under elevated tank; drilled in 1927 by W. G. Hamlin; depth, 223 feet; diameter, 12 inches, cased to 79 feet; deep-well turbine and 10-horsepower electric motor; static water level reported, 80 feet below land surface in 1939 ; yield, 100 gallons a minute.

Pumpage: Minimum, 36,000 gallons a day; maximum, 134,000 g $\$$ llons a day. Storage: Elevated tank, 50,000 gallons.

Number of customers: 160.

Treatment: None.

Analysis

[Collected Mar. 21, 1947. $\mathrm{pH}$ is 7.4. Analyzed by J. H. Rowley]

\begin{tabular}{|c|c|c|c|c|c|}
\hline & $\begin{array}{l}\text { Parts per } \\
\text { million }\end{array}$ & $\begin{array}{c}\text { Equivalents } \\
\text { per million }\end{array}$ & & $\begin{array}{l}\text { Parts per } \\
\text { million }\end{array}$ & $\begin{array}{l}\text { Equivalents } \\
\text { per million }\end{array}$ \\
\hline $\begin{array}{l}\text { Silica }\left(\mathrm{SiO}_{2}\right) \\
\text { Iron }(\mathrm{Fe}) \\
\text { Calcium }(\mathrm{Ca}) \\
\text { Magnesium }(\mathrm{Mg}) \\
\text { Sodium }(\mathrm{Na}) \\
\text { Potassium }(\mathrm{K}) \\
\text { Bicarbonate }\left(\mathrm{HCO}_{3}\right)\end{array}$ & $\begin{array}{l}42 \\
39 \\
39 \\
39 \\
10 \\
342\end{array}$ & $\begin{array}{r}1.95 \\
3.21 \\
1.70 \\
.26 \\
5.61\end{array}$ & $\begin{array}{l}\text { Sulfate }\left(\mathrm{SO}_{4}\right) \\
\text { Chloride }(\mathrm{Cl}) \\
\text { Fluoride (F) } \\
\left.\text { Nitrate (NO})_{3}\right) \\
\text { Dissolved solids } \\
\text { Total hardness as } \mathrm{CaCO}_{3}\end{array}$ & $\begin{array}{l}3 E \\
20 \\
3.2 \\
3.2 \\
386 \\
25 \varepsilon\end{array}$ & 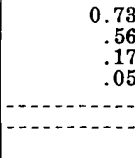 \\
\hline
\end{tabular}

\section{RALLS}

Population in 1940: 1,512.

Source of information: Water superintendent, Mar. 6, 1945.

Ownership: Municipal.

Source of supply: Two wells.

Well 1. At elevated tank; drilled in 1924; depth, 225 feet; diameter, 12 inches; deep-well turbine pump and 10-horsepower electric motor; yield, 75 gallons a minute.

Well 2. Five blocks west of well 1; drilled in 1927; depth, 285 feet; diameter, 8 inches; deep-well turbine pump and 15-horsep?wer electric motor; yield, 145 gallons a minute.

Pumpage: Average, 125,000 gallons a day.

Storage: Two ground storage reservoirs, 50,000 gallons each; elevated tank. 50,000 gallons.

Number of customers : 213 in 1936.

Treatment: None.

Analysis, well 2

[Collected Aug., 1944. pH is 7.7. Analyzed by State Health Dept.]

\begin{tabular}{|c|c|c|c|c|c|}
\hline & $\begin{array}{c}\text { Parts per } \\
\text { million }\end{array}$ & $\begin{array}{l}\text { Equivalents } \\
\text { per million }\end{array}$ & & $\begin{array}{l}\text { Parts per } \\
\text { million }\end{array}$ & $\begin{array}{l}\text { Equivalents } \\
\text { per million }\end{array}$ \\
\hline $\begin{array}{l}\text { Iron }(\mathrm{Fe}) \\
\text { Calcium }(\mathrm{Ca}) \\
\text { Magnesium }(\mathrm{Mg}) \\
\text { Sodium }(\mathrm{Na}) \\
\text { Bicarbonate }\left(\mathrm{H} \mathrm{CO}_{3}\right) \\
\text { Sulfate }\left(\mathrm{SO}_{4}\right)\end{array}$ & $\begin{array}{l}0.15 \\
42 \\
36 \\
61 \\
372 \\
48\end{array}$ & $\begin{array}{r}2.10 \\
2.96 \\
2.65 \\
6.10 \\
1.00\end{array}$ & $\begin{array}{l}\text { Chloride (Cl) } \\
\text { Fluoride (F) } \\
\left.\text { Nitrate (NO }{ }_{3}\right) \\
\text { Dissolved solids } \\
\text { Total hardness as } \mathrm{CaCO}_{3}\end{array}$ & $\begin{array}{c}21 \\
3.5 \\
4.4 \\
394 \\
253\end{array}$ & \begin{tabular}{r}
0.59 \\
.18 \\
.07 \\
\hdashline-18 \\
-0.0
\end{tabular} \\
\hline
\end{tabular}




\section{CULBERSON COUNTY}

\section{VAN HORN}

Population in 1940: 1,250.

Source of information: G. N. Langdon, mayor, Aug. 13, 1948.

Ownership: Municipal.

Source of supply: Two wells.

Well 1. Drilled in 1948 by Layne-Texas Co.; depth, 600 feet; diameter, 12 inches; deep-well turbine pump and 75-horsepower elec ${ }^{+}$ric motor; static water level, 490 feet below land surface Aug. 1948.

Well 2. Drilled in 1931; depth, 602 feet; diameter, 12 inches; deep-well turbine pump and 40-horsepower electric motor; static water level, 490 feet below land surface May 1944; yield, 170 gallons a minute with drawdown of 40 feet; temperature, $80^{\circ} \mathrm{F}$.

Pumpage: Average, 100,000 gallons a day.

Storage: Ground reservoir, 117,000 gallons; elevated tank, 50,000 gallons; elevated tank, 102,000 gallons.

Number of customers: 320 .

Treatment: None.

Analysis, well 2

[Collected July 24, 1943. $\mathrm{pH}$ is 8.2. Analyzed by J. H. Rowley]

\begin{tabular}{|c|c|c|c|c|c|}
\hline & $\begin{array}{l}\text { Parts per } \\
\text { million }\end{array}$ & $\begin{array}{l}\text { Equivalents } \\
\text { per million }\end{array}$ & & $\begin{array}{c}\text { Parts per } \\
\text { million }\end{array}$ & $\begin{array}{l}\text { Equivalents } \\
\text { per million }\end{array}$ \\
\hline $\begin{array}{l}\text { Silica }\left(\mathrm{SiO}_{2}\right) \\
\text { Iron }(\mathrm{Fe}) \\
\text { Calcium }(\mathrm{Ca}) \\
\text { Magnesium }(\overline{\mathrm{Mg}}) \\
\text { Sodium }(\mathrm{Na}) \\
\text { Potassium }(\mathrm{K}) \\
\text { Bicarbonate }\left(\mathrm{H}_{\mathrm{CO}}\right)_{3}\end{array}$ & $\begin{array}{l}26 \\
19.02 \\
8.0 \\
112.0 \\
956.2\end{array}$ & $\begin{array}{r}.95 \\
.66 \\
4.87 \\
.24 \\
4.20\end{array}$ & $\begin{array}{l}\text { Sulfate }\left(\mathrm{SO}_{4}\right) \\
\text { Chloride }(\mathrm{Cl}) \\
\text { Fluoride }(\mathrm{F}) \\
\text { Nitrate (NO} \\
\text { Dissol ed solids } \\
\text { Total hardness as } \mathrm{CaCO}\end{array}$ & $\begin{array}{r}78 \\
22 \\
2.8 \\
8.0 \\
413 \\
80\end{array}$ & $\begin{array}{r}1.62 \\
.62 \\
.15 \\
.13 \\
-.0 .- \\
-0.0\end{array}$ \\
\hline
\end{tabular}

Driller's log, well 2

\begin{tabular}{|c|c|c|c|c|c|}
\hline & $\begin{array}{c}\text { Thickness } \\
\text { (feet) }\end{array}$ & $\begin{array}{l}\text { Depth } \\
\text { (feet) }\end{array}$ & & $\underset{\text { (feet) }}{\text { Thickness }}$ & $\begin{array}{c}\text { Depth } \\
\text { (feet) }\end{array}$ \\
\hline $\begin{array}{l}\text { Top soil and sand } \\
\text { Sand and clay } \\
\text { Clay and some sand }\end{array}$ & $\begin{array}{l}20 \\
30 \\
30\end{array}$ & $\begin{array}{l}20 \\
50 \\
80\end{array}$ & $\begin{array}{l}\text { Hard adobe clay } \\
\text { Clay and gravel } \\
\text { River-bed gravel (water) }\end{array}$ & $\begin{array}{r}414 \\
12 \\
96\end{array}$ & $\begin{array}{l}494 \\
506 \\
602\end{array}$ \\
\hline
\end{tabular}

\section{DALLAM COUNTY}

\section{DALHART}

Population in 1940: 4,682.

Source of information: V. R. Wilson, water superintendent, Mar. 16, 1948.

Owner: Southwestern Public Service Co.

Source of supply: Five wells at Southwestern Public Service Co. p?wer plant.

Well 11. Drilled in 1929 by Layne-Texas Co.; depth, 547 feet; diameter, 10 inches, cased to 520 feet; deep-well turbine pump and 60-horsepower eleciric motor; static water level, 244 feet below land surfac? No. 1929; yield, 366 gallons a minute with drawdown of 37 feet. 
Well 12. Drilled in 1938 by L. E. McDade; depth, 526 feet; diameter, 121/2 inches, cased to 489 feet, 36 feet perforated; deep-well turbine pump and 50-horsepower electric motor; static water level, 264 feet below land surface Dec. 1938; yield, 340 gallons a minvte with drawdown of 78 feet after pumping 24 hours.

Well 13. Drilled in 1942 by H. H. Heiskell; depth, 542 feet; diameter, $12 \frac{1}{2}$ inches, 57 feet of perforations; deep-well turbine pump and 50horsepower electric motor; static water level, 257.7 feet below land surface Aug. 1942; yield, 400 gallons a minute with drawdown of 74 feet.

Well 14. Drilled in 1943 by H. H. Heiskell; depth, 555 feet; diameter, 26 inches; deep-well turbine pump and electric motor; static water level, 264 feet below land surface; yield, 195 gallons a minute with drawdown of 58 feet.

Well 15. Drilled in 1943 by H. H. Heiskell; depth, 550 feet; diameter, 121/2 inches; deep-well turbine pump and electric motor; static water level, 267 feet below land surface; yield, 43 gallons a minute vith drawdown of 104 feet after pumping 137 hours.

Pumpage: Maximum, 1,650,000 gallons a day; minimum, 550,000 gallons a day.

Storage: Elevated tank, 200,000 gallons; two ground reservoirs, 200,000 gallons each.

Number of customers: 1,598 .

Treatment: Chlorination.

Analysis, well 18

[Collected Mar. 16, 1948. pH is 7.2. Analyzed by D. E. Weaver]

\begin{tabular}{|c|c|c|c|c|c|}
\hline & $\begin{array}{l}\text { Parts per } \\
\text { million }\end{array}$ & $\begin{array}{l}\text { Equivalents } \\
\text { per million }\end{array}$ & & $\begin{array}{c}\text { Parts per } \\
\text { million }\end{array}$ & $\begin{array}{l}\text { Equivalents } \\
\text { per million }\end{array}$ \\
\hline $\begin{array}{l}\text { Silica }\left(\mathrm{SiO}_{2}\right) \\
\text { Iron }(\mathrm{Fe}) \\
\text { Calcium }(\mathrm{Ca}) \\
\text { Magnesium }(\mathrm{Mg}) \\
\text { Sodium }(\mathrm{Na}) \\
\text { Potassium }(\mathrm{K}){ }^{-} \mathrm{H} \\
\text { Bicarbonate }\left(\mathrm{H} \mathrm{CO}_{3}\right)\end{array}$ & $\begin{array}{l}30 \\
38 \\
31 \\
20 \\
1.6 \\
238\end{array}$ & $\begin{array}{r}1.90 \\
\mathbf{2 . 5 5} \\
.87 \\
.04 \\
\mathbf{3 . 9 0}\end{array}$ & $\begin{array}{l}\text { Sulfate }\left(\mathrm{SO}_{4}\right) \\
\text { Chloride }(\mathrm{Cl}) \\
\text { Fluoride (F) } \\
\text { Nitrate (NO} \\
\text { Dissol ed solids } \\
\text { Total hardness as } \mathrm{CaCO}\end{array}$ & $\begin{array}{c}43 \\
13 \\
1.4 \\
6.0 \\
302 \\
222\end{array}$ & $\begin{array}{r}0.90 \\
.37 \\
.07 \\
.10 \\
-10 \\
\\
\end{array}$ \\
\hline
\end{tabular}

Drillers' logs

\begin{tabular}{|c|c|c|c|c|}
\hline & $\begin{array}{c}\text { Thickness } \\
\text { (feet) }\end{array}$ & $\begin{array}{c}\text { Depth } \\
\text { (feet) }\end{array}$ & $\begin{array}{c}\text { Thickness } \\
\text { (feet) }\end{array}$ & $\begin{array}{l}\text { Depth } \\
\text { (feet) }\end{array}$ \\
\hline
\end{tabular}

Well 11

\begin{tabular}{|c|c|c|c|c|c|}
\hline $\begin{array}{l}\text { Surface soil. } \\
\text { Sand } \\
\text { Sandy clay } \\
\text { Rock. } \\
\text { Packed sand, gravel. } \\
\text { Packed sand. } \\
\text { Sand. } \\
\text { Clay } \\
\text { Sand. }\end{array}$ & $\begin{array}{r}19 \\
31 \\
2 \\
6 \\
24 \\
61 \\
10 \\
20 \\
12\end{array}$ & $\begin{array}{r}19 \\
50 \\
52 \\
58 \\
82 \\
143 \\
153 \\
173 \\
185\end{array}$ & $\begin{array}{l}\text { Clay, } \\
\text { Sand } \\
\text { Sand } \\
\text { Sand, boulders, and sandrock } \\
\text { Hard sand, clay streak. } \\
\text { Sand } \\
\text { Water sand } \\
\text { No record. }\end{array}$ & $\begin{array}{r}10 \\
7 \\
\mathbf{5 5} \\
\mathbf{2 2} \\
\mathbf{3} \\
\mathbf{9} \\
\mathbf{7 0} \\
\mathbf{5 3} \\
\mathbf{1 3 3}\end{array}$ & $\begin{array}{l}195 \\
202 \\
257 \\
279 \\
282 \\
291 \\
361 \\
414 \\
547\end{array}$ \\
\hline
\end{tabular}




\begin{tabular}{|c|c|c|c|c|c|}
\hline & $\underset{\text { (feet) }}{\text { Thickness }}$ & $\begin{array}{c}\text { Depth } \\
\text { (feet) }\end{array}$ & & $\underset{\text { (feet) }}{\text { Thickness }}$ & $\begin{array}{l}\text { Depth } \\
\text { (feet) }\end{array}$ \\
\hline \multicolumn{6}{|c|}{ Well 12} \\
\hline $\begin{array}{l}\text { Surface soil } \\
\text { Sand and clay } \\
\text { Sand and gravel } \\
\text { Gravel, hard rock } \\
\text { Sand, gravel, reak water. } \\
\text { Clay, } \\
\text { Gravel, little rater }\end{array}$ & $\begin{array}{r}4 \\
181 \\
10 \\
10 \\
67 \\
18 \\
6\end{array}$ & $\begin{array}{l}4 \\
185 \\
195 \\
205 \\
272 \\
290 \\
296\end{array}$ & $\begin{array}{l}\text { Hard-packed sand. } \\
\text { Fine sand, some gravel, clay, } \\
\quad \text { ater. } \\
\text { Yellow clay... } \\
\text { Fine to coarse sand, thin } \\
\text { layers sandrock, water } \\
\text { cavities. }\end{array}$ & $\begin{array}{r}35 \\
26 \\
\\
50 \\
119\end{array}$ & $\begin{array}{l}331 \\
357 \\
\\
407 \\
526\end{array}$ \\
\hline \multicolumn{6}{|c|}{ Well 13} \\
\hline $\begin{array}{l}\text { Surface soil } \\
\text { Sandy clay } \\
\text { Caliche } \\
\text { Sandy clay } \\
\text { Sand, little rater } \\
\text { Clay } \\
\text { Dry sand } \\
\text { Sandy clay } \\
\text { Clay and gravel } \\
\text { Sandy clay } \\
\text { Dry sand and gravel } \\
\text { Sandy clay } \\
\text { Brown clay } \\
\text { Muddy u ater sand } \\
\text { Yellow clay -. } \\
\text { Water sand, gravel } \\
\text { Broken sand, clay }\end{array}$ & $\begin{array}{r}3 \\
47 \\
15 \\
30 \\
3 \\
7 \\
5 \\
12 \\
8 \\
20 \\
75 \\
10 \\
5 \\
32 \\
16 \\
12 \\
10\end{array}$ & $\begin{array}{r}3 \\
50 \\
65 \\
95 \\
98 \\
105 \\
110 \\
122 \\
130 \\
150 \\
225 \\
235 \\
240 \\
272 \\
288 \\
300 \\
310\end{array}$ & 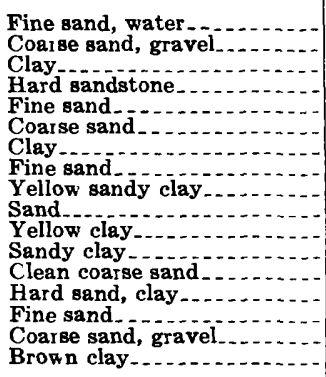 & $\begin{array}{r}10 \\
15 \\
18 \\
7 \\
10 \\
22 \\
5 \\
8 \\
10 \\
25 \\
5 \\
15 \\
11 \\
19 \\
30 \\
20 \\
2\end{array}$ & $\begin{array}{l}\mathbf{3 2 0} \\
\mathbf{3 3 5} \\
\mathbf{3 5 3} \\
\mathbf{3 6 0} \\
\mathbf{3 7 0} \\
\mathbf{3 9 2} \\
\mathbf{3 9 7} \\
405 \\
415 \\
440 \\
445 \\
\mathbf{4 6 0} \\
471 \\
490 \\
\mathbf{5 2 0} \\
\mathbf{5 4 0} \\
\mathbf{5 4 2}\end{array}$ \\
\hline
\end{tabular}

\begin{tabular}{|c|c|c|c|c|c|}
\hline \multicolumn{6}{|c|}{ Well 14} \\
\hline $\begin{array}{l}\text { Sand and clay } \\
\text { Hard caliche. } \\
\text { Sand, clay } \\
\text { Coarse sand, gravel } \\
\text { Loose gravel, sand. }\end{array}$ & $\begin{array}{r}68 \\
17 \\
175 \\
70 \\
45\end{array}$ & $\begin{array}{r}68 \\
85 \\
260 \\
330 \\
\mathbf{3 7 5}\end{array}$ & $\begin{array}{l}\text { Clay, gravel. } \\
\text { Yellow clay, sand } \\
\text { Coarse water sand } \\
\text { Hard shale, sand, gravel } \\
\text { Coarse sand, gravel. }\end{array}$ & $\begin{array}{l}60 \\
20 \\
42 \\
33 \\
25\end{array}$ & $\begin{array}{l}435 \\
455 \\
497 \\
530 \\
555\end{array}$ \\
\hline \multicolumn{6}{|c|}{ Well 15} \\
\hline $\begin{array}{l}\text { Top soil } \\
\text { Caliche } \\
\text { Red sand, } \\
\text { Caliche } \\
\text { White clay } \\
\text { Hard brown sand. } \\
\text { Dry sand, clay }\end{array}$ & $\begin{array}{r}3 \\
7 \\
10 \\
27 \\
33 \\
5 \\
60\end{array}$ & $\begin{array}{r}3 \\
10 \\
20 \\
47 \\
80 \\
85 \\
145\end{array}$ & $\begin{array}{l}\text { Dry sand. } \\
\text { Coar se sand, hard shells. } \\
\text { Coarse sand, gravel. } \\
\text { Clay, gravel. } \\
\text { Clean sand, gravel. } \\
\text { Gravel, coarse sand, shells... }\end{array}$ & $\begin{array}{r}103 \\
52 \\
102 \\
15 \\
51 \\
52\end{array}$ & $\begin{array}{l}248 \\
300 \\
402 \\
417 \\
468 \\
550\end{array}$ \\
\hline
\end{tabular}

\section{TEXIINE}

Population in 1940: 385.

Source of information: Mr. Greer, Mar. 17, 1948.

Owner: Southwestern Public Service Co.

Source of supply: Two wells at power plant.

Well 1 (East well). Drilled in 1922; depth, 260 feet; diameter, 8 inches; static water level, 76 feet below land surface; yield, 62 gallons a minute; not used for a year; new pump to be installed.

Well 2 (West well). Drilled in 1922; depth, 297 feet; diameter, 8 inches; deep-well turbine pump and electric motor; static water lerel, 76 feet below land surface; yield, 250 gallons a minute, drawdown 20 feet.

Pumpage: Average, 150,000 gallons a day.

Storage: Elevated tank, 150,000 gallons.

Number of customers: 145.

Treatment: None. 
Analysis, well 2

[Collected Mar. 17, 1948. pH is 7.2. Analyzed by D. E. Weaver]

\begin{tabular}{|c|c|c|c|c|c|}
\hline & $\begin{array}{c}\text { Parts per } \\
\text { million }\end{array}$ & $\begin{array}{l}\text { Equivalents } \\
\text { per million }\end{array}$ & & $\begin{array}{c}\text { Parts per } \\
\text { rillion }\end{array}$ & $\begin{array}{l}\text { Equivalents } \\
\text { per million }\end{array}$ \\
\hline $\begin{array}{l}\text { Silica }\left(\mathrm{SiO}_{2}\right) \\
\text { Iron (Fe) } \\
\text { Calcium }(\mathrm{Ca}) \\
\text { Magnesium }(\mathrm{Mg}) \\
\text { Sodium (Na) } \\
\text { Potassium }(\mathrm{K}) \\
\text { Bicarbonate }\left(\mathrm{H}_{\mathrm{CO}} \mathrm{CO}_{3}\right)\end{array}$ & $\begin{array}{c}12 \\
2.2 \\
32 \\
27 \\
30 \\
3.6 \\
244\end{array}$ & $\begin{array}{r}1.60 \\
2.22 \\
1.80 \\
.09 \\
4.00\end{array}$ & $\begin{array}{l}\text { Sulfate ( } \mathrm{SO} \text { ) } \\
\text { Chloride (Cl) } \\
\text { Fluoride (F) } \\
\text { Nitrate (NO } \mathrm{N}_{3} \text { ) } \\
\text { Dissolved solids... } \\
\text { Total hardness as } \mathrm{CaCO}_{3}\end{array}$ & $\begin{array}{l}42 \\
8 \\
1.0 \\
298^{.0} \\
191\end{array}$ & $\begin{array}{r}0.87 \\
.23 \\
.05 \\
.00 \\
-0 \\
-0 .-\end{array}$ \\
\hline
\end{tabular}

Driller's log of railroad well in Texline

\begin{tabular}{|c|c|c|c|c|c|}
\hline & $\begin{array}{c}\text { Thickness } \\
\text { (feet) }\end{array}$ & $\begin{array}{l}\text { Depth } \\
\text { (feet) }\end{array}$ & & $\underset{\text { (feet) }}{\text { Thickness }}$ & $\begin{array}{l}\text { Depth } \\
\text { (feet) }\end{array}$ \\
\hline $\begin{array}{l}\text { Clay and sand } \\
\text { Packed sand } \\
\text { Quic'rsand } \\
\text { Hard rock } \\
\text { Blue clay }\end{array}$ & $\begin{array}{r}25 \\
45 \\
20 \\
10 \\
130\end{array}$ & $\begin{array}{r}25 \\
70 \\
90 \\
100 \\
230\end{array}$ & $\begin{array}{l}\text { Water sand } \\
\text { Hard rock } \\
\text { Soft blue clay } \\
\text { Coarse sand } \\
\text { Hard rock }\end{array}$ & $\begin{array}{r}20 \\
5 \\
5 \\
25 \\
10\end{array}$ & $\begin{array}{l}250 \\
255 \\
260 \\
285 \\
295\end{array}$ \\
\hline
\end{tabular}

\section{DAWSON COUNTY}

\section{LAMESA}

Population in 1940: 6,038.

Source of information: G. M. Roberts, city manager, Sept. 12, 1947.

Ownership: Municipal.

Source of supply: Eighteen wells.

Well 1. Drilled in 1929; depth, 300 feet; diameter, 10 to 8 inches; deepwell turbine pump and 10-horsepower electric motor; yiald, 175 gallons a minute.

Well 2. Drilled in 1929; depth, 160 feet; diameter 10 to 8 inches; deepwell turbine pump and 10-horsepower electric motor; yield, 100 gallons a minute; temperature, $661 / 2^{\circ} \mathrm{F}$.

Well 3. Drilled in 1929; depth, 160 feet; diameter 10 to 8 inches; deepwell turbine pump and 10-horsepower electric motor; yield, 125 gallons a minute.

Well 4. Drilled in 1935; depth, 150 feet; diameter, 10 to 8 inches; deepwell turbine pump and 5-horsepower electric motor; sta.tic water level, 72.2 feet below land surface on Dec. 21, 1945, and 82.33 feet below land surface on Sept. 12, 1947; yield, 60 gallons a minute; temperature, $67^{\circ} \mathrm{F}$.

Well 5. Drilled in 1924; depth, 300 feet; diameter 10 in nhes; deep-well turbine pump and 15-horsepower electric motor; yield, 125 gallons a minute.

Well 6. Drilled in 1924; depth, 300 feet; diameter, 6 inches; deep-well turbine pump and 15-horsepower electric motor; yield, 125 gallons a minute.

Well 7. Drilled in 1940; depth, 160 feet; diameter, 10 irches; deep-well turbine pump and 10-horsepower electric motor; static water level, 66 feet below land surface on Sept. 17, 1941, and 76 feet bel?w land surface on Feb. 5, 1946; yield 175 gallons a minute.

Well 8. Drilled in 1940; depth, 151 feet; diameter, 10 to 8 inches; deepwell turbine pump and 10-horsepower electric motor; yield, 125 gallons a minute.

Well 9. Drilled in 1940; depth, 160 feet; diameter 10 to 8 inches; deep- 
well turbine pump and 15-horsepower electric motor; yield, 35 gallons a minute.

Well 10. Drilled in 1945; depth, 163 feet; diameter, 12 inches; static water level, 95.2 feet below land surface on Sept. 13, 1947; deep-well turbine pump and 10-horsepower electric motor; yield, 20 gallons a minute.

Well 11. Drilled in 1945; depth, 160 feet; diameter, 12 inches; deep-well turbine pump and 5-horsepower electric motor; yield, 100 gallons a minute; temperature, $6612^{\circ} \mathrm{F}$.

Well 12. Drilled in 1945; depth, 160 feet; diameter, 12 incl $9 \mathrm{~s}$; static water level, 69 feet below land surface on March 13, 1946; deep-well turbine pump and 5-horsepower electric motor; yield, 100 gallons a minute.

Well 13. Drilled in 1946; depth, 168 feet; diameter 12 to 10 inches; deepwell turbine pump and $7 \frac{1}{2}$-horsepower electric motor; yield, 175 gallons a minute.

Well 14. Drilled in 1946; depth, 197 feet; diameter, 12 inches; deep-well turbine pump and 71/2-horsepower electric motor; yield, 130 gallons a minute.

Well 15. Drilled in 1946; depth, 220 feet; diameter, 12 inches; deep-well turbine pump and 10-horsepower electric motor; yield, 100 gallons a minute.

Well 16. Drilled in 1946; depth, 255 feet; diameter, 12 inches; deep-well turbine pump and 10-horsepower electric motor; yield, 75 gallons a minute.

Well 17. Drilled in 1947; depth, 182 feet; diameter, 14 inches; deep-well turbine pump and 71/2-horsepower electric motor; yield, 90 gallons a minute.

Well 18. Drilled in 1947; depth, 138 feet; diameter, 12 inches; pump not installed.

Pumpage (estimated): Minimum, 450,000 gallons a day; maximum, 1,500,000 gallons a day.

Storage: Ground reservoir, 250,000 gallons; two ground reservoirs, 200,000 gallons each; ground reservoir, 100,000 gallons; elevated tank, 200,000 gallons; elevated tank, 100,000 gallons.

Number of customers: Estimated, 2000.

Treatment: Chlorination.

\section{Analyses}

[Collected Sept. 13, 1947. pH: well 6, 8.0; well 13, 7.5. Analyzed by B. C. Dryer]

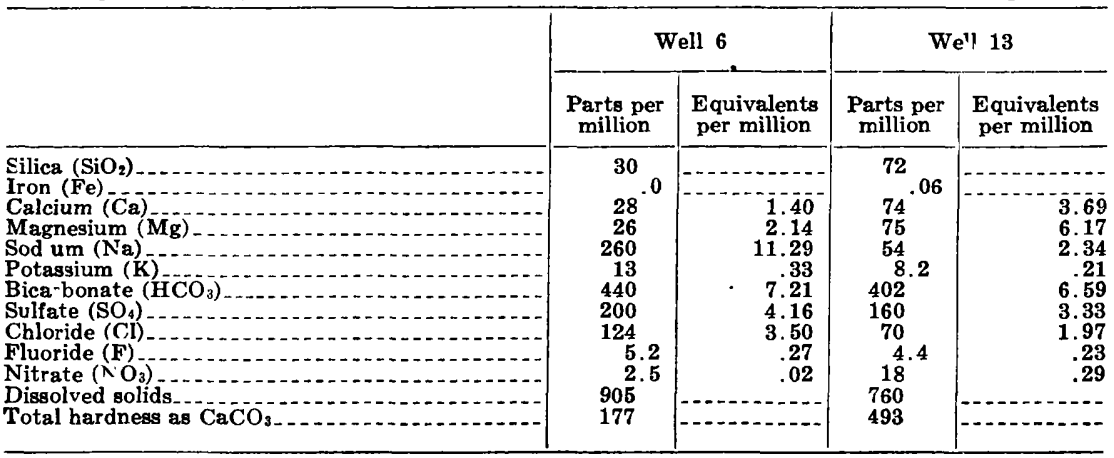




\begin{tabular}{|c|c|c|c|c|c|}
\hline \multirow{2}{*}{. } & \multicolumn{2}{|c|}{$\begin{array}{l}\text { Composite sample } \\
\text { from } 13 \text { wells }\end{array}$} & & \multicolumn{2}{|c|}{$\begin{array}{l}\text { Composite sample } \\
\text { from } 13 \text { wells }\end{array}$} \\
\hline & $\begin{array}{l}\text { Parts per } \\
\text { million }\end{array}$ & $\begin{array}{c}\text { Equivalents } \\
\text { per million }\end{array}$ & & $\begin{array}{l}\text { Parts per } \\
\text { million }\end{array}$ & $\begin{array}{l}\text { Equivalents } \\
\text { per million }\end{array}$ \\
\hline $\begin{array}{l}\text { Silica }\left(\mathrm{SiO}_{2}\right) \\
\text { Iron }(\mathrm{Fe}) \\
\text { Calcium }(\mathrm{Ca}) \\
\text { Magnesium }(\mathrm{M} \mathbf{g}) \\
\text { Sodium (Na) } \\
\text { Potassium }(\mathrm{K}) \\
\left.\text { Bicarbonate ( } \mathrm{HCO}_{3}\right)^{-}\end{array}$ & $\begin{array}{l}37 \\
38 \\
57 \\
164 \\
25 \\
388\end{array}$ & $\begin{array}{r}1.90 \\
4.69 \\
7.15 \\
.64 \\
6.36\end{array}$ & $\begin{array}{l}\text { Sulfate }\left(\mathrm{SO}_{4}\right) \\
\text { Chloride }(\mathrm{Cl}) \\
\text { Fluoride (F) } \\
\left.\text { Nitrate (NO})_{3}\right) \\
\text { Dissolved solids. } \\
\text { Total hardness as } \mathrm{CaCO}_{3}\end{array}$ & $\begin{array}{l}193 \\
130 \\
5.6 \\
2.5 \\
843 \\
330\end{array}$ & $\begin{array}{r}4.02 \\
3.67 \\
.29 \\
.04 \\
-0 .- \\
\end{array}$ \\
\hline
\end{tabular}

\section{Driller's logs}

\begin{tabular}{|c|c|c|c|c|c|}
\hline & $\begin{array}{c}\text { Thickness } \\
\text { (feet) }\end{array}$ & $\begin{array}{l}\text { Depth } \\
\text { (feet) }\end{array}$ & & $\underset{\text { (feet) }}{\text { Thickness }}$ & $\begin{array}{l}\text { Depth } \\
\text { (feet) }\end{array}$ \\
\hline \multicolumn{6}{|c|}{ Well 4} \\
\hline $\begin{array}{l}\text { Caliche } \\
\text { Róck } \\
\text { Sand } \\
\text { Water sand } \\
\text { Sandrock_. }\end{array}$ & $\begin{array}{r}30 \\
10 \\
8 \\
10 \\
\mathbf{2}\end{array}$ & $\begin{array}{l}30 \\
40 \\
48 \\
58 \\
60\end{array}$ & $\begin{array}{l}\text { Sand } \\
\text { Water sand } \\
\text { Sand } \\
\text { Water sand } \\
\text { Red beds. }\end{array}$ & $\begin{array}{r}20 \\
10 \\
52 \\
8 \\
-\end{array}$ & $\begin{array}{r}80 \\
90 \\
142 \\
150 \\
150\end{array}$ \\
\hline \multicolumn{6}{|c|}{ Well 5} \\
\hline $\begin{array}{l}\text { Red clay } \\
\text { Caliche } \\
\text { Cap rock. } \\
\text { Sand } \\
\text { Water sand. }\end{array}$ & $\begin{array}{r}4 \\
46 \\
10 \\
30 \\
10\end{array}$ & $\begin{array}{r}\mathbf{4} \\
\mathbf{5 0} \\
\mathbf{6 0} \\
90 \\
\mathbf{1 0 0}\end{array}$ & $\begin{array}{l}\text { Pand } \\
\text { Red beds and thin-bedded } \\
\text { blue shale streaks. }\end{array}$ & $\begin{array}{r}90 \\
10 \\
100\end{array}$ & $\begin{array}{l}190 \\
200 \\
300\end{array}$ \\
\hline \multicolumn{6}{|c|}{ Well 7} \\
\hline $\begin{array}{l}\text { Surface soil } \\
\text { Sand and caliche } \\
\text { Pack sand, } \\
\text { Hard sand } \\
\text { Sand and caliche rock } \\
\text { Sand and rock, light } \\
\text { Sand and rock, dark } \\
\text { Coarse sand and fine gravel } \\
\text { Fine-grained sand, dry } \\
\text { Fine-grained sand and fine } \\
\text { gravel, dry. } \\
\text { Packed sand. }\end{array}$ & $\begin{array}{r}3 \\
4 \\
8 \\
8 \\
8 \\
10 \\
7 \\
9 \\
4 \\
7 \\
\\
5\end{array}$ & $\begin{array}{r}3 \\
7 \\
15 \\
23 \\
31 \\
41 \\
48 \\
57 \\
61 \\
68 \\
73\end{array}$ & $\begin{array}{l}\text { Coarse-grained sand, water-- } \\
\text { Sandy clay } \\
\text { Fine-grained sand, water. } \\
\text { Brown clay } \\
\text { Fine packed sand, } \\
\text { Coarse-grained sand, water.- } \\
\text { Packed sand, tight. } \\
\text { Pink clay. } \\
\text { Coarse-grained sand, water.- } \\
\text { Coarse water sand and some } \\
\text { pea gravel. }\end{array}$ & $\begin{array}{r}8 \\
2 \\
16 \\
4 \\
12 \\
20 \\
5 \\
11 \\
6 \\
3\end{array}$ & $\begin{array}{r}81 \\
83 \\
99 \\
103 \\
115 \\
135 \\
140 \\
151 \\
157 \\
160\end{array}$ \\
\hline
\end{tabular}

Well 8

\begin{tabular}{|c|c|c|c|c|c|}
\hline $\begin{array}{l}\text { Surface soil } \\
\text { Packed sand. } \\
\text { Sand. } \\
\text { Hard sand. } \\
\text { Hard limestone. } \\
\text { Hard sand } \\
\text { Gravel, medium } \\
\text { Sand. }\end{array}$ & $\begin{array}{r}3 \\
5 \\
7 \\
-\quad 5 \\
4 \\
21 \\
8 \\
10\end{array}$ & $\begin{array}{r}3 \\
8 \\
15 \\
20 \\
24 \\
45 \\
53 \\
63\end{array}$ & $\begin{array}{l}\text { Sand and fine gravel, water.- } \\
\text { Clean sand and fine gravel, } \\
\text { water. } \\
\text { Packed sand. } \\
\text { Pink clay. } \\
\text { Packed sand. } \\
\text { Coarse sand and gravel }\end{array}$ & $\begin{array}{r}27 \\
8 \\
12 \\
9 \\
14 \\
18\end{array}$ & $\begin{array}{r}90 \\
98 \\
110 \\
119 \\
133 \\
151\end{array}$ \\
\hline
\end{tabular}

\begin{tabular}{|c|c|c|c|c|c|}
\hline \multicolumn{6}{|c|}{ Well 9} \\
\hline $\begin{array}{l}\text { Surface soil } \\
\text { Hard caliche } \\
\text { Packed sand and rock. } \\
\text { Sand and rock. } \\
\text { Coarse sand and gravel } \\
\text { Sand and fine gravel. } \\
\text { Packed sand } \\
\text { Coarse sand and gravel, water }\end{array}$ & $\begin{array}{r}\mathbf{3} \\
7 \\
6 \\
\mathbf{3 0} \\
\mathbf{9} \\
\mathbf{5} \\
\mathbf{5} \\
\mathbf{1 0}\end{array}$ & $\begin{array}{r}3 \\
10 \\
16 \\
46 \\
55 \\
60 \\
65 \\
75\end{array}$ & $\begin{array}{l}\text { Sandy clay } \\
\text { Sand and gravel. } \\
\text { Packed sand. } \\
\text { Pink clay. } \\
\text { Sand, water. } \\
\text { Sand and pea gravel, water.- } \\
\text { Coarse sand and pea gravel. } \\
\text { Red beds... }\end{array}$ & $\begin{array}{r}2 \\
18 \\
15 \\
10 \\
10 \\
10 \\
12 \\
8\end{array}$ & $\begin{array}{r}77 \\
95 \\
110 \\
120 \\
130 \\
140 \\
152 \\
160\end{array}$ \\
\hline
\end{tabular}




\begin{tabular}{l|l|l|l|}
\hline Thickness & $\begin{array}{c}\text { Depth } \\
\text { (feet) }\end{array}$
\end{tabular}$\left|\begin{array}{c}\text { Thickness } \\
\text { (feet) }\end{array}\right| \begin{gathered}\text { Depth } \\
\text { (feet) }\end{gathered}$

\begin{tabular}{|c|c|c|c|c|c|}
\hline \multicolumn{6}{|c|}{ Well 10} \\
\hline $\begin{array}{l}\text { Surface soil } \\
\text { Sand and shale } \\
\text { Gravel. } \\
\text { Sand, water } \\
\text { Pink shale. }\end{array}$ & $\begin{array}{r}3 \\
52 \\
8 \\
45 \\
12\end{array}$ & $\begin{array}{r}35 \\
55 \\
63 \\
108 \\
120\end{array}$ & $\begin{array}{l}\text { Sand, dry } \\
\text { Sand, medium fine grained, } \\
\text { water } \\
\text { Red clay }\end{array}$ & $\begin{array}{r}8 \\
20 \\
15\end{array}$ & $\begin{array}{l}128 \\
148 \\
163\end{array}$ \\
\hline \multicolumn{6}{|c|}{ Well 11} \\
\hline $\begin{array}{l}\text { Surface soil } \\
\text { Shale } \\
\text { Caliche. } \\
\text { Sand } \\
\text { Sard white rock } \\
\text { Sand and gravel } \\
\text { Sand }\end{array}$ & $\begin{array}{r}4 \\
11 \\
10 \\
5 \\
15 \\
15 \\
20\end{array}$ & $\begin{array}{r}4 \\
15 \\
25 \\
30 \\
45 \\
60 \\
80\end{array}$ & $\begin{array}{l}\text { Sand, dry } \\
\text { Sand, water } \\
\text { Pink shale. } \\
\text { Sand, dry } \\
\text { Sand, water. } \\
\text { Red clay }\end{array}$ & $\begin{array}{r}10 \\
17 \\
28 \\
10 \\
15 \\
5\end{array}$ & $\begin{array}{r}90 \\
107 \\
135 \\
145 \\
160 \\
165\end{array}$ \\
\hline
\end{tabular}

Well 13

\begin{tabular}{|c|c|c|c|c|c|}
\hline $\begin{array}{l}\text { Top soil, caliche and clay. } \\
\text { Coarse gravel } \\
\text { Sand and fine gravel. }\end{array}$ & $\begin{array}{r}58 \\
7 \\
10\end{array}$ & $\begin{array}{l}58 \\
65 \\
75\end{array}$ & $\begin{array}{l}\text { Clay } \\
\text { Red beds fine gravel. }\end{array}$ & $\begin{array}{l}55 \\
20 \\
18\end{array}$ & $\begin{array}{l}130 \\
150 \\
168\end{array}$ \\
\hline
\end{tabular}

Well 14

\begin{tabular}{|c|c|c|c|c|c|}
\hline $\begin{array}{l}\text { Surface soil } \\
\text { Shale. } \\
\text { Rock } \\
\text { Sandy shale. } \\
\text { Sand and gravel. }\end{array}$ & $\begin{array}{r}4 \\
6 \\
11 \\
29 \\
75\end{array}$ & $\begin{array}{r}4 \\
10 \\
21 \\
50 \\
125\end{array}$ & $\begin{array}{l}\text { Red shale. } \\
\text { Yellow, sandy shale } \\
\text { Sand and gravel } \\
\text { Red shale. } \\
\text { Rock. }\end{array}$ & $\begin{array}{r}23 \\
17 \\
27 \\
4 \\
1\end{array}$ & $\begin{array}{l}148 \\
165 \\
192 \\
196 \\
197\end{array}$ \\
\hline
\end{tabular}

Well 15

\begin{tabular}{|c|c|c|c|c|c|}
\hline $\begin{array}{l}\text { Surface soil } \\
\text { Caliche. } \\
\text { Sandy shale. } \\
\text { Sand } \\
\text { Sand and gravel, water... }\end{array}$ & $\begin{array}{r}4 \\
36 \\
55 \\
10 \\
\mathbf{3 0}\end{array}$ & $\begin{array}{r}4 \\
40 \\
95 \\
105 \\
135\end{array}$ & $\begin{array}{l}\text { Caliche } \\
\text { Red shale } \\
\text { Blue sandy shale. } \\
\text { Sand } \\
\text { Red shale. }\end{array}$ & $\begin{array}{r}25 \\
20 \\
5 \\
33 \\
2\end{array}$ & $\begin{array}{l}160 \\
180 \\
185 \\
218 \\
220\end{array}$ \\
\hline
\end{tabular}

\section{Well 16}

\begin{tabular}{|c|c|c|c|c|c|}
\hline $\begin{array}{l}\text { Surface soil } \\
\text { Rock. } \\
\text { Sandy shale.. } \\
\text { Rock } \\
\text { Sandy shale } \\
\text { Sand and gravel } \\
\text { Hard rock }\end{array}$ & $\begin{array}{r}2 \\
18 \\
35 \\
20 \\
26 \\
20 \\
8\end{array}$ & $\begin{array}{r}2 \\
20 \\
55 \\
75 \\
101 \\
121 \\
129\end{array}$ & $\begin{array}{l}\text { Sand and gravel } \\
\text { Caliche. } \\
\text { Sand } \\
\text { Caliche. } \\
\text { Red shale. } \\
\text { Sand. } \\
\text { Yellow clay. }\end{array}$ & $\begin{array}{r}6 \\
35 \\
15 \\
17 \\
20 \\
31 \\
2\end{array}$ & $\begin{array}{l}135 \\
170 \\
185 \\
202 \\
222 \\
253 \\
255\end{array}$ \\
\hline
\end{tabular}

\begin{tabular}{|c|c|c|c|c|c|}
\hline \multicolumn{6}{|c|}{$\checkmark$ ell 17} \\
\hline $\begin{array}{l}\text { Surface soil } \\
\text { Hard rock } \\
\text { Sandy shale. } \\
\text { Rock } \\
\text { Sandy shale. } \\
\text { Sand and gravel. }\end{array}$ & $\begin{array}{r}3 \\
19 \\
36 \\
19 \\
21 \\
20\end{array}$ & $\begin{array}{r}3 \\
22 \\
58 \\
77 \\
98 \\
118\end{array}$ & $\begin{array}{l}\text { Hard rock } \\
\text { Sand and gravel } \\
\text { Caliche. } \\
\text { Sand } \\
\text { Caliche. }\end{array}$ & $\begin{array}{r}9 \\
6 \\
34 \\
6 \\
9\end{array}$ & $\begin{array}{l}127 \\
133 \\
167 \\
173 \\
182\end{array}$ \\
\hline
\end{tabular}

\section{Well 18}

\begin{tabular}{|c|c|c|c|c|c|}
\hline $\begin{array}{l}\text { Unknown } \\
\text { Solid rock } \\
\text { Coarse gravel } \\
\text { Water-bearing sand. }\end{array}$ & $\begin{array}{r}70 \\
17 \\
8 \\
18\end{array}$ & $\begin{array}{r}70 \\
87 \\
95 \\
113\end{array}$ & $\begin{array}{l}\text { Solid rock } \\
\text { Open cavity } \\
\text { Water-bearing sand } \\
\text { Red beds... }\end{array}$ & $\begin{array}{r}8 \\
3 \\
11 \\
3\end{array}$ & $\begin{array}{l}121 \\
124 \\
135 \\
138\end{array}$ \\
\hline
\end{tabular}




\section{DEAF SMITH COUNTY}

Population in 1940: 2,584.

\section{HEREFORD}

Source of information: O. Carroll, water superintendent, March 1945.

Ownership: Municipal.

Source of supply: Two wells.

Well 2. At pumping station 7 blocks southeast of post offce; dug to $\mathbf{5 0}$ feet and drilled from 50 to 200 feet; diameter, 14 inches; deep-well turbine pump and 50-horsepower electric motor; statis water level reported, 60 feet below land surface in 1938; yield, 1,000 gallons a - minute.

Well 3. 150 feet northeast of well 2; drilled in 1939 by Bradford Supply Co.; depth, 160 feet; diameter, 20 inches; deep-well turbine pump and 50 -horsepower electric motor; static water level reported, 65 feet below land surface in 1939 and 75 feet below land surface in 1941; yield, 1,325 gallons a minute.

Pumpage (estimated) : 600,000 gallons a day.

Storage: Ground reservoir, 120,000 gallons; elevated tank, 175,000 gallons. Number of customers: 750 .

Treatment: None.

\section{Analyses}

[Collected Mar., 1945. pH: well 2, 7.6; well 3, 7.8. Analyzed by B. C. Dwyer and J. H. Rowley]

\begin{tabular}{|c|c|c|c|c|}
\hline & \multicolumn{2}{|c|}{ Well 2} & \multicolumn{2}{|c|}{ Well 3} \\
\hline & $\begin{array}{l}\text { Parts per } \\
\text { million }\end{array}$ & $\begin{array}{l}\text { Equivalents } \\
\text { per million }\end{array}$ & $\begin{array}{l}\text { Part: per } \\
\text { million }\end{array}$ & $\begin{array}{l}\text { Equivalents } \\
\text { per million }\end{array}$ \\
\hline 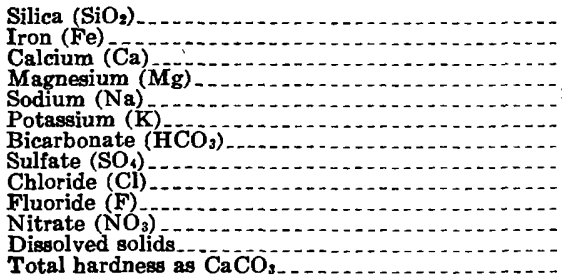 & $\begin{array}{l}59 \\
46 \\
46 \\
66 \\
32 \\
9.5 \\
340 \\
139 \\
22 \\
3.5 \\
7.0 \\
557 \\
386\end{array}$ & $\begin{array}{r}\mathbf{2} .30 \\
5.43 \\
1.40 \\
.24 \\
5.57 \\
2.89 \\
.62 \\
.18 \\
.11 \\
-2 .-\end{array}$ & $\begin{array}{l}6: .08 \\
54 \\
5 ? \\
60 \\
15 \\
311 \\
207 \\
30 \\
3.6 \\
5.5 \\
648 \\
37 ?\end{array}$ & $\begin{array}{r}2.70 \\
4.85 \\
2.61 \\
.38 \\
4.90 \\
4.31 \\
.85 \\
.19 \\
.09 \\
.0\end{array}$ \\
\hline
\end{tabular}

\section{DICKENS COUNTY}

\section{DICKENS}

Population in 1940: 465.

Source of information: Cecil Meadors, mayor, Feb. 5, 1946.

Ownership: Municipal.

Source of supply: Four wells.

Well 1. At 7th and Davis Streets; drilled in 1936 by L. A. Feples; depth, 90 feet; diameter, 6 inches; deep-well turbine pump and 3-horsepower electric motor; static water level, 77.0 feet below land surface in 1936; yield, 10 gallons a minute.

Well 2. At O'Neal Street and State highway; drilled in 1936 by L. A. Peeples; depth, 156 feet; diameter, 6 inches; deep-well turbine pump and 3-horsepower electric motor; yield, 18 gallons a minute. 
Well 3. Forty feet east of well 2; drilled in 1945; depth, 150 feet; diameter, 6 inches; deep-well turbine pump and 3-horsepower electric motor; yield, 30 gallons a minute.

Well 4. One block north and two blocks west of court house; drilled in 1935 by L. A. Peeples; depth, 110 feet; diameter, 12 to 8 inches; deepwell turbine pump and electric motor; static water level, 89.82 feet below land surface on Sept. 16, 1947 ; yieid, 50 gallons a mirute; temperature, $661 / 2^{\circ} \mathrm{F}$.

Pumpage: No record.

Storage: Standpipe, 52,000 gallons.

Number of customers: 115 .

Treatment: None.

\section{Analyses}

[Collected Feb. 21, 1946. pH: well 1, 7.6; well 2, 7.4; well 3, 7.5; well 4, 7.4. Analyzed by C. B. Cibulka]

\begin{tabular}{|c|c|c|c|c|}
\hline \multirow[t]{2}{*}{. } & \multicolumn{2}{|c|}{ Well 1} & \multicolumn{2}{|c|}{ Well 2} \\
\hline & $\begin{array}{l}\text { Parts per } \\
\text { million }\end{array}$ & $\begin{array}{l}\text { Equivalents } \\
\text { per million }\end{array}$ & $\begin{array}{c}\text { Parts per } \\
\text { million }\end{array}$ & $\begin{array}{l}\text { Equivalent } \\
\text { per millions }\end{array}$ \\
\hline \multirow[t]{3}{*}{ 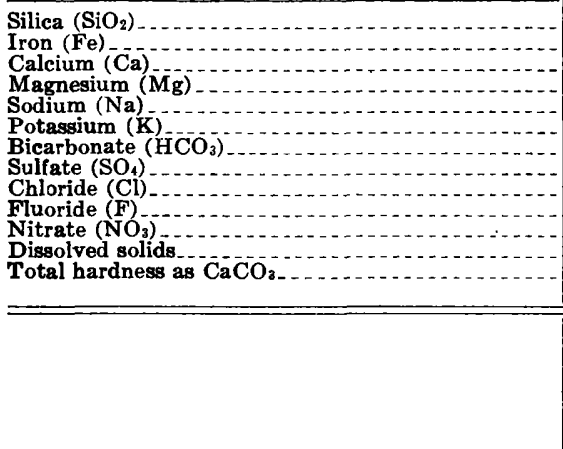 } & $\begin{array}{l}16 \\
2.2 \\
68 \\
17 \\
39 \\
3.1 \\
258 \\
47 \\
44 \\
1.0 \\
4.7 \\
373 \\
240\end{array}$ & $\begin{array}{r}3.39 \\
1.40 \\
1.71 \\
.08 \\
4.23 \\
.98 \\
1.24 \\
.05 \\
.08\end{array}$ & $\begin{array}{l}14 \\
74 \\
20 \\
38 \\
4.6 \\
303 \\
49 \\
30 \\
4.4 \\
399 \\
266\end{array}$ & $\begin{array}{r}3.69 \\
1.64 \\
1.65 \\
4.97 \\
1.02 \\
1.07 \\
.02 \\
.07 \\
\hdashline\end{array}$ \\
\hline & \multicolumn{2}{|c|}{ Well 3} & \multicolumn{2}{|c|}{ Well 4} \\
\hline & $\begin{array}{l}\text { Parts per } \\
\text { million }\end{array}$ & $\begin{array}{l}\text { Equivalents } \\
\text { per million }\end{array}$ & $\begin{array}{l}\text { Parts per } \\
\text { million }\end{array}$ & $\begin{array}{l}\text { Equivalents } \\
\text { per million }\end{array}$ \\
\hline 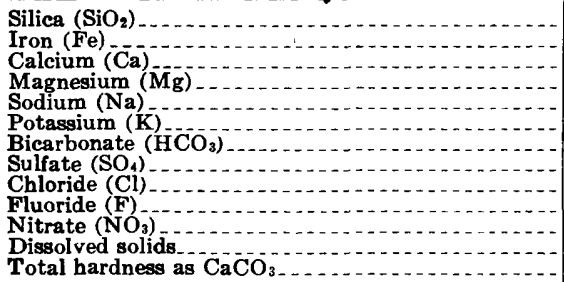 & $\begin{array}{l}18 \\
76 \\
76^{.39} \\
20 \\
41 \\
3.5 \\
282 \\
61 \\
47 \\
3.6 \\
414 \\
272\end{array}$ & $\begin{array}{r}3.79 \\
1.64 \\
1.79 \\
.09 \\
4.62 \\
1.27 \\
1.33 \\
.03 \\
.06 \\
\hdashline\end{array}$ & $\begin{array}{l}16 \\
8^{16} \\
25 \\
23 \\
3.06 \\
3.8 \\
246 \\
55 \\
60 \\
3^{30} \\
450 \\
318\end{array}$ & $\begin{array}{r}4.29 \\
2.06 \\
1.04 \\
4.10 \\
4.03 \\
1.15 \\
1.69 \\
.04 \\
.48\end{array}$ \\
\hline
\end{tabular}

Driller's logs

\begin{tabular}{|c|c|c|c|c|c|}
\hline & $\begin{array}{c}\text { Thickness } \\
\text { (feet) }\end{array}$ & $\begin{array}{l}\text { Depth } \\
\text { (feet) }\end{array}$ & & $\begin{array}{c}\text { Thickress } \\
\text { (feet) }\end{array}$ & $\begin{array}{c}\text { Depth } \\
\text { (feet) }\end{array}$ \\
\hline \multicolumn{6}{|c|}{ Well 1} \\
\hline $\begin{array}{l}\text { Gray sand } \\
\text { Soft sand rock } \\
\text { Gray sand } \\
\text { Red sticky clay } \\
\text { Coarse-grained yellow sand, } \\
\text { dry. } \\
\text { Soft sand rock. } \\
\text { Coarse - grained sand and } \\
\text { gravel, dry. }\end{array}$ & $\begin{array}{r}7 \\
3 \\
5 \\
12 \\
26 \\
1 \\
20\end{array}$ & $\begin{array}{r}7 \\
10 \\
15 \\
27 \\
53 \\
54 \\
74\end{array}$ & $\begin{array}{l}\text { Sand rock } \\
\text { Coarse-grained sand and } \\
\text { gravel. } \\
\text { Rock } \\
\text { Sand and gravel. } \\
\text { Hard rock... } \\
\text { Sand and gravel. }\end{array}$ & $\begin{array}{l}2 \\
3 \\
1 \\
4\end{array}$ & $\begin{array}{l}82 \\
85 \\
86 \\
90\end{array}$ \\
\hline
\end{tabular}




\begin{tabular}{|c|c|c|c|c|c|}
\hline & $\underset{\text { (feet) }}{\text { Thickness }}$ & $\begin{array}{c}\text { Depth } \\
\text { (feet) }\end{array}$ & & $\begin{array}{c}\text { Thickness } \\
\text { (feet) }\end{array}$ & $\underset{\text { (feet) }}{\text { Depth }}$ \\
\hline \multicolumn{6}{|c|}{ Well 2} \\
\hline $\begin{array}{l}\text { Top soil } \\
\text { Red sticky clay } \\
\text { Blue clay and sand } \\
\text { Dry sand } \\
\text { Hard rock. } \\
\text { Sand } \\
\text { Hard rock } \\
\text { Dry sand. }\end{array}$ & $\begin{array}{r}4 \\
8 \\
15 \\
29 \\
1 \\
3 \\
1 \\
22\end{array}$ & $\begin{array}{l}4 \\
12 \\
27 \\
56 \\
57 \\
60 \\
61 \\
83\end{array}$ & $\begin{array}{l}\text { Sticky, dense blue clay } \\
\text { Fine-grained water sand. } \\
\text { Sticky, dense blue clay. } \\
\text { Blue water sand } \\
\text { Blue clay. } \\
\text { Coarse-grained blue water } \\
\text { sand and gravel. }\end{array}$ & $\begin{array}{r}11 \\
6 \\
22 \\
13 \\
2 \\
19\end{array}$ & $\begin{array}{r}94 \\
100 \\
122 \\
135 \\
137 \\
156\end{array}$ \\
\hline
\end{tabular}

\section{SPUR}

Population in $1940: 2,136$.

Source of information: J. H. Cowan, city secretary, Feb. 18, 1946.

Ownership: Municipal.

Source of supply: Four wells.

Well 1. Dug in 1940; depth, 51 feet; diameter, 18 inches; turbine pump and 15-horsepower electric motor; static water level, 29.8 feet below land surface Feb. 18, 1946; yield, 250 gallons a minute; temperature, $641^{\circ} \mathrm{F}$.

Well 2. Dug in 1943; depth, 40 feet; diameter, 18 inches; turbine pump and 15-horsepower electric motor; static water level, 13.48 feet below land surface Feb. 18, 1946; yield, 200 gallons a minute.

Well 3. Dug in 1945; depth, 49 feet; diameter, 18 inches; deep-well turbine pump and 15-horsepower electric motor; static water level, 15.34 feet below land surface Feb. 18, 1946; yield, 200 gallons a minute.

Well 4. Dug in 1942; depth, 32 feet; diameter, 18 inches; deepened to 45 feet in 1947; deepwell turbine pump and 15-horsepower electric motor; yield, 150 gallons a minute; temperature, $6312^{\circ} \mathrm{F}$.

\section{Average pumpage in gallons a day}

\begin{tabular}{|c|c|c|c|c|c|c|}
\hline & 1942 & 1943 & 1944 & 1945 & 1946 & 1947 \\
\hline $\begin{array}{l}\text { January } \\
\text { February } \\
\text { Mareh } \\
\text { April } \\
\text { May } \\
\text { June } \\
\text { July } \\
\text { August } \\
\text { September } \\
\text { October } \\
\text { November } \\
\text { December }\end{array}$ & $\begin{array}{l}115,000 \\
107,000 \\
123,000 \\
111,000 \\
-215,000 \\
246,000 \\
161,000 \\
133,000 \\
137,000 \\
130,000 \\
106,000\end{array}$ & $\begin{array}{l}109,000 \\
105,000 \\
120,000 \\
140,000 \\
161,000 \\
170,000 \\
169,000 \\
254,000 \\
-166,000 \\
176,000 \\
131,000\end{array}$ & $\begin{array}{l}135,000 \\
130,000 \\
135,000 \\
171,000 \\
175,000 \\
178,000 \\
207,000 \\
238,000 \\
169,000 \\
145,000 \\
137,000 \\
128,000\end{array}$ & $\begin{array}{l}124,000 \\
124,000 \\
137,000 \\
169,000 \\
258,000 \\
219,000 \\
249,000 \\
304,000 \\
230,000 \\
134,000 \\
132,000 \\
131,000\end{array}$ & $\begin{array}{l}140,000 \\
138,000 \\
193,000 \\
274,000 \\
251,000 \\
340,000 \\
416,000 \\
425,000 \\
225,000 \\
193,000 \\
170,000 \\
200,000\end{array}$ & \begin{tabular}{r}
163,000 \\
171,000 \\
200,000 \\
245,000 \\
224,000 \\
371,000 \\
393,000 \\
413,000 \\
\\
\hdashline..-- \\
\end{tabular} \\
\hline
\end{tabular}

Storage: Concrete reservoir and elevated tank, total capacity, 200,000 gallons.

Number of customers: 730 .

Treatment: Chlorination. 


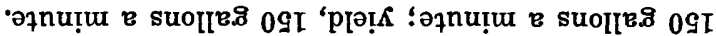

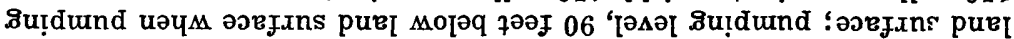

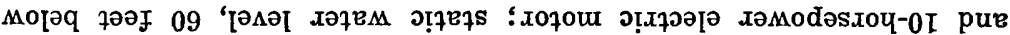

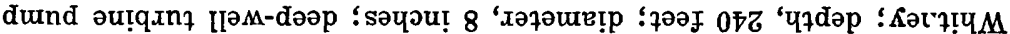

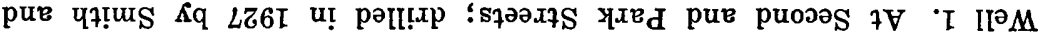

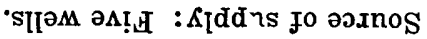
'[ed!̣ọun

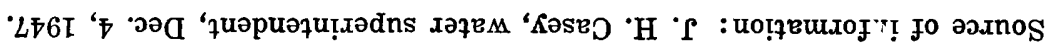

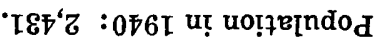

NOANGYV'T

\section{XINกOว XHTNOA}

\begin{tabular}{|c|c|c|c|c|c|}
\hline $\begin{array}{l}z 8 \\
7 z\end{array}$ & $\begin{array}{l}8 \\
9 \tau\end{array}$ & puвs Јәาะ & $\begin{array}{l}8 \\
9 \\
8\end{array}$ & $\begin{array}{l}\boldsymbol{z} \\
\mathbf{8} \\
\mathbf{g}\end{array}$ & 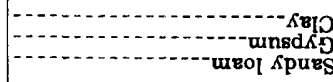 \\
\hline
\end{tabular}

8 IIPM

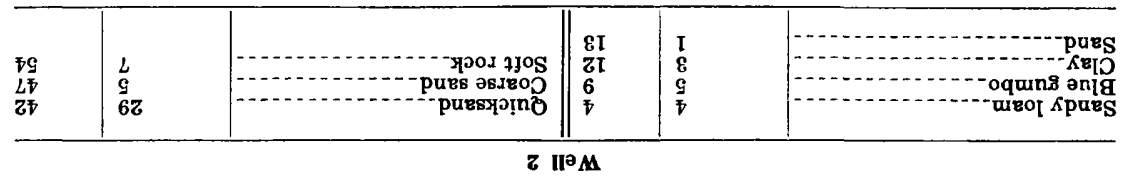

\begin{tabular}{|c|c|c|c|c|c|}
\hline $\begin{array}{l}89 \\
87 \\
78\end{array}$ & $\begin{array}{l}9 \\
91 \\
z\end{array}$ & 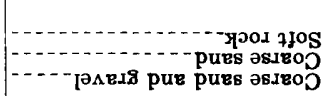 & $\begin{array}{l}08 \\
\text { II } \\
9 \\
\text { I }\end{array}$ & $\begin{array}{l}6 \mathrm{I} \\
9 \\
9 \\
1\end{array}$ & - \\
\hline
\end{tabular}

I IIPM

\begin{tabular}{|c|c|c|c|}
\hline 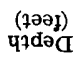 & 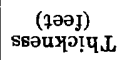 & $\begin{array}{l}\text { (7әә) } \\
\text { чzdəव }\end{array}$ & 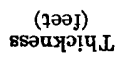 \\
\hline
\end{tabular}

sfool s,dallud

\begin{tabular}{|c|c|c|c|c|}
\hline 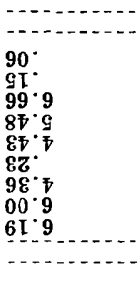 & 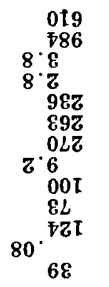 & 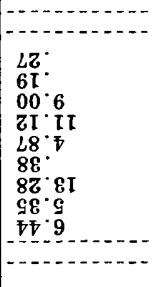 & 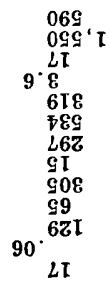 & 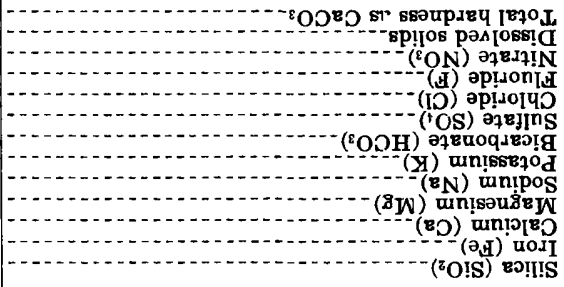 \\
\hline 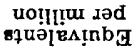 & $\begin{array}{l}\text { uolltwu } \\
\text { sad sqded }\end{array}$ & 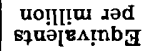 & $\begin{array}{c}\text { uo!II! } \\
\text { dəd squed }\end{array}$ & \\
\hline \multicolumn{2}{|c|}{$\nabla[]^{\partial} M$} & \multicolumn{2}{|c|}{$\boldsymbol{I} \mathrm{II}^{\partial} \mathbf{M}$} & \\
\hline
\end{tabular}

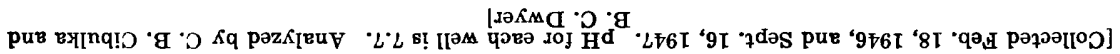




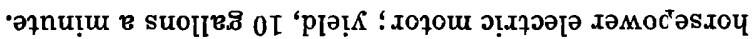

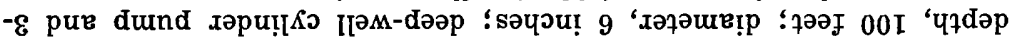

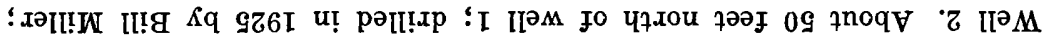

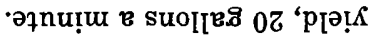

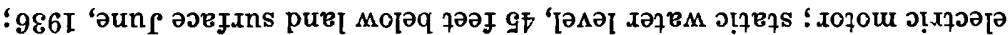

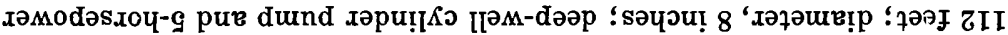

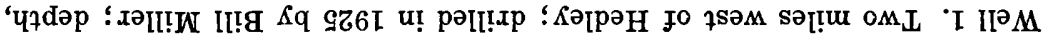

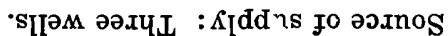

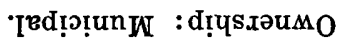
'876I ‘ 97

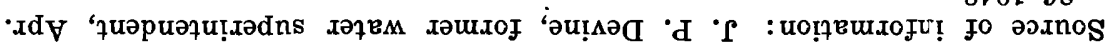
¿\&89:076I U!̣ uo!̣? [ndod

\section{ХF'ТसНH}

\begin{tabular}{|c|c|c|c|c|c|}
\hline $\begin{array}{l}Z 0 \& \\
8 Z I \\
0 Z I \\
80 I\end{array}$ & $\begin{array}{l}08 \mathrm{I} \\
8 \\
8 \mathrm{Z}\end{array}$ & 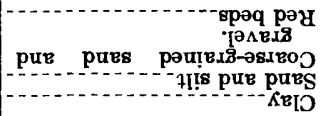 & $\begin{array}{l}98 \\
08 \\
79 \\
09 \\
7\end{array}$ & $\begin{array}{l}9 \\
9 z \\
\square \\
97 \\
7\end{array}$ & 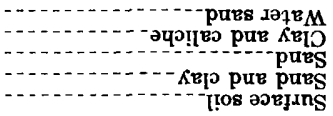 \\
\hline $\begin{array}{l}\text { (7әวЈ) } \\
\text { 47dวa }\end{array}$ & $\begin{array}{c}\text { (१әวЈ) } \\
\text { sรวนYว!ฺป }\end{array}$ & & $\begin{array}{c}(72 \partial \mathrm{J}) \\
\text { ч7dәа }\end{array}$ & $\begin{array}{c}\text { (ұәәј) } \\
\text { ssәนมว!บป }\end{array}$ & \\
\hline
\end{tabular}

z nam 'bol s, «an?.นa

\begin{tabular}{|c|c|c|c|c|c|}
\hline $\begin{array}{ll}82 \\
0 D^{\prime}\end{array}$ & $\begin{array}{l}\begin{array}{l}79 \\
298 \\
9 I \\
0\end{array} \\
97 \\
6 I\end{array}$ & 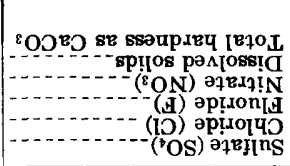 & \begin{tabular}{l}
$9 \mathbf{g}^{\circ} \mathbf{7}$ \\
$\mathbf{8 0}$ \\
$\mathbf{9 Z}$ \\
06 \\
$\mathbf{6 8} \cdot \mathbf{0}$ \\
\hdashline
\end{tabular} & 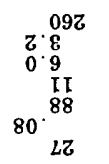 & 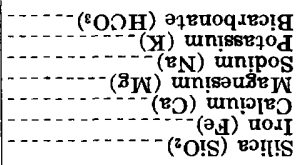 \\
\hline 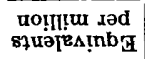 & $\begin{array}{l}\text { uot!ltu } \\
\text { səd s7x d }\end{array}$ & & 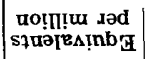 & $\begin{array}{l}\text { uot!l!w } \\
\text { xad sfited }\end{array}$ & \\
\hline
\end{tabular}

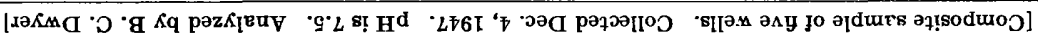

$$
\text { s?sfๆpuV }
$$

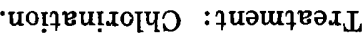

$$
\begin{aligned}
& .008 \text { : sxəuropsno jo xəquin } \\
& \text { suoI[?] }
\end{aligned}
$$

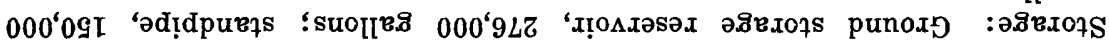

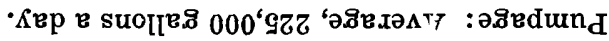

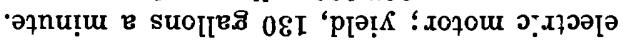

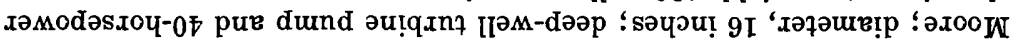

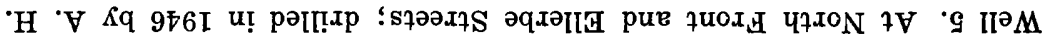

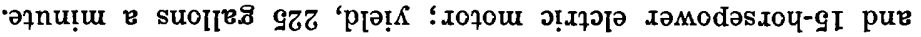

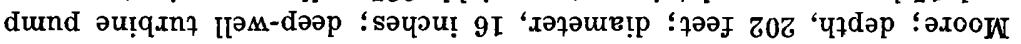

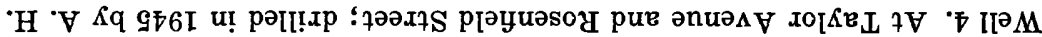

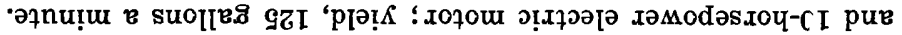

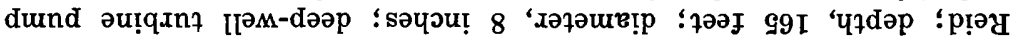

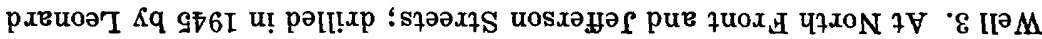

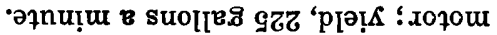

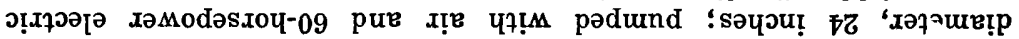

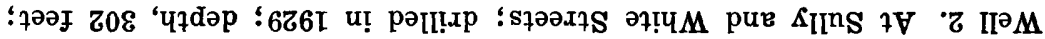


•əznu!̣u в suol[8.8 $29 \mathrm{I}$ 'p $\mathrm{p}$

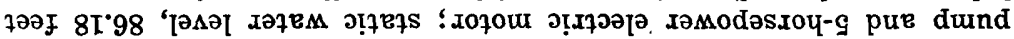

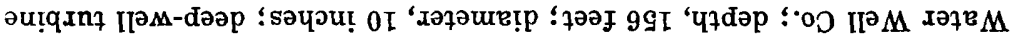

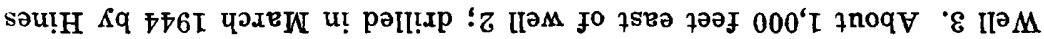

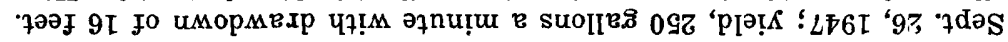

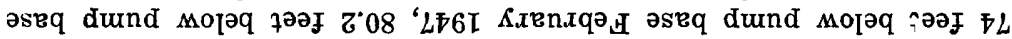

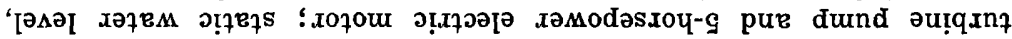

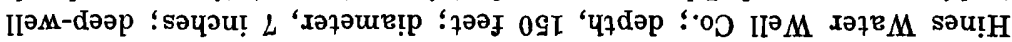
$\Lambda q$ Ð

ๆәәэ 89 јо имормвхр чт!м

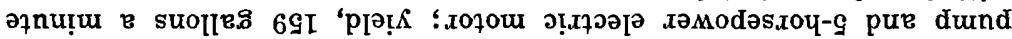

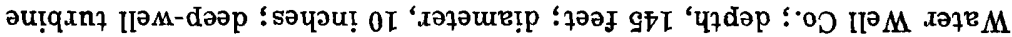

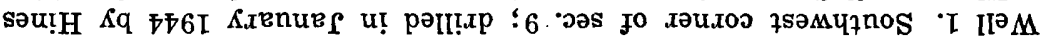

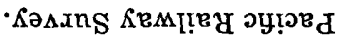
pue sexəI 'I 'L 'ZT 'भाq '

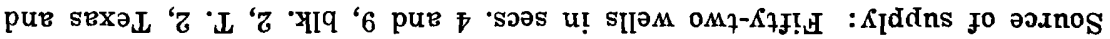

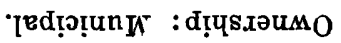

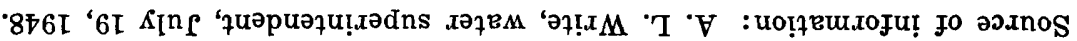

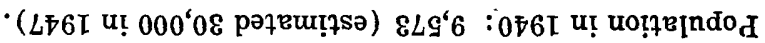
PSSTCO

\section{XINกOว \&OLD田}

\begin{tabular}{|c|c|c|c|c|c|}
\hline $\begin{array}{l}\text { ZII } \\
86 \\
06\end{array}$ & $\begin{array}{l}7 \mathrm{I} \\
8 \\
8 \mathrm{I}\end{array}$ & 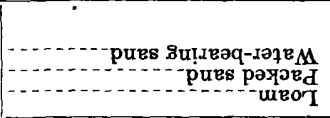 & $\begin{array}{l}2 L \\
99 \\
08 \\
7\end{array}$ & $\begin{array}{l}\text { ZI } \\
98 \\
98 \\
7\end{array}$ & 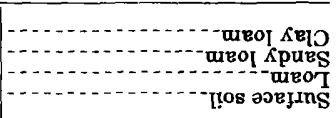 \\
\hline 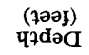 & $\begin{array}{c}\text { (7әәر) } \\
\text { sรวนมวเบ. }\end{array}$ & & 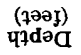 & $\begin{array}{c}\text { (7әәر) } \\
\text { ssวนมวเบป }\end{array}$ & \\
\hline
\end{tabular}

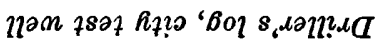

\begin{tabular}{|c|c|c|c|c|c|}
\hline $\begin{array}{ll}8 \varepsilon^{\circ} \\
\mathbf{9 g} \cdot 0\end{array}$ & \begin{tabular}{|l}
$8 I Z$ \\
888 \\
$0 \%$ \\
$9 \cdot I$ \\
$8 I$ \\
$I 8$
\end{tabular} & 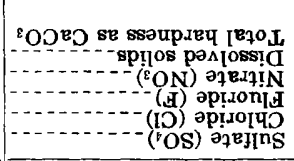 & 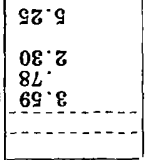 & 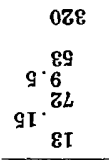 & 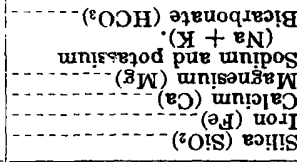 \\
\hline $\begin{array}{c}\text { uo!̣!!u sad } \\
\text { squอ[вa!̣b G }\end{array}$ & $\begin{array}{c}\text { uo!̣lI!u } \\
\text { دəd squed }\end{array}$ & & 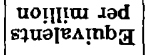 & $\begin{array}{l}\text { uotil!u } \\
\text { sad squed }\end{array}$ & \\
\hline
\end{tabular}

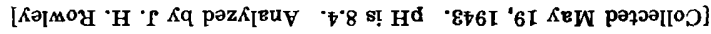

I 11 วn 's?̣sh

:OUONT: : UaUqREIL

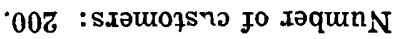
suolle.8

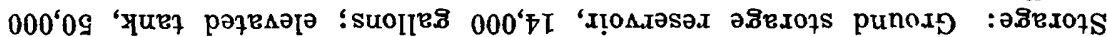

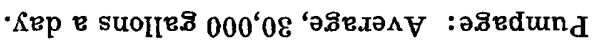

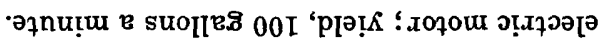

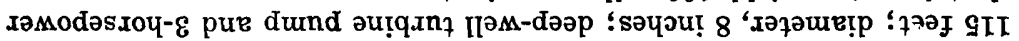

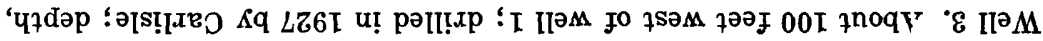




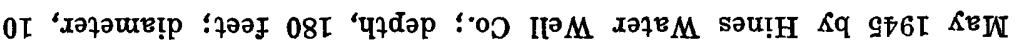

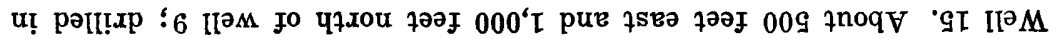

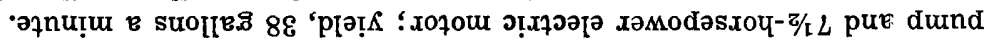

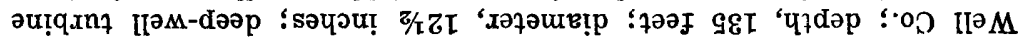

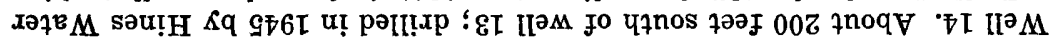

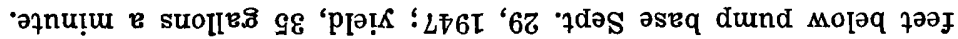

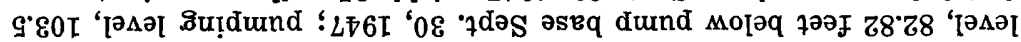

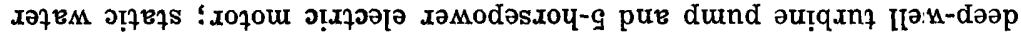

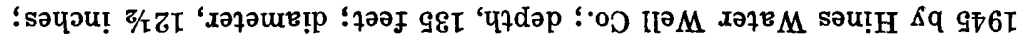

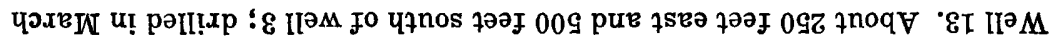

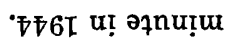

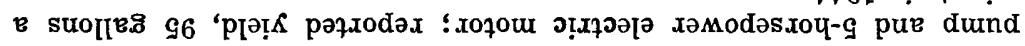

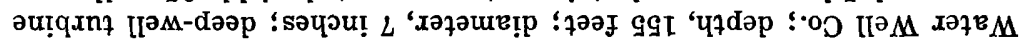

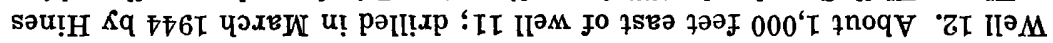

- 976 I u! әұnu!̣u

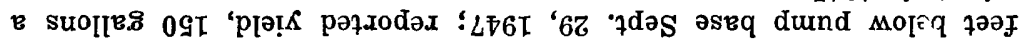
$0^{\circ} 60$ I '† $ә$

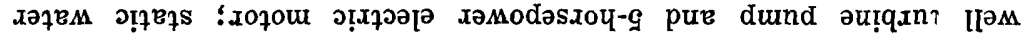

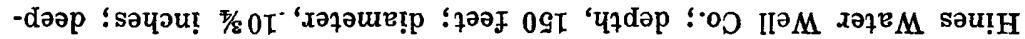

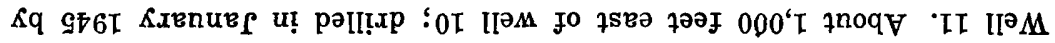

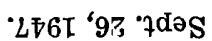

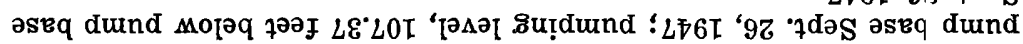

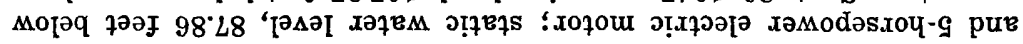

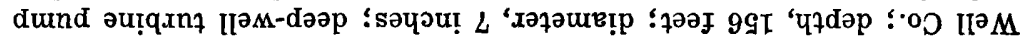

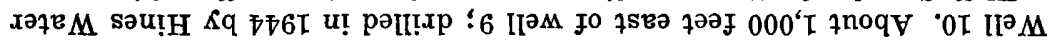

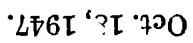

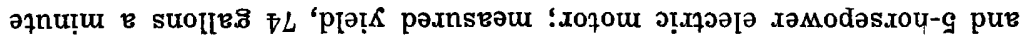

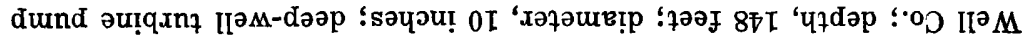

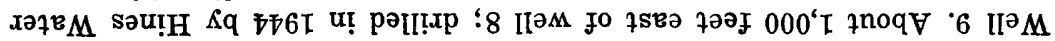

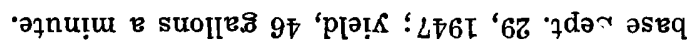
dund мојәq ұәәғ \&I'60I '

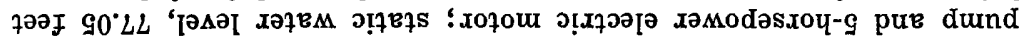

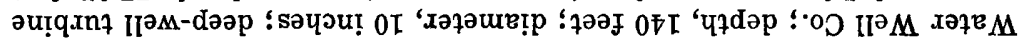

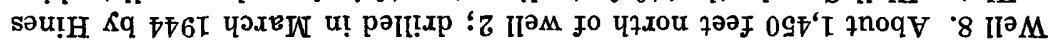

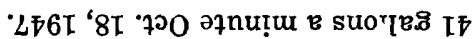

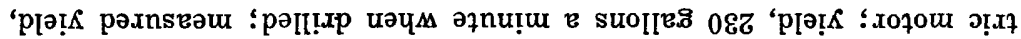

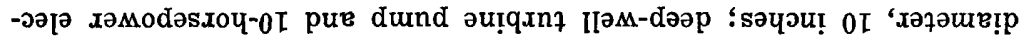

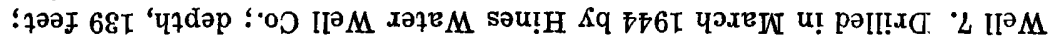

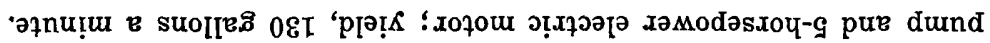

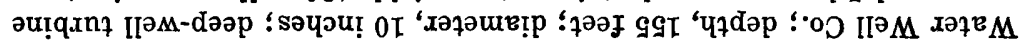

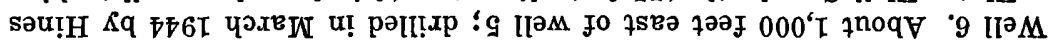

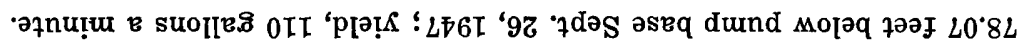

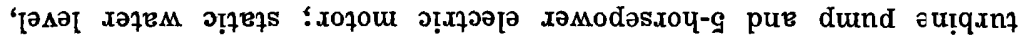

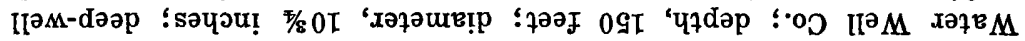

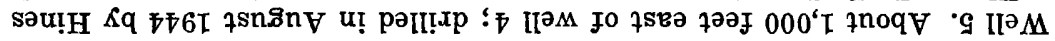

7әәэ $6 \&$ го имор

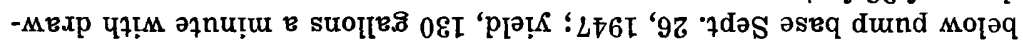

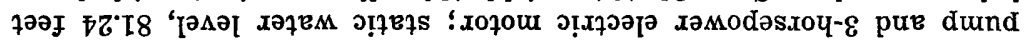

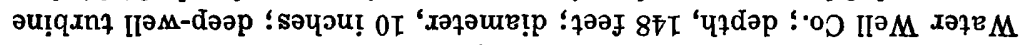

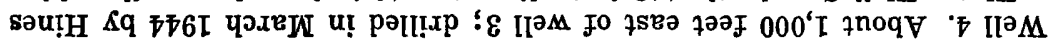


inches; deep-well turbine pump and 5-horsepower electric motor; static water level, 87.14 feet below pump base Sept. 26, 1947; pumping level, 132.7 feet below pump base Sept. 29, 1947.

Well 16. About 1,000 feet north of well 15; drilled in May 1945 by Hines Water Well Co.; depth, 150 feet; diameter, $103 / 4$ inches; deen-well turbine pump and 15-horsepower electric motor; static water level, 88.76 feet below pump base Sept. 26, 1947; pumping level, 140.13 feet below pump base Sept. 29, 1947; yield, 95 gallons a minute.

Well 17. About 1,000 feet north of well 16 ; drilled in June 1945 by Hines Water Well Co.; depth, 165 feet; diameter, 10 inches; deep-well turbine pump and 15-horsepower electric motor; static water level, 78.9 feet below pump base Sept. 26, 1947; pumping level, 103.5 feet b:low pump base Sept. 29, 1947; yield, 110 gallons a minute in 1945 .

Well 18. About 1,100 feet west of northeast corner of sec. 4; drilled in June 1945 by Hines Water Well Co.; depth, 175 feet; diameter, 10 inches; deep-well turbine pump and 10-horsepower electwic motor; static water level, 47.16 feet below pump base Sept. 26, 1947; pumping level, 95.8 feet below pump base Sept. 29, 1947; yield, 140 gallons a minute in $\mathbf{1 9 4 5}$.

Well 19. About 1,500 feet east of well 17; drilled in August 1945 by Hines Water Well Co.; depth, 155 feet; diameter, 10 inches; deep-well turbine pump and 71/2-horsepower electric motor; static water level, 81.32 feet below pump base Sept. 26, 1947; pumping level, 138.8 feet b?low pump base Sept. 29, 1947; yield, 90 gallons a minute in 1945 .

Well 20. About 1,200 feet north and 1,450 feet east of the southeast corner of sec. 4; drilled in August 1945 by Hines Water Well Co.; depth, 175 feet; diameter, 10 inches; deep-well turbine pump and 10-horsepower electric motor; yield, 120 gallons a minute in 1945 .

Well 21. About 1,350 feet north of well 20; drilled in January 1946 by Hines Water Well Co.; depth, 160 feet; diameter, 10 inches; deep-well turbine pump and 10-horsepower electric motor; static water level, 62.30 feet below pump base Sept. 26, 1947; yield, 180 gallon * a minute in 1946.

Well 22. About 417 feet east and 316 feet south of the northvrest corner of sec. 45; drilled in December 1945 by Bethel and Matthews; depth, 164 feet; diameter, 10 inches; deep-well turbine pump and 25-horsepower electric motor; static water level, 56.35 feet below pump base Sept. 29, 1947; pumping level, 119.4 feet below pump base Sept. 26, 1947; yield, 300 gallons a minute in January 1946.

Well 23. About 378 feet east and 61 feet north of the southwost corner of $\mathrm{NW}^{1 / 4}$ sec. 45; drilled in January 1946 by Bethel and Matthews; depth, 156 feet; diameter, 10 inches; deep-well turbine pump and 15horsepower electric motor; static water level, 54.65 feet below pump base Sept. 29, 1947; pumping level, 99.42 feet below pump base Sept. 29, 1947; yield, 180 gallons a minute in 1946 .

Well 24. About 1,200 feet northeast of well 23; drilled in January 1946 by Bethel and Matthews; depth, 156 feet; diameter, 10 inches; deepwell turbine pump and 10-horsepower electric motor; static vrater level, 53.75 feet below pump base Sept. 29, 1947; pumping level, 97.35 feet below pump base Sept. 29, 1947; yield, 221 gallons a minute. 
Well 25. About 258 feet west and 279 feet north of the southeast corner of the NW $1 / 4$ sec. 45 ; drilled in February 1946 by Bethel and Matthews; depth, 164 feet; diameter, 10 inches; deep-well turbine rump and 15horsepower electric motor; static water level, 55.21 feet below pump base Sept. 26, 1947; reported yield, 254 gallons a minute.

Well 26. About $269^{\circ}$ feet west and 1,150 feet south of the northeast corner of the NW1/4 sec. 45; drilled in February 1946 by Bethel and Matthews; depth, 174 feet; diameter, 10\%/4 inches; deep-well turbine pump and 15-horsepower electric motor; static water level, $54.89 \mathrm{fee}^{+}$below pump base Sept. 29, 1947; pumping level, 92.12 feet below pump base Sept. 29, 1947; yield, 257 gallons a minute.

Well 27. About 831 feet north of well 23; drilled in Febriary 1946 by Bethel and Matthews; depth, 158 feet; diameter, 10 $3 / 4$ incr es; deep-well turbine pump and 10-horsepower electric motor; static water level, 58.35 feet below pump base Sept. 29, 1947; yield, 192 gallons a minute.

Well 28. About 1,040 feet west of well 20; drilled in May 1946 by Bethel and Matthews; depth, 150 feet; diameter, 10 inches; deer-well turbine pump and 10-horsepower electric motor; static water level, 79.19 feet below pump base Sept. 26, 1947; pumping level, 121.0 feet below pump base Sept. 29, 1947; reported yield, 170 gallons a minute.

Well 29. About 1,260 feet south of well 18; drilled in May 1946 by Bethel and Matthews; depth, 150 feet; diameter, 10 inches; deer-well turbine pump and 71/2-horsepower electric motor; static water lerel, 46.77 feet below pump base Sept. 29, 1947; pumping level, 68.4 feet below pump base Sept. 29, 1947; reported yield, 250 gallons a minute.

Well 30. About 1,000 feet west of northeast corner of sec. 44; drilled in November 1946 by Bethel and Matthews; depth, 160 fent; diameter, $103 / 4$ inches; deep-well turbine pump and 15-horsepower electric motor; static water level, 60.09 feet below pump base Sept. 29, 1947; pumping level, 94.9 feet below pump base Sept. 29, 1947; yield, 211 gallons a minute.

Well 31. Drilled in December 1946 by Bethel and Matthews; depth, 160 feet; diameter, $103 / 4$ inches; deep-well turbine pump and 15-horsepower electric motor; static water level, 55.01 feet below pump base Sept. 29, 1947; pumping level, 89.44 feet below pump base Sept. 29, 1947; yield, 210 gallons a minute December 1946.

Well 32. About 1,500 feet west of well 30; drilled in December 1946 by Bethel and Matthews; depth, 160 feet; diameter, 103/4 inches; deepwell turbine pump and 25-horsepower electric motor; static water level, 65.31 feet below pump base Sept. 29, 1947; pumping level, 107.6 feet below pump base Sept. 29, 1947; yield, 251 gallons $\Sigma$. minute.

Well 33. About 1,300 feet south of well 32; drilled in December 1946 by Bethel and Matthews; depth, 147 feet; diameter, 103/4 incres; deep-well turbine pump and 15-horsepower electric motor; static water level, 54.12 feet below pump base Sept. 29, 1947; pumping level, 102.52 feet below pump base Sept. 29, 1947; yield, 178 gallons a mintte.

Well 34. About 1,500 feet west of well 32; drilled in January 1947 by Bethel and Matthews; depth, 150 feet; diameter, 103/4 incres; deep-well turbine pump and 25-horsepower electric motor; static water level, 58.86 feet below pump base Sept. 29, 1947; pumping level, 100.25 feet below pump base Sept. 29, 1947; yield, 91 gallons a minute. 
Well 35. About 1,300 feet south of well 34; drilled in January 1947 by Bethel and Matthews; depth, 140 feet; diameter, 103/4 inches; deep-well turbine pump and electric motor; static water level, 58.21 feet below pump base Sept. 29, 1947; yield, 152 gallons a minute.

Well 36. About 100 feet west and 100 feet south of the northwest corner of the NW $1 / 4$ sec. 45; drilled in January 1947 by Bethel an 7 Matthews; depth, 180 feet; diameter, $103 / 4$ inches; deep-well turbine pump and 25horsepower electric motor; static water level, 54.54 feet below pump base Sept. 26, 1947; yield, 310 gallons a minute.

Well 37. About 1,050 feet southeast of well 22; drilled in January 1927 by Bethel and Matthews; depth, 166 feet; diameter, $103 / 4$ inches; deepwell turbine pump and 25-horsepower electric motor; static water level, 56.67 feet below pump base Sept. 29, 1947; pumping level, 119.8 feet below pump base Sept. 29, 1947; yield, 223 gallons a minute January 1947.

Well 40. About 1,250 feet southwest of well 34; drilled in A pril 1948 by Bethel and Matthews; depth, 140 feet; well yielded insuf'icient water and was abandoned. See driller's log.

Well 41. Along the southern edge of sec. 45 about 3,400 feet from the east line; drilled in April 1948 by Bethel and Matthews; depth, 170 feet; diameter, 15 to $103 / 4$ inches; deep-well turbine pump and 20horsepower electric motor; static water level, 60 feet below land surface April 30, 1948; pumping level, 115 feet below land surface after pumping 6 hours at a rate of 150 gallons a minute; yield. 150 gallons a minute.

Well 42. About 1,200 feet north of well 41; drilled in May 1948 by Bethel and Matthews; depth, 170 feet; diameter, 16 to $103 / 4$ inches; deep-well turbine pump and 25-horsepower electric motor; static water level reported, 60 feet below land surface when drilled; pumping level, 125 feet below land surface after $4 \frac{1 / 2}{1 / 2}$ hours of pumping at a rate of 223 gallons a minute; yield, 223 gallons a minute.

Well 43. About 1,500 feet east of well 42; drilled in May 1948 by Bethel and Matthews; depth, 130 feet; diameter, 20 to 103/4 inches; deep-well turbine pump and 15-horsepower electric motor.

Well 44. About 1,300 feet north of well 43; drilled in June 1948 by Bethel and Matthews; depth, 175 feet; diameter, 16 to $103 / 4$ inches; deep-well turbine pump and 15-horsepower electric motor; yield, 150 gallons a minute.

Well 45. About 1,700 feet south of the north line and about 2,000 feet west of the east line of sec. 45; drilled in June 1948 by Bethel and Matthews; depth, 180 feet; diameter, 16 to $10 \% 4$ inches; doep-well turbine pump and 25-horsepower electric motor.

Well 46. About 1,000 feet south of the north line and 600 feet west of the east line of sec. 45; drilled in June 1948 by Bethel and Matthews; depth, 180 feet; diameter, 16 to $103 / 4$ inches; deep-well trurbine pump and 25-horsepower electric motor; yield, 125 gallons a minute after pumping 8 hours.

Well 47. About 1,200 feet west of well 1; drilled in June 1948 by Bethel and Matthews; depth, 150 feet; diameter 16 inches; we!1 abandoned because it did not yield sufficient water. See driller's log. 
Well 48. About 0.2 mile west and 0.8 mile south of the intersection of Highways 80 and 51 in Odessa; drilled in July 1948 by Bethel and Matthews; depth, 130 feet; diameter, 15 to $103 / 4$ inches; deep-well turbine pump and electric motor; drawdown, 46 feet after pumping 1 hour at a rate of 146 gallons a minute.

Well 49. About 2,200 feet south and 660 feet west of the intersection of Crane Avenue and Clement Street in Odessa; drilled in August 1948 by Bethel and Matthews; depth, 120 feet; diameter, 16 to $103 / 4$ inches; deep-well turbine pump and electric motor; static water level reported, 29 feet below land surface June 1948.

Well 50. About 2,200 feet south of the intersection of Crane Avenue and Clement Street; drilled in August 1948 by Bethel and Matthews; depth, 120 feet; diameter, 16 to $10 \%$ inches; deep-well turbine pump and electric motor; static water level, 22 feet below land surface A ugust 1948; yield, 243 gallons a minute after 1 hour of pumping.

Well 51. About 1,100 feet south of the intersection of Crane Avenue and Clements Street; drilled in August 1948 by Bethel and Matthews; depth, 125 feet; diameter, 16 to $103 / 4$ inches; deep-well turbine pump and electric motor; static water level, 30 feet below land surface August 1948; yield, 164 gallons a minute after 6 hours of pumping.

Well 52. About 450 feet east of well 1; drilled in August 1948 by Bethel and Matthews; depth, 128 feet; diameter, 16 to $103 / 4$ incher; deep-well turbine pump and electric motor.

Well 53. About 1,100 feet east of well 49; drilled in August 1948 by Bethel and Matthews; depth, 125 feet; diameter, 16 to $103 / 4$ inches; deep-well turbine pump and electric motor.

Well 54. About 1,110 feet east of well 52; drilled in Augrast 1948 by Bethel and Matthews; depth, 120 feet; diameter, 16 to $10 \%$ inches; deep-well turbine pump and electric motor.

Average pumpage in gallons a day

\begin{tabular}{|c|c|c|c|}
\hline & 1946 & 1947 & 1948 \\
\hline 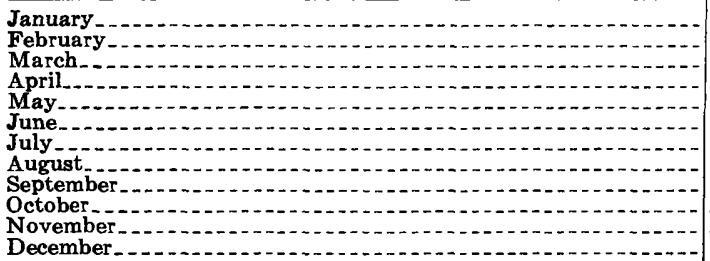 & 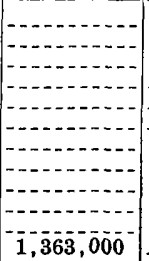 & \begin{tabular}{|l|}
$1,417,000$ \\
$1,508,000$ \\
$1,707,000$ \\
$3,719,070$ \\
$3,477,070$ \\
$2,983,070$ \\
$2,287,070$ \\
$1,641,070$
\end{tabular} & 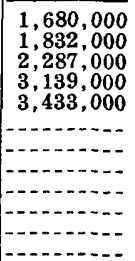 \\
\hline
\end{tabular}

Storage: Surface reservoir, 100,000 gallons; surface reservoir, $§ 00,000$ gallons; surface reservoir, 420,000 gallons; two surface reservoirs, 440,000 gallons each; surface reservoir, 500,000 gallons; elevated tank, 100,000 gallons; elevated tank, 500,000 gallons.

Number of customers: 4,141 .

Treatment: Chlorination. 


\section{Analyses}

[Collected Sept. 22, 1948. pH : well 2, 7.5 ; well 28, 7.7 ; well 32, 7.3 ; wel 44, 7.7. Analyzed by D. E. Weaver]

\begin{tabular}{|c|c|c|c|c|}
\hline & \multicolumn{2}{|c|}{ Well 2} & \multicolumn{2}{|c|}{ Well 28} \\
\hline & $\begin{array}{l}\text { Parts per } \\
\text { million }\end{array}$ & $\begin{array}{l}\text { Equivalents } \\
\text { per million }\end{array}$ & $\begin{array}{l}\text { Parts per } \\
\text { million }\end{array}$ & $\begin{array}{l}\text { Equivalents } \\
\text { per million }\end{array}$ \\
\hline \multirow[t]{3}{*}{ 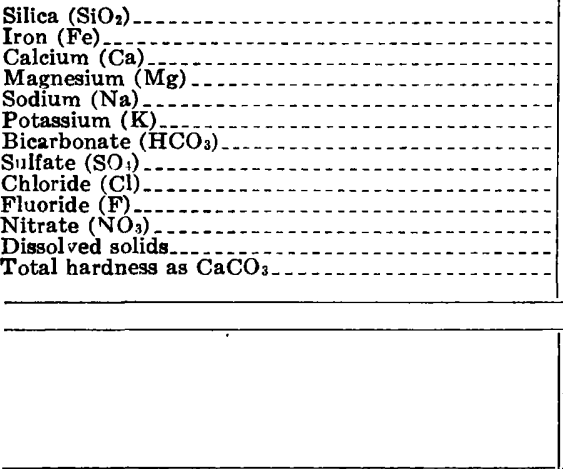 } & $\begin{array}{l}38 \\
132 \\
34 \\
81 \\
4.10 \\
186 \\
255 \\
155 \\
1.4 \\
14 \\
877 \\
470\end{array}$ & 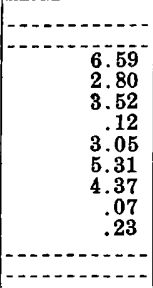 & $\begin{array}{l}32 \\
72 \\
17 \\
37 \\
1.6 \\
214 \\
72 \\
42 \\
1.8 \\
17 \\
406 \\
250\end{array}$ & $\begin{array}{r}r .59 \\
1.40 \\
.09 \\
.04 \\
3.51 \\
1.50 \\
1.18 \\
.09 \\
.27 \\
\hdashline-2 .\end{array}$ \\
\hline & \multicolumn{2}{|c|}{ Well 32} & \multicolumn{2}{|c|}{ Well 44} \\
\hline & $\begin{array}{l}\text { Parts per } \\
\text { million }\end{array}$ & $\begin{array}{l}\text { Equivalents } \\
\text { per million }\end{array}$ & $\begin{array}{l}\text { Parts per } \\
\text { million }\end{array}$ & $\begin{array}{l}\text { Equivalents } \\
\text { per million }\end{array}$ \\
\hline 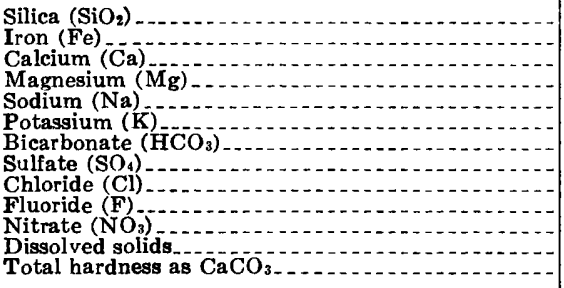 & $\begin{array}{c}32 \\
74 \\
15 \\
31 \\
2.05 \\
214 \\
66 \\
39 \\
1.0 \\
16 \\
402 \\
246\end{array}$ & $\begin{array}{r}3.69 \\
1.23 \\
1.35 \\
.05 \\
3.51 \\
1.37 \\
1.10 \\
.05 \\
.26\end{array}$ & $\begin{array}{l}44 \\
6^{46} .05 \\
14 \\
25 \\
3.6 \\
220 \\
44 \\
26 \\
2.0 \\
12 \\
362 \\
222\end{array}$ & $\begin{array}{r}3.29 \\
1.15 \\
1.09 \\
.09 \\
3.61 \\
.92 \\
.73 \\
.11 \\
.19 \\
\hdashline .0 .\end{array}$ \\
\hline
\end{tabular}

\section{Drillers' logs}

\begin{tabular}{l|l|l|l|l}
\hline & $\begin{array}{c}\text { Thickness } \\
\text { 'feet) }\end{array}$ & $\begin{array}{c}\text { Depth } \\
\text { (feet) }\end{array}$ & $\begin{array}{c}\text { Thickness } \\
\text { (feet) }\end{array}$ & $\begin{array}{l}\text { Depth } \\
\text { (feet) }\end{array}$ \\
\hline
\end{tabular}

Well 1

\begin{tabular}{|c|c|c|c|c|c|}
\hline $\begin{array}{l}\text { Surface soil } \\
\text { Caliche } \\
\text { Brown sand } \\
\text { Sand and gravel } \\
\text { Brown sand }\end{array}$ & $\begin{array}{r}4 \\
41 \\
20 \\
10 \\
35\end{array}$ & $\begin{array}{r}4 \\
45 \\
65 \\
75 \\
110\end{array}$ & $\begin{array}{l}\text { White sand } \\
\text { Brown sand } \\
\text { Sand. } \\
\text { Blue shale and red beds. }\end{array}$ & $\begin{array}{r}17 \\
7 \\
\mathbf{3} \\
\mathbf{8}\end{array}$ & $\begin{array}{l}127 \\
134 \\
137 \\
145\end{array}$ \\
\hline
\end{tabular}

Well 2

\begin{tabular}{|c|c|c|c|c|c|}
\hline $\begin{array}{l}\text { Surface soil } \\
\text { Caliche } \\
\text { Brown sand, water } \\
\text { Sand and gravel, water. }\end{array}$ & $\begin{array}{r}4 \\
36 \\
25 \\
53\end{array}$ & $\begin{array}{r}4 \\
40 \\
65 \\
118\end{array}$ & $\begin{array}{l}\text { Coarse sand and gravel } \\
\text { White sand. } \\
\text { Blue shale and red beds. }\end{array}$ & $\begin{array}{r}17 \\
5 \\
10\end{array}$ & $\begin{array}{l}135 \\
140 \\
150\end{array}$ \\
\hline
\end{tabular}

\section{Well 3}

\begin{tabular}{|c|c|c|c|c|c|}
\hline $\begin{array}{l}\text { Soil } \\
\text { Caliche } \\
\text { Brown sand, dry } \\
\text { Hard sand. }\end{array}$ & $\begin{array}{r}10 \\
35 \\
25 \\
6\end{array}$ & $\begin{array}{l}10 \\
45 \\
70 \\
76\end{array}$ & $\begin{array}{l}\text { Sand, water } \\
\text { Blue shale. } \\
\text { Red beds... }\end{array}$ & $\begin{array}{r}72 \\
3 \\
5\end{array}$ & $\begin{array}{l}148 \\
151 \\
156\end{array}$ \\
\hline
\end{tabular}




\begin{tabular}{c|c|c|}
\hline $\begin{array}{c}\text { Thickness } \\
\text { (feet) }\end{array}$ & $\begin{array}{c}\text { Depth } \\
\text { (feet) }\end{array}$
\end{tabular}$\left|\begin{array}{c}\text { Tr ickness } \\
\text { (feet) }\end{array}\right| \begin{gathered}\text { Depth } \\
\text { (feet) }\end{gathered}$

\begin{tabular}{|c|c|c|c|c|c|}
\hline \multicolumn{6}{|c|}{ Well 4} \\
\hline $\begin{array}{l}\text { Surface soil } \\
\text { Caliche } \\
\text { Hard sand } \\
\text { Sand, dry } \\
\text { Sand, water. }\end{array}$ & $\begin{array}{r}5 \\
37 \\
6 \\
22 \\
4\end{array}$ & $\begin{array}{r}5 \\
42 \\
48 \\
70 \\
74\end{array}$ & $\begin{array}{l}\text { Hard sand } \\
\text { Sand, water. } \\
\text { Bl:1e shale... } \\
\text { Red beds....... }\end{array}$ & $\begin{array}{r}8 \\
58 \\
3 \\
5\end{array}$ & $\begin{array}{r}82 \\
140 \\
143 \\
148\end{array}$ \\
\hline
\end{tabular}

\section{Well 5}

\begin{tabular}{|c|c|c|c|c|c|}
\hline $\begin{array}{l}\text { Surface soil } \\
\text { Caliche } \\
\text { Sand } \\
\text { Yellow sand, little water. }\end{array}$ & $\begin{array}{l}10 \\
25 \\
25 \\
11\end{array}$ & $\begin{array}{l}10 \\
\mathbf{3 5} \\
60 \\
71\end{array}$ & $\begin{array}{l}\text { Yellow sand } \\
\text { Sand, water. } \\
\text { Red beds. }\end{array}$ & $\begin{array}{r}19 \\
\mathbf{5 5} \\
\mathbf{5}\end{array}$ & $\begin{array}{r}90 \\
145 \\
150\end{array}$ \\
\hline
\end{tabular}

\section{Well 6}

\begin{tabular}{|c|c|c|c|c|c|}
\hline $\begin{array}{l}\text { Surface soil } \\
\text { Caliche } \\
\text { Brown sand. }\end{array}$ & $\begin{array}{r}\mathbf{5} \\
\mathbf{4 5} \\
\mathbf{2 0}\end{array}$ & $\begin{array}{r}5 \\
50 \\
70\end{array}$ & $\begin{array}{l}\text { Sand, water } \\
\text { Brown sand, water } \\
\text { Red beds. }\end{array}$ & $\begin{array}{r}70 \\
10 \\
5\end{array}$ & $\begin{array}{l}140 \\
150 \\
155\end{array}$ \\
\hline
\end{tabular}

\section{Well 8}

\begin{tabular}{|c|c|c|c|c|c|}
\hline $\begin{array}{l}\text { Surface soil } \\
\text { Caliche } \\
\text { Sand, water } \\
\text { Sand and gravel. }\end{array}$ & $\begin{array}{r}-9 \\
54 \\
5 \\
30\end{array}$ & $\begin{array}{r}9 \\
63 \\
68 \\
98\end{array}$ & $\begin{array}{l}\text { Sand } \\
\text { Blue shale. } \\
\text { Red beds.... }\end{array}$ & $\begin{array}{r}32 \\
4 \\
6\end{array}$ & $\begin{array}{l}130 \\
134 \\
140\end{array}$ \\
\hline
\end{tabular}

\section{Well 9}

\begin{tabular}{|c|c|c|c|c|c|}
\hline $\begin{array}{l}\text { Surface soil } \\
\text { Caliche } \\
\text { Hard sand } \\
\text { Brown sand, dry }\end{array}$ & $\begin{array}{r}4 \\
36 \\
10 \\
10\end{array}$ & $\begin{array}{r}4 \\
40 \\
50 \\
60\end{array}$ & $\begin{array}{l}\text { Hard sand. } \\
\text { Sand, water. } \\
\text { Blue shale. } \\
\text { Red beds. }\end{array}$ & $\begin{array}{r}12 \\
66 \\
7 \\
3\end{array}$ & $\begin{array}{r}72 \\
138 \\
145 \\
148\end{array}$ \\
\hline
\end{tabular}

\section{Well 10}

\begin{tabular}{|c|c|c|c|c|c|}
\hline $\begin{array}{l}\text { Surface soil } \\
\text { Caliche } \\
\text { Hard sand } \\
\text { Sand, water. }\end{array}$ & $\begin{array}{r}6 \\
34 \\
25 \\
5\end{array}$ & $\begin{array}{r}6 \\
40 \\
65 \\
70\end{array}$ & $\begin{array}{l}\text { White sand, water. } \\
\text { Yellow sand } \\
\text { Blue shale. } \\
\text { Red beds. }\end{array}$ & $\begin{array}{r}25 \\
50 \\
6 \\
5\end{array}$ & $\begin{array}{r}95 \\
145 \\
151 \\
156\end{array}$ \\
\hline
\end{tabular}

\begin{tabular}{|c|c|c|c|c|c|}
\hline \multicolumn{6}{|c|}{ Well 11} \\
\hline $\begin{array}{l}\text { Surface soil } \\
\text { Caliche } \\
\text { Sand, water }\end{array}$ & $\begin{array}{r}5 \\
60 \\
75\end{array}$ & $\begin{array}{r}5 \\
65 \\
140\end{array}$ & $\begin{array}{l}\text { Blue shale. } \\
\text { Red beds. }\end{array}$ & $\begin{array}{l}5 \\
\mathbf{5}\end{array}$ & $\begin{array}{l}145 \\
150\end{array}$ \\
\hline
\end{tabular}

\begin{tabular}{|c|c|c|c|c|c|}
\hline \multicolumn{6}{|c|}{ Well 12} \\
\hline $\begin{array}{l}\text { Surface soil. } \\
\text { Caliche } \\
\text { Brown sand } \\
\text { Sand, water }\end{array}$ & $\begin{array}{r}5 \\
35 \\
20 \\
8\end{array}$ & $\begin{array}{r}5 \\
40 \\
60 \\
68\end{array}$ & $\begin{array}{l}\text { White sand } \\
\text { Gravel and sand } \\
\text { Sand, water } \\
\text { Red beds. }\end{array}$ & $\begin{array}{r}12 \\
5 \\
64 \\
6\end{array}$ & $\begin{array}{r}80 \\
85 \\
149 \\
155\end{array}$ \\
\hline \multicolumn{6}{|c|}{ Well 13} \\
\hline 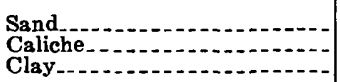 & $\begin{array}{l}15 \\
\mathbf{2 5} \\
\mathbf{3 5}\end{array}$ & $\begin{array}{l}15 \\
40 \\
75\end{array}$ & $\begin{array}{l}\text { Sand, water } \\
\text { Blue shale. } \\
\text { Red beds. }\end{array}$ & $\begin{array}{r}45 \\
10 \\
5\end{array}$ & $\begin{array}{l}120 \\
130 \\
135\end{array}$ \\
\hline
\end{tabular}




\begin{tabular}{l|l|l|l|l|l|}
\hline $\begin{array}{c}\text { Thickness } \\
\text { (feet) }\end{array}$ & $\begin{array}{c}\text { Depth } \\
\text { (feet) }\end{array}$ & $\begin{array}{c}\text { Thickness } \\
\text { (feet) }\end{array}$ & $\begin{array}{c}\text { Depth } \\
\text { (feet) }\end{array}$ \\
\hline
\end{tabular}

\begin{tabular}{|c|c|c|c|c|c|}
\hline \multicolumn{6}{|c|}{ Well 14} \\
\hline $\begin{array}{l}\text { Surface soil } \\
\text { Caliche } \\
\text { Clay, } \\
\text { Sand, dry } \\
\text { Sand, water }\end{array}$ & $\begin{array}{r}2 \\
33 \\
10 \\
20 \\
30\end{array}$ & $\begin{array}{l}2 \\
35 \\
45 \\
65 \\
95\end{array}$ & $\begin{array}{l}\text { Blue shale. } \\
\text { Sand, water. } \\
\text { Blue shale. } \\
\text { Red beds. }\end{array}$ & $\begin{array}{r}5 \\
26 \\
4 \\
5\end{array}$ & $\begin{array}{l}100 \\
126 \\
130 \\
135\end{array}$ \\
\hline
\end{tabular}

\section{Well 16}

\begin{tabular}{|c|c|c|c|c|c|}
\hline $\begin{array}{l}\text { Surface soil } \\
\text { Caliche. } \\
\text { Red sand } \\
\text { Yellow sand }\end{array}$ & $\begin{array}{r}5 \\
35 \\
20 \\
5\end{array}$ & $\begin{array}{r}5 \\
40 \\
60 \\
65\end{array}$ & $\begin{array}{l}\text { White sand } \\
\text { Brown sand. } \\
\text { Sand and gravel } \\
\text { Blue shale and red beds. }\end{array}$ & $\begin{array}{l}20 \\
35 \\
18 \\
12\end{array}$ & $\begin{array}{r}85 \\
120 \\
138 \\
150\end{array}$ \\
\hline
\end{tabular}

\section{Well 17}

\begin{tabular}{|c|c|c|c|c|c|}
\hline $\begin{array}{l}\text { Surface soil } \\
\text { Caliche. } \\
\text { Clay } \\
\text { Sand } \\
\text { Blue shale. }\end{array}$ & $\begin{array}{r}3 \\
42 \\
15 \\
60 \\
5\end{array}$ & $\begin{array}{r}3 \\
45 \\
60 \\
120 \\
125\end{array}$ & $\begin{array}{l}\text { Yellow clay } \\
\text { Sand. } \\
\text { Gravel } \\
\text { Blue shale. } \\
\text { Red beds. } \ldots \ldots\end{array}$ & $\begin{array}{r}\mathbf{5} \\
20 \\
\mathbf{3} \\
\mathbf{3} \\
\mathbf{9}\end{array}$ & $\begin{array}{l}130 \\
150 \\
153 \\
156 \\
165\end{array}$ \\
\hline
\end{tabular}

\begin{tabular}{|c|c|c|c|c|c|}
\hline \multicolumn{6}{|c|}{ Well 18} \\
\hline $\begin{array}{l}\text { Surface soil } \\
\text { Caliche. } \\
\text { Clay } \\
\text { Brown sand } \\
\text { Blue shale. } \\
\text { Sand, water }\end{array}$ & $\begin{array}{r}3 \\
42 \\
15 \\
30 \\
5 \\
25\end{array}$ & $\begin{array}{r}3 \\
45 \\
60 \\
90 \\
95 \\
120\end{array}$ & $\begin{array}{l}\text { Blue shale. } \\
\text { Sand, water. } \\
\text { Gravel } \\
\text { Red beds } \\
\text { No record }\end{array}$ & $\begin{array}{r}5 \\
25 \\
3 \\
12 \\
10\end{array}$ & $\begin{array}{l}125 \\
150 \\
153 \\
165 \\
175\end{array}$ \\
\hline
\end{tabular}

\section{Well 19}

\begin{tabular}{|c|c|c|c|c|c|}
\hline $\begin{array}{l}\text { Surface soil } \\
\text { Caliche } \\
\text { Clay } \\
\text { Brown shale } \\
\text { Brown sand. } \\
\text { Sand and gravel }\end{array}$ & $\begin{array}{r}3 \\
32 \\
5 \\
10 \\
15 \\
10\end{array}$ & $\begin{array}{r}3 \\
35 \\
40 \\
50 \\
65 \\
75\end{array}$ & $\begin{array}{l}\text { Sand } \\
\text { Blie shale } \\
\text { Hard sand. } \\
\text { Sand and gravel } \\
\text { Red beds. }\end{array}$ & $\begin{array}{r}25 \\
15 \\
10 \\
22 \\
8\end{array}$ & $\begin{array}{l}100 \\
115 \\
125 \\
147 \\
155\end{array}$ \\
\hline
\end{tabular}

\section{Well 21}

\begin{tabular}{|c|c|c|c|c|c|}
\hline $\begin{array}{l}\text { Surface soil } \\
\text { Caliehe... } \\
\text { Brown shale. } \\
\text { Sand and gravel } \\
\text { Sand. }\end{array}$ & $\begin{array}{r}4 \\
41 \\
15 \\
40 \\
10\end{array}$ & $\begin{array}{r}4 \\
45 \\
60 \\
100 \\
110\end{array}$ & $\begin{array}{l}\text { Blue shale } \\
\text { Sand and gravel } \\
\text { Bl.re shale. } \\
\text { Red beds }\end{array}$ & $\begin{array}{r}5 \\
30 \\
5 \\
10\end{array}$ & $\begin{array}{l}115 \\
145 \\
150 \\
160\end{array}$ \\
\hline
\end{tabular}

\section{Well 22}

\begin{tabular}{|c|c|c|c|c|c|}
\hline $\begin{array}{l}\text { Caliehe } \\
\text { Hard sand. } \\
\text { Gravel, water } \\
\text { Hard sand. }\end{array}$ & $\begin{array}{r}50 \\
5 \\
10 \\
5\end{array}$ & $\begin{array}{l}50 \\
55 \\
65 \\
70\end{array}$ & $\begin{array}{l}\text { Sand and gravel } \\
\text { Blue shale. } \\
\text { Red beds. }\end{array}$ & $\begin{array}{r}90 \\
3 \\
1\end{array}$ & $\begin{array}{l}160 \\
163 \\
164\end{array}$ \\
\hline
\end{tabular}

\section{Well 23}

\begin{tabular}{|c|c|c|c|c|c|}
\hline $\begin{array}{l}\text { Surface soil } \\
\text { Caliche } \\
\text { Sand, dry } \\
\text { Hard sand, dry } \\
\text { Sand and gravel, water. }\end{array}$ & $\begin{array}{r}4 \\
31 \\
8 \\
7 \\
65\end{array}$ & $\begin{array}{r}4 \\
35 \\
43 \\
50 \\
115\end{array}$ & $\begin{array}{l}\text { Sand, water } \\
\text { Sand and gravel } \\
\text { Sand. } \\
\text { Blue shale. } \\
\text { Red rock. }\end{array}$ & $\begin{array}{r}5 \\
20 \\
11 \\
\mathbf{3} \\
\mathbf{2}\end{array}$ & $\begin{array}{l}120 \\
140 \\
151 \\
154 \\
156\end{array}$ \\
\hline
\end{tabular}

\footnotetext{
$947535-51-5$
} 


\begin{tabular}{|c|c|c|c|c|c|}
\hline & $\begin{array}{c}\text { Thickness } \\
\text { (feet) }\end{array}$ & $\begin{array}{l}\text { Depth } \\
\text { (feet) }\end{array}$ & & $\begin{array}{c}\text { Thickness } \\
\text { (feet) }\end{array}$ & $\begin{array}{l}\text { Depth } \\
\text { (feet) }\end{array}$ \\
\hline \multicolumn{6}{|c|}{ Well 24} \\
\hline $\begin{array}{l}\text { Surface soil } \\
\text { Caliche } \\
\text { Sand } \\
\text { Sand and gravel. }\end{array}$ & $\begin{array}{r}3 \\
47 \\
15 \\
68\end{array}$ & $\begin{array}{r}3 \\
50 \\
65 \\
133\end{array}$ & $\begin{array}{l}\text { Blue shale } \\
\text { Gravel. } \\
\text { Red beds }\end{array}$ & $\begin{array}{r}14 \\
7 \\
2\end{array}$ & $\begin{array}{l}147 \\
154 \\
156\end{array}$ \\
\hline \multicolumn{6}{|c|}{ Well 25} \\
\hline $\begin{array}{l}\text { Surface soil } \\
\text { Caliche } \\
\text { Sand and gravel }\end{array}$ & $\begin{array}{r}3 \\
52 \\
95\end{array}$ & $\begin{array}{r}3 \\
55 \\
150\end{array}$ & $\begin{array}{l}\text { Brown sand } \\
\text { Blue shale } \\
\text { Red rock }\end{array}$ & $\begin{array}{l}8 \\
4 \\
2\end{array}$ & $\begin{array}{l}158 \\
162 \\
164\end{array}$ \\
\hline
\end{tabular}

\begin{tabular}{|c|c|c|c|c|c|}
\hline $\begin{array}{l}\text { Surface soil } \\
\text { Caliche } \\
\text { White sand } \\
\text { Red sand }\end{array}$ & $\begin{array}{r}3 \\
37 \\
10 \\
10\end{array}$ & $\begin{array}{r}3 \\
40 \\
50 \\
60\end{array}$ & $\begin{array}{l}\text { Sand and gravel } \\
\text { Brown sand } \\
\text { Blie ghale } \\
\text { Red beds }\end{array}$ & $\begin{array}{r}80 \\
28 \\
2 \\
4\end{array}$ & $\begin{array}{l}140 \\
168 \\
170 \\
174\end{array}$ \\
\hline
\end{tabular}

\begin{tabular}{|c|c|c|c|c|c|}
\hline \multicolumn{6}{|c|}{ Well 27} \\
\hline $\begin{array}{l}\text { Surface soil } \\
\text { Caliche } \\
\text { White sand }\end{array}$ & $\begin{array}{r}3 \\
37 \\
10\end{array}$ & $\begin{array}{r}3 \\
40 \\
50\end{array}$ & $\begin{array}{l}\text { Sand and gravel } \\
\text { Blue shale. } \\
\text { Red beds }\end{array}$ & $\begin{array}{r}102 \\
4 \\
2\end{array}$ & $\begin{array}{l}152 \\
156 \\
158\end{array}$ \\
\hline
\end{tabular}

Well 28

\begin{tabular}{|c|c|c|c|c|c|}
\hline $\begin{array}{l}\text { Caliche } \\
\text { Rock } \\
\text { Caliche } \\
\text { Rock }\end{array}$ & $\begin{array}{r}28 \\
17 \\
25 \\
5\end{array}$ & $\begin{array}{l}28 \\
45 \\
70 \\
75\end{array}$ & $\begin{array}{l}\text { Sand and gravel } \\
\text { Gravel } \\
\text { Blue shale. } \\
\text { Red beds }\end{array}$ & $\begin{array}{r}15 \\
55 \\
1 \\
4\end{array}$ & $\begin{array}{r}90 \\
145 \\
146 \\
150\end{array}$ \\
\hline
\end{tabular}

Well 29

\begin{tabular}{|c|c|c|c|c|c|}
\hline $\begin{array}{l}\text { Surface soil } \\
\text { Hard caliche } \\
\text { Caliche } \\
\text { Gravel, water }\end{array}$ & $\begin{array}{r}15 \\
15 \\
15 \\
5\end{array}$ & $\begin{array}{l}15 \\
30 \\
45 \\
50\end{array}$ & $\begin{array}{l}\text { Yellow clay } \\
\text { Sand, water } \\
\text { Blue shale } \\
\text { Red beds.... }\end{array}$ & $\begin{array}{r}8 \\
72 \\
18 \\
2\end{array}$ & $\begin{array}{r}58 \\
130 \\
148 \\
150\end{array}$ \\
\hline
\end{tabular}

\section{Well 30}

Surface soil.

Sand, dry.

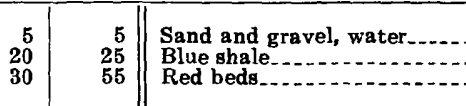

\begin{tabular}{r|r}
97 & 152 \\
4 & 156 \\
4 & 160
\end{tabular}

\section{Well 32}

Surface soil

Hard caliche

Sand, dry

\begin{tabular}{r|}
5 \\
20 \\
40
\end{tabular}

\begin{tabular}{r|}
5 \\
25 \\
65
\end{tabular}
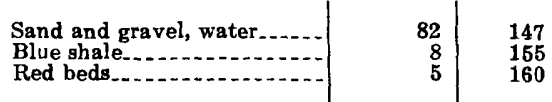

Well 33

\begin{tabular}{|c|c|c|c|c|c|}
\hline $\begin{array}{l}\text { Caliche } \\
\text { Hard sand } \\
\text { Sand and gravel, water }\end{array}$ & $\begin{array}{l}15 \\
40 \\
85\end{array}$ & $\begin{array}{r}15 \\
55 \\
140\end{array}$ & $\begin{array}{l}\text { Blue shale } \\
\text { Red beds. }\end{array}$ & $\begin{array}{l}2 \\
5\end{array}$ & $\begin{array}{l}142 \\
147\end{array}$ \\
\hline \multicolumn{6}{|c|}{ Well 34} \\
\hline $\begin{array}{l}\text { Caliche } \\
\text { Sand } \\
\text { Sand and gravel, water. }\end{array}$ & $\begin{array}{l}35 \\
25 \\
79\end{array}$ & $\begin{array}{r}35 \\
60 \\
139\end{array}$ & $\begin{array}{l}\text { Blue shale. } \\
\text { Red beds....... }\end{array}$ & $\begin{array}{l}6 \\
5\end{array}$ & $\begin{array}{l}145 \\
150\end{array}$ \\
\hline
\end{tabular}




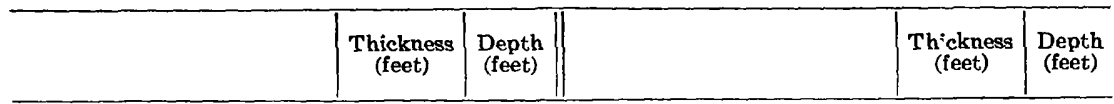

Well 35

\begin{tabular}{|c|c|c|c|c|c|}
\hline $\begin{array}{l}\text { Caliche } \\
\text { Yellow sand } \\
\text { Sand and gravel, water }\end{array}$ & $\begin{array}{l}30 \\
25 \\
75\end{array}$ & $\begin{array}{r}30 \\
55 \\
130\end{array}$ & $\begin{array}{l}\text { Blue shale } \\
\text { Red beds. }\end{array}$ & $\begin{array}{l}\mathbf{5} \\
\mathbf{5}\end{array}$ & $\begin{array}{l}135 \\
140\end{array}$ \\
\hline
\end{tabular}

\begin{tabular}{|c|c|c|c|c|c|}
\hline \multicolumn{6}{|c|}{ Well 36} \\
\hline $\begin{array}{l}\text { Caliche } \\
\text { Hard sand } \\
\text { Sand and gravel, water. }\end{array}$ & $\begin{array}{r}35 \\
20 \\
105\end{array}$ & $\begin{array}{r}35 \\
55 \\
160\end{array}$ & Blue shale & $\begin{array}{r}15 \\
5\end{array}$ & $\begin{array}{l}175 \\
180\end{array}$ \\
\hline
\end{tabular}

Well 37

\begin{tabular}{|c|c|c|c|c|c|}
\hline $\begin{array}{l}\text { Surface soil } \\
\text { Caliche. } \\
\text { Hard rock. } \\
\text { Yellow sand } \\
\text { Sand and gravel }\end{array}$ & $\begin{array}{r}4 \\
11 \\
20 \\
20 \\
25\end{array}$ & $\begin{array}{r}4 \\
15 \\
35 \\
55 \\
80\end{array}$ & $\begin{array}{l}\text { Sand } \\
\text { Hard brown lime. } \\
\text { Sand and gravel. } \\
\text { BIue shale } \\
\text { Red beds. }\end{array}$ & $\begin{array}{r}3 \\
15 \\
58 \\
5 \\
5\end{array}$ & $\begin{array}{r}83 \\
98 \\
156 \\
161 \\
166\end{array}$ \\
\hline
\end{tabular}

Well 40

\begin{tabular}{|c|c|c|c|c|c|}
\hline $\begin{array}{l}\text { Surface soil } \\
\text { Caliche. } \\
\text { Hard rock. } \\
\text { Hard yellow sand } \\
\text { Hard brown sand } \\
\text { White sand, water. }\end{array}$ & $\begin{array}{r}5 \\
11 \\
4 \\
30 \\
10 \\
30\end{array}$ & $\begin{array}{r}5 \\
16 \\
20 \\
50 \\
60 \\
90\end{array}$ & $\begin{array}{l}\text { Hard brown sand } \\
\text { Hard rock } \\
\text { Blue shale. } \\
\text { Hard brown sand } \\
\text { Red beds.......... }\end{array}$ & $\begin{array}{l}10 \\
10 \\
10 \\
10 \\
10\end{array}$ & $\begin{array}{l}100 \\
110 \\
120 \\
130 \\
140\end{array}$ \\
\hline
\end{tabular}

Well 41

\begin{tabular}{|c|c|c|c|c|c|}
\hline $\begin{array}{l}\text { Surface soil } \\
\text { Sand. } \\
\text { Caliche sand } \\
\text { Brown sandy lime } \\
\text { Hard sandy }\end{array}$ & $\begin{array}{r}5 \\
\mathbf{1 5} \\
\mathbf{3 0} \\
\mathbf{1 5} \\
\mathbf{5}\end{array}$ & $\begin{array}{l}\mathbf{5} \\
20 \\
\mathbf{5 0} \\
\mathbf{6 5} \\
70\end{array}$ & $\begin{array}{l}\text { Hard lime } \\
\text { Gravel and sand, water } \\
\text { Blue shale } \\
\text { Gravel and sand. } \\
\text { Red beds....... }\end{array}$ & $\begin{array}{r}10 \\
45 \\
5 \\
35 \\
5\end{array}$ & $\begin{array}{r}80 \\
125 \\
130 \\
165 \\
170\end{array}$ \\
\hline
\end{tabular}

Well 42

\begin{tabular}{|c|c|c|c|c|c|}
\hline $\begin{array}{l}\text { Surface soil } \\
\text { Caliche } \\
\text { Yellow sand }\end{array}$ & $\begin{array}{r}3 \\
\mathbf{3 7} \\
\mathbf{3 0}\end{array}$ & $\begin{array}{r}3 \\
40 \\
70\end{array}$ & $\begin{array}{l}\text { Gravel and sand } \\
\text { Blue shale. } \\
\text { Red beds }\end{array}$ & $\begin{array}{r}85 \\
10 \\
5\end{array}$ & $\begin{array}{l}155 \\
165 \\
170\end{array}$ \\
\hline
\end{tabular}

Well 43

\begin{tabular}{|c|c|c|c|c|c|}
\hline $\begin{array}{l}\text { Caliche } \\
\text { Yellow sand and gravel } \\
\text { Hard white sand }\end{array}$ & $\begin{array}{l}\mathbf{5 5} \\
\mathbf{2 5} \\
\mathbf{2 0}\end{array}$ & $\begin{array}{r}55 \\
80 \\
100\end{array}$ & $\begin{array}{l}\text { Sand } \\
\text { Blue } \\
\text { Red beds. }\end{array}$ & $\begin{array}{r}15 \\
10 \\
5\end{array}$ & $\begin{array}{l}115 \\
125 \\
130\end{array}$ \\
\hline
\end{tabular}

Well 44

\begin{tabular}{|c|c|c|c|c|c|}
\hline $\begin{array}{l}\text { Surface soil } \\
\text { Caliche. } \\
\text { Hard sand. } \\
\text { Gravel, water } \\
\text { Hard red sand. }\end{array}$ & $\begin{array}{l}5 \\
\mathbf{3 5} \\
20 \\
15 \\
15\end{array}$ & $\begin{array}{r}5 \\
40 \\
60 \\
75 \\
90\end{array}$ & $\begin{array}{l}\text { Gravel and sand } \\
\text { Blue shale... } \\
\text { Gravel } \\
\text { Blue shale... } \\
\text { Red beds... }\end{array}$ & $\begin{array}{r}45 \\
10 \\
20 \\
5 \\
5\end{array}$ & $\begin{array}{l}135 \\
145 \\
165 \\
170 \\
175\end{array}$ \\
\hline
\end{tabular}

Well 45

\begin{tabular}{|c|c|c|c|c|c|}
\hline $\begin{array}{l}\text { Surface soil } \\
\text { Hard caliche } \\
\text { Hard sand. } \\
\text { Gravel, water. }\end{array}$ & $\begin{array}{r}4 \\
56 \\
15 \\
15\end{array}$ & $\begin{array}{r}4 \\
60 \\
75 \\
90\end{array}$ & $\begin{array}{l}\text { Hard sand. } \\
\text { Gravel, nater. } \\
\text { Red beds. }\end{array}$ & $\begin{array}{r}20 \\
65 \\
5\end{array}$ & $\begin{array}{l}110 \\
175 \\
180\end{array}$ \\
\hline
\end{tabular}




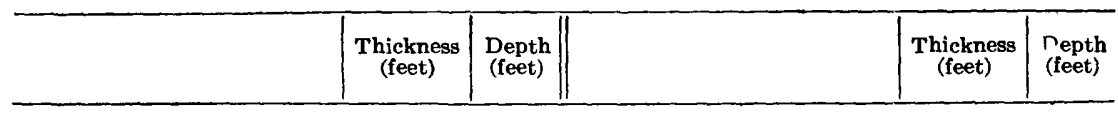

\begin{tabular}{|c|c|c|c|c|c|}
\hline \multicolumn{6}{|c|}{ Well 46} \\
\hline $\begin{array}{l}\text { Surface soil } \\
\text { Hard caliche } \\
\text { Yellow sand } \\
\text { Gravel, water }\end{array}$ & $\begin{array}{r}2 \\
48 \\
25 \\
50\end{array}$ & $\begin{array}{r}2 \\
50 \\
75 \\
125\end{array}$ & $\begin{array}{l}\text { Sand and gravel } \\
\text { Blue shale. } \\
\text { Gravel } \\
\text { Red beds. }\end{array}$ & $\begin{array}{r}30 \\
10 \\
10 \\
5\end{array}$ & $\begin{array}{l}155 \\
165 \\
175 \\
180\end{array}$ \\
\hline
\end{tabular}

\begin{tabular}{|c|c|c|c|c|c|}
\hline \multicolumn{6}{|c|}{ Well 47} \\
\hline $\begin{array}{l}\text { Surface soil } \\
\text { Caliche } \\
\text { White sand } \\
\text { Rock } \\
\text { Brown sand }\end{array}$ & $\begin{array}{r}4 \\
12 \\
29 \\
10 \\
55\end{array}$ & $\begin{array}{r}4 \\
16 \\
45 \\
55 \\
110\end{array}$ & $\begin{array}{l}\text { Blue shale } \\
\text { Brown sand } \\
\text { Blue shale } \\
\text { Red beds }\end{array}$ & $\begin{array}{r}15 \\
15 \\
5 \\
5\end{array}$ & $\begin{array}{l}125 \\
140 \\
145 \\
150\end{array}$ \\
\hline
\end{tabular}

\section{Well 48}

\begin{tabular}{|c|c|c|c|c|c|}
\hline $\begin{array}{l}\text { Surface soil } \\
\text { Caliche } \\
\text { Hard rock } \\
\text { Sand and gravel, water. } \\
\text { Sand, water. }\end{array}$ & $\begin{array}{r}2 \\
18 \\
35 \\
10 \\
10\end{array}$ & $\begin{array}{l}2 \\
20 \\
55 \\
65 \\
75\end{array}$ & $\begin{array}{l}\text { White sand } \\
\text { Brown sand } \\
\text { Blue shale. } \\
\text { Red beds. }\end{array}$ & $\begin{array}{r}20 \\
25 \\
5 \\
5\end{array}$ & $\begin{array}{r}95 \\
120 \\
125 \\
130\end{array}$ \\
\hline
\end{tabular}

\begin{tabular}{|c|c|c|c|c|c|}
\hline \multicolumn{6}{|c|}{ Well 49} \\
\hline $\begin{array}{l}\text { Surface soil } \\
\text { Caliche } \\
\text { Brown sand }\end{array}$ & $\begin{array}{r}5 \\
30 \\
7\end{array}$ & $\begin{array}{r}5 \\
35 \\
42\end{array}$ & $\begin{array}{l}\text { Sand, water } \\
\text { Blue shale }\end{array}$ & $\begin{array}{r}70 \\
8\end{array}$ & $\begin{array}{l}112 \\
120\end{array}$ \\
\hline
\end{tabular}

Well 50

\begin{tabular}{|c|c|c|c|c|c|}
\hline $\begin{array}{l}\text { Surface soil. } \\
\text { Caliche } \\
\text { Brown sand. }\end{array}$ & $\begin{array}{r}5 \\
21 \\
10\end{array}$ & $\begin{array}{r}5 \\
26 \\
36\end{array}$ & $\begin{array}{l}\text { Sand, nater } \\
\text { Bli.e shale. } \\
\text { Red keds...... }\end{array}$ & $\begin{array}{r}72 \\
8 \\
4\end{array}$ & $\begin{array}{l}108 \\
116 \\
120\end{array}$ \\
\hline
\end{tabular}

Well 51

\begin{tabular}{l|r|r|r|r|r}
\hline Surface soil & 5 \\
Caliche & 5 & 5 \\
Brown sand & 30 \\
\end{tabular}

Well 52

\begin{tabular}{|c|c|c|c|c|c|}
\hline $\begin{array}{l}\text { Surface soil } \\
\text { Caliche } \\
\text { Red sand.... }\end{array}$ & $\begin{array}{r}5 \\
30 \\
6\end{array}$ & $\begin{array}{r}5 \\
35 \\
41\end{array}$ & $\begin{array}{l}\text { Sand, water } \\
\text { Blue shale } \\
\text { Red beds......... }\end{array}$ & $\begin{array}{r}77 \\
7 \\
3\end{array}$ & $\begin{array}{l}118 \\
125 \\
128\end{array}$ \\
\hline
\end{tabular}

\section{Well 53}

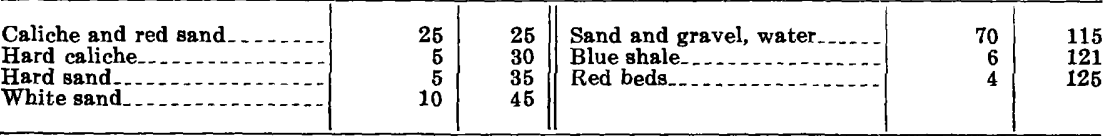

\section{Well 54}

\begin{tabular}{|c|c|c|c|c|c|}
\hline $\begin{array}{l}\text { Caliche } \\
\text { Red sand } \\
\text { Sand and gravel, water }\end{array}$ & $\begin{array}{l}25 \\
15 \\
72\end{array}$ & $\begin{array}{r}25 \\
40 \\
112\end{array}$ & $\begin{array}{l}\text { Blue shale } \\
\text { Red beds. }\end{array}$ & $\begin{array}{l}\mathbf{3} \\
\mathbf{5}\end{array}$ & $\begin{array}{l}115 \\
120\end{array}$ \\
\hline
\end{tabular}




\section{EDWARDS COUNTY}

Population in 1940: 1,339.

\section{ROCKSPRINGS}

Source of information: T. Osborne, water superintendent, Sept. 20, 1948.

Ownership: Municipal.

Source of supply: Three wells four blocks south of city hall.

West well. Drilled about 1928; depth, 475 feet; diameter, 8 inches; deepwell turbine pump and 15-horsepower electric motor; static water level reported, 426 feet below land surface in 1945; yield, 100 gallons a minute.

East well. About 65 feet from west well; drilled in 1931 by Layne-Texas Co.; depth, 602 feet; diameter, 8 inches; deep-well turbine pump and 10-horsepower electric motor; static water level, $443 \mathrm{fee}^{+}$below land surface in 1946; yield, 45 gallons a minute.

New well. About 125 feet east of east well; drilled in 1946 by Thompson and Carr; depth, 480 feet; diameter, 10 inches; deep-well turbine pump and 15-horsepower electric motor; static water level, 426 fe t below land surface in 1946 ; yield, 60 gallons a minute.

Pumpage (estimated) : 200,000 gallons a day.

Storage: Concrete ground storage reservoir, 150,000 gallons; $e^{\top}$ evated tank, 50,000 gallons.

Number of customers: 370 .

Treatment: Chlorination.

Analyses

[Collected Sept. 20, 1948. pH of each nell is 7.6. Analyzed by D. E. Weaver]

\begin{tabular}{|c|c|c|c|c|c|c|}
\hline & \multicolumn{2}{|c|}{ West well } & \multicolumn{2}{|c|}{ East well } & \multicolumn{2}{|c|}{ New well } \\
\hline & $\begin{array}{c}\text { Parts } \\
\text { per } \\
\text { million }\end{array}$ & $\begin{array}{l}\text { Equiva- } \\
\text { lents per } \\
\text { million }\end{array}$ & $\begin{array}{c}\text { Parts } \\
\text { per } \\
\text { million }\end{array}$ & $\begin{array}{l}\text { Equiva- } \\
\text { lents per } \\
\text { million }\end{array}$ & $\begin{array}{l}\text { Parts } \\
\text { pe* } \\
\text { mill on }\end{array}$ & $\begin{array}{l}\text { Equiva- } \\
\text { lents per } \\
\text { million }\end{array}$ \\
\hline $\begin{array}{l}\text { Silica }\left(\mathrm{SiO}_{2}\right) \\
\text { Iron }(\mathrm{Fe}) \\
\text { Calcium }(\mathrm{Ca}) \\
\text { Magnesium }(\mathrm{M} \mathrm{M}) \\
\text { Sodium }(\mathrm{Na}) \\
\text { Potassium }(\mathrm{K}) \\
\text { Bicarbonate }\left(\mathrm{HCO}_{3}\right) \\
\text { Sulfate }\left(\mathrm{SO}_{4}\right) \\
\text { Chloride }(\mathrm{Cl}) \\
\text { Fluoride }(\mathrm{F}) \\
\text { Nitrate (NO } \\
\text { Dissol ed solids. } \\
\text { Total hardness as } \mathrm{CaCO}_{3}\end{array}$ & $\begin{array}{l}13 \\
48 \\
16 \\
4.05 \\
2.8 \\
208.8 \\
7.7 \\
11.4 \\
3.2 \\
215 \\
186\end{array}$ & $\begin{array}{r}2.396 \\
1.316 \\
.041 \\
.072 \\
3.409 \\
.160 \\
.310 \\
.021 \\
.052 \\
\end{array}$ & $\begin{array}{c}12 \\
47 \\
4.05 \\
13 \\
4.9 \\
3.2 \\
216 \\
9.5 \\
10.5 \\
2.8 \\
215 \\
191\end{array}$ & $\begin{array}{r}2.346 \\
1.480 \\
.034 \\
.082 \\
3.541 \\
.198 \\
.282 \\
.021 \\
.045 \\
\end{array}$ & $\begin{array}{l}12.05 \\
47^{\circ} \\
16 \\
5.2 \\
2.8 \\
20 \varepsilon \\
7.2 \\
11.2 \\
20 \varepsilon^{2.5} \\
18 \varepsilon\end{array}$ & $\begin{array}{r}\mathbf{2} .346 \\
1.316 \\
.080 \\
.072 \\
\mathbf{3} .409 \\
.150 \\
.310 \\
.021 \\
.040 \\
\end{array}$ \\
\hline
\end{tabular}

\section{EL PASO COUNTY}

\section{EI PASO}

Population in 1940: 96,810 .

Source of information: E. J. Umbenhauer, water superintendent, April 1947. Ownership: Municipal.

Source of supply: Fifteen wells and Rio Grande.

Well 3. Montana and Chelsea Streets; drilled in 1922 by Layne-Texas Co.; dep'h, 862 feet; diameter, 26 inches; deep-well turbiro pump and 100-horsepower electric motor; static water level, 113.62 feet below land surface February 31, 1944; drawdown, 40.69 feet after pumping 100 hours, July 3, 1936; yield, 1,250 gallons a minute. 
Well 4. Madison and White Oak Streets; drilled in $1924 \mathrm{by}$ Layne-Texas

Co.; depth, 882 feet; diameter, 24 inches; deep-well turtine pump and 100-horsepower electric motor; static water level, 70.11 feet below land surface February 1944; drawdown, 75 feet when pumping 730 gallons a minute; yield, 730 gallons a minute.

Well 8. One mile north of Mesa Pumping Plant; drilled in 1928 by LayneTexas Co.; depth, 715 feet; diameter, 20 inches; deep-well turbine pump and 150-horsepower electric motor; static water level, 207.58 feet below land surface February 1944 ; yield, 1,370 gallons a minute; temperature, $82^{\circ} \mathrm{F}$.

Well 9. Luna and Pera Streets; drilled in 1928; depth, 802 feet; diameter, 24 inches; deep-well turbine pump and 50-horsepower electric motor; static water level, 31.44 feet below land surface April 1944; yield, 700 gallons a minute.

Well 11. 1.7 miles east of Mesa Pumping Plant; drilled in 1930 by C. R. Jensen; depth, 736 feet; diameter, 20 inches; deep-well turbine pump and 150-horsepower electric motor; static water level, 206.03 feet below land surface May 1, 1939; yield, 1,170 gallons a minute.

Well 14. San Antonio and Walnut Streets; drilled in 1937 by LayneTexas Co.; depth, 703 feet; diameter, 36 inches; deep-well turbine pump and 100-horsepower electric motor; static water level, 32.78 feet below land surface July 7, 1939; drawdown, 31.77 feet when pumping 1,500 gallons a minute in 1937; yield, 1,500 gallons a minute.

Well 15. About 1 mile east of Mesa Pumping Plant; drillad in 1938 by Layne-Texas Co.; depth, 1,055 feet; diameter, 24 incl is; deep-well turbine pump and electric motor; static water level, 222.28 feet below land surface when drilled; drawdown, 42.39 feet when rumping 1,800 gallons a minute; yield, 1,800 gallons a minute.

Well 17. San Antonio and Tornillo Streets; drilled in 1938 by C. R. Jensen; depth, 750 feet; diameter, 121/2 inches; deep-well turbine pump and electric motor; static water level, 39.13 feet below land surface February 1944 ; yield, 1,320 gallons a minute.

Well 18. Haddock Addition; drilled in 1938 by C. R. Jensen; depth, 902 feet; diameter, 24 inches; deep-well turbine pump and electric motor; static water level, 26.65 feet below land surface Feb. 28, 1940; drawdown, 59.5 feet when pumping 1,180 gallons a minute; yield, 1,180 gallons a minute.

Well 19. 1.3 miles east-southeast of Mesa Pumping Plant; drilled in 1940 by C. R. Jensen; depth, 425 feet; diameter, 24 inches; dee pump and electric motor; yield, 1,100 gallons a minute.

Well 20. One mile north of Mesa Well Field; drilled in $1: 41$ by LayneTexas Co.; depth, 909 feet; diameter, 24 inches; deep-well turbine pump and 250-horsepower electric motor; static water level, $20 € .48$ feet below land surface June 21, 1941; yield, 1,550 gallons a minute.

Well 21. Two miles north of Mesa Well Field; drilled in 1941 by LayneTexas Co.; depth, 806 feet; diameter, 24 inches; deep-well turbine pump and 250-horsepower electric motor; static water level, 196.91 feet below land surface June 25, 1941; drawdown, 51.09 feet after pumping 24 hours at a rate of 1,585 gallons a minute; yield, 1,585 gal'ons a minute. 
Shallow wells 1, 2, and 3. At Rio Grande surface water treating plant located along canal; depths, about 50 feet; diameters, 24 inches; deepwell turbine pumps and electric motors; combined yield, about 3,000,000 gallons a day; water pumped to treating plant.

Surface water. River treating plant for taking surface water from canal on Rio Grande has a capacity of $10,000,000$ gallons a dar.

Average pumpage in gallons a day

\begin{tabular}{|c|c|c|c|}
\hline & \multicolumn{2}{|c|}{ Ground water } & \multirow{2}{*}{$\begin{array}{l}\text { Surface water } \\
\text { Rio Grande }\end{array}$} \\
\hline & Deep wells & Shallow wells & \\
\hline $\begin{array}{l}19445456 \\
1946 \\
1947\end{array}$ & $\begin{array}{r}9,400,000 \\
10,610,000 \\
10,800,000 \\
8,500,000\end{array}$ & $2,000,000$ (est.) & $\begin{array}{r}\mathbf{3}, 940,000 \\
4,110,000 \\
\mathbf{5}, 120,000 \\
\end{array}$ \\
\hline
\end{tabular}

Storage: Seven ground reservoirs, total capacity 70,000,000 gallons; elevated tank, 50,000 gallons.

Number of customers: 18,500 .

Treatment: Well water, chlorination; surface water, screening, grit separation, chlorination, aeration. primary settling, secondary chlcrination, coagulation with activated carbon, soda, ash and lime, flash mixing, flocculation, secondary settling, carbonation, and filtration.

\section{Analyses}

[Collected Apr. 21 and 22, 1949. Analyzed by D. E. Weaver and J. R. Avrett]

\begin{tabular}{|c|c|c|c|c|}
\hline & \multicolumn{2}{|c|}{ Well 3} & \multicolumn{2}{|c|}{ Well 4} \\
\hline & $\begin{array}{l}\text { Parts per } \\
\text { million }\end{array}$ & $\begin{array}{l}\text { Equivalents } \\
\text { per million }\end{array}$ & $\begin{array}{c}\text { Parts per } \\
\text { million }\end{array}$ & $\begin{array}{c}\text { Equivalents } \\
\text { per million }\end{array}$ \\
\hline \multirow[t]{3}{*}{ 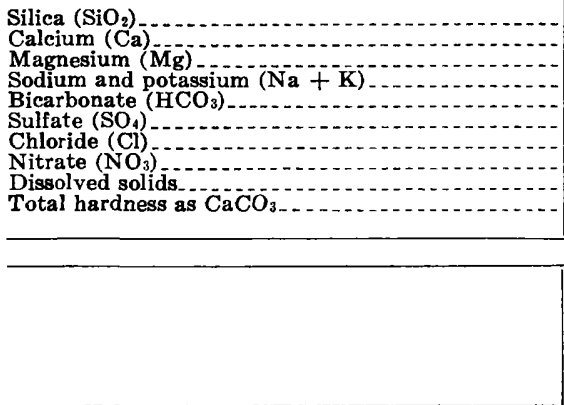 } & $\begin{array}{c}36 \\
56 \\
21 \\
172 \\
161 \\
76 \\
274 \\
2.2 \\
743 \\
226\end{array}$ & \begin{tabular}{r}
2.80 \\
1.73 \\
$\mathbf{7} .46$ \\
2.64 \\
1.58 \\
7.73 \\
.04 \\
\hdashline \\
\end{tabular} & 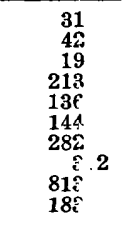 & \begin{tabular}{r}
2.10 \\
1.56 \\
9.26 \\
2.23 \\
3.00 \\
7.95 \\
.05 \\
\hdashline \\
\end{tabular} \\
\hline & \multicolumn{2}{|c|}{ Well 8} & \multicolumn{2}{|c|}{ Well 9} \\
\hline & $\begin{array}{l}\text { Parts per } \\
\text { million }\end{array}$ & $\begin{array}{l}\text { Equivalents } \\
\text { per million }\end{array}$ & $\begin{array}{l}\text { Parts per } \\
\text { millicn }\end{array}$ & $\begin{array}{l}\text { Equivalents } \\
\text { per million }\end{array}$ \\
\hline 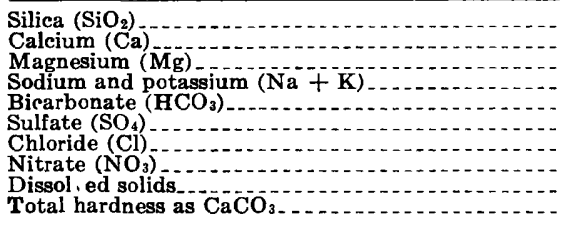 & $\begin{array}{r}42 \\
45 \\
18 \\
55 \\
198 \\
64 \\
64 \\
48 \\
11 \\
386 \\
186\end{array}$ & \begin{tabular}{r}
2.25 \\
1.48 \\
2.38 \\
3.25 \\
1.33 \\
1.35 \\
.18 \\
\hdashline \\
\end{tabular} & $\begin{array}{c}32 . \\
18 \\
7.1 \\
76 \\
181 \\
79 \\
135 \\
6 \\
524 \\
76\end{array}$ & $\begin{array}{r}0.95 \\
.58 \\
6.96 \\
2.97 \\
1.60 \\
\mathbf{3} .92 \\
.00 \\
\end{array}$ \\
\hline
\end{tabular}




\begin{tabular}{|c|c|c|c|c|}
\hline & \multicolumn{2}{|c|}{ Well 12} & \multicolumn{2}{|c|}{ Well 15} \\
\hline & $\begin{array}{l}\text { Parts per } \\
\text { million }\end{array}$ & $\begin{array}{l}\text { Equivalents } \\
\text { per million }\end{array}$ & $\begin{array}{l}\text { Parts por } \\
\text { millior }\end{array}$ & $\begin{array}{l}\text { Equivalents } \\
\text { fer million }\end{array}$ \\
\hline \multirow[t]{3}{*}{ 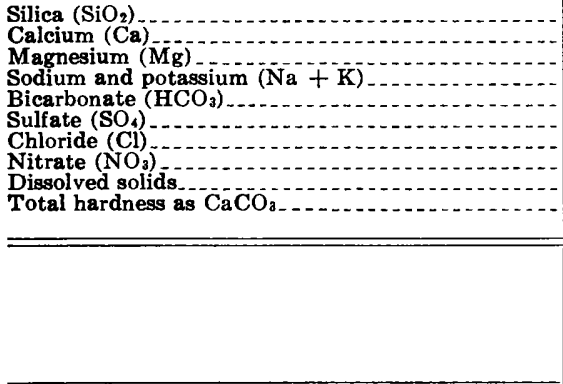 } & $\begin{array}{r}37 \\
43 \\
14 \\
60 \\
198 \\
55 \\
50 \\
6.0 \\
372 \\
160\end{array}$ & $\begin{array}{r}2.14 \\
1.15 \\
2.62 \\
3.25 \\
1.15 \\
1.41 \\
.10\end{array}$ & $\begin{array}{l}36 \\
24 \\
10 \\
129 \\
197 \\
91 \\
86 \\
4.4 \\
482 \\
101\end{array}$ & \begin{tabular}{r}
1.20 \\
.82 \\
5.60 \\
3.23 \\
1.89 \\
2.43 \\
.07 \\
\hdashline \\
\end{tabular} \\
\hline & \multicolumn{2}{|c|}{ Well 17} & \multicolumn{2}{|c|}{ Well 18} \\
\hline & $\begin{array}{c}\text { Parts per } \\
\text { million }\end{array}$ & $\begin{array}{c}\text { Equivalents } \\
\text { per million }\end{array}$ & $\begin{array}{c}\text { Parts per } \\
\text { million }\end{array}$ & $\begin{array}{l}\text { Equivalents } \\
\text { per million }\end{array}$ \\
\hline \multirow[t]{3}{*}{ 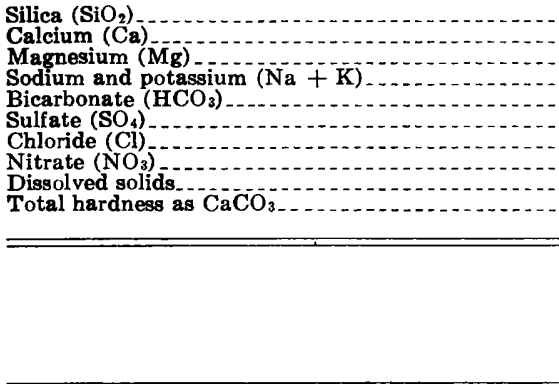 } & 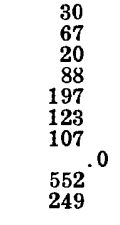 & $\begin{array}{r}\mathbf{3} . \mathbf{3 4} \\
\mathbf{1} .64 \\
\mathbf{3} .83 \\
\mathbf{3 . 2 3} \\
\mathbf{2 . 5 6} \\
\mathbf{3 . 0 2} \\
.00 \\
\hdashline\end{array}$ & $\begin{array}{r}29 \\
55 \\
21 \\
146 \\
159 \\
89 \\
225 \\
{ }^{675} \\
224\end{array}$ & $\begin{array}{r}2.75 \\
1.73 \\
6.33 \\
2.61 \\
1.85 \\
6.35 \\
.00 \\
\hdashline-0\end{array}$ \\
\hline & \multicolumn{2}{|c|}{ Well 19} & \multicolumn{2}{|c|}{ Well 20} \\
\hline & $\begin{array}{l}\text { Parts per } \\
\text { million }\end{array}$ & $\begin{array}{c}\text { Equivalents } \\
\text { per million }\end{array}$ & $\begin{array}{l}\text { Parts per } \\
\text { million }\end{array}$ & $\begin{array}{l}\text { Equivalents } \\
\text { per million }\end{array}$ \\
\hline $\begin{array}{l}\text { Silica }\left(\mathrm{SiO}_{2}\right) \\
\text { Calcium }(\mathrm{Ca}) \\
\text { Magnesium }(\overline{\mathrm{Mg}}) \\
\text { Sodium and potassium }(\mathrm{Na}+\mathrm{K}) \\
\text { Bicarbonate }\left(\mathrm{HCO}_{3}\right) \\
\left.\text { Sulfate ( } \mathrm{SO}_{4}\right) \\
\text { Chloride }(\mathrm{Cl}) \\
\text { Nitrate }\left(\mathrm{NO}_{3}\right) \\
\text { Dissolved solids } \\
\text { Total hardness as } \mathrm{CaCO} \mathrm{CO}_{3}\end{array}$ & $\begin{array}{r}40 \\
24 \\
9.8 \\
134 \\
199 \\
103 \\
83 \\
5.6 \\
502 \\
100\end{array}$ & $\begin{array}{r}1.20 \\
.81 \\
5.82 \\
3.26 \\
2.14 \\
2.34 \\
.09 \\
-2 .--\end{array}$ & $\begin{array}{r}29 \\
34 \\
13 \\
43 \\
178 \\
37 \\
30 \\
7.2 \\
288 \\
138\end{array}$ & \begin{tabular}{r}
1.70 \\
1.07 \\
1.89 \\
2.92 \\
.77 \\
.85 \\
.12 \\
\hdashline \\
\end{tabular} \\
\hline
\end{tabular}

Drillers' logs

\begin{tabular}{|c|c|c|c|c|c|}
\hline & $\underset{\text { (feet) }}{\text { Thickness }}$ & $\begin{array}{l}\text { Depth } \\
\text { (feet) }\end{array}$ & & $\begin{array}{c}\text { Thickness } \\
\text { (feet) }\end{array}$ & $\begin{array}{c}\text { Depth } \\
\text { (feet) }\end{array}$ \\
\hline \multicolumn{6}{|c|}{ Well 11} \\
\hline $\begin{array}{l}\text { Soil } \\
\text { Caliche } \\
\text { Sand } \\
\text { Clay } \\
\text { Gravel } \\
\text { Clay } \\
\text { Sand } \\
\text { Clay } \\
\text { Sand } \\
\text { Clay } \\
\text { Sand } \\
\text { Clay } \\
\text { Sand } \\
\text { Slayd and boulders } \\
\text { Rock } \\
\text { Sand } \\
\text { Clay }\end{array}$ & $\begin{array}{r}4 \\
11 \\
55 \\
10 \\
20 \\
6 \\
58 \\
6 \\
30 \\
20 \\
40 \\
15 \\
33 \\
4 \\
38 \\
1 \\
17 \\
4\end{array}$ & $\begin{array}{r}4 \\
15 \\
70 \\
80 \\
100 \\
106 \\
164 \\
170 \\
200 \\
220 \\
260 \\
275 \\
308 \\
312 \\
350 \\
351 \\
368 \\
372\end{array}$ & $\begin{array}{l}\text { Sand and boulders } \\
\text { Clay,-. } \\
\text { Sand, gravel, and boulders. } \\
\text { Clay, } \\
\text { Rock., gravel, and boulders. } \\
\text { Sand, } \\
\text { Clay,- } \\
\text { Sand, gravel, and boulders. } \\
\text { Clay, gravel, and boulders. } \\
\text { Sand, } \\
\text { Clay.- gravel, and boulders. } \\
\text { Sand, } \\
\text { Sayd, gravel, and boulders. } \\
\text { Clay, } \\
\text { Sand, gravel, and boulders. } \\
\text { Sand, }\end{array}$ & $\begin{array}{r}38 \\
4 \\
54 \\
3 \\
1 \\
22 \\
5 \\
29 \\
5 \\
57 \\
12 \\
26 \\
3 \\
57 \\
4 \\
16 \\
50\end{array}$ & $\begin{array}{l}\mathbf{4 1 0} \\
\mathbf{4 1 4} \\
\mathbf{4 6 8} \\
\mathbf{4 7 1} \\
\mathbf{4 7 2} \\
\mathbf{4 9 4} \\
\mathbf{4 9 9} \\
528 \\
533 \\
590 \\
602 \\
\mathbf{6 2 8} \\
\mathbf{6 3 1} \\
688 \\
\mathbf{6 9 2} \\
\mathbf{7 0 8} \\
\mathbf{7 5 8}\end{array}$ \\
\hline
\end{tabular}




\begin{tabular}{|c|c|c|c|c|c|}
\hline & $\begin{array}{c}\text { Thickness } \\
\text { (feet) }\end{array}$ & $\begin{array}{l}\text { Depth } \\
\text { (feet) }\end{array}$ & & $\underset{(\text { feet })}{T h}$ & $\begin{array}{l}\text { Depth } \\
\text { (feet) }\end{array}$ \\
\hline \multicolumn{6}{|c|}{ Well 15} \\
\hline $\begin{array}{l}\text { Sandy soil. } \\
\text { Caliche. } \\
\text { Coarse sand and gravel } \\
\text { Clay } \\
\text { Sand } \\
\text { Clayers of sand and ci } \\
\text { Sandy } \\
\text { Clay } \\
\text { Sandy clay } \\
\text { Clay } \\
\text { Sand (water sample) } \\
\text { Clay } \\
\text { Sand } \\
\text { Clayd sand } \\
\text { Sand } \\
\text { Clay } \\
\text { Sand } \\
\text { Sandy clay } \\
\text { Sand } \\
\text { Clay } \\
\text { Sand (water sample) } \\
\text { Clay }\end{array}$ & $\begin{array}{r}2 \\
6 \\
70 \\
20 \\
20 \\
100 \\
8 \\
18 \\
14 \\
12 \\
17 \\
25 \\
15 \\
17 \\
6 \\
82 \\
15 \\
4 \\
10 \\
54 \\
18 \\
6 \\
9 \\
2\end{array}$ & $\begin{array}{r}2 \\
8 \\
78 \\
98 \\
118 \\
218 \\
226 \\
244 \\
258 \\
270 \\
287 \\
312 \\
327 \\
344 \\
350 \\
432 \\
447 \\
451 \\
461 \\
\mathbf{5 1 5} \\
\mathbf{5 3 3} \\
\mathbf{5 3 9} \\
\mathbf{5 4 8} \\
\mathbf{5 5 0}\end{array}$ & $\begin{array}{l}\text { Sand } \\
\text { Clay } \\
\text { Cland } \\
\text { Sand } \\
\text { Clay } \\
\text { Sand } \\
\text { Clay } \\
\text { Sandy clay } \\
\text { Sand } \\
\text { Clay } \\
\text { Sandy clay } \\
\text { Sandy clay } \\
\text { Sand } \\
\text { Clay (water sample) } \\
\text { Sand (static head, 226 ft.) } \\
\text { Clay } \\
\text { Sandy clay } \\
\text { Sand } \\
\text { Clay and boulders. } \\
\text { Sand } \\
\text { Sandy clay }\end{array}$ & \begin{tabular}{r|}
23 \\
27 \\
24 \\
3 \\
34 \\
16 \\
1 \\
1 \\
8 \\
12 \\
15 \\
27 \\
8 \\
10 \\
10 \\
44 \\
12 \\
21 \\
28 \\
15 \\
50 \\
10 \\
60 \\
25
\end{tabular} & $\begin{array}{r}573 \\
600 \\
624 \\
627 \\
651 \\
667 \\
668 \\
676 \\
688 \\
703 \\
730 \\
738 \\
748 \\
758 \\
802 \\
814 \\
835 \\
863 \\
878 \\
928 \\
938 \\
998 \\
1,023 \\
1,078\end{array}$ \\
\hline
\end{tabular}

Population in 1940: 2,100.

FABENS

Source of information: Mrs. L. W. Moore, Bookkeeper, April 9, 1948.

Owner: Fabens Water Co.

Source of supply: Three wells.

Well 1. Drilled in 1938 by Burdick and Burdick; depth, 218 feet; diameter, 6 inches; deep-well turbine pump and 15-horsepower electric motor; static water level, 26.24 feet below land surface July 8, 1938; yield, 200 gallons a minute.

Well 2. Drilled in 1938 by Burdick and Burdick; depth, 218 feet; diameter, 6 inches; deep-well turbine pump and 15-horsepower electric motor; yield, 200 gallons a minute.

Well 3. Drilled in May 1947; depth, 247 feet; diameter, 6 inches; deepwell turbine pump and electric motor; yield, 200 gallons a minute.

Pumpage: Average, 140,000 gallons a day.

Storage: Ground storage reservoir, 110,000 gallons; elevated tank, 50,000 gallons.

Number of customers: 590 .

Treatment: Chlorination.

Analyses

[Collected A pr. 9, 1948. pH: well 1, 8.1; well 3, 8.2. Analyzed by H. D. S nith]

\begin{tabular}{|c|c|c|c|c|}
\hline & \multicolumn{2}{|c|}{ Well 1} & \multicolumn{2}{|c|}{ Well 3} \\
\hline & $\begin{array}{l}\text { Parts per } \\
\text { million }\end{array}$ & $\begin{array}{l}\text { Equivalents } \\
\text { per million }\end{array}$ & $\begin{array}{l}\text { Parts fer } \\
\text { million }\end{array}$ & $\begin{array}{l}\text { Equivalents } \\
\text { per million }\end{array}$ \\
\hline 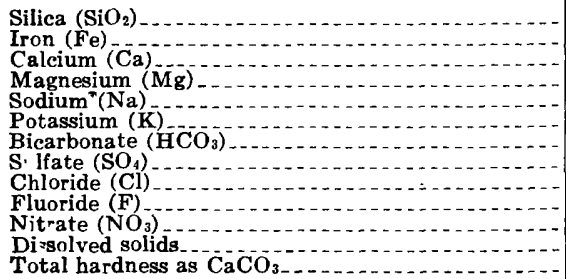 & $\begin{array}{l}31 \\
52 \\
12 \\
109 \\
10 \\
220 \\
115 \\
91 \\
.3 \\
529 \\
180\end{array}$ & $\begin{array}{r}0.60 \\
.99 \\
4.74 \\
.26 \\
3.61 \\
2.39 \\
2.57 \\
.02 \\
.00 \\
\hdashline .0\end{array}$ & $\begin{array}{l}32 \\
54 \\
12 \\
105 \\
8.4 \\
236 \\
110 \\
80 \\
.3 \\
2.5 \\
184\end{array}$ & $\begin{array}{r}2.70 \\
.99 \\
4.58 \\
.21 \\
3.87 \\
2.29 \\
2.26 \\
.02 \\
.04 \\
-0\end{array}$ \\
\hline
\end{tabular}


Population in 1940: 250.

TORNILLO

Source of information: O. T. Smith, owner, Aug. 3, 1948.

Owner: O. T. Smith.

Source of supply: Well drilled by Jack Dougherty; depth, 320 feet; diameter,

6 inches; Hi-lift pump and 3-horsepower electric motor; statis water level,

41.5 feet below land surface Aug. 3, 1948; yield, 50 gallons a minute.

Pumpage (estimated) : 30,000 gallons a day.

Storage: Elevated tank, 21,000 gallons.

Number of customers: 82.

Treatment: Chlorination.

Analysis

[Collected Sept. 14, 1948. pH is 7.8. Analyzed by H. D. Smith]

\begin{tabular}{|c|c|c|c|c|c|}
\hline & $\begin{array}{l}\text { Parts per } \\
\text { million }\end{array}$ & $\begin{array}{l}\text { Equivalent } \\
\text { I er million }\end{array}$ & & $\begin{array}{l}\text { Parta per } \\
\text { million }\end{array}$ & $\begin{array}{l}\text { Equivalents } \\
\text { per million }\end{array}$ \\
\hline $\begin{array}{l}\text { Silica }\left(\mathrm{SiO}_{2}\right) \\
\text { Iron }(\mathrm{Fe}) \\
\text { Calcium }(\mathrm{Ca}) \\
\text { Magnesium }(\mathrm{Mg}) \\
\text { Sodium }(\mathrm{Na}) \\
\text { Potassium }(\mathrm{K}) \\
\text { Bicarbonate }\left(\mathrm{H} \overline{\mathrm{CO}}_{3}\right)\end{array}$ & $\begin{array}{l}30 \\
120^{.30} \\
38 \\
358 \\
8.8 \\
200\end{array}$ & $\begin{array}{r}5.99 \\
3.12 \\
15.55 \\
.23 \\
3.28\end{array}$ & $\begin{array}{l}\text { Sulfate }\left(\mathrm{SO}_{4}\right) \\
\text { Chloride }(\mathrm{Cl}) \\
\text { Fluoride (F) } \\
\left.\text { Nitrate (NO} \mathrm{N}_{3}\right) \\
\text { Dissolved solids } \\
\text { Total hardness as } \mathrm{CaCO}_{3}\end{array}$ & $\begin{array}{r}2 \varepsilon 7 \\
5 \varepsilon 8 \\
1.0 \\
3.2 \\
1,4 \varepsilon n \\
456\end{array}$ & $\begin{array}{r}4.93 \\
16.58 \\
.05 \\
.05 \\
-0 .- \\
\end{array}$ \\
\hline
\end{tabular}

\section{YST.ETA}

[Includes adjacent Rio Grande territory]

Population in 1940: 2,100 (includes the city of Ysleta only).

Source of information: Edward Lang, water superintendent, Sept. 20, 1948. Owner: El Paso Water Control and Improvement District No. 1.

Source of supply: Four wells.

Well 1. Drilled in November 1946 by Layne-Texas Co.; depth, 704 feet; diameter, 14 inches; deep-well turbine pump and 30-horsepower electric motor; static water level, 102 feet below land surface Dec. 18, 1946; yield on test, 380 gallons a minute with drawdown of 62 feet; present yield, 400 gallons a minute.

Well 2. Drilled June 1947 by Layne-Texas Co.; depth, 689 feet; diameter, 14 inches; deep-well turbine pump and 25-horsepower electric motor; static water level, 20 feet below land surface; yield on test, 180 gallons a minute with drawdown of $\mathbf{4 2}$ feet; present yield, 535 gallons a minute.

Well 3. Drilled in March 1947 by Layne-Texas Co.; derth, 786 feet; diameter, 14 inches; deep-well turbine pump and 25-horsepower electric motor; static water level, 50 feet below land surface Feb. 28, 1947; yield on test, 350 gallons a minute with drawdown of 50 feet; present yield, 460 gallons a minute.

Well 4. Drilled in June 1947 by Layne-Texas Co.; drilled tc 600 feet and plugged back to 219 feet; diameter, 14 inches; deep-well turbine pump and 25-horsepower electric motor; static water level, 52 feet below land surface June 5, 1947; yield on test, 243 gallons a minute with drawdown of 26 feet; well not in use.

Pumpage: Average, 600,000 gallons a day.

Storage: Four elevated tanks, 200,000 gallons each.

Number of customers: 2,100.

Treatment: Chlorination. 
Analysis, well 2

[Collected Sept. 20, 1948. pH is 7.8. Analyzed by J. R. Avrett]

\begin{tabular}{|c|c|c|c|c|c|}
\hline & $\begin{array}{c}\text { Parts per } \\
\text { million }\end{array}$ & $\begin{array}{l}\text { Equivalents } \\
\text { per million }\end{array}$ & & $\begin{array}{c}\text { Parts per } \\
\text { million }\end{array}$ & $\begin{array}{l}\text { Equivalents } \\
\text { per million }\end{array}$ \\
\hline $\begin{array}{l}\text { Silica }\left(\mathrm{SiO}_{2}\right) \\
\text { Iron }(\mathrm{Fe}) \\
\text { Calcium }(\mathrm{Ca}) \\
\text { Magnesium }(\mathrm{Mg}) \\
\text { Sodium }(\mathrm{Na}) \\
\text { Potassium }(\mathrm{K}) \\
\text { Bicarbonate }\left(\mathrm{H} \mathrm{CO}_{3}\right)\end{array}$ & $\begin{array}{l}28 \\
26 \\
10 \\
122 \\
164.2\end{array}$ & $\begin{array}{r}1.30 \\
.82 \\
5.30 \\
.18 \\
2.69\end{array}$ & $\begin{array}{l}\text { Sulfate }\left(\mathrm{SO}_{4}\right) \\
\text { Chloride }(\mathrm{Cl}) \\
\text { Fluoride (F) } \\
\left.\text { Nitrate (NO } \mathrm{N}_{3}\right) \\
\text { Dissolved solids } \\
\text { Total hardness as } \mathrm{CaCO}{ }_{3}\end{array}$ & $\begin{array}{r}88 \\
107 \\
1.2 \\
475 \\
106\end{array}$ & $\begin{array}{r}1.83 \\
3.02 \\
.04 \\
.02 \\
-0 .- \\
-1.0\end{array}$ \\
\hline
\end{tabular}

\section{FISHER COUNTY}

\section{ROBY}

Population in 1940: 947.

Source of information: J. Ammons, city commissioner, Sept. 15, 1947.

Ownership: Municipal.

Source of supply: Pond on Cottonwood Creek.

Pumpage (estimated): Summer, 130,000 gallons a day; winter, 65,000 gallons a day.

Storage: Standpipe, 65,000 gallons.

Number of customers: 300 . (Only a few customers use this source of supply for drinking water.)

Treatment: None.

\section{Analysis}

[Collected Sept. 15, 1947. $\mathrm{pH}$ is 7.8. Analyzed by B. C. Dwyer]

\begin{tabular}{|c|c|c|c|c|c|}
\hline & $\begin{array}{l}\text { Parts per } \\
\text { million }\end{array}$ & $\begin{array}{l}\text { Equivalents } \\
\text { per million }\end{array}$ & & $\begin{array}{c}\text { Parts per } \\
\text { million }\end{array}$ & $\begin{array}{l}\text { Equivalents } \\
\text { per million }\end{array}$ \\
\hline $\begin{array}{l}\text { Silica }\left(\mathrm{SiO}_{2}\right) \\
\text { Iron ( } \mathrm{Fe}) \\
\text { Calcium }(\mathrm{Ca}) \\
\text { Magnesium }(\mathrm{Mg}) \\
\text { Sodium (Na) } \\
\text { Potassium }(\mathrm{K}) \\
\text { Bicarbonate }\left(\mathrm{HCO}_{3}\right)\end{array}$ & $\begin{array}{l}16 \\
628 \\
180 \\
389 \\
13 \\
304\end{array}$ & $\begin{array}{r}31.35 \\
14.80 \\
16.90 \\
.33 \\
4.98\end{array}$ & $\begin{array}{l}\text { Sulfate }\left(\mathrm{SO}_{4}\right) \\
\text { Chloride (Cl) } \\
\text { Fluoride (F) } \\
\left.\text { Nitrate (NO})_{3}\right) \\
\text { Dissolved solids } \\
\text { Total hardness as } \mathrm{CaCO}_{3}\end{array}$ & $\begin{array}{r}2,170 \\
456 \\
20^{.8} \\
4,020 \\
2,310\end{array}$ & $\begin{array}{r}45.18 \\
12.86 \\
.04 \\
.32 \\
-1 .- \\
\end{array}$ \\
\hline
\end{tabular}

\section{ROTAN}

Population in 1940: 2,029.

Source of information: John Price, alderman, Sept. 15, 1947.

Ownership: Municipal.

Source of supply: Nine wells at Camp Springs, $171 / 2$ miles west of Rotan, and one well in Rotan.

Well 1. In Camp Springs tract; dug; depth, 97 feet; diameter, 4 feet; jet pump and 1 1/4-horsepower electric motor; pumping level, 83.04 feet below land surface Sept. 15, 1947; yield, 10 gallons a minute; temperature, $70^{\circ} \mathrm{F}$.

Well 2. In Camp Springs tract; drilled; depth, 60 feet; diameter, 10 inches; jet pump and 2-horsepower electric motor; yield, 20 gallons a minute; temperature, $70^{\circ} \mathrm{F}$.

Well 3. In Camp Springs tract; drilled; depth, 60 feet; diameter, 10 inches; jet pump and 2-horsepower electric motor; yield, 25 gallons a minute.

Well 4. In Camp Springs tract; drilled by Frank Aaron; dep+h, 238 feet; diameter, 10 inches; deep-well turbine pump and 5-horsepower electric 
motor; static water level, 146.7 feet below land surface; yield, 5 gallons a minute.

Well 5. In Camp Springs tract; drilled; depth, 120 feet; diameter, 8 inches; jet pump and 2-horsepower electric motor.

Well 7. In Camp Springs tract; drilled by Frank Aaron; dopth, 190 feet; diameter, 8 inches; deep-well turbine pump and 5-horsepower electric motor; yield, $\mathbf{4 0}$ gallons a minute.

Well 8. In Camp Springs tract; drilled by Frank Aaron in 1941; depth, 205 feet; diameter, 10 inches; deep-well turbine pump and diesel engine; yield, 150 gallons a minute.

Well 9. In Camp Springs tract; drilled by Sam Henderson in 1944; depth, 160 feet; diameter, 10 inches; deep-well turbine pump and diesel engine; water level, 95 feet below land surface Sept. 15, 1947; yield, 85 gallons a minute.

Well 9a. In Camp Springs tract; dug in 1934; depth, 40 feet; diameter, 12 feet; jet pump and 1-horsepower electric motor; water level, 30.1 feet below land surface Sept. 15, 1947; yield, 10 gallons a minute.

Rotan well. South of Rotan gin, 0.6 mile south of city hall; depth, 55 feet; diameter, 24 feet; deep-well turbine pump and 25-horsenower electric motor; static water level, 25.6 feet below land surface Sept. 15, 1947; yield, 200 gallons a minute.

Average pumpage in gallons a day

\begin{tabular}{ll|r|r|r}
\hline & & 1945 & 1946 & 1947 \\
\hline January & & 196 \\
March &
\end{tabular}

Storage: Concrete ground reservoir, 54,000 gallons; steel ground storage tank, 100,000 gallons.

Number of customers: 758.

Treatment: None.

\section{Analyses}

[Collected Sept. 15, 1947. pH: well 1, 7.8; nell 2, 7.1. Analyzed by B. C. Dwyer]

\begin{tabular}{|c|c|c|c|c|}
\hline & \multicolumn{2}{|c|}{ Well 1} & \multicolumn{2}{|c|}{ Well 2} \\
\hline & $\begin{array}{l}\text { Parts per } \\
\text { million }\end{array}$ & $\begin{array}{l}\text { Equivalents } \\
\text { per million }\end{array}$ & $\begin{array}{l}\text { Parts per } \\
\text { million }\end{array}$ & $\begin{array}{l}\text { Equivalents } \\
\text { per million }\end{array}$ \\
\hline 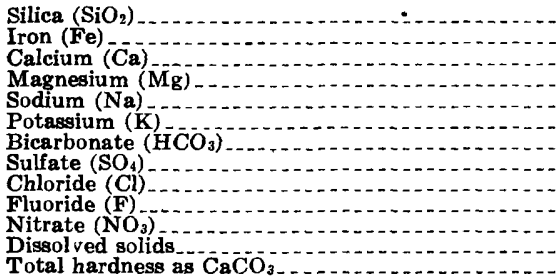 & $\begin{array}{l}11 \\
65 \\
34 \\
28 \\
2.04 \\
2.8 \\
304 \\
61 \\
34 \\
1.8 \\
388 \\
302\end{array}$ & $\begin{array}{r}3.24 \\
2.80 \\
1.20 \\
.07 \\
4.98 \\
1.27 \\
.96 \\
.09 \\
.01\end{array}$ & $\begin{array}{l}8.0 \\
\qquad 6.06 \\
13 \\
6.4 \\
2.0 \\
118 \\
179 \\
16 \\
3.4 \\
38^{3.8} \\
2 £ 3\end{array}$ & $\begin{array}{r}4.79 \\
1.07 \\
.28 \\
.05 \\
1.93 \\
3.73 \\
.45 \\
.02 \\
.06\end{array}$ \\
\hline
\end{tabular}




\section{FLOYD COUNTY}

\section{DOUGHERTY}

Population in 1940: 200.

Source of information: J. E. Newton, water superintendent, Nov. 17, 1945.

Owner: F. M. Dougherty.

Source of supply: Well drilled by D. L. Handley; depth, about 300 feet; diameter, $5 \%$ inches; deep-well turbine pump and oil engine; yield, 37 gallons a minute.

Pumpage: No record.

Storage: Concrete ground reservoir, 50,000 gallons; elevated tank, 35,000 gallons.

Number of customers: 30 .

Treatment: None.

\section{Analysis}

[Collected Nov. 17, 1945. pH is 8.3. Analyzed by J. H. Rowley]

\begin{tabular}{|c|c|c|c|c|c|}
\hline & $\begin{array}{l}\text { Parts per } \\
\text { million }\end{array}$ & $\begin{array}{l}\text { Equivalent } \\
\text { per millior }\end{array}$ & & $\begin{array}{c}\text { Parts per } \\
\text { million }\end{array}$ & $\begin{array}{l}\text { Equivalents } \\
\text { per million }\end{array}$ \\
\hline $\begin{array}{l}\text { Silica }\left(\mathrm{SiO}_{2}\right) \\
\text { Iron }(\mathrm{Fe}) \\
\text { Calcium }(\mathrm{Ca}) \\
\text { Magnesium }(\mathrm{M} \mathrm{g}) \\
\text { Sodium }(\mathrm{Na}) \\
\text { Potassium }(\mathrm{K}) \\
\text { Bicarbonate }\left(\mathrm{H} \mathrm{C}^{2} \mathrm{O}_{3}\right)^{-}\end{array}$ & $\begin{array}{l}36 \\
41 \\
36 \\
44 \\
10 \\
346\end{array}$ & $\begin{array}{r}2.05 \\
2.96 \\
1.92 \\
.26 \\
5.67\end{array}$ & $\begin{array}{l}\text { Sulfate }\left(\mathrm{SO}_{4}\right) \\
\text { Chloride }(\mathrm{Cl}) \\
\text { Fluoride (F) } \\
\text { Nitrate (NO} 3) \\
\text { Dissol ed solids } \\
\text { Total hardness as } \mathrm{CaCO}_{3}\end{array}$ & $\begin{array}{c}38 \\
18 \\
2.8 \\
4.1 \\
400 \\
250\end{array}$ & $\begin{array}{r}0.79 \\
.51 \\
.15 \\
.07 \\
.0 . \\
\end{array}$ \\
\hline
\end{tabular}

\section{FLOYDADA}

Population in 1940: 2,689.

Source of information: Floyd Johnson, water superintendent, Feb. 28, 1945.

Ownership: Municipal.

Source of supply: Three wells.

Well 1. Drilled in 1928 by D. L. McDonald; depth, 212 feet; d ameter, 16 inches; deep-well turbine pump and electric motor; water level reported, 136 feet below land surface in 1938; yield, 380 gallons a minute; temperature, $64^{\circ} \mathrm{F}$.

Well 2. Drilled in 1928 by D. L. McDonald; depth, 200 feet; d:ameter, 16 inches; deep-well turbine pump and 30-horsepower electric motor; yield, 308 gallons a minute.

Well 3. Drilled in 1920 by D. L. McDonald; depth, 160 feet; dameter, 30 inches; deep-well turbine pump and 10-horsepower electric motor; yield, 150 gallons a minute.

Pumpage: Average, 280,000 gallons a day.

Storage: Ground storage reservoir, 200,000 gallons; elevated tank, 50,000 gallons.

Number of customers: 777 .

Treatment: None. 


\section{Analyses}

[Collected Feb. 28, 1945. pH: well 1, 7.5; well 3, 7.7. Analyzed by J. H. Rowley]

\begin{tabular}{|c|c|c|c|c|}
\hline & \multicolumn{2}{|c|}{ Well 1} & \multicolumn{2}{|c|}{ Well 3} \\
\hline & $\begin{array}{l}\text { Parts per } \\
\text { million }\end{array}$ & $\begin{array}{l}\text { Equivalents } \\
\text { per million }\end{array}$ & $\begin{array}{l}\text { Parts per } \\
\text { million }\end{array}$ & $\begin{array}{l}\text { Equivalents } \\
\text { per million }\end{array}$ \\
\hline 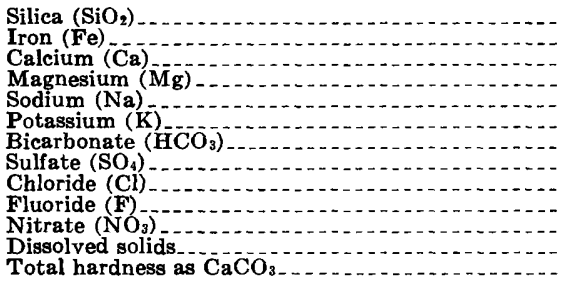 & $\begin{array}{l}53 \\
39 \\
41 \\
26 \\
9.3 \\
296 \\
39 \\
26 \\
4.4 \\
3.5 \\
387 \\
266\end{array}$ & $\begin{array}{r}3.37 \\
1.12 \\
.24 \\
4.85 \\
.81 \\
.73 \\
.23 \\
.06\end{array}$ & $\begin{array}{c}56 \\
36 \\
37 \\
37 \\
41 \\
8.7 \\
312 \\
39 \\
23 \\
4.0 \\
4.0 \\
404 \\
242\end{array}$ & $\begin{array}{r}1.80 \\
3.07 \\
1.78 \\
.22 \\
5.11 \\
.81 \\
.65 \\
.21 \\
.06\end{array}$ \\
\hline
\end{tabular}

\section{Driller's log, well 3}

\begin{tabular}{|c|c|c|c|c|c|}
\hline & $\begin{array}{c}\text { Thickness } \\
\text { (feet) }\end{array}$ & $\begin{array}{l}\text { Depth } \\
\text { (feet) }\end{array}$ & & $\begin{array}{c}\text { Thickness } \\
\text { (feet) }\end{array}$ & $\underset{\text { (feet) }}{\text { Depth }}$ \\
\hline $\begin{array}{l}\text { Top soil, caliche, and red clay } \\
\text { Clay and rock } \\
\text { Clay and sandrock } \\
\text { Water sand } \\
\text { Clay and sandrock } \\
\text { Water sand }\end{array}$ & $\begin{array}{r}27 \\
14 \\
69 \\
4 \\
6 \\
5\end{array}$ & $\begin{array}{r}27 \\
41 \\
110 \\
114 \\
120 \\
125\end{array}$ & $\begin{array}{l}\text { Soft packed red clay } \\
\text { Sandrock. } \\
\text { Clay and sand } \\
\text { Clay and sandrock } \\
\text { Water sand. }\end{array}$ & $\begin{array}{r}11 \\
8 \\
6 \\
4 \\
6\end{array}$ & $\begin{array}{l}136 \\
144 \\
150 \\
154 \\
160\end{array}$ \\
\hline
\end{tabular}

\section{IOCKNEY}

Population in 1940: 1,350 .

Source of information: W. W. Miller, water superintendent, Fєh. 28, 1945.

Ownership: Municipal.

Source of supply: Well drilled in 1927 by D. L. Handley; cepth, 282 feet; diameter, 16 inches; deep-well turbine pump and oil engire; static water level, 65.67 feet below land surface Feb. 28, 1947; pumping level, 87.65 feet below land surface when pumping 500 gallons a minute; yiel $\lambda, 500$ gallons a minute; temperature, $64^{\circ} \mathrm{F}$.

Pumpage: Average, 120,000 gallons a day.

Storage: Concrete ground reservoir, 50,000 gallons; elevated steel tank, 75,000 gallons.

Number of customers: 235.

Treatment: None.

\section{Analysis}

[Collected Feb. 28, 1945. pH is 7.4. Analyzed by J. H. Rowlev]

\begin{tabular}{|c|c|c|c|c|c|}
\hline & $\begin{array}{c}\text { Parts per } \\
\text { million }\end{array}$ & $\begin{array}{l}\text { Equivalents } \\
\text { per million }\end{array}$ & & $\begin{array}{c}\text { Prrts per } \\
\text { nillion }\end{array}$ & $\begin{array}{l}\text { Equivalents } \\
\text { per million }\end{array}$ \\
\hline $\begin{array}{l}\text { Sil ca }\left(\mathrm{SiO}_{2}\right) \\
\text { Iron }(\mathrm{Fe}) \\
\text { Calcium }(\mathrm{Ca}) \\
\text { Magnesium }(\mathrm{M}) \\
\text { Sodium }(\mathrm{Na}) \\
\text { Potassium }(\mathrm{K}) \\
\text { Bicarbonate }\left(\mathrm{H}^{2} \overline{C O}_{3}\right)^{2}\end{array}$ & $\begin{array}{l}57 \\
46 \\
45 \\
52 \\
13 \\
376\end{array}$ & $\begin{array}{r}2.30 \\
3.70 \\
2.25 \\
.33 \\
6.16\end{array}$ & $\begin{array}{l}\text { Sulfate }\left(\mathrm{SO}_{4}\right) \\
\text { Chloride }(\mathrm{Cl}) \\
\text { Fluoride }(\mathrm{F}) \\
\text { Nitrate }(\mathrm{NO})_{3} \\
\text { Dissolved solids } \\
\text { Total hardness as } \mathrm{CaCO}_{3}\end{array}$ & $\begin{array}{c}38 \\
47 \\
3.6 \\
6.8 \\
494 \\
300\end{array}$ & \begin{tabular}{r}
0.79 \\
1.33 \\
.19 \\
.11 \\
\\
\hdashline-1.2 \\
\end{tabular} \\
\hline
\end{tabular}




\section{SOUTH PLAINS}

Population in 1940: 250.

Source of information: Raymond Upton, owner, Nov. 17, 1945.

Owner: Raymond Upton.

Source of supply: Well drilled by D. L. Handley; depth, 181 feet; diameter, 6 inches; deep-well cylinder and gasoline engine; yield, less than 5 ? gallons a minute.

Pumpage: No record.

Storage: Elevated tank, 35,000 gallons.

Number of customers: 15.

Treatment: None.

\section{Analysis}

[Collected Nov. 17, 1945. $\mathrm{pH}$ is 6.9. Analyzed by J. H. Rowley]

\begin{tabular}{|c|c|c|c|c|c|}
\hline & $\begin{array}{l}\text { Parts per } \\
\text { million }\end{array}$ & $\begin{array}{l}\text { Equivalents } \\
\text { per million }\end{array}$ & & $\begin{array}{l}\text { Parts per } \\
\text { million }\end{array}$ & $\begin{array}{l}\text { Equivalents } \\
\text { per million }\end{array}$ \\
\hline $\begin{array}{l}\text { Silica }\left(\mathrm{SiO}_{2}\right) \\
\text { Iron }(\mathrm{Fe}) \\
\text { Calcium }(\mathrm{Ca}) \\
\text { Magnesium }(\mathrm{Mg}) \\
\text { Sodium (Na) } \\
\text { Potassium }(\mathrm{K}) \\
\text { Bicarbonate }\left(\mathrm{H} \mathrm{CO}_{3}\right)\end{array}$ & $\begin{array}{l}50 \\
42 \\
30 \\
32 \\
911 \\
9.9\end{array}$ & $\begin{array}{r}2.10 \\
2.47 \\
1.38 \\
.25 \\
5.10\end{array}$ & $\begin{array}{l}\text { Sulfate }\left(\mathrm{SO}_{4}\right) \\
\text { Chloride }(\mathrm{Cl}) \\
\text { Fluoride (F) } \\
\left.\text { Nitrate (NO } \mathrm{N}_{3}\right) \\
\text { Dissolved solids } \\
\text { Total hardness as } \mathrm{CaCO}_{3}\end{array}$ & $\begin{array}{r}26 \\
14 \\
2.8 \\
1.2 \\
361 \\
228\end{array}$ & $\begin{array}{r}0.54 \\
.39 \\
.15 \\
.02 \\
.02 \\
-15\end{array}$ \\
\hline
\end{tabular}

\section{GAINES COUNTY}

\section{SEAGRAVES}

Population in 1940: 3,225.

Source of information: Don Elder, water superintendent, Oct. 24, 1๕ 45.

Ownership: Municipal.

Source of supply: Four wells.

Well 1. At pump station; drilled in 1929 by J. E. Stokes; deptr, 192 feet; diameter, 10 inches; deep-well turbine pump and 3-horsepower electric motor; static water level, 75 feet below land surface in 1938; yield, 60 gallons a minute.

Well 2. At pump station; drilled in 1929 by J. E. Stokes; depth, 145 feet; diameter, 10 inches; deep-well turbine pump and 5-horsepower electric motor; static water level, 75 feet below land surface in 1938; yield, 150 gallons a minute.

Well 3. At pump station; drilled in 1938 by W. A. Willis; deptr, 183 feet; diameter, 10 inches; deep-well turbine pump and 15-horsepower electric motor; static water level, 83.3 feet below land surface Aug. 9, 1938; yield, 200 gallons a minute.

Well 4. At pump station; drilled in 1941 by Paul Pierson; deptr, 180 feet; diameter, 12 inches; deep-well turbine pump and 15-horspower electric motor; yield, 200 gallons a minute. 
Average pumpage in gallons a day

\begin{tabular}{|c|c|c|c|}
\hline & 1943 & 1944 & 1945 \\
\hline 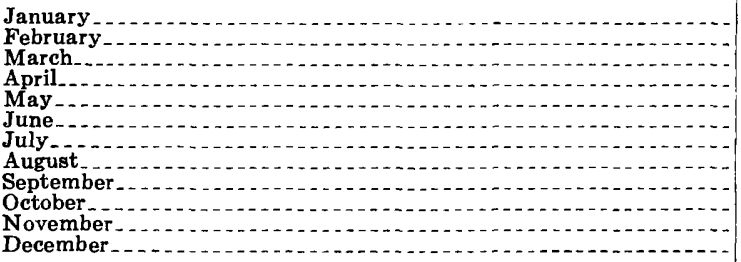 & $\begin{array}{r}95,800 \\
86,500 \\
107,200 \\
165,200 \\
158,000 \\
218,100 \\
237,600 \\
317,600 \\
193,500 \\
127,500 \\
116,300 \\
95,800\end{array}$ & $\begin{array}{r}103,500 \\
98,300 \\
134,100 \\
176,500 \\
192,800 \\
乏 10,900 \\
270,300 \\
279,800 \\
171,500 \\
127,400 \\
126,100 \\
198,000\end{array}$ & $\begin{array}{r}121,700 \\
156,000 \\
178,400 \\
240,300 \\
308,800 \\
358,200 \\
255,400 \\
362,400 \\
\end{array}$ \\
\hline
\end{tabular}

Storage: Concrete ground reservoir, 65,000 gallons; elevated tank, 55,000 gallons.

Number of customers: 450 .

Treatment: None.

\section{Analyses}

[Collected May 23, 1944. pH is 7.6. Analyzed by W. W. Hastings]

\begin{tabular}{|c|c|c|c|c|c|}
\hline & \multicolumn{2}{|c|}{ Well 1} & & \multicolumn{2}{|c|}{ Well 1} \\
\hline & $\begin{array}{c}\text { Parts per } \\
\text { million }\end{array}$ & $\begin{array}{c}\text { Equivalents } \\
\text { per million }\end{array}$ & & $\begin{array}{c}\text { Parts per } \\
\text { mi"ion }\end{array}$ & $\begin{array}{l}\text { Equivalents } \\
\text { per million }\end{array}$ \\
\hline $\begin{array}{l}\text { Silica }\left(\mathrm{SiO}_{2}\right) \\
\text { Iron }(\mathrm{Fe}) \\
\text { Calcium }(\mathrm{Ca}) \\
\text { Magnesium }(\mathrm{Mg}) \\
\text { Sodium }(\mathrm{Na}) \\
\text { Potassium }(\mathrm{K}) \\
\text { Bicarbonate }\left(\mathrm{H}^{2} \mathrm{CO}_{3}\right)^{-}\end{array}$ & $\begin{array}{l}{ }_{61}^{44} \\
90 \\
89 \\
13 \\
261\end{array}$ & $\begin{array}{r}\mathbf{3} .04 \\
7.40 \\
\mathbf{3 . 8 8} \\
.33 \\
4.28\end{array}$ & $\begin{array}{l}\text { Sulfate }\left(\mathrm{SO}_{4}\right) \\
\text { Chloride (Cl) } \\
\text { Fluoride (F) } \\
\left.\text { Nitrate (NO})_{3}\right) \\
\text { Dissol ed solids. } \\
\text { Total hardness as } \mathrm{CaCO}\end{array}$ & $\begin{array}{l}265 \\
162 \\
2.8 \\
8.0 \\
863 \\
522\end{array}$ & \begin{tabular}{r}
5.52 \\
4.57 \\
.15 \\
.13 \\
\hdashline..- \\
-15
\end{tabular} \\
\hline
\end{tabular}

[Collected Oct. 24, 1945. pH: wells 3 and 4, each 8.4. Analyzed by C. B. Cibulka]

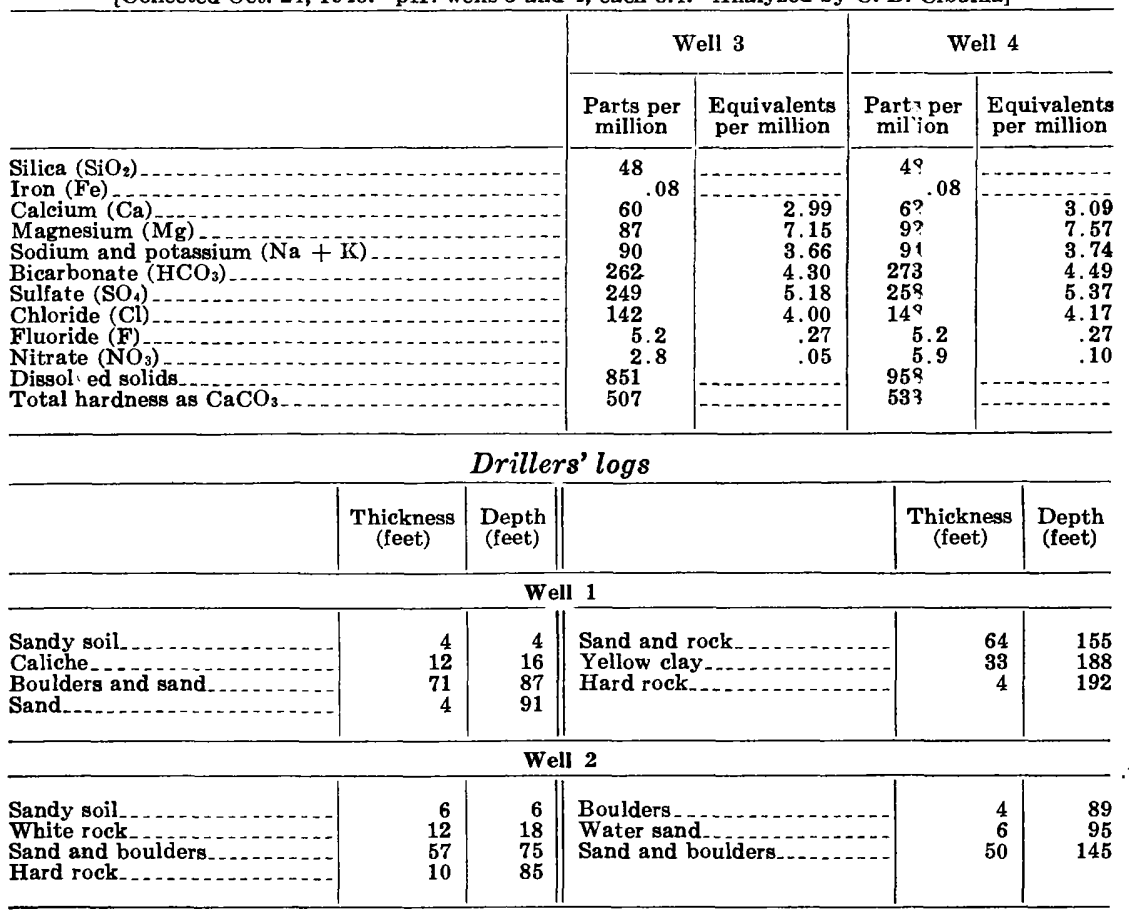




\begin{tabular}{|c|c|c|c|c|c|}
\hline & $\begin{array}{c}\text { Thickness } \\
\text { (feet) }\end{array}$ & $\begin{array}{c}\text { Depth } \\
\text { (feet) }\end{array}$ & & $\begin{array}{c}\text { Thickness } \\
\text { (feet) }\end{array}$ & $\begin{array}{l}\text { Depth } \\
\text { (feet) }\end{array}$ \\
\hline \multicolumn{6}{|c|}{ Well 3} \\
\hline $\begin{array}{l}\text { Sandy clay } \\
\text { Caliche. } \\
\text { Sand } \\
\text { Hard sandstone. } \\
\text { Hard sand. } \\
\text { Soft sand, a ater. }\end{array}$ & $\begin{array}{l}7 \\
13 \\
25 \\
10 \\
38 \\
52\end{array}$ & $\begin{array}{r}7 \\
20 \\
45 \\
55 \\
93 \\
145\end{array}$ & $\begin{array}{l}\text { Hard sandrock } \\
\text { Sand, water. } \\
\text { Gravel, water } \\
\text { Hard sandrock } \\
\text { Shale. }\end{array}$ & $\begin{array}{r}10 \\
17 \\
6 \\
4 \\
1\end{array}$ & $\begin{array}{l}155 \\
172 \\
178 \\
182 \\
183\end{array}$ \\
\hline
\end{tabular}

Population in 1940: 1,761.

\section{SEMINOLE}

Source of information: J. S. Combs, city secretary, Nov. 7, 1945.

Ownership: Municipal.

Source of supply: Three wells.

Well 1. Half a block south of courthouse; drilled in 1939; deptr, 145 feet; diameter, $10 \% / 4$ to 8 inches; deep-well turbine pump and 20-1 orsepower electric motor; static water level, 56 feet below land surfare Nov. 8, 1939; drawdown, 47 feet while pumping 145 gallons a minute; yield, 150 gallons a minute.

Well 2. Four blocks south and six blocks west of courthouse; drilled in 1940 by J. E. Stokes; depth, 188 feet; diameter, 12 inches; deep-well turbine pump and 25-horsepower electric motor; yield, 200 gallons a minute.

Well 3. Two blocks south and eight blocks west of courthouse; drilled in 1945 by Gibbons and Taylor; depth, 250 feet; diameter, 13 $3 / 8$ to 103/4 inches; deep-well turbine pump and gasoline engine; static water level, 102.8 feet below land surface Oct. 22, 1945; measured drawdown, 55 feet while pumping 280 gallons a minute.

Pumpage (estimated) : 250,000 gallons a day.

Storage: Concrete ground reservoir, 100,000 gallons; elevated tank, 50,000 gallons.

Number of customers: 400 .

Treatment: None.

\section{Analyses}

[Collected Nov. 7, 1945. pH: well 1, 8.4.; well 2, 8.5, Analyzed by C. B. Cibulka]

\begin{tabular}{|c|c|c|c|c|}
\hline & \multicolumn{2}{|c|}{ Well 1} & \multicolumn{2}{|c|}{ Well 2} \\
\hline & $\begin{array}{c}\text { Parts per } \\
\text { million }\end{array}$ & $\begin{array}{l}\text { Equivalents } \\
\text { per million }\end{array}$ & $\begin{array}{l}\text { Parts per } \\
\text { million }\end{array}$ & $\begin{array}{l}\text { Equivalents } \\
\text { per milion }\end{array}$ \\
\hline 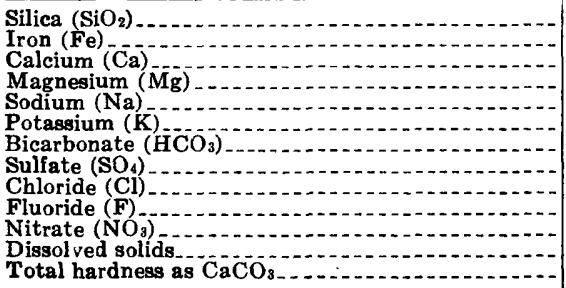 & $\begin{array}{l}45 \\
57 \\
45 \\
45 \\
68 \\
11 \\
265 \\
123 \\
89 \\
4 \cdot 4 \\
8.3 \\
610 \\
328\end{array}$ & \begin{tabular}{r}
2.85 \\
3.70 \\
2.95 \\
.28 \\
4.35 \\
2.56 \\
2.51 \\
.23 \\
.13 \\
\hdashline-2. \\
\end{tabular} & $\begin{array}{l}56 \\
65^{.08} \\
54 \\
79 \\
11 \\
266 \\
173 \\
109 \\
4.8 \\
6.4 \\
732 \\
384\end{array}$ & $\begin{array}{r}3.24 \\
4.44 \\
\mathbf{3 . 4 3} \\
.28 \\
4.37 \\
3.60 \\
3.07 \\
.25 \\
.10 \\
-1 .-\end{array}$ \\
\hline
\end{tabular}

$947535-51-6$ 


\section{Drillers' logs}

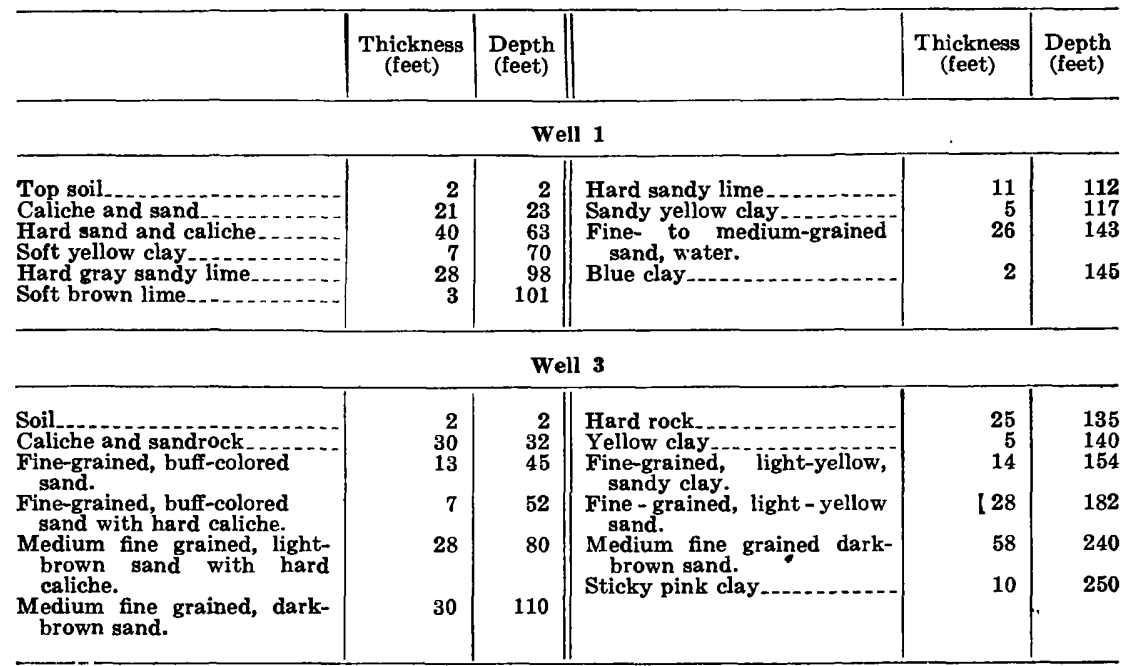

\section{GARZA COUNTY}

Population in 1940: 2,046.

\section{POST}

Source of information: A. R. Carr, former water superintendant, Sept. 21, 1948.

Ownership: Municipal.

Source of supply: Seventeen wells.

East field, 3.5 miles west of Post:

Well 121. Drilled in 1912; depth, 97.5 feet; diameter, 23 to 9 inches; deepwell turbine pump and 2-horsepower electric motor; yield; 30 gallons a minute.

Well 124. Dug in 1912; depth, 98 feet; diameter, $105 / 8$ inch 9 ; deep-well cylinder pump and 3-horsepower electric motor; yield, 20 rallons a minute.

Well 126. Drilled in 1912; depth, 98 feet; diameter, $10 \%$ inches; deepwell cylinder pump and 3-hórsepower electric motor; yielc', 20 gallons a minute.

Well 135. Drilled in 1916 by J. L. Williams; depth, 94 feet; ciameter, $10 \%$ inches; deep-well turbine pump and 2-horsepower electric motor; static water level, 80 feet below land surface in 1916; yield, 20 gallons a minute.

West field, 5 miles west of Post:

Well 140. Dug in 1917 and later drilled deeper; depth, 120 feet; diameter of dug well, 20 to 10 inches; deep-well turbine pump and 10-horsepower electric motor; yield, 120 gallons a minute.

Well 146. Drilled in 1926 by D. L. McDonald; depth, 80 feet; diameter, 24 inches; deep-well cylinder pump and 5-horsepower eloctric motor; yield, 25 gallons a minute. 
Well 147. Drilled in 1937 by L. A. Peeples; depth, 97 feet; dis meter, 20 to 18 inches; deep-well cylinder pump and 5-horsepower elestric motor; yield, 20 gallons a minute.

Well 148. Drilled in 1937 by L. A. Peeples; depth, 201 feet; diameter, 15 to 12 inches; deep-well cylinder pump and 5-horsepower electric motor; yield, 35 gallons a minute.

Well 152. Drilled in 1940 by Layne-Texas Co.; depth, 143 feet; diameter, $10 \%$ inches; deep-well turbine pump and 3-horsepower elentric motor; static water level reported, 80 feet below land surface April 1940; yield, 25 gallons a minute with 5 -foot drawdown.

Northwest field, 6 miles northwest of Post:

Well 149. Drilled in 1939 by L. A. Peeples; deep-well turbin? pump and 3-horsepower electric motor.

Well 150. Drilled in 1939 by L. A. Peeples; depth, 115 feet; diameter, 12 inches; deep-well turbine pump and 5-horsepower electric motor.

Well 151. Drilled in 1940 by Layne-Texas Co.; depth, 110 feet; pump jack and 3-horsepower electric motor; static water level reported, 85 feet below land surface in 1940; gravel-walled; yield, 35 gallors a minute.

Well 153. Drilled in 1941 by Layne-Texas Co.; depth, 95 feet; diameter, $103 / 4$ inches; deep-well turbine pump and 71/2-horseporver electric motor; static water level reported, 73 feet below land surfac? July 1941; yield, 60 gallons a minute.

Well 154. Drilled in 1944 by Layne-Texas Co.; depth, 98 feet; diameter, $103 / 4$ inches; deep-well turbine pump and 5-horsepower ele tric motor; static water level reported, 66 feet below land surface July 1944; gravel-walled; yield, 94 gallons a minute.

Well 155. Drilled in 1945 by Layne-Texas Co.; depth, 971/2 feet; diameter, $103 / 4$ inches; deep-well turbine pump and 5-horsepower elentric motor; static water level reported, 63 feet below land surface in June 1945; gravel-walled; yield, 85 gallons a minute.

Well 156. Drilled in 1946 by Layne-Texas Co.; depth, 95 feet; diameter, $103 / 4$ inches; deep-well turbine pump and 5-horsepower ele tric motor; static water level reported, 64 feet below land surface July 1946; gravel-walled; yield, 77 gallons a minute.

Well 157. Drilled in 1946 by Layne-Texas Co.; depth, 101 feet; diameter, $103 / 4$ inches; deep-well turbine pump and 5-horsepower ele?tric motor; static water level reportéd, 591/2 feet below land surface July 1946: yield, 133 gallons a minute.

Pumpage: Average, 300,000 gallons a day.

Storage: Ground storage reservoir, $1,600,000$ gallons.

Number of customers: 850 .

Treatment: Chlorination.

\section{Analysis}

[Composite sample. Collected Nov. 29, 1946. pH is 7.8. Analyzed by C. B. Cibulka]

\begin{tabular}{|c|c|c|c|c|c|}
\hline & $\begin{array}{c}\text { Parts per } \\
\text { million }\end{array}$ & $\begin{array}{l}\text { Equivalentc } \\
\text { per million }\end{array}$ & 网 & $\begin{array}{l}\text { Parts } \text { Fer }^{a} \\
\text { million }\end{array}$ & $\begin{array}{l}\text { Equivalents } \\
\text { per million }\end{array}$ \\
\hline $\begin{array}{l}\text { Silica }\left(\mathrm{SiO}_{2}\right) \\
\text { Iron }(\mathrm{Fe}) \\
\text { Calcium }(\mathrm{Ca}) \\
\text { Magnesium }(\mathrm{Mg}) \\
\text { Sodium }(\mathrm{Na}) \\
\text { Potassium }(\mathrm{K}) \\
\text { Bicarbonate }\left(\mathrm{HCO}_{3}\right)^{-}\end{array}$ & $\begin{array}{l}56 \\
44 \\
43 \\
48 \\
78 \\
6.4 \\
344\end{array}$ & $\begin{array}{r}2.20 \\
3.54 \\
3.39 \\
.16 \\
5.64\end{array}$ & $\begin{array}{l}\text { Sulfate }\left(\mathrm{SO}_{4}\right) \\
\text { Chloride (Cl) } \\
\text { Fluoride (F) } \\
\left.\text { Nitrate (NO})_{3}\right) \\
\text { Dissolved solids. } \\
\text { Total hardness as } \mathrm{CaCO}_{3}\end{array}$ & $\begin{array}{l}81 \\
58 \\
5.6 \\
2.0 \\
544 \\
287\end{array}$ & $\begin{array}{r}1.69 \\
1.64 \\
.29 \\
.03 \\
-0.0 \\
\end{array}$ \\
\hline
\end{tabular}




\section{Drillers' $\log 8$}

\begin{tabular}{|c|c|c|c|c|c|}
\hline & $\begin{array}{c}\text { Thickness } \\
\text { (feet) }\end{array}$ & $\begin{array}{l}\text { Depth } \\
\text { (feet) }\end{array}$ & & $\begin{array}{c}\text { Thickness } \\
\text { (feet) }\end{array}$ & $\begin{array}{l}\text { Depth } \\
\text { (feet) }\end{array}$ \\
\hline \multicolumn{6}{|c|}{ Well 126} \\
\hline $\begin{array}{l}\text { Soil and subsoil } \\
\text { White dirt } \\
\text { White rock } \\
\text { Hard red rock }\end{array}$ & $\begin{array}{r}4 \\
20 \\
4 \\
6\end{array}$ & $\begin{array}{r}4 \\
24 \\
28 \\
34\end{array}$ & $\begin{array}{l}\text { Soft red rock } \\
\text { Dry, gray sand } \\
\text { Water sand strata. } \\
\text { Red clay. }\end{array}$ & $\begin{array}{l}14 \\
25 \\
25 \\
1 / 2\end{array}$ & $\begin{array}{l}48 \\
73 \\
98 \\
981 / 2\end{array}$ \\
\hline \multicolumn{6}{|c|}{ Well 135} \\
\hline $\begin{array}{l}\text { Soil } \\
\text { Red clay } \\
\text { Red sand. } \\
\text { Sandstone. }\end{array}$ & $\begin{array}{r}4 \\
16 \\
30 \\
7\end{array}$ & $\begin{array}{r}4 \\
20 \\
50 \\
\mathbf{5 7}\end{array}$ & $\begin{array}{l}\text { Red sand } \\
\text { Sand and } \mathrm{w} \text { ater } \\
\text { Red clay }\end{array}$ & $\begin{array}{r}23 \\
12 \\
2\end{array}$ & $\begin{array}{l}80 \\
92 \\
94\end{array}$ \\
\hline \multicolumn{6}{|c|}{ Well 147} \\
\hline $\begin{array}{l}\text { Top soil } \\
\text { Caliche. } \\
\text { Clay } \\
\text { Sandrock. } \\
\text { Packed sand. }\end{array}$ & $\begin{array}{r}3 \\
24 \\
15 \\
2 \\
\mathbf{2 1}\end{array}$ & $\begin{array}{r}3 \\
27 \\
42 \\
44 \\
65\end{array}$ & $\begin{array}{l}\text { Fire dry sand } \\
\text { Fire nater sand } \\
\text { Sand and gravel. } \\
\text { Gravel. } \\
\text { Clay }\end{array}$ & $\begin{array}{l}6 \\
5 \\
9 \\
4 \\
8\end{array}$ & $\begin{array}{l}71 \\
76 \\
85 \\
89 \\
97\end{array}$ \\
\hline
\end{tabular}

Well 148

\begin{tabular}{|c|c|c|c|c|c|}
\hline $\begin{array}{l}\text { Top soil } \\
\text { Clay and white rock. } \\
\text { Sand rock } \\
\text { Clay } \\
\text { Caliche rock } \\
\text { Clay }\end{array}$ & $\begin{array}{r}3 \\
43 \\
4 \\
16 \\
5 \\
4\end{array}$ & $\begin{array}{r}3 \\
\mathbf{4 6} \\
\mathbf{5 0} \\
\mathbf{6 6} \\
\mathbf{7 1} \\
\mathbf{7 5}\end{array}$ & $\begin{array}{l}\text { Sand and gravel (water) } \\
\text { Clay } \\
\text { Packed sand, dry } \\
\text { Dry sandrock } \\
\text { Dry clay } \\
\text { Dry packed sand }\end{array}$ & $\begin{array}{r}14 \\
28 \\
57 \\
2 \\
5 \\
20\end{array}$ & $\begin{array}{r}89 \\
117 \\
174 \\
176 \\
181 \\
201\end{array}$ \\
\hline
\end{tabular}

Well 149

\begin{tabular}{l|r|r||r|r}
\hline Top soil & 4 & 4 & $\begin{array}{r}\text { Sandy clay, caving } \\
\text { Coarse sand, soft clay }\end{array}$ \\
Clay and caliche. & 21 & 25 & $\mathbf{7 3}$ \\
Caliche. & 22 & 47 & 110 \\
\hline
\end{tabular}

Well 150

\begin{tabular}{|c|c|c|c|c|c|}
\hline $\begin{array}{l}\text { Top soil } \\
\text { Clay and caliche rock } \\
\text { Caliche } \\
\text { Red clay } \\
\text { Packed sand }\end{array}$ & $\begin{array}{r}4 \\
19 \\
22 \\
6 \\
9\end{array}$ & $\begin{array}{r}4 \\
23 \\
45 \\
51 \\
60\end{array}$ & $\begin{array}{l}\text { Rock } \\
\text { Coarse sand and gravel. } \\
\text { Red clay, very sticky }\end{array}$ & $\begin{array}{r}2 \\
8 \\
21 \\
24\end{array}$ & $\begin{array}{r}62 \\
70 \\
91 \\
115\end{array}$ \\
\hline
\end{tabular}

\begin{tabular}{|c|c|c|c|c|c|}
\hline \multicolumn{6}{|c|}{ Well 151} \\
\hline $\begin{array}{l}\text { Soil } \\
\text { Caliche } \\
\text { Sand and caliche } \\
\text { Sand and clay } \\
\text { Clay } \\
\text { Caliche and hard red clay }\end{array}$ & $\begin{array}{r}3 \\
11 \\
3 \\
3 \\
8 \\
5 \\
12 \\
10\end{array}$ & $\begin{array}{r}3 \\
14 \\
17 \\
20 \\
28 \\
33 \\
45 \\
55\end{array}$ & $\begin{array}{l}\text { Hard red clay } \\
\text { Quicksand and clay. } \\
\text { Sand, gravel, boulders and } \\
\text { clay. } \\
\text { Hard layers. } \\
\text { Sand, gravel, and clay } \\
\text { Red clay }\end{array}$ & $\begin{array}{r}22 \\
10 \\
15 \\
1 \\
1 \\
2 \\
5\end{array}$ & $\begin{array}{r}77 \\
87 \\
102 \\
103 \\
105 \\
110\end{array}$ \\
\hline
\end{tabular}

\section{Well 152}

\begin{tabular}{|c|c|c|c|c|c|}
\hline $\begin{array}{l}\text { Surface soil } \\
\text { Clay } \\
\text { Caliche } \\
\text { Clay and caliche. } \\
\text { Hard caliche } \\
\text { Soft layers fine sand } \\
\text { Hard layers. }\end{array}$ & $\begin{array}{r}6 \\
10 \\
18 \\
3 \\
11 \\
2 \\
1\end{array}$ & $\begin{array}{r}6 \\
16 \\
34 \\
37 \\
48 \\
50 \\
51\end{array}$ & $\begin{array}{l}\text { Fine soft sand } \\
\text { Hard layers. } \\
\text { Fine hard sand-brick dust, } \\
\text { \& mall amount. } \\
\text { Hard sand, more clay } \\
\text { Hard red clay and caliche. } \\
\text { Clay, little sand }\end{array}$ & $\begin{array}{r}3 \\
4 \\
33 \\
9 \\
35 \\
8\end{array}$ & $\begin{array}{r}54 \\
58 \\
91 \\
100 \\
135 \\
143\end{array}$ \\
\hline
\end{tabular}




\begin{tabular}{l|l|c||c|c|c|}
\hline & $\begin{array}{c}\text { Thickness } \\
\text { (feet) }\end{array}$ & $\begin{array}{c}\text { Depth } \\
\text { (feet) }\end{array}$ & $\begin{array}{c}\text { Thicl zess } \\
\text { (fe t) }\end{array}$ & $\begin{array}{c}\text { Depth } \\
\text { (feet) }\end{array}$ \\
\hline
\end{tabular}

\begin{tabular}{|c|c|c|c|c|c|}
\hline \multicolumn{6}{|c|}{ Well 153} \\
\hline $\begin{array}{l}\text { Sand and clay } \\
\text { Clay and caliche } \\
\text { Sand }\end{array}$ & $\begin{array}{r}20 \\
28 \\
6\end{array}$ & $\begin{array}{l}20 \\
48 \\
54\end{array}$ & $\begin{array}{l}\text { Clay and caliche } \\
\text { Sand clay }\end{array}$ & $\begin{array}{l}20 \\
20 \\
1\end{array}$ & $\begin{array}{l}74 \\
94 \\
95\end{array}$ \\
\hline
\end{tabular}

\begin{tabular}{|c|c|c|c|c|c|}
\hline \multicolumn{6}{|c|}{ Well 154} \\
\hline $\begin{array}{l}\text { Surface dirt } \\
\text { Yellow sandy clay } \\
\text { Rock with soft streaks }\end{array}$ & $\begin{array}{r}4 \\
35 \\
17\end{array}$ & $\begin{array}{l}4 \\
39 \\
56\end{array}$ & $\begin{array}{l}\text { Fine sand, hard streaks } \\
\text { Yellow sand, good } \\
\text { Yellow clay }\end{array}$ & $\begin{array}{r}10 \\
31 \\
1\end{array}$ & $\begin{array}{l}66 \\
97 \\
98\end{array}$ \\
\hline
\end{tabular}

\begin{tabular}{|c|c|c|c|c|c|}
\hline \multicolumn{6}{|c|}{ Well 155} \\
\hline $\begin{array}{l}\text { Top soil } \\
\text { Soft caliche } \\
\text { Caliche, hard streaks } \\
\text { Hard caliche and lime rock } \\
\text { Red clay } \\
\text { Clay with hard layers }\end{array}$ & $\begin{array}{r}3 \\
14 \\
7 \\
9 \\
12 \\
11\end{array}$ & $\begin{array}{r}3 \\
17 \\
24 \\
33 \\
45 \\
66\end{array}$ & $\begin{array}{l}\text { Sand and clay, lost little } \\
\text { water. } \\
\text { Good sand, lost much mud. } \\
\text { Clay and rock. } \\
\text { Soft sticky clay }\end{array}$ & $\begin{array}{r}11 \\
25 \\
3 \\
9\end{array}$ & $\begin{array}{r}67 \\
92 \\
95 \\
104\end{array}$ \\
\hline
\end{tabular}

\section{Well 156}

\begin{tabular}{|c|c|c|c|c|c|}
\hline $\begin{array}{l}\text { Surface soil } \\
\text { Sandy clay, streaks of caliche } \\
\text { Hard caliche. } \\
\text { Caliche, Boft streaks } \\
\text { Hard caliche rock }\end{array}$ & $\begin{array}{r}3 \\
11 \\
3 \\
16 \\
6\end{array}$ & $\begin{array}{r}3 \\
14 \\
17 \\
33 \\
39\end{array}$ & $\begin{array}{l}\text { Red clay streaks, sandy } \\
\text { Sandy clay } \\
\text { Sand and fine gravel. } \\
\text { Red clay, sticky }\end{array}$ & $\begin{array}{r}11 \\
17 \\
25 \\
8\end{array}$ & $\begin{array}{l}50 \\
67 \\
92 \\
95\end{array}$ \\
\hline
\end{tabular}

\begin{tabular}{|c|c|c|c|c|c|}
\hline \multicolumn{6}{|c|}{ Well 157} \\
\hline $\begin{array}{l}\text { Surface soil } \\
\text { Sandy clay, caliche, } \\
\text { streaks. } \\
\text { Hard caliche. }\end{array}$ & $\begin{array}{r}3 \\
12 \\
22\end{array}$ & $\begin{array}{r}3 \\
15 \\
37\end{array}$ & $\begin{array}{l}\text { Hard sandy clay, caliche } \\
\text { streaks. } \\
\text { Fine sand. } \\
\text { Sand and gravel. } \\
\text { Sandy clay }\end{array}$ & $\begin{array}{r}27 \\
3 \\
30 \\
4\end{array}$ & $\begin{array}{r}64 \\
67 \\
97 \\
101\end{array}$ \\
\hline
\end{tabular}

\section{GRAY COUNTY}

\section{LFFORS}

Population in 1940: 809.

Source of information: C. W. Graham, water superintendent, Dec. 2, 1948.

Ownership: Municipal.

Source of supply: Two wells.

Well 1. In block 10, Thut Heights Addition; drilled in 1940 by J. T. McCarty; depth, 130 feet; diameter, 15 inches; deep-well turbine pump and 10-horsepower electric motor.

Well 2. In block 11, Thut Heights Addition; drilled by Drake Lard; depth, 130 feet; diameter, 15 inches; deep-well turbine pump and 10-horsepower electric motor.

Pumpage (estimated) : 40,000 gallons a day.

Storage: Elevated tank, 5,500 gallons.

Number of customers: 230.

Treatment: None. 


\section{Analysis well 1}

[Collected Oct. 5, 1948. pH is 7.5. Analyzed by J. R. Avrett]

\begin{tabular}{|c|c|c|c|c|c|}
\hline & $\begin{array}{l}\text { Parts per } \\
\text { million }\end{array}$ & $\begin{array}{l}\text { Equivalents } \\
\text { per million }\end{array}$ & & $\begin{array}{l}\text { Pants per } \\
\text { million }\end{array}$ & $\begin{array}{l}\text { Equivalents } \\
\text { per million }\end{array}$ \\
\hline $\begin{array}{l}\text { Silica }\left(\mathrm{SiO}_{2}\right) \\
\text { Iron }(\mathrm{Fe}) \\
\text { Calcium }\left(\mathrm{C}_{2}\right) \\
\text { Magnesium }(\mathrm{Mg}) \\
\text { Sodium }(\mathrm{Na}) \\
\text { Potassium }(\mathrm{K}) \\
\text { Bicarbonate }\left(\mathrm{HCO}_{3}\right)\end{array}$ & $\begin{array}{l}21 \\
3.8 \\
96 \\
17 \\
42 \\
3.6 \\
214\end{array}$ & $\begin{array}{r}4.79 \\
1.40 \\
1.83 \\
.09 \\
3.51\end{array}$ & $\begin{array}{l}\text { Sulfate }\left(\mathrm{SO}_{4}\right) \\
\text { Chloride }(\mathrm{Cl}) \\
\text { Fluoride (F) } \\
\left.\text { Nitrate (NO})_{3}\right) \\
\text { Dissolved solids } \\
\text { Total hardness as } \mathrm{CaCO}\end{array}$ & $\begin{array}{r}15 \\
144 \\
5.7 \\
514 \\
\mathbf{3 1 0}\end{array}$ & $\begin{array}{r}0.31 \\
4.06 \\
.04 \\
.08 \\
\\
\end{array}$ \\
\hline
\end{tabular}

\section{MCLFAN}

Population in 1940: 1,489 .

Source of information: Pete Fulbright, water superintendent, June 25, 1948.

Ownership: Municipal.

Source of supply: Three wells.

Well 1. Drilled in 1927 by Edwards Well Co.; depth, 156 feet; diameter, 16 inches; 30-foot screen on bottom; deep-well turbine pump and 20horsepower electric motor; static water level, 105 feet $k$ slow land surface; yield, 300 gallons a minute.

Well 2. Drilled in 1936 by T. J. McCarty; depth, 167 feet; diameter, $12 \frac{1}{2}$ inches; deep-well turbine pump and 25-horsepower electric motor; static water level, 110 feet; yield, 250 gallons a minute.

Well 3. Drilled in 1943 by Layne-Texas Co. for U. S. Prisoner of War Camp; depth, 209 feet; diameter, 103/4 inches; deep-well turbine pump and 20-horsepower electric motor; static water level, 115 feet below land surface; drawdown of 19 feet when pumping 220 gallons a minute Sept. 23, 1943; yield, 250 gallons a minute.

Pumpage: Average, 500,000 gallons a day.

Storage: Ground storage reservoir, 210,000 gallons; elevateł tank, 60,000 gallons.

Number of customers: 463.

Treatment: None.

\section{Analysis well s}

[Collected June 25, 1948. $\mathrm{pH}$ is 7.3. Analyzed by D. E. Weave-]

\begin{tabular}{|c|c|c|c|c|c|}
\hline & $\begin{array}{l}\text { Parts per } \\
\text { million }\end{array}$ & $\begin{array}{l}\text { Equivalents } \\
\text { per million }\end{array}$ & & $\begin{array}{l}\text { Parts per } \\
\text { million }\end{array}$ & $\begin{array}{l}\text { Equivalents } \\
\text { per million }\end{array}$ \\
\hline $\begin{array}{l}\text { Silica }\left(\mathrm{SiO}_{2}\right) \\
\text { Iron }(\mathrm{Fe}) \\
\text { Calcium }(\mathrm{Ca}) \\
\text { Magnesium }(\mathrm{Mg}) \\
\text { Sodium }(\mathrm{Na}) \\
\text { Potassium }(\mathrm{K}) \\
\text { Bicarbonate }\left(\mathrm{H} \mathrm{HCO}_{3}\right)^{-}\end{array}$ & $\begin{array}{c}28 \\
1.4 \\
70 \\
6.3 \\
27 \\
1.2 \\
252\end{array}$ & $\begin{array}{r}3.49 \\
.52 \\
1.17 \\
.03 \\
4.13\end{array}$ & $\begin{array}{l}\text { Sulfate }\left(\mathrm{SO}_{4}\right) \\
\text { Chloride }(\mathrm{Cl}) \\
\text { Fluoride (F) } \\
\text { Nitrate }\left(\mathrm{NO}_{3}\right) \\
\text { Dissolved solids } \\
\text { Total hardness as } \mathrm{CaCO}_{3}\end{array}$ & $\begin{array}{l}16 \\
24 \\
30.2 \\
200\end{array}$ & $\begin{array}{r}0.33 \\
.68 \\
.00 \\
.05 \\
-0 . \\
\end{array}$ \\
\hline
\end{tabular}




\section{Drillers' logs}

\begin{tabular}{|c|c|c|c|c|c|}
\hline & $\begin{array}{c}\text { Thickness } \\
\text { (feet) }\end{array}$ & $\begin{array}{c}\text { Depth } \\
\text { (feet) }\end{array}$ & & $\begin{array}{c}\text { Thickness } \\
\text { (feet) }\end{array}$ & $\begin{array}{l}\text { Depth } \\
\text { (feet) }\end{array}$ \\
\hline \multicolumn{6}{|c|}{ Well 1} \\
\hline $\begin{array}{l}\text { Clay, and clay } \\
\text { Sand ane, loose sand. } \\
\text { Hard packed sand } \\
\text { Soft, loose sand } \\
\text { Sandrock } \\
\text { Soft, loose sand } \\
\text { Sand, gravel, little clay } \\
\text { Medium sand, gravel }\end{array}$ & $\begin{array}{r}5 \\
25 \\
5 \\
42 \\
18 \\
1 \\
14 \\
4 \\
4\end{array}$ & $\begin{array}{r}5 \\
\mathbf{3 0} \\
\mathbf{3 5} \\
77 \\
95 \\
96 \\
110 \\
114 \\
118\end{array}$ & $\begin{array}{l}\text { Hard sandstone } \\
\text { Fair water sand and gravel. } \\
\text { Coarse sand and gravel } \\
\text { Clay } \\
\text { Medium water sand } \\
\text { Soft sandrock. } \\
\text { Good water sand } \\
\text { Medium sandrock } \\
\text { Hard sandrock }\end{array}$ & $\begin{array}{r}4 \\
10 \\
3 \\
6 \\
5 \\
1 \\
4 \\
4 \\
1\end{array}$ & $\begin{array}{l}122 \\
132 \\
135 \\
141 \\
146 \\
147 \\
151 \\
155 \\
156\end{array}$ \\
\hline \multicolumn{6}{|c|}{ Well 3} \\
\hline $\begin{array}{l}\text { Soil and clay. } \\
\text { Sand } \\
\text { Hard sand and streaks of clay } \\
\text { and gypsum. } \\
\text { Coarse sand and fine gravel... }\end{array}$ & $\begin{array}{l}20 \\
36 \\
18 \\
\\
55\end{array}$ & $\begin{array}{r}20 \\
56 \\
74 \\
129\end{array}$ & $\begin{array}{l}\text { Rock } \\
\text { Good coarse sand, fine gravel } \\
\text { Rock and gravel } \\
\text { Coarse white sand } \\
\text { Red clay } \ldots\end{array}$ & $\begin{array}{r}1 \\
17 \\
3 \\
53 \\
6\end{array}$ & $\begin{array}{l}130 \\
147 \\
150 \\
203 \\
209\end{array}$ \\
\hline
\end{tabular}

PAMPA

Population in 1940: 12,895.

Source of information: E. S. Lowery, water superintendent, Nov. 2), 1947.

Ownership: Municipal.

Source of supply: Five wells; another well being drilled.

Well 1, South. Drilled by D. L. McDonald in 1939; depth, 450 feet; diameter, 16 inches; deep-well turbine pump and 125-horsepower electric motor; pump set at 420 feet; static water level, 347 feet below land surface in 1947; drawdown, 23 feet after pumping 600 gallons a minute for 36 hours; yield, 600 gallons a minute.

Well 2, South. Drilled by D. L. McDonald in 1939; depth, 450 feet; diameter, 16 inches; deep-well turbine pump and 125-horseporer electric motor; pump set at 420 feet; static water level, 347 feet below land surface in 1947; drawdown of 23 feet after pumping 600 gallors a minute for 36 hours; yield, 600 gallons a minute.

Well 1, North. Drilled by D. L. McDonald in 1935; depth, 395 feet; diameter, 18 inches; deep-well turbine pump and 60-horsepover electric motor; yield, 315 gallons a minute.

Well 3, North. Drilled by D. L. McDonald; depth, 412 feet; d:ameter, 18 inches; deep-well turbine pump and 75-horsepower electric motor; static water level, 358 feet below land surface in 1947; drawdown of 22 feet after pumping 460 gallons a minute for 36 hours; yield, 460 gallons a minute.

Well 4, North. Drilled by D. L. McDonald in 1945; depth, 414 feet; diameter, 151/2 inches; deep-well turbine pump and 75-horsepower electric motor, pump set at 400 feet; static water level, 333 feet below land surface in 1947; drawdown of 40 feet after pumping 520 gallons a minute for 36 hours; yield, 520 gallons a minute.

Pumpage: Maximum, 3,000,000 gallons a day; minimum, 1,000,00? gallons a day; average, 1,500,000 gallons a day. 
Storage: Ground storage reservoir, 100,000 gallons; four ground storage reservoirs, 3,360,000 gallons, 440,000 gallons, 220,000 gallons, and 220,000 gallons, respectively.

Number of customers : 4,000 .

Treatment: None.

\section{Analysis}

[Composite sample of all wells, collected Nov. 20, 1947. pH is 7.4. Analyzed by B. C. Dwyer]

\begin{tabular}{|c|c|c|c|c|c|}
\hline & $\begin{array}{c}\text { Parts per } \\
\text { million }\end{array}$ & $\begin{array}{l}\text { Equivalents } \\
\text { per million }\end{array}$ & & $\begin{array}{l}\text { Parts per } \\
\text { million }\end{array}$ & $\begin{array}{l}\text { Equivalents } \\
\text { per million }\end{array}$ \\
\hline $\begin{array}{l}\text { Silica }\left(\mathrm{SiO}_{2}\right) \\
\text { Iron }(\mathrm{Fe}) \\
\text { Calcium }(\mathrm{Ca}) \\
\text { Magnesium }(\mathrm{Mg}) \\
\text { Sodium }(\mathrm{Na}) \\
\text { Potassium }(\mathrm{K}) \\
\text { Bicarbonate }\left(\mathrm{HCO}_{3}\right)\end{array}$ & $\begin{array}{l}12 \\
59 \\
27 \\
113 \\
12 \\
230\end{array}$ & $\begin{array}{r}2.94 \\
2.22 \\
4.91 \\
.48 \\
3.77\end{array}$ & $\begin{array}{l}\text { Sulfate }\left(\mathrm{SO}_{4}\right) \\
\text { Chloride }(\mathrm{Cl}) \\
\text { Fluoride (F) } \\
\text { Nitrate (NO }) \\
\text { Dissolved solids. } \\
\text { Total hardness as } \mathrm{CaCO}\end{array}$ & $\begin{array}{l}141 \\
132 \\
1.2 \\
4.0 \\
638 \\
258\end{array}$ & \begin{tabular}{r}
2.94 \\
3.72 \\
.06 \\
.06 \\
\\
\hdashline.-- \\
\end{tabular} \\
\hline
\end{tabular}

\section{HALE COUNTY}

Population in 1940: 847.

\section{ABERNATHY}

Source of information: 0 . Rutledge, water superintendent, Nov. 16, 1945.

Ownership: Municipal.

Source of supply: Two wells.

Well 1. Drilled in 1934; depth, 200 feet; deep-well turbine pump and 25horsepower electric motor; yield, 300 gallons a minute.

Well 2. Drilled in 1944 by Clowe and Clowan; depth, 226 feet; diameter 15 inches; deep-well turbine pump and 40-horsepower e?ectric motor; yield, 600 gallons a minute.

Pumpage: Average, 150,000 gallons a day in winter; 550,000 gallons a day in summer.

Storage: Elevated tank, 150,000 gallons.

Number of customers: 275.

Treatment: None.

\section{Analysis}

[Composite sample of wells 1 and 2. Collected Nov. 16, 1945. pH is 7.2. Analyzed by C. B. Cibulka]

\begin{tabular}{|c|c|c|c|c|c|}
\hline & $\begin{array}{l}\text { Parts per } \\
\text { million }\end{array}$ & $\begin{array}{l}\text { Equivalents } \\
\text { per million }\end{array}$ & & $\begin{array}{c}\text { Part? per } \\
\text { mill ion }\end{array}$ & $\begin{array}{l}\text { Equivalents } \\
\text { per million }\end{array}$ \\
\hline $\begin{array}{l}\text { Silica }\left(\mathrm{SiO}_{2}\right) \\
\text { Iron }(\mathrm{Fe}) \\
\text { Calcium }(\mathrm{Ca}) \\
\text { Magnesium }(\overline{\mathrm{Mg}}) \\
\text { Sodium (Na) } \\
\text { Potassium (K) } \\
\left.\left.\text { Bicarbonate ( } \mathrm{H}_{\mathrm{C}} \mathrm{CO}\right)_{3}\right)\end{array}$ & $\begin{array}{l}37 \\
49 \\
33 \\
23 \\
10 \\
301\end{array}$ & $\begin{array}{r}2.45 \\
2.71 \\
.98 \\
.26 \\
4.93\end{array}$ & $\begin{array}{l}\text { Sulfate }\left(\mathrm{SO}_{4}\right) \\
\text { Chloride (Cl) } \\
\text { Fluoride (F) } \\
\left.\text { Nitrate (NO }{ }_{2}\right) \\
\text { Dissolved solids. } \\
\text { Total hardness as } \mathrm{CaCO}_{8}\end{array}$ & $\begin{array}{l}21 \\
20 \\
2.2 \\
4.0 \\
358 \\
258\end{array}$ & $\begin{array}{r}0.44 \\
.85 \\
.12 \\
.06 \\
-0.0 \\
\end{array}$ \\
\hline
\end{tabular}

Driller's log, well 2

\begin{tabular}{|c|c|c|c|c|c|}
\hline & $\begin{array}{c}\text { Thickness } \\
\text { (feet) }\end{array}$ & $\begin{array}{l}\text { Depth } \\
\text { (feet) }\end{array}$ & & $\begin{array}{c}\text { Thickness } \\
\text { (feet) }\end{array}$ & $\begin{array}{l}\text { Depth } \\
\text { (feet) }\end{array}$ \\
\hline $\begin{array}{l}\text { Surface soil. } \\
\text { Caliche } \\
\text { Red clay } \\
\text { Red sardy clay } \\
\text { Sand } \\
\text { Dry red sand }\end{array}$ & $\begin{array}{r}5 \\
5 \\
50 \\
10 \\
10 \\
30\end{array}$ & $\begin{array}{r}5 \\
10 \\
60 \\
70 \\
80 \\
110\end{array}$ & $\begin{array}{l}\text { Red clay } \\
\text { Red sandy clay, little water.- } \\
\text { Sand and gravel, water } \\
\text { Red sandy clay } \\
\text { Rock } \\
\text { Yellow sandy clay }\end{array}$ & $\begin{array}{r}25 \\
15 \\
20 \\
2 \\
25 \\
29\end{array}$ & $\begin{array}{l}135 \\
150 \\
170 \\
172 \\
197 \\
226\end{array}$ \\
\hline
\end{tabular}




\section{HALG CENTER}

Population in 1940: 836.

Source of information: R. C. Davis, water superintendent, Mar. 3, 1945.

Ownership: Municipal.

Source of supply: Two wells.

Well 1. Drilled in 1926 by W. G. Sears; depth, 120 feet; diameter, 12 inches; deep-well turbine pump and 50-horsepower electric motor; static water level, 51 feet below land surface; drawdown reported, 40 feet below land surface after pumping 543 gallons a minute for 12 hours; yield, 550 gallons a minute; temperature, $64^{\circ} \mathrm{F}$.

Well 2. Drilled in 1936 by Garms and Mounts; depth, 123 feet; diameter, 12 inches; deep-well turbine pump and 25-horsepower electric motor; yield, 350 gallons a minute.

Pumpage: Average, 150,000 gallons a day.

Storage: Elevated tank, 50,000 gallons.

Number of customers: 214.

Treatment: None.

Analysis, well 2

[Collected Mar. 3, 1945. $\mathrm{pH}$ is 7.6. Analyzed by J. H. Rowley]

\begin{tabular}{|c|c|c|c|c|c|}
\hline & $\begin{array}{c}\text { Parts per } \\
\text { million }\end{array}$ & $\begin{array}{l}\text { Equivalent } \\
\text { per million }\end{array}$ & & $\begin{array}{c}\text { Parts per } \\
\text { million }\end{array}$ & $\begin{array}{l}\text { Equivalents } \\
\text { per million }\end{array}$ \\
\hline $\begin{array}{l}\text { Silica }\left(\mathrm{SiO}_{2}\right) \\
\text { Iron }(\mathrm{Fe}) \\
\text { Calcium }(\mathrm{Ca}) \\
\text { Magnesium }(\overline{\mathrm{Mg}}) \\
\text { Sodium }(\mathrm{Na}) \\
\text { Potassium }(\mathrm{K}) \\
\text { Bicarbonate }\left(\mathrm{HCO}_{3}\right)^{-}\end{array}$ & $\begin{array}{l}54 \\
50 \\
52 \\
21 \\
8.02 \\
349\end{array}$ & $\begin{array}{r}2.50 \\
4.28 \\
.91 \\
.23 \\
5.72\end{array}$ & $\begin{array}{l}\text { Sulfate }\left(\mathrm{SO}_{4}\right) \\
\text { Chloride (Cl) } \\
\text { Fluoride (F) } \\
\text { Nitrate (NOz) } \\
\text { Dissolved solids } \\
\text { Total hardness as } \mathrm{CaCO}\end{array}$ & $\begin{array}{c}43 \\
35 \\
4.4 \\
5.2 \\
445 \\
339\end{array}$ & $\begin{array}{r}0.90 \\
.99 \\
.23 \\
.08 \\
-0 \\
-0 .-\end{array}$ \\
\hline
\end{tabular}

\section{PETERSBURG}

Population in 1940: 496.

Source of information: L. A. Peeples, well driller, Nov. 30, 1945.

Ownership: Municipal.

Source of supply: Well drilled in 1945 by L. A. Peeples; depth, 222 feet; diameter, 12 inches; deep-well turbine pump and electric motor; yield, 600 gallons a minute; temperature, $631 / 2^{\circ}$ F. Municipal supply still under process of construction.

Pumpage: No record.

\section{Analysis}

[Collected Nov. 30, 1945. pH is 8.2. Analyzed by J. H. Rowley]

\begin{tabular}{|c|c|c|c|c|c|}
\hline & $\begin{array}{l}\text { Parts per } \\
\text { million }\end{array}$ & $\begin{array}{l}\text { Equivalents } \\
\text { per million }\end{array}$ & & $\begin{array}{c}\text { Parts per } \\
\text { million }\end{array}$ & $\begin{array}{l}\text { Equivalents } \\
\text { per mi lion }\end{array}$ \\
\hline 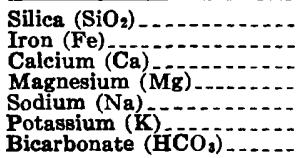 & $\begin{array}{l}42 \\
36 \\
40 \\
34 \\
11 \\
334\end{array}$ & $\begin{array}{r}\mathbf{1} .80 \\
\mathbf{3} .29 \\
\mathbf{1 . 5 0} \\
.28 \\
\mathbf{5 . 4 7}\end{array}$ & $\begin{array}{l}\text { Sulfate (SOH) } \\
\text { Chloride (CI) } \\
\text { Fluoride (F) } \\
\text { Nitrate (NOs) } \\
\text { Dissolved solids. } \\
\text { Total hardness as } \mathrm{CaCO}_{3}\end{array}$ & $\begin{array}{c}34 \\
17 \\
3.6 \\
1.2 \\
383 \\
254\end{array}$ & $\begin{array}{r}0.71 \\
.48 \\
.19 \\
.02 \\
-0.9 \\
\end{array}$ \\
\hline
\end{tabular}


Population in 1940: 8,492 .

\section{PLAINVIEW}

Source of information: S. E. Bolles, water superintendent, Feb. 28, 1945.

Ownership: Municipal.

Source of supply: Three wells.

Well 1. Drilled in 1937 by L. A. Peeples; depth, 301 fest; diameter, 18 inches; deep-well turbine pump and electric motor; yie' $d, 852$ gallons a minute with drawdown of 60 feet in 1937; temperature, $64^{\circ} \mathrm{F}$.

Well 2. Drilled in 1937 by L. A. Peeples; depth, 301 fe?t; diameter, 18 inches; deep-well turbine pump and electric motor; yie'd, 628 gallons a minute with drawdown of 60 feet in 1937.

Well 3. Drilled in 1937 by L. A. Peeples; depth, 301 feet; diameter, 8 inches; deep-well turbine pump and electric motor; yield, 1,086 gallons a minute with drawdown of 63 feet in 1937.

Pumpage: Average, 1,050,000 gallons a day.

Storage: Concrete ground storage reservoir, 500,000 gallons; elevated tank, 200,000 gallons.

Number of customers: 2,127 .

Treatment: None.

Analysis, well 1

[Collected Feb. 28, 1945. pH is 7.4. Analyzed by J. H. Rowley]

\begin{tabular}{|c|c|c|c|c|c|}
\hline & $\begin{array}{c}\text { Parts per } \\
\text { million }\end{array}$ & $\mid \begin{array}{l}\text { Equivalents } \\
\text { per million }\end{array}$ & & $\begin{array}{l}\text { Prrts per } \\
\text { million }\end{array}$ & $\begin{array}{l}\text { Equivalents } \\
\text { per million }\end{array}$ \\
\hline $\begin{array}{l}\text { Silica }\left(\mathrm{SiO}_{2}\right) \\
\text { Iron }(\mathrm{Fe}) \\
\text { Calcium }(\mathrm{Ca}) \\
\text { Magnesium }(\mathrm{Mg}) \\
\text { Sodium }(\mathrm{Na}) \\
\text { Potassium }(\mathrm{K}) \\
\text { Bicarbonate }\left(\mathrm{HCO}_{2}\right)\end{array}$ & $\begin{array}{c}60 \\
44 \\
37 \\
28 \\
8.50 \\
329\end{array}$ & $\begin{array}{r}2.20 \\
3.04 \\
1.23 \\
.22 \\
5.39\end{array}$ & $\begin{array}{l}\text { Sulfate }\left(\mathrm{SO}_{4}\right) \\
\text { Chloride }(\mathrm{Cl}) \\
\text { Fluoride }(\mathrm{F}) \\
\text { Nitrate }\left(\mathrm{NO}_{3}\right) \\
\text { Dissolved solids. } \\
\text { Total hardness as } \mathrm{CaCO}_{3}\end{array}$ & $\begin{array}{c}28 \\
18 \\
3.6 \\
1.2 \\
390 \\
262\end{array}$ & \begin{tabular}{r}
0.58 \\
.51 \\
.19 \\
.02 \\
\hdashline.- \\
.-
\end{tabular} \\
\hline
\end{tabular}

Driller's log, well 1

\begin{tabular}{|c|c|c|c|c|c|}
\hline & $\begin{array}{c}\text { Thickness } \\
\text { (feet) }\end{array}$ & $\begin{array}{l}\text { Depth } \\
\text { (feet) }\end{array}$ & & $\begin{array}{c}\text { Thickness } \\
\text { (feet) }\end{array}$ & $\underset{\text { (feet) }}{\text { Depth }}$ \\
\hline $\begin{array}{l}\text { Top soil } \\
\text { Red caliche. } \\
\text { Hard caliche and nodules. } \\
\text { Pink gravel } \\
\text { Sandy caliche. } \\
\text { Red shale } \\
\text { Sand and gravel } \\
\text { Packed sand } \\
\text { Rock }\end{array}$ & $\begin{array}{r}5 \\
29 \\
9 \\
16 \\
2 \\
9 \\
15 \\
25 \\
6\end{array}$ & $\begin{array}{r}5 \\
\mathbf{3 4} \\
43 \\
\mathbf{5 9} \\
61 \\
70 \\
85 \\
110 \\
116\end{array}$ & $\begin{array}{l}\text { White sand } \\
\text { Red sand } \\
\text { Hard sand } \\
\text { Sand. } \\
\text { Fine-grained sand } \\
\text { Gritty sand } \\
\text { Coarse-grained sand. }\end{array}$ & $\begin{array}{r}21 \\
10 \\
14 \\
15 \\
18 \\
9 \\
10 \\
88\end{array}$ & $\begin{array}{l}137 \\
147 \\
161 \\
176 \\
194 \\
203 \\
213 \\
301\end{array}$ \\
\hline
\end{tabular}

\section{HALL COUNTY}

Population in 1940: 603.

\section{ESTELLINE}

Source of information: H. J. Rogers, co-owner, Mar. 28, 1948.

Cwners: A. J. Rogers and H. J. Rogers.

Source of supply: Two wells.

Well 1. At elevated tank; dug in 1929; depth, 47 feet; diameter, 8 feet; deep-well cylinder pump and 5 -horsepower electric mot`r; static water 
level, 37.8 feet below land surface Mar. 8, 1947; yield, 40 gallons a minute.

Well 2. About 250 feet south of well 1; drilled; depth, 58 feet; diameter, 8 inches; deep-well cylinder pump and 5-horsepower electric motor; yield, 45 gallons a minute.

Average pumpage, in gallons a day

\begin{tabular}{|c|c|c|c|}
\hline & 1945 & 1949 & 1947 \\
\hline 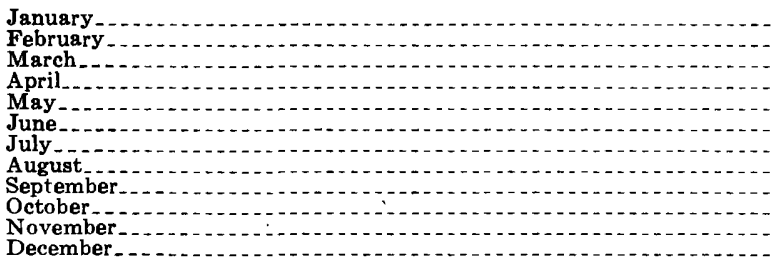 & 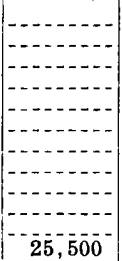 & $\begin{array}{l}23,800 \\
26,200 \\
19,300 \\
27,000 \\
26,300 \\
37,200 \\
34,200 \\
48,800 \\
29,000 \\
21,300 \\
24,800 \\
22,400\end{array}$ & 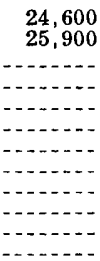 \\
\hline
\end{tabular}

Storage: Ground reservoir, 15,000 gallons; elevated tank, 50,000 gallons.

Number of customers: 150.

Treatment: Chlorination.

\section{Analysis}

[Composite sample of wells 1 and 2. Collected Mar. 28, 1947. pH is 7.6. Analyzed by B. C. Dwyer]

\begin{tabular}{|c|c|c|c|c|c|}
\hline & $\begin{array}{c}\text { Parts per } \\
\text { million }\end{array}$ & $\begin{array}{l}\text { Equivalents } \\
\text { per million }\end{array}$ & & $\begin{array}{l}\text { Parts per } \\
\text { million }\end{array}$ & $\begin{array}{l}\text { Equivalents } \\
\text { per million }\end{array}$ \\
\hline 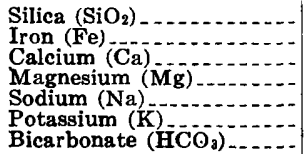 & $\begin{array}{c}8.8 \\
47.09 \\
28 \\
97 \\
5.9 \\
358\end{array}$ & $\begin{array}{r}2.35 \\
2.30 \\
4.23 \\
5.15 \\
5.87\end{array}$ & $\begin{array}{l}\text { Sulfate }\left(\mathrm{SO}_{4}\right) \\
\text { Chloride }(\mathrm{Cl}) \\
\text { Fluoride (F) } \\
\left.\text { Nitrate (NO }{ }_{3}\right) \\
\text { Dissolved solids } \\
\text { Total hardness as } \mathrm{CaCO}_{3}\end{array}$ & $\begin{array}{l}77 \\
34 \\
1.0 \\
34 \\
538 \\
232\end{array}$ & $\begin{array}{r}1.60 \\
.96 \\
.05 \\
.55 \\
-5\end{array}$ \\
\hline
\end{tabular}

\section{MEMPHIS}

Population in 1940: 3,869 .

Source of information: Roy Fultz, Manager, June 1943.

Owner: Community Public Service Co.

Source of supply: Forty-one shallow wells in three well fields aborat 6 miles northwest of the city in Donley County, and one well on the Milam farm in Hall County. The Memphis well field in Donley County consist: of three batteries of shallow dug wells ranging in depth from 12 to 25 feet. The east battery consists of 9 wells, the middle battery 16 wells, and the west battery 16 wells. Wells flow into collecting reservoirs. The water then flows through pipe lines by gravity into the city of Memphis. Combined yield of all wells, about 200 gallons a minute.

Milam Farm well. Stand-by well; drilled in 1941; depth, 30 feet; diameter, 12 inches; deep-well turbine pump and electric motor; static water level, 10.2 feet below land surface in 1941; yield, 45 gallons a minute. 
Average pumpage, in gallons a day

\begin{tabular}{|c|c|c|c|}
\hline & 1941 & 1942 & 1943 \\
\hline 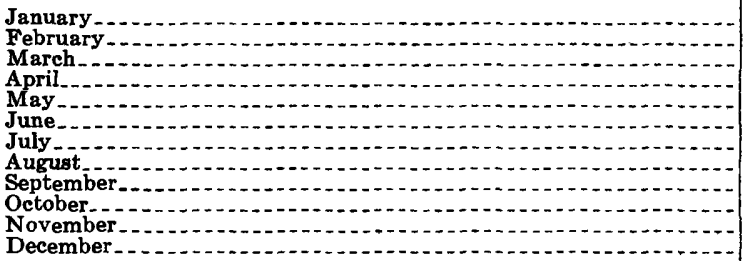 & $\begin{array}{l}141,935 \\
182,142 \\
119,354 \\
153,333 \\
138,709 \\
126,666 \\
154,838 \\
190,322 \\
173,333 \\
164,, 16 \\
203,333 \\
203,225\end{array}$ & $\begin{array}{l}135,483 \\
196,428 \\
151,612 \\
176,666 \\
148,387 \\
186,666 \\
219,354 \\
222,580 \\
223,333 \\
170,967 \\
230,000 \\
161,290\end{array}$ & 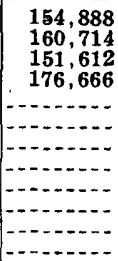 \\
\hline
\end{tabular}

Storage: Ground storage reservoir at Donley County well field, 510,000 gallons; elevated tank.

Treatment: Chlorination.

\section{Analysis}

[Composite sample of 41 wells in Donley County. Collected May 20, 1943. pH iv 8.4. Analyzed by J. H. Rowley]

\begin{tabular}{|c|c|c|c|c|c|}
\hline & $\begin{array}{c}\text { Parts per } \\
\text { million }\end{array}$ & $\begin{array}{l}\text { Equivalents } \\
\text { per million }\end{array}$ & & $\begin{array}{c}\text { Parts per } \\
\text { m'llion }\end{array}$ & $\begin{array}{l}\text { Equivalents } \\
\text { per million }\end{array}$ \\
\hline $\begin{array}{l}\text { Silica }\left(\mathrm{SiO}_{2}\right) \\
\text { Iron }(\mathrm{Fe}) \\
\text { Calcium }(\mathrm{Ca}) \\
\text { Magnesium }(\mathrm{Mg}) \\
\text { Sodium and potassium } \\
(\mathrm{Na}+\mathrm{K}) \text {. } \\
\text { Bicarbonate }\left(\mathrm{HCO}_{3}\right)\end{array}$ & $\begin{array}{l}26 \\
73 \\
10 \\
19 \\
260\end{array}$ & $\begin{array}{r}\mathbf{3 . 6 4} \\
.82 \\
.81 \\
4.26\end{array}$ & $\begin{array}{l}\text { Sulfate }\left(\mathrm{SO}_{4}\right) \\
\text { Chloride }(\mathrm{Cl}) \\
\text { Fluoride }(\mathrm{F}) \\
\text { Nitrate (NOs) } \\
\text { Dissolved solids } \\
\text { Total hardness as } \mathrm{CaCO}_{3}\end{array}$ & $\begin{array}{r}20 \\
17 \\
5.6 \\
309 \\
223\end{array}$ & $\begin{array}{r}0.42 \\
.48 \\
.03 \\
.08 \\
-0.0 \\
\end{array}$ \\
\hline
\end{tabular}

Population in 1940: 930.

\section{TURKEY}

Source of information: G. O. Coker, water superintendent, Mar. 28, 1947.

Ownership: Municipal.

Source of supply: Two wells.

Well 1. Four and one-half miles southwest of Turkey; drilled in 1928

by S. H. Kimball; depth, 100 feet; diameter, 12 inches; deep-well turbine pump and 15-horsepower electric motor; static water level, 40 feet below land surface; yield, 200 gallons a minute.

Well 2. About 300 feet southwest of well 1 ; drilled in 1928 by S. H. Kimball; depth, 100 feet; diameter, 12 inches; deep-well turbine pump and 15-horsepower electric motor; yield, 150 gallons a minute.

Pumpage: Average, 75,000 gallons a day.

Storage: Ground storage reservoir, 200,000 gallons; elevated tank, 50,000 gallons.

Number of customers: 260.

Treatment: Chlorination.

\section{Analysis}

[Composite sample of wells 1 and 2. Collected Mar. 28, 1947. pH is 7.4. Analyzed by B. C. Dwyer]

\begin{tabular}{|c|c|c|c|c|c|}
\hline & $\begin{array}{c}\text { Parts per } \\
\text { million }\end{array}$ & $\begin{array}{l}\text { Equivalents } \\
\text { per million }\end{array}$ & & $\begin{array}{l}\text { Par's per } \\
\text { milion }\end{array}$ & $\begin{array}{l}\text { Equivalents } \\
\text { per million }\end{array}$ \\
\hline $\begin{array}{l}\text { Silica }\left(\mathrm{SiO}_{2}\right) \\
\text { Iron }(\mathrm{Fe}) \\
\text { Calcium }(\mathrm{Ca}) \\
\text { Magnesium }(\mathrm{Mg}) \\
\text { Sodium }(\mathrm{Na}) \\
\text { Potassium }(\mathrm{K})^{-} \\
\text {Bicarbonate }\left(\mathrm{HCO}_{8}\right)^{-}\end{array}$ & $\begin{array}{c}26 \\
1.0 \\
152 \\
56 \\
93 \\
8.5 \\
262\end{array}$ & $\begin{array}{r}7.49 \\
4.61 \\
4.05 \\
4.22 \\
4.29\end{array}$ & $\begin{array}{l}\text { Sulfate }\left(\mathrm{SO}_{4}\right) \\
\text { Chloride }(\mathrm{Cl}) \\
\text { Fluoride }(\mathrm{F}) \\
\text { Nitrate }(\mathrm{NO}) \\
\text { Dissolved solids } \\
\text { Total hardness as } \mathrm{CaCO}\end{array}$ & $\begin{array}{c}394 \\
140 \\
1.6 \\
10 \\
1,090 \\
610\end{array}$ & 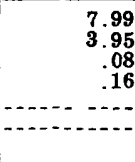 \\
\hline
\end{tabular}




\section{HANSFORD COUNTY}

Population in 1940: 350.

\section{GRUVER}

Source of information: Earl Lowe, Southwestern Public Service Co., June $23,1948$.

Owner: Southwestern Public Service Co.

Source of supply: Two wells.

Well 1. Drilled in 1931 by A. H. Masiran; depth, 342 feet; diameter, 10 inches; deep-well turbine pump and 10-horsepower electric motor; static water level, 158.5 feet below land surface in July 1931; drawdown of 19 feet in 1948.

Well 2. Drilled in May 1948 by H. H. Heiskell; depth, 413 feet; diameter, 13 inches; deep-well turbine pump and 20-horsepower electric motor; static water level, 184 feet below land surface; drawdown of 37 feet when pumping 490 gallons a minute.

Pumpage: Average, 100,000 gallons a day.

Storage: Elevated tank, 50,000 gallons; ground storage reservoir to be installed, 100,000 gallons.

Number of customers : 250.

Treatment: None.

Analysis, well 2

[Collected June 23, 1948. pH is 7.5. Analyzed by H. D. Smith]

\begin{tabular}{|c|c|c|c|c|c|}
\hline & $\begin{array}{c}\text { Parts per } \\
\text { million }\end{array}$ & $\begin{array}{l}\text { Equivalents } \\
\text { per million }\end{array}$ & & $\begin{array}{c}\text { Parts per } \\
\text { million }\end{array}$ & $\begin{array}{l}\text { Equivalents } \\
\text { per million }\end{array}$ \\
\hline $\begin{array}{l}\text { Silica }\left(\mathrm{SiO}_{2}\right) \\
\text { lron }(\mathrm{Fe}) \\
\text { Calcium }(\mathrm{Ca}) \\
\text { Magnesium }(\mathrm{Mg}) \\
\text { Sodium }(\mathrm{Na}) \\
\text { Potassium }(\mathrm{K}) \\
\text { Bicarbonate }\left(\mathrm{H} \mathrm{CO}_{3}\right)\end{array}$ & $\begin{array}{l}33 \\
1.8 \\
31 \\
42 \\
8.4 \\
302\end{array}$ & $\begin{array}{r}2.20 \\
2.55 \\
1.83 \\
.21 \\
4.95\end{array}$ & $\begin{array}{l}\text { Sulfate }\left(\mathrm{SO}_{4}\right) \\
\text { Chloride }(\mathrm{Cl}) \\
\text { Fluoride }(\mathrm{F}) \\
\text { Nitrate }\left(\mathrm{NO}_{3}\right) \\
\text { Dissolved solids } \\
\text { Total hardness as } \mathrm{CaCU}\end{array}$ & $\begin{array}{r}67 \\
11 \\
2.2 \\
7.8 \\
376 \\
238\end{array}$ & \begin{tabular}{r}
1.39 \\
.31 \\
.12 \\
.13 \\
\hdashline$-1-$ \\
\end{tabular} \\
\hline
\end{tabular}

Driller's log, well 1

\begin{tabular}{|c|c|c|c|c|c|}
\hline & $\begin{array}{c}\text { Thickness } \\
\text { (feet) }\end{array}$ & $\underset{\text { (feet) }}{\text { Depth }}$ & & $\begin{array}{l}\text { Trickness } \\
\text { (feet) }\end{array}$ & $\begin{array}{l}\text { Depth } \\
\text { (feet) }\end{array}$ \\
\hline $\begin{array}{l}\text { Brown sandy clay } \\
\text { Light-brown sandy clay } \\
\text { Broken sand, rock and clay. } \\
\text { Very fine water sand. } \\
\text { Light-brown sandy clay. } \\
\text { Fine sand. } \\
\text { Broken rock, sand. }\end{array}$ & $\begin{array}{r}10 \\
110 \\
60 \\
10 \\
35 \\
36 \\
57\end{array}$ & $\begin{array}{r}10 \\
120 \\
180 \\
190 \\
225 \\
261 \\
318\end{array}$ & $\begin{array}{l}\text { Red clay } \\
\text { Very hard sandrock } \\
\text { Red clay } \\
\text { Very hard sandrock. } \\
\text { Sand and gravel, water. } \\
\text { Very hard sar drock. }\end{array}$ & $\begin{array}{r}2 \\
2 \\
2 \\
2 \\
14 \\
2\end{array}$ & $\begin{array}{l}320 \\
322 \\
324 \\
326 \\
340 \\
342\end{array}$ \\
\hline
\end{tabular}

\section{HITCHLAND}

Population in 1940: 100.

Source of information: E. P. Siler, station agent, June 23, 1948.

Owner: C. R. I. \& P. Railroad Co.

Source of supply: Well, which supplies the railroad as well as the city.

Well 1. Drilled by D. L. McDonald; depth, 484 feet; diameter, 10 inches; deep-well turbine pump and 25-horsepower electric motor; static water level, $1 \diamond 6.0$ feet below land surface August 3, 1936.

Pumpage: Average, 25,000 gallons a day.

Storage: Elevated tank, 48,000 gallons.

Number of customers: 7 .

Treatment: None. 
Analysis, well 1

[Collected June 23, $1948 \mathrm{pH}$ is 7.5. Analyzed by H. D. Smith]

\begin{tabular}{|c|c|c|c|c|c|}
\hline & $\begin{array}{l}\text { Parts per } \\
\text { million }\end{array}$ & $\begin{array}{l}\text { Equivalents } \\
\text { per million }\end{array}$ & & $\begin{array}{c}\text { Parts per } \\
\text { million }\end{array}$ & $\begin{array}{l}\text { Equivalents } \\
\text { Jer million }\end{array}$ \\
\hline $\begin{array}{l}\text { Silica }\left(\mathrm{SiO}_{2}\right) \\
\text { Iron (Fe) } \\
\text { Calcium }(\mathrm{Ca}) \\
\text { Magnesium }(\mathrm{M} \mathbf{g}) \\
\text { Sodium }(\mathrm{Na}) \\
\text { Potassium }(\mathrm{K}) \\
\text { Bicarbonate }\left(\mathrm{H}^{2} \mathrm{CO}_{3}\right)\end{array}$ & $\begin{array}{l}35 \\
51 \\
32 \\
26 \\
8.8 \\
254\end{array}$ & $\begin{array}{r} \\
2.55 \\
2.63 \\
1.13 \\
\times \quad 4.23 \\
* 16\end{array}$ & $\begin{array}{l}\text { Sulfate }\left(\mathrm{SO}_{4}\right) \\
\text { Chloride }(\mathrm{Cl}) \\
\text { Fluoride (F) } \\
\left.\text { Nitrate (NO})_{3}\right) \\
\text { Dissolved solids } \\
\text { Total hardness as } \mathrm{CaCO}\end{array}$ & $\begin{array}{c}87 \\
10 \\
1.8 \\
8.3 \\
\mathbf{3 7 3} \\
\mathbf{2 5 9}\end{array}$ & $\begin{array}{r}1.81 \\
.28 \\
.09 \\
.13 \\
-0 .-\end{array}$ \\
\hline
\end{tabular}

\section{Driller's log, well 1}

\begin{tabular}{|c|c|c|c|c|c|}
\hline & $\underset{\text { (feet) }}{\text { Thickness }}$ & $\begin{array}{c}\text { Depth } \\
\text { (feet) }\end{array}$ & & $\underset{\text { ('eet) }}{\text { Thickness }}$ & $\begin{array}{l}\text { Depth } \\
\text { (feet) }\end{array}$ \\
\hline $\begin{array}{l}\text { Soil } \\
\text { Soft caliche } \\
\text { Hard caliche } \\
\text { Yellow clay } \\
\text { Rock }\end{array}$ & $\begin{array}{l}\mathbf{2} \\
\mathbf{8} \\
\mathbf{3} \\
\mathbf{7} \\
\mathbf{3}\end{array}$ & $\begin{array}{r}2 \\
10 \\
13 \\
20 \\
23\end{array}$ & $\begin{array}{l}\text { Sandy clay } \\
\text { Rock } \\
\text { Sandy clay } \\
\text { Clean sand. }\end{array}$ & $\begin{array}{r}132 \\
3 \\
32 \\
85\end{array}$ & $\begin{array}{l}155 \\
158 \\
190 \\
275\end{array}$ \\
\hline
\end{tabular}

Population in 1940: 200.

\section{MORSE}

Source of information: Earl Lowe, superintendent, Southwestern Public Service Co., June 23, 1948.

Owner: Southwestern Public Service Co.

Source of supply: Well, drilled in 1931 by W. M. Brown; depth, 354 feet; deep-well turbine pump and $7 \frac{1}{2}$-horsepower electric motor; static water level, 159 feet below land surface in spring of 1948; drawdowr of 20 feet. Pumpage: No record.

Storage: Elevated tank, 4,500 gallons.

Number of customers: 15.

Treatment: None.

\section{Analysis}

[Collected June 23, 1948. pH is 7.8. Analyzed by H. D. Smith]

\begin{tabular}{|c|c|c|c|c|c|}
\hline & $\begin{array}{l}\text { Parts per } \\
\text { million }\end{array}$ & $\begin{array}{l}\text { Equivalents } \\
\text { per million }\end{array}$ & & $\begin{array}{c}\text { Parts per } \\
\text { million }\end{array}$ & $\begin{array}{l}\text { Equivalents } \\
\text { per million }\end{array}$ \\
\hline $\begin{array}{l}\text { Silica }\left(\mathrm{SiO}_{2}\right) \\
\text { Iron }(\mathrm{Fe}) \\
\text { Calcium }(\mathrm{Ca}) \\
\text { Magnesium }(\mathrm{Mg}) \\
\text { Sodium (Na) } \\
\text { Potassium }(\mathrm{K}) \\
\text { Bicarbonate (HCOs) }\end{array}$ & $\begin{array}{c}44 \\
1.2 \\
36 \\
28 \\
12 \\
9.6 \\
242\end{array}$ & $\begin{array}{r}\mathbf{1} .797 \\
2.303 \\
.522 \\
.246 \\
\mathbf{3 . 9 6 7}\end{array}$ & $\begin{array}{l}\text { Sulfate }\left(\mathrm{SO}_{4}\right) \\
\text { Chloride (Cl) } \\
\text { Fluoride (F) } \\
\text { Nitrate (NO } \\
\text { Dissolved solids } \\
\text { Total hardness as } \mathrm{CaCO}_{3}\end{array}$ & $\begin{array}{l}24 \\
8.4 \\
2.2 \\
5.8 \\
260 \\
205\end{array}$ & $\begin{array}{r}0.500 \\
.237 \\
.116 \\
.094 \\
-0.0 \\
\end{array}$ \\
\hline
\end{tabular}

Population in 1940: 1,105.

\section{SPEARMAN}

Source of information: Leo Ducas, city manager, June 23, 1948. Ownership: Municipal. 
Source of supply: Three wells.

Well 1. Drilled in 1924 by Ed Wilbanks; depth, 348 feet; diameter, 10 inches; deep-well turbine pump and 25-horsepower elsctric motor; static water level, 256 feet below land surface May 23, 1936; drawdown of 25.2 feet after pumping 180 gallons a minute for 72 hours in 1936.

Well 2. Drilled; depth, 282 feet; diameter, 8 inches; deej-well turbine pump and 20-horsepower electric motor; static water level, 256.8 feet below land surface Aug. 14, 1936; drawdown of 22.9 feet after pumping 180 gallons a minute for 15 minutes.

Well 3. Drilled in June 1947 by Ed. Wilbanks; depth, 405 feet; deep-well turbine pump and 120-horsepower natural-gas engine; yield, 650 gallons a minute.

Pumpage: Average, 250,000 gallons a day.

Storage: Ground storage reservoir, 165,000 gallons; elevated tank, 75,000 gallons.

Number of customers: 550 .

Treatment: Chlorination.

Analysis, well 3

[Collected June 23, 1948, $\mathrm{pH}$ is 7.5. Analyzed by D. E. Weaver]

\begin{tabular}{|c|c|c|c|c|c|}
\hline & $\begin{array}{l}\text { Parts per } \\
\text { million }\end{array}$ & $\begin{array}{l}\text { Equivalents } \\
\text { per million }\end{array}$ & & $\begin{array}{l}\text { Parts per } \\
\text { mil"on }\end{array}$ & $\begin{array}{l}\text { Equivalents } \\
\text { per million }\end{array}$ \\
\hline $\begin{array}{l}\text { Silica }\left(\mathrm{SiO}_{2}\right) \\
\text { Iron }(\mathrm{Fe}) \\
\text { Calcium }(\mathrm{Ca}) \\
\text { Magnesium }(\mathrm{M} \mathrm{g}) \\
\text { Sodium }(\mathrm{Na}) \\
\text { Potassium }(\mathrm{K}) \\
\text { Bicarbonate }\left(\mathrm{HCO}_{3}\right)^{-}\end{array}$ & $\begin{array}{l}42 \\
52 \\
27 \\
16 \\
1.6 \\
256\end{array}$ & $\begin{array}{r}2.60 \\
2.22 \\
.70 \\
.04 \\
4.20\end{array}$ & $\begin{array}{l}\text { Sulfate }\left(\mathrm{SO}_{4}\right) \\
\text { Chloride }(\mathrm{Cl}) \\
\text { Fluoride (F) } \\
\left.\text { Nitrate (N }{ }_{3}\right) \\
\text { Dissolved solids } \\
\text { Total hardness as } \mathrm{CaCO}\end{array}$ & $\begin{array}{l}43 \\
9.2 \\
1.2 \\
7.0 \\
3 ? 4 \\
241\end{array}$ & $\begin{array}{r}0.90 \\
.26 \\
.06 \\
.11 \\
\\
\end{array}$ \\
\hline
\end{tabular}

Driller's log, well 1

\begin{tabular}{|c|c|c|c|c|c|}
\hline & $\begin{array}{c}\text { Thickness } \\
\text { (feet) }\end{array}$ & $\begin{array}{l}\text { Depth } \\
\text { (feet) }\end{array}$ & & $\begin{array}{c}\text { Thickness } \\
\text { (feet) }\end{array}$ & $\begin{array}{c}\text { Depth } \\
\text { (feet) }\end{array}$ \\
\hline $\begin{array}{l}\text { Surface soil } \ldots \ldots \\
\text { Clay } \\
\text { Cap rock }\end{array}$ & $\begin{array}{r}3 \\
40 \\
15\end{array}$ & $\begin{array}{r}3 \\
43 \\
58\end{array}$ & $\begin{array}{l}\text { Packed sand } \\
\text { Clay } \\
\text { Coarse white sand. }\end{array}$ & $\begin{array}{r}202 \\
20 \\
68\end{array}$ & $\begin{array}{l}260 \\
280 \\
\mathbf{3 4 8}\end{array}$ \\
\hline
\end{tabular}

\section{HARTLEY COUNTY}

\section{CHANNING}

Population in 1940: 475.

Source of information: D. S. Perkins, Southwestern Public Service Co., June 24, 1948.

Owner: Southwestern Public Service Co.

Source of supply: Three wells.

Well 1. Drilled in 1910 by R. L. Hood; depth, 328 feet; diameter, 6 inches; deep-well turbine pump and 10-horsepower electric motor; static water level, 288 feet below land surface; yield, 24 gallons a minute.

Well 2. Drilled; depth, 350 feet; diameter, 4 inches; dee?-well turbine pump and 5-horsepower electric motor; yield, 15 gallons a minute. 
Well 3. Drilled in September 1946 by H. H. Heiskell; derth, 400 feet; diameter, 10 inches, casing perforated from 334 feet to $3 \subseteq 5$ feet; deepwell turbine pump and 20-horsepower electric motor; static water level, 290 feet below land surface in September 1946; yie!d, 80 gallons a minute.

Pumpage: 25,000 gallons a day.

Storage: Ground storage reservoir, 20,000 gallons; elevated tank, 50,000 gallons.

Number of customers: 100.

Treatment: None.

Analysis, well 3

[Collected June 24, 1948. $\mathrm{pH}$ is 7.4. Analyzed by D. E. Weaver]

\begin{tabular}{|c|c|c|c|c|c|}
\hline & $\begin{array}{l}\text { Parts per } \\
\text { million }\end{array}$ & $\begin{array}{l}\text { Equivalent } \\
\text { per million }\end{array}$ & & $\begin{array}{l}\text { Parts per } \\
\text { million }\end{array}$ & $\begin{array}{l}\text { Equivalents } \\
\text { per million }\end{array}$ \\
\hline $\begin{array}{l}\text { Silica }\left(\mathrm{SiO}_{2}\right) \\
\text { Iron }(\mathrm{Fe}) \\
\text { Calcium }(\mathrm{Ca}) \\
\text { Magnesium }(\mathrm{Mg}) \\
\text { Sodium }(\mathrm{Na}) \\
\text { Potassium }(\mathrm{K}) \\
\text { Bicarbonate }\left(\mathrm{H}^{2} \mathrm{CO}_{3}\right)\end{array}$ & $\begin{array}{l}32 \\
43.49 \\
22 \\
9.0 \\
2.4 \\
202\end{array}$ & $\begin{array}{r}2.146 \\
1.809 \\
.391 \\
.061 \\
3.311\end{array}$ & $\begin{array}{l}\text { Sulfate }\left(\mathrm{SO}_{4}\right) \\
\text { Chloride }(\mathrm{Cl}) \\
\text { Fluoride (F) } \\
\left.\text { Nitrate (NO}{ }_{3}\right) \\
\text { Dissolved solids } \\
\text { Total hardness as } \mathrm{CaCO}\end{array}$ & $\begin{array}{l}21 \\
13 \\
13 \\
26 ? \\
19 ?\end{array}$ & \begin{tabular}{r}
0.437 \\
.367 \\
.011 \\
.210 \\
\\
\hdashline \\
\end{tabular} \\
\hline
\end{tabular}

\section{HARTLEY}

Population in 1940: 150.

Source of information: R. M. Williams, Dec. 3, 1948.

Owner: E. E. Williams.

Source of supply: Well, drilled by R. Mitchell; depth, 400 feet; diameter, $61 / 4$ inches; deep-well turbine pump and 15 -horsepower electric motor.

Pumpage (estimated): 10,000 gallons a day.

Storage: Elevated tank, capacity unknown.

Treatment: None.

\section{Analysis}

[Collected Oct. 4, 1948, pH is 7.5. Analyzed by J. R. Avrett]

\begin{tabular}{|c|c|c|c|c|c|}
\hline & $\begin{array}{c}\text { Parts per } \\
\text { million }\end{array}$ & $\begin{array}{l}\text { Equivalents } \\
\text { per million }\end{array}$ & & $\begin{array}{c}\text { Parts per } \\
\text { million }\end{array}$ & $\begin{array}{l}\text { Equivalents } \\
\text { per million }\end{array}$ \\
\hline $\begin{array}{l}\text { Silica }\left(\mathrm{SiO}_{2}\right) \\
\text { Iron }(\mathrm{Fe}) \\
\text { Calcium }(\mathrm{Ca}) \\
\text { Magnesium }(\mathrm{Mg}) \\
\text { Sodium }(\mathrm{Na}) \\
\text { Potassium }(\mathrm{K}) \\
\text { Bicarbonate }\left(\mathrm{H} \mathrm{CO}_{3}\right)\end{array}$ & $\begin{array}{l}28 \\
34 \\
29 \\
29 \\
{ }_{27}^{8} .0\end{array}$ & $\begin{array}{r}1.70 \\
2.38 \\
1.26 \\
.20 \\
4.46\end{array}$ & $\begin{array}{l}\text { Sulfate }\left(\mathrm{SO}_{4}\right) \\
\text { Chloride }(\mathrm{Cl}) \\
\text { Fluoride (F) } \\
\left.\text { Nitrate (NO})_{3}\right) \\
\text { Dissolved solids as } \mathrm{CaCO}_{3} \\
\text { Total hardness as }\end{array}$ & $\begin{array}{r}34 \\
8.0 \\
1.2 \\
7.5 \\
314 \\
20.1\end{array}$ & 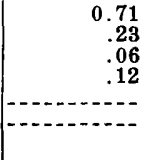 \\
\hline
\end{tabular}

\section{HEMPHILL COUNTY}

\section{Canadian}

Population in 1940: 2,151.

Source of information: J. C. Reagan, water superintendent, June 24, 1948. Ownership: Municipal. 
Source of supply: Five wells, three at pumping station and two northeast of city along the flood plains of the Canadian River.

Well 1. At pumping plant; drilled in 1925; depth, 72 feet; diameter, $121 / 2$ inches; deep-well turbine pump and $7 \frac{1}{2}$-horsepower electric motor; static water level, 22 feet below land surface in 1935, 35 feet below land surface in 1948; yield, 100 gallons a minute.

Well 2. At pumping plant; drilled in 1932; depth, 69 feet; diameter, 10 inches; deep-well turbine pump and 5-horsepower electric mintor; yield, 150 gallons a minute.

Well 3. At city pumping plant; drilled in 1936; depth, 84 feet; diameter, 10 inches; deep-well turbine pump and 10-horsepower electric motor; yield, 350 gallons a minute.

Well 4 (owner's new well 1). Along the Canadian flood plairs northeast of Canadian; drilled in 1945; depth, 94 feet; diameter, 12 ir shes; deepwell turbine pump and 25-horsepower electric motor; yie'd, 405 gallons a minute.

Well 5 (owner's new well 2). About 340 feet from well 4; drilled in 1947; depth, 109 feet; diameter, 121/2 inches; deep-well turbine pứmp and 20-horsepower electric motor; pumping level, 70 feet below land surface; yield, 700 gallons a minute.

Pumpage: Average, 300,000 gallons a day.

Storage: Ground storage reservoir, 189,000 gallons; ground storag” reservoir, 104,000 gallons; standpipe, 100,000 gallons.

Number of customers: 700 .

Treatment: Chlorination.

\section{Analyses}

[Collected June 24, 1948. pH: well 3, 7.4; well 4, 7.5; well 5, 7.3. Analyzed by D. E. Weaver and H. D. Smith]

\begin{tabular}{|c|c|c|c|c|c|c|}
\hline & \multicolumn{2}{|c|}{ Well 3} & \multicolumn{2}{|c|}{ Well 4} & \multicolumn{2}{|c|}{ Well 5} \\
\hline & $\begin{array}{c}\text { Parts } \\
\text { per } \\
\text { million }\end{array}$ & $\begin{array}{l}\text { Equiva- } \\
\text { lents Fer } \\
\text { million }\end{array}$ & $\begin{array}{l}\text { Parts } \\
\text { Fer } \\
\text { million }\end{array}$ & $\begin{array}{l}\text { Equiva- } \\
\text { lents fer } \\
\text { million }\end{array}$ & $\begin{array}{l}\text { Parts } \\
\text { Fer } \\
\text { million }\end{array}$ & $\begin{array}{l}\text { Equiva- } \\
\text { lents per } \\
\text { million }\end{array}$ \\
\hline 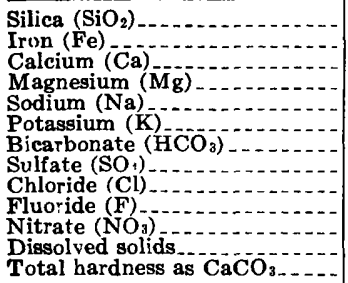 & $\begin{array}{l}29 \\
78 \\
34 \\
186 \\
5.65 \\
\mathbf{5 3 6} \\
104 \\
245 \\
1.6 \\
14 \\
841 \\
334\end{array}$ & $\begin{array}{r}\mathbf{3} .89 \\
\mathbf{2} .80 \\
8.07 \\
.14 \\
5.51 \\
2.17 \\
\mathbf{6 . 9 1} \\
.08 \\
.23 \\
- \\
-2 .-1\end{array}$ & $\begin{array}{l}33 \\
52 \\
27 \\
87 \\
4.05 \\
250 \\
44 \\
123 \\
1.2 \\
3.2 \\
504 \\
241\end{array}$ & 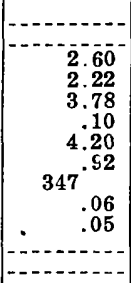 & $\begin{array}{l}32 \\
{ }^{33} .05 \\
27 \\
68 \\
3.6 \\
258 \\
39 \\
99 \\
3.0 \\
357 \\
244\end{array}$ & $\begin{array}{r}2.65 \\
2.22 \\
2.96 \\
.09 \\
4.23 \\
.81 \\
2.79 \\
.04 \\
.05 \\
\end{array}$ \\
\hline
\end{tabular}

\section{HOCKLEY COUNTY}

\section{ANTON}

Population in 1940: 548.

Source of information: A. H. Vincent, city secretary Mar. 13, 1917. Ownership: Municipal. 
Source of supply: Well; drilled in 1936 by Panhandle Construction Co.; depth, 115 feet; diameter, 12 inches; deep-well turbine pump and 15-horsepower electric motor; yield, 185 gallons a minute.

Pumpage: Average, 220,000 gallons a day.

Storage: Elevated tank, 50,000 gallons.

Number of customers: 146.

Treatment: None.

\section{Analysis}

[Collected Mar. 13, 1947. pH is 7.2. Analyzed by J. H. Rowley]

\begin{tabular}{|c|c|c|c|c|c|}
\hline & $\begin{array}{c}\text { Parts per } \\
\text { million }\end{array}$ & $\begin{array}{l}\text { Equivalents } \\
\text { per million }\end{array}$ & & $\begin{array}{c}\text { Parts per } \\
\text { m'llion }\end{array}$ & $\begin{array}{l}\text { Equivalents } \\
\text { per million }\end{array}$ \\
\hline $\begin{array}{l}\text { Silica }\left(\mathrm{SiO}_{2}\right) \\
\text { 1 ron }(\mathrm{Fe}) \\
\text { Calcium }(\mathrm{Ca}) \\
\text { Magnesium }(\mathrm{Mg}) \\
\text { Sodium (Na) } \\
\text { Potassium (K) } \\
\text { Bicarbonate (HCO }\end{array}$ & $\begin{array}{l}62 \\
104 \\
107 \\
83 \\
18 \\
337\end{array}$ & $\begin{array}{r}\mathbf{5 . 1 9} \\
\mathbf{3 . 5 9} \\
\mathbf{5 6} \\
\mathbf{5 . 5 2}\end{array}$ & $\begin{array}{l}\text { Sulfate }\left(\mathrm{SO}_{4}\right) \\
\text { Chloride }(\mathrm{Cl}) \\
\text { Fluoride (F) } \\
\text { Nitrate (NO} \text { (N) } \\
\text { Dissolved solids. } \\
\text { Total hardness as } \mathrm{CaCO}_{3}\end{array}$ & $\begin{array}{c}360 \\
166 \\
2.8 \\
12 \\
1,080 \\
700\end{array}$ & $\begin{array}{r}7.50 \\
4.68 \\
.15 \\
.19 \\
\\
-0\end{array}$ \\
\hline
\end{tabular}

\section{Driller's log}

\begin{tabular}{|c|c|c|c|c|c|}
\hline & $\begin{array}{c}\text { Thickness } \\
\text { (feet) }\end{array}$ & $\begin{array}{l}\text { Depth } \\
\text { (feet) }\end{array}$ & & $\begin{array}{c}\text { Thickness } \\
\text { (feet) }\end{array}$ & $\begin{array}{l}\text { Depth } \\
\text { (feet) }\end{array}$ \\
\hline $\begin{array}{l}\text { Top soil } \\
\text { Gray caliche } \\
\text { White caliche } \\
\text { Fine sand and clay } \\
\text { Sand (water) }\end{array}$ & $\begin{array}{r}10 \\
10 \\
8 \\
8 \\
6\end{array}$ & $\begin{array}{l}10 \\
20 \\
28 \\
36 \\
42\end{array}$ & $\begin{array}{l}\text { Fine sand, with some clay. } \\
\text { Red clay } \\
\text { Packed sand and clay } \\
\text { Sand (water) }\end{array}$ & $\begin{array}{r}14 \\
9 \\
17 \\
33\end{array}$ & $\begin{array}{r}56 \\
65 \\
82 \\
115\end{array}$ \\
\hline
\end{tabular}

\section{IEVELLAND}

Population in 1940: 3,091.

Source of information: L. E. Mabe, mayor, Mar. 15, 1947.

Ownership: Municipal.

Source of supply: Four wells.

Well 1. Drilled in 1927; depth, 220 feet; diameter, 12 inches; deep-well turbine pump and 50-horsepower electric motor; yield, 500 gallons a minute.

Well 2. Drilled in 1930; depth, 208 feet; diameter, 10 inches; deep-well turbine pump and 30-horsepower electric motor; yield, 390 gallons a minute.

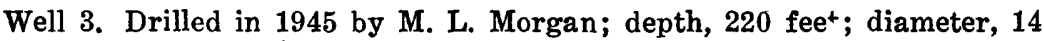
inches; deep-well turbine pump and 30-horsepower electric motor; yield, 500 gallons a minute.

Well 4. Drilled in 1946 by M. L. Morgan; depth, $231 \mathrm{fee}^{+}$; diameter, 14 inches; deep-well turbine pump and 30-horsepower eloctric motor.

Pumpage: Maximum in summer, 1,440,000 gallons a day; minimum in winter, 480,000 gallons a day.

Storage: Elevated tank, 75,000 gallons.

Number of customers: 1,416 .

Treatment: Chlorination. 
Analysis, well 1

[Collected Mar. 5, 1945. pH is 7.3. Analyzed by J. H. Rowley]

\begin{tabular}{|c|c|c|c|c|c|}
\hline & $\begin{array}{c}\text { Parts per } \\
\text { million }\end{array}$ & $\left|\begin{array}{l}\text { Equivalents } \\
\text { per million }\end{array}\right|$ & & $\begin{array}{c}\text { Parts per } \\
\text { million }\end{array}$ & $\begin{array}{l}\text { Equivalents } \\
\text { per million }\end{array}$ \\
\hline $\begin{array}{l}\text { Silica }\left(\mathrm{SiO}_{2}\right) \\
\text { Iron }(\mathrm{Fe}) \\
\text { Calcium }(\mathrm{Ca}) \\
\text { Magnesium }(\mathrm{Mg}) \\
\text { Sodium }(\mathrm{Na}) \\
\text { Potassium }(\mathrm{K}) \\
\left.\text { Bicarbonate }(\mathrm{H})_{3}\right)^{2}\end{array}$ & $\begin{array}{l}49 \\
63 \\
69 \\
46 \\
13 \\
320\end{array}$ & $\begin{array}{r}3.14 \\
5.67 \\
2.01 \\
.33 \\
5.25\end{array}$ & $\begin{array}{l}\text { Sulfate }\left(\mathrm{SO}_{4}\right) \\
\text { Chloride }(\mathrm{Cl}) \\
\text { Fluoride (F) } \\
\left.\text { Nitrate (NO }{ }_{3}\right) \\
\text { Dissolved solids } \\
\text { Total hardness as } \mathrm{CaCO}\end{array}$ & $\begin{array}{c}193 \\
60 \\
3.4 \\
673 \\
440\end{array}$ & \begin{tabular}{r}
4.02 \\
1.69 \\
.18 \\
.01 \\
\hdashline-1. \\
\end{tabular} \\
\hline
\end{tabular}

Drillers' logs

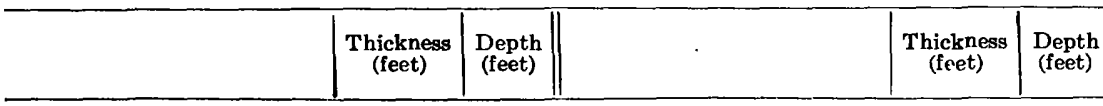

Well 1

\begin{tabular}{|c|c|c|c|c|c|}
\hline $\begin{array}{l}\text { Top soil. } \\
\text { Sand } \\
\text { Soft clay } \\
\text { Hard rock } \\
\text { Soft formation } \\
\text { Rock. } \\
\text { Soft sand formation }\end{array}$ & $\begin{array}{r}2 \\
10 \\
33 \\
6 \\
4 \\
4 \\
17\end{array}$ & $\begin{array}{r}2 \\
12 \\
\mathbf{4 5} \\
\mathbf{5 1} \\
\mathbf{5 5} \\
\mathbf{5 9} \\
\mathbf{7 6}\end{array}$ & $\begin{array}{l}\text { Hard rock } \\
\text { Extra soft sand } \\
\text { Extra hard rock } \\
\text { Sand (water) } \\
\text { Soft sandy clay } \\
\text { Sand (water) }\end{array}$ & $\begin{array}{r}3 \\
17 \\
7 \\
20 \\
28 \\
47\end{array}$ & $\begin{array}{r}79 \\
96 \\
103 \\
123 \\
151 \\
198\end{array}$ \\
\hline
\end{tabular}

Well 4

\begin{tabular}{|c|c|c|c|c|c|}
\hline $\begin{array}{l}\text { Top soil } \\
\text { Mixed elay and caliche. } \\
\text { Red sand, } \\
\text { Sand, clay } \\
\text { Caliche and rock. } \\
\text { Caliche } \\
\text { Sandrock } \\
\text { Lime rock } \\
\text { Loose sand and rock } \\
\text { Sandy clay }\end{array}$ & $\begin{array}{r}5 \\
12 \\
6 \\
9 \\
11 \\
9 \\
8 \\
10 \\
15 \\
14\end{array}$ & $\begin{array}{r}\mathbf{5} \\
17 \\
23 \\
\mathbf{3 2} \\
\mathbf{4 3} \\
\mathbf{5 2} \\
\mathbf{6 0} \\
70 \\
\mathbf{8 5} \\
\mathbf{9 9}\end{array}$ & $\begin{array}{l}\text { Sand and gravel, water. } \\
\text { Packed sand. } \\
\text { Brown clay } \\
\text { Sand, water } \\
\text { Sand and gravel } \\
\text { Brown clay } \\
\text { Sand and gravel, water } \\
\text { Gravel. } \\
\text { Clay }\end{array}$ & $\begin{array}{r}8 \\
11 \\
6 \\
6 \\
\mathbf{6} \\
13 \\
2 \\
7 \mathbf{7} \\
\mathbf{5} \\
\mathbf{6}\end{array}$ & $\begin{array}{l}107 \\
118 \\
124 \\
130 \\
143 \\
145 \\
220 \\
225 \\
231\end{array}$ \\
\hline
\end{tabular}

\section{HOWARD COUNTY}

\section{BIG SPRING}

Population in 1940: 12,604 .

Source of information: R. C. Hester, production superintendent, Aug. 21, 1947.

Ownership: Municipal.

Source of supply: Twenty-eight wells in four well fields and tro surface water lakes.

Two wells in City Park field, 2.5 miles southwest of city; 13 wells in section 17 well field; 7 wells in section 33 well field; and 7 wells in O'Barr well field in Glasscock County, 21 miles southwest of Big Spring.

Moss Creek Reservoir. Eight miles east of Big Spring; capacity, 2,500 acre-feet.

Powell Creek Reservoir. Twelve miles southwest of Big Spring; capacity 1,600 acre-feet. 
Well records

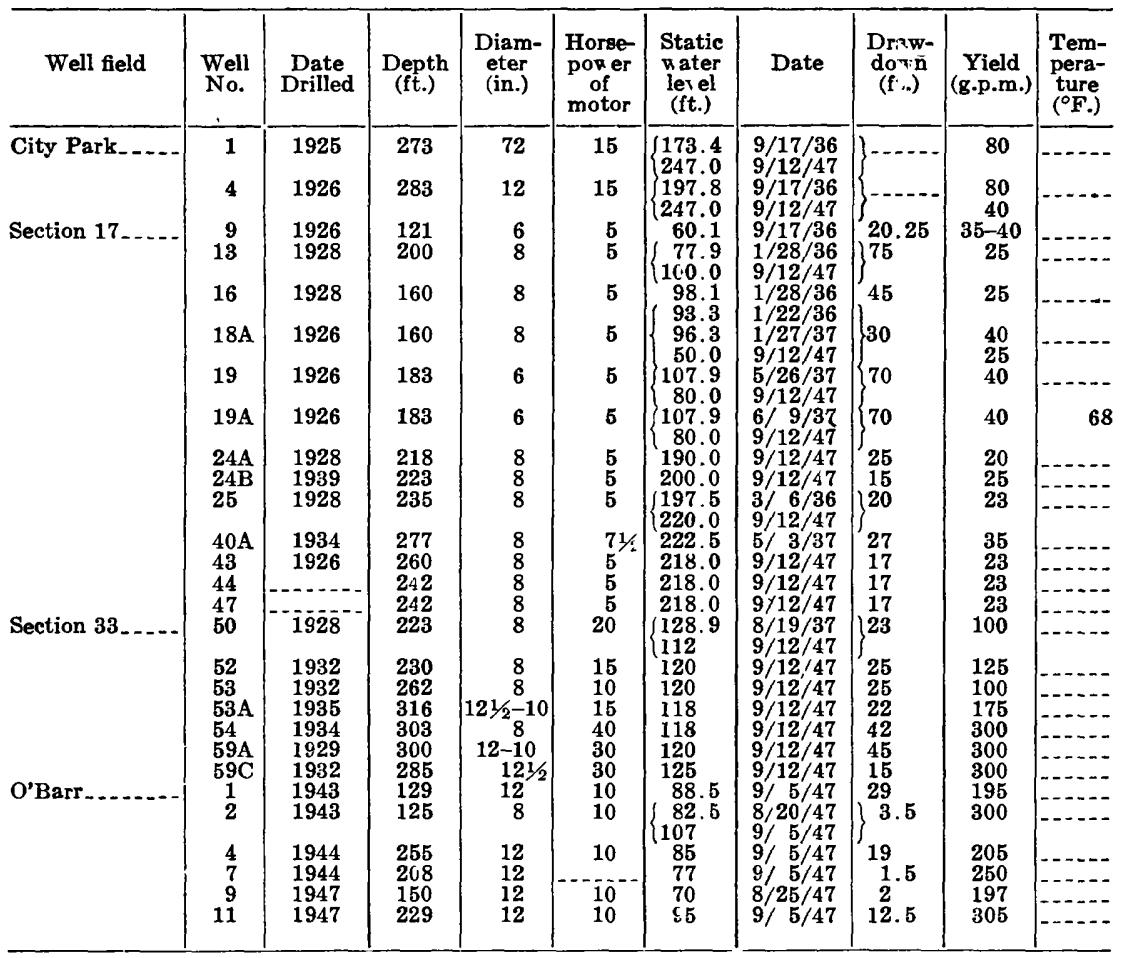

Average pumpage, in gallons a day

\begin{tabular}{|c|c|c|c|c|c|}
\hline & Lakes & $\begin{array}{l}\text { Section } 17 \\
n \text { ell field }\end{array}$ & $\begin{array}{c}\text { Section } 33 \\
n \text { ell field }\end{array}$ & $\begin{array}{l}\text { City Park } \\
\text { nell Leld }\end{array}$ & $\begin{array}{l}\text { O'Barr } \text { nell } \\
\text { field }\end{array}$ \\
\hline \multicolumn{6}{|c|}{1945} \\
\hline $\begin{array}{l}\text { January } \\
\text { February } \\
\text { March } \\
\text { April } \\
\text { May } \\
\text { June. } \\
\text { July } \\
\text { August. } \\
\text { Septemier } \\
\text { October } \\
\text { Nov ember. } \\
\text { December }\end{array}$ & $\begin{array}{r}78,000 \\
561,000 \\
14 \overline{2}, 000 \\
165,000 \\
128,000 \\
616,000 \\
942,000 \\
918,060 \\
1,314,000 \\
1,344,000 \\
1,268,000\end{array}$ & $\begin{array}{r}407,000 \\
398,000 \\
389,000 \\
362,000 \\
347,000 \\
350,000 \\
295,060 \\
293,000 \\
299,000 \\
143,000 \\
64,000 \\
\end{array}$ & 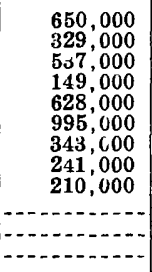 & $\begin{array}{r}45,000 \\
25,000 \\
35,060 \\
33,000 \\
10 ?, 000 \\
54,000 \\
47,000 \\
43,000 \\
13,000 \\
35,000 \\
\end{array}$ & $\begin{array}{r}116,000 \\
737,000 \\
1,493,000 \\
1,553,000 \\
1,490,000 \\
884,000 \\
1,122,000 \\
918,000\end{array}$ \\
\hline \multicolumn{6}{|c|}{1946} \\
\hline $\begin{array}{l}\text { January } \\
\text { February. } \\
\text { March } \\
\text { April. } \\
\text { May. } \\
\text { June. } \\
\text { July } \\
\text { Aurust. } \\
\text { September } \\
\text { October. } \\
\text { November } \\
\text { December. }\end{array}$ & $\begin{array}{r}1,250,000 \\
2,301,000 \\
1,383,000 \\
1,323,000 \\
1,266,000 \\
1,248,000 \\
1,348,000 \\
1,261,000 \\
1,243,000 \\
716,000 \\
1,255,000 \\
996,000\end{array}$ & \begin{tabular}{|r|}
1,000 \\
244,000 \\
324,000 \\
324,000 \\
323,000 \\
320,000 \\
64,000 \\
161,000
\end{tabular} & $\begin{array}{r}\mathbf{5 7}, 000 \\
327,000 \\
472,000 \\
622,000 \\
246,000 \\
377,000 \\
88,000 \\
193,000\end{array}$ & $\begin{array}{r}5,000 \\
-6 \overline{7}, 000 \\
7 \overline{5}, 000 \\
75,000 \\
7 \overline{5}, 000 \\
75,000 \\
7 \overline{5}, 000 \\
7 \overline{5}, 000 \\
65,000 \\
67,000 \\
73,000\end{array}$ & $\begin{array}{r}168,000 \\
750,000 \\
869,000 \\
808,000 \\
1,139,000 \\
758,00 \\
51,000 \\
216,000 \\
4,000\end{array}$ \\
\hline
\end{tabular}




\begin{tabular}{l|l|c|c|c|c}
\hline Lakes & $\begin{array}{c}\text { Section 17 } \\
\text { well field }\end{array}$ & $\begin{array}{c}\text { Section 33 } \\
\text { well field }\end{array}$ & $\begin{array}{c}\text { City Park } \\
\text { well fielt }\end{array}$ & $\begin{array}{c}\text { O'Barr well } \\
\text { field }\end{array}$ \\
\hline
\end{tabular}

1947

\begin{tabular}{|c|c|c|c|c|c|}
\hline $\begin{array}{l}\text { January } \\
\text { February } \\
\text { March. } \\
\text { April } \\
\text { May } \\
\text { June. } \\
\text { July }\end{array}$ & $\begin{array}{l}1,207,000 \\
1,300,000 \\
1,260,000 \\
1,236,000 \\
1,292,000 \\
1,409,000 \\
1,551,000\end{array}$ & $\begin{array}{r}160,000 \\
29,000 \\
241,000 \\
314,000 \\
311,000 \\
322,000 \\
316,000\end{array}$ & $\begin{array}{r}163,000 \\
6,000 \\
57,000 \\
364,000 \\
578,000\end{array}$ & $\begin{array}{l}71,000 \\
72,000 \\
75,000 \\
75,000 \\
73,000 \\
75,000 \\
58,000\end{array}$ & $\begin{array}{r}16,000 \\
100,000 \\
58,000 \\
613,000 \\
619,000 \\
1,106,000 \\
968,000\end{array}$ \\
\hline
\end{tabular}

Storage: Three ground reservoirs, each 1,000,000 gallons; two elevated tanks, each 200,000 gallons.

Number of customers: 3,723 .

Treatment: Well water, chlorination; lake water, coagulation, filtration, and chlorination.

\section{Analyses}

\section{O'Barr field}

[Collected Aug. 22, 1947. pH of each well is 7.2. Analyzed by B. C. Dwyer]

\begin{tabular}{|c|c|c|c|c|}
\hline & \multicolumn{2}{|c|}{ Well 1} & \multicolumn{2}{|c|}{ Well 4} \\
\hline & $\begin{array}{l}\text { Parts per } \\
\text { million }\end{array}$ & $\begin{array}{l}\text { Equivalents } \\
\text { per million }\end{array}$ & $\begin{array}{l}\text { Parts per } \\
\text { millicn }\end{array}$ & $\begin{array}{l}\text { Equivalents } \\
\text { per million }\end{array}$ \\
\hline 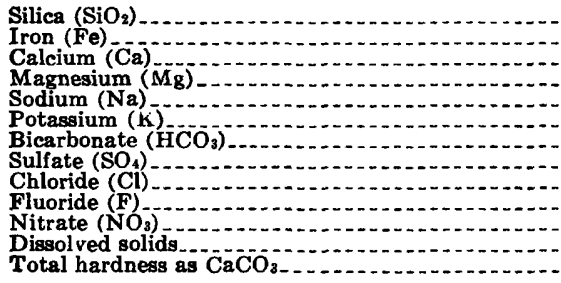 & $\begin{array}{l}15 \\
94 \\
20 \\
11 \\
4.04 \\
318 \\
29 \\
36 \\
5.6 \\
372 \\
316\end{array}$ & \begin{tabular}{r}
4.69 \\
1.64 \\
.49 \\
.12 \\
5.21 \\
.60 \\
1.02 \\
.03 \\
.08 \\
\hdashline .0. \\
\end{tabular} & $\begin{array}{l}20 \\
78 \\
14 \\
14 \\
24 \\
4.1 \\
258 \\
32 \\
40 \\
1.2 \\
5.0 \\
345 \\
252\end{array}$ & $\begin{array}{r}3.89 \\
1.15 \\
1.03 \\
.10 \\
4.23 \\
1.67 \\
.06 \\
.08 \\
\hdashline\end{array}$ \\
\hline
\end{tabular}

City Park well field, well 4

[Collected Aug. 21, 1947. pH is 7.2. Analyzed by B. C. Dwyer]

\begin{tabular}{|c|c|c|c|c|c|}
\hline . & $\begin{array}{l}\text { Parts per } \\
\text { million }\end{array}$ & $\begin{array}{l}\text { Equivalents } \\
\text { per million }\end{array}$ & & $\underset{\text { million }}{\text { Parts per }}$ & $\begin{array}{l}\text { Equivalents } \\
\text { per million }\end{array}$ \\
\hline $\begin{array}{l}\text { Silica }\left(\mathrm{SiO}_{2}\right) \\
\text { Iron }(\mathrm{Fe}) \\
\text { Calcium }(\mathrm{Ca}) \\
\text { Magnesium }(\mathrm{Mg}) \\
\text { Sodium }(\mathrm{Na}) \\
\text { Potassium }(\mathrm{K}) \\
\left.\text { Bicarbonate }(\mathrm{HCO})_{8}\right)\end{array}$ & $\begin{array}{c}15 \\
100 \\
10 \\
28 \\
5.8 \\
294\end{array}$ & $\begin{array}{r}4.99 \\
.82 \\
1.26 \\
.15 \\
4.82\end{array}$ & $\begin{array}{l}\text { Sulfate (SO, } \\
\text { Chloride (Cl) } \\
\text { Fluoride (F) } \\
\text { Nitrate (NO})_{3} \text { ) } \\
\text { Dissolved solids.. } \\
\text { Total hardness as } \mathrm{CaCO}_{3}\end{array}$ & $\begin{array}{c}4 f \\
44 \\
1.0 \\
5.7 \\
40 \Sigma \\
290\end{array}$ & $\begin{array}{r}0.96 \\
1.24 \\
.05 \\
.09 \\
\end{array}$ \\
\hline
\end{tabular}




\begin{tabular}{|c|c|c|c}
\multicolumn{2}{|c|}{ Raw water } & \multicolumn{2}{c}{ Finirhed water } \\
\hline $\begin{array}{c}\text { Parts per } \\
\text { million }\end{array}$ & $\begin{array}{c}\text { Equivalents } \\
\text { per million }\end{array}$ & $\begin{array}{c}\text { Parts per } \\
\text { million }\end{array}$ & $\begin{array}{c}\text { Equivalents } \\
\text { per million }\end{array}$ \\
\hline
\end{tabular}

\section{Moss Creek Reservoir}

Collected: Raw sample, Aug. 9, 1945; finished sample, Aug. 21, 1947. pH, finished sample, is 7.7. Analyzed by C. B. Cibulka and B. C. Dwyer]

\begin{tabular}{|c|c|c|c|c|}
\hline 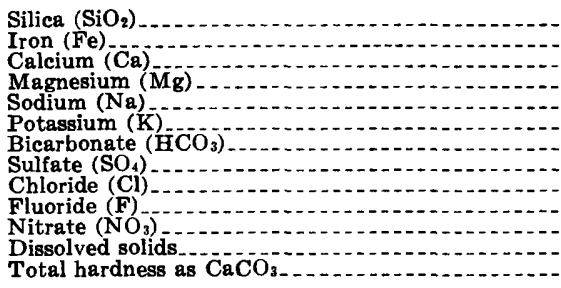 & 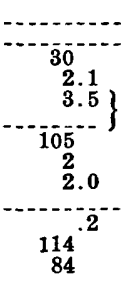 & $\begin{array}{r}1.497 \\
.173 \\
.152 \\
1.721 \\
.042 \\
.056 \\
.003\end{array}$ & $\begin{array}{c}3.4 \\
.^{\circ} \\
40 \\
6.9 \\
20 \\
3.9 \\
162 \\
17 \\
14 \\
1.2 \\
186 \\
128\end{array}$ & $\begin{array}{r}.597 \\
.567 \\
.857 \\
.010 \\
2.655 \\
.354 \\
.395 \\
.011 \\
.016\end{array}$ \\
\hline & \multicolumn{2}{|c|}{ Raw water } & \multicolumn{2}{|c|}{ Finished water } \\
\hline & $\begin{array}{l}\text { Parts per } \\
\text { million }\end{array}$ & $\begin{array}{l}\text { Equivalents } \\
\text { per million }\end{array}$ & $\begin{array}{c}\text { Parts per } \\
\text { million }\end{array}$ & $\begin{array}{l}\text { Equivalents } \\
\text { per million }\end{array}$ \\
\hline
\end{tabular}

Powell Creek Reservoir

[Collected Aug. 21, 1947. pH: raw water, 7.5; finished water, 7.9. Analyzed by B. C. Dwyer]

\begin{tabular}{|c|c|c|c|c|}
\hline 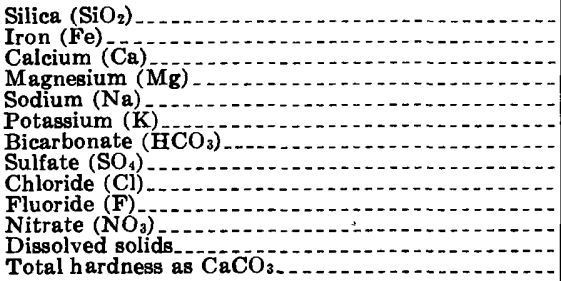 & $\begin{array}{l}7.1 \\
32.10 \\
4.1 \\
12.5 \\
3.5 \\
13.8 \\
10^{138} \\
97\end{array}$ & 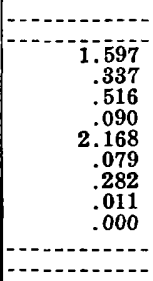 & $\begin{array}{c}1.0 \\
57^{.00} \\
8.7 \\
14 \\
6.4 \\
156 \\
52 \\
22 \\
1.6 \\
240 \\
178\end{array}$ & $\begin{array}{r}\overline{\mathbf{2}} .845 \\
.715 \\
.589 \\
.164 \\
\mathbf{2 . 5 6 2} \\
1.083 \\
.620 \\
.032 \\
.016 \\
\end{array}$ \\
\hline
\end{tabular}

\section{Drillers' logs}

\begin{tabular}{l|l|l|l|l|l}
\hline (feet) & $\begin{array}{c}\text { Depth } \\
\text { (feet) }\end{array}$ & $\begin{array}{c}\text { Thickness } \\
\text { (eet) }\end{array}$ & $\begin{array}{c}\text { Depth } \\
\text { (feet) }\end{array}$ \\
\hline
\end{tabular}

O'Barr field, well 1

\begin{tabular}{|c|c|c|c|c|c|}
\hline $\begin{array}{l}\text { Top soil. } \\
\text { of gravel with small amount } \\
\text { Red clay } \\
\text { Gravel with small amount of } \\
\text { clay. } \\
\text { Cavern, first showing of water } \\
\text { Conglomerate. }\end{array}$ & $\begin{array}{r}6 \\
18 \\
4 \\
64 \\
3 \\
3\end{array}$ & $\begin{array}{r}6 \\
24 \\
28 \\
82 \\
85 \\
88\end{array}$ & $\begin{array}{l}\text { Gravel with some yellow clay } \\
\text { Gravel with some white clay } \\
\text { Gravel with some yellow clay } \\
\text { Apparent cavern (probably } \\
\text { water). } \\
\text { Conglomerate. } \\
\text { Dark-red clay. }\end{array}$ & $\begin{array}{r}11 \\
9 \\
10 \\
2 \\
2 \\
7\end{array}$ & $\begin{array}{r}99 \\
108 \\
118 \\
120 \\
122 \\
129\end{array}$ \\
\hline
\end{tabular}

\section{O'Barr field, well 2}

\begin{tabular}{|c|c|c|c|c|c|}
\hline $\begin{array}{l}\text { Top soil } \\
\text { Red sandy clay } \\
\text { Red clay } \\
\text { Pink clay } \\
\text { Soft white lime and clay }\end{array}$ & $\begin{array}{r}3 \\
7 \\
24 \\
6 \\
30\end{array}$ & $\begin{array}{r}3 \\
10 \\
34 \\
40 \\
70\end{array}$ & $\begin{array}{l}\text { Very porous white lime and } \\
\text { clay, water bearing. } \\
\text { Conglomerate. } \\
\text { Soft yellow lime and clay. } \\
\text { Gravel and sand, some clay. } \\
\text { Red beds. }\end{array}$ & $\begin{array}{r}8 \\
7 \\
15 \\
19 \\
183\end{array}$ & $\begin{array}{r}78 \\
85 \\
100 \\
119 \\
302\end{array}$ \\
\hline
\end{tabular}




\begin{tabular}{|c|c|c|c|c|c|}
\hline & $\begin{array}{c}\text { Thickness } \\
\text { (feet) }\end{array}$ & $\begin{array}{c}\text { Depth } \\
\text { (feet) }\end{array}$ & & $\underset{\text { (feet) }}{\text { Thickness }}$ & $\begin{array}{c}\text { Depth } \\
\text { (feet) }\end{array}$ \\
\hline \multicolumn{6}{|c|}{ O'Barr field, well 4} \\
\hline $\begin{array}{l}\text { Top soil. } \\
\text { Reddish-brown clay } \\
\text { Gravel and light-gray clay. } \\
\text { Coarse sand and gravel, clay } \\
\text { Brownish - yellow mud and } \\
\text { gravel, water. } \\
\text { Sand and gravel } \\
\text { Conglomerate. } \\
\text { Sand and gravel } \\
\text { Conglomerate... }\end{array}$ & $\begin{array}{r}24 \\
11 \\
8 \\
19 \\
10 \\
40 \\
42 \\
37 \\
19\end{array}$ & $\begin{array}{r}24 \\
35 \\
43 \\
62 \\
72 \\
112 \\
154 \\
191 \\
210\end{array}$ & $\begin{array}{l}\text { Sand and gravel, some clay } \\
\text { balls. } \\
\text { Soft buff sand. } \\
\text { Yellow sand } \\
\text { Reddish-buff sand and gravel } \\
\text { Shale. } \\
\text { Sandy clay } \\
\text { Conglomerate } \\
\text { Yellow and red gravelly clay } \\
\text { and shale. }\end{array}$ & $\begin{array}{r}7 \\
23 \\
25 \\
\mathbf{3} \\
16 \\
\mathbf{3} \\
17 \\
11\end{array}$ & $\begin{array}{l}217 \\
\\
240 \\
245 \\
248 \\
264 \\
267 \\
284 \\
295\end{array}$ \\
\hline \multicolumn{6}{|c|}{ O'Barr field, well 7} \\
\hline $\begin{array}{l}\text { Top soil. } \\
\text { Sandy clay } \\
\text { Clayey gravel } \\
\text { Conglomerate. and boulders, } \\
\text { Clay, sand, and } \\
\text { water. } \\
\text { Conglomerate. } \\
\text { Soft yellow sand } \\
\text { Coarse sand and gravel. } \\
\text { Yellow sandy and gravelly } \\
\text { clay. }\end{array}$ & $\begin{array}{r}10 \\
29 \\
6 \\
15 \\
14 \\
\\
5 \\
11 \\
64 \\
3\end{array}$ & $\begin{array}{r}10 \\
\mathbf{3 9} \\
\mathbf{4 5} \\
60 \\
74 \\
79 \\
\mathbf{7 0} \\
\mathbf{1 5 4} \\
157\end{array}$ & $\begin{array}{l}\text { Coarse sand and gravel, } \\
\text { some conglomerate. } \\
\text { Sandy and gravelly clay.-.-- } \\
\text { Sand and gravel, some } \\
\text { boulders and clay. } \\
\text { Yellowish-brown to blue } \\
\text { sandy mud. } \\
\text { Dark-red gravelly and sandy } \\
\text { clay with thin beds of } \\
\text { sandstone and streaks of } \\
\text { yellow and blue clay. }\end{array}$ & $\begin{array}{r}11 \\
6 \\
10 \\
8 \\
23\end{array}$ & $\begin{array}{r}168 \\
174 \\
184 \\
192\end{array}$ \\
\hline
\end{tabular}

Population in 1940: 574 .

\section{COAHOMA}

Source of information: R. A. Marshall, city secretary, Aug. 22, 1947.

Ownership: Municipal.

Source of supply: Two wells.

Well 2. Dug in 1940; depth, 50 feet; diameter, 4 feet; deep-well turbine pump and 71/2-horsepower electric motor; yield, 125 gallors a minute.

Well 3. About 150 feet east of well 2; drilled in 1946 by Ollie Williams; depth, 54 feet; diameter, 8 inches; deep-well turbine pump and 5horsepower electric motor; yield, 70 gallons a minute.

Pumpage (estimated) : 80,000 gallons a day.

Storage: Elevated tank, 55,000 gallons.

Number of customers: 140.

Treatment: None.

\section{Analysis, well 2}

[Collected Aug. 22, 1947. pH is 7.2. Analyzed by B. C. Dwyer]

\begin{tabular}{|c|c|c|c|c|c|}
\hline & $\begin{array}{l}\text { Parts per } \\
\text { million }\end{array}$ & $\begin{array}{l}\text { Equivalents } \\
\text { per million }\end{array}$ & & $\begin{array}{l}\text { Parts per } \\
\text { million }\end{array}$ & $\begin{array}{l}\text { Equivalents } \\
\text { per million }\end{array}$ \\
\hline $\begin{array}{l}\text { Silica }\left(\mathrm{SiO}_{2}\right) \\
\text { Iron }(\mathrm{Fe}) \\
\text { Calcium }(\mathrm{Ca}) \\
\text { Magnesium }(\mathrm{Mg}) \\
\text { Sodium }(\mathrm{Na}) \\
\text { Potassium }(\mathrm{K}) \\
\text { Bicarbonate }\left(\mathrm{HCO}_{3}\right)^{-}\end{array}$ & $\begin{array}{r}63 \\
249 \\
94 \\
177 \\
20 \\
260\end{array}$ & $\begin{array}{r}7.43 \\
7.69 \\
7.51 \\
4.26\end{array}$ & $\begin{array}{l}\text { Sulfate }\left(\mathrm{SO}_{4}\right) \\
\text { Chloride }(\mathrm{Cl}) \\
\text { Fluoride (F) } \\
\text { Nitrate }\left(\mathrm{NO}_{3}\right) \\
\text { Dissolved solids } \\
\text { Total hardness as } \mathrm{CaCO}\end{array}$ & $\begin{array}{c}33 E \\
59 \mathrm{C} \\
1.4 \\
2 \epsilon \\
1,68 \mathrm{C} \\
1,01 \mathrm{C}\end{array}$ & \begin{tabular}{r}
6.97 \\
16.64 \\
.07 \\
.42 \\
\hdashline-1. \\
\end{tabular} \\
\hline
\end{tabular}

Population in 1940: 400.

\section{FORSAN}

Source of information: Tillman Shoults, owner, Aug. 22, 1947. Owner: Tillman Shoults. 
Source of supply: Well; drilled; depth, 280 feet; diameter, 6 ir hes; deepwell cylinder pump and 3-horsepower electric motor.

Pumpage: No record.

Storage: Elevated tank, 3,200 gallons.

Number of customers: 35.

Treatment: None.

\section{Analysis}

[Collected Aug. 22, 1947. pH is 7.8. Analyzed by B. C. Dwyer]

\begin{tabular}{|c|c|c|c|c|c|}
\hline & $\begin{array}{c}\text { Parts per } \\
\text { million }\end{array}$ & $\begin{array}{l}\text { Equivalente } \\
\text { per million }\end{array}$ & & $\begin{array}{l}\text { Parts per } \\
\text { millicn }\end{array}$ & $\begin{array}{l}\text { Equivalents } \\
\text { per million }\end{array}$ \\
\hline $\begin{array}{l}\text { Silica }\left(\mathrm{SiO}_{2}\right) \\
\text { Iron (Fe) } \\
\text { Calcium }(\mathrm{Ca}) \\
\text { Magnesium }(\mathrm{Mg}) \\
\text { Sodium (Na) } \\
\text { Potassium (K) } \\
\text { Bicarbonate }\left(\mathrm{H}_{\mathbf{C}} \mathrm{CO}_{2}\right)\end{array}$ & $\begin{array}{l}10 \\
72.64 \\
72.6 \\
72.0\end{array}$ & $\begin{array}{r}.62 \\
1.81 \\
.18 \\
3.84\end{array}$ & $\begin{array}{l}\text { Sulfate }\left(\mathrm{SO}_{4}\right) \\
\text { Chloride }(\mathrm{Cl}) \\
\text { Fluoride (F) } \\
\text { Nitrate (NO}) \\
\text { Dissolved solids } \\
\text { Total hardness as } \mathrm{CaCO}_{3}\end{array}$ & $\begin{array}{r}42 \\
46 \\
1.0 \\
8.8 \\
352 \\
210\end{array}$ & $\begin{array}{r}\mathbf{0 . 8 7} \\
1.30 \\
.05 \\
.14 \\
-14 \\
-0 .- \\
\end{array}$ \\
\hline
\end{tabular}

\section{HUDSPETH COUNTY}

Population in 1940: 450.

\section{FORT HANCOCK}

Source of information: J. A. Walton, pump operator, Sept. 20, 1948.

Owner: Texas and New Orleans Railroad.

Source of Supply: Well in southern part of town; drilled; der ${ }^{+h}, 50$ feet; diameter, 12 inches; triplex pump and 25-horsepower gasoline engine; static water level, 9 feet below land surface; yield, 200 gallons a minute.

Pumpage: 100,000 gallons a day.

Storage: Elevated tank, 20,000 gallons.

Number of customers: 73 .

Treatment: None.

Analysis

[Collected Sept. 20, 1948. $\mathrm{pH}$ is 7.5. Analyzed by H. D. Smith]

\begin{tabular}{|c|c|c|c|c|c|}
\hline & $\begin{array}{c}\text { Parts per } \\
\text { million }\end{array}$ & $\begin{array}{l}\text { Equivalents } \\
\text { per million }\end{array}$ & & $\begin{array}{c}\text { Parts per } \\
\text { millicn }\end{array}$ & $\begin{array}{l}\text { Equivalents } \\
\text { per million }\end{array}$ \\
\hline $\begin{array}{l}\text { Silica }\left(\mathrm{SiO}_{2}\right) \\
\text { Iron }(\mathrm{Fe}) \\
\text { Calcium }(\mathrm{Ca}) \\
\text { Magnesium }(\mathrm{Mg}) \\
\text { Sodium }(\mathrm{Na}) \\
\text { Potagsium }(\mathrm{K}) \\
\text { Bicarbonate }\left(\mathrm{HC} \mathrm{CO}_{3}\right)^{-}\end{array}$ & $\begin{array}{l}88 \\
186 \\
45 \\
457 \\
9.6 \\
262\end{array}$ & $\begin{array}{r}-9.28 \\
3.70 \\
19.89 \\
.25 \\
4.29\end{array}$ & $\begin{array}{l}\text { Sulfate }\left(\mathrm{SO}_{4}\right) \\
\text { Chloride }(\mathrm{Cl}) \\
\text { Fluoride (F) } \\
\text { Nitrate (NO } \\
\text { Dissolved solids } \\
\text { Total hardness as } \mathrm{CaCO}_{8}\end{array}$ & $\begin{array}{r}502 \\
648 \\
1.0 \\
2.8 \\
2,020 \\
649\end{array}$ & $\begin{array}{r}10.45 \\
18.28 \\
.05 \\
.05 \\
\\
\end{array}$ \\
\hline
\end{tabular}

Population in 1940: 723.

\section{SIERRA BLANCA}

Source of information: Wm. Melbreth, owner, Sept. 20, 1948.

Owner: Wm. Melbreth.

Source of supply: Two wells.

Well 1. Drilled in 1925 by M. C. Craley; depth, 1,000 feet; diameter, 6 inches; deep-well cylinder pump and 20-horsepower electric motor; static water level reported, 920 feet below land surfac?; yield, 40 gallons a minute.

Well 2. Drilled in 1942; depth, 1,000 feet; diameter, 6 inches; deep-well cylinder pump and 15-horsepower electric motor; yield, 35 gallons a minute. 
Pumpage: Average, 36,000 gallons a day.

Storage: Elevated tank, 180,000 gallons.

Number of customers: 150.

Treatment: Chlorination.

Analysis, well 2

[Collected July 23, 1943. $\mathrm{pH}$ is 7.8. Analyzed by J. H. Rowley]

\begin{tabular}{|c|c|c|c|c|c|}
\hline & $\begin{array}{l}\text { Parts per } \\
\text { million }\end{array}$ & $\begin{array}{l}\text { Equivalents } \\
\text { per million }\end{array}$ & & $\begin{array}{c}\text { Parts per } \\
\text { millic n }\end{array}$ & $\begin{array}{l}\text { Equivalents } \\
\text { per million }\end{array}$ \\
\hline $\begin{array}{l}\text { Silica }\left(\mathrm{SiO}_{2}\right) \\
\text { Iron (Fe) } \\
\text { Calcium }(\mathrm{Ca}) \\
\text { Magnesium }(\mathrm{Mg}) \\
\text { Sodium (Na) } \\
\text { Potassium (K) } \\
\text { Bicarbonate }\left(\mathrm{HCO}_{3}\right) \\
\end{array}$ & $\begin{array}{l}20 \\
1.1 \\
68 \\
19 \\
496 \\
22 \\
340\end{array}$ & $\begin{array}{r}3.39 \\
1.56 \\
21.57 \\
.56 \\
5.57\end{array}$ & $\begin{array}{l}\text { Sulfate }\left(\mathrm{SO}_{4}\right) \\
\text { Chloride }(\mathrm{Cl}) \\
\text { Fluoride (F) } \\
\text { Nitrate (NO}) \\
\text { Dissolved solids } \\
\text { Total hardness as } \mathrm{CaCO}_{3}\end{array}$ & $\begin{array}{c}373 \\
468 \\
5.3 \\
16 \\
1,655 \\
248\end{array}$ & $\begin{array}{r}7.77 \\
13.20 \\
.28 \\
.26 \\
\\
\\
-2 .\end{array}$ \\
\hline
\end{tabular}

\section{HUTCHINSON COUNTY}

Population in 1940: 10,018.

\section{BORGER}

Source of information: R. R. Darrell, water superintendent, June 23, 1948.

Owner: Phillips Petroleum Co.

Source of supply: Eight wells at the Plains Water Station in Carson County 13 miles southwest of Borger.

Well 1. Drilled in 1926 by D. L. McDonald; depth, 410 feet; diameter, 18 inches; deep-well turbine pump and 100-horsepower ele?tric motor; static water level, 235 feet below land surface in 1926; yicld; 700 gallons a minute; temperature, $62^{\circ} \mathrm{F}$.

Well 2. Drilled in June 1927 by D. L. McDonald; depth, 384 feet; diameter, 20 inches; deep-well turbine pump and 100-horseporer electric

- motor.

Well 3. Drilled in February 1927 by D. L. McDonald; depth, 371 feet; diameter, 18 inches; deep-well turbine pump and 100-horsepower electric motor; yield, 700 gallons a minute.

Well 4. Drilled in May 1927 by D. L. McDonald; depth, 376 feet; diameter, 20 inches; deep-well turbine pump and 100-horsepower elertric motor.

Well 5. Drilled in September 1929 by D. L. McDonald; depth, 495 feet; diameter, 20 inches; deep-well turbine pump and 100-horsepower electric motor; static water level, 260 feet below land surface Sept. 10, 1929, and 280.5 feet below land surface July 30, 1947; yield, 700 gallons a minute.

W.ell 6. Drilled in 1936 by D. L. McDonald; depth, 535 feet; diameter, 20 inches; deep-well turbine pump and 100-horsepower electric motor; static water level, 243 feet below land surface Aug. 1, 1936; yield, 740 gallons a minute with drawdown of 24.5 feet a minute.

Well 7. Drilled in 1937 by D. L. McDonald; depth, 403 feet; diameter, 20 inches; static water level, 195 feet below land surface Apr. 18, 1937; yield, 700 gallons a minute.

Well 8. Drilled in November 1937; depth, 459 feet; diameter, 20 inches; deep-well turbine pump and 100-horsepower electric motor; yield, 800 gallons a minute. 


\section{Average pumpage, in gallons a day}

\begin{tabular}{|c|c|c|c|c|c|}
\hline $\begin{array}{l}\text { January } \\
\text { February } \\
\text { March } \\
\text { April }\end{array}$ & $\begin{array}{l}698,745 \\
692,000 \\
695,000 \\
760,000\end{array}$ & $\begin{array}{l}\text { May } \\
\text { June } \\
\text { July } \\
\text { August. }\end{array}$ & $\begin{array}{r}793,000 \\
1,041,000 \\
1,260,000 \\
1,479,000\end{array}$ & $\begin{array}{l}\text { September } \\
\text { October } \\
\text { November } \\
\text { December }\end{array}$ & $\begin{array}{r}1,278,000 \\
1,011,000 \\
820,000 \\
758,000\end{array}$ \\
\hline
\end{tabular}

Storage: Elevated tank, 20,000 gallons.

Treatment: None.

\section{Analysis}

[Composite sample, collected Nov. 14, 1947. pH is 8.0. Analyzed by B. C. Dwyer]

\begin{tabular}{|c|c|c|c|c|c|}
\hline & $\begin{array}{c}\text { Parts per } \\
\text { million }\end{array}$ & $\begin{array}{l}\text { Equivalents } \\
\text { per million }\end{array}$ & & $\begin{array}{l}\text { Parts per } \\
\text { million }\end{array}$ & $\begin{array}{l}\text { Equivalents } \\
\text { per million }\end{array}$ \\
\hline $\begin{array}{l}\text { Silica }\left(\mathrm{SiO}_{2}\right) \\
\text { Iron }(\mathrm{Fe}) \\
\text { Calcium }(\mathrm{Ca}) \\
\text { Magnesium }(\mathrm{Mg}) \\
\text { Sodium (Na) } \\
\text { Potassium }(\mathrm{K}) \\
\text { Bicarbonate }\left(\mathrm{H}_{\mathrm{CO}}\right)\end{array}$ & $\begin{array}{l}28 \\
46 \\
20 \\
24 \\
7.04 \\
74.6\end{array}$ & $\begin{array}{r}\mathbf{2} . \overrightarrow{30} \\
\mathbf{1} .64 \\
1.03 \\
.19 \\
4.07\end{array}$ & $\begin{array}{l}\text { Sulfate }\left(\mathrm{SO}_{4}\right) \\
\text { Chloride }(\mathrm{Cl}) \\
\text { Fluoride (F) } \\
\left.\text { Nitrate (NO})_{3}\right) \\
\text { Dissolved solids } \\
\text { Total hardness as } \mathrm{CaCO}_{3}\end{array}$ & $\begin{array}{l}24 \\
18 \\
39.5 \\
197\end{array}$ & $\begin{array}{r}.50 \\
.51 \\
.02 \\
.06 \\
\\
-0.0\end{array}$ \\
\hline
\end{tabular}

\section{Driller's log, well 5}

\begin{tabular}{|c|c|c|c|c|c|}
\hline & $\begin{array}{c}\text { Thickness } \\
\text { (feet) }\end{array}$ & $\begin{array}{l}\text { Depth } \\
\text { (feet) }\end{array}$ & & $\begin{array}{c}\text { Tickness } \\
\text { (feet) }\end{array}$ & $\begin{array}{c}\text { Depth } \\
\text { (feet) }\end{array}$ \\
\hline $\begin{array}{l}\text { Sandrock } \\
\text { Hard sandrock } \\
\text { Soft sandrock } \\
\text { Hard sandrock } \\
\text { Medium sandrock. } \\
\text { Hard sandrock } \\
\text { Soft sandrock } \\
\text { Hard sandrock } \\
\text { Medium sandrock } \\
\text { Soft sandrock. } \\
\text { Soft sandrock } \\
\text { Clay and sand }\end{array}$ & $\begin{array}{l}23 \\
22 \\
23 \\
21 \\
23 \\
22 \\
20 \\
64 \\
12 \\
10 \\
10 \\
11\end{array}$ & $\begin{array}{r}23 \\
45 \\
68 \\
89 \\
112 \\
134 \\
154 \\
218 \\
230 \\
240 \\
250 \\
261\end{array}$ & $\begin{array}{l}\text { Clay and sand } \\
\text { Soft sandrock } \\
\text { Clay } \\
\text { Clay and sand } \\
\text { Sandrock. } \\
\text { Soft sandrock. } \\
\text { Soft sandrock } \\
\text { Soft sandrock } \\
\text { Red and blue clay and sand } \\
\text { Clay and sandrock } \\
\text { Clay and sandrock. }\end{array}$ & $\begin{array}{r}22 \\
10 \\
12 \\
36 \\
6 \\
20 \\
22 \\
21 \\
21 \\
23 \\
22\end{array}$ & $\begin{array}{r}283 \\
293 \\
305 \\
341 \\
347 \\
367 \\
389 \\
410 \\
431 \\
454 \\
476\end{array}$ \\
\hline
\end{tabular}

\section{PRINGLE}

Population in 1940: 20.

Source of information: S. H. Chisum, county surveyor, June 24, 1948.

Ownership: Municipal.

Source of supply: Well, drilled in 1922; depth, 230 feet; static water level, 215 feet; windmill.

Storage: Elevated tank, 2,500 gallons.

Number of customers: 4.

Treatment: None.

\section{Analysis}

[Collected June 24, 1948. $\mathrm{pH}$ is 7.8. Analyzed by D. E. Weaver]

\begin{tabular}{|c|c|c|c|c|c|}
\hline & $\begin{array}{l}\text { Parts per } \\
\text { million }\end{array}$ & $\mid \begin{array}{l}\text { Equivalents } \\
\text { per million }\end{array}$ & & $\begin{array}{c}\text { Parts per } \\
\text { milli?n }\end{array}$ & $\begin{array}{l}\text { Equivalents } \\
\text { per million }\end{array}$ \\
\hline $\begin{array}{l}\text { Silica }\left(\mathrm{SiO}_{2}\right) \\
\text { Iron }(\mathrm{Fe}) \\
\text { Calcium }(\mathrm{Ca}) \\
\text { Magnesium }(\mathrm{Mg}) \\
\text { Sodium }(\mathrm{Na}) \\
\text { Potassium }(\mathrm{K}) \\
\text { Bicarbonate }\left(\mathrm{HCO}_{3}\right)^{-}\end{array}$ & $\begin{array}{l}46 \\
38.52 \\
29 \\
4.1 \\
226 \\
1.6\end{array}$ & $\begin{array}{r}1.897 \\
2.385 \\
.178 \\
.041 \\
3.704\end{array}$ & $\begin{array}{l}\text { Sulfate }\left(\mathrm{SO}_{4}\right) \\
\text { Chloride }(\mathrm{Cl}) \\
\text { Fluoride (F) } \\
\text { Nitrate (NO} \\
\text { Dissolved solids } \\
\text { Total hardness as } \mathrm{CaCO_{3 }}\end{array}$ & $\begin{array}{l}20 \\
6.8 \\
1.2 \\
5.8 \\
273 \\
214\end{array}$ & $\begin{array}{r}0.416 \\
.192 \\
.068 \\
.094 \\
\\
-0.0 \\
\end{array}$ \\
\hline
\end{tabular}


Population in 1940: 635 .

\section{STINNETT}

Source of information: F. B. Early, city commissioner, June 24, 1948.

Owner: Phillips Petroleum Co.

Source of supply: Eight wells at the Plains Water Station in Carson County,

13 miles southwest of Borger, owned by the Phillips Petroleum Co., which supply Phillips Petroleum Co. and the cities of Borger and Stinnett.

Pumpage: See under Borger.

Storage: See under Borger.

Number of customers: 120.

Treatment: None.

For analysis of water see under Borger.

\section{IRION COUNTY}

\section{BARNHART}

Population in 1940: 250 .

Source of information: Floyd Burks, owner, Sept. 10, 1947.

Owner: Floyd Burks.

Source of supply: Two wells.

Well 1. Drilled about 1927; depth, 600 feet; diameter, 6 inches; deep-well cylinder pump and 5-horsepower electric motor; pumping level, 292.8 feet below land surface Sept. 10, 1947.

Well 2. Drilled about 1930; depth, 400 feet; diameter, 6 inches; deepwell cylinder pump and 5-horsepower electric motor; punping level, 250.8 feet below land surface Sept. 10, 1947.

Pumpage (estimated) : 11,000 gallons a day.

Storage: Concrete ground reservoir, 19,000 gallons.

Number of customers: 46.

Treatment: None.

\section{Analysis}

[Composite sample of wells 1 and 2. Collected Aug. 20, 1947. pH is 7.9. Analyzed by B. C. Dwyer

\begin{tabular}{|c|c|c|c|c|c|}
\hline . & $\begin{array}{l}\text { Parts per } \\
\text { million }\end{array}$ & $\begin{array}{l}\text { Equivalents } \\
\text { per million }\end{array}$ & & $\begin{array}{l}\text { Parts per } \\
\text { million }\end{array}$ & $\begin{array}{l}\text { Equivalents } \\
\text { per million }\end{array}$ \\
\hline $\begin{array}{l}\text { Silica }\left(\mathrm{SiO}_{2}\right) \\
\text { Iron }(\mathrm{Fe}) \\
\text { Calcium }(\mathrm{Ca}) \\
\text { Magnesium }(\mathrm{Mg}) \\
\text { Sodium }(\mathrm{Na}) \\
\text { Potassium }(\mathrm{K}) \\
\text { Bicarbonate }\left(\mathrm{HC} \mathrm{CO}_{2}\right)^{-}\end{array}$ & $\begin{array}{l}10 \\
74 \\
35 \\
45 \\
7.14 \\
298\end{array}$ & $\begin{array}{r}3.69 \\
2.88 \\
1.96 \\
.18 \\
4.88\end{array}$ & $\begin{array}{l}\text { Sulfate }\left(\mathrm{SO}_{4}\right) \\
\text { Chloride }(\mathrm{Cl}) \\
\text { Fluoride }(\mathrm{F}) \\
\text { Nitrate (NO} \\
\text { Dissolved solids } \\
\text { Total hardness as } \mathrm{CaCO}_{3}\end{array}$ & $\begin{array}{c}88 \\
66 \\
1.6 \\
3.8 \\
486 \\
328\end{array}$ & $\begin{array}{r}1.83 \\
1.86 \\
.08 \\
.06 \\
-0.0\end{array}$ \\
\hline
\end{tabular}

\section{MERTZON}

Population in 1940: 869 .

Source of information: John Clark, owner, Aug. 20, 1947.

Owner: John Clark.

Source of supply: Three wells.

Well 1. East well; depth, 159 feet; diameter, 8 inches; deep-well cylinder pump and 2-horsepower gasoline engine; static water level, 104.3 feet below land surface Aug. 20, 1947. 
Well 2. South well; drilled about 1932; depth, 159 feet; diameter, 5 inches; deep-well cylinder pump and windmill; static water level, 104.2 feet below land surface Aug. 20, 1947.

Well 3. West well; drilled in 1941; depth, 150 feet; diameter, 6 inches; deep-well cylinder pump and 3-horsepower gasoline engine; static water level, 105.9 feet below land surface Aug. 20, 1947.

Pumpage (estimated): Average, 4,800 gallons a day.

Storage: Two concrete ground reservoirs, 40,000 gallons each; two elevated tanks, 1,900 and 1,600 gallons, respectively.

Number of customers: 28.

Treatment: None.

Analysis, well 3

[Collected Aug. 20, 1947. pH is 7.8. Analyzed by B. C. Dwyer]

\begin{tabular}{|c|c|c|c|c|c|}
\hline & $\begin{array}{c}\text { Parts per } \\
\text { million }\end{array}$ & $\begin{array}{l}\text { Equivalent } \\
\text { per million }\end{array}$ & & $\begin{array}{c}\text { Partr per } \\
\text { mill }\end{array}$ & $\begin{array}{l}\text { Equivalents } \\
\text { per million }\end{array}$ \\
\hline $\begin{array}{l}\text { Silica }\left(\mathrm{SiO}_{2}\right) \\
\text { Iron }(\mathrm{Fe}) \\
\text { Calcium }(\mathrm{Ca}) \\
\text { Magnesium }(\mathrm{Mg}) \\
\text { Sodium (Na) } \\
\text { Potassium }(\mathbf{K}) \\
\text { Bicarbonate }\left(\mathbf{H} \mathrm{CO}_{8}\right)\end{array}$ & $\begin{array}{l}5.0 \\
100 \\
54 \\
168 \\
11 \\
380\end{array}$ & $\begin{array}{r}4.99 \\
4.44 \\
7.30 \\
.28 \\
5.41\end{array}$ & $\begin{array}{l}\text { Sulfate (SOH) } \\
\text { Chloride (Cl) } \\
\text { Fluoride (F) } \\
\text { Nitrate (NO }{ }_{8} \text { ) } \\
\text { Dissolved solids } \\
\text { Total hardness as } \mathrm{CaCO}_{3}\end{array}$ & $\begin{array}{l}367 \\
182 \\
1.4 \\
992 \\
472\end{array}$ & $\begin{array}{r}6.39 \\
5.18 \\
.07 \\
.01 \\
\\
\end{array}$ \\
\hline
\end{tabular}

\section{JEFF DAVIS COUNTY}

\section{VALENTINE}

Population in 1940: 499.

Source of information: George W. Newton, water superintend ^nt, Aug. 13, 1948.

Ownership: Municipal.

Source of supply: Well; drilled in 1944 by E. Harrell; depth, 87 feet; diameter, 8 inches; deep-well turbine pump and 25-horsepower electric motor; static water level, 270 feet below land surface; yield, 80 gallons a minute; temperature, $82^{\circ} \mathrm{F}$.

Pumpage (estimated) : 20,000 gallons a day.

Storage: Ground reservoir, 30,000 gallons; elevated tank, 23,Cn0 gallons.

Number of customers: 125.

Treatment: None.

\section{Analysis}

[Collected Aug. 13, 1948. $\mathrm{pH}$ is 8.2. Analyzed by H. D. Smith]

\begin{tabular}{|c|c|c|c|c|c|}
\hline & $\begin{array}{l}\text { Parts per } \\
\text { million }\end{array}$ & $\begin{array}{l}\text { Equivalent } \\
\text { per million }\end{array}$ & & $\begin{array}{l}\text { Parti per } \\
\text { million }\end{array}$ & $\begin{array}{l}\text { Equivalents } \\
\text { per million }\end{array}$ \\
\hline $\begin{array}{l}\text { Silica }\left(\mathrm{SiO}_{2}\right) \\
\text { Iron }(\mathrm{Fe}) \\
\text { Calcium }(\mathrm{Ca}) \\
\text { Magnesium }(\mathrm{Mg}) \\
\text { Sodium }(\mathrm{Na}) \\
\text { Potassium }(\mathrm{K}) \\
\text { Bicarbonate }\left(\mathrm{H} \mathrm{C} \mathrm{O}_{\mathrm{s}}\right)^{-}\end{array}$ & $\begin{aligned} & 28 \\
& 4.35 \\
& 71.8 \\
& 71.6 \\
& 152\end{aligned}$ & $\begin{array}{r}0.215 \\
.066 \\
8.098 \\
.194 \\
2.498\end{array}$ & $\begin{array}{l}\text { Sulfate }\left(\mathrm{SO}_{4}\right) \\
\text { Chloride }(\mathrm{Cl}) \\
\text { Fluoride (F) } \\
\left.\text { Nitrate (NO})_{3}\right) \\
\text { Dissolved solids. } \\
\text { Total hardness as } \mathrm{CaCO}_{3}\end{array}$ & $\begin{array}{c}27 \\
13 \\
1.3 \\
4.5 \\
2.1 \\
14\end{array}$ & $\begin{array}{r}0.562 \\
.367 \\
.068 \\
.078 \\
-07 \\
-0 .-\end{array}$ \\
\hline
\end{tabular}




\section{KENT COUNTY}

\section{JAYTON}

Population in 1940: 770 .

Source of information: Lee Rice, water superintendent, Sept. 15, 1947.

Ownership: Municipal.

Source of supply: Two wells, 2 miles west of town.

Well 1. Dug in 1934; depth, 35 feet; diameter, 16 feet; twr deep-well cylinder pumps and 5-horsepower electric motor; static water level, 22.8 feet below land surface Sept. 15, 1947; yield, 60 gallons a minute.

Well 2. Drilled in 1945 by A. T. Leach; depth, 45 feet; diameter, 10 inches; deep-well turbine pump and 10-horsepower electric motor; yield, 160 gallons a minute.

Pumpage (estimated) : 125,000 gallons a day.

Storage: Elevated tank, 50,000 gallons.

Number of customers: 148.

Treatment: None.

Analysis, well 2

[Collected Sept. 15, 1947. $\mathrm{pH}$ is 7.6. Analyzed by B. C. Dwyer]

\begin{tabular}{|c|c|c|c|c|c|}
\hline & $\begin{array}{l}\text { Parts per } \\
\text { million }\end{array}$ & $\begin{array}{l}\text { Equivalent } \\
\text { per million }\end{array}$ & & $\begin{array}{l}\text { Parts per } \\
\text { million }\end{array}$ & $\begin{array}{l}\text { Equivalents } \\
\text { per million }\end{array}$ \\
\hline 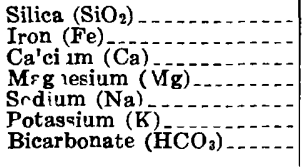 & $\begin{array}{r}23 \\
117^{.04} \\
11 \\
9 \cdot 4 \\
3.8 \\
282\end{array}$ & $\begin{array}{r}5.84 \\
.90 \\
.41 \\
.10 \\
4.62\end{array}$ & $\begin{array}{l}\text { Sulfate }\left(\mathrm{SO}_{4}\right) \\
\text { Chloride (Cl) } \\
\text { Fluoride (F) } \\
\left.\text { Nitrate (NO }{ }_{3}\right) \\
\text { Dissolved solids } \\
\text { Total hardness as } \mathrm{CaCO}\end{array}$ & $\begin{array}{l}104 \\
10 \\
4^{-4.8} \\
\mathbf{3 3 7}\end{array}$ & $\begin{array}{r}2.17 \\
.28 \\
.02 \\
.16 \\
\hdashline-0 \\
\hdashline-0 .-\end{array}$ \\
\hline
\end{tabular}

\section{LAMB COUNTY}

\section{AMHERST}

Population in 1940: 749 .

Source of information: E. D. House, water superintendent, Mar. 1, 1945.

Ownership: Municipal.

Source of supply: Two wells.

Well 1. Drilled in 1936 by J. M. Whitfield; depth, 210 feet; diameter $121 / 2$ inches; deep-well turbine pump and 25-horsepower electric motor; yield, 250 gallons a minute.

Well 2. Drilled in 1942; depth, 218 feet; diameter, 12 inches; deep-well turbine pump and electric motor; yield, 250 gallons a minute.

Pumpage (estimated) : 150,000 gallons a day.

Storage: Elevated tank, 75,000 gallons.

Number of customers: 200.

Treatment: None.

Analysis, well 1

[Collected Mar. 1, 1945. pH is 7.7. Analyzed by M. L. Begley]

\begin{tabular}{|c|c|c|c|c|c|}
\hline & $\begin{array}{l}\text { Parts per } \\
\text { million }\end{array}$ & $\begin{array}{l}\text { Equivalent } \\
\text { per million }\end{array}$ & & $\begin{array}{l}\text { Parts per } \\
\text { million }\end{array}$ & $\begin{array}{l}\text { Equivalents } \\
\text { per million }\end{array}$ \\
\hline $\begin{array}{l}\text { Silica }\left(\mathrm{SiO}_{2}\right) \\
\text { Iron }(\mathrm{Fe}) \\
\text { Calcium }(\mathrm{Ca}) \\
\text { Magnesium }(\mathrm{Mg}) \\
\text { Sodium }(\mathrm{Na}) \\
\text { Potassium }(\mathrm{K}) \\
\text { Bicarbonate }\left(\mathrm{HC} \mathrm{CO}_{3}\right)\end{array}$ & $\begin{array}{l}51 \\
66 \\
52 \\
41 \\
15 \\
294\end{array}$ & $\begin{array}{rr}3.29 \\
4 \\
4 \\
4 \\
1.28 \\
1.38 \\
4 \\
4.82\end{array}$ & $\begin{array}{l}\text { Sulfate }\left(\mathrm{SO}_{4}\right) \\
\text { Chloride (Cl) } \\
\text { Fluoride (F) } \\
\left.\text { Nitrate (NO } \mathrm{N}_{3}\right) \\
\text { Dissolved solids } \\
\text { Total hardness as } \mathrm{CaCO}{ }_{3}\end{array}$ & $\begin{array}{l}93 \\
98 \\
2.0 \\
6.3 \\
577 \\
\mathbf{3 7 8}\end{array}$ & \begin{tabular}{r}
1.94 \\
2.76 \\
.11 \\
.10 \\
\hdashline..-- \\
-10
\end{tabular} \\
\hline
\end{tabular}


Population in 1940: 3,817 .

\section{LITTLEFTELD}

Source of information: W. G. Street, water superintendent, 11ar. 1, 1945.

Ownership: Municipal.

Source of supply: Two wells.

Well 1. Known as East well; drilled; depth, 130 feet; diameter, 151/2 inches; deep-well turbine pump and electric motor; yie'd, 400 gallons a minute.

Well 2. Known as West well; drilled; depth, 130 feet; diameter, 121/2 inches; deep-well turbine pump and electric motor; yield, 400 gallons a minute; temperature, $64^{\circ} \mathrm{F}$.

Pumpage: Average, 400,000 gallons a day.

Storage: Concrete ground reservoir, 160,000 gallons; elevated tank, 75,000 gallons.

Number of customers: 1,050 .

Treatment: None.

Analysis, well 2

[Collected Mar. 1, 1945. pH is 7.7. Analyzed by M. L. Begley'

\begin{tabular}{|c|c|c|c|c|c|}
\hline & $\begin{array}{c}\text { Parts per } \\
\text { million }\end{array}$ & $\begin{array}{l}\text { Equivalents } \\
\text { per million }\end{array}$ & & $\begin{array}{c}\text { Parts per } \\
\text { million }\end{array}$ & $\begin{array}{l}\text { Equivalents } \\
\text { per million }\end{array}$ \\
\hline $\begin{array}{l}\text { Silica }\left(\mathrm{SiO}_{2}\right) \\
\text { Iron }(\mathrm{Fe}) \\
\text { Calcium }(\mathrm{Ca}) \\
\text { Magnesium }(\mathrm{Mg}) \\
\text { Sodium }(\mathrm{Na}) \\
\text { Potassium }(\mathrm{K}) \\
\text { Bicarbonate }\left(\mathrm{HCO}_{3}\right)\end{array}$ & $\begin{array}{l}42 \\
62 \\
39 \\
46 \\
14 \\
303\end{array}$ & $\begin{array}{r}3.09 \\
3.21 \\
2.00 \\
.36 \\
-4.97\end{array}$ & $\begin{array}{l}\text { Sulfate }\left(\mathrm{SO}_{4}\right) \\
\text { Chloride (Cl) } \\
\text { Fluoride (F) } \\
\text { Nitrate (NO})_{3} \text { ) } \\
\text { Dissolved solids } \\
\text { Total hardness as } \mathrm{CaCO}\end{array}$ & $\begin{array}{c}77 \\
69 \\
2.0 \\
1.8 \\
502 \\
315\end{array}$ & $\begin{array}{r}1.60 \\
1.95 \\
.11 \\
.03 \\
-0.0 \\
\end{array}$ \\
\hline
\end{tabular}

Driller's $\log 8$

\begin{tabular}{|c|c|c|c|c|c|}
\hline & $\begin{array}{c}\text { Thickness } \\
\text { (feet) }\end{array}$ & $\begin{array}{l}\text { Depth } \\
\text { (feet) }\end{array}$ & & $\begin{array}{c}\text { Thickness } \\
\text { (feet) }\end{array}$ & $\begin{array}{l}\text { Depth } \\
\text { (feet) }\end{array}$ \\
\hline \multicolumn{6}{|c|}{ Well I } \\
\hline $\begin{array}{l}\text { Subsoil. } \\
\text { Chalk } \\
\text { Pink clayy } \\
\text { Pink sandy clay } \\
\text { Rock } \\
\text { Calichere } \\
\text { Pink sand and calicher } \\
\text { Pink sand, seeps } \\
\text { Water sand and gravel }\end{array}$ & $\begin{array}{r}3 \\
3 \\
14 \\
6 \\
5 \\
9 \\
20 \\
9 \\
13\end{array}$ & $\begin{array}{r}3 \\
6 \\
20 \\
26 \\
31 \\
40 \\
60 \\
69 \\
82\end{array}$ & $\begin{array}{l}\text { Water sand and gravel with } \\
\text { clay balls. } \\
\text { White water sand } \\
\text { Water gravel } \\
\text { Yellow clay } \\
\text { Water sand } \\
\text { Water sand and gravel } \\
\text { Yellow clay }\end{array}$ & $\begin{array}{r}5 \\
11 \\
2 \\
19 \\
5 \\
12 \\
12\end{array}$ & $\begin{array}{r}87 \\
98 \\
100 \\
119 \\
124 \\
136 \\
148\end{array}$ \\
\hline
\end{tabular}

\section{Well 2}

\begin{tabular}{|c|c|c|c|c|c|}
\hline $\begin{array}{l}\text { Subsoil } \\
\text { Chalk. } \\
\text { Pink clay } \\
\text { Pink sandy clay } \\
\text { Rock chalk } \\
\text { Caliche. } \\
\text { Pink sand and caliche. }\end{array}$ & $\begin{array}{r}4 \\
5 \\
13 \\
5 \\
3 \\
8 \\
19\end{array}$ & $\begin{array}{r}4 \\
9 \\
22 \\
27 \\
\mathbf{3 0} \\
\mathbf{3 8} \\
\mathbf{5 7}\end{array}$ & $\begin{array}{l}\text { Pink sand, seeps } \\
\text { Water sand and gravel. } \\
\text { Pink clay. } \\
\text { White water sand } \\
\text { Yellow clay } \\
\text { Water sand and gravel. } \\
\text { Yellow clay. }\end{array}$ & $\begin{array}{r}11 \\
12 \\
14 \\
4 \\
17 \\
13 \\
2\end{array}$ & $\begin{array}{r}68 \\
80 \\
94 \\
98 \\
115 \\
128 \\
130\end{array}$ \\
\hline
\end{tabular}

Population in 1940: 782.

\section{OLTON}

Source of information: B. A. Dodson, water superintendent, I^ar. 1, 1945.

Ownership: Municipal. 
Source of supply: Well at elevated tank; drilled in 1933; depth. 200 feet; diameter 12 inches; deep-well turbine pump and electric motor; yield, 250 gallons a minute.

Pumpage (estimated) : 20,000 gallons a day.

Storage: Elevated tank, 50,000 gallons.

Number of customers: 180.

Treatment: None.

\section{Analysis}

[Collected Mar. 1, 1945. pH is 7.7. Analyzed by M. L. Begley]

\begin{tabular}{|c|c|c|c|c|c|}
\hline & $\begin{array}{c}\text { Parts per } \\
\text { million }\end{array}$ & $\begin{array}{l}\text { Equivalents } \\
\text { per million }\end{array}$ & & $\begin{array}{l}\text { Parts per } \\
\text { million }\end{array}$ & $\begin{array}{l}\text { Equivalents } \\
\text { per million }\end{array}$ \\
\hline $\begin{array}{l}\text { Silica }\left(\mathrm{SiO}_{2}\right) \\
\text { Iron }(\mathrm{Fe}) \\
\text { Calcium }(\mathrm{Ca}) \\
\text { Magnesium }(\overline{\mathrm{M}} \mathrm{g}) \\
\text { Sodium }(\mathrm{Na}) \\
\text { Potassium }(\mathrm{K}) \\
\text { Bicarbonate }(\mathrm{H} \overline{\mathrm{CO}}))\end{array}$ & $\begin{array}{l}33 \\
59 \\
29 \\
12 \\
7.9 \\
289\end{array}$ & $\begin{array}{r}2.94 \\
2.38 \\
.50 \\
.20 \\
4.74\end{array}$ & $\begin{array}{l}\text { Sulfate }\left(\mathrm{SO}_{4}\right) \\
\text { Chloride }(\mathrm{Cl}) \\
\text { Fluoride (F) } \\
\text { Nitrate (NO}) \\
\text { Dissolved solids } \\
\text { Total hardness as } \mathrm{CaCO}_{3}\end{array}$ & $\begin{array}{r}22 \\
20 \\
2.8 \\
6.8 \\
335 \\
266\end{array}$ & $\begin{array}{r}0.46 \\
.56 \\
.15 \\
.11 \\
\\
-15 \\
-.-1-\end{array}$ \\
\hline
\end{tabular}

\section{SUDAN}

Population in 1940: 974.

Source of information: Frank White, water superintendent, Mar. 1, 1945.

Ownership: Municipal.

Source of supply: Five wells.

Well 1. Drilled in 1935 by H. J. McCarty; depth, 134 feet; diameter, 10 inches; deep-well turbine pump and electric motor.

Well 2. Drilled; depth, 134 feet; diameter, 10 inches; deep-well turbine pump and electric motor.

Well 3. Drilled; depth, 134 feet; diameter, 10 inches; deep-well turbine pump and electric motor.

Well 4. Drilled; depth, 150 feet; diameter, 10 inches; deep-well turbine pump and electric motor.

Well 5. Drilled; depth, 150 feet; diameter, 10 inches; deep-well turbine pump and electric motor.

Pumpage (estimated) : 100,000 gallons a day.

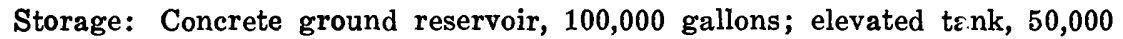
gallons.

Number of customers: 298.

Treatment: None.

Analysis, well 5

[Collected Mar. 1, 1945. pH is 7.7. Analyzed by M. L. Begley]

\begin{tabular}{|c|c|c|c|c|c|}
\hline & $\begin{array}{l}\text { Parts per } \\
\text { million }\end{array}$ & $\begin{array}{l}\text { Equivalent } \\
\text { per million }\end{array}$ & & $\begin{array}{c}\text { Parts pe* } \\
\text { million }\end{array}$ & $\begin{array}{l}\text { Equivalents } \\
\text { per million }\end{array}$ \\
\hline $\begin{array}{l}\text { Silica }\left(\mathrm{SiO}_{2}\right) \\
\text { Iron }(\mathrm{Fe}) \\
\text { Calcium }(\mathrm{Ca}) \\
\text { Magnesium }(\mathrm{Mg}) \\
\text { Sodium }(\mathrm{Na}) \\
\text { Potassium }(\mathrm{K}) \\
\text { Bicarbonate }\left(\mathrm{H} \mathrm{HOO}_{3}\right)\end{array}$ & $\begin{array}{l}42 \\
82.00 \\
23 \\
30 \\
7.6 \\
282\end{array}$ & $\begin{array}{r}4.09 \\
1.89 \\
1.31 \\
4.19 \\
4.62\end{array}$ & $\begin{array}{l}\text { Sulfate }\left(\mathrm{SO}_{4}\right) \\
\text { Chloride }(\mathrm{Cl}) \\
\text { Fluoride }(\mathrm{F}) \\
\text { Nitrate }\left(\mathrm{NO}_{3}\right) \\
\text { Dissolved solids. } \\
\text { Total hardness as } \mathrm{CaCO}_{3}\end{array}$ & $\begin{array}{c}66 \\
50 \\
1.2 \\
1.5 \\
453 \\
299\end{array}$ & \begin{tabular}{r}
1.37 \\
1.41 \\
.06 \\
.02 \\
\hdashline-0. \\
-0.0
\end{tabular} \\
\hline
\end{tabular}




\section{LIPSCOMB COUNTY}

Population in 1940: 386.

BOOKER

Source of information: E. E. McKee, water superintendent, June 24, 1948.

Ownership: Municipal.

Source of supply: Two wells at city water tower.

Well 1. Drilled in 1928 by Dade; depth, 310 feet; diameter, 8 inches; deep-well turbine pump and 20-horsepower electric motor; yield, 100 gallons a minute.

Well 2. Drilled in 1940; depth, 315 feet; diameter, 121/2 inches; deepwell turbine pump and 40-horsepower electric motor; static water level, 95 feet below land surface in 1940; yield, 250 gallons a minute with drawdown of 21 feet.

Pumpage: Average, 40,000 gallons a day.

Storage: Elevated tank, 50,000 gallons.

Number of customers: 215.

Treatment: None.

Analysis, well 1

[Collected June 24, 1948. pH is 7.3. Analyzed by D. E. Weaver?

\begin{tabular}{|c|c|c|c|c|c|}
\hline & $\begin{array}{c}\text { Parts per } \\
\text { million }\end{array}$ & $\begin{array}{l}\text { Equivalent } \\
\text { per million }\end{array}$ & & $\begin{array}{c}\text { Parts per } \\
\text { million }\end{array}$ & $\begin{array}{l}\text { Equivalents } \\
\text { per million }\end{array}$ \\
\hline $\begin{array}{l}\text { Silica }\left(\mathrm{SiO}_{2}\right) \\
\text { Iron }(\mathrm{Fe}) \\
\text { Calcium }(\mathrm{Ca}) \\
\text { Magnesium }(\mathrm{Mg}) \\
\text { Sodium }(\mathrm{Na}) \\
\text { Potassium }(\mathrm{K}) \\
\text { Bicarbonate }\left(\mathrm{HCOO}_{3}\right)\end{array}$ & $\begin{array}{l}45 \\
69 \\
30 \\
26 \\
3.65 \\
272\end{array}$ & $\begin{array}{r}3.44 \\
2.47 \\
1.13 \\
.09 \\
4.46\end{array}$ & $\begin{array}{l}\text { Sulfate }\left(\mathrm{SO}_{4}\right) \\
\text { Chloride }(\mathrm{Cl}) \\
\text { Fluoride (F) } \\
\text { Nitrate (NO} \\
\text { Dissolved solids. } \\
\text { Total hardness as } \mathrm{CaCO}_{3}\end{array}$ & $\begin{array}{l}34 \\
64 \\
5.4 \\
411 \\
2.1\end{array}$ & $\begin{array}{r}0.71 \\
1.81 \\
.02 \\
.08 \\
-0 .-2 \\
-0.0\end{array}$ \\
\hline
\end{tabular}

\section{FOLLETT}

Population in 1940: 431.

Source of information: Carl Fleming, water superintendent, June 24, 1948.

Ownership: Municipal.

Source of supply: Two wells.

Well 1. Drilled in 1928; depth, 369 feet; diameter, 6 inches; deep-well turbine pump and 15-horsepower electric motor; static water level, 170 feet below land surface; yield, 80 gallons a minute.

Well 2. Drilled in 1930; depth, 370 feet; diameter, 8 inches; deep-well turbine pump and 25-horsepower electric motor; yield, 100 gallons a minute.

Pumpage (estimated): Summer, 150,000 gallons a day; winter, 40,000 gallons a day.

Storage: Ground storage reservoir, 70,000 gallons; elevated tank, 50,000 gallons.

Number of customers: $\mathbf{1 5 0}$.

Treatment: None. 


\section{Analysis}

[Composite sample, collected June $24,1948 . \mathrm{pH}$ is 8.0 . Analyzed by H. D. S rith]

\begin{tabular}{|c|c|c|c|c|c|}
\hline & $\begin{array}{c}\text { Parts per } \\
\text { million }\end{array}$ & $\begin{array}{l}\text { Equivalent } \\
\text { per millior }\end{array}$ & . & $\begin{array}{c}\text { Parts per } \\
\text { million }\end{array}$ & $\begin{array}{l}\text { Equivalents } \\
\text { per million }\end{array}$ \\
\hline $\begin{array}{l}\text { Silica }\left(\mathrm{SiO}_{2}\right) \\
\text { Iron }(\mathrm{Fe}) \\
\text { Calcium }(\mathrm{Ca}) \\
\text { Magnesium }(\mathrm{Mg}) \\
\text { Sodium }(\mathrm{Na}) \\
\text { Potassium }(\mathrm{K}) \\
\text { Bicarbonate }\left(\mathrm{H} \mathrm{CO}_{3}\right)\end{array}$ & $\begin{array}{l}50 \\
47 \\
25 \\
34 \\
13 \\
270\end{array}$ & $\begin{array}{r}2.3 \overline{5} \\
2.06 \\
1.48 \\
.38 \\
4.43\end{array}$ & $\begin{array}{l}\text { Sulfate }\left(\mathrm{SO}_{4}\right) \\
\text { Chloride }(\mathrm{Cl}) \\
\text { Fluoride (F) } \\
\left.\text { Nitrate (NO } \mathrm{NO}_{3}\right) \\
\text { Dissolved solids } \\
\text { Total hardness as } \mathrm{CaCO}_{3}\end{array}$ & $\begin{array}{l}21 \\
45 \\
1.0 \\
4.5 \\
358 \\
220\end{array}$ & \begin{tabular}{r}
0.44 \\
1.27 \\
.05 \\
.07 \\
\hdashline-.0 \\
\end{tabular} \\
\hline
\end{tabular}

\section{HIGGINS}

Population in 1940: 741.

Source of information: Ted Lorenz, water superintendent, June 24, 1948.

Ownership: Municipal.

Source of supply: Two wells 100 feet apart near city light plant.

Well 1. Drilled in 1926; depth, 130 feet; diameter, 10 inches; deep-well turbine pump and 20-horsepower electric motor; yield on test, 240 gallons a minute for 99 minutes; on continuous pumping, pumpage decreased to less than 200 gallons a minute.

Well 2. Drilled in 1938; depth, 133 feet; diameter, 10 inches; deep-well turbine pump and 10-horsepower electric motor; yield, 200 gallons a minute.

Pumpage (estimated): Summer, 150,000 gallons a day; winter, 75,000 gallons a day.

Storage: Ground storage reservoir, 55,000 gallons; elevated tank, 250,000 gallons. (System tied into the Santa Fe Railroad reservo'r, 185,000 gallons.)

Number of customers: 217.

Treatment: None.

Analysis, well 2

[Collected June 24, 1948. pH is 7.5. Analyzed by H. D. Smith]

\begin{tabular}{|c|c|c|c|c|c|}
\hline & $\begin{array}{c}\text { Parts per } \\
\text { million }\end{array}$ & $\begin{array}{l}\text { Equivalent } \\
\text { per million }\end{array}$ & & $\begin{array}{c}\text { Parts pe- } \\
\text { million }\end{array}$ & $\begin{array}{l}\text { Equivalents } \\
\text { per million }\end{array}$ \\
\hline $\begin{array}{l}\text { Silica }\left(\mathrm{SiO}_{2}\right) \\
\text { Iron (Fe) } \\
\text { Calcium }(\mathrm{Ca}) \\
\text { Magnesium }(\mathrm{Mg}) \\
\text { Sodium }(\mathrm{Na}) \\
\text { Potassium (K) } \\
\text { Bicarbonate (HCOs) }\end{array}$ & $\begin{array}{l}76 \\
71 \\
19 \\
286 \\
4.8\end{array}$ & $\begin{array}{r}3.64 \\
.90 \\
.33 \\
.12 \\
4.69\end{array}$ & $\begin{array}{l}\text { Sulfate }\left(\mathrm{SO}_{4}\right) \\
\text { Chloride }(\mathrm{Cl}) \\
\text { Fluoride (F) } \\
\text { Nitrate (NO}) \\
\text { Dissolved solids. } \\
\text { Total hardness as CaCO, }\end{array}$ & $\begin{array}{r}9.6 \\
14.6 \\
292 \\
222\end{array}$ & $\begin{array}{r}\mathbf{0 . 2 0} \\
.39 \\
.03 \\
.12 \\
\\
-.-1 \\
\end{array}$ \\
\hline
\end{tabular}

\section{LUBBOCK COUNTY}

\section{IDALOU}

Population in 1940: 503.

Source of information: Mrs. J. T. Carlton, city secretary, Mar. 12, 1947.

Ownership: Municipal.

Source of supply: Well at elevated tank; drilled in 1925 by L. A. Peeples; depth, 125 feet; diameter, 15 inches; deep-well turbine pump and 10-horsepower electric motor.

Pumpage (estimated) : 30,000 gallons a day.

Storage: Elevated tank, 50,000 gallons.

Number of customers: 153.

Treatment: None. 


\section{Analysis}

[Collected Mar. 12, 1947. pH is 7.2. Analyzed by B. C. Dwyer]

\begin{tabular}{|c|c|c|c|c|c|}
\hline & $\begin{array}{c}\text { Parts per } \\
\text { million }\end{array}$ & $\begin{array}{l}\text { Equivalent } \\
\text { per million }\end{array}$ & & $\begin{array}{l}\text { Part? per } \\
\text { milion }\end{array}$ & $\begin{array}{l}\text { Equivalents } \\
\text { per million }\end{array}$ \\
\hline $\begin{array}{l}\text { Silica }\left(\mathrm{SiO}_{2}\right) \\
\text { Iron }(\mathrm{Fe}) \\
\text { Calcium }(\mathrm{Ca}) \\
\text { Magnesium }(\mathrm{Mg}) \\
\text { Sodium }(\mathrm{Na}) \\
\text { Potassium }(\mathrm{K})^{-} \\
\text {Bicarbonate }\left(\mathrm{H}_{\mathrm{CO}} \mathrm{CO}_{3}\right)\end{array}$ & $\begin{array}{l}32 \\
54 \\
68 \\
36 \\
9 \\
972 \\
37\end{array}$ & $\begin{array}{r}\mathbf{2} .70 \\
5.59 \\
1.58 \\
.23 \\
6.10\end{array}$ & $\begin{array}{l}\text { Sulfate }\left(\mathrm{SO}_{4}\right) \\
\text { Chloride (Cl) } \\
\text { Fluoride (F) } \\
\text { Nitrate (NO} \\
\text { Dissolved solids. } \\
\text { Total hardness as } \mathrm{CaCO}_{3}\end{array}$ & $\begin{array}{c}67 \\
78 \\
6.0 \\
5.5 \\
565 \\
414\end{array}$ & \begin{tabular}{r}
1.39 \\
2.20 \\
.32 \\
.09 \\
\hdashline-0 \\
\end{tabular} \\
\hline
\end{tabular}

\section{LUBBOCK}

Population in 1940: 31,853 .

Source of information: A. L. King, city engineer, Sept. 25, 1945.

Ownership: Municipal.

Source of supply: Nineteen wells.

Well 1. Three-quarters of a mile northeast of Lubbock post office; drilled in 1925 by Grant Baker; depth, 98 feet; diameter, 24 inches; deep-well turbine pump and 30-horsepower electric motor; static water level, 28.4 feet below land surface Sept. 25, 1944; drawdowr reported, 50 feet while pumping 60 gallons a minute in 1925; yield, 600 gallons a minute.

Well 2. Drilled in 1917 by T. P. Wright; depth, 300 feet; diameter, 24 inches; deep-well turbine pump and 25-horsepower electric motor; static water level, 81.3 feet below land surface Sept. 26, 1944; drawdown reported, 57 feet while pumping 617 gallons a minute in 1932.

Well 3. Drilled in 1925 by D. L. McDonald; depth, 210 feet; diameter, 24 inches; deep-well turbine pump and 15-horsepower electric motor; static water level, 72.3 feet below land surface Sept. 25, 1944.

Well 4. Drilled in 1928 by B. B. Baron; depth, 156 feet; diameter, 24 inches; deep-well turbine pump and 30-horsepower electric motor; static water level, 67.42 feet below land surface Oct. 2, 1934.

Well 5. Drilled in 1929 by Coy Rodgers; depth, 150 feet; diameter, 24 inches; deep-well turbine pump and 20-horsepower electric motor; static water level, 72.2 feet below land surface Sept. 26. 1944; drawdown, 49 feet while pumping 440 gallons a minute in 1929.

Well 6. Drilled in 1931 by D. L. McDonald; depth, 142 feet; diameter, 18 inches; deep-well turbine pump and 20-horsepower electric motor; static water level, 80.0 feet below land surface Sept. 25, 1944; drawdown, 64 feet while pumping 430 gallons a minute in 1932.

Well 7. Drilled in 1931 by D. L. McDonald; depth, 158 feet; diameter, 18 inches; deep-well turbine.pump and 40-horsepower electric motor; static water level, 68.1 feet below land surface Sept. 2\&. 1944; drawdown, 56 feet while pumping 780 gallons a minute.

Well 8. Drilled in 1931 by D. L. McDonald; depth, 157 feet; diameter, 18 inches; deep-well turbine pump and 15-horsepower electric motor; static water level, 76.4 feet below land surface Sept. 28, 1944. 
Well 9. Drilled in 1937 by B. B. Baron; depth, 151 feet; diameter, 22 to 18 inches; deep-well turbine pump and 50-horsepower electric motor; static water level, 66.5 feet below land surface Sept. 28, 1944; drawdown, 55 feet while pumping 650 gallons a minute in 1937 .

Well 10. Drilled in 1938 by Crawford and Anderson; depth, 151 feet; diameter, 24 to 18 inches; deep-well turbine pump and 40-horsepower electric motor; static water level, 67.4 feet below land surface Sept. 25, 1944 ; yield, 850 gallons a minute March 1938.

Well 11. Drilled in 1938 by Crawford and Anderson; depth, 145 feet; diameter 24 to 18 inches; deep-well turbine pump and 25-horsepower electric motor; static water level, 61.7 feet below land surface Sept. 28, 1944; drawdown, 36 feet while pumping 500 gallons a minute in 1938.

Well 12. Drilled in 1938 by Crawford and Anderson; depth, 145 feet; diameter 22 to 18 inches; deep-well turbine pump and 30 -horsepower electric motor; static water level, 58.4 feet below land surface Sept. 28, 1944; drawdown 53 feet while pumping 430 gallons a minute in 1938.

Well 13. Drilled in 1939 by Crawford and Anderson; depth, 150 feet; diameter, 22 to 18 inches; deep-well turbine pump and 40.horsepower electric motor; static water level, 53.7 feet below land surface Sept. 25, 1944; drawdown 35 feet while pumping 640 gallons a minute in 1939.

Well 14. Drilled in 1940 by Crawford and Anderson; depth, 135 feet; diameter, 22 to 18 inches; deep-well turbine pump and 25 -horsepower electric motor; static water level, 59.4 feet below land surface Sept. 26, 1944; drawdown 65 feet while pumping 535 gallons a minute in 1940.

Well 15. Drilled in 1940 by Crawford and Anderson; depth, 135 feet; diameter 22 to 18 inches; deep-well turbine pump and 40 -horsepower electric motor; static water level, 60.2 feet below land surface Sept. 25, 1944; drawdown, 41 feet while pumping 890 gallons a minute in 1940.

Well 16. Drilled in 1941 by L. A. Peeples; depth, 135 feet; diameter, 22 to 18 inches; deep-well turbine pump and 25-horsepower electric motor; static water level, 54.6 feet below land surface Sept. 28, 1944; drawdown, 78 feet while pumping 640 gallons a minute in 1941.

Well 17. Drilled in 1941 by L. A. Peeples; depth, 125 feet; diameter, 22 to 18 inches; deep-well turbine pump and 40-horsepower electric motor; static water level, 42.3 feet below land surface Sept. 30, 1944; drawdown, 45 feet while pumping 825 gallons a minute in 1943.

Well 18. Drilled in 1943 by Geo. Anderson; depth, 110 feet; diameter, 22 to 18 inches; deep-well turbine pump and 25-horsepower electric motor; static water level, 25.6 feet below land surface Sept. 30, 1944; drawdown, 65 feet while pumping 700 gallons a minute in 1943 .

Well 19. Drilled in 1945 by L. A. Peeples; depth, 145 feet; diameter, 22 to 18 inches; deep-well turbine pump and electric motor; static water level, 26.5 feet below land surface Jan. 29, 1945; drawdown, 23 feet while pumping 1,050 gallons a minute. 
Average pumpage, in gallons a day

\begin{tabular}{|c|c|c|c|}
\hline & 1942 & 1943 & 1944 \\
\hline 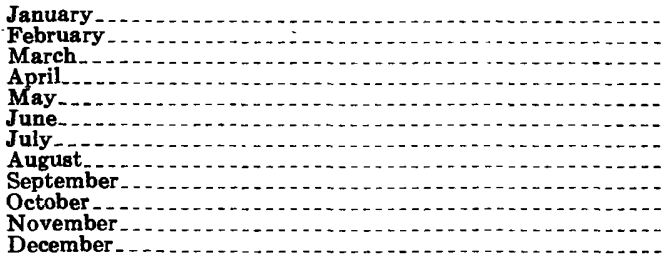 & $\begin{array}{l}2,440,000 \\
2,620,000 \\
3,040,000 \\
3,150,000 \\
5,100,000 \\
6,150,000 \\
5,730,000 \\
4,840,000 \\
2,780,000 \\
2,480,000 \\
2,830,000 \\
2,690,000\end{array}$ & $\begin{array}{l}2,850,000 \\
3,170,000 \\
4,500,000 \\
4,670,000 \\
4,43 C, 000 \\
5,28 C, 000 \\
6,16 C, 000 \\
8,22 C, 000 \\
4,70 C, 000 \\
3,48 C, 000 \\
3,08 C, 000 \\
2,72 C, 000\end{array}$ & $\begin{array}{l}2,630,000 \\
2,750,000 \\
4,507,000 \\
4,070,000 \\
4,400,000 \\
6,170,000 \\
5,000,000 \\
5,960,000 \\
4,100,000 \\
3,420,000 \\
3,090,000 \\
2,750,000\end{array}$ \\
\hline
\end{tabular}

Storage: Six concrete ground storage reservoirs, total capanity, $7,435,000$ gallons.

Number of customers: 8,700 .

Treatment: None.

\section{Analyses}

[Collected: Well 2, Feb. 15, 1944; well 5, Oct. 2, 1944. pH, well 2, is 8.1. Analyzed by J. H. Rowley]

\begin{tabular}{|c|c|c|c|c|}
\hline & \multicolumn{2}{|c|}{ Well 2} & \multicolumn{2}{|c|}{ Well 5} \\
\hline & $\begin{array}{l}\text { Parts per } \\
\text { million }\end{array}$ & $\begin{array}{l}\text { Equivalents } \\
\text { per million }\end{array}$ & $\begin{array}{l}\text { Parts per } \\
\text { million }\end{array}$ & $\begin{array}{l}\text { Equivalents } \\
\text { per million }\end{array}$ \\
\hline 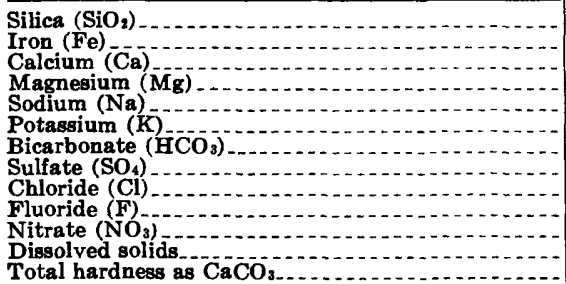 & $\begin{array}{l}52 \\
57.02 \\
65 \\
79 \\
23 \\
318 \\
169 \\
110 \\
5.4 \\
7.5 \\
746 \\
410\end{array}$ & $\begin{array}{r}\mathbf{2} .85 \\
\mathbf{5} .35 \\
\mathbf{3 . 4 4} \\
.59 \\
\mathbf{5 . 2 1} \\
\mathbf{3 . 5 2} \\
\mathbf{3 . 1 0} \\
.28 \\
.12 \\
\hdashline\end{array}$ & \begin{tabular}{r}
60 \\
60 \\
11.8 \\
318 \\
153 \\
150 \\
\hdashline$\$ 3.8$ \\
$6 \$ 4$ \\
379
\end{tabular} & $\begin{array}{r}-2.65 \\
4.93 \\
5.11 \\
5.21 \\
3.19 \\
4.23 \\
.06\end{array}$ \\
\hline
\end{tabular}

[Collected: Wells 6 and 13, Sept. 22, 1944; well 16, Sept. 25, 1944; well 19, Feb. 15, 1945. pH, well 19, is 7.5. Analyzed by J. H. Row ley]

\begin{tabular}{|c|c|c|c|c|}
\hline & \multicolumn{2}{|c|}{ Well 6} & \multicolumn{2}{|c|}{ Well 13} \\
\hline & $\begin{array}{l}\text { Parts per } \\
\text { million }\end{array}$ & $\begin{array}{l}\text { Equivalents } \\
\text { per million }\end{array}$ & $\begin{array}{l}\text { Part? per } \\
\text { milion }\end{array}$ & $\begin{array}{l}\text { Equivalents } \\
\text { per million }\end{array}$ \\
\hline 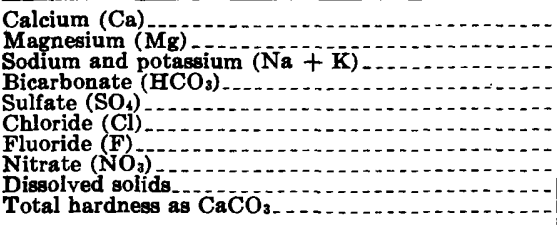 & \begin{tabular}{|l}
78 \\
86 \\
114 \\
354 \\
319 \\
114 \\
3.3 \\
4.1 \\
893 \\
548
\end{tabular} & $\begin{array}{r}\mathbf{3 . 8 9} \\
\mathbf{7 . 0 7} \\
\mathbf{4 . 9 4} \\
\mathbf{5 . 8 0} \\
\mathbf{6 . 6 4} \\
\mathbf{3 . 2 2} \\
.17 \\
.07 \\
\\
\end{array}$ & $\begin{array}{c}7 \hat{\zeta} \\
94 \\
165 \\
345 \\
421 \\
149 \\
3.2 \\
1.5 \\
1,08 ? \\
57 ?\end{array}$ & $\begin{array}{r}\mathbf{3 . 7 9} \\
7.73 \\
7.21 \\
\mathbf{5} .65 \\
8.77 \\
4.12 \\
.17 \\
.02 \\
\\
\end{array}$ \\
\hline
\end{tabular}




\begin{tabular}{|c|c|c|c|c|}
\hline & \multicolumn{2}{|c|}{ Well 16} & \multicolumn{2}{|c|}{ Well 19} \\
\hline & $\begin{array}{l}\text { Parts per } \\
\text { million }\end{array}$ & $\begin{array}{l}\text { Equivalents } \\
\text { per million }\end{array}$ & $\begin{array}{l}\text { Parts per } \\
\text { million }\end{array}$ & $\begin{array}{l}\text { Equivalents } \\
\text { per million }\end{array}$ \\
\hline 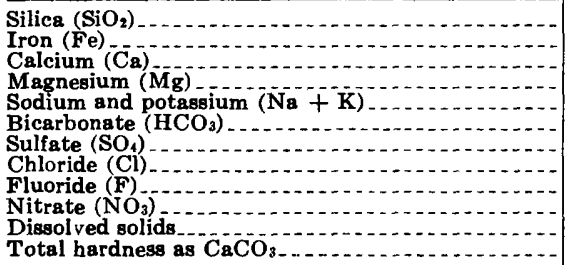 & $\begin{array}{r}45 \\
58 \\
59 \\
249 \\
120 \\
98 \\
3.5 \\
2.8 \\
619 \\
351\end{array}$ & $\begin{array}{r}4.25 \\
4.77 \\
2.55 \\
4.08 \\
2.50 \\
2.76 \\
.18 \\
.05 \\
-12\end{array}$ & $\begin{array}{c}64 \\
69 \\
80 \\
150 \\
317 \\
272 \\
190 \\
4.1 \\
5.1 \\
990 \\
501\end{array}$ & $\begin{array}{r}\mathbf{3 . 4 4} \\
6.58 \\
6.50 \\
\mathbf{5 . 2 0} \\
\mathbf{5 . 6 6} \\
\mathbf{5 . 3 6} \\
.22 \\
.08\end{array}$ \\
\hline
\end{tabular}

\section{Drillers' logs}

\begin{tabular}{|c|c|c|c|c|c|}
\hline & $\underset{\text { (feet) }}{\text { Thickness }}$ & $\begin{array}{l}\text { Depth } \\
\text { (feet) }\end{array}$ & 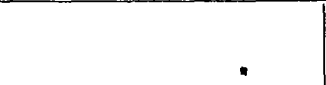 & $\underset{\text { (feet) }}{\text { Thickness }}$ & $\begin{array}{l}\text { Depth } \\
\text { (feet) }\end{array}$ \\
\hline \multicolumn{6}{|c|}{ Well 15} \\
\hline $\begin{array}{l}\text { Caliche } \\
\text { Sandy red rock and red clay } \\
\text { Hard sandrock } \\
\text { Red water sand. } \\
\text { Red sandrock. } \\
\text { Water sand. }\end{array}$ & $\begin{array}{r}20 \\
\mathbf{3 2} \\
\mathbf{3} \\
8 \\
5 \\
\mathbf{1 7}\end{array}$ & $\begin{array}{l}20 \\
52 \\
55 \\
63 \\
68 \\
85\end{array}$ & $\begin{array}{l}\text { Red clay } \\
\text { Water sand } \\
\text { Red clay } \\
\text { Gray clay } \\
\text { Caliche rock }\end{array}$ & $\begin{array}{r}21 \\
22 \\
2 \\
20 \\
10\end{array}$ & $\begin{array}{l}106 \\
128 \\
130 \\
150 \\
160\end{array}$ \\
\hline \multicolumn{6}{|c|}{ Well 16} \\
\hline $\begin{array}{l}\text { Top soil } \\
\text { Caliche clay and sandy } \\
\text { caliche. } \\
\text { Caliche rock (water at } 39 \\
\text { feet). } \\
\text { Red sand, water }\end{array}$ & $\begin{array}{r}4 \\
31 \\
15 \\
11\end{array}$ & $\begin{array}{r}4 \\
35 \\
50 \\
61\end{array}$ & $\begin{array}{l}\text { Rock } \\
\text { Red sand, water. } \\
\text { Red clay, } \\
\text { Sand and gravel, water. } \\
\text { Red clay ...... } \\
\text { Clayey fine-grained sand.... }\end{array}$ & $\begin{array}{r}2 \\
12 \\
4 \\
\mathbf{9 2} \\
24 \\
18\end{array}$ & $\begin{array}{r}63 \\
75 \\
79 \\
111 \\
135 \\
153\end{array}$ \\
\hline \multicolumn{6}{|c|}{ Well 19} \\
\hline $\begin{array}{l}\text { Top soil and caliche clay } \\
\text { Caliche clay and small rock. } \\
\text { Gray sand, water } \\
\text { Sandy red clay } \\
\text { Red sand, honeycomb rock } \\
\text { and some gravel, water. }\end{array}$ & $\begin{array}{l}20 \\
10 \\
13 \\
40 \\
11\end{array}$ & $\begin{array}{l}20 \\
\mathbf{3 0} \\
\mathbf{4 3} \\
\mathbf{8 3} \\
\mathbf{9 4}\end{array}$ & $\begin{array}{l}\text { Coarse sand and gravel, } \\
\text { water. } \\
\text { Sandy red clay } \\
\text { Dry packed sand } \\
\text { White caliche rock. }\end{array}$ & $\begin{array}{r}13 \\
33 \\
11 \\
\mathbf{3}\end{array}$ & $\begin{array}{l}107 \\
140 \\
151 \\
154\end{array}$ \\
\hline
\end{tabular}

Population in 1940: 3,587 .

\section{SLATON}

Source of information: City secretary, Feb. 7, 1944.

Ownership: Municipal.

Source of supply: Three wells in well field about 1 mile north of town.

Well 1. Drilled in 1925 by W. M. Edwards; depth, 135 feet; diameter, 18 inches; deep-well turbine pump and 15-horsepower electric motor; yield, 235 gallons a minute.

Well 2. Drilled by D. W. McDonald; depth, 125 feet; diameter, 18 inches; deep-well turbine pump and 15-horsepower electric motor; yield, 140 gallons a minute. 
Well 3. Drilled; depth, 206 feet; diameter, 18 inches; deep-well turbine pump and 40-horsepower electric motor; static water level, 99.6 feet below land surface Jan. 18, 1937; yield, 360 gallons a minute; temperature, $65^{\circ} \mathrm{F}$.

Pumpage (estimated) : 150,000 gallons a day.

Storage: Ground reservoir at well field, 50,000 gallons; elevated tank, 50,000 gallons.

Number of customers: 950 .

Treatment: None.

Analysis, well 1

[Collected Feb. 17, 1944. pH is 8.3. Analyzed by J. H. Rowle:r]

\begin{tabular}{|c|c|c|c|c|c|}
\hline & $\begin{array}{c}\text { Parts per } \\
\text { million }\end{array}$ & $\begin{array}{l}\text { Equivalent } \\
\text { per million }\end{array}$ & & $\begin{array}{c}\text { P irts per } \\
\text { million }\end{array}$ & $\begin{array}{l}\text { Equivalents } \\
\text { per million }\end{array}$ \\
\hline 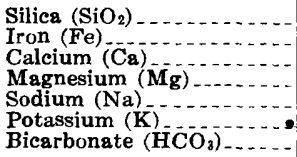 & $\begin{array}{l}51 \\
42 \\
51 \\
88 \\
15 \\
342\end{array}$ & $\begin{array}{r}2.10 \\
4.19 \\
3.81 \\
5.38 \\
5.60\end{array}$ & $\begin{array}{l}\text { Sulfate }\left(\mathrm{SO}_{4}\right) \\
\text { Chloride }(\mathrm{Cl}) \\
\text { Fluoride (F) } \\
\text { Nitrate (NO} \\
\text { Dissolved solids } \\
\text { Total hardness as } \mathrm{CaCO}\end{array}$ & $\begin{array}{r}121 \\
71 \\
5.9 \\
3.0 \\
611 \\
\mathbf{3 1 4}\end{array}$ & $\begin{array}{r}2.52 \\
2.00 \\
.31 \\
.05 \\
\\
\\
\end{array}$ \\
\hline
\end{tabular}

Driller's log, well 1

\begin{tabular}{|c|c|c|c|c|c|}
\hline & $\begin{array}{c}\text { Thickness } \\
\text { (feet) }\end{array}$ & $\begin{array}{l}\text { Depth } \\
\text { (feet) }\end{array}$ & & $\begin{array}{c}\text { Thickness } \\
\text { (feet) }\end{array}$ & $\begin{array}{l}\text { Depth } \\
\text { (feet) }\end{array}$ \\
\hline $\begin{array}{l}\text { No record. } \\
\text { Fine soft sand, little water } \\
\text { Stiff red clay } \\
\text { Sand and clay } \\
\text { Sand, some water }\end{array}$ & $\begin{array}{r}84 \\
5 \\
\mathbf{3} \\
14 \\
10\end{array}$ & $\begin{array}{r}84 \\
89 \\
92 \\
106 \\
116\end{array}$ & $\begin{array}{l}\text { Hard red clay. } \\
\text { Fine-grained sand, water. } \\
\text { Sand, water } \\
\text { Large gravel, rock and coarse } \\
\text { sand, water. }\end{array}$ & $\begin{array}{l}\mathbf{3} \\
\mathbf{4} \\
\mathbf{9} \\
\mathbf{3}\end{array}$ & $\begin{array}{l}119 \\
123 \\
132 \\
135\end{array}$ \\
\hline
\end{tabular}

\section{LYNN COUNTY}

\section{O'DONNELL}

Population in 1940: 1,187.

Source of information: Rochelle Howard, water superintendent, Nov. 29, 1946.

Ownership: Municipal.

Source of supply: Nine wells.

Well 1. At elevated tank; depth, 82 feet; deep-well cylinder pump and 3/4-horsepower electric motor; static water level reported, 70 feet below land surface; yield reported, about 3 gallons a minute in November 1946.

Well 2. At elevated tank; depth, 82 feet; deep-well cylinder pump and $11 / 2$-horsepower electric motor; static water level report:d, 70 feet below land surface; yield reported, about 2 gallons a minute in November 1946.

Well 3. At elevated tank; depth, 90 feet; deep-well cylinder pump and $3 / 4$-horsepower electric motor; static water level reported, 70 feet below land surface; yield reported, about 3 gallons a minute in November 1946.

Well 4. In northeast corner of city limits; depth, 82 feet; deep-well cylinder and 3/4-horsepower electric motor; static water level reported, 70 feet below land surface; yield reported, about 4 gallons a minute in November 1946. 
Well 5. In northeast corner of city limits; depth, 82 feet; deep-well cylinder pump and $3 / 4$-horsepower electric-motor; static water level reported, 70 feet below land surface; yield reported, about 5 gallons a minute in November 1946.

Well 6. At intersection of U. S. Highway 87 bypass and business routes north of O'Donnell; drilled in 1938; depth, 62 feet; deep-we'l cylinder pump and 2-horsepower electric motor; static water level reported, 58 feet below land surface; yield reported, about 8 gallons a minute in November 1946.

Well 7. West of intersection of U. S. Highway 87 bypass and business routes north of O'Donnell; drilled in 1945; depth, 64 feet; deep-well turbine pump and 3-horsepower electric motor; static woter level reported, 52 feet below land surface; yield reported, 25 gallons a minute in November 1946.

Well 8. West of intersection of U. S. Highway 87 bypass and business routes north of O'Donnell; drilled in April 1945; depth, 62 feet; deepwell turbine pump and 3-horsepower electric motor; static water level reported, 52 feet below land surface.

Well 9. West of elevated tank; drilled in 1934 by Charlie Nunally; depth, 67 feet; diameter, $5 \%$ inches; Hi-lift pump and 3-horsepower electric motor; static water level reported, 22 feet below land surface; yield reported, about 50 gallons a minute in November 1946.

Pumpage: Minimum, 50,000 gallons a day; maximum, 150,000 gallons a day. Storage: Elevated tank, 55,000 gallons; ground storage reservcir, 76,900 gallons.

Number of customers: 300 .

Treatment: Chlorination.

\section{Analysis}

[Composite sample of all wells. Collected Nov. 29, 1946. pH is 7.4. Analyzed by C. F Cibulka]

\begin{tabular}{|c|c|c|c|c|c|}
\hline & $\begin{array}{l}\text { Parts per } \\
\text { million }\end{array}$ & $\begin{array}{l}\text { Equivalents } \\
\text { per million }\end{array}$ & & $\begin{array}{c}\text { Parts per } \\
\text { million }\end{array}$ & $\begin{array}{l}\text { Equivalents } \\
\text { per million }\end{array}$ \\
\hline $\begin{array}{l}\text { Iron (Fe) } \\
\text { Calcium }(\mathrm{Ca}) \\
\text { Magnesium }(\mathrm{Mg}) \\
\text { Sodium }(\mathrm{Na}) \\
\text { Potassium }(\mathrm{K}) \\
\left.\text { Bicarbonate }(\mathrm{HCO})^{2}\right)\end{array}$ & $\begin{array}{l}0.17 \\
116 \\
127 \\
296 \\
40 \\
392\end{array}$ & $\begin{array}{r}5.79 \\
10.44 \\
12.88 \\
1.02 \\
6.43\end{array}$ & $\begin{array}{l}\text { Sulfate }\left(\mathrm{SO}_{4}\right) \\
\text { Chloride }(\mathrm{Cl}) \\
\text { Fluoride (F) } \\
\text { Nitrate (NO} \\
\text { Dissolved solids } \\
\text { Total hardness as } \mathrm{CaCO}_{3}\end{array}$ & $\begin{array}{r}507 \\
452 \\
5.6 \\
6.2 \\
1,890 \\
812\end{array}$ & \begin{tabular}{r}
10.56 \\
12.75 \\
.29 \\
.10 \\
\hdashline-1.5 \\
\end{tabular} \\
\hline
\end{tabular}

\section{TAHOKA}

Population in 1940: 2,129.

Source of information: G. H. Hines, water superintendent, Nov. 2\%, 1946.

Ownership: Municipal.

Source of supply: Eight wells.

Well 1. About 3 miles north of elevated tank; drilled in 1937 by L. A. Peeples; depth, 80 feet; diameter, 10 inches; deep-well turkine pump and 15-horsepower electric motor; static water level, 52 feet kelow land surface; yield, 150 gallons a minute.

Well 2. About 3 miles north of elevated tank; drilled in 1937 by L. A. Peeples; depth, 80 feet; diameter, 10 inches; deep-well turbine pump and 15-horsepower electric motor; yield, 150 gallons a minute. 
Well 3. Northeast of well 1; drilled in 1939 by L. A. Peeples; depth, 80 feet; diameter, 10 inches; deep-well turbine pump and 71/2-horsepower electric motor; yield, 100 gallons a minute.

Well 4. Three miles north of elevated tank; drilled ir 1939 by L. A. Peeples; depth, 80 feet; diameter, 10 inches; deep-well turbine pump and $71 / 2$-horsepower electric motor; yield, 100 gallons a minute.

Well 5. Three miles north of elevated tank, northeast of well 3; drilled in 1941 by L. A. Peeples; depth, 80 feet; diameter, 10 inches; deepwell turbine pump and $7 \frac{1}{2}$-horsepower electric motor; Jield, 100 gallons a minute.

Well 6. Four miles north of elevated tank; drilled in January 1946 by L. M. Bankson; depth, 80 feet; diameter, 10 inches; well not equipped for service on Nov. 29, 1946.

Well 7. Four miles north of elevated tank; drilled in January 1946 by L. M. Bankson; depth, 80 feet; diameter, 10 inches; well not equipped for service on Nov. 29, 1946.

Well 8. Four miles north of elevated tank; drilled in November 1946 by

G. C. Paulk; depth, 92 feet; diameter, 10 inches; pumping equipment not installed Nov. 29, 1946.

Pumpage (estimated) : Average, 300,000 gallons a day.

Storage: Ground storage reservoir, 255,000 gallons; elevated tank, 55,000 gallons.

Number of customers: 678.

Treatment: Chlorination.

\section{Analysis}

[Composite sample of five wells. Collected Nov. 29, 1946. pH is 7.8. Analyzed by C. B. Cibulka]

\begin{tabular}{|c|c|c|c|c|c|}
\hline & $\begin{array}{l}\text { Parts per } \\
\text { million }\end{array}$ & $\begin{array}{l}\text { Equivalents } \\
\text { per million }\end{array}$ & & $\begin{array}{l}\text { Farts per } \\
\text { million }\end{array}$ & $\begin{array}{l}\text { Equivalents } \\
\text { per million }\end{array}$ \\
\hline 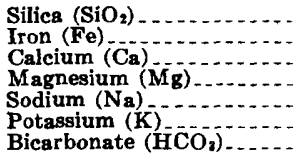 & $\begin{array}{l}37 \\
48 \\
45 \\
26 \\
5.2 \\
372\end{array}$ & $\begin{array}{r}-4.40 \\
3.70 \\
1.12 \\
.13 \\
6.10\end{array}$ & $\begin{array}{l}\text { Sulfate }\left(\mathrm{SO}_{4}\right) \\
\text { Chloride }(\mathrm{Cl}) \\
\text { Fluoride (F) } \\
\text { Nitrate }\left(\mathrm{NO}_{3}\right) \\
\text { Dissolved solids } \\
\text { Total hardness as } \mathrm{CaCO}\end{array}$ & $\begin{array}{r}5.4 \\
28 \\
5.6 \\
3.5 \\
387 \\
305\end{array}$ & $\begin{array}{r}0.11 \\
.79 \\
.29 \\
.06 \\
-.0 \\
-0 .-2\end{array}$ \\
\hline
\end{tabular}

Driller's log, well 1

\begin{tabular}{|c|c|c|c|c|c|}
\hline & $\begin{array}{c}\text { Thickness } \\
\text { (feet) }\end{array}$ & $\begin{array}{c}\text { Depth } \\
\text { (feet) }\end{array}$ & & $\begin{array}{c}\text { Thickness } \\
\text { (feet) }\end{array}$ & $\begin{array}{c}\text { Depth } \\
\text { (feet) }\end{array}$ \\
\hline $\begin{array}{l}\text { Top goil } \\
\text { Caliche } \\
\text { Sandrock }\end{array}$ & $\begin{array}{r}6 \\
40 \\
3\end{array}$ & $\begin{array}{r}6 \\
46 \\
49\end{array}$ & $\begin{array}{l}\text { Hard rock } \\
\text { Sand and gravel } \\
\text { Yellow clay at } 80 \text { feet }\end{array}$ & $\begin{array}{r}10 \\
21 \\
-\end{array}$ & $\begin{array}{l}59 \\
80\end{array}$ \\
\hline
\end{tabular}

\section{MARTIN COUNTY}

Population in 1940: 1,245 .

\section{STANTON}

Source of information: G. B. Shelburne, Dec. 18, 1946.

Ownership: Municipal.

Source of supply: Four wells.

Well 1. Half a block north of elevated tank; drilled in 1927; depth, 142 feet; diameter, 6 inches; deep-well cylinder pump and 71/2-horsepower electric motor; yield, 50 gallons a minute. 
Well 2. A quarter of a block northwest of elevated tank; drilled in 1930; depth, 135 feet; diameter, 8 inches; deep-well turbine pump and 71/2horsepower electric motor; static water level, 65 feet below land surface; yield, 150 gallons a minute.

Well 3. Three-quarters of a block northeast of elevated tank and half a block east of well 1; drilled in 1943 by Skeen Bros.; depth, 149 feet; diameter, 8 inches; deep-well turbine pump and 71/2-horsepower electric motor; yield, 80 gallons a minute.

Well 4. Six hundred feet southwest of elevated tank; drilled in 1946 by L. Graves; depth, 160 feet; diameter, 8 inches; deep-well turkine pump and 10-horsepower electric motor; yield, 150 gallons a minute.

Average pumpage, in gallons a day

\begin{tabular}{|c|c|c|c|c|c|}
\hline \multicolumn{6}{|c|}{1945} \\
\hline $\begin{array}{l}\text { January } \\
\text { February } \\
\text { March } \\
\text { April }\end{array}$ & $\begin{array}{l}34,931 \\
43,421 \\
35,185 \\
66,160\end{array}$ & $\begin{array}{l}\text { May } \\
\text { June } \\
\text { July } \\
\text { August }\end{array}$ & $\begin{array}{r}93,265 \\
139,407 \\
65,865 \\
110,326\end{array}$ & $\begin{array}{l}\text { September } \\
\text { October } \\
\text { November } \\
\text { December.... }\end{array}$ & $\begin{array}{l}94,126 \\
49,760 \\
54,201 \\
52,508\end{array}$ \\
\hline
\end{tabular}

Storage: Ground storage reservoir, 50,000 gallons; elevated $\operatorname{tank}, \mathbf{7 5 , 0 0 0}$ gallons.

Number of customers: 310 .

Treatment: None.

\section{Analysis}

[Composite sample of wells 1, 2, and 3. Collected Dec. 18, 1946. pH is 7.4. Analyzed by C. B. Cibulka]

\begin{tabular}{|c|c|c|c|c|c|}
\hline & $\begin{array}{c}\text { Parts per } \\
\text { million }\end{array}$ & $\begin{array}{l}\text { Equivalents } \\
\text { per million }\end{array}$ & & $\begin{array}{c}\text { Parts per } \\
\text { million }\end{array}$ & $\begin{array}{l}\text { Equivalents } \\
\text { per million }\end{array}$ \\
\hline $\begin{array}{l}\text { Silica }\left(\mathrm{SiO}_{2}\right) \\
\text { Iron }(\mathrm{Fe}) \\
\text { Calcium }(\mathrm{Ca}) \\
\text { Magnesium }(\mathrm{Mg}) \\
\text { Sodium }(\mathrm{Na}) \\
\text { Potassium }(\mathrm{K}) \\
\text { Bicarbonate }\left(\mathrm{HCO}_{z}\right)^{-}\end{array}$ & $\begin{array}{r}36 \\
128 \\
65 \\
178 \\
13 \\
292\end{array}$ & $\begin{array}{r}\mathbf{6} . \mathbf{3 9} \\
\mathbf{5} \cdot \mathbf{3 5} \\
\mathbf{7 . 7 3} \\
. \mathbf{3 3} \\
4.79\end{array}$ & $\begin{array}{l}\text { Sulfate }\left(\mathrm{SO}_{4}\right) \\
\text { Chloride }(\mathrm{Cl}) \\
\text { Fluoride (F) } \\
\left.\text { Nitrate (NO})_{3}\right) \\
\text { Dissolved solids. } \\
\text { Total hardness as } \mathrm{CaCO}_{3}\end{array}$ & \begin{tabular}{|c|}
426 \\
208 \\
3.2 \\
6.4 \\
1,210 \\
587
\end{tabular} & 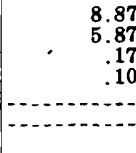 \\
\hline
\end{tabular}

\section{Driller's log, well 1}

\begin{tabular}{|c|c|c|c|c|c|}
\hline & $\begin{array}{c}\text { Thickness } \\
\text { (feet) }\end{array}$ & $\underset{\text { (feet) }}{\text { Depth }}$ & , & $\begin{array}{c}\text { Thickress } \\
\text { (feet) }\end{array}$ & $\begin{array}{c}\text { Depth } \\
\text { (feet) }\end{array}$ \\
\hline $\begin{array}{l}\text { Top soil. } \\
\text { Caliche } \\
\text { Boulders } \\
\text { Dry sand } \\
\text { Boulders } \\
\text { Dry sand }\end{array}$ & $\begin{array}{r}4 \\
20 \\
5 \\
7 \\
5 \\
5\end{array}$ & $\begin{array}{l}4 \\
24 \\
29 \\
36 \\
41 \\
46\end{array}$ & $\begin{array}{l}\text { Boulders } \\
\text { Dry red sand } \\
\text { Quicksand, water } \\
\text { Red clay } \\
\text { Water sand and gravel. } \\
\text { Lime rock. }\end{array}$ & $\begin{array}{r}10 \\
12 \\
18 \\
5 \\
21 \\
30\end{array}$ & $\begin{array}{r}56 \\
68 \\
86 \\
91 \\
112 \\
142\end{array}$ \\
\hline
\end{tabular}

\section{MIDLAND COUNTY}

\section{MIDLAND}

Population in 1940: 9,352 .

Source of information: A. B. Cole, water superintendent, Dec. 18, 1946.

Ownership: Municipal. 
Source of supply: Fourteen wells in two well fields.

Rosedale well 1. Two and three-quarter miles east of city hall; drilled in 1940 by Chas. Skeen; depth, 137 feet; diameter, 16 inches; deep-well turbine pump and 15-horsepower electric motor; static water level, 35 feet below land surface; yield, 130 gallons a minute when drilled.

Rosedale well 2. About 23/4 miles east of city hall; drilled in January 1940 by T. Hines; depth, 127 feet; diameter, 16 inches; deep-well turbine pump and 20-horsepower electric motor; static water level, 35 feet below land surface; yield, 160 gallons a minute when drilled.

Rosedale well 3. About $2 \% / 4$ miles east of city hall; drilled in June 1940 by T. Hines; depth, 109 feet; diameter, 16 inches; deep-well turbine pump and 20-horsepower electric motor; yield, 190 gallons a minute when drilled.

Rosedale well 4. About $23 / 4$ miles east of city hall; drilled in June 1940 by Chas. Skeen; depth, 127 feet; diameter, 16 inches; deep-well turbine pump and 15-horsepower electric motor; yield, 295 gallons a minute when drilled.

Rosedale well 7. Two and three-quarter miles east of city hall; drilled in 1942 by Chas. Skeen; depth, 130 feet; diameter, 16 inches; deep-well turbine pump and 15-horsepower electric motor; yield, 225 gallons a minute.

Rosedale well 8. Two and three-quarter miles east of city hall; drilled in May 1941 by Chas. Skeen; depth, 147 feet; diameter, 16 inches; deepwell turbine pump and 15-horsepower electric motor; yield, 225 gallons a minute.

Cloverdale well 1. Four miles east of city hall; drilled in 1927 by LayneTexas Co.; depth, 110 feet; diameter, 20 inches; deep-well turbine pump and 20-horsepower electric motor; static water level, 35 feet below land surface; yield, 500 gallons a minute with drawdown of 15 feet.

Cloverdale well 3. Four miles east of city hall; drilled is 1934 by Watson and Hines; depth, 115 feet; diameter, 151/2 inches; deep-well turbine pump and 10-horsepower electric motor; yield, 225 gallons a minute.

Cloverdale well 4. Four miles east of city hall; drilled ir 1935 by Watson and Hines; depth, 125 feet; diameter, 151/2 inches; deep-well turbine pump and 20-horsepower electric motor; yield, 400 gallons a minute with drawdown of 25 feet.

Cloverdale well 5. Four miles east of city hall; drilled ir 1935 by Watson and Hines; depth, 128 feet; diameter, 16 inches; deep-well turbine pump and 10-horsepower electric motor; yield, 200 rallons a minute.

Cloverdale well 6. Four miles east of city hall; drilled ir 1936 by Watson and Hines; depth, 128 feet; diameter, 17 inches; deep-well turbine pump and 20-horsepower electric motor; yield, 400 gallons a minute with drawdown of 37 feet.

Cloverdale well 7. Four miles east of city hall; drilled ir 1937 by Watson and Hines; depth, 115 feet; diameter, 16 inches; deep-well turbine pump and 15-horsepower electric motor; yield, 300 gallons a minute with drawdown of $\mathbf{5 6}$ feet.

Cloverdale well 8. Four miles east of city hall; drilled ir 1940 by Watson and Hines; depth, 135 feet; diameter, 16 inches; deep-rrell turbine pump and 15-horsepower electric motor; yield, 300 gallons a minute. 
Cloverdale well 9. Four miles east of city hall; drilled in 1946 by $\mathrm{T}$. Hines; depth, 130 feet; diameter, 16 inches; deep-well turbine pump and 15-horsepower electric motor; yield, 300 gallons a minute.

Pumpage (estimated): Average, 2,500,000 gallons a day.

Storage: Ground storage reservoir, 1,000,000 gallons; ground storage reservoir, 500,000 gallons; ground storage reservoir, 300,000 gallons; elevated tank, 2,225,000 gallons; elevated tank, 300,000 gallons; elevated tank, 100,000 gallons.

Number of customers: 3,000 .

Treatment: Chlorination.

\section{Analyses}

[Collected Dec. 18, 1946. pH, each well, is 7.4. Analyzed by C. B. Cibulka]

\begin{tabular}{|c|c|c|c|c|}
\hline \multirow{2}{*}{ • } & \multicolumn{2}{|c|}{$\begin{array}{l}\text { Rosedale well field } \\
\text { (Composite sample } \\
\text { from } 6 \text { wells) }\end{array}$} & \multicolumn{2}{|c|}{$\begin{array}{l}\text { Cloverdale well field } \\
\text { (Composite sample } \\
\text { from } 8 \text { wells) }\end{array}$} \\
\hline & $\begin{array}{l}\text { Parts per } \\
\text { million }\end{array}$ & $\begin{array}{l}\text { Equivalents } \\
\text { per million }\end{array}$ & $\begin{array}{l}\text { Parts per } \\
\text { million }\end{array}$ & $\begin{array}{l}\text { Equivalents } \\
\text { per million }\end{array}$ \\
\hline 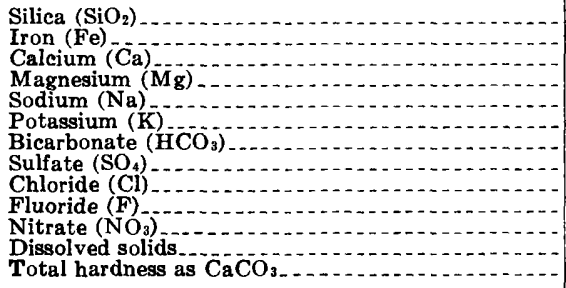 & $\begin{array}{r}53 \\
153 \\
94 \\
219 \\
14 \\
244 \\
540 \\
344 \\
1.8 \\
15 \\
1,550 \\
768\end{array}$ & $\begin{array}{r}7.64 \\
7.73 \\
9.54 \\
.36 \\
4.00 \\
11.24 \\
9.70 \\
.09 \\
.24 \\
-9 .-\end{array}$ & $\begin{array}{l}53 \\
154 \\
95 \\
217 \\
15 \\
244 \\
541 \\
342 \\
3.27 \\
14 \\
1,550 \\
775\end{array}$ & $\begin{array}{r}7.69 \\
7.81 \\
9.43 \\
.38 \\
4.00 \\
11.26 \\
9.65 \\
.17 \\
.23 \\
\hdashline\end{array}$ \\
\hline
\end{tabular}

Drillers' logs

\begin{tabular}{l|r|r||r|r|r}
\hline & $\begin{array}{c}\text { Thickness } \\
\text { (feet) }\end{array}$ & $\begin{array}{l}\text { Depth } \\
\text { (feet) }\end{array}$ & $\begin{array}{c}\text { Thickness } \\
\text { (feet) }\end{array}$ & $\begin{array}{c}\text { Depth } \\
\text { (feet) }\end{array}$ \\
\hline
\end{tabular}

Rosedale well 2

\begin{tabular}{|c|c|c|c|c|c|}
\hline $\begin{array}{l}\text { Top soil } \\
\text { Caliche. } \\
\text { Sand and caliche } \\
\text { Sand and gravel. } \\
\text { Water sand } \\
\text { Sand and gravel. }\end{array}$ & $\begin{array}{l}10 \\
15 \\
10 \\
12 \\
18 \\
15\end{array}$ & $\begin{array}{l}10 \\
25 \\
35 \\
47 \\
65 \\
80\end{array}$ & $\begin{array}{l}\text { Yellow sand } \\
\text { Hard gravel } \\
\text { Sand } \\
\text { Brown lime. } \\
\text { Red beds. }\end{array}$ & $\begin{array}{r}\mathbf{5} \\
30 \\
5 \\
5 \\
2\end{array}$ & $\begin{array}{r}85 \\
115 \\
120 \\
125 \\
127\end{array}$ \\
\hline
\end{tabular}

Rosedale well 3

\begin{tabular}{|c|c|c|c|c|c|}
\hline $\begin{array}{l}\text { Top soil } \\
\text { Caliehe } \\
\text { Water sand } \\
\text { Lime } \\
\text { Hard shells }\end{array}$ & $\begin{array}{r}3 \\
42 \\
5 \\
10 \\
10\end{array}$ & $\begin{array}{r}3 \\
45 \\
50 \\
60 \\
70\end{array}$ & $\begin{array}{l}\text { Sand } \\
\text { Rock } \\
\text { Sand and gravel } \\
\text { Red beds. }\end{array}$ & $\begin{array}{r}6 \\
4 \\
23 \\
6\end{array}$ & $\begin{array}{r}70 \\
30 \\
168 \\
109\end{array}$ \\
\hline
\end{tabular}




\begin{tabular}{|c|c|c|c|c|}
\hline & $\begin{array}{c}\text { Thickness } \\
\text { (feet) }\end{array}$ & $\begin{array}{l}\text { Depth } \\
\text { (feet) }\end{array}$ & $\begin{array}{c}\text { Thickness } \\
\text { (feet) }\end{array}$ & $\begin{array}{l}\text { Depth } \\
\text { (feet) }\end{array}$ \\
\hline
\end{tabular}

\begin{tabular}{|c|c|c|c|c|c|}
\hline \multicolumn{6}{|c|}{ Rosedale well 4} \\
\hline $\begin{array}{l}\text { Top soil } \\
\text { Caliche } \\
\text { Water sand } \\
\text { Sand and gravel }\end{array}$ & $\begin{array}{r}4 \\
39 \\
9 \\
23\end{array}$ & $\begin{array}{r}4 \\
43 \\
52 \\
75\end{array}$ & $\begin{array}{l}\text { Sand and blue shale } \\
\text { Gravel } \\
\text { Red beds. }\end{array}$ & $\begin{array}{r}\mathbf{2 5} \\
\mathbf{2 5} \\
\mathbf{2}\end{array}$ & $\begin{array}{l}100 \\
125 \\
127\end{array}$ \\
\hline
\end{tabular}

\section{Rosedale well 8}

Top soil.

Gray clay

Caliche and clay

Chalk rock and caliche, very white.

Caliche (hard rock at 27 feet)

Hard rock

Gray clay (i foot of hard rock)

Sand and gravel (1 foot of hard rock; tested 40 gallons a minute).

Sand and gravel, little clay

Gravel (1 foot of hard rock)

Packed sand and clay.

\begin{tabular}{|c|c|c|}
\hline $\begin{array}{r}5 \\
\mathbf{5} \\
\mathbf{5} \\
10\end{array}$ & $\begin{array}{r}5 \\
10 \\
15 \\
25\end{array}$ & $\begin{array}{l}\text { Gravel, water, some sand.-- } \\
\text { Water sand ( } 1 \text { foot of clay) -- } \\
\text { Water sand and gravel. } \\
\text { Gray clay (some red clay)..- }\end{array}$ \\
\hline $\begin{array}{l}\mathbf{5} \\
\mathbf{5} \\
\mathbf{5}\end{array}$ & $\begin{array}{l}\mathbf{3 0} \\
\mathbf{3 5} \\
\mathbf{4 0}\end{array}$ & $\begin{array}{l}\text { Clay and gravel. } \\
\text { Shelly sandrock. } \\
\text { Sandrock and clay } \\
\text { Hard gravel and clay. }\end{array}$ \\
\hline 5 & 45 & $\begin{array}{l}\text { Hard sandrock } \\
\text { Water sand } \\
\text { Gravel, sand, and some clay. }\end{array}$ \\
\hline & $\begin{array}{l}\mathbf{5 0} \\
\mathbf{5 5} \\
\mathbf{6 0}\end{array}$ & $\begin{array}{l}\text { Sand and clay } \\
\text { Red beds }\end{array}$ \\
\hline
\end{tabular}

\section{Cloverdale well 3}

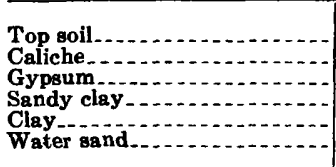

\begin{tabular}{r|r||r|}
10 & 10 \\
10 & 20 \\
10 & 30 \\
15 & 45 & \\
5 & 50 & \\
5 & 55 &
\end{tabular}

Gypsum
Ward limer sand and gravel
Red rock.
Water sand and gravel.

\begin{tabular}{r|r}
10 & 65 \\
6 & 71 \\
30 & 101 \\
2 & 103 \\
12 & 115 \\
15 & 130 \\
\hline
\end{tabular}

\section{Cloverdale well 4}

\begin{tabular}{|c|c|c|c|c|c|}
\hline $\begin{array}{l}\text { Caliche } \\
\text { Lime. } \\
\text { Water gand. } \\
\text { Hard lime... } \\
\text { Gravel } \\
\text { Red rock. } \\
\text { Gravel }\end{array}$ & $\begin{array}{r}35 \\
11 \\
2 \\
\mathbf{1} \\
12 \\
13 \\
5 \\
7\end{array}$ & $\begin{array}{l}35 \\
46 \\
48 \\
60 \\
73 \\
78 \\
\mathbf{8 5}\end{array}$ & $\begin{array}{l}\text { Blue shale } \\
\text { Lime } \\
\text { Red rock } \\
\text { Hard gravel } \\
\text { Gravel. } \\
\text { Clay }\end{array}$ & $\begin{array}{r}8 \\
1 \\
1 \\
12 \\
\mathbf{5} \\
13\end{array}$ & $\begin{array}{r}93 \\
94 \\
95 \\
107 \\
112 \\
125\end{array}$ \\
\hline
\end{tabular}

\section{Cloverdale well 5}

\begin{tabular}{|c|c|c|c|c|c|}
\hline $\begin{array}{l}\text { Caliche. } \\
\text { Lime } \\
\text { Sand. } \\
\text { Lime } \\
\text { Gravel } \\
\text { Red rock } \\
\text { Gravel }\end{array}$ & $\begin{array}{r}35 \\
11 \\
4 \\
10 \\
13 \\
5 \\
7\end{array}$ & $\begin{array}{l}\mathbf{3 5} \\
\mathbf{4 6} \\
\mathbf{5 0} \\
\mathbf{6 0} \\
\mathbf{7 3} \\
\mathbf{7 8} \\
\mathbf{8 5}\end{array}$ & $\begin{array}{l}\text { Blue shale } \\
\text { Red rock. } \\
\text { Hard gravel } \\
\text { Gravel and shale } \\
\text { Gravel } \\
\text { Clay }\end{array}$ & $\begin{array}{r}8 \\
2 \\
10 \\
2 \\
5 \\
16\end{array}$ & $\begin{array}{r}93 \\
95 \\
105 \\
107 \\
112 \\
128\end{array}$ \\
\hline
\end{tabular}

\section{Cloverdale well 6}

\begin{tabular}{|c|c|c|c|c|c|}
\hline $\begin{array}{l}\text { Caliche } \\
\text { Sand and clay } \\
\text { Water sand. }\end{array}$ & $\begin{array}{l}\mathbf{2 5} \\
\mathbf{2 5} \\
\mathbf{1 0}\end{array}$ & $\begin{array}{l}\mathbf{2 5} \\
\mathbf{5 0} \\
\mathbf{6 0}\end{array}$ & $\begin{array}{l}\text { Lime and gand. } \\
\text { Sand and gravel } \\
\text { Blue clay }\end{array}$ & $\begin{array}{r}14 \\
50 \\
4\end{array}$ & $\begin{array}{r}74 \\
124 \\
128\end{array}$ \\
\hline
\end{tabular}

\section{Cloverdale well 8}

\begin{tabular}{|c|c|c|c|c|c|}
\hline $\begin{array}{l}\text { Top soil } \\
\text { Caliche. } \\
\text { Hard sand. } \\
\text { Hard lime } \\
\text { Limene } \\
\text { Shale }\end{array}$ & $\begin{array}{r}8 \\
17 \\
5 \\
8 \\
18 \\
4\end{array}$ & $\begin{array}{r}8 \\
25 \\
30 \\
\mathbf{3 8} \\
\mathbf{5 6} \\
\mathbf{6 0}\end{array}$ & $\begin{array}{l}\text { Water sand } \\
\text { Shale } \\
\text { Hard shell } \\
\text { Sand and gravel } \\
\text { Red beds...... }\end{array}$ & $\begin{array}{r}20 \\
5 \\
2 \\
42 \\
6\end{array}$ & $\begin{array}{r}80 \\
85 \\
87 \\
129 \\
135\end{array}$ \\
\hline
\end{tabular}




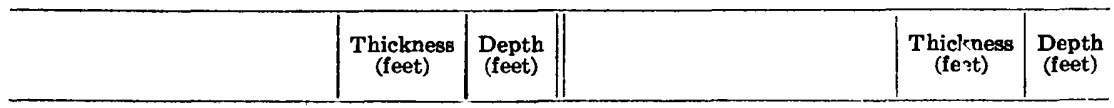

Cloverdale well 9

\begin{tabular}{|c|c|c|c|c|c|}
\hline $\begin{array}{l}\text { Sandy soil } \\
\text { Mixed clay and gypsum } \\
\text { Rock. } \\
\text { Clay } \\
\text { Dry packed sand } \\
\text { Mixed sand and clay } \\
\text { Rock }\end{array}$ & $\begin{array}{r}10 \\
\mathbf{2 7} \\
\mathbf{8} \\
\mathbf{3} \\
\mathbf{2} \\
\mathbf{5} \\
\mathbf{2}\end{array}$ & $\begin{array}{l}10 \\
37 \\
45 \\
48 \\
50 \\
55 \\
57\end{array}$ & $\begin{array}{l}\text { Mixed sand and clay, water. } \\
\text { Mixed fine sand and clay } \\
\text { Yellow clay } \\
\text { Mixed clay and sand } \\
\text { Yellow clay } \\
\text { Red beds. }\end{array}$ & $\begin{array}{r}6 \\
22 \\
17 \\
13 \\
14 \\
1\end{array}$ & $\begin{array}{r}68 \\
85 \\
102 \\
115 \\
129 \\
130\end{array}$ \\
\hline
\end{tabular}

\section{MITCHELL COUNTY}

\section{COLORADO CITY}

Population in $1940: 5,213$.

Source of information: H. F. McCorcle, pump supervisor, May 29, 1946.

Ownership: Municipal.

Scource of supply: Sixteen wells.

Well 1. About 2,800 feet northeast of 17th and Chestnut Streets; drilled in 1927 by W. T. Vedder; depth, 223 feet; diameter, 15 inches; deepwell turbine pump and 10-horsepower electric motor; static vrater level, 124.8 feet below land surface Mar. 7, 1946; yield, 60 gallons a minute with a pumping level of 156 feet below land surface Mar. 19, 1946.

Well 2. About 1,150 feet west of well 1; drilled in 1921 by W. T. Vedder; depth, 220 feet; diameter, 15 inches; deep-well turbine pump and 20 horsepower electric motor; static water level, 133.5 feet below land surface Mar. 5, 1946; yield, 75 gallons a minute with a pumping level of 162 feet below land surface Mar. 6, 1946.

Well 3. About 2,050 feet south of well 2; drilled; depth, 22: feet; diameter, 8 inches; deep-well cylinder pump and 10-horsepower electric motor; static water level, 116.4 feet below land surface Mar. 5, 1946; yield, 23 gallons a minute.

Well 4. About 800 feet south of well 3 ; drilled in 1925 by Claude Bell; depth, 220 feet; deep-well turbine pump and 10-horsepower electric motor; static water level, 119.6 feet below land surface Mar. 5, 1946; yield, 21 gallons a minute with pumping level of 176 feet below land surface Mar. 9, 1946.

Well 5. About 500 feet south of well 4; drilled; depth, about 220 feet; deep-well turbine pump and 10-horsepower electric motor; static water level, 110.3 feet below land surface Mar. 7, 1946; yield, $7 \mathrm{E}$ gallons a minute with pumping level of 150 feet below land surface Mareh 1946 .

Well 6. About 425 feet northwest of well 5 ; drilled; depth, about 220 feet; deep-well cylinder pump and electric motor; static water level, 117.8 feet below land surface Mar. 5, 1946; yield, 22 gallons a minute with pumping level of 130.5 feet below land surface March 1946.

Well 7. About 400 feet northwest of well 6; drilled; depth, about 220 feet; deep-well cylinder pump and 10-horsepower electric motor; yield, 32 gallons a minute.

Well 8. About 400 feet north of well 7; drilled; depth, about 220 feet; deep-well cylinder pump and 10-horsepower electric motor; static water level, 110.9 feet below land surface Mar. 5, 1946; yield $1 \varepsilon$ gallons a minute with pumping level of 196.8 feet below land surface March 1946. 
Well 9. About 400 feet north of well 7; drilled; depth, atout 220 feet; deep-well cylinder pump and 10-horsepower electric motor; static water level, 112.9 feet below land surface Mar. 5, 1946; yield, 30 gallons a minute with pumping level of 200 feet below land surfac? March 1946.

Well 10. About 800 feet southeast of well 9 ; drilled in 1920 ; depth, 240 feet; diameter, 15 inches; deep-well turbine pump and 15-horsepower electric motor; static water level, 122 feet below land surface Mar. 6, 1946; yield, 75 gallons a minute with pumping level of $\mathbf{1 5 2}$ feet below land surface.

Well 11. About 2,750 feet northwest of well 10; drilled in 1936; depth, 233 feet; deep-well turbine pump and electric motor; static water level, 137.4 feet below land surface Mar. 7, 1946; yield, 90 gal'ons a minute with pumping level of 162 feet below land surface.

Well 12. About 1,550 feet northeast of well 11; drilled in 1944 by Olin House; depth, 255 feet; diameter, 10 inches; deep-well turbine pump and 10-horsepower electric motor; static water level, 143.3 feet below land surface Mar. 5, 1945; yield, 95 gallons a minute with pumping level of 192.5 feet below land surface March 1946 .

Well 13. About 1,150 feet west of well 12; drilled in 1944 by Olin House; depth, 249 feet; diameter, 10 inches; deep-well turbine pump and 10horsepower electric motor; static water level, 128.2 feet below land surface Mar. 5, 1946; yield, 55 gallons a minute with pumoing level of 183 feet below land surface March 1946.

Well 14. About 1,250 feet south of well 13; drilled in 1944 by Olin House; depth, 238 feet; diameter, 10 inches; deep-well turbine pump and 10horsepower electric motor; static water level, 127.9 fe,t below land surface Mar. 5, 1946; yield, 35 gallons a minute with pumping level of 171 feet below land surface March 1946.

Well 16. In City Park about 950 feet southwest of State Highway 18 and Texas \& Pacific Railroad; drilled in 1922; depth, 256 feet; deepwell cylinder pump and 5-horsepower electric motor; stat; water level, 77.2 feet below land surface Mar. 8, 1946; yield, 25 gallons a minute.

Well 17. About 500 feet east of well 16; drilled in 1922; denth, 256 feet; diameter, 8 inches; deep-well cylinder pump and 5-horsenower electric motor; static water level, 84.2 feet below land surface Mar. 8, 1946; yield, 20 gallons a minute.

Average pumpage, in gallons a day

\begin{tabular}{|c|c|c|c|c|c|}
\hline $\begin{array}{l}\text { January } \\
\text { February } \\
\text { March } \\
\text { April }\end{array}$ & $\begin{array}{l}171,700 \\
188,800 \\
170,300 \\
205,800\end{array}$ & $\begin{array}{l}\text { May } \\
\text { June } \\
\text { July } \\
\text { August.... }\end{array}$ & $\begin{array}{l}262,300 \\
276,200 \\
281,900 \\
433,600\end{array}$ & $\begin{array}{l}\text { September. } \\
\text { October } \\
\text { November } \\
\text { December }\end{array}$ & $\begin{array}{l}278,800 \\
244,200 \\
230,000 \\
156,700\end{array}$ \\
\hline
\end{tabular}

Storage: Four ground storage reservoirs: 1,000,000 gallons, 169,000 gallons, 125,000 gallons, and 125,000 gallons, respectively; elevated tank, 100,000 gallons; standpipe, 70,000 gallons.

Number of customers: 1,500 .

Treatment: Chlorination. 


\section{Analysis}

[Composite sample of 14 wells. Collected May 29, 1946. pH is 7.9. Analyzed by C. B. Cibulka]

\begin{tabular}{|c|c|c|c|c|c|}
\hline - & $\begin{array}{c}\text { Parts per } \\
\text { million }\end{array}$ & $\begin{array}{l}\text { Equivalents } \\
\text { per million }\end{array}$ & & $\begin{array}{l}\text { Parts per } \\
\text { mill on }\end{array}$ & $\begin{array}{l}\text { Equivalents } \\
\text { per million }\end{array}$ \\
\hline $\begin{array}{l}\text { Silica }\left(\mathrm{SiO}_{2}\right) \\
\text { Iron }(\mathrm{Fe}) \\
\text { Calcium }(\mathrm{Ca}) \\
\text { Magnesium }(\mathrm{Mg}) \\
\text { Sodium }(\mathrm{Na}) \\
\text { Potassium }(\mathrm{K}) \\
\text { Bicarbonate }\left(\mathrm{H} \mathrm{CO}_{3}\right)^{-}\end{array}$ & $\begin{array}{l}16 \\
94 \\
49 \\
90 \\
26 \\
336\end{array}$ & $\begin{array}{r}4.69 \\
4.08 \\
3.91 \\
.67 \\
5.51\end{array}$ & $\begin{array}{l}\text { Sulfate }\left(\mathrm{SO}_{4}\right) \\
\text { Chloride (CI) } \\
\text { Fluoride (F) } \\
\text { Nitrate (NO } \\
\text { Dissolved solids } \\
\text { Total hardness as } \mathrm{CaCO}_{3}\end{array}$ & $\begin{array}{l}29 ? \\
54 \\
1.4 \\
83 ? \\
43 ?\end{array}$ & $\begin{array}{r}6.20 \\
1.52 \\
.07 \\
.00 \\
-0 .- \\
\end{array}$ \\
\hline
\end{tabular}

Drillers' logs

\begin{tabular}{|c|c|c|c|c|c|}
\hline & $\underset{\text { (feet) }}{\text { Thickness }}$ & $\underset{\text { (feet) }}{\text { Depth }}$ & & $\begin{array}{c}\text { Thickness } \\
\text { (feet) }\end{array}$ & $\begin{array}{l}\text { Depth } \\
\text { (feet) }\end{array}$ \\
\hline \multicolumn{6}{|c|}{ Well 12} \\
\hline $\begin{array}{l}\text { Surface sand } \\
\text { Sandy clay } \\
\text { Sand and gravel. } \\
\text { Red clay } \\
\text { Sandrock } \\
\text { Blue clay } \\
\text { Sandrock }\end{array}$ & $\begin{array}{l}10 \\
10 \\
20 \\
30 \\
10 \\
25 \\
40\end{array}$ & $\begin{array}{r}10 \\
20 \\
40 \\
70 \\
80 \\
105 \\
145\end{array}$ & $\begin{array}{l}\text { Water sand } \\
\text { Blue clay } \\
\text { Soft rock } \\
\text { Hard rock. } \\
\text { Water-bearing sand } \\
\text { Red beds... }\end{array}$ & $\begin{array}{r}20 \\
17 \\
2 \\
3 \\
68.5 \\
.5\end{array}$ & $\begin{array}{l}165 \\
182 \\
184 \\
187 \\
255 \\
256\end{array}$ \\
\hline \multicolumn{6}{|c|}{ Well 13} \\
\hline $\begin{array}{l}\text { Surface sand } \\
\text { Red clay } \\
\text { Gravel } \\
\text { Sandrock } \\
\text { Red clay. } \\
\text { Blue clay }\end{array}$ & $\begin{array}{r}5 \\
10 \\
5 \\
10 \\
40 \\
70\end{array}$ & $\begin{array}{r}5 \\
15 \\
20 \\
30 \\
70 \\
140\end{array}$ & $\begin{array}{l}\text { Water sand } \\
\text { Blue clay. } \\
\text { Rock } \\
\text { Water sand } \\
\text { Red beds.... }\end{array}$ & $\begin{array}{r}20 \\
10 \\
3 \\
76 \\
-2-1\end{array}$ & $\begin{array}{l}160 \\
170 \\
173 \\
249 \\
249\end{array}$ \\
\hline \multicolumn{6}{|c|}{ Well 14} \\
\hline $\begin{array}{l}\text { Top sand. } \\
\text { Sandstone. } \\
\text { Red clay } \\
\text { Sandstone. } \\
\text { Red clay }\end{array}$ & $\begin{array}{l}10 \\
20 \\
40 \\
15 \\
25\end{array}$ & $\begin{array}{r}10 \\
30 \\
70 \\
85 \\
110\end{array}$ & $\begin{array}{l}\text { Blue clay } \\
\text { Water sand } \\
\text { Blue clay } \\
\text { Sandstone and conglomerate }\end{array}$ & $\begin{array}{r}28 \\
7 \\
23 \\
70\end{array}$ & $\begin{array}{l}138 \\
145 \\
168 \\
238\end{array}$ \\
\hline
\end{tabular}

\section{MOORE COUNTY}

\section{DUMAS}

Population in 1940: 2,117 (estimated 5,000 in 1947).

Source of information: J. W. Mills, city clerk, Nov. 21, 1947.

Ownership: Municipal.

Source of supply: Four wells.

Well 1. Driłled in 1931 by Leo McDade; depth, 565 feet; d:ameter, 151/2 inches; deep-well turbine pump and 25-horsepower electric motor; static water level, 260 feet below land surface.

Well 2. Drilled in 1931; depth, 576 feet; diameter, 151/2 inck es; deep-well turbine pump and 50-horsepower electric motor.

Well 3. Drilled in 1944 by H. H. Heiskell; depth, 600 feet; diameter, $151 / 2$ inches; deep-well turbine pump and 50-horsepower electric motor; yield, 400 gallons a minute. 
Well 4. Drilled in 1946; depth, 360 feet; diameter, $15 \frac{1}{2}$ inches; deepwell turbine pump and 50-horsepower electric motor; yield, 200 gallons a minute.

Pumpage: Maximum 894,000 gallons a day; minimum, 235,000 gallons a day. Storage: Ground storage reservoir, 200,000 gallons; elevated tank, 50,000 gallons.

Treatment: Chlorination.

\section{Analysis, well 2}

[Collected Mar. 17, 1948. pH is 7.1. Analyzed by D. E. Weaver]

\begin{tabular}{|c|c|c|c|c|c|}
\hline & $\begin{array}{l}\text { Parts per } \\
\text { million }\end{array}$ & $\begin{array}{l}\text { Equivalents } \\
\text { per million }\end{array}$ & & $\begin{array}{l}\text { Parts pe } \\
\text { million }\end{array}$ & $\begin{array}{l}\text { Equivalents } \\
\text { per million }\end{array}$ \\
\hline $\begin{array}{l}\text { Silica }\left(\mathrm{SiO}_{2}\right) \\
\text { Iron }(\mathrm{Fe}) \\
\text { Calcium }(\mathrm{Ca}) \\
\text { Magnesium }(\mathrm{Mg}) \\
\text { Sodium }(\mathrm{Na}) \\
\text { Potassium }(\mathrm{K}) \\
\text { Bicarbonate }\left(\mathrm{HC} \mathrm{OO}_{8}\right)^{-}\end{array}$ & $\begin{array}{l}16 \\
43 \\
19 \\
95 \\
10 \\
339\end{array}$ & $\begin{array}{r}\mathbf{2} .15 \\
1.56 \\
4.13 \\
.26 \\
5.56\end{array}$ & $\begin{array}{l}\text { Sulfate }\left(\mathrm{SO}_{4}\right) \\
\text { Chloride }(\mathrm{Cl}) \\
\text { Fluoride (F) } \\
\left.\text { Nitrate (NO })_{3}\right) \\
\text { Dissolved solids } \\
\text { Total hardness as } \mathrm{CaCO}_{3}\end{array}$ & $\begin{array}{l}98 \\
11 \\
1.8 \\
496 \\
186\end{array}$ & $\begin{array}{r}2.04 \\
.31 \\
.09 \\
.00 \\
-0 .- \\
\end{array}$ \\
\hline
\end{tabular}

\section{MOTLEY COUNTY}

\section{MATADOR}

Population in 1940: 1,376 .

Source of information: H.O. Stanfield, water superintendent, Mar. 7, 1945.

Ownership: Municipal.

Source of supply: Three wells.

Well 1. Drilled in 1928 by Air Made Well Co.; depth, 277 feet; diameter, 28 to $65 \%$ inches; deep-well turbine pump and 71/2-horsepower electric motor; static water level, 72 feet below land surface August 1944; yield, 100 gallons a minute.

Well 3. About 1,250 feet north of well 1; drilled in 1939; denth, 293 feet; diameter, 12 inches; deep-well turbine pump and 71/2-horsepower electric motor; static water level, 85 feet below land surface March 1945; yield, 135 gallons a minute with drawdown of 35 feet; temperature, $641 / 2^{\circ} \mathrm{F}$.

Well 5. Drilled in 1946 by G. G. Sawtelle; depth, 145 feet; dinmeter, 121/2 to $6 \%$ inches; deep-well turbine pump and $7 \frac{1}{2}$-horsepnwer electric motor; static water level, 77.7 feet below land surface Sopt. 16, 1947; yield, 100 gallons a minute.

Average pumpage, in gallons a day

\begin{tabular}{|c|c|c|c|c|}
\hline & 1944 & 1945 & I 946 & 1947 \\
\hline 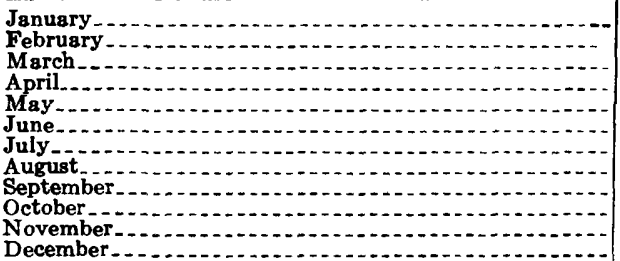 & $\begin{array}{r}62,800 \\
56,000 \\
56,600 \\
66,900 \\
102,500 \\
148,500 \\
140,700 \\
78,500 \\
68,700 \\
61,000 \\
52,700\end{array}$ & 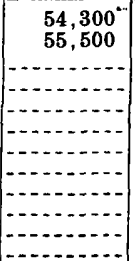 & $\begin{array}{r}75,000 \\
5,600 \\
\Upsilon 5,700 \\
129,000 \\
113,200 \\
192,000 \\
179,600 \\
181,000 \\
87,300 \\
73,000 \\
77,700 \\
76,200\end{array}$ & \begin{tabular}{r}
79,600 \\
94,500 \\
76,700 \\
96,700 \\
95,000 \\
187,200 \\
226,000 \\
234,200 \\
\hdashline \\
\hdashline \\
-0
\end{tabular} \\
\hline
\end{tabular}


Storage: Ground reservoir, 100,000 gallons; elevated tank, 50,000 gallons. Number of customers: 340 .

Treatment: None.

\section{Analyses}

[Wells 1 and 3, collected Mar. 7, 1945, pH: well 1, 7.0; well 3, 7.2. Analyzed by J. H. Rowley. Well 5, collected Sept. 16, 1947. $\mathrm{pH}$ is 7.8. Analyzed by B. C. Dwyer]

\begin{tabular}{|c|c|c|c|c|c|c|}
\hline \multirow{2}{*}{$f$} & \multicolumn{2}{|c|}{ Well 1} & \multicolumn{2}{|c|}{ Well 3} & \multicolumn{2}{|c|}{ Well 5} \\
\hline & $\begin{array}{l}\text { Parts per } \\
\text { million }\end{array}$ & $\begin{array}{l}\text { Equivalents } \\
\text { per million }\end{array}$ & $\begin{array}{l}\text { Parts per } \\
\text { million }\end{array}$ & $\begin{array}{l}\text { Equivalents } \\
\text { per million }\end{array}$ & $\begin{array}{l}\text { Parts per } \\
\text { million }\end{array}$ & $\begin{array}{l}\text { Equivalents } \\
\text { per million }\end{array}$ \\
\hline 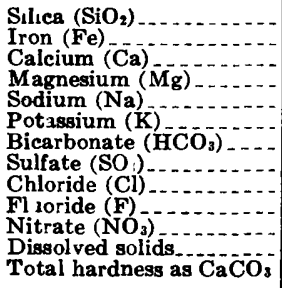 & $\begin{array}{l}25 \\
127 \\
35 \\
142 \\
7.05 \\
354 \\
139 \\
204 \\
70^{.5} \\
925 \\
461\end{array}$ & $\begin{array}{r}6.34 \\
2.88 \\
6.18 \\
.20 \\
5.80 \\
2.89 \\
5.75 \\
.03 \\
1.13 \\
\cdots\end{array}$ & $\begin{array}{l}23 \\
69 \\
20 \\
145 \\
5.3 \\
392 \\
92 \\
102 \\
16 \\
685 \\
254\end{array} .9$ & $\begin{array}{r}3.44 \\
1.64 \\
6.32 \\
.14 \\
6.43 \\
1.92 \\
2.88 \\
.05 \\
.26 \\
\hdashline-.-1\end{array}$ & $\begin{array}{l}20 \\
97 \\
25 \\
126 \\
10 \\
370 \\
110 \\
138 \\
2_{21}^{.06} \\
7_{49}^{345} \\
345\end{array}$ & $\begin{array}{r}4.84 \\
2.06 \\
5.46 \\
.26 \\
6.06 \\
2.29 \\
3.89 \\
.04 \\
.34 \\
\\
\end{array}$ \\
\hline
\end{tabular}

Driller's log, well 1

\begin{tabular}{|c|c|c|c|c|c|}
\hline & $\begin{array}{c}\text { Thickness } \\
\text { (feet) }\end{array}$ & $\begin{array}{l}\text { Depth } \\
\text { (feet) }\end{array}$ & & $\underset{\text { (feet) }}{\text { Thichness }}$ & $\begin{array}{l}\text { Depth } \\
\text { (feet) }\end{array}$ \\
\hline $\begin{array}{l}\text { Sandy clay } \\
\text { Sand } \\
\text { Slay and gravel and clay } \\
\text { Yellow sand } \\
\text { Fine-grained white sand. }\end{array}$ & $\begin{array}{r}4 \\
4 \\
12 \\
40 \\
25 \\
4\end{array}$ & $\begin{array}{r}4 \\
8 \\
20 \\
60 \\
85 \\
89\end{array}$ & $\begin{array}{l}\text { Fine-grained yellow sand } \\
\text { Sand and gravel } \\
\text { Red clay and sand } \\
\text { Sand and gravel } \\
\text { Red shale. }\end{array}$ & $\begin{array}{r}13 \\
3 \\
10 \\
14 \\
148\end{array}$ & $\begin{array}{l}102 \\
105 \\
115 \\
129 \\
277\end{array}$ \\
\hline
\end{tabular}

\section{ROARING SPRINGS}

Population in 1940: 514.

Source of information: J. D. Mitchell, city secretary, Sept. 16, 1947.

Owner: Roaring Springs Townsite Co.

Source of supply: Well on bank of Dutchman's Creek, 1 mile north of Roaring Springs; dug in 1913; depth, 24 feet; diameter 24 to 18 feet; deep-well turbine pump and 10-horsepower electric motor; yield, 250 gallons a minute.

Pumpage (estimated) : 70,000 gallons a day.

Storage: Elevated tank, 50,000 gallons.

Number of customers: 110 .

Treatment: None.

\section{Analysis}

[Collected December 1947. pH is 7.5. Analyzed by B. C. Dwyer]

\begin{tabular}{|c|c|c|c|c|c|}
\hline & $\begin{array}{c}\text { Parts per } \\
\text { million }\end{array}$ & $\begin{array}{l}\text { Equivalents } \\
\text { per million }\end{array}$ & & $\begin{array}{c}\text { Parts per } \\
\text { million }\end{array}$ & $\begin{array}{l}\text { Equivalents } \\
\text { per million }\end{array}$ \\
\hline $\begin{array}{l}\text { Silica }\left(\mathrm{SiO}_{2}\right) \\
\text { Iron }(\mathrm{Fe}) \\
\text { Calcium }(\mathrm{Ca}) \\
\text { Magnesium }(\mathrm{Mg}) \\
\text { Sodium }(\mathrm{Na}) \\
\text { Potassium }(\mathrm{K}) \\
\text { Bicarbonate }(\mathrm{H} \overline{\mathrm{CO}} \mathrm{g})\end{array}$ & $\begin{array}{l}18 \\
80^{31} \\
31 \\
41 \\
522 \\
32.3\end{array}$ & $\begin{array}{r}3.99 \\
2.55 \\
1.80 \\
.14 \\
5.28\end{array}$ & $\begin{array}{l}\text { Sulfate }\left(\mathrm{SO}_{4}\right) \\
\text { Chloride }(\mathrm{Cl}) \\
\text { Fluoride (F) } \\
\text { Nitrate }\left(\mathrm{NO}_{3}\right) \\
\text { Dissolved solids } \\
\text { Total hardness as } \mathrm{CaCO}_{3}\end{array}$ & $\begin{array}{l}67 \\
60 \\
2.0 \\
474 \\
327\end{array}$ & $\begin{array}{r}1.39 \\
1.69 \\
.11 \\
.01 \\
\\
-1 .- \\
\end{array}$ \\
\hline
\end{tabular}




\section{NOLAN COUNTY}

Population in 1940: $1,166$.

\section{ROSCOE}

Source of information: D. L. Kesler, pump operator, June 20, 1946.

Ownership: Municipal.

Source of supply: Three wells.

Well 1. Drilled in 1930; depth, 172 feet; diameter, 10 inches; deep-well turbine pump and 15-horsepower electric motor; static water level reported, 125 feet below land surface when drilled; yield. 135 gallons a minute in June, 1946.

Well 2. About 100 feet east of well 1; drilled in 1944 by Jack Stewart; depth, 170 feet; diameter, 8 inches; deep-well turbine pump and 5horsepower electric motor; yield, 18 gallons a minute.

Well 3. Drilled in June 1946 by Olin House; depth, 180 feet; diameter, 8 inches; static water level, 88 feet below land surface June 25, 1946; no pumping equipment stalled June 25, 1946.

Pumpage: No record.

Storage: Ground storage reservoir, 100,000 gallons; elevated tank, 55,000 gallons.

Number of customers: 275.

Treatment: Chlorination.

\section{Analyses}

[Collected June 20, 1946. pH: well 1, 7.6; well 2, 7.4. Analyzed by C. B. Cibulka]

\begin{tabular}{|c|c|c|c|c|}
\hline & \multicolumn{2}{|c|}{ Well 1} & \multicolumn{2}{|c|}{ Well 2} \\
\hline & $\begin{array}{l}\text { Parts per } \\
\text { million }\end{array}$ & $\begin{array}{l}\text { Equivalents } \\
\text { per million }\end{array}$ & $\begin{array}{l}\text { Parts per } \\
\text { million }\end{array}$ & $\begin{array}{l}\text { Equivalents } \\
\text { per million }\end{array}$ \\
\hline 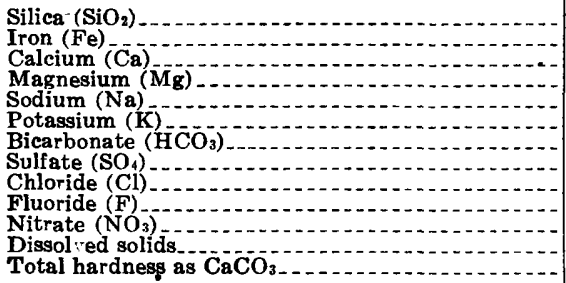 & $\begin{array}{l}19 \\
92 \\
22 \\
22 \\
11 \\
5.3 \\
256 \\
55 \\
50 \\
1.8 \\
10 \\
420 \\
320\end{array}$ & \begin{tabular}{r}
4.59 \\
1.81 \\
.48 \\
.13 \\
4.20 \\
1.15 \\
1.41 \\
.09 \\
.16 \\
\hdashline \\
-1.0 \\
\end{tabular} & $\begin{array}{l}21 \\
88^{.00} \\
21 \\
17 \\
4.9 \\
243 \\
55 \\
60 \\
1.8 \\
6.1 \\
437 \\
306\end{array}$ & $\begin{array}{r}4.39 \\
1.73 \\
1.76 \\
.13 \\
1.98 \\
1.69 \\
.09 \\
.10 \\
\end{array}$ \\
\hline
\end{tabular}

Driller's log, well s

\begin{tabular}{|c|c|c|c|c|c|}
\hline & $\begin{array}{c}\text { Thickness } \\
\text { (feet) }\end{array}$ & $\begin{array}{l}\text { Depth } \\
\text { (feet) }\end{array}$ & & $\begin{array}{c}\text { Thickness } \\
\text { (feet) }\end{array}$ & $\begin{array}{l}\text { Depth } \\
\text { (feet) }\end{array}$ \\
\hline $\begin{array}{l}\text { Top soil } \\
\text { Caliche } \\
\text { Sandstone. } \\
\text { Red sandy clay } \\
\text { Sandstone... }\end{array}$ & $\begin{array}{r}2 \\
10 \\
8 \\
24 \\
51\end{array}$ & $\begin{array}{r}2 \\
12 \\
20 \\
44 \\
95\end{array}$ & $\begin{array}{l}\text { Sand and gravel, water } \\
\text { Sandstone } \\
\text { Sand and gravel } \\
\text { Sandstone } \\
\text { Red shale. }\end{array}$ & $\begin{array}{r}\mathbf{6} \\
\mathbf{2 7} \\
\mathbf{5} \\
\mathbf{3 3} \\
\mathbf{1 4}\end{array}$ & $\begin{array}{l}101 \\
128 \\
133 \\
166 \\
180\end{array}$ \\
\hline
\end{tabular}

\section{SWEETWATER}

Population in 1940: 10,367 .

Source of information: Roy Duckett, water superintendent, Jun: 2, 1946.

Ownership: Municipal. 
Source of supply: Two impounding reservoirs.

Lake Trammel. On Sweetwater Creek about 8 miles south of S-reetwater; earth filled; dam built in 1914; lake-surface area about 240 ac:"es.

Lake Sweetwater. On Bitter Creek about 6 miles southeast of Sweetwater; earth filled; dam built in 1930; lake-surface area about 800 acres.

Average pumpage, in gallons a day

\begin{tabular}{|c|c|c|c|c|}
\hline & & 1945 & & 946 \\
\hline & Lake Trammel & Lake Sw eetwater & Lake Trammel & Lø ke Sweetwater \\
\hline 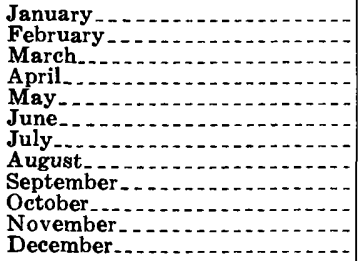 & $\begin{array}{l}926,200 \\
948,000 \\
980,000 \\
856,100 \\
934,900 \\
769,400\end{array}$ & $\begin{array}{r}0.137,400 \\
1,135,800 \\
1,453,100 \\
958,900 \\
720,600 \\
659,500\end{array}$ & $\begin{array}{r}859,200 \\
951,200 \\
770,600 \\
884,700 \\
814,200 \\
1,144,700 \\
\\
\\
\end{array}$ & 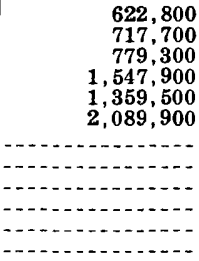 \\
\hline
\end{tabular}

Storage: Elevated tank, 750,000 gallons; stand pipe, 280,000 gallons.

Number of customers: 2,500.

Treatment: Prechlorination, aeration, coagulation, sedimentation, filtration, and postchlorination.

\section{Analysis of finished water}

[Composite sample, collected July 2, 1946. pH is 7.6. Analyzed by C. B. Cibulka]

\begin{tabular}{|c|c|c|c|c|c|}
\hline & $\begin{array}{l}\text { Parts per } \\
\text { million }\end{array}$ & $\begin{array}{l}\text { Equivalents } \\
\text { per million }\end{array}$ & & $\begin{array}{c}\text { Parts per } \\
\text { million }\end{array}$ & $\begin{array}{l}\text { Equivalents } \\
\text { per million }\end{array}$ \\
\hline $\begin{array}{l}\text { Silica }\left(\mathrm{SiO}_{2}\right) \\
\text { Iron }(\mathrm{Fe}) \\
\text { Calcium }(\mathrm{Ca}) \\
\text { Magnesium }(\mathrm{Mg}) \\
\text { Sodium }(\mathrm{Na}) \\
\text { Potassium }(\mathrm{K}) \\
\text { Bicarbonate ( } \mathrm{HCO} \text { ) }\end{array}$ & $\begin{array}{c}4.3 \\
49^{.00} \\
12 \\
13 \\
5.1 \\
162\end{array}$ & $\begin{array}{r}.987 \\
.057 \\
.130 \\
2.655\end{array}$ & $\begin{array}{l}\text { Sulfate }\left(\mathrm{SO}_{4}\right) \\
\text { Chloride (Cl) } \\
\text { Fluoride (F) } \\
\left.\text { Nitrate (NO }{ }_{3}\right) \\
\text { Dissolved solids } \\
\text { Total hardness as } \mathrm{CaCO}\end{array}$ & ${ }_{232^{.2}}^{38}$ & $\begin{array}{r}.791 \\
.677 \\
.010 \\
.003 \\
-0.0 \\
\end{array}$ \\
\hline
\end{tabular}

\section{OCHILTREE COUNTY}

\section{PERRYTON}

Population in 1940: 2,325 .

Source of information: Melvin Anderson, water superintendent, June 24, 1948. Ownership: Municipal.

Source of supply: Three wells.

Well 1. Drilled; depth, 295 feet; diameter, 12 inches; deep-well turbine pump and 50-horsepower electric motor; yield, 250 gallons a minute.

Well 2. Drilled in May 1946 by H. H. Heiskell; depth, 420 feet; diameter, 16 inches; deep-well turbine pump and electric motor; static water level, 248 feet below land surface May 1946; yield, 500 gallon` a minute.

Well 3. Drilled in June 1948 by George Brothers; depth, 420 feet; diameter, 16 inches; deep-well turbine pump and 60-horseporer electric motor; static water level, 265 feet below land surface June 1948; yield, 550 gallons a minute. 
Pumpage: Average, 600,000 gallons a day.

Storage: Ground storage reservoir, 215,000 gallons; elevated tank, 35,000 gallons.

Number of customers: 1,015 .

Treatment: None.

\section{Analyses}

[Collected June 24, 1948. pH: well 1, 7.5; well 2, 7.6. Analyzed by D. E. Weaver]

\begin{tabular}{|c|c|c|c|c|}
\hline \multirow[b]{2}{*}{$\checkmark$} & \multicolumn{2}{|c|}{ Well 1} & \multicolumn{2}{|c|}{ Well 2} \\
\hline & $\begin{array}{l}\text { Parts per } \\
\text { million }\end{array}$ & $\begin{array}{l}\text { Equivalents } \\
\text { per million }\end{array}$ & $\begin{array}{l}\text { Parts per } \\
\text { million }\end{array}$ & $\begin{array}{l}\text { Equivalents } \\
\text { per million }\end{array}$ \\
\hline 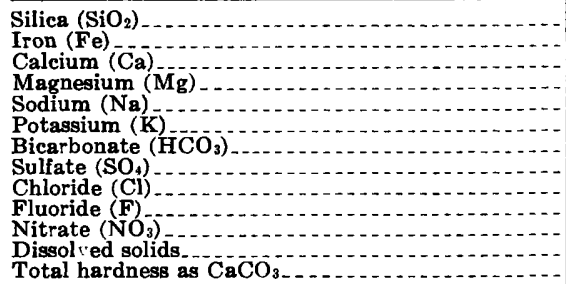 & $\begin{array}{l}44 \\
40^{\circ} .00 \\
27 \\
25 \\
5.2 \\
242 \\
12 \\
32 \\
2.4 \\
7.0 \\
301 \\
211\end{array}$ & 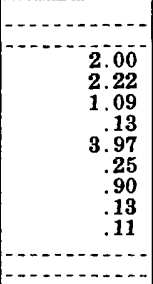 & $\begin{array}{l}46 \\
42 \\
28 \\
28 \\
27 \\
6.4 \\
246 \\
33 \\
25 \\
2.4 \\
6.8 \\
338 \\
220\end{array}$ & $\begin{array}{r}2.10 \\
2.30 \\
1.17 \\
.16 \\
4.03 \\
.69 \\
.71 \\
.13 \\
.11 \\
\end{array}$ \\
\hline
\end{tabular}

Driller's logs

\begin{tabular}{l|l|l|l|l|}
\hline Thickness & $\begin{array}{c}\text { Depth } \\
\text { (feet) }\end{array}$ & $\begin{array}{c}\text { Thickness } \\
\text { (feet) }\end{array}$ & $\begin{array}{c}\text { Depth } \\
\text { (feet) }\end{array}$ \\
\hline
\end{tabular}

Well 2

\begin{tabular}{|c|c|c|c|c|c|}
\hline $\begin{array}{l}\text { Surface soil } \\
\text { Sand and clay } \\
\text { Caliche } \\
\text { Sand, clay, and caliche... } \\
\text { Sand, clay, and gravel }\end{array}$ & $\begin{array}{r}7 \\
18 \\
75 \\
95 \\
40\end{array}$ & $\begin{array}{r}7 \\
25 \\
100 \\
195 \\
235\end{array}$ & $\begin{array}{l}\text { Sandy clay } \\
\text { Sand } \\
\text { Sand and gravel. } \\
\text { Sand } \\
\text { Clay and red rock }\end{array}$ & $\begin{array}{r}15 \\
70 \\
20 \\
80 \\
-\end{array}$ & $\begin{array}{l}\mathbf{2 5 0} \\
\mathbf{3 2 0} \\
\mathbf{3 4 0} \\
420 \\
420\end{array}$ \\
\hline
\end{tabular}

Well 3

\begin{tabular}{|c|c|c|c|c|c|}
\hline $\begin{array}{l}\text { Surface soil } \\
\text { Clay and caliche } \\
\text { Sandy clay } \\
\text { Fine sand, dry } \\
\text { Coarse sand and gravel } \\
\text { Sandy clay, lime shells. }\end{array}$ & $\begin{array}{r}11 \\
117 \\
13 \\
28 \\
41 \\
110\end{array}$ & $\begin{array}{r}11 \\
128 \\
141 \\
169 \\
210 \\
320\end{array}$ & $\begin{array}{l}\text { Coarse sand and gravel } \\
\text { Clay } \\
\text { Coarse sand and gravel } \\
\text { Clay } \\
\text { Red shale rock. }\end{array}$ & $\begin{array}{r}35 \\
30 \\
22 \\
13 \\
---\end{array}$ & $\begin{array}{l}355 \\
385 \\
407 \\
420 \\
420\end{array}$ \\
\hline
\end{tabular}

\section{OLDHAM COUNTY}

Population in 1940: 187.

\section{ADRIAN}

Source of information: John Horton, water manager, Nov. 20, 1947.

Ownership: Municipal.

Source of supply: Well, drilled in 1947 by John Hohenshelt; depth, 496 feet; diameter, 7 inches; deep-well cylinder pump and 5-horsepower electric motor; static water level, 455 feet below land surface; yield, 18 gallons a minute; temperature, $64^{\circ} \mathrm{F}$.

Pumpage (estimated) : 10,000 gallons a day.

Storage: Elevated tank, 20,000 gallons.

Treatment: None. 
Analysis

[Collected Nov. 20, 1948. Analyzed by D. E. Weaver]

\begin{tabular}{|c|c|c|c|c|c|}
\hline & $\begin{array}{l}\text { Parts per } \\
\text { million }\end{array}$ & $\begin{array}{l}\text { Equivalents } \\
\text { per million }\end{array}$ & & $\begin{array}{l}\text { Parts per } \\
\text { million }\end{array}$ & $\begin{array}{l}\text { Equivalents } \\
\text { per million }\end{array}$ \\
\hline $\begin{array}{l}\text { Silica }\left(\mathrm{SiO}_{2}\right) \\
\text { Calcium }(\mathrm{Ca}) \\
\text { Magnesium }(\mathrm{Mg}) \\
\text { Sodium and Potassium } \\
\quad(\mathrm{Na}+\mathrm{K}) \text {. } \\
\text { Bicarbonate }\left(\mathrm{HCO}_{3}\right)\end{array}$ & $\begin{array}{r}17 \\
4 \\
4 \\
371 \\
556\end{array}$ & $\begin{array}{r}0.20 \\
16.15 \\
9.11\end{array}$ & $\begin{array}{l}\text { Sulfate }\left(\mathrm{SO}_{4}\right) \\
\text { Chloride }(\mathrm{Cl}) \\
\text { Nitrate (NO}) \\
\text { Dissolved golids } \\
\text { Total hardness as } \mathrm{CaCO}_{3}\end{array}$ & $\begin{array}{c}280 \\
60 \\
3.2 \\
1,010 \\
26\end{array}$ & $\begin{array}{r}5.83 \\
1.69 \\
.05 \\
-r .-5\end{array}$ \\
\hline
\end{tabular}

Driller's log

\begin{tabular}{|c|c|c|c|c|c|}
\hline & $\underset{\text { (feet) }}{\text { Thickness }}$ & $\underset{\text { (feet) }}{\text { Depth }}$ & & $\underset{\text { (feet) }}{\text { Thickness }}$ & $\begin{array}{l}\text { Depth } \\
\text { (feet) }\end{array}$ \\
\hline $\begin{array}{l}\text { No record } \\
\text { Hard sand } \\
\text { Pinkish red clay, no sand. } \\
\text { Very fine dry sand, multi- } \\
\text { colored layers. }\end{array}$ & $\begin{array}{r}160 \\
40 \\
160 \\
70\end{array}$ & $\begin{array}{l}160 \\
200 \\
\mathbf{3 6 0} \\
\mathbf{4 3 0}\end{array}$ & $\begin{array}{l}\text { Blue shale } \\
\text { Water sand, first water. } \\
\text { Blue shale. }\end{array}$ & $\begin{array}{r}\mathbf{2 5} \\
\mathbf{3 0} \\
\mathbf{9}\end{array}$ & $\begin{array}{l}455 \\
435 \\
494\end{array}$ \\
\hline
\end{tabular}

\section{VFGA}

Population in 1940: 515.

Source of information: R. W. Armitage, city secretary, Nov. 20, 1947.

Ownership: Municipal.

Source of supply: Two wells.

Well 1. Drilled in 1930 by John Hohenshelt; depth, 300 feet; diameter, 7 inches; deep-well turbine pump and electric motor; static water level, 200 feet below land surface; yield 125 gallons a minute.

Well 2. About 75 feet from well 1; drilled in 1943 by H. H. Heiskel; depth, 330 feet; diameter, 10 inches; deep-well turbine pump and electric motor; yield, 125 gallons a minute.

Pumpage: No record.

Storage: Elevated tank, 50,000 gallons.

Treatment: None.

\section{Analysis}

[Composite sample, collected Nov. 20, 1947. Analyzed by D. E. Weaver]

\begin{tabular}{|c|c|c|c|c|c|}
\hline & $\begin{array}{c}\text { Parts per } \\
\text { million }\end{array}$ & $\begin{array}{l}\text { Equivalents } \\
\text { per million }\end{array}$ & & $\begin{array}{l}\text { Parts pe- } \\
\text { million }\end{array}$ & $\begin{array}{l}\text { Equivalents } \\
\text { per million }\end{array}$ \\
\hline $\begin{array}{l}\mathrm{Silica}\left(\mathrm{SiO}_{2}\right) \\
\mathrm{Calcium}(\mathrm{Ca}) \\
\text { Magnesium }(\mathrm{Mg}) \\
\text { Sodium and potassium } \\
\quad(\mathrm{Na}+\mathrm{K}) \text {. } \\
\text { Bicarbonate }\left(\mathrm{HCO}_{3}\right)\end{array}$ & $\begin{array}{r}29 \\
31 \\
52 \\
81 \\
\\
218\end{array}$ & $\begin{array}{r}1.55 \\
4.23 \\
3.51 \\
3.58\end{array}$ & $\begin{array}{l}\text { Sulfate }\left(\mathrm{SO}_{4}\right) \\
\text { Chloride }(\mathrm{Cl}) \\
\left.\text { Nitrate (NO }{ }_{3}\right) \\
\text { Dissolved solids } \\
\text { Total hardness as } \mathrm{CaCO}_{3}\end{array}$ & $\begin{array}{r}20 c \\
2 \varepsilon \\
5 c \\
59 \varepsilon \\
29 \varepsilon\end{array}$ & $\begin{array}{r}4.16 \\
.79 \\
.81 \\
-9.9 \\
-9.8\end{array}$ \\
\hline
\end{tabular}

\section{WHLDORADO}

Population in 1940: 60 .

Source of information: A. F. Moore, co-owner, Nov. 20, 1947.

Owners: W. E. O'Neil, C. C. Kellogg, and A. F. Moore.

Source of supply: Well; drilled about 1900; depth, 251 feet; cliameter, 5 inches; deep-well cylinder pump and windmill and gasoline engire; temperature, $61^{\circ} \mathrm{F}$.

Pumpage: No record.

Storage: Elevated tank, 5,000 gallons.

Treatment: None. 


\section{Analysis}

[Collected Nov. 20, 1947. Analyzed by D. E. Weaver]

\begin{tabular}{|c|c|c|c|c|c|}
\hline & $\begin{array}{l}\text { Parts per } \\
\text { million }\end{array}$ & $\begin{array}{l}\text { Equlvalents } \\
\text { per million }\end{array}$ & & $\begin{array}{c}\text { Parts per } \\
\text { million }\end{array}$ & $\begin{array}{l}\text { Equivalents } \\
\text { per million }\end{array}$ \\
\hline $\begin{array}{l}\text { Silica }\left(\mathrm{SiO}_{2}\right) \\
\text { Calcium }(\mathrm{Ca}) \\
\text { Magnesium }(\mathrm{Mg}) \\
\text { Sodium and potassium } \\
\text { ( } \mathrm{Na}+\mathrm{K}) \text {. } \\
\text { Bicarbonate }\left(\mathrm{HCO}_{3}\right)\end{array}$ & $\begin{array}{r}55 \\
29 \\
20 \\
28 \\
224\end{array}$ & $\begin{array}{r}1.447 \\
1.645 \\
1.232 \\
3.680\end{array}$ & $\begin{array}{l}\text { Sulfate }\left(\mathrm{SO}_{4}\right) \\
\text { Chloride }(\mathrm{Cl}) \\
\text { Nitrate }\left(\mathrm{NO}_{3}\right) \\
\text { Dissolved solids } \\
\text { Total hardness as } \mathrm{CaCO}_{3}\end{array}$ & $\begin{array}{r}17 \\
6.0 \\
7.5 \\
227 \\
154\end{array}$ & $\begin{array}{r}0.354 \\
.169 \\
.121 \\
\\
-1.0 \\
\end{array}$ \\
\hline
\end{tabular}

\section{PARMER COUNTY}

\section{FARWELL}

Population in 1940: 1,250 .

Source of information: B.N. Graham, City secretary, Mar. 2, 1945.

Owner: Texico-Farwell Water Works, Inc.

Source of supply: Two wells.

Well 1. North well; drilled; depth, 300 feet; diameter, 6 inches; deepwell turbine pump and electric motor; yield, 65 gallons a minute.

Well 2. South well; drilled; depth, 318 feet; diameter, 12 inches; deepwell turbine pump and electric motor; static water level, if feet below land surface in 1939; yield, 250 gallons a minute; temperature, $65^{\circ} \mathrm{F}$. Pumpage: Average, 200,000 gallons a day.

Storage: Elevated tank, 50,000 gallons.

Number of customers: 200.

Treatment: None.

\section{Analysis, well 2}

[Collected Mar. 2, 1945. $\mathrm{pH}$ is 7.8. Analyzed by M. L. Begley]

\begin{tabular}{|c|c|c|c|c|c|}
\hline & $\begin{array}{l}\text { Parts per } \\
\text { million }\end{array}$ & $\begin{array}{l}\text { Equivalents } \\
\text { per million }\end{array}$ & & $\begin{array}{l}\text { Parts per } \\
\text { million }\end{array}$ & $\begin{array}{l}\text { Equivalents } \\
\text { per million }\end{array}$ \\
\hline 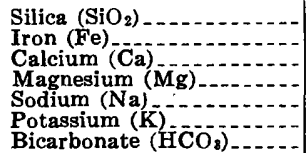 & $\begin{array}{l}32 \\
33 \\
25 \\
27 \\
7.04 \\
230\end{array}$ & $\begin{array}{r}1.65 \\
2.06 \\
1.16 \\
3.18 \\
3.77\end{array}$ & $\begin{array}{l}\text { Sulfate }\left(\mathrm{SO}_{4}\right) \\
\text { Chloride }(\mathrm{CI}) \\
\text { Fluoride }(\mathrm{F}) \\
\text { Nitrate }\left(\mathrm{NO}_{3}\right) \\
\text { Dissolved solids } \\
\text { Total hardness as } \mathrm{CaCO}_{3}\end{array}$ & $\begin{array}{l}2 \pi \\
16 \\
2.8 \\
7.2 \\
290 \\
186\end{array}$ & 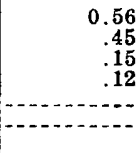 \\
\hline
\end{tabular}

\section{FRIONA}

Population in 1940: 803.

Source of information: Mr. Wilson, City clerk, Oct. 6; 1948.

Ownership: Municipal.

Source of supply: Two wells.

Well 1. Drilled in 1929 by Leo McDade; depth, 216 feet; diameter, 10 inches; deep-well turbine pump and 20-horsepower elentric motor; yield, 150 gallons a minute.

Well 2. Drilled in 1935 by L. G. Simpson; depth, 192 feet; diameter, 10 inches; deep-well turbine pump and 15-horsepower electric motor; yield, 100 gallons a minute.

Pumpage: 57,000 gallons a day.

Storage: Elevated tank, capacity unknown.

Number of customers: 348 .

Treatment: Chlorination. 


\section{Analysis}

[Composite sample, collected Oct. 6, 1948. pH is 7.7. Analyzed by J. R. Avrett]

\begin{tabular}{|c|c|c|c|c|c|}
\hline & $\begin{array}{c}\text { Parts per } \\
\text { million }\end{array}$ & $\begin{array}{l}\text { Equivalents } \\
\text { per million }\end{array}$ & & $\begin{array}{l}\text { Parts per } \\
\text { mill on }\end{array}$ & $\begin{array}{l}\text { Equivalents } \\
\text { per million }\end{array}$ \\
\hline $\begin{array}{l}\text { Silica }\left(\mathrm{SiO}_{2}\right) \\
\text { Iron (Fe) } \\
\text { Calcium }(\mathrm{Ca}) \\
\text { Magnesium }(\mathrm{Mg}) \\
\text { Sodium (Na) } \\
\text { Potassium }(\mathrm{K}) \\
\text { Bicarbonate }\left(\mathrm{H} \mathrm{C} \mathrm{O}_{3}\right)\end{array}$ & $\begin{array}{l}37 \\
32 \\
32 \\
28 \\
6.00 \\
252\end{array}$ & $\begin{array}{r}1.60 \\
2.63 \\
1.22 \\
4.17\end{array}$ & $\begin{array}{l}\text { Sulfate }\left(\mathrm{SO}_{4}\right) \\
\text { Chloride (Cl) } \\
\text { Fluoride (F) } \\
\left.\text { Nitrate (NO} \mathrm{O}_{3}\right) \\
\text { Dissolved solids } \\
\text { Total hardness as } \mathrm{CaCO}_{3}\end{array}$ & $\begin{array}{l}29 \\
22 \\
2.4 \\
9.5 \\
317 \\
212\end{array}$ & $\begin{array}{r}0.58 \\
.62 \\
.13 \\
.15 \\
\\
\end{array}$ \\
\hline
\end{tabular}

Driller's log, well 1

\begin{tabular}{|c|c|c|c|c|c|}
\hline & $\underset{\text { (feet) }}{\text { Thickness }}$ & $\begin{array}{l}\text { Depth } \\
\text { (feet) }\end{array}$ & & $\underset{\text { (feet) }}{\text { Thickness }}$ & $\begin{array}{c}\text { Depth } \\
\text { (feet) }\end{array}$ \\
\hline $\begin{array}{l}\text { Red clay } \\
\text { White chalky sandstone.. } \\
\text { Packed sand. }\end{array}$ & $\begin{array}{l}\mathbf{2 5} \\
\mathbf{3 6} \\
\mathbf{2 9}\end{array}$ & $\begin{array}{l}25 \\
61 \\
90\end{array}$ & $\begin{array}{l}\text { Brown packed sand (water } \\
\text { at } 155 \text { feet). } \\
\text { Porous water sand } \\
\text { Sandstone. }\end{array}$ & $\begin{array}{r}125 \\
11 \\
4\end{array}$ & $\begin{array}{l}215 \\
226 \\
230\end{array}$ \\
\hline
\end{tabular}

\section{PECOS COUNTY}

\section{FORT STOCKTON}

Population in 1940: 3,294 .

Source of information: Cleve Nunn, manager of utilities, Oct. 21, 1946.

Ownership: Municipal.

Source of supply: Three wells.

Well 1. Drilled; depth, 160 feet; diameter, 6 inches; deep-well turbine

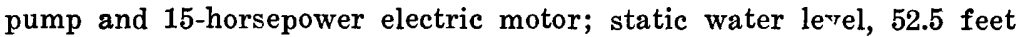
below land surface Oct. 21, 1946; yield, 450 gallons a minute.

Well 2. South well; drilled in 1932 by Arthur Powell; derth, 193 feet; diameter, 13 inches; deep-well turbine pump and 25-horserower electric motor; static water level, 51.4 feet.below land surface Oct.. 21, 1946; yield, 750 gallons a minute.

Well 3. Middle well; drilled in 1946 by R. A. Cleveland; depth, 203 feet; diameter, 12 inches; deep-well turbine pump and 25-horsepower electric motor; static water level, 51.8 feet below land surface $C$.t. 21, 1946; yield, 500 gallons a minute.

Average pumpage in gallons a day, 1946

\begin{tabular}{|c|c|c|c|c|c|}
\hline $\begin{array}{l}\text { January } \\
\text { February } \\
\text { March }\end{array}$ & $\begin{array}{l}315,000 \\
299,000 \\
350,000\end{array}$ & $\begin{array}{l}\text { April } \\
\text { May } \\
\text { June }\end{array}$ & $\begin{array}{l}589,000 \\
435,000 \\
688,000\end{array}$ & $\begin{array}{l}\text { July } \\
\text { August } \\
\text { September }\end{array}$ & $\begin{array}{l}673,000 \\
728,000 \\
490,000\end{array}$ \\
\hline
\end{tabular}

Storage: Concrete ground reservoir, 100,000 gallons; two elevate tanks, total capacity, 175,000 gallons.

Number of customers: 794.

Treatment: Chlorination and ammoniation. 


\section{Analyses}

[Collected Oct. 21, 1946. pH : well 2, 7.0; well 3, 7.2. Analyzed by C. B. Cibulka]

\begin{tabular}{|c|c|c|c|c|}
\hline & \multicolumn{2}{|c|}{ Well 2} & \multicolumn{2}{|c|}{ Well 3} \\
\hline & $\begin{array}{l}\text { Parts per } \\
\text { million }\end{array}$ & $\begin{array}{l}\text { Equivalents } \\
\text { per million }\end{array}$ & $\begin{array}{l}\text { Parts per } \\
\text { million }\end{array}$ & $\begin{array}{l}\text { Equivalents } \\
\text { per million }\end{array}$ \\
\hline 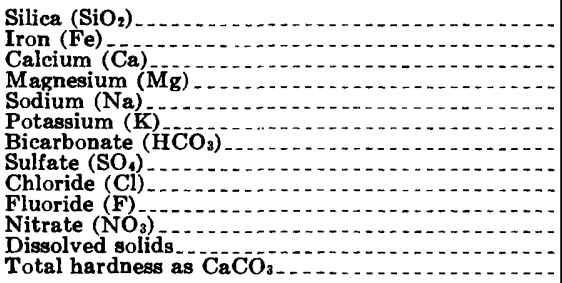 & $\begin{array}{l}16 \\
156 \\
52 \\
238 \\
38 \\
276 \\
427 \\
350 \\
1.8 \\
1.420^{.4} \\
604\end{array}$ & $\begin{array}{r}7.79 \\
4.28 \\
10.34 \\
.97 \\
4.52 \\
8.89 \\
9.87 \\
.09 \\
.01\end{array}$ & $\begin{array}{l}16 \\
156 \\
53 \\
235 \\
39 \\
274 \\
424 \\
352 \\
1.8 \\
1,410 \\
608\end{array}$ & $\begin{array}{r}7.79 \\
4.36 \\
10.20 \\
1.00 \\
4.49 \\
8.83 \\
9.93 \\
.09 \\
.01 \\
\end{array}$ \\
\hline
\end{tabular}

Population in 1940: 75 .

\section{IMPERLAL}

Source of information: Mrs. E. E. Scarbrough, wife of former owner, Sept. $29,1948$.

Owner: Imperial Water Co.

Source of supply: Wells of the Byrd-Frost Water Co. about 15 miles north of Imperial in Crane County.

Pumpage: 10,000 gallons a day.

Storage: Elevated tank, 8,000 gallons.

Number of customers: $\mathbf{7 5}$.

Treatment: None.

\section{Analysis}

[Tap sample, collected Oct. 26, 1946. pH is 7.4. Analyzed by C. B. Cibul '₹a]

\begin{tabular}{|c|c|c|c|c|c|}
\hline & $\begin{array}{l}\text { Parts per } \\
\text { million }\end{array}$ & $\begin{array}{l}\text { Equivalents } \\
\text { per million }\end{array}$ & & $\begin{array}{l}\text { Parts p’r } \\
\text { millior }\end{array}$ & $\begin{array}{l}\text { Equivalents } \\
\text { per million }\end{array}$ \\
\hline $\begin{array}{l}\text { Silica }\left(\mathrm{SiO}_{2}\right) \\
\text { Iron }(\mathrm{Fe}) \\
\text { Calcium }(\mathrm{Ca}) \\
\text { Magnesium }(\mathrm{Mg}) \\
\text { Sodium }(\mathrm{Na}) \\
\text { Potassium }(\mathrm{K}) \\
\left.\text { Bicarbonate }(\mathrm{H})^{-} \mathrm{O}_{2}\right)\end{array}$ & $\begin{array}{l}39 \\
64.05 \\
5.7 \\
24 \\
3.6 \\
178\end{array}$ & $\begin{array}{r}\mathbf{3} .194 \\
.469 \\
1.043 \\
.092 \\
2.918\end{array}$ & $\begin{array}{l}\text { Sulfate }\left(\mathrm{SO}_{4}\right) \\
\text { Chloride (CI) } \\
\text { Fluoride (F) } \\
\text { Nitrate (NO })_{\mathrm{a}} \text { ) } \\
\text { Dissolved solids, } \\
\text { Total hardness as } \mathrm{CaCO}\end{array}$ & $\begin{array}{l}32 \\
32 \\
1.0 \\
16 \\
293 \\
183\end{array}$ & $\begin{array}{r}0.666 \\
.903 \\
.053 \\
.258 \\
-0 .- \\
-0.0\end{array}$ \\
\hline
\end{tabular}

\section{IRAAN}

Population in 1940: 1,000.

Source of information: Geo. L. Munnich, Dec. 13, 1946.

Owner: Iraan Ice, Water and Gas Co.

Source of supply: Two wells about 0.4 mile south of ice plant.

Well 1. Drilled in 1936 by Fred Slaughter; depth, 210 feet; diameter, 8 inches; deep-well cylinder pump and 3-horsepower electric motor; static water level, 115.6 feet below land surface Dec. 13, 1946; yield, 25 gallons a minute.

Well 2. Drilled by Sam Parker; depth, 210 feet; diameter, 7 irches; deepwell turbine pump and 5 -horsepower electric motor; yield, 75 gallons a minute. 
Average pumpage, in gallons a day

\begin{tabular}{|c|c|c|c|c|c|}
\hline $\begin{array}{l}\text { January } \\
\text { February. } \\
\text { March } \\
\text { April }\end{array}$ & $\begin{array}{l}21,300 \\
29,600 \\
29,300 \\
30,300\end{array}$ & $\begin{array}{l}\text { May } \\
\text { June } \\
\text { July } \\
\text { August_. }\end{array}$ & $\begin{array}{l}\mathbf{3 0}, \mathbf{3 0 0} \\
\mathbf{3 5}, 000 \\
\mathbf{3 6}, 000 \\
41,500\end{array}$ & $\begin{array}{l}\text { September } \\
\text { October } \\
\text { November } \\
\text { December }\end{array}$ & $\begin{array}{r}28,200 \\
20,900 \\
22,700 \\
-\ldots\end{array}$ \\
\hline
\end{tabular}

Storage: Concrete ground reservoir on top of hill, 19,000 gallons.

Number of customers: 174.

Treatment: None.

Analysis, well 1

[Collected Feb. 11, 1947. pH is 7.3. Analyzed by B. C. Dwyer]

\begin{tabular}{|c|c|c|c|c|c|}
\hline & $\begin{array}{c}\text { Parts pe' } \\
\text { million }\end{array}$ & $\begin{array}{l}\text { Equivalents } \\
\text { per million }\end{array}$ & & $\begin{array}{c}\text { Parts per } \\
\text { million }\end{array}$ & $\begin{array}{l}\text { Equivalents } \\
\text { per million }\end{array}$ \\
\hline $\begin{array}{l}\text { Silica }\left(\mathrm{SiO}_{2}\right) \\
\text { Iron }(\mathrm{Fe}) \\
\text { Calcium }(\mathrm{Ca}) \\
\text { Magnesium }(\mathrm{Mg}) \\
\text { Sodium }(\mathrm{Na}) \\
\text { Potassium }(\mathrm{K}) \\
\text { Bicarbonate }\left(\mathrm{HC} \mathrm{CO}_{2}\right)^{-}\end{array}$ & $\begin{array}{l}12 \\
152 \\
74 \\
221 \\
24 \\
292\end{array}$ & $\begin{array}{r}7.59 \\
6.09 \\
9.61 \\
.61 \\
4.79\end{array}$ & $\begin{array}{l}\text { Sulfate (SO4) } \\
\text { Chloride (Cl) } \\
\text { Fluoride (F) } \\
\text { Nitrate (NOs) } \\
\text { Dissolved solids } \\
\text { Total hardness as } \mathrm{CaCO}_{3}\end{array}$ & $\begin{array}{r}239 \\
500 \\
1.2 \\
6.2 \\
1,37 \mathrm{C} \\
684\end{array}$ & $\begin{array}{r}4.85 \\
14.10 \\
.06 \\
.10 \\
-.-\end{array}$ \\
\hline
\end{tabular}

Driller's log, well 2

\begin{tabular}{|c|c|c|c|c|c|}
\hline & $\begin{array}{c}\text { Thickness } \\
\text { (feet) }\end{array}$ & $\begin{array}{c}\text { Depth } \\
\text { (feet) }\end{array}$ & & $\begin{array}{c}\text { Ty jickness } \\
\text { (feet) }\end{array}$ & $\begin{array}{l}\text { Depth } \\
\text { (feet) }\end{array}$ \\
\hline $\begin{array}{l}\text { Gray lime } \\
\text { Yellow lime } \\
\text { Yellow sandy lime }\end{array}$ & $\begin{array}{l}38 \\
52 \\
18\end{array}$ & $\begin{array}{r}38 \\
90 \\
108\end{array}$ & $\begin{array}{l}\text { Yellow lime } \\
\text { Yellow sand and lime } \\
\text { Sand, water... }\end{array}$ & $\begin{array}{r}68 \\
4 \\
30\end{array}$ & $\begin{array}{l}176 \\
180 \\
210\end{array}$ \\
\hline
\end{tabular}

\section{POTTER COUNTY}

Population in 1940: 51,686 .

\section{AMARILLO}

Source of information: M. V. Moss, city manager, June 23, 1948.

Ownership: Municipal.

Source of supply: Thirty-two wells southwest of Amarillo.

Palo Duro well 1. Drilled in 1927; depth, 200 feet; diameter, 10 inches; deep-well turbine pump and electric motor.

Palo Duro well 2. Drilled in 1927; depth, 200 feet; diameter, 10 inches; deep-well turbine pump and electric motor.

Palo Duro well 3. Drilled in 1927; depth, 200 feet; diameter, 10 inches; deep-well turbine pump and electric motor; pumping level, 95 feet below land surface January 1943, 117 feet below land surface August 1945; static water level, 61.0 feet below land surface Jenuary 1943; yield, 720 gallons a minute Jan. 30, 1942.

Palo Duro well 4. Drilled in 1927; depth, 200 feet; diameter, 10 inches; deep-well turbine pump and electric motor; pumping level, 95 feet below land surface January 1943, 130 feet below land surface August 1945; static water level, 61.0 feet below land surface Jønuary 1943; yield, 594 gallons a minute Jan. 30, 1942. 
Palo Duro well 5. Drilled in 1927; depth, 200 feet; diameter, 10 inches; deep-well turbine pump and electric motor; pumping level, 70 feet below land surface January 1945, 121.0 feet below land surface August 1945; static water level, 35.0 feet below land surface Jaruary 1943; yield, 454 gallons a minute Jan. 30, 1942.

Palo Duro well 6. Drilled in 1927; depth, 200 feet; diameter, 10 inches; deep-well turbine pump and electric motor; pumping level, 100.0 feet below land surface January 1943, 128 feet below land surface August 1945; static water level, 88.0 feet below land surface Jaruary 1943; yield, 751 gallons a minute Jan. 30, 1942.

Palo Duro well 7. Drilled in 1927; depth, 200 feet; diameter, 10 inches; deep-well turbine pump and electric motor; pumping level, 52 feet below land surface January 1943, 114.0 feet below land surface August 1945; static water level, 38.0 feet below land surface Jaruary 1943; yield, 860 gallons a minute Jan. $30,1942$.

Palo Duro well 8. Drilled in 1927; depth, 200 feet; diameter, 10,inches; deep-well turbine pump and electric motor; pumping level, 86.0 feet below land surface January 1943, 130 feet below land surface August 1945; static water level, 61.0 feet below land surface Jaruary 1943; yield, 820 gallons a minute Jan. 30,1942 .

Palo Duro well 9. Drilled in 1927; depth, 200 feet; diameter, 10 inches; deep-well turbine pump and electric motor; yield, 598 gallons a minute Jan. 30, 1942.

Palo Duro well 10. Drilled in 1927; depth, 200 feet; diameter, 10 inches; deep-well turbine pump and electric motor; yield, 450 gallons a minute Jan. 30, 1942.

Greely well 1. Drilled in 1944 by H. H. Heiskell; depth, 313 feet; diameter, 16 inches; deep-well turbine pump and 50-horsepower electric motor; static water level, 120.0 feet July 1944; pumping level, $18^{\urcorner}$feet July 1944 and 212 feet May 1946.

Greely well 2. Drilled in 1944 by H. H. Heiskell; depth, 264 feet; diameter, 16 inches; deep-well turbine pump and electric motor; static water level, 106.0 feet below land surface May 12, 1946; pumping level, 158 feet below land surface May 1946; yield, 1,115 gallons a minute with drawdown of 15 feet May 13, 1944.

Greely well 3. Drilled in 1944; depth, 283 feet; diameter, 16 inches; deep-well turbine pump and 60-horsepower electric motor; static water level, 135.0 feet below land surface April 1945; pumping level, 156 feet below land surface April 1945 and 187 feet below lend surface May 1946.

Greely well 4. Drilled in 1946 by H. H. Heiskell; depth, 305 feet; diameter, 16 inches; deep-well turbine pump and 60-horsepovrer electric motor; yield, 800 gallons a minute.

Greely well 5. Drilled in 1946 by H. H. Heiskell; depth, 262 feet; diameter, 16 inches; deep-well turbine pump and electric motor; static water level, 126.0 feet below land surface Apr. 1, 1946; yield, 1,340 gallons a minute with drawdown of 44 feet Apr. 2, 1946.

Greely well 7. Drilled in May 1946 by H. H. Heiskell; deptl . 303 feet; diameter, 16 inches; deep-well turbine pump and 60-horsepower electric motor; yield, 900 gallons a minute. 
Greely well 8. Drilled in 1946 by H. H. Heiskell; depth, 280 feet; diameter, 16 inches; deep-well turbine pump and 60-horsepower electric motor; yield, 600 gallons a minute.

Bush well. Drilled in 1944 by H. H. Heiskell; depth, 260 feet; diameter, 16 inches; deep-well turbine pump and 60-horsepower electric motor; static water level, 135.0 feet below land surface May 1944; pumping level, 182 feet below land surface May 1946; yield, 1,060 gallons a minute with drawdown of 24 feet May 5, 1944.

Bush well 1. Drilled in 1943 by H. H. Heiskell; depth, 296 feet; diameter, 16 inches; deep-well turbine pump and 60-horsepower electric motor; static water level, 150 feet below land surface May 25, 1943; pumping level, 198 feet below land surface May 1946; yield, 880 gallons a minute with drawdown of 36 feet May 26, 1943.

Bush well 2. Drilled in 1944 by H. H. Heiskell; depth, 250 feet; diameter, 16 inches; deep-well turbine pump and 75-horsepower electric motor; static water level, 153 feet below land surface June 1945; pumping level, 180 feet below land surface June 1945, 190 feet below land surface May 1946; yield, 1,100 gallons a minute with drawdown of 33 feet April 1944.

Bush well 3. Drilled in 1944 by H. H. Heiskell; depth, 239 feet; diameter, 16 inches; deep-well turbine pump and 60-horsepower electric motor; static water level, 132 feet below land surface Apr. 22, 1944; pumping level, 200 feet below land surface May 1946; yield,.720 gallons a minute with drawdown of 49 feet Apr. 23, 1944.

Bush well 4. Drilled in 1943 by H. H. Heiskell; depth, 305 feet; diameter, 16 inches; deep-well turbine pump and 60-horsepower electric motor; static water level, 141 feet below land surface May 9, 1943; pumping level, 205 feet below land surface May 1946; yield, 880 gallons a minute with drawdown of 49 feet May 10, 1943.

Bush well 5. Drilled in 1943 by H. H. Heiskell; depth, 263 feet; diameter, 16 inches; deep-well turbine pump and 50-horsepower electric motor; static water level, 137 feet below land surface Apr. 23, 1943; pumping level, 192 feet below land surface May 1946; yield, 1,090 gallons a minute with drawdown of 18.5 feet Apr. 24, 1943.

McDonald well 1. Drilled in 1929 by D. L. McDonald; depth, 270 feet; diameter, 18 inches; deep-well turbine pump and 75-horsepower electric motor; static water level, 162.0 feet below land surface July 30, 1931; pumping level, 240 feet below land surface May 1946; yield, 750 gallons a minute with drawdown of 42 feet Sept. 1, 1941.

McDonald well 2. Drilled in 1929 by D. L. McDonald; depth, 270 feet; diameter, 18 inches; deep-well turbine pump and 75-horsepower electric motor; static water level, 160.0 feet below land surface Aug. 24, 1929; pumping level, 240.0 feet below land surface May 1946; yield, 750 gallons a minute.

McDonald well 3. Drilled in 1929 by D. L. McDonald; depth, 270 feet; diameter, 18 inches; deep-well turbine pump and 75-horsepower electric motor; static water level, 156 feet below land surface Aug. 13, 1929; pumping level, 230 feet below land surface May 1946; yield, 750 gallons a minute with drawdown of 37 feet.

McDonald well 4. Drilled in 1929 by D. L. McDonald; depth, 322 feet; diameter, 18 inches; deep-well turbine pump and 75-horsepower electric motor; static water level, 162.0 feet below land surface Aug. 1, 
1931; pumping level, 229 feet below land surface May 1936; yield, 750 gallons a minute with drawdown of 46 feet Sept. 2, 1931.

McDonald well 5. Drilled in 1929 by D. L. McDonald; dep+h, 336 feet; diameter, 18 inches; deep-well turbine pump and 75-horsep 7 wer electric motor; static water level, 163.0 feet below land surface $A$ ug. 1,1931 ; pumping level, 239 feet below land surface May 1946; yield, 750 gallons a minute with drawdown of 44 feet.

McDonald well 6. Drilled in 1947 by H. H. Heiskell; depth, 280 feet; diameter, 16 inches; deep-well turbine pump and 50-horsep motor; yield, 750 gallons a minute.

Bassett well 1. Drilled in 1947 by H. H. Heiskell; depth, 265 feet; diameter, 16 inches; deep-well turbine pump and 50-horsepower electric motor; yield, 750 gallons a minute.

Bassett well 2. Drilled in 1947 by H. H. Heiskell; depth, 280 feet; diameter, 16 inches; deep-well turbine pump and 50-horsepower electric motor; yield, 750 gallons a minute.

Brinkman well 1. Drilled in 1944 by H. H. Heiskell; depłh, 277 feet; diameter, 16 inches; deep-well turbine pump and 50-horsep motor; static water level, 170 feet below land surface Jenuary 1945; pumping level, 210 feet below land surface May 1946; yield. 700 gallons a minute with drawdown of 50 feet.

Average pumpage, in gallons a day

\begin{tabular}{|c|c|c|c|c|c|c|c|}
\hline & 1942 & 1943 & 1944 & 1945 & 1946 & 1947 & 1948 \\
\hline 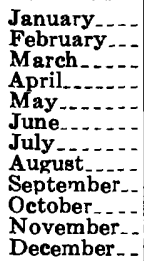 & $\begin{array}{r}\mathbf{3}, 400,000 \\
\mathbf{3 , 3 6 0 , 0 0 0} \\
\mathbf{3}, 360,000 \\
\mathbf{3 , 7 3 0 , 0 0 0} \\
\mathbf{6 , 5 0 0 , 0 0 0} \\
7,410,000 \\
10,300,000 \\
\mathbf{6 , 1 5 0 , 0 0 0} \\
\mathbf{5 , 1 9 0 , 0 0 0} \\
4,400,000 \\
4,550,000 \\
4,430,000\end{array}$ & $\begin{array}{r}4,600,000 \\
5,220,000 \\
\mathbf{5 , 9 8 0 , 0 0 0} \\
7,480,000 \\
\mathbf{6 , 6 3 0 , 0 0 0} \\
10,930,000 \\
7,820,000 \\
11,500,000 \\
8,960,000 \\
\mathbf{6 , 6 4 0 , 0 0 0} \\
\mathbf{6 , 1 9 0 , 0 0 0} \\
\mathbf{5 , 3 0 0 , 0 0 0}\end{array}$ & $\begin{array}{r}\mathbf{5 , 4 7 0 , 0 0 0} \\
\mathbf{5 , 5 0 0 , 0 0 0} \\
\mathbf{5 , 7 8 0 , 0 0 0} \\
\mathbf{6 , 5 0 0 , 0 0 0} \\
7,660,000 \\
\mathbf{8 , 1 4 0 , 0 0 0} \\
9,000,000 \\
10,950,000 \\
\mathbf{8 , 1 5 0 , 0 0 0} \\
6,680,000 \\
6, \mathbf{3 1 0 , 0 0 0} \\
\mathbf{5 , 8 4 0 , 0 0 0}\end{array}$ & $\begin{array}{r}5,980,000 \\
6,570,000 \\
7,970,000 \\
7,780,000 \\
11,430,000 \\
11,900,000 \\
12,250,000 \\
11,800,000 \\
11,500,000 \\
7,020,000 \\
6,910,000 \\
6,000,000\end{array}$ & $\begin{array}{r}5,810,000 \\
6,210,000 \\
7,210,000 \\
10,200,000 \\
11,640,000 \\
13,180,000 \\
15,160,000 \\
15,400,000 \\
8,540,000 \\
6,780,000 \\
6,230,000 \\
6,240,000\end{array}$ & $\begin{array}{r}6,290000 \\
6,460000 \\
6,700.000 \\
7,500.000 \\
7,690.000 \\
11,900000 \\
16,180000 \\
15,260000 \\
13,820000 \\
10,020.000 \\
6,390000 \\
\mathbf{5 , 2 1 0} 000\end{array}$ & $\begin{array}{r}\mathbf{5}, 040,000 \\
\mathbf{5 , 1 4 0 , 0 0 0} \\
6,220,000 \\
11,300,000 \\
10,950,000 \\
- \\
\\
\end{array}$ \\
\hline
\end{tabular}

Storage: Three ground storage reservoirs; 5,000,000 gallons each; three elevated tanks, 1,000,000 gallons each; one elevated tank, 50C,000 gallons. Number of customers: 16,812 .

Treatment: None.

\section{Analyses}

[All samples collected June 23, 1948. pH: Palo Duro well, 7.5; Greeley well, 7.6; Bush well and Brinkman well, each 7.4. Palo Duro well and Greeley well analyzed by H. D. Smith; Bush well and Brinkman well analyzed by D. E. Weaver and H. D. Smith]

\begin{tabular}{|c|c|c|c|c|}
\hline & \multicolumn{2}{|c|}{ Palo Duro well 2} & \multicolumn{2}{|c|}{ Greeley well 1} \\
\hline & $\begin{array}{l}\text { Parts per } \\
\text { million }\end{array}$ & $\begin{array}{l}\text { Equivalents } \\
\text { per million }\end{array}$ & $\begin{array}{c}\text { Parts per } \\
\text { million }\end{array}$ & $\begin{array}{l}\text { Equivalents } \\
\text { per million }\end{array}$ \\
\hline 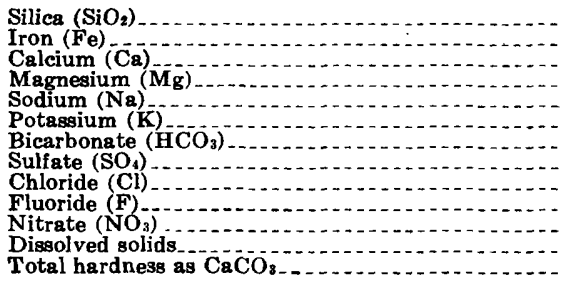 & $\begin{array}{l}52 \\
44 \\
43 \\
43 \\
23 \\
6.4 \\
354 \\
32 \\
6.7 \\
4.4 \\
5.1 \\
376 \\
287\end{array}$ & \begin{tabular}{r}
$\mathbf{2} .20$ \\
3.54 \\
1.00 \\
.16 \\
$\mathbf{5 . 8 0}$ \\
.67 \\
.19 \\
.23 \\
.08 \\
\hdashline-1.0 \\
\end{tabular} & $\begin{array}{l}80 \\
32.00 \\
43 \\
25 \\
8.0 \\
328 \\
34 \\
7.0 \\
3.2 \\
2.8 \\
372 \\
257\end{array}$ & $\begin{array}{r}1.60 \\
3.54 \\
1.09 \\
.20 \\
5.38 \\
.71 \\
.20 \\
.17 \\
.05 \\
\hdashline\end{array}$ \\
\hline
\end{tabular}




\begin{tabular}{|c|c|c|c|c|}
\hline & \multicolumn{2}{|c|}{ Bush well 1} & \multicolumn{2}{|c|}{ Brinkman well 1} \\
\hline & $\begin{array}{l}\text { Parts per } \\
\text { million }\end{array}$ & $\begin{array}{l}\text { Equivalents } \\
\text { per million }\end{array}$ & $\begin{array}{l}\text { Parts pe- } \\
\text { million }\end{array}$ & $\begin{array}{l}\text { Equivalents } \\
\text { per million }\end{array}$ \\
\hline 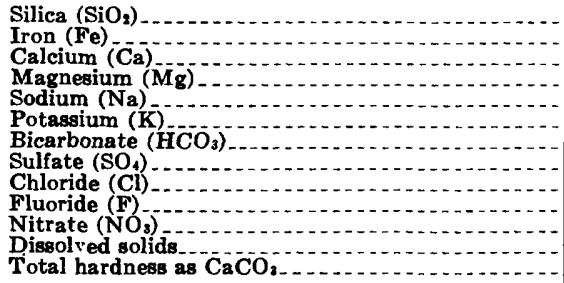 & $\begin{array}{l}67 \\
58 \\
26 \\
24 \\
6.00 \\
288 \\
50 \\
9.0 \\
2.4 \\
3.5 \\
382 \\
252\end{array}$ & $\begin{array}{r}2.89 \\
2.14 \\
1.04 \\
.15 \\
4.72 \\
1.04 \\
.25 \\
.13 \\
.06 \\
-1.2\end{array}$ & $\begin{array}{c}62 \\
48 \\
48 \\
27 \\
16 \\
9.2 \\
294 \\
25 \\
4.1 \\
2.8 \\
2.8 \\
341 \\
231\end{array}$ & $\begin{array}{r}2.40 \\
2.22 \\
.70 \\
.24 \\
4.82 \\
.52 \\
.12 \\
.15 \\
.05\end{array}$ \\
\hline
\end{tabular}

\section{Drillers' logs}

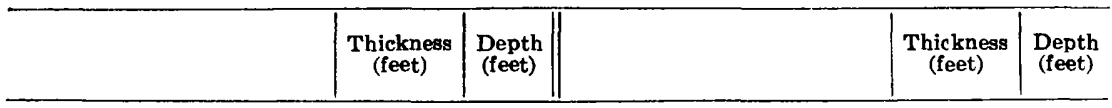

\section{Greeley well 1}

\begin{tabular}{|c|c|c|c|c|c|}
\hline $\begin{array}{l}\text { Soil } \\
\text { Caliche } \\
\text { Red sand } \\
\text { Saliche. } \\
\text { Sand and caliche. }\end{array}$ & $\begin{array}{r}3 \\
17 \\
20 \\
40 \\
20\end{array}$ & $\begin{array}{r}3 \\
20 \\
40 \\
80 \\
100\end{array}$ & $\begin{array}{l}\text { Red water sand } \\
\text { Water sand } \\
\text { Water sand and shells. } \\
\text { Water sand } \\
\text { Fine water sand }\end{array}$ & $\begin{array}{l}40 \\
60 \\
20 \\
20 \\
40\end{array}$ & $\begin{array}{l}140 \\
200 \\
220 \\
240 \\
280\end{array}$ \\
\hline
\end{tabular}

Greeley well 2

\begin{tabular}{|c|c|c|c|c|c|}
\hline $\begin{array}{l}\text { Soil } \\
\text { Red and yellow caliche. } \\
\text { Yellow sand, caliche. } \\
\text { Sand and shells } \\
\text { Red sand and shells. }\end{array}$ & $\begin{array}{l}3 \\
17 \\
40 \\
20 \\
10\end{array}$ & $\begin{array}{r}3 \\
20 \\
60 \\
80 \\
90\end{array}$ & $\begin{array}{l}\text { Water sand, shells } \\
\text { Red water sand and shells } \\
\text { Hard shell, sand. } \\
\text { Brown and yellow clay }\end{array}$ & $\begin{array}{r}30 \\
120 \\
20 \\
4\end{array}$ & $\begin{array}{r}120 \\
240 \\
+\quad 260 \\
264\end{array}$ \\
\hline
\end{tabular}

\section{Greeley well 3}

\begin{tabular}{|c|c|c|c|c|c|}
\hline Sand and caliche & $\begin{array}{r}3 \\
17 \\
60\end{array}$ & $\begin{array}{r}3 \\
20 \\
80\end{array}$ & $\begin{array}{l}\text { Sand and shells } \\
\text { Water sand } \\
\text { Red shale. }\end{array}$ & $\begin{array}{r}180 \\
19 \\
4\end{array}$ & $\begin{array}{l}260 \\
279 \\
283\end{array}$ \\
\hline
\end{tabular}

\section{Greeley well 4}

\begin{tabular}{|c|c|c|c|c|c|}
\hline $\begin{array}{l}\text { Soil } \\
\text { White caliche } \\
\text { Brown caliche } \\
\text { White caliche }\end{array}$ & $\begin{array}{r}3 \\
7 \\
12 \\
58\end{array}$ & $\begin{array}{r}3 \\
10 \\
22 \\
80\end{array}$ & $\begin{array}{l}\text { Muddy sand and shells } \\
\text { Red sand, coarse shells } \\
\text { Red sand, clay and shells... } \\
\text { Red beds. }\end{array}$ & $\begin{array}{r}70 \\
130 \\
15 \\
10\end{array}$ & $\begin{array}{l}150 \\
280 \\
295 \\
305\end{array}$ \\
\hline
\end{tabular}

\section{Greeley well 5}

\begin{tabular}{|c|c|c|c|c|c|}
\hline $\begin{array}{l}\text { Soil } \\
\text { Sand and clay } \\
\text { Saliche } \\
\text { Sand and caliche } \\
\text { Sand and shells. }\end{array}$ & $\begin{array}{r}7 \\
43 \\
30 \\
40 \\
10\end{array}$ & $\begin{array}{r}7 \\
50 \\
80 \\
120 \\
130\end{array}$ & $\begin{array}{l}\text { Sand } \\
\text { Sand and shells } \\
\text { Sand } \\
\text { Sand and shells } \\
\text { Red roek. }\end{array}$ & $\begin{array}{r}20 \\
20 \\
30 \\
62 \\
1\end{array}$ & $\begin{array}{l}150 \\
170 \\
200 \\
262 \\
263\end{array}$ \\
\hline
\end{tabular}




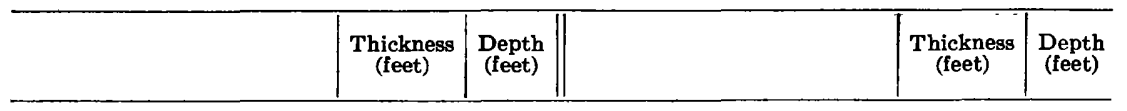

Bush well 1

\begin{tabular}{|c|c|c|c|c|c|}
\hline $\begin{array}{l}\text { Soil } \\
\text { Brown clay } \\
\text { Brown caliche } \\
\text { Brown caliche } \\
\text { Hard shell and gravel. }\end{array}$ & $\begin{array}{r}\mathbf{3} \\
\mathbf{5} \\
\mathbf{3 2} \\
\mathbf{3 5} \\
\mathbf{6}\end{array}$ & $\begin{array}{r}3 \\
8 \\
40 \\
75 \\
81\end{array}$ & $\begin{array}{l}\text { Brown sandy clay } \\
\text { Fine-grained sand } \\
\text { Sand and shell } \\
\text { Clay and shell } \\
\text { Red and blue shale }\end{array}$ & $\begin{array}{l}47 \\
32 \\
90 \\
10 \\
\mathbf{3 6}\end{array}$ & $\begin{array}{l}128 \\
160 \\
250 \\
260 \\
296\end{array}$ \\
\hline
\end{tabular}

Bush well 2

\begin{tabular}{|c|c|c|c|c|c|}
\hline $\begin{array}{l}\text { Soil } \\
\text { Gray caliche } \\
\text { Haray fine-grained sand. } \\
\text { Hay sand. }\end{array}$ & $\begin{array}{r}3 \\
17 \\
20 \\
60\end{array}$ & $\begin{array}{r}3 \\
20 \\
40 \\
100\end{array}$ & $\begin{array}{l}\text { Brown sand and shells } \\
\text { Clean water sand } \\
\text { Red and blue sand and shells } \\
\text { Gray sand. }\end{array}$ & $\begin{array}{r}54 \\
76 \\
11 \\
9\end{array}$ & $\begin{array}{l}154 \\
230 \\
241 \\
250\end{array}$ \\
\hline
\end{tabular}

Bush well 3

\begin{tabular}{|c|c|c|c|c|c|}
\hline $\begin{array}{l}\text { Soil } \\
\text { Gray caliche, sand } \\
\text { Red caliche, sand. } \\
\text { Dry sand, clay, and shells }\end{array}$ & $\begin{array}{r}5 \\
15 \\
40 \\
16\end{array}$ & $\begin{array}{r}5 \\
20 \\
60 \\
76\end{array}$ & $\begin{array}{l}\text { Dry sand, clay, and shells } \ldots \\
\text { Clean water sand. } \\
\text { Red shales and shells }\end{array}$ & $\begin{array}{r}54 \\
100 \\
9\end{array}$ & $\begin{array}{l}\mathbf{1 3 0} \\
\mathbf{2 3 0} \\
\mathbf{2 3 9}\end{array}$ \\
\hline
\end{tabular}

Bush well 4

\begin{tabular}{|c|c|c|c|c|c|}
\hline $\begin{array}{l}\text { Soil } \\
\text { Brown caliche clay } \\
\text { Sandy clay } \\
\text { Brown clay and caliche } \\
\text { Brown clay and sandy clay } \\
\text { Brown sand.. } \\
\text { Fine water sand. } \\
\text { Sand and shells. }\end{array}$ & $\begin{array}{l}4 \\
23 \\
13 \\
44 \\
31 \\
18 \\
60 \\
17\end{array}$ & $\begin{array}{r}4 \\
27 \\
40 \\
\mathbf{8 4} \\
115 \\
133 \\
193 \\
210\end{array}$ & $\begin{array}{l}\text { Sand and hard shells } \\
\text { Loose red sand } \\
\text { Hard shell, red and blue clay } \\
\text { Red, blue and white clay } \\
\text { Fine gray sand. } \\
\text { Hard shell. } \\
\text { Loose gray sand. } \\
\text { Red beds }\end{array}$ & $\begin{array}{r}19 \\
24 \\
22 \\
11 \\
\mathbf{3} \\
\mathbf{2} \\
\mathbf{6} \\
\mathbf{8}\end{array}$ & $\begin{array}{l}229 \\
253 \\
275 \\
286 \\
289 \\
291 \\
297 \\
305\end{array}$ \\
\hline
\end{tabular}

McDonald well 1

\begin{tabular}{|c|c|c|c|c|c|}
\hline $\begin{array}{l}\text { Top soil (clay and sand) } \\
\text { Caliche } \\
\text { Yellowish clay } \\
\text { Red sandy clay } \\
\text { Light sandy clay } \\
\text { Gray clayey sand } \\
\text { Soft red sandy clay } \\
\text { Honey combed sandrock } \\
\text { Red cavey sand. } \\
\text { Sandrock. } \\
\text { Soft honeycombed sandrock }\end{array}$ & $\begin{array}{r}4 \\
8 \\
58 \\
12 \\
13 \\
45 \\
5 \\
18 \\
6 \\
1 \\
12\end{array}$ & $\begin{array}{r}4 \\
12 \\
70 \\
82 \\
95 \\
140 \\
145 \\
163 \\
169 \\
170 \\
182\end{array}$ & $\begin{array}{l}\text { Sandrock } \\
\text { Sand and sand boulders } \\
\text { Sandy clay } \\
\text { Red cavey sand. } \\
\text { Very fine-grained sand } \\
\text { White clay } \\
\text { Red sand } \\
\text { White clay } \\
\text { Clean red sand } \\
\text { Red clay }\end{array}$ & $\begin{array}{r}4 \\
8 \\
19 \\
10 \\
15 \\
2 \\
11 \\
3 \\
5 \\
11\end{array}$ & $\begin{array}{l}186 \\
194 \\
213 \\
223 \\
238 \\
240 \\
251 \\
254 \\
259 \\
270\end{array}$ \\
\hline
\end{tabular}

MeDonald well 2

\begin{tabular}{|c|c|c|c|c|c|}
\hline $\begin{array}{l}\text { Soil } \\
\text { Red clay } \\
\text { Caliche } \\
\text { Yellow clay } \\
\text { Yellow sandy clay } \\
\text { Gray sandy clay } \\
\text { Light-gray sandy clay } \\
\text { Soft red sandy clay } \\
\text { Honeycombed sandrock. } \\
\text { Dirty gray sand. } \\
\text { Clean red sand.. }\end{array}$ & $\begin{array}{r}4 \\
13 \\
4 \\
47 \\
19 \\
5 \\
8 \\
48 \\
3 \\
9 \\
9\end{array}$ & $\begin{array}{r}4 \\
17 \\
21 \\
68 \\
87 \\
92 \\
100 \\
148 \\
151 \\
160 \\
163\end{array}$ & $\begin{array}{l}\text { Gray clayey sand } \\
\text { Loose sand boulders } \\
\text { Clean red sand. } \\
\text { Gray clayey sand. } \\
\text { Gray sand and sandrock. } \\
\text { Gray clayey sand. } \\
\text { Soft sandrock } \\
\text { Coarse sand and honey- } \\
\text { combed sandrock. } \\
\text { No record. }\end{array}$ & $\begin{array}{r}7 \\
6 \\
4 \\
3 \\
3 \\
14 \\
4 \\
6 \\
60\end{array}$ & $\begin{array}{l}170 \\
176 \\
180 \\
183 \\
186 \\
200 \\
204 \\
210 \\
270\end{array}$ \\
\hline
\end{tabular}

\section{MeDonald well 3}

\begin{tabular}{|c|c|c|c|c|c|}
\hline $\begin{array}{l}\text { Soil } \\
\text { Yellow clay clay } \\
\text { Gray sandy clay } \\
\text { Red sandy clay, soft } \\
\text { Red sandy clay, } \\
\text { Sandrock } \\
\text { Gray sand and rock } \\
\text { Coarse sand and sandrock. }\end{array}$ & $\begin{array}{r}4 \\
46 \\
12 \\
45 \\
43 \\
4 \\
2 \\
14\end{array}$ & $\begin{array}{r}4 \\
50 \\
62 \\
107 \\
150 \\
154 \\
156 \\
170\end{array}$ & $\begin{array}{l}\text { Honeycombed sand } \\
\text { Red sandstone } \\
\text { Red sand with thin clay } \\
\text { strata. } \\
\text { Red cavey sand } \\
\text { Clayey gray sand } \\
\text { Red caving sand } \\
\text { No record. }\end{array}$ & $\begin{array}{r}6 \\
14 \\
8 \\
10 \\
4 \\
3 \\
55\end{array}$ & $\begin{array}{l}176 \\
190 \\
198 \\
\\
208 \\
212 \\
215 \\
270\end{array}$ \\
\hline
\end{tabular}




\begin{tabular}{l|r|r||l|r|r}
\hline & $\begin{array}{c}\text { Thickness } \\
\text { (feet) }\end{array}$ & $\begin{array}{c}\text { Depth } \\
\text { (feet) }\end{array}$ & & $\begin{array}{r}\text { Thi,kness } \\
\text { (feet) }\end{array}$ & $\begin{array}{c}\text { Depth } \\
\text { (feet) }\end{array}$ \\
\hline
\end{tabular}

Brinkman well 1

\begin{tabular}{|c|c|c|c|c|c|}
\hline $\begin{array}{l}\text { Soil } \\
\text { Caliche } \\
\text { Brown caliche } \\
\text { Sand and shells } \\
\text { Fine-grained sand and shells. }\end{array}$ & $\begin{array}{r}4 \\
6 \\
110 \\
53 \\
\mathbf{3 5}\end{array}$ & $\begin{array}{r}4 \\
10 \\
120 \\
173 \\
208\end{array}$ & $\begin{array}{l}\text { Fine water sand, and shells } \\
\text { Red and blue shale and } \\
\text { shells. } \\
\text { Red, gray, and blue shells. - - }\end{array}$ & $\begin{array}{r}42 \\
6\end{array}$ & $\begin{array}{l}\mathbf{2 5} \\
\mathbf{2 5}\end{array}$ \\
\hline
\end{tabular}

\section{PRESIDIO COUNTY}

Population in 1940: 3,805 .

\section{MARFA}

Source of information: H. F. Dyer, water superintendent, July 1948.

Ownership: Municipal.

Source of supply: Three wells on city lot at standpipe.

Well 2. Drilled in 1928 by Layne-Texas Co.; depth, 881 feet; diameter, $15 \frac{1}{2}$ inches to 281 feet and 10 inches from 281 to 881 feet; deep-well turbine pump and 125-horsepower electric motor; yield, 1,000 gallons a minute.

Well 3. Drilled in 1936 by J. H. Cass; depth, 889 feet; d:æmeter, 10 inches to 306 feet and 8 inches from 306 to 889 feet; deep-well turbine pump and 100-horsepower electric motor; yield, 800 gallons a minute.

Well 4. Drilled in 1945 by Emmitt Harrell; depth, 1,100 feet; diameter, 16 inches to 314 feet and 11 inches from 314 to 1,100 feet; deep-well turbine pump and 65-horsepower electric motor; yield, 301 gallons a minute.

Pumpage (estimated): 1,000,000 gallons a day, which includes water sold to Fort D. A. Russell.

Storage: Ground reservoir, 83,000 gallons; elevated tank; 325,000 gallons. Number of customers: 1,200 .

Treatment: None.

Analysis, well 2

[Collected July 19, 1948. pH is 7.6. Analyzed by H. D. Smith]

\begin{tabular}{|c|c|c|c|c|c|}
\hline & $\begin{array}{l}\text { Parts per } \\
\text { million }\end{array}$ & $\begin{array}{l}\text { Equivalents } \\
\text { per million }\end{array}$ & & $\begin{array}{l}\text { Parts per } \\
\text { millior }\end{array}$ & $\begin{array}{l}\text { Equivalen's } \\
\text { per million }\end{array}$ \\
\hline 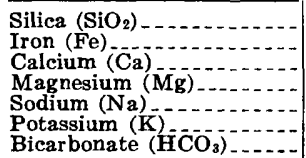 & $\begin{array}{l}72 \\
26 \\
2.05 \\
59 \\
13 \\
192\end{array}$ & $\begin{array}{r}1.298 \\
.181 \\
2.570 \\
.333 \\
3.147\end{array}$ & $\begin{array}{l}\text { Sulfate }\left(\mathrm{SO}_{4}\right) \\
\text { Chloride }(\mathrm{Cl}) \\
\text { Fluoride }(\mathrm{F}) \\
\text { Nitrate }\left(\mathrm{NO}_{3}\right) \\
\text { Dissolved solids } \\
\text { Total hardness as } \mathrm{CaCO}_{3}\end{array}$ & $\begin{array}{c}26 \\
17 \\
2.8 \\
4.2 \\
328 \\
74\end{array}$ & $\begin{array}{r}0.541 \\
.479 \\
.147 \\
.068 \\
-0.0 \\
\end{array}$ \\
\hline
\end{tabular}




\section{Drillers' logs}

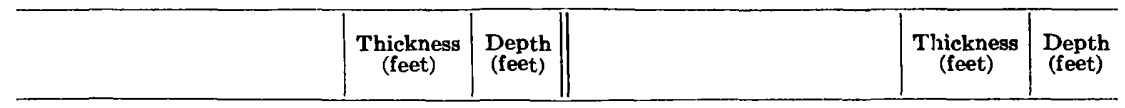

\begin{tabular}{|c|c|c|c|c|c|}
\hline \multicolumn{6}{|c|}{ Well 3} \\
\hline $\begin{array}{l}\text { Silt, sand, and gravel } \\
\text { Clay } \\
\text { Sand } \\
\text { Water sand } \\
\text { Hard sand } \\
\text { Sand and gravel. } \\
\text { Black rock } \\
\text { Pink clay } \\
\text { Sandy clay } \\
\text { Water sand } \\
\text { Sand } \\
\text { White clay } \\
\text { Hard rock }\end{array}$ & $\begin{array}{r}18 \\
20 \\
168 \\
34 \\
10 \\
49 \\
60 \\
3 \\
49 \\
15 \\
24 \\
46 \\
31\end{array}$ & $\begin{array}{r}18 \\
38 \\
206 \\
240 \\
250 \\
299 \\
359 \\
362 \\
411 \\
426 \\
450 \\
496 \\
527\end{array}$ & $\begin{array}{l}\text { Brown clay } \\
\text { Sandy shale } \\
\text { Broken lime } \\
\text { Sand and shale } \\
\text { Lava wash } \\
\text { Water sand } \\
\text { Brown clay } \\
\text { Water sand } \\
\text { Brown clay } \\
\text { Coarse sand } \\
\text { Sandrock. } \\
\text { Sandy clay } \\
\text { Water sand and gravel }\end{array}$ & $\begin{array}{r}\mathbf{5 3} \\
\mathbf{6} \\
14 \\
\mathbf{5 0} \\
\mathbf{4 7} \\
\mathbf{2 1} \\
29 \\
\mathbf{2 3} \\
\mathbf{2} \\
8 \\
\mathbf{3 2} \\
11\end{array}$ & $\begin{array}{l}\mathbf{5 8 0} \\
\mathbf{5 8 6} \\
\mathbf{6 0 0} \\
\mathbf{6 5 0} \\
\mathbf{6 9 7} \\
\mathbf{7 1 8} \\
\mathbf{7 4 7} \\
\mathbf{7 7 0} \\
\mathbf{7 7 2} \\
780 \\
812 \\
823 \\
\mathbf{8 8 9}\end{array}$ \\
\hline
\end{tabular}

\begin{tabular}{|c|c|c|c|c|c|}
\hline \multicolumn{6}{|c|}{ Well 4} \\
\hline $\begin{array}{l}\text { Soil and clay } \\
\text { Sand and gravel } \\
\text { Brown rock. } \\
\text { Brown sand } \\
\text { Red rock } \\
\text { Sand and gravel } \\
\text { Sticky clay } \\
\text { Clay and some gravel } \\
\text { Clay } \\
\text { Clay and gravel. }\end{array}$ & $\begin{array}{r}30 \\
268 \\
60 \\
212 \\
8 \\
222 \\
30 \\
20 \\
10 \\
10\end{array}$ & $\begin{array}{r}30 \\
298 \\
358 \\
\mathbf{5 7 0} \\
\mathbf{5 7 8} \\
800 \\
830 \\
850 \\
860 \\
870\end{array}$ & $\begin{array}{l}\text { Sand and gravel } \\
\text { Clay } \\
\text { Gravel } \\
\text { Clay } \\
\text { Brown lava rock. } \\
\text { Clay and lava rock } \\
\text { Brown lava rock } \\
\text { Clay and lava rock } \\
\text { Broken clay and rock. }\end{array}$ & $\begin{array}{r}\mathbf{5} \\
\mathbf{9} \\
\mathbf{5} \\
1 \\
75 \\
\mathbf{2 5} \\
\mathbf{1 4} \\
\mathbf{2 9} \\
\mathbf{6 7}\end{array}$ & $\begin{array}{r}875 \\
884 \\
889 \\
890 \\
965 \\
990 \\
1004 \\
1083 \\
1100\end{array}$ \\
\hline
\end{tabular}

\section{PREsidio}

Population in 1940: 1,500 .

Source of information: A. H. Spangle, owner, July 1948.

Source of supply: Two wells; depth, 48 feet; diameter, 12 inches; piston-type pumps and 15-horsepower electric motors.

Pumpage (estimated): 130,000 gallons a day.

Storage: None; pumped directly into pipeline.

Number of customers: 115.

Treatment: None.

\section{Analysis}

[Composite sample, collected July 19, 1948. pH is 7.4. Analyed by H. D. Smith]

\begin{tabular}{|c|c|c|c|c|c|}
\hline & $\begin{array}{l}\text { Parts per } \\
\text { million }\end{array}$ & $\begin{array}{l}\text { Equivalents } \\
\text { per million }\end{array}$ & & $\begin{array}{l}\text { Parta per } \\
\text { mill ion }\end{array}$ & $\begin{array}{l}\text { Equivalents } \\
\text { per million }\end{array}$ \\
\hline $\begin{array}{l}\text { Silica }\left(\mathrm{SiO}_{2}\right) \\
\text { Iron }(\mathrm{Fe}) \\
\text { Calcium }(\mathrm{Ca}) \\
\text { Magnesium }(\mathrm{Mg}) \\
\text { Sodium }(\mathrm{Na}) \\
\text { Potassium }(\mathrm{K}) \\
\text { Bicarbonate }\left(\mathrm{HCOO}_{3}\right)\end{array}$ & $\begin{array}{l}60 \\
78.05 \\
9.8 \\
63 \\
8.4 \\
260\end{array}$ & $\begin{array}{r}3.89 \\
.81 \\
2.72 \\
.21 \\
4.26\end{array}$ & $\begin{array}{l}\text { Sulfate (SOA) } \\
\text { Chloride (Cl) } \\
\text { Fluoride (F) } \\
\text { Nitrate (NO } \mathrm{N}_{3} \text { ) } \\
\text { Dissolved solids. } \\
\text { Total hardness as } \mathrm{CaCO}_{2}\end{array}$ & $\begin{array}{r}114 \\
29 \\
1.2 \\
9.6 \\
524 \\
23\end{array}$ & $\begin{array}{r}2.37 \\
.79 \\
.06 \\
.15 \\
\\
\hdashline-1 .-\end{array}$ \\
\hline
\end{tabular}

\section{RANDALL COUNTY}

\section{CANYON}

Population in 1940: 2,600.

Source of information: C. L. Key, water superintendent, Dec. 4. 1947.

Ownership: Municipal. 
Source of supply: Four wells.

Pomona well 1. Drilled in 1930 by Omer Kersey; depth, 490 feet; diameter, $15 \frac{1}{2}$ to $12 \frac{1}{2}$ inches; deep-well turbine pump and electric motor; static water level, 250 feet below land surface; pumping lerel, 425 feet below land surface; yield, 200 gallons a minute; temperature, $66^{\circ} \mathrm{F}$.

Pomona well 2. Drilled in 1930 by Omer Kersey; depth, 520 feet; diameter, $15 \frac{1}{2}$ to $12 \frac{1}{2}$ inches; deep-well turbine pump and electric motor; yield, 250 gallons a minute.

Utility well. Drilled; depth, 490 feet; diameter, $15 \frac{1}{12}$ to $12 \frac{1}{2}$ inches; deepwell turbine pump and electric motor; pumping level, 335 feet below land surface while pumping 130 gallons a minute and 351 feet below land surface while pumping 185 gallons a minute; yield, 150 gallons a minute.

Muncey well. Drilled in 1943 by Muncey Bros.; depth, 504 fee $^{+}$; diameter, 14 to 10 inches; deep-well turbine pump and electric motor; yield, 450 gallons a minute.

\section{Average pumpage, in gallons a day}

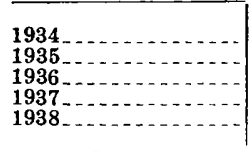

\begin{tabular}{|c|c|c|c|c|}
\hline $\begin{array}{l}161,000 \\
185,000 \\
171,000 \\
181,000 \\
187,000\end{array}$ & $\begin{array}{l}1939 \ldots \ldots \\
1940 \\
1941 \\
1942 \\
1943 \ldots \ldots\end{array}$ & $\begin{array}{l}213,000 \\
251,000 \\
249,000 \\
189,000 \\
235,000\end{array}$ & $\begin{array}{l}1944 \ldots 1 \\
1945 \\
1946\end{array}$ & $\begin{array}{l}296,000 \\
246,000 \\
384,000 \\
430,000\end{array}$ \\
\hline
\end{tabular}

Storage: Ground storage reservoir, 750,000 gallons; elevated tank, 250,000 gallons.

Treatment: None.

\section{Analysis}

[Composite sample of four wells, collected Dec. 4, 1947. $\mathrm{pH}$ is 8.0. Analyzed by D. E. Weaver]

\begin{tabular}{|c|c|c|c|c|c|}
\hline & $\begin{array}{l}\text { Parts per } \\
\text { million }\end{array}$ & $\begin{array}{l}\text { Equivalents } \\
\text { per million }\end{array}$ & & $\begin{array}{l}\text { Parts piłr } \\
\text { millior }\end{array}$ & $\begin{array}{l}\text { Equivalents } \\
\text { per million }\end{array}$ \\
\hline $\begin{array}{l}\text { Silica }\left(\mathrm{SiO}_{2}\right) \\
\text { Iron }(\mathrm{Fe}) \\
\text { Calcium }(\mathrm{Ca}) \\
\text { Magnesium }(\mathrm{Mg}) \\
\text { Sodium }(\mathrm{Na}) \\
\text { Potassium }(\mathrm{K}) \\
\text { Bicarbonate }\left(\mathrm{H} \overline{C O}_{3}\right)\end{array}$ & $\begin{array}{r}11 \\
7.05 \\
7.7 \\
5.8 \\
135 \\
3.2 \\
341\end{array}$ & $\begin{array}{r}.38 \\
.48 \\
5.86 \\
.08 \\
5.59\end{array}$ & $\begin{array}{l}\text { Sulfate }\left(\mathrm{SO}_{4}\right) \\
\text { Chloride }(\mathrm{Cl}) \\
\text { Fluoride (F) } \\
\left.\text { Nitrate (NO})_{3}\right) \\
\text { Dissolved solids. } \\
\text { Total hardness as } \mathrm{CaCO}\end{array}$ & $\begin{array}{c}29 \\
17 \\
2.2 \\
390 \\
43\end{array}$ & \begin{tabular}{r}
0.60 \\
.48 \\
.12 \\
.01 \\
\hdashline.- \\
\end{tabular} \\
\hline
\end{tabular}

\section{Driller's logs}

\begin{tabular}{|c|c|c|c|c|c|}
\hline & $\underset{\text { (feet) }}{\text { Thickness }}$ & $\begin{array}{c}\text { Depth } \\
\text { (feet) }\end{array}$ & & $\begin{array}{c}\text { Thickness } \\
\text { (f ?et) }\end{array}$ & $\begin{array}{c}\text { Depth } \\
\text { (feet) }\end{array}$ \\
\hline \multicolumn{6}{|c|}{ Pomona well 1} \\
\hline $\begin{array}{l}\text { Soil. } \\
\text { Gypsum } \\
\text { Pink shale } \\
\text { Gypsum } \\
\text { Red sandy shale. } \\
\text { Brown shale } \\
\text { Blue sandy shale } \\
\text { Red shale } \\
\text { Blue shale } \\
\text { Pink sandy shale. }\end{array}$ & $\begin{array}{r}3 \\
62 \\
19 \\
3 \\
38 \\
20 \\
53 \\
34 \\
13 \\
\mathbf{2 0}\end{array}$ & $\begin{array}{r}3 \\
65 \\
84 \\
87 \\
87 \\
125 \\
145 \\
198 \\
232 \\
245 \\
245 \\
265\end{array}$ & $\begin{array}{l}\text { Light-brown shale } \\
\text { White water sand } \\
\text { Red shale } \\
\text { Hard sandy shale } \\
\text { Brown shale } \\
\text { White water sand } \\
\text { Blue shale } \\
\text { Water sand } \\
\text { Blue sandy shale }\end{array}$ & $\begin{array}{r}72 \\
20 \\
6 \\
30 \\
17 \\
33 \\
3 \\
39 \\
\mathbf{3}\end{array}$ & $\begin{array}{l}337 \\
357 \\
363 \\
393 \\
410 \\
443 \\
446 \\
485 \\
488\end{array}$ \\
\hline
\end{tabular}

$947535-51-10$ 


\begin{tabular}{|c|c|c|c|c|c|}
\hline 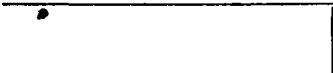 & $\begin{array}{c}\text { Thickness } \\
\text { (feet) }\end{array}$ & $\begin{array}{l}\text { Depth } \\
\text { (feet) }\end{array}$ & & $\underset{\text { (feet) }}{\text { Thickness }}$ & $\begin{array}{l}\text { Depth } \\
\text { (feet) }\end{array}$ \\
\hline \multicolumn{6}{|c|}{ Pomona well 2} \\
\hline $\begin{array}{l}\text { No record } \\
\text { Light-brown shale. } \\
\text { Sand, no water. } \\
\text { White water sand } \\
\text { Red shale... }\end{array}$ & $\begin{array}{r}265 \\
60 \\
10 \\
15 \\
13\end{array}$ & $\begin{array}{l}\mathbf{2 6 5} \\
\mathbf{3 2 5} \\
\mathbf{3 3 5} \\
\mathbf{3 5 0} \\
\mathbf{3 6 3}\end{array}$ & $\begin{array}{l}\text { H S S (Hard sandstone ?) } \\
\text { Brown shale } \\
\text { Sand, no water } \\
\text { White water sand } \\
\text { Blue sandy shale. }\end{array}$ & $\begin{array}{r}\mathbf{3 0} \\
\mathbf{2 7} \\
\mathbf{5} \\
\mathbf{1 5} \\
\mathbf{5 0}\end{array}$ & $\begin{array}{l}393 \\
420 \\
425 \\
440 \\
490\end{array}$ \\
\hline \multicolumn{6}{|c|}{ Utility well } \\
\hline $\begin{array}{l}\text { Soil } \\
\text { Pink clay } \\
\text { Caliche and little lime rock } \\
\text { sand. } \\
\text { Sand and caliche, red and } \\
\text { gray. } \\
\text { White shale, first water } \\
\text { Red shale. } \\
\text { Brown shale. } \\
\text { Blue shale. } \\
\text { Red shale. }\end{array}$ & $\begin{array}{r}5 \\
10 \\
30 \\
10 \\
10 \\
25 \\
25 \\
45 \\
30 \\
105\end{array}$ & $\begin{array}{r}5 \\
15 \\
45 \\
55 \\
65 \\
90 \\
115 \\
160 \\
190 \\
295\end{array}$ & $\begin{array}{l}\text { Brown shale } \\
\text { White sandstone. } \\
\text { Red shale } \\
\text { Gray sandstone } \\
\text { Blue shale } \\
\text { Gray sandstone } \\
\text { Red shale and a little gray } \\
\text { sandstone. } \\
\text { White sandstone } \\
\text { good). } \\
\text { Red shale. }\end{array}$ & $\begin{array}{r}\mathbf{3 3} \\
\mathbf{2 2} \\
\mathbf{3 4} \\
\mathbf{3 0} \\
\mathbf{3} \\
\mathbf{2 3} \\
\mathbf{1 6} \\
\mathbf{2 9} \\
\mathbf{5}\end{array}$ & $\begin{array}{l}328 \\
350 \\
384 \\
414 \\
417 \\
440 \\
456 \\
485 \\
490\end{array}$ \\
\hline
\end{tabular}

\section{REAGAN COUNTY}

\section{BIG LAKE}

Population in 1940: 763.

Source of information: Ross Clark, city secretary, Aug. 21, 1947.

Ownership: Municipal.

Source of supply: Two wells.

Well 2. Half a block south of the county courthouse near el avated tank; depth, 535 feet; diameter, 6 inches; deep-well turbine prmp and 25horsepower electric motor; pump set at 390 feet; pumpin? level, 329.3 feet below land surface while pumping 140 gallons a minute Sept. 11, 1947; temperature, $69^{\circ} \mathrm{F}$.

Well 3. Two blocks south of county courthouse; drilled in 1944 by W. A. Schooler; depth, 535 feet; diameter, 7 inches; cased to kottom; deepwell turbine pump and 25-horsepower electric motor, pump set at 410 feet; pumping level, 242.6 feet below land surface while pumping 150 gallons a minute Sept. 11, 1947 ; temperature, $69^{\circ} \mathrm{F}$.

\section{Average pumpage, in gallons a day}

\begin{tabular}{|c|c|c|c|c|c|c|c|c|}
\hline & 1946 & 1947 & & 1946 & 1947 & & 1946 & 1947 \\
\hline $\begin{array}{l}\text { January } \\
\text { February } \\
\text { March. } \\
\text { April. }\end{array}$ & $\begin{array}{l}41,840 \\
41,700 \\
57,000\end{array}$ & $\begin{array}{l}46,800 \\
54,600 \\
50,000 \\
55,400\end{array}$ & $\begin{array}{l}\text { May... } \\
\text { June... } \\
\text { July. } \\
\text { August. }\end{array}$ & $\begin{array}{l}69,900 \\
72,200 \\
86,800 \\
85,000\end{array}$ & $\begin{array}{r}79,600 \\
108,000 \\
123,200 \\
\end{array}$ & $\begin{array}{l}\text { September... } \\
\text { October..... } \\
\text { November ..- } \\
\text { December... }\end{array}$ & $\begin{array}{r}111,200 \\
58,200 \\
56,700 \\
44,800\end{array}$ & 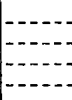 \\
\hline
\end{tabular}

Storage: Elevated tank, 65,000 gallons; concrete ground tank, 10^,000 gallons. Number of customers: 315 .

Treatment: Occasional chlorination. 


\section{Analyses}

[Collected Sept. 11, 1947. pH: well 2, 7.3; well 3, 7.4. Analyzed by B. C. Dwyer]

\begin{tabular}{|c|c|c|c|c|}
\hline \multirow[t]{2}{*}{ - } & \multicolumn{2}{|c|}{ Well 2} & \multicolumn{2}{|c|}{ T'ell 3} \\
\hline & $\begin{array}{l}\text { Parts per } \\
\text { million }\end{array}$ & $\begin{array}{l}\text { Equivalents } \\
\text { per million }\end{array}$ & $\begin{array}{l}\text { Parts per } \\
\text { million }\end{array}$ & $\begin{array}{l}\text { Equivalents } \\
\text { per million }\end{array}$ \\
\hline 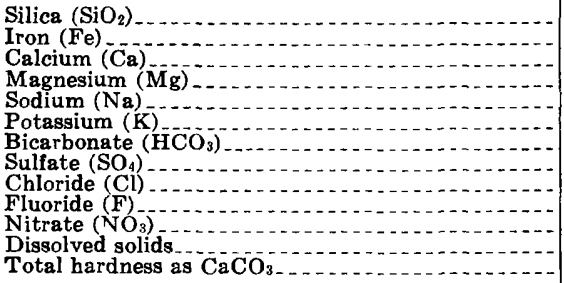 & $\begin{array}{l}6.0 \\
182^{.09} \\
109 \\
289 \\
24 \\
274 \\
933 \\
254 \\
2.4 \\
1,940^{.8} \\
902\end{array}$ & $\begin{array}{r}9.08 \\
8.96 \\
12.56 \\
.61 \\
4.49 \\
19.42 \\
7.16 \\
.13 \\
.01 \\
\end{array}$ & $\begin{array}{c}6.0 \\
167^{.0 \mathrm{~S}} \\
108 \\
306 \\
28 \\
274 \\
947 \\
244 \\
2.6 \\
1^{.0} \\
1,940 \\
861\end{array}$ & $\begin{array}{r}8.34 \\
8.88 \\
13.30 \\
.72 \\
4.49 \\
19.72 \\
6.88 \\
.14 \\
.01\end{array}$ \\
\hline
\end{tabular}

\section{SANTA RITA}

Population in 1940: 75 .

Source of information: M. B. Rogers, clerk, Sept. 11, 1947.

Owner: Group No. 1 Oil Co.

Source of supply: Two wells about 2 miles northeast of camp.

Well 1. Drilled by Signal Gasoline Co.; depth, 325 feet; diameter, 12 inches; cylinder pump and 5-horsepower electric motor; static water level, 192.9 feet below land surface Sept. 11, 1947; yield reported, 15 gallons a minute; water level measured while well 2 was pumping.

Well 2. Drilled by Signal Gasoline Co.; depth, 460 feet; diameter, 12 inches; deep-well turbine pump and $7 \frac{1}{2}$-horsepower electric motor; pump set at 230 feet; water level reported, 190 feet below lard surface; yield reported, 44 gallons a minute; temperature, $691 / 2^{\circ} \mathrm{F}$.

Pumpage: Maximum, 63,000 gallons a day; minimum, 30,000 gallıns a day; average, 42,000 gallons a day.

Storage: Two wooden ground tanks, 67,000 gallons each; one steel ground tank, 462,000 gallons.

Number of customers: 35 .

Treatment: None.

Analysis, well 2

[Collected Sept. 11, 1947. pH is 7.7. Analyzed by B. C. Dwyer]

\begin{tabular}{|c|c|c|c|c|c|}
\hline & $\begin{array}{c}\text { Parts per } \\
\text { million }\end{array}$ & $\begin{array}{l}\text { Equivalents } \\
\text { per million }\end{array}$ & & $\begin{array}{c}\text { Parts pe } \\
\text { million }\end{array}$ & $\begin{array}{l}\text { Equivalents } \\
\text { per million }\end{array}$ \\
\hline $\begin{array}{l}\text { Silica }\left(\mathrm{SiO}_{2}\right) \\
\text { Iron }(\mathrm{Fe}) \\
\text { Calcium }(\mathrm{Ca}) \\
\text { Magnesium }(\mathrm{Mg}) \\
\text { Sodium }(\mathrm{Na}) \\
\text { Potassium }(\mathrm{K}) \\
\text { Bicarbonate }\left(\mathrm{H}^{2} \mathrm{CO}_{3}\right)\end{array}$ & $\begin{array}{l}10 \\
149 \\
93 \\
574 \\
21 \\
252\end{array}$ & $\begin{array}{r}7.44 \\
7.65 \\
24.97 \\
.54 \\
4.13\end{array}$ & $\begin{array}{l}\text { Sulfate }\left(\mathrm{SO}_{4}\right) \\
\text { Chloride (Cl) } \\
\text { Fluoride (F) } \\
\text { Nitrate (NO} \\
\text { Dissolved solids. } \\
\text { Total hardness as } \mathrm{CaCO}\end{array}$ & $\begin{array}{c}704 \\
760 \\
2.6 \\
15 \\
2,450 \\
754\end{array}$ & $\begin{array}{r}14.66 \\
21.43 \\
.14 \\
.24 \\
-.24 \\
-. .2-\end{array}$ \\
\hline
\end{tabular}

\section{TEXON}

Population in 1940: 1,123.

Source of information: W. J. Grissett, assistant superintendent, Aug. 20, 1947.

Owner: Big Lake Oil Co. 
Source of supply: Six wells.

Well 1. Three miles north of Texon; drilled in 1932; depth, 359 feet; diameter, 7 inches; deep-well cylinder pump and electric motor; yield, 53 gallons a minute.

Well 2. Three miles north of Texon; drilled in 1932; depth, 360 feet; diameter, 7 inches; deep-well cylinder pump and electric motor; yield, 53 gallons a minute.

Well 3. Three miles north of Texon; drilled in 1938; depth, 359 feet; diameter, 7 inches; deep-well cylinder pump and electric motor; yield, 53 gallons a minute.

Well 4. At gasoline plant one-fourth mile northwest of Texon; drilled in 1925; depth, 400 feet; diameter, 7 inches; deep-well cylinder pump; yield, 18 gallons a minute.

Well 5. At gasoline plant one-fourth mile northwest of Texon; drilled in 1925; depth, 400 feet; diameter, 7 inches; deep-well cylinder pump; yield, 7 gallons a minute.

Well 6. One and a half miles southwest of Texon; drilled about 1927; depth, 450 feet; diameter 7 inches; deep-well cylinder pump and electric motor; yield, 11 gallons a minute.

Pumpage (estimated) : 140,000 gallons a day.

Storage: Seven steel tanks, total capacity 111,000 gallons.

Number of customers: 1,123 .

Treatment: None.

\section{Analyses}

[Collected: well 1, Aug. 20, 1947; wells 3 and 5, Sept. 11, 1947. pH: well 1, 7.8; wells 3 and 5, each 7.7. Analyzed by B. C. Dwyer]

\begin{tabular}{|c|c|c|c|c|c|c|}
\hline & \multicolumn{2}{|c|}{ Well 1} & \multicolumn{2}{|c|}{ Well 3} & \multicolumn{2}{|c|}{ Well 5} \\
\hline & $\begin{array}{l}\text { Parts per } \\
\text { million }\end{array}$ & $\begin{array}{l}\text { Equivalents } \\
\text { per million }\end{array}$ & $\begin{array}{l}\text { Parts per } \\
\text { million }\end{array}$ & $\begin{array}{c}\text { Equivalents } \\
\text { per million }\end{array}$ & $\begin{array}{l}\text { Parts per } \\
\text { million }\end{array}$ & $\begin{array}{l}\text { Equivalents } \\
\text { per million }\end{array}$ \\
\hline 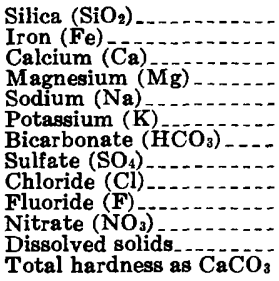 & $\begin{array}{c}17 \\
155 \\
90 \\
387 \\
6.32 \\
260 \\
903 \\
310 \\
2.4 \\
11 \\
2,010 \\
757\end{array}$ & $\begin{array}{r}7.74 \\
7.40 \\
16.81 \\
.16 \\
4.26 \\
18.80 \\
8.74 \\
.13 \\
.18 \\
\hdashline\end{array}$ & $\begin{array}{c}11 \\
136 \\
84 \\
238 \\
16 \\
246 \\
813 \\
112 \\
2.6 \\
11 \\
1,540 \\
685\end{array}$ & $\begin{array}{r}6.79 \\
6.91 \\
10.33 \\
.41 \\
4.03 \\
16.93 \\
3.16 \\
.14 \\
.18 \\
\\
\end{array}$ & $\begin{array}{r}8.0 \\
3.9 \\
156 \\
103 \\
285 \\
19 \\
262 \\
985 \\
144 \\
2.6 \\
9.0 \\
1,840 \\
813\end{array}$ & $\begin{array}{r}7.79 \\
8.47 \\
12.40 \\
4.49 \\
4.29 \\
20.51 \\
4.06 \\
.14 \\
.15\end{array}$ \\
\hline
\end{tabular}

Drillers' logs

\begin{tabular}{|c|c|c|c|c|c|}
\hline & $\underset{\text { (feet) }}{\text { Thickness }}$ & $\begin{array}{c}\text { Depth } \\
\text { (feet) }\end{array}$ & & $\underset{\text { (feet) }}{\text { Thickness }}$ & $\begin{array}{l}\text { Depth } \\
\text { (feet) }\end{array}$ \\
\hline \multicolumn{6}{|c|}{ Well 1} \\
\hline $\begin{array}{l}\text { Surface soil- } \\
\text { Yellow lime } \\
\text { White lime- } \\
\text { Yellow lime } \\
\text { Sandy lime } \\
\text { Brown sandy rock }\end{array}$ & $\begin{array}{r}10 \\
40 \\
105 \\
20 \\
25 \\
20\end{array}$ & $\begin{array}{r}10 \\
50 \\
155 \\
175 \\
200 \\
220\end{array}$ & $\begin{array}{l}\text { Sand, water-.... } \\
\text { Red beds } \\
\text { White sand } \\
\text { Red beds } \\
\text { White sand. } \\
\text { Red beds }\end{array}$ & $\begin{array}{r}46 \\
14 \\
58 \\
4 \\
14 \\
3\end{array}$ & $\begin{array}{l}266 \\
280 \\
338 \\
342 \\
356 \\
\mathbf{3 5 9}\end{array}$ \\
\hline
\end{tabular}




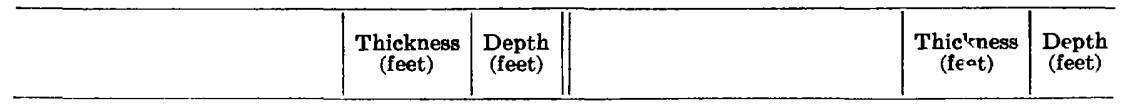

\begin{tabular}{|c|c|c|c|c|c|}
\hline \multicolumn{6}{|c|}{ Well 2} \\
\hline $\begin{array}{l}\text { Surface soil } \\
\text { Lime } \\
\text { Sand } \\
\text { Lime } \\
\text { Sand } \\
\text { Rime beds } \\
\text { Broken lime and red rock. }\end{array}$ & $\begin{array}{r}17 \\
200 \\
18 \\
3 \\
7 \\
13 \\
14 \\
28\end{array}$ & $\begin{array}{r}17 \\
217 \\
235 \\
238 \\
245 \\
258 \\
272 \\
300\end{array}$ & $\begin{array}{l}\text { Sand, water } \\
\text { Sandy lime } \\
\text { Lime and red rock } \\
\text { Hard sandrock } \\
\text { Sand, water } \\
\text { Hard sandrock. } \\
\text { Red rock. }\end{array}$ & $\begin{array}{r}12 \\
8 \\
10 \\
15 \\
5 \\
8 \\
2\end{array}$ & $\begin{array}{l}\mathbf{3 1 2} \\
\mathbf{3 2 0} \\
\mathbf{3 3 0} \\
\mathbf{3 4 5} \\
\mathbf{3 5 0} \\
\mathbf{3 5 8} \\
\mathbf{3 6 0}\end{array}$ \\
\hline
\end{tabular}

Well 3

\begin{tabular}{|c|c|c|c|c|c|}
\hline $\begin{array}{l}\text { Surface soil. } \\
\text { White lime } \\
\text { Yellow lime } \\
\text { White lime and sand } \\
\text { Sand, water } \\
\text { Red beds }\end{array}$ & $\begin{array}{r}12 \\
38 \\
125 \\
45 \\
46 \\
8\end{array}$ & $\begin{array}{r}12 \\
50 \\
175 \\
220 \\
266 \\
274\end{array}$ & $\begin{array}{l}\text { Sandrock } \\
\text { Sand, water } \\
\text { Red rock. } \\
\text { Sand, rock } \\
\text { Red rock }\end{array}$ & $\begin{array}{r}6 \\
58 \\
4 \\
14 \\
\mathbf{3}\end{array}$ & $\begin{array}{l}280 \\
338 \\
342 \\
356 \\
359\end{array}$ \\
\hline
\end{tabular}

\section{REEVES COUNTY}

\section{BALMORHEA}

Population in 1940: 1,000.

Source of information: W. E. Gould, owner, Dec. 11, 1946.

Owner: W. E. Gould.

Source of supply: Two wells.

Well 1. Four and a half miles west of city; drilled in 1928; depth, 79 feet; diameter, 6 inches; deep-well cylinder and 2-horsepow or electric motor.

Well 2. One and a half miles west of city; drilled in 1928; depth, 67 feet; diameter, 6 inches; deep-well cylinder and 2-horsepower elec'ric motor; yield, 15 gallons a minute.

Pumpage (estimated): Average, 21,000 gallons a day.

Storage: Two concrete storage reservoirs, 7,000 gallons each.

Number of customers: 60 .

Treatment: Chlorination.

\section{Analysis}

[Composite sample of wells 1 and 2. Collected Dec. 11, 1946. pH is 7.0. Analyzed by C. B. Cibulka]

\begin{tabular}{|c|c|c|c|c|c|}
\hline & $\begin{array}{l}\text { Parts per } \\
\text { million }\end{array}$ & $\begin{array}{l}\text { Equivalents } \\
\text { per million }\end{array}$ & & $\begin{array}{l}\text { Parts pe } \\
\text { million }\end{array}$ & $\begin{array}{l}\text { Equivalents } \\
\text { per million }\end{array}$ \\
\hline $\begin{array}{l}\text { Silica }\left(\mathrm{SiO}_{2}\right) \\
\text { Iron }(\mathrm{Fe}) \\
\text { Calcium }(\mathrm{Ca}) \\
\text { Magnesium }(\mathrm{Mg}) \\
\text { Sodium }(\mathrm{Na}) \\
\text { Potassium }(\mathrm{K}) \\
\text { Bicarbonate }\left(\mathrm{HCO}_{3}\right)^{-}\end{array}$ & $\begin{array}{l}32 \\
178 \\
29 \\
274 \\
27.78 \\
376\end{array}$ & $\begin{array}{r}8.88 \\
2.38 \\
11.92 \\
.17 \\
6.16\end{array}$ & $\begin{array}{l}\text { Sulfate }\left(\mathrm{SO}_{4}\right) \\
\text { Chloride }(\mathrm{Cl}) \\
\text { Fluoride (F) } \\
\left.\text { Nitrate (NO }{ }_{2}\right) \\
\text { Dissolved solids. } \\
\text { Total hardness as } \mathrm{CaCO}_{3}\end{array}$ & $\begin{array}{c}517 \\
220 \\
12 \\
1,450 \\
563\end{array} .8$ & $\begin{array}{r}10.76 \\
6.20 \\
.04 \\
.19 \\
\\
\end{array}$ \\
\hline
\end{tabular}

\section{PECOS}

Population in 1940: 4,855.

Source of information: J. H. Oglesby, water superintendent, Dec. 17, 1946. Ownership: Municipal. 
Source of supply: Five wells, $91 / 2$ miles southeast of city.

Well 1. Drilled in 1933 by Tom Simmonds; depth, 187 f set; diameter, 10 inches; deep-well turbine pump and 20-horsepower electric motor; static water level, 87.8 feet below land surface Oct. 10, 1940; yield, 250 gallons a minute.

Well 2. Drilled in 1935 by Tom Simmonds; depth, 211 feet; diameter, 10 inches; deep-well turbine pump and 20-horsepower electric motor; yield, 300 gallons a minute.

Well 3. Drilled in 1935 by Tom Simmonds; depth, 300 feet; diameter, 10 inches; deep-well turbine pump and 20-horsepower electric motor; yield, 200 gallons a minute.

Well 4. Drilled in 1942 by D. M. Bassett; depth, 191 feet; diameter, 10 inches; deep-well turbine pump and 20 -horsepower electric motor; yield, 300 gallons a minute.

Well 5. Drilled in 1942 by D. M. Bassett; depth, 170 feet; diameter, 10 inches; deep-well turbine pump and 20-horsepower electric motor; yield, 300 gallons a minute.

Average pumpage, in gallons a day

\begin{tabular}{|c|c|c|c|c|c|c|c|c|}
\hline & 1945 & 1946 & & 1945 & 1946 & & 1945 & 1946 \\
\hline $\begin{array}{l}\text { January... } \\
\text { February.. } \\
\text { March... } \\
\text { April }\end{array}$ & 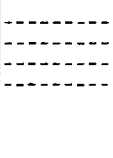 & $\begin{array}{l}452,600 \\
451,100 \\
571,500 \\
687,100\end{array}$ & $\begin{array}{l}\text { May_- } \\
\text { June._- } \\
\text { July_-:- } \\
\text { August_. }\end{array}$ & - & $\begin{array}{l}719,300 \\
890,100 \\
968,600 \\
972,900\end{array}$ & $\begin{array}{l}\text { September } \\
\text { October-- } \\
\text { November- } \\
\text { December - }\end{array}$ & 40 & $\begin{array}{c}696,300 \\
546,100 \\
481,000 \\
425,700 \\
\text { first } 15 \\
\text { days of } \\
\text { month }\end{array}$ \\
\hline
\end{tabular}

Storage: Concrete storage reservoir, 1,500,000 gallons; ground storage reservoir, 200,000 gallons; elevated tank, 110,000 gallons.

Number of customers: 1,336 .

Treatment: None.

\section{Analysis}

[Composite sample of five wells. Cellected Sept. 4, 1946. pH is 7.0. Analyzed by C. B. Cibulka]

\begin{tabular}{|c|c|c|c|c|c|}
\hline & $\begin{array}{c}\text { Parts per } \\
\text { million }\end{array}$ & $\begin{array}{l}\text { Equivalents } \\
\text { per million }\end{array}$ & & $\begin{array}{c}\text { Parts per } \\
\text { million }\end{array}$ & $\begin{array}{l}\text { Equivalents } \\
\text { per million }\end{array}$ \\
\hline $\begin{array}{l}\text { Silica }\left(\mathrm{SiO}_{2}\right) \\
\text { Iron }(\mathrm{Fe}) \\
\text { Calcium }(\mathrm{Ca}) \\
\text { Magnesium }(\mathrm{Mg}) \\
\text { Sodium and Potassium } \\
\text { (Na + K). } \\
\text { Bicarbonate }\left(\mathrm{HCO}_{3}\right)^{-}\end{array}$ & $\begin{array}{l}27 \\
91 \\
94 \\
62 \\
214\end{array}$ & $\begin{array}{r}4.54 \\
1.97 \\
2.69 \\
3.51\end{array}$ & $\begin{array}{l}\text { Sulfate }\left(\mathrm{SO}_{4}\right) \\
\text { Chloride }(\mathrm{Cl}) \\
\text { Fluoride }(\mathrm{F}) \\
\text { Nitrate }\left(\mathrm{NO}_{3}\right) \\
\text { Dissolved solids } \\
\text { Total hardness as } \mathrm{CaCO}_{3}\end{array}$ & $\begin{array}{l}163 \\
72 \\
2.0 \\
10 \\
578 \\
370\end{array}$ & \begin{tabular}{r}
3.39 \\
2.03 \\
.11 \\
.16 \\
\hdashline-16 \\
\end{tabular} \\
\hline
\end{tabular}

Drillers' logs

\begin{tabular}{|c|c|c|c|c|c|}
\hline & 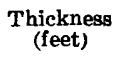 & $\underset{\text { (feet) }}{\text { Depth }}$ & & $\begin{array}{c}\text { Thickness } \\
\text { (feet) }\end{array}$ & \multirow[t]{2}{*}{$\underset{\text { (feet) }}{\text { Depth }}$} \\
\hline \multicolumn{5}{|c|}{ Well 1} & \\
\hline $\begin{array}{l}\text { Top soil } \\
\text { Red sandrock } \\
\text { Conglomerate } \\
\text { Red sandrock. } \\
\text { Conglomerate } \\
\text { Yellow clay } \\
\text { Conglomerate } \\
\text { Sand and honeycomb, water. }\end{array}$ & $\begin{array}{r}10 \\
20 \\
10 \\
5 \\
20 \\
5 \\
14 \\
19\end{array}$ & $\begin{array}{r}10 \\
30 \\
40 \\
45 \\
65 \\
70 \\
84 \\
103\end{array}$ & $\begin{array}{l}\text { Yellow clay } \\
\text { Red sand, shale } \\
\text { Red sand, water } \\
\text { Light sand. } \\
\text { Dry sand. } \\
\text { Red sandrock and clay } \\
\text { Sand and honeycomb, water } \\
\text { Red sandrock. }\end{array}$ & $\begin{array}{rr} & 16 \\
1 & 17 \\
& 6 \\
& 5 \\
& 13 \\
& 17 \\
& 6\end{array}$ & $\begin{array}{l}119 \\
136 \\
142 \\
147 \\
160 \\
177 \\
183 \\
187\end{array}$ \\
\hline
\end{tabular}




\begin{tabular}{|c|c|c|c|c|}
\hline . & $\underset{\text { (feet) }}{\text { Thickness }}$ & $\begin{array}{c}\text { Depth } \\
\text { (feet) }\end{array}$ & $\begin{array}{c}\text { Thicleness } \\
\text { (feet) }\end{array}$ & $\begin{array}{c}\text { Depth } \\
\text { (feet) }\end{array}$ \\
\hline
\end{tabular}

\section{Well 2}

\begin{tabular}{|c|c|c|c|c|}
\hline $\begin{array}{l}\text { Top soil, sand, and caliche... } \\
\text { Brown sandstone and clay. } \\
\text { Hard white sandstone. } \\
\text { Yellow clay with little sand } \\
\text { and very small amount of } \\
\text { water. } \\
\text { Red sand, water. }\end{array}$ & $\begin{array}{r}24 \\
6 \\
23 \\
27\end{array}$ & $\begin{array}{l}24 \\
30 \\
53 \\
80\end{array}$ & $\begin{array}{l}\text { Yellow sand, loose rock } \\
\text { Yellow clay and rock } \\
\text { Red sandstone, water } \\
\text { Red shale. } \\
\text { Red sandstone, water } \\
\text { Red sand and clay. }\end{array}$ & $\begin{array}{r}14 \\
3 \\
44 \\
11 \\
26 \\
20\end{array}$ \\
\hline
\end{tabular}

\section{Well 3}

\begin{tabular}{|c|c|c|c|c|c|}
\hline $\begin{array}{l}\text { Top soil and caliche } \\
\text { Red sandstone, yellow clay. } \\
\text { Brown rock, clay in seams, } \\
\text { little water at } 80 \text { feet. } \\
\text { Red sandstone. } \\
\text { Red sandstone and gravel. } \\
\text { Red sandstone. } \\
\text { Red clay } \\
\text { Red sandstone, water. } \\
\text { Red clay. } \\
\text { Very hard red sandstone. } \\
\text { Red clay. }\end{array}$ & $\begin{array}{r}30 \\
6 \\
44 \\
10 \\
20 \\
8 \\
1 \\
29 \\
8 \\
10 \\
3\end{array}$ & $\begin{array}{r}30 \\
36 \\
80 \\
90 \\
110 \\
118 \\
119 \\
148 \\
156 \\
166 \\
169\end{array}$ & $\begin{array}{l}\text { Red standstone, water } \\
\text { Red clay } \\
\text { Sand and black gravel, water } \\
\text { Red clay } \\
\text { Red sandstone, gravel, water } \\
\text { Red sandstone, } \\
\text { Red clay } \\
\text { Red gandstone. } \\
\text { Red clay } \\
\text { Red sandstone. } \\
\text { Red clay }\end{array}$ & $\begin{array}{r}7 \\
28 \\
6 \\
4 \\
5 \\
11 \\
2 \\
13 \\
2 \\
28 \\
25\end{array}$ & $\begin{array}{l}176 \\
204 \\
210 \\
214 \\
219 \\
230 \\
232 \\
245 \\
247 \\
275 \\
300\end{array}$ \\
\hline
\end{tabular}

\begin{tabular}{l|r|r||r|r|r}
\hline & & & \\
\hline
\end{tabular}

\section{TOYAH}

Population in 1940: 464.

Source of information: O. G. McPherson, Dec. 11, 1946.

Owner: Texas and Pacific Railroad.

Source of supply: Impounding reservoir on Big Aguja Canyon, 47 miles southwest of Toyah.

Average pumpage, in gallons a day, 1946

\begin{tabular}{|c|c|c|c|c|c|}
\hline $\begin{array}{l}\text { March } \\
\text { April } \\
\text { May }\end{array}$ & $\begin{array}{l}17,635 \\
22,393 \\
20,226\end{array}$ & $\begin{array}{l}\text { June } \\
\text { July } \\
\text { August...... }\end{array}$ & $\begin{array}{l}25,080 \\
24,542 \\
19,000\end{array}$ & $\begin{array}{l}\text { September } \\
\text { October }\end{array}$ & $\begin{array}{l}11,217 \\
11,800\end{array}$ \\
\hline
\end{tabular}

Storage: Ground storage reservoir, 200,000 gallons; ground storage reservoir, 180,000 gallons; elevated tank, 150,000 gallons; elevated tark, 100,000 gallons. 


\section{Analyses}

[Collected Dec. 11, 1946. pH: raw water, 7.5; finished water, 7.2. Analyzed by C. B. Cibulka]

\begin{tabular}{|c|c|c|c|c|}
\hline & \multicolumn{2}{|c|}{ Raw water } & \multicolumn{2}{|c|}{ Finished water } \\
\hline & $\begin{array}{l}\text { Parts per } \\
\text { million }\end{array}$ & $\begin{array}{l}\text { Equivalents } \\
\text { per million }\end{array}$ & $\begin{array}{l}\text { Parts per } \\
\text { million }\end{array}$ & $\begin{array}{l}\text { Equivalents } \\
\text { per million }\end{array}$ \\
\hline 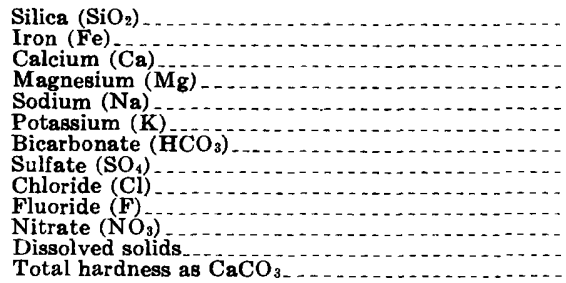 & $\begin{array}{l}32 \\
37^{.68} \\
4.6 \\
17 \\
3.6 \\
158 \\
6.7 \\
10 \\
1.0 \\
191^{.8} \\
111\end{array}$ & $\begin{array}{r}1.847 \\
.378 \\
.760 \\
.092 \\
2.590 \\
.139 \\
.282 \\
.053 \\
.013 \\
\end{array}$ & $\begin{array}{l}32.69 \\
35.69 \\
5.5 \\
17 \\
3.4 \\
152 \\
6.9 \\
12.9 \\
1.0 \\
189^{-5} \\
110\end{array}$ & $\begin{array}{r}.747 \\
.452 \\
.748 \\
.089 \\
2.491 \\
.144 \\
.338 \\
.053 \\
.008 \\
-0 .-\end{array}$ \\
\hline
\end{tabular}

\section{ROBERTS COUNTY}

Population in 1940: 713.

\section{MIAMI}

Source of information: C. Hubbard, plant operator, June 24, 1948.

Owner: Southwestern Public Service Co.

Source of supply: Well; drilled in 1947; depth, 113 feet; diameter, 18 inches; deep-well turbine pump and electric motor; static water level. 45 feet below land surface January 1947; yield, 320 gallons a minute with drawdown of 45 f eet.

Pumpage (estimated): 65,000 gallons a day.

Storage: Ground reservoir on hill, 186,000 gallons.

Number of customers: 183.

Treatment: None.

\section{Analysis}

[Collected June 24, 1948. pH is 7.9. Analyzed by H. D. Smith]

\begin{tabular}{|c|c|c|c|c|c|}
\hline & $\begin{array}{c}\text { Parts per } \\
\text { million }\end{array}$ & $\begin{array}{l}\text { Equivalents } \\
\text { per million }\end{array}$ & & $\begin{array}{c}\text { Parts per } \\
\text { million }\end{array}$ & $\begin{array}{l}\text { Equivalents } \\
\text { per million }\end{array}$ \\
\hline $\begin{array}{l}\text { Silica }\left(\mathrm{SiO}_{2}\right) \\
\text { Iron }(\mathrm{Fe}) \\
\text { Calcium }(\mathrm{Ca}) \\
\text { Magnesium }(\mathrm{Mg}) \\
\text { Sodium }(\mathrm{Na}) \\
\text { Potassium }(\mathrm{K}) \\
\text { Bicarbonate (HCOO}) \\
\end{array}$ & $\begin{array}{r}28 \\
59.00 \\
8.6 \\
2.9 \\
6.0 \\
214\end{array}$ & $\begin{array}{r}2.945 \\
.707 \\
.126 \\
.153 \\
3.508\end{array}$ & $\begin{array}{l}\text { Sulfate }\left(\mathrm{SO}_{4}\right) \\
\text { Chloride }(\mathrm{Cl}) \\
\text { Fluoride (F) } \\
\left.\text { Nitrate (NO})_{3}\right) \\
\text { Dissolved solids } \\
\text { Total hardness as } \mathrm{CaCO}_{3}\end{array}$ & $\begin{array}{r}5.7 \\
5.2 \\
.6 \\
8.3 \\
246 \\
183\end{array}$ & $\begin{array}{r}0.119 \\
.147 \\
.032 \\
.134 \\
-0 .- \\
\end{array}$ \\
\hline
\end{tabular}

\section{SCHLEICHER COUNTY}

\section{EIDORADO}

Population in 1940: 1,530 .

Source of information: L. B. Burk, water superintendent, Sept. 21, 1948.

Ownership: Municipal.

Source of supply: Two wells.

Well 1. Near city hall; drilled in December 1938 by V. P. O'Harrow; depth, 361 feet; diameter, 8 inches; deep-well turbine pump and 50horsepower electric motor; static water level, 280 fest below land surface when drilled; yield, 400 gallons a minute. 
Well 2. Three blocks north and half a block east of city hall; drilled in 1939 by V. P. O'Harrow; depth, 361 feet; diameter, 8 inches; deepwell turbine pump and 40 -horsepower electric motor; yield 225 gallons a minute when drilled.

Pumpage (estimated) : 125,000 gallons a day.

Storage: Concrete ground reservoir, 100,000 gallons; elevated tank, 50,000 gallons.

Number of customers: 450 .

Treatment: Chlorination.

\section{Analyses}

[Collected Sept. 21, 1948. pH: well 1, 7.5; well 2, 7.2. Analyzed by D. E. Wexver]

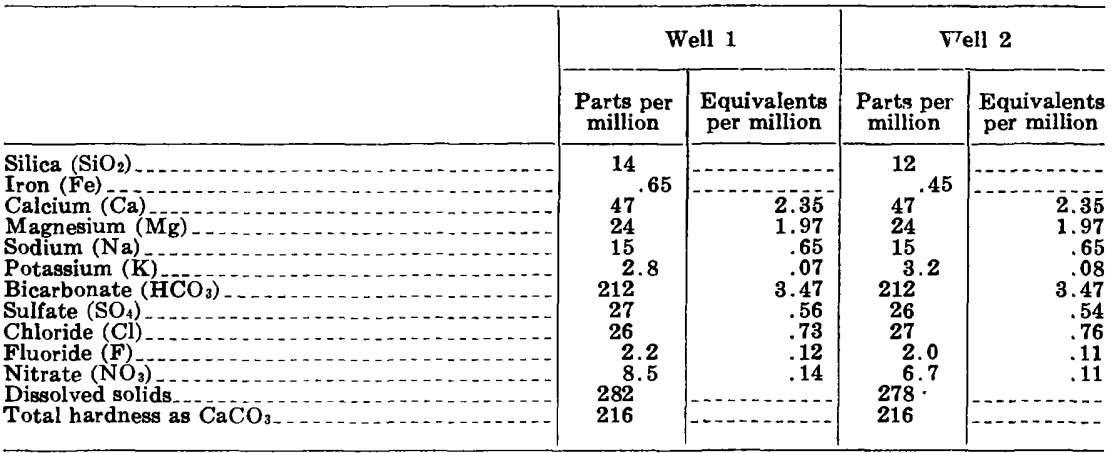

Driller's log, well 1

\begin{tabular}{|c|c|c|c|c|c|}
\hline & $\begin{array}{c}\text { Thickness } \\
\text { (feet) }\end{array}$ & $\begin{array}{l}\text { Depth } \\
\text { (feet) }\end{array}$ & & $\underset{\text { (feet) }}{\text { Thickness }}$ & $\begin{array}{l}\text { Depth } \\
\text { (feet) }\end{array}$ \\
\hline $\begin{array}{l}\text { Surface soil } \\
\text { Caliche and boulders } \\
\text { Hard lime layers } \\
\text { Caliche } \\
\text { Hard lime } \\
\text { Hard lime layers in caliche. } \\
\text { Soft lime } \\
\text { Hard lime layers } \\
\text { Caliche. } \\
\text { Hard layers and caliche } \\
\text { Hard lime. } \\
\text { Lime layers and caliche. } \\
\text { Lime and flint layers. }\end{array}$ & $\begin{array}{r}2 \\
6 \\
42 \\
2 \\
15 \\
19 \\
16 \\
5 \\
3 \\
9 \\
25 \\
87 \\
5\end{array}$ & $\begin{array}{r}2 \\
8 \\
50 \\
52 \\
67 \\
86 \\
102 \\
107 \\
110 \\
119 \\
144 \\
231 \\
236\end{array}$ & $\begin{array}{l}\text { Hard lime layers and caliche } \\
\text { Hard lime } \\
\text { Hard gray lime } \\
\text { Soft gray lime. } \\
\text { Soft lime. } \\
\text { Lime } \\
\text { Soft lime and flint } \\
\text { Lime } \\
\text { Soft mixed rock. } \\
\text { Hard rock. } \\
\text { Soft porous lime, water } \\
\text { Hard light-gray lime...... }\end{array}$ & $\begin{array}{r}32 \\
12 \\
29 \\
5 \\
8 \\
8 \\
5 \\
3 \\
6 \\
1 \\
4 \\
12\end{array}$ & $\begin{array}{l}\mathbf{2 6 8} \\
\mathbf{2 8 0} \\
\mathbf{3 0 9} \\
\mathbf{3 1 4} \\
\mathbf{3 2 2} \\
\mathbf{3 3 0} \\
\mathbf{3 3 5} \\
\mathbf{3 3 8} \\
\mathbf{3 4 4} \\
\mathbf{3 4 5} \\
\mathbf{3 4 9} \\
\mathbf{3 6 1}\end{array}$ \\
\hline
\end{tabular}

\section{SCURRY COUNTY}

\section{HERMLEIGH}

Population in 1940: 404.

Source of information: Geo. O. Hale, pump operator, June 20, 1946.

Ownership: Municipal.

Source of supply: Three wells.

Well 1. Drilled in 1928; depth, 220 feet; diameter, 6 inches; deep-well cylinder pump and 5-horsepower electric motor; yield, 3 gallons a minute. 
Well 2. Drilled in 1928; depth, 240 feet; diameter, 6 inches; deep-well cylinder pump and 3/4-horsepower electric motor; yield, 3 gallons a minute.

Well 3. Drilled; depth, 260 feet; diameter, 6 inches; deer-well cylinder pump and 1-horsepower electric motor; yield, 3 gallons a minute.

Pumpage: No record.

Storage: Ground storage reservoir, 150,000 gallons (not used); elevated tank, 150,000 gallons.

Number of customers: 54 .

Treatment: None.

\section{Analysis}

[Composite sample of three wells. Collected June 20, 1946. pH is 8.1. Analyzed by C. B. Cibulka]

\begin{tabular}{|c|c|c|c|c|c|}
\hline & $\begin{array}{c}\text { Parts per } \\
\text { million }\end{array}$ & $\begin{array}{l}\text { Equivalents } \\
\text { per million }\end{array}$ & & $\begin{array}{l}\text { Parts per } \\
\text { million }\end{array}$ & $\begin{array}{l}\text { Equivalents } \\
\text { per million }\end{array}$ \\
\hline $\begin{array}{l}\text { Silica }\left(\mathrm{SiO}_{2}\right) \\
\text { Iron }(\mathrm{Fe}) \\
\text { Calcium }(\mathrm{Ca}) \\
\text { Magnesium }(\mathrm{Mg}) \\
\text { Sodium }(\mathrm{Na}) \\
\text { Potassium }(\mathrm{K}) \\
\text { Bicarbonate }\left(\mathrm{HCOO}_{3}\right)^{-}\end{array}$ & $\begin{array}{l}17 \\
84 \\
21 \\
94 \\
4.2 \\
274\end{array}$ & $\begin{array}{r}4.19 \\
1.73 \\
4.07 \\
.11 \\
4.49\end{array}$ & $\begin{array}{l}\text { Sulfate }\left(\mathrm{SO}_{4}\right) \\
\text { Chloride }(\mathrm{Cl}) \\
\text { Fluoride }(\mathrm{F}) \\
\text { Nitrate }\left(\mathrm{NO}_{3}\right) \\
\text { Dissolved solids } \\
\text { Total hardness as } \mathrm{CaCO}_{3}\end{array}$ & $\begin{array}{l}222 \\
32 \\
1.8 \\
636 \\
236\end{array}$ & \begin{tabular}{r}
4.62 \\
.90 \\
.09 \\
.01 \\
\hdashline-0.0 \\
\end{tabular} \\
\hline
\end{tabular}

Population in 1940: 3,815 .

\section{SNYDER}

Source of information: Carl Keller, May 20, 1946.

Ownership: Municipal.

Source of supply: Four wells.

Well 1. At city hall; drilled in 1925 by D. D. Doty; depth, 160 feet; diameter, 17 inches; deep-well turbine pump and 20-horsepower electric motor; yield, 250 gallons a minute in May 1946; temperature, $70^{\circ} \mathrm{F}$.

Well 2. About 100 feet east of well 1; drilled in 1926 by D. D. Doty; depth, 165 feet; diameter, 17 inches; deep-well turbine pump and 15horsepower electric motor; static water level, $66 \mathrm{fee}^{+}$below land surface May 23, 1946; yield, 250 gallons a minute with dr:wdown of 40 feet after 4 hours of pumping May 23, 1946.

Well 3. About 0.8 mile north of courthouse; drilled in 1928 by D. D. Doty; depth, 187 feet; diameter, 17 inches; no pumping equipment as of May 26, 1946; static water level, 45 feet below land surface May $22,1946$.

Well 4. About 0.3 mile east of courthouse; drilled in 1945 by D. D. Doty; depth, 205 feet; diameter, 17 to 12 inches; deer-well turbine pump and 15-horsepower electric motor; static water level, 30 feet below land surface May 24, 1946; yield, 125 gallons a minute; temperature, $69^{\circ} \mathrm{F}$.

Pumpage (estimated): Average, 375,000 gallons a day.

Storage: Concrete ground reservoir, 150,000 gallons; elevated tank, 100,000 gallons.

Number of customers: 925 .

Treatment: None. 


\section{Analyses}

[Collected May 29, 1946. pH: wells 1 and 2, 7.5; well 4, 7.6. Analyzed by C. B. Cibulka]

\begin{tabular}{|c|c|c|c|c|c|c|}
\hline & \multicolumn{2}{|c|}{ Well 1} & \multicolumn{2}{|c|}{ Well 2} & \multicolumn{2}{|c|}{ Well 4} \\
\hline & $\begin{array}{l}\text { Parts per } \\
\text { million }\end{array}$ & $\begin{array}{l}\text { Equivalents } \\
\text { per million }\end{array}$ & $\begin{array}{l}\text { Parts per } \\
\text { million }\end{array}$ & $\begin{array}{l}\text { Equivalents } \\
\text { per million }\end{array}$ & $\begin{array}{l}\text { Parts per } \\
\text { million }\end{array}$ & $\begin{array}{l}\text { Equivalents } \\
\text { per million }\end{array}$ \\
\hline 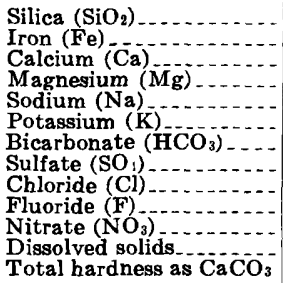 & $\begin{array}{l}27 \\
60 \\
23 \\
43 \\
45 \\
5.04 \\
308 \\
32 \\
34 \\
1.6 \\
12 \\
391 \\
244\end{array}$ & $\begin{array}{r}2.99 \\
1.89 \\
1.94 \\
.13 \\
5.05 \\
.67 \\
.96 \\
.08 \\
.19 \\
\hdashline .96\end{array}$ & $\begin{array}{l}27 \\
79 \\
28 \\
28 \\
49 \\
4.8 \\
316 \\
51 \\
66 \\
1.6 \\
20 \\
496 \\
312\end{array}$ & $\begin{array}{r}3.94 \\
2.30 \\
2.14 \\
.12 \\
5.18 \\
1.06 \\
1.86 \\
.08 \\
.32\end{array}$ & $\begin{array}{l}27 \\
56 \\
19 \\
19 \\
40 \\
3.8 \\
292 \\
24 \\
24 \\
1.8 \\
9.4 \\
349 \\
218\end{array}$ & $\begin{array}{r}2.80 \\
1.56 \\
1.75 \\
.10 \\
4.79 \\
.50 \\
.68 \\
.09 \\
.15 \\
\hdashline\end{array}$ \\
\hline
\end{tabular}

\section{Driller's logs}

\begin{tabular}{|c|c|c|c|c|}
\hline & $\begin{array}{c}\text { Thickness } \\
\text { (feet) }\end{array}$ & $\begin{array}{l}\text { Depth } \\
\text { (feet) }\end{array}$ & $\begin{array}{c}\text { Thiskness } \\
\text { (feet) }\end{array}$ & $\begin{array}{l}\text { Depth } \\
\text { (feet) }\end{array}$ \\
\hline
\end{tabular}

Well 1

\begin{tabular}{|c|c|c|c|c|c|}
\hline $\begin{array}{l}\text { Chalk rock } \\
\text { Hard rock. } \\
\text { Sandy clay } \\
\text { Rock } \\
\text { Sandy clay } \\
\text { Sandrock } \\
\text { Hard rock } \\
\text { Soft rock } \\
\text { Sandrock }\end{array}$ & $\begin{array}{r}15 \\
2 \\
8 \\
3 \\
3 \\
10 \\
4 \\
10 \\
4\end{array}$ & $\begin{array}{l}15 \\
17 \\
25 \\
28 \\
31 \\
41 \\
45 \\
55 \\
59\end{array}$ & $\begin{array}{l}\text { Red clay } \\
\text { Sand, } \\
\text { Chalk and sand } \\
\text { Clay, } \\
\text { Rock, water, } \\
\text { Red clay } \\
\text { Rock, clay } \\
\text { Red }\end{array}$ & $\begin{array}{r}31 \\
30 \\
8 \\
2 \\
7 \\
27 \\
11 \\
8\end{array}$ & $\begin{array}{r}90 \\
120 \\
128 \\
130 \\
137 \\
164 \\
175 \\
183\end{array}$ \\
\hline
\end{tabular}

Well 2

\begin{tabular}{|c|c|c|c|c|c|}
\hline $\begin{array}{l}\text { Soft clay and sand } \\
\text { Sand rock, water } \\
\text { Blue clay, } \\
\text { Hard rock, } \\
\text { Red and blue clay. } \\
\text { Hard rock. } \\
\text { Sand, water. } \\
\text { Hard rock. } \\
\text { Soft sand, water } \\
\text { Red clay, } \\
\text { Sandrock. } \\
\text { Soft sand. }\end{array}$ & $\begin{array}{r}35 \\
12 \\
13 \\
1 \\
31 \\
1 \\
4 \\
1 \\
5 \\
18 \\
4 \\
\mathbf{2}\end{array}$ & $\begin{array}{r}35 \\
47 \\
60 \\
61 \\
92 \\
93 \\
97 \\
98 \\
103 \\
121 \\
125 \\
127\end{array}$ & $\begin{array}{l}\text { Hard rock. } \\
\text { Sand, } \\
\text { Sandrock } \\
\text { Rock, shale. } \\
\text { Soft sand } \\
\text { Hard rock } \\
\text { Cave } \\
\text { Hard rock } \\
\text { Cave } \\
\text { Hard rock } \\
\text { Yellow clay }\end{array}$ & $\begin{array}{r}7 \\
6 \\
10 \\
4 \\
2 \\
3 \\
1 \\
1 \\
1 \\
3 \\
14\end{array}$ & $\begin{array}{l}134 \\
140 \\
150 \\
154 \\
156 \\
159 \\
160 \\
161 \\
162 \\
165 \\
179\end{array}$ \\
\hline
\end{tabular}

Well 3

Soil and clay

Chalk and gravel...........

Clay and gravel.

Sand

Sand and gravel

Yellow sandrock

Clay.

Hard rock.

Soft rave

Soft cave..................

Hard rock

Soft sand.

Hard rock

Sandy clay

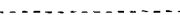

Red rock, sand............

\begin{tabular}{|c|c|c|}
\hline $\begin{array}{l}\text { Hard rock. } \\
\text { Clay, } \\
\text { Rock, soft. } \\
\text { Sand, water } \\
\text { Hard rock. } \\
\text { Soft sand } \\
\text { Hard rock. } \\
\text { Sandrock. } \\
\text { Water sand } \\
\text { Rock, hard } \\
\text { Soft rock } \\
\text { Hard rock. } \\
\text { Soft rock. } \\
\text { Hard rock. } \\
\text { Red clay }\end{array}$ & $\begin{array}{r}2 \\
2 \\
1 \\
7 \\
4 \\
9 \\
2 \\
4 \\
10 \\
7 \\
5 \\
4 \\
2 \\
4 \\
15\end{array}$ & $\begin{array}{l}111 \\
113 \\
114 \\
121 \\
125 \\
134 \\
136 \\
140 \\
150 \\
157 \\
162 \\
166 \\
168 \\
172 \\
187\end{array}$ \\
\hline
\end{tabular}




\section{SHERMAN COUNTY}

Population in 1940: 877.

\section{STRATFORD}

Source of information: Chester Guthrie, manager, Southwestern Public Service Co., June 24, 1948.

Owner: Southwestern Public Service Co.

Source of supply: Two wells.

Well 2. Drilled; depth, 300 feet; diameter, 8 inches; deer-well turbine pump and electric motor; static water level, 220 feet below land surface; yield, 170 gallons a minute with drawdown of 30 feet.

Well 3. Drilled in 1930 by L. E. McDade; depth, 300 feet; diameter, 10 inches; deep-well turbine pump and 30-horsepower electric motor; static water level, 216 feet below land surface; yield, 225 gallon` a minute.

Pumpage: Average, 125,000 gallons a day.

Storage: Ground reservoir, 20,000 gallons; elevated tank, 85,000 gallons.

Number of customers: 470.

Treatment: Chlorination.

Analysis, well 3

[Collected June 24, 1948. $\mathrm{pH}$ is 7.6. Analyzed by D. E. Weaver]

\begin{tabular}{|c|c|c|c|c|c|}
\hline & $\begin{array}{c}\text { Parts per } \\
\text { million }\end{array}$ & $\begin{array}{l}\text { Equivalents } \\
\text { per million }\end{array}$ & & $\begin{array}{l}\text { Parts per } \\
\text { mill on }\end{array}$ & $\begin{array}{l}\text { Equivalents } \\
\text { per million }\end{array}$ \\
\hline 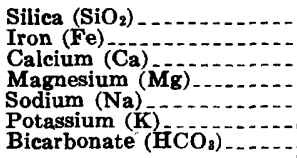 & $\begin{array}{l}31 \\
36 \\
36 \\
14 \\
5.2 \\
244\end{array}$ & $\begin{array}{r}1.80 \\
2.96 \\
.61 \\
.13 \\
4.00\end{array}$ & $\begin{array}{l}\text { Sulfate }\left(\mathrm{SO}_{4}\right) \\
\text { Chloride }(\mathrm{Cl}) \\
\text { Fluoride (F) } \\
\left.\text { Nitrate (NO })_{8}\right) \\
\text { Dissolved solids. } \\
\text { Total hardness as } \mathrm{CaCO}_{3}\end{array}$ & $\begin{array}{c}49 \\
10 \\
1.2 \\
9.5 \\
314 \\
23 ?\end{array}$ & $\begin{array}{r}1.00 \\
.28 \\
.06 \\
.15 \\
-15 \\
\end{array}$ \\
\hline
\end{tabular}

Driller's log, well 3

\begin{tabular}{|c|c|c|c|c|c|}
\hline & $\begin{array}{c}\text { Thickness } \\
\text { (feet) }\end{array}$ & $\begin{array}{l}\text { Depth } \\
\text { (feet) }\end{array}$ & & $\begin{array}{c}\text { Thickness } \\
\text { (feet) }\end{array}$ & $\begin{array}{l}\text { Depth } \\
\text { (feet) }\end{array}$ \\
\hline $\begin{array}{l}\text { Top soil } \\
\text { Soft sandstone } \\
\text { Yellow clay } \\
\text { Caliche. }\end{array}$ & $\begin{array}{l}4 \\
25 \\
60 \\
60\end{array}$ & $\begin{array}{r}4 \\
29 \\
89 \\
149\end{array}$ & $\begin{array}{l}\text { Packed sand. } \\
\text { Loose, sliding sand } \\
\text { Water, quicksand }\end{array}$ & $\begin{array}{l}\mathbf{4 0} \\
\mathbf{3 0} \\
\mathbf{8 1}\end{array}$ & $\begin{array}{l}189 \\
219 \\
300\end{array}$ \\
\hline
\end{tabular}

\section{STONEWALL COUNTY}

\section{ASPERMONT}

Population in 1940: 1,041 .

Source of information: Marvin B. Austin, water superintendent, Sept. 15, 1947.

Ownership: Municipal.

Source of supply: Lake Sellers; built in 1913 and enlarged in 1934; capacity, 500 acre-feet.

Pumpage: Average, 140,000 gallons a day.

Storage: Settling basin at treating plant, 100,000 gallons; e'evated tank, 65,000 gallons.

Number of customers: 250.

Treatment: Coagulation, sedimentation, prechlorination and postchlorination. 


\section{Analyses}

[Collected Sept. 15, 1947. pH: raw water, 7.7; finished water, 6.8. Analyzed by B. C. Dwyer]

\begin{tabular}{|c|c|c|c|c|}
\hline & \multicolumn{2}{|c|}{ Raw water } & \multicolumn{2}{|c|}{ Finished water } \\
\hline & $\begin{array}{c}\text { Parts per } \\
\text { million }\end{array}$ & $\begin{array}{l}\text { Equivalents } \\
\text { per million }\end{array}$ & $\begin{array}{l}\text { Parts pe } \\
\text { million }\end{array}$ & $\begin{array}{l}\text { Equivalents } \\
\text { per million }\end{array}$ \\
\hline 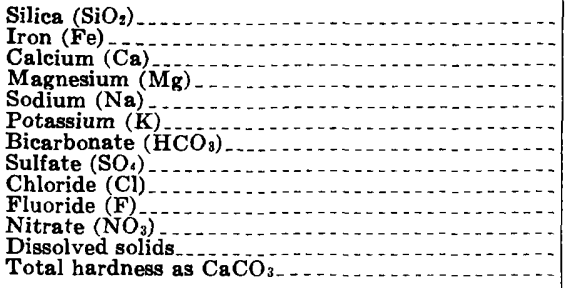 & $\begin{array}{l}28 \\
91 \\
19 \\
19 \\
19 \\
2.2 \\
250 \\
46 \\
50 \\
1.0 \\
30 \\
460 \\
305\end{array}$ & 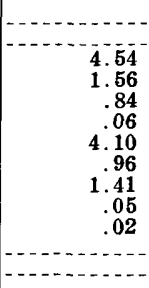 & 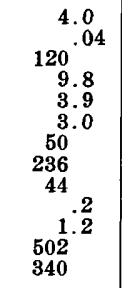 & \begin{tabular}{r}
5.99 \\
.81 \\
.17 \\
.08 \\
.82 \\
4.96 \\
1.24 \\
.01 \\
.02 \\
\hdashline-2 \\
\end{tabular} \\
\hline
\end{tabular}

\section{SUTTON COUNTY}

\section{SO.NORA}

Population in 1940: 2,528.

Source of information: R. M. McCarver, water superintendent, July 22, 1947. Ownership: Municipal.

Source of supply: Three wells.

Well 1. About 1,500 feet northwest of city hall; drilled in 1938; depth, 303 feet; diameter, 10 inches; deep-well turbine pump and 40-horsepower electric motor; yield, 400 gallons a minute.

Well 2. East of well 1; drilled in 1938 by Layne-Texas Co.; depth, 303 feet; diameter, $103 / 4$ inches; deep-well turbine pump and 40-horsepower electric motor; static water level, 276 feet below land surfare in 1938; yield, 420 gallons a minute in 1938.

Well 3. Drilled in 1947 by Layne-Texas Co.; depth, 302 feet; diameter, $103 / 4$ inches; deep-well turbine pump and 60-horsepower electric motor; static water level, 276 feet below land surface in June 1947; yield, 600 gallons a minute.

Pumpage (estimated): Average, 375,000 gallons a day.

Storage: Three concrete ground storage reservoirs, 50,000 gallons, 150,000 gallons, and 200,000 gallons, respectively. All reservoirs located on hill.

Number of customers: 800 .

Treatment: Chlorination.

Analysis of finished water

[Composite sample from 3 wells. Collected July 22, 1947. pH is 7.5. Analyzed by F C. Dryer]

\begin{tabular}{|c|c|c|c|c|c|}
\hline & $\begin{array}{c}\text { Parts per } \\
\text { million }\end{array}$ & $\begin{array}{l}\text { Equivalent: } \\
\text { per million }\end{array}$ & & $\begin{array}{c}\text { Parts pe- } \\
\text { million }\end{array}$ & $\begin{array}{l}\text { Equivalents } \\
\text { per million }\end{array}$ \\
\hline $\begin{array}{l}\text { Silica }\left(\mathrm{SiO}_{2}\right) \\
\text { Iron }(\mathrm{Fe}) \\
\text { Calcium }(\mathrm{Ca}) \\
\text { Magnesium }(\mathrm{Mg}) \\
\text { Sodium }(\mathrm{Na}) \\
\text { Potassium }(\mathrm{K}) \\
\text { Bicarbonate }\left(\mathrm{H}^{2} \mathrm{CO}_{3}\right)\end{array}$ & $\begin{array}{l}11 \\
75 \\
20 \\
4.00 \\
4.8 \\
304\end{array}$ & $\begin{array}{r}3.74 \\
1.64 \\
.21 \\
.11 \\
4.98\end{array}$ & 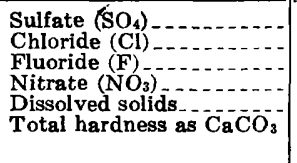 & $\begin{array}{r}8.8 \\
16.2 \\
5.2 \\
297 \\
269\end{array}$ & $\begin{array}{r}0.18 \\
.45 \\
.01 \\
.08 \\
\\
\end{array}$ \\
\hline
\end{tabular}


Driller's log, well 2

\begin{tabular}{|c|c|c|c|c|c|}
\hline & $\begin{array}{c}\text { Thickness } \\
\text { (feet) }\end{array}$ & $\begin{array}{l}\text { Depth } \\
\text { (feet) }\end{array}$ & & $\begin{array}{c}\text { Thickness } \\
\text { (feet) }\end{array}$ & $\begin{array}{l}\text { Depth } \\
\text { (feet) }\end{array}$ \\
\hline 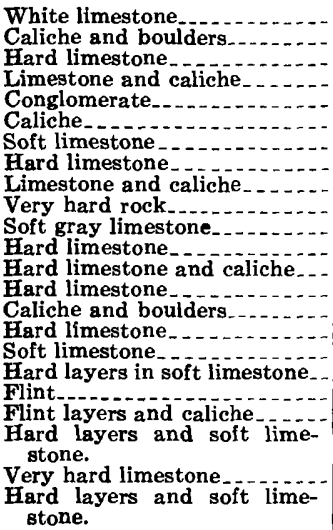 & $\begin{array}{l}10 \\
2.5 \\
1.5 \\
5 \\
10 \\
3 \\
9 \\
5 \\
8 \\
4 \\
1.5 \\
26.5 \\
2 \\
13 \\
1 \\
14 \\
5 \\
10 \\
1.5 \\
10.5 \\
6 \\
2 \\
19\end{array}$ & $\begin{array}{l}10 \\
12.5 \\
14 \\
19 \\
29 \\
32 \\
41 \\
46 \\
54 \\
58 \\
59.5 \\
86 \\
88 \\
101 \\
102 \\
116 \\
121 \\
131 \\
132.5 \\
143 \\
149 \\
151 \\
170\end{array}$ & 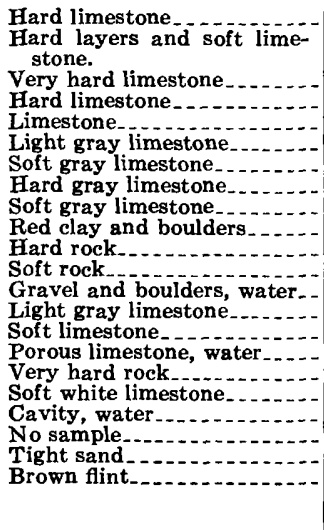 & $\begin{array}{c}1.5 \\
22.5 \\
6 \\
12 \\
6 \\
10 \\
8 \\
17 \\
1 \\
20.5 \\
1.5 \\
4.5 \\
2 \\
3 \\
3 \\
2.5 \\
2.5 \\
3.5 \\
1.5 \\
2 \\
2 \\
1\end{array}$ & $\begin{array}{l}171.5 \\
194 \\
200 \\
212 \\
218 \\
228 \\
236 \\
253 \\
254 \\
274.5 \\
275.5 \\
280 \\
282 \\
285 \\
288 \\
290.5 \\
293 \\
296.5 \\
298 \\
300 \\
302 \\
303\end{array}$ \\
\hline
\end{tabular}

\section{SWISHER COUNTY}

Population in 1940: 576.

\section{HAPPY}

Source of information: Wm. F. Miller, water superintendent, Mar. 6, 1945.

Ownership: Municipal.

Source of supply: Two wells.

Well 1. About 20 feet north of elevated tank; drilled in 1928 by Leo

McDade; depth, 179 feet; diameter, 6 inches; deep-well turbine pump and 10-horsepower electric motor; yield, 25 gallons a minute; temperature, $6212^{\circ} \mathrm{F}$.

Well 2. About 40 feet south of well 1; drilled in 1928 by Travis Gillum; depth, 179 feet; diameter, 6 inches; deep-well turbine pump and 3horsepower electric motor; yield, 25 gallons a minute; temperature, $6212^{\circ} \mathrm{F}$.

Pumpage: No record.

Storage: Concrete ground reservoir, 150,000 gallons; elevated tank, 50,000 gallons.

Number of customers: 100.

Treatment: None.

\section{Analysis, well 2}

[Collected Mar. 6, 1945. pH is 7.9. Analyzed by M. L. Begley]

\begin{tabular}{|c|c|c|c|c|c|}
\hline & $\begin{array}{l}\text { Parts per } \\
\text { million }\end{array}$ & $\begin{array}{l}\text { Equivalents } \\
\text { per million }\end{array}$ & & $\begin{array}{l}\text { Parts per } \\
\text { million }\end{array}$ & $\begin{array}{l}\text { Equivalents } \\
\text { per million }\end{array}$ \\
\hline $\begin{array}{l}\text { Silica }\left(\mathrm{SiO}_{2}\right) \\
\text { Iron }(\mathrm{Fe}) \\
\text { Calcium }(\mathrm{Ca}) \\
\text { Magnesium }(\mathrm{Mg}) \\
\text { Sodium }(\mathrm{Na}) \\
\text { Potassium }(\mathrm{K}) \\
\text { Bicarbonate }\left(\mathrm{H} \mathrm{HOO}_{3}\right)^{-}\end{array}$ & $\begin{array}{l}38 \\
47 \\
27 \\
24 \\
2.04 \\
5.9 \\
283\end{array}$ & $\begin{array}{r}2.35 \\
2.22 \\
1.06 \\
4.15 \\
4.64\end{array}$ & $\begin{array}{l}\text { Sulfate }\left(\mathrm{SO}_{4}\right) \\
\text { Chloride }(\mathrm{Cl}) \\
\text { Fluoride (F) } \\
\left.\text { Nitrate (NO})_{3}\right) \\
\text { Dissolved solids. } \\
\text { Total hardness as } \mathbf{C a C O}\end{array}$ & $\begin{array}{l}26 \\
10 \\
2.0 \\
13 \\
332 \\
228\end{array}$ & 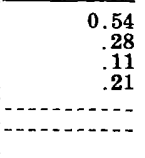 \\
\hline
\end{tabular}




\section{TULIA}

Population in 1940: 2,055.

Source of information: L. L. Schenault, water superintendent, Mar. 3, 1945. Ownership: Municipal.

Source of supply: Three wells.

Well 1. Drilled in 1922 by R. J. Stalling; depth, 198 feet; diameter, 18 inches; deep-well turbine pump and 40-horsepower electric motor; yield, 400 gallons a minute.

Well 2. Drilled in 1926 by G. E. Higgins; depth, 167 feet; diameter, 26 inches; deep-well turbine pump and 50-horsepower electric motor; static water level, 78.5 feet below land surface Mar. 3, 1945; yield, 515 gallons a minute.

Well 3. Drilled in 1914 by R. J. Stalling; depth, 140 feet; diameter, 18 inches; deep-well turbine pump and 25-horsepower elentric motor; yield, 200 gallons a minute.

Pumpage: No record.

Storage: Ground storage reservoir, 265,000 gallons; elevated tank, 50,000 gallons.

Number of customers: 700 .

Treatment: None.

Analysis, well 2

[Collected Mar. 3, 1945. $\mathrm{pH}$ is 7.8. Analyzed by M. L. Begley]

\begin{tabular}{|c|c|c|c|c|c|}
\hline & $\begin{array}{l}\text { Parts per } \\
\text { million }\end{array}$ & $\begin{array}{l}\text { Equivalents } \\
\text { per million }\end{array}$ & & $\begin{array}{l}\text { Parts per } \\
\text { million }\end{array}$ & $\begin{array}{l}\text { Equivalents } \\
\text { per million }\end{array}$ \\
\hline $\begin{array}{l}\text { Silica }\left(\mathrm{SiO}_{2}\right) \\
\text { Iron }(\mathrm{Fe}) \\
\text { Calcium }(\mathrm{Ca}) \\
\text { Magnesium }(\mathrm{Mg}) \\
\text { Sodium }(\mathrm{Na}) \\
\text { Potassium }(\mathrm{K}) \\
\text { Bicarbonate }\left(\mathrm{HCO}_{2}\right)\end{array}$ & $\begin{array}{l}\mathbf{5 8} \\
\mathbf{3 6} \\
\mathbf{3 1} \\
\mathbf{5 3} \\
\mathbf{8} . \mathbf{0} \\
\mathbf{3 1 9}\end{array}$ & $\begin{array}{r}1.80 \\
2.55 \\
2.29 \\
.22 \\
\mathbf{5 . 2 3}\end{array}$ & $\begin{array}{l}\text { Sulfate }\left(\mathrm{SO}_{4}\right) \\
\text { Chloride (Cl) } \\
\text { Fluoride (F) } \\
\text { Nitrate (NO} \\
\text { Dissolved solids } \\
\text { Total hardness as } \mathrm{CaCO}_{3}\end{array}$ & $\begin{array}{l}3 \varepsilon \\
2 \varepsilon \\
5.2 \\
2.8 \\
37 \% \\
21 \varepsilon\end{array}$ & $\begin{array}{r}0.69 \\
.62 \\
.27 \\
.05 \\
-.0- \\
-0.0\end{array}$ \\
\hline
\end{tabular}

\section{TERRELL COUNTY}

Population in 1940: 2,150.

\section{SANDERSON}

Source of information: James Caroline, water superintendent, Sept. 28, 1948.

Owner: Community Public Service Co.

Source of supply: Twelve wells.

Well 1. At ice plant near Texas and New Orleans Railroad on Wilson Street; drilled; depth reported, 470 feet; deep-well cylinder and pump jack and 3-horsepower electric motor; yield, 9 gallons a ninute.

Well 2. Near well 1; drilled; depth, 415 feet; deep-well cylinder and pump jack and 3-horsepower electric motor; yield, 8 gallons a minute.

Well 3. Near elevated tank; drilled; depth, 470 feet; deep-well turbine pump and 71/2-horsepower electric motor; yield, 22 gallons a minute.

Well 5. About 50 feet west of Wilson Street near the corner of Pine and Wilson Streets; drilled in 1940 by Layne-Texas Co.; depth, 525 feet; diameter, $12 \%$ to $65 \%$ inches; deep-well cylinder and electric motor; yield, 3 gallons a minute.

Well 7. Three blocks north and one block east of the courthruse; drilled by C. V. Cox; depth, 491 feet; diameter, 12 to 8 inches; deep-well cylinder and pump jack and electric motor; yield, 13 gallons a minute. 
Well 8. Northeast of well 7; drilled in June 1943 by Brownie Locke; depth, 485 feet; diameter, 12 inches; deep-well cylinder and pump jack and 5-horsepower electric motor; yield, 10 gallons a minute.

Well 10. Northeast of well 8; drilled in July 1945 by Huffman and Brazell; depth, 525 feet; diameter, 10 to 81/4 inches; deer-well cylinder and pump jack and 3-horsepower electric motor; yield", 9 gallons a minute.

Well 11. About 850 yards northeast of well 10; drilled; depth, 545 feet; diameter, 8 inches; deep-well cylinder and pump jack and $71 / 2$-horsepower electric motor; yield, 13 gallons a minute.

Well 14. West of well 11; drilled in April 1946 by Huffmar and Brazell; depth, 525 feet; diameter, 81/4 inches; deep-well cylind $r$ and pump jack; yield, 7 gallons a minute.

Well 15. Northeast of well 11; drilled in April 1946 by Huffman and Brazell; depth, 580 feet; diameter, $8 \%$ inches; deep-well cylinder and pump jack and electric motor; yield, 8 gallons a minute.

Well 16. About 4,200 feet northwest of power plant; drilled; depth, 840 feet; diameter, 7 inches; deep-well cylinder and pump jack and electric motor; yield on test, 49 gallons a minute.

Well 17. South of well 16; drilled; depth, unknown; diameter, unknown; deep-well cylinder and pump jack and 5-horsepower eloctric motor; yield, 17 gallons a minute.

Pumpage (estimated) : 150,000 gallons a day.

Storage: Concrete reservoir, rock reservoir, and steel tank; all elevated on hill; capacity, unknown.

Number of customers (estimated) : 350 .

Treatment: Hypochlorination.

\section{Analysis}

[Composite sample, collected Sept. 28, 1948. $\mathrm{pH}$ is 8.3. Analyzed by D. F. Weaver]

\begin{tabular}{|c|c|c|c|c|c|}
\hline & $\begin{array}{l}\text { Parts per } \\
\text { million }\end{array}$ & $\begin{array}{l}\text { Equivalents } \\
\text { per million }\end{array}$ & & $\begin{array}{c}\text { Parts per } \\
\text { millon }\end{array}$ & $\begin{array}{l}\text { Equivalents } \\
\text { per million }\end{array}$ \\
\hline $\begin{array}{l}\text { Silica }\left(\mathrm{SiO}_{2}\right) \\
\text { Iron }(\mathrm{Fe}) \\
\text { Calcium }(\mathrm{Ca}) \\
\text { Magnesium }(\mathrm{Mg}) \\
\text { Sodium }(\mathrm{Na}) \\
\text { Potassium }(\mathrm{K}) \\
\text { Bicarbonate }\left(\mathrm{H} \mathrm{CO}_{s}\right)^{-}\end{array}$ & $\begin{array}{l}12 \\
56 \\
22 \\
3.65 \\
3.1 \\
230 \\
230\end{array}$ & $\begin{array}{r}2.795 \\
1.809 \\
.135 \\
.061 \\
3.775\end{array}$ & 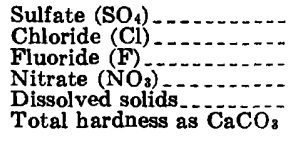 & $\begin{array}{l}2 ? \\
13 \\
3.6 \\
23 ? \\
23 ?\end{array}$ & $\begin{array}{r}0.541 \\
.367 \\
.032 \\
.052 \\
.052 \\
\end{array}$ \\
\hline
\end{tabular}

Drillers' logs

\begin{tabular}{|c|c|c|c|c|c|}
\hline & $\begin{array}{c}\text { Thickness } \\
\text { (feet) }\end{array}$ & $\frac{\text { Depth }}{\text { (feet) }}$ & & $\begin{array}{c}\text { Tickness } \\
\text { (feet) }\end{array}$ & $\begin{array}{l}\text { Depth } \\
\text { (feet) }\end{array}$ \\
\hline \multicolumn{6}{|c|}{ Well 5} \\
\hline $\begin{array}{l}\text { Hard rock } \\
\text { White lime } \\
\text { White and gray lime } \\
\text { Yellow lime } \\
\text { Gray-blue lime } \\
\text { Yellow lime } \\
\text { Gray lime } \\
\text { Blue lime } \\
\text { Yellow sandrock } \\
\text { White sandrock }\end{array}$ & $\begin{array}{l}24 \\
61 \\
35 \\
40 \\
15 \\
40 \\
50 \\
35 \\
20 \\
20\end{array}$ & $\begin{array}{r}24 \\
85 \\
120 \\
160 \\
175 \\
215 \\
265 \\
300 \\
320 \\
\mathbf{8 4 0}\end{array}$ & $\begin{array}{l}\text { Yellow sandrock } \\
\text { White sandrock } \\
\text { Sandrock } \\
\text { Dark lime rock } \\
\text { Blue lime rock } \\
\text { Sandy blue rock } \\
\text { Fine white sandrock } \\
\text { Blue sandrock } \\
\text { Gray sandrock }\end{array}$ & $\begin{array}{r}10 \\
10 \\
30 \\
5 \\
15 \\
30 \\
10 \\
50 \\
25\end{array}$ & $\begin{array}{l}350 \\
360 \\
390 \\
395 \\
410 \\
440 \\
450 \\
500 \\
525\end{array}$ \\
\hline
\end{tabular}




\begin{tabular}{|c|c|c|c|}
\hline $\begin{array}{c}\text { Thickness } \\
\text { (feet) }\end{array}$ & $\begin{array}{c}\text { Depth } \\
\text { (feet) }\end{array}$ & $\begin{array}{c}\text { Thickness } \\
\text { (feet) }\end{array}$ & $\begin{array}{c}\text { Depth } \\
\text { (feet) }\end{array}$ \\
\hline
\end{tabular}

Well 7

\begin{tabular}{|c|c|c|c|c|c|}
\hline $\begin{array}{l}\text { Gravel } \\
\text { Yellow lime. } \\
\text { Gray lime } \\
\text { Yellow lime. } \\
\text { Blue lime } \\
\text { Yellow lime } \\
\text { Gray sandrock } \\
\text { Yellow sand } \\
\text { Blue sand } \\
\text { Yellow sand }\end{array}$ & $\begin{array}{r}6 \\
54 \\
75 \\
95 \\
\mathbf{3 0} \\
\mathbf{3 0} \\
15 \\
\mathbf{2 0} \\
\mathbf{5} \\
15\end{array}$ & $\begin{array}{r}6 \\
60 \\
135 \\
230 \\
260 \\
290 \\
305 \\
325 \\
330 \\
345\end{array}$ & $\begin{array}{l}\text { Gray sand. } \\
\text { First water } \\
\text { Yellow sand. } \\
\text { Blue sand } \\
\text { Gray sand } \\
\text { Yellow sand } \\
\text { Soapstone } \\
\text { Soapstone } \\
\text { Rock } \\
\text { Soapstone and sand }\end{array}$ & $\begin{array}{r}15 \\
5 \\
10 \\
15 \\
45 \\
15 \\
15 \\
13 \\
9 \\
4\end{array}$ & $\begin{array}{l}360 \\
365 \\
375 \\
390 \\
435 \\
450 \\
465 \\
478 \\
487 \\
491\end{array}$ \\
\hline
\end{tabular}

Well 8

\begin{tabular}{|c|c|c|c|c|c|}
\hline $\begin{array}{l}\text { Rock and caliche } \\
\text { Gray lime } \\
\text { Brown lime. } \\
\text { Blue lime } \\
\text { Gray lime } \\
\text { Yellow lime. }\end{array}$ & $\begin{array}{r}10 \\
100 \\
20 \\
20 \\
30 \\
40\end{array}$ & $\begin{array}{r}10 \\
110 \\
130 \\
150 \\
180 \\
220\end{array}$ & $\begin{array}{l}\text { Gray lime } \\
\text { Blue lime } \\
\text { Gray sandrock } \\
\text { Yellow sandrock strips of } \\
\text { soapstone. }\end{array}$ & $\begin{array}{r}60 \\
30 \\
40 \\
135\end{array}$ & $\begin{array}{l}280 \\
310 \\
350 \\
485\end{array}$ \\
\hline
\end{tabular}

Well 10

\begin{tabular}{|c|c|c|c|c|c|}
\hline $\begin{array}{l}\text { Caliche } \\
\text { Broken lime } \\
\text { Lime shells and caliche. } \\
\text { Gray lime } \\
\text { White lime } \\
\text { Yellow lime, sandy } \\
\text { Gray lime } \\
\text { Blue lime } \\
\text { White sand } \\
\text { Yellow sand } \\
\text { White sand. }\end{array}$ & $\begin{array}{l}25 \\
65 \\
35 \\
40 \\
20 \\
20 \\
50 \\
45 \\
15 \\
45 \\
10\end{array}$ & $\begin{array}{r}25 \\
90 \\
125 \\
165 \\
185 \\
\mathbf{2 0 5} \\
255 \\
300 \\
315 \\
360 \\
370\end{array}$ & $\begin{array}{l}\text { Yellow lime, sandy } \\
\text { Blue shale.. } \\
\text { Sand } \\
\text { Blue shale.. } \\
\text { Sand } \\
\text { Blue sand } \\
\text { Blue lime } \\
\text { Blue lime, sandy } \\
\text { Blue lime } \\
\text { Blue shale....... }\end{array}$ & $\begin{array}{r}20 \\
15 \\
10 \\
45 \\
10 \\
10 \\
5 \\
11 \\
14 \\
15\end{array}$ & $\begin{array}{l}390 \\
405 \\
415 \\
460 \\
470 \\
480 \\
485 \\
496 \\
510 \\
525\end{array}$ \\
\hline
\end{tabular}

Well 11

\begin{tabular}{|c|c|c|c|c|c|}
\hline $\begin{array}{l}\text { Soil and caliche. } \\
\text { Broken lime } \\
\text { Yellow lime } \\
\text { White lime } \\
\text { Yellow lime } \\
\text { Blue lime } \\
\text { White lime } \\
\text { Yellow sand }\end{array}$ & $\begin{array}{l}11 \\
89 \\
65 \\
45 \\
50 \\
40 \\
10 \\
45\end{array}$ & $\begin{array}{r}11 \\
100 \\
165 \\
210 \\
260 \\
300 \\
310 \\
355\end{array}$ & $\begin{array}{l}\text { Lime, sandy (water) } \ldots . . . \\
\text { Gray lime } \\
\text { Gray lime } \\
\text { Sand (water) } \\
\text { Sandy lime. } \\
\text { Sand } \\
\text { Blue shale... }\end{array}$ & $\begin{array}{r}45 \\
25 \\
40 \\
5 \\
40 \\
10 \\
25\end{array}$ & $\begin{array}{l}400 \\
425 \\
465 \\
470 \\
510 \\
520 \\
545\end{array}$ \\
\hline
\end{tabular}

Well 14

\begin{tabular}{|c|c|c|c|c|c|}
\hline $\begin{array}{l}\text { Broken lime } \\
\text { Grey lime } \\
\text { Yellow lime. } \\
\text { Lime } \\
\text { Gray lime } \\
\text { Yellow caliche. } \\
\text { Caliche lime } \\
\text { Yellow lime } \\
\text { Gray lime } \\
\text { Yellow broken lime. } \\
\text { Gray lime } \\
\text { Yellow broken lime. } \\
\text { Sandy lime. }\end{array}$ & $\begin{array}{r}15 \\
25 \\
10 \\
85 \\
25 \\
15 \\
5 \\
65 \\
49 \\
9 \\
7 \\
20 \\
20\end{array}$ & $\begin{array}{r}\mathbf{1 5} \\
40 \\
\mathbf{5 0} \\
135 \\
160 \\
175 \\
\mathbf{1 8 0} \\
\mathbf{2 4 5} \\
\mathbf{2 9 4} \\
\mathbf{3 0 3} \\
\mathbf{3 1 0} \\
\mathbf{3 3 0} \\
\mathbf{3 5 0}\end{array}$ & $\begin{array}{l}\text { Blue shale } \\
\text { Yellow sandy shale. } \\
\text { Broken sand } \\
\text { Blue shale } \\
\text { Broken sand. } \\
\text { Blue shale. } \\
\text { Broken lime } \\
\text { Blue sandy shale. } \\
\text { Broken lime } \\
\text { Blue shale. } \\
\text { Brown sand, some green } \\
\text { shale. }\end{array}$ & $\begin{array}{r}10 \\
25 \\
10 \\
10 \\
7 \\
8 \\
10 \\
10 \\
20 \\
30 \\
35\end{array}$ & $\begin{array}{l}360 \\
385 \\
395 \\
405 \\
412 \\
420 \\
430 \\
440 \\
460 \\
490 \\
525\end{array}$ \\
\hline
\end{tabular}




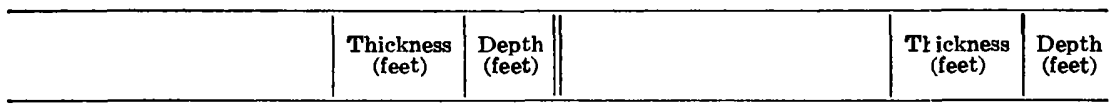

Well 15

\begin{tabular}{|c|c|c|c|c|c|}
\hline $\begin{array}{l}\text { Caliche } \\
\text { Yellow lime. } \\
\text { Gray lime. } \\
\text { Lime. } \\
\text { Broken lime. } \\
\text { Gray lime. } \\
\text { Lime } \\
\text { Red shale, sandy } \\
\text { Lime. } \\
\text { Yellow lime } \\
\text { Gray lime } \\
\text { Yellow broken lime } \\
\text { Broken sand. jard } \\
\text { Gellow sand, hard lime. }\end{array}$ & $\begin{array}{r}25 \\
5 \\
10 \\
60 \\
5 \\
20 \\
55 \\
4 \\
16 \\
50 \\
65 \\
5 \\
10 \\
20 \\
25\end{array}$ & $\begin{array}{r}25 \\
\mathbf{3 0} \\
40 \\
100 \\
105 \\
125 \\
180 \\
184 \\
200 \\
250 \\
\mathbf{3 1 5} \\
320 \\
\mathbf{3 3 0} \\
350 \\
\mathbf{3 7 5}\end{array}$ & $\begin{array}{l}\text { Blue shale. } \\
\text { Yellow lime } \\
\text { Blue shale. } \\
\text { Broken lime } \\
\text { Sandy blue shale } \\
\text { Blue shale and lime shells.-- } \\
\text { Blue sandy shale. } \\
\text { Sandy lime. } \\
\text { Sandy lime } \\
\text { Sandy blue shale. } \\
\text { Blue shale. } \\
\text { Red beds. } \\
\text { Blue shale. } \\
\text { Lime. } \\
\text { Unknown. }\end{array}$ & $\begin{array}{r}5 \\
10 \\
5 \\
15 \\
55 \\
30 \\
5 \\
10 \\
15 \\
20 \\
5 \\
5 \\
5 \\
10 \\
10\end{array}$ & $\begin{array}{l}\mathbf{3 8 0} \\
\mathbf{3 9 0} \\
\mathbf{3 9 5} \\
\mathbf{4 1 0} \\
\mathbf{4 6 5} \\
\mathbf{4 9 5} \\
\mathbf{5 0 0} \\
\mathbf{5 1 0} \\
\mathbf{5 2 5} \\
\mathbf{5 4 5} \\
\mathbf{5 5 0} \\
\mathbf{5 5 5} \\
\mathbf{5 6 0} \\
\mathbf{5 7 0} \\
\mathbf{5 8 0}\end{array}$ \\
\hline
\end{tabular}

Well i6

\begin{tabular}{|c|c|c|c|c|c|}
\hline $\begin{array}{l}\text { Caliche } \\
\text { Gravel } \\
\text { Caliche. } \\
\text { Lime. } \\
\text { Grown lime and shale. } \\
\text { Lime and shells } \\
\text { Lime and caliche } \\
\text { Blue lime } \\
\text { Yellow caliche } \\
\text { Sand and gravel } \\
\text { Yellow caliche. } \\
\text { Sand, water. } \\
\text { Water sand and gravel } \\
\text { Blue shale... } \\
\text { Sandy lime.... }\end{array}$ & $\begin{array}{r}50 \\
10 \\
5 \\
20 \\
20 \\
10 \\
75 \\
70 \\
50 \\
45 \\
5 \\
25 \\
15 \\
15 \\
10 \\
25\end{array}$ & $\begin{array}{r}50 \\
60 \\
65 \\
85 \\
105 \\
115 \\
190 \\
260 \\
310 \\
355 \\
360 \\
385 \\
400 \\
415 \\
425 \\
450\end{array}$ & $\begin{array}{l}\text { Lime, shells, and shale } \\
\text { Broken sand. } \\
\text { Blue shale. } \\
\text { Lime, shells, and shale } \\
\text { Lime, lime } \\
\text { Sandy limels, and shale } \\
\text { Lime, shells, } \\
\text { Lime } \\
\text { Gray lime } \\
\text { Brown lime } \\
\text { Brown lime. } \\
\text { Sandy (?) } \\
\text { Blue lime. } \\
\text { Brown lime. } \\
\text { Blue sand. }\end{array}$ & $\begin{array}{r}65 \\
10 \\
15 \\
10 \\
5 \\
15 \\
160 \\
30 \\
20 \\
20 \\
3 \\
10 \\
12 \\
10 \\
5\end{array}$ & $\begin{array}{l}515 \\
525 \\
540 \\
550 \\
\mathbf{5 5 5} \\
\mathbf{5 7 0} \\
730 \\
760 \\
780 \\
800 \\
\mathbf{8 0 3} \\
813 \\
825 \\
835 \\
\mathbf{8 4 0}\end{array}$ \\
\hline
\end{tabular}

\section{TERRY COUNTY}

Population in 1940: 4,009.

\section{BROWNFIELD}

Source of information: E. E. Jones, water superintendent, Mas 24, 1944.

Ownership: Municipal.

Source of supply: Three wells.

Well 1. Dug and drilled; depth, 107 feet; deep-well turbina pump and electric motor; yield, 300 gallons a minute.

Well 2. Drilled in 1932; depth, 117 feet; deep-well turbin? pump and electric motor; yield, 400 gallons a minute.

Well 3. Drilled in 1941 by Paul Pierson; depth, 132 feet; diameter, 13 inches; deep-well turbine pump and 20-horsepower electric motor; yield, 400 gallons a minute.

Pumpage: No record.

Storage: Two concrete storage reservoirs, total capacity, 140,000 gallons; two elevated tanks, total capacity, 250,000 gallons.

Number of customers: 1,000 .

Treatment: None. 
Analysis, well 1

[Collected May 24, 1944. $\mathrm{pH}$ is 7.9. Analyzed by W. W. Hastings]

\begin{tabular}{|c|c|c|c|c|c|}
\hline & $\begin{array}{l}\text { Parts per } \\
\text { million }\end{array}$ & $\begin{array}{l}\text { Equivalents } \\
\text { per million }\end{array}$ & & $\begin{array}{l}\text { Parts Fer } \\
\text { million }\end{array}$ & $\begin{array}{l}\text { Equivalents } \\
\text { per million }\end{array}$ \\
\hline $\begin{array}{l}\text { Silica }\left(\mathrm{SiO}_{2}\right) \\
\text { Iron }(\mathrm{Fe}) \\
\text { Calcium }(\mathrm{Ca}) \\
\text { Magnesium }(\mathrm{Mg}) \\
\text { Sodium }(\mathrm{Na}) \\
\text { Potassium }(\mathrm{K}) \\
\text { Bicarbonate }\left(\mathrm{H} \mathrm{CO}_{3}\right)\end{array}$ & $\begin{array}{l}46 \\
64 \\
61 \\
64 \\
15 \\
291\end{array}$ & $\begin{array}{r}\mathbf{3} .19 \\
\mathbf{5} .02 \\
\mathbf{2 . 7 8} \\
. \mathbf{3 8} \\
4.77\end{array}$ & $\begin{array}{l}\text { Sulfate }\left(\mathrm{SO}_{4}\right) \\
\text { Chloride }(\mathrm{Cl}) \\
\text { Fluoride (F) } \\
\left.\text { Nitrate (NO})_{3}\right) \\
\text { Dissolved solids } \\
\text { Total hardness as } \mathrm{CaCO}\end{array}$ & $\begin{array}{r}169 \\
98 \\
3.0 \\
10 \\
740 \\
410\end{array}$ & $\begin{array}{r}3.52 \\
2.76 \\
.16 \\
.16\end{array}$ \\
\hline
\end{tabular}

Driller's log, well 8

\begin{tabular}{|c|c|c|c|c|c|}
\hline & $\underset{\text { (feet) }}{\text { Thickness }}$ & $\underset{\text { (feet) }}{\text { Depth }}$ & & $\begin{array}{c}\text { Thickness } \\
\text { (feet) }\end{array}$ & $\underset{\text { (feet) }}{\text { Depth }}$ \\
\hline $\begin{array}{l}\text { Soil, clay, and sand } \\
\text { Soft red sand } \\
\text { Hard sand } \\
\text { Soft white sand (dry) } \\
\text { Caliche_. } \\
\text { Water sand. }\end{array}$ & $\begin{array}{r}33 \\
36 \\
5 \\
12 \\
8 \\
4\end{array}$ & $\begin{array}{l}33 \\
69 \\
74 \\
86 \\
94 \\
98\end{array}$ & $\begin{array}{l}\text { Chert, crystalline sandstone.- } \\
\text { Water sand, probably some } \\
\text { water. } \\
\text { Chert, } \\
\text { Gravel, water. } \\
\text { Red rock (red bed), clay... }\end{array}$ & $\begin{array}{r}7 \\
1 \\
4 \\
20 \\
2\end{array}$ & $\begin{array}{l}105 \\
106 \\
110 \\
130 \\
132\end{array}$ \\
\hline
\end{tabular}

\section{TOM GREEN COUNTY}

\section{Christoval}

Population in 1940: 544.

Source of information: H. H. Shaw, owner, Aug. 19, 1947.

Owner: H. A. Shaw.

Source of supply: Dug well; depth, 40 feet; dimensions, 4 by 7 feet at top and 8 by 16 feet at bottom; lined with concrete to a depth of 3 ) feet below the surface; centrifugal pump and 3-horsepower electric motor; static water level, 23 feet below land surface; pumping level, 24.52 feet below land surface Aug. 19, 1947; yield, 100 gallons a minute.

Pumpage: Average, 80,000 gallons a day.

Storage: Two concrete storage reservoirs, 14,000 gallons each.

Number of customers: 40 .

Treatment: None.

\section{Analysis}

[Collected Aug. 19, 1947. pH is 7.0. Analyzed by B. C. Dwyer]

\begin{tabular}{|c|c|c|c|c|c|}
\hline • & $\begin{array}{l}\text { Parts per } \\
\text { million }\end{array}$ & $\begin{array}{l}\text { Equivalents } \\
\text { per million }\end{array}$ & & $\begin{array}{l}\text { Parts for } \\
\text { million }\end{array}$ & $\begin{array}{l}\text { Equivalents } \\
\text { per million }\end{array}$ \\
\hline 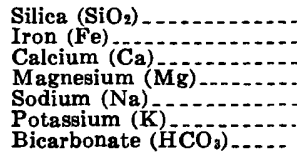 & $\begin{array}{l}15 \\
104^{.04} \\
16 \\
5.1 \\
1.2 \\
350\end{array}$ & $\begin{array}{r}5.19 \\
1.32 \\
.22 \\
.03 \\
5.74\end{array}$ & 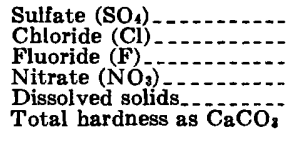 & $\begin{array}{l}16 \\
22 \\
3.2 \\
355 \\
326\end{array}$ & $\begin{array}{r}0.33 \\
.62 \\
.01 \\
.06 \\
-0 .-0 \\
-0.0\end{array}$ \\
\hline
\end{tabular}

\section{SAN ANGELO}

Population in 1940: 25,802.

Source of information: Walter Beaty, water superintendent, Aug. 19, 1947.

Owner: West Texas Utilities Co.

Source of supply: Four reservoirs.

Lake Concho. At south end of city near filtration plant; constructed in 1902 ; capacity, 459 acre-feet. 
Lake Ben Ficklin. Above Lake Concho; constructed in 1918; capacity, 398 acre-feet.

Lake Metcalfe. Constructed in 1902; capacity, 425 acre-feet.

Lake Nosworthy. Constructed in 1930; capacity, 10,500 acr?-feet.

Average pumpage, in gallons a day

\begin{tabular}{|c|c|c|c|c|c|c|c|c|}
\hline & 1946 & 1947 & & 1946 & 1947 & & 1946 & 1947 \\
\hline $\begin{array}{l}\text { January } \\
\text { February } \\
\text { March... } \\
\text { April. }\end{array}$ & $\begin{array}{l}\mathbf{3 , 1 7 2 , 0 0 0} \\
\mathbf{3}, \mathbf{8 1 8 , 0 0 0} \\
4,180,000 \\
6,470,000\end{array}$ & $\begin{array}{l}2,896,000 \\
3,488,000 \\
2,835,000 \\
5,437,000\end{array}$ & $\begin{array}{l}\text { May_... } \\
\text { June_... } \\
\text { July_... } \\
\text { August._. }\end{array}$ & $\begin{array}{r}6,742,000 \\
9,315,000 \\
10,634,000 \\
11,065,000\end{array}$ & $\begin{array}{r}\mathbf{5 , 1 2 4 , 0 0 0} \\
\mathbf{9}, 353,000 \\
11,770,000 \\
\end{array}$ & $\begin{array}{l}\text { September - } \\
\text { October-- } \\
\text { November- } \\
\text { December - }\end{array}$ & \begin{tabular}{|l|}
$\mathbf{6}, 750,000$ \\
$\mathbf{3}, \mathbf{4 7 6}, 000$ \\
$\mathbf{3}, 617,000$ \\
$\mathbf{3 , 0 3 8}, 000$
\end{tabular} & 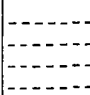 \\
\hline
\end{tabular}

Storage: Ground storage reservoir, 630,000 gallons; two elerated tanks, 250,000 gallons each.

Number of customers: 9,702 .

Treatment: Coagulation, sedimentation, filtration, and chlorination.

\section{Analyses}

[Collected Aug. 18, 1947. pH: raw and finished water, each 7.5. Analyzed by B. C. Dwyer]

\begin{tabular}{|c|c|c|c|c|}
\hline \multirow[b]{2}{*}{ Lake Nosworthy } & \multicolumn{2}{|c|}{ Raw water } & \multicolumn{2}{|c|}{ Fin'shed water } \\
\hline & $\begin{array}{c}\text { Parts per } \\
\text { million }\end{array}$ & $\begin{array}{c}\text { Equivalents } \\
\text { per million }\end{array}$ & $\begin{array}{c}\text { Parts por } \\
\text { million }\end{array}$ & $\begin{array}{c}\text { Equivalents } \\
\text { per million }\end{array}$ \\
\hline 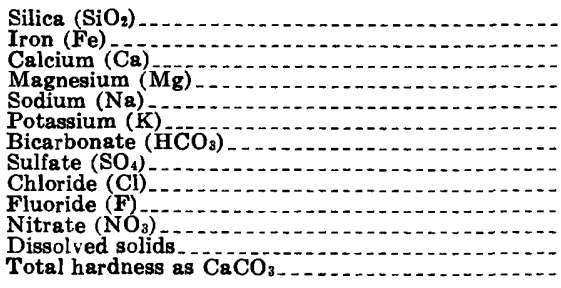 & $\begin{array}{l}11 \\
55^{.37} \\
20 \\
43 \\
7.0 \\
228 \\
29 \\
74 \\
.6 \\
352^{.0} \\
220\end{array}$ & $\begin{array}{r}\mathbf{2 . 7 5} \\
1.64 \\
\mathbf{1 . 8 9} \\
\mathbf{3 . 1 8} \\
.60 \\
2.09 \\
.03 \\
.00 \\
\end{array}$ & 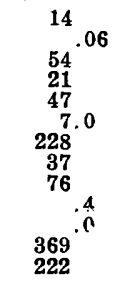 & \begin{tabular}{r}
2.70 \\
1.73 \\
2.06 \\
.18 \\
3.74 \\
.77 \\
2.14 \\
.02 \\
.00 \\
\hdashline-1.7 \\
\end{tabular} \\
\hline
\end{tabular}

Population in 1940: 450.

\section{SANATORIUM}

Source of information: T. E. Heskew, chief engineer, Aug. 19, 1947. .

Owner: State of Texas.

Source of supply: Two wells northeast of Sanatorium power plant, half a mile apart; auxiliary supply from North Concho River.

Well 1. Dug in 1930; depth, 80 feet; diameter, 12 feet; deep-well turbine pump and 40-horsepower electric motor; static water level, 18.5 feet below land surface Aug. 19, 1947; yield, 250 gallons a minute; reported decline considerable after several hours of pumping.

Well 2. Dug in 1938; depth, 80 feet; diameter, 12 feet; deep-well turbine pump and 40-horsepower electric motor; pumping level, 53.8 ;eet below land surface Aug. 19, 1947; yield, 500 gallons a minute.

Pumpage: Maximum, 375,000 gallons a day: minimum, 225,000 gallons a day. Storage: Elevated tank, 100,000 gallons.

Number of customers: 1,350 patients and staff members.

Treatment: None. 
Analysis, well 2

[Collected Aug. 19, 1947. $\mathrm{pH}$ is 7.2. Analyzed by B. C. Dwyer]

\begin{tabular}{|c|c|c|c|c|c|}
\hline$\cdot$ & $\begin{array}{l}\text { Parts per } \\
\text { million }\end{array}$ & $\begin{array}{l}\text { Equivalents } \\
\text { per million }\end{array}$ & & $\begin{array}{l}\text { Parts } p \text {;r } \\
\text { millior }\end{array}$ & $\begin{array}{l}\text { Equivalents } \\
\text { per million }\end{array}$ \\
\hline $\begin{array}{l}\text { Silica }\left(\mathrm{SiO}_{2}\right) \\
\text { Iron }(\mathrm{Fe}) \\
\text { Calcium }(\mathrm{Ca}) \\
\text { Magnesium }(\mathrm{Mg}) \\
\text { Sodium }(\mathrm{Na}) \\
\text { Potassium }(\mathrm{K}) \\
\text { Bicarbonate (HCO }\end{array}$ & $\begin{array}{l}20 \\
90 \\
52 \\
35 \\
7.04 \\
390^{-0}\end{array}$ & $\begin{array}{r}4.49 \\
4.28 \\
1.53 \\
6.18 \\
6.39\end{array}$ & $\begin{array}{l}\text { Sulfate (SO } \\
\text { Chloride (CI) } \\
\text { Fluoride (F) } \\
\text { Nitrate (NO } \\
\text { Dissolved solids } \\
\text { Total hardness as } \mathrm{CaCO}_{3}\end{array}$ & $\begin{array}{l}100 \\
68 \\
3.6 \\
3.8 \\
568 \\
438\end{array}$ & $\begin{array}{r}2.08 \\
1.92 \\
.03 \\
.06 \\
-06 \\
-0 .-\end{array}$ \\
\hline
\end{tabular}

Driller's log, well 1

\begin{tabular}{|c|c|c|c|c|c|}
\hline & $\begin{array}{c}\text { Thickness } \\
\text { (feet) }\end{array}$ & $\begin{array}{l}\text { Depth } \\
\text { (feet) }\end{array}$ & & $\underset{\text { (f ’et) }}{\text { Thickness }}$ & $\begin{array}{l}\text { Depth } \\
\text { (feet) }\end{array}$ \\
\hline $\begin{array}{l}\text { Soil } \\
\text { Goose gravel } \\
\text { Gravel and clay } \\
\text { Hard conglomerate, water at } \\
\quad 32 \text { feet. }\end{array}$ & $\begin{array}{r}4 \\
20 \\
4 \\
32\end{array}$ & $\begin{array}{r}4 \\
24 \\
28 \\
60\end{array}$ & $\begin{array}{l}\text { Coarse tight sand } \\
\text { Hard rock } \\
\text { Yellow shale. } \\
\text { Blue shale. }\end{array}$ & $\begin{array}{r}4 \\
5 \\
10 \\
1\end{array}$ & $\begin{array}{l}64 \\
69 \\
79 \\
80\end{array}$ \\
\hline
\end{tabular}

\section{UPTON COUNTY}

Population in 1940: 2,595.

\section{MCCAMEY}

Source of information: E. B. Heinze, city secretary, Feb. 10, 1947.

Ownership: Municipal.

Source of supply: Two wells 15 miles southeast of McCamey in Peros County.

Well 1. Drilled in 1929 by Layne-Texas Co.; depth, 272 feet; diameter $15 \frac{1}{2}$ to 8 inches; deep-well turbine pump and 30-horseporrer electric motor; static water level, 167.6 feet below land surface Fel . 11, 1947; yield, 280 gallons a minute.

Well 2. Drilled in 1929 by Layne-Texas Co.; depth, 354 feet; diameter, 16 to 8 inches; deep-well turbine pump and 30-horsepowor electric motor; static water level, 148.1 feet below land surface Fel 10, 1947; yield, 280 gallons a minute.

Average pumpage, in gallons a day

\begin{tabular}{|c|c|c|c|c|c|c|c|c|}
\hline & 1946 & 1947 & & 1946 & 1947 & & $194 \mathrm{~S}$ & 1947 \\
\hline $\begin{array}{l}\text { January... } \\
\text { February } \\
\text { March. } \\
\text { April. }\end{array}$ & $\begin{array}{l}112,300 \\
124,000 \\
140,000 \\
160,900\end{array}$ & \begin{tabular}{|c|}
123,000 \\
\\
\end{tabular} & $\begin{array}{l}\text { May_... } \\
\text { June_. } \\
\text { July.... } \\
\text { August_- }\end{array}$ & $\begin{array}{l}198,000 \\
252,000 \\
290,000 \\
293,600\end{array}$ & $-1-1$ & $\begin{array}{l}\text { September- } \\
\text { October--- } \\
\text { November- } \\
\text { December - }\end{array}$ & $\begin{array}{r}199, C 00 \\
162, C 00 \\
115,600 \\
98,100\end{array}$ & 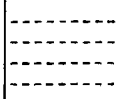 \\
\hline
\end{tabular}

Storage: Two ground storage reservoirs, 100,000 gallons each; elevated tank, 100,000 gallons.

Number of customers: 693.

Treatment: None. 


\section{Analysis, well 2}

[Collected Feb. 10, 1947. $\mathrm{pH}$ is 7.2. Analyzed by B. C. Dwyer]

\begin{tabular}{|c|c|c|c|c|c|}
\hline & $\begin{array}{l}\text { Parts per } \\
\text { million }\end{array}$ & $\begin{array}{l}\text { Equivalents } \\
\text { per million }\end{array}$ & & $\begin{array}{c}\text { Parts per } \\
\text { million }\end{array}$ & $\begin{array}{l}\text { Equivalents } \\
\text { per million }\end{array}$ \\
\hline 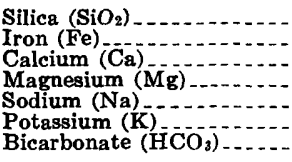 & $\begin{array}{l}14 \\
71 \\
25 \\
30 \\
5.4 \\
250\end{array}$ & $\begin{array}{r}\mathbf{3} .54 \\
2.06 \\
1.30 \\
.14 \\
4.10\end{array}$ & $\begin{array}{l}\text { Sulfate }\left(\mathrm{SO}_{4}\right) \\
\text { Chloride (Cl) } \\
\text { Fluoride (F) } \\
\text { Nitrate (NO} \mathrm{N}_{3} \text { ) } \\
\text { Dissolved solids } \\
\text { Total hardness as } \mathrm{CaCO}_{3}\end{array}$ & $\begin{array}{l}7 € \\
44 \\
1.2 \\
3.3 \\
397 \\
28 C\end{array}$ & \begin{tabular}{r}
1.58 \\
1.24 \\
.06 \\
.06 \\
\hdashline-0. \\
-0.0
\end{tabular} \\
\hline
\end{tabular}

\section{Driller's logs}

\begin{tabular}{l|l|l|l|}
\hline $\begin{array}{c}\text { Thickness } \\
\text { (feet) }\end{array}$ & $\begin{array}{c}\text { Depth } \\
\text { (feet) }\end{array}$ & $\begin{array}{c}\text { Ti ickness } \\
\text { (feet) }\end{array}$ & $\begin{array}{c}\text { Depth } \\
\text { (feet) }\end{array}$ \\
\hline
\end{tabular}

Well 1

\begin{tabular}{|c|c|c|c|c|c|}
\hline $\begin{array}{l}\text { Caliche } \\
\text { Pink clay } \\
\text { White and yellow clay } \\
\text { Red sand with some clay } \\
\text { Pink sand. }\end{array}$ & $\begin{array}{r}155 \\
9 \\
11 \\
28 \\
5\end{array}$ & $\begin{array}{l}155 \\
164 \\
175 \\
203 \\
208\end{array}$ & $\begin{array}{l}\text { Red sand with little clay } \\
\text { Red clay } \\
\text { Clay, } \\
\text { Rock } \\
\text { Sand. }\end{array}$ & $\begin{array}{r}\mathbf{5 7} \\
\mathbf{1} \\
\mathbf{2} \\
\mathbf{1} \\
\mathbf{3}\end{array}$ & $\begin{array}{r}265 \\
266 \\
268 \\
-\quad 269 \\
272\end{array}$ \\
\hline
\end{tabular}

Well 2

\begin{tabular}{|c|c|c|c|c|c|}
\hline $\begin{array}{l}\text { Soil } \\
\text { Caliche and gravel } \\
\text { Gravel. } \\
\text { Caliche and gravel } \\
\text { Gravel, water. } \\
\text { Caliche. } \\
\text { Caliche and gravel }\end{array}$ & $\begin{array}{r}16 \\
129 \\
15 \\
10 \\
10 \\
40 \\
50\end{array}$ & $\begin{array}{r}16 \\
145 \\
160 \\
170 \\
180 \\
220 \\
270\end{array}$ & $\begin{array}{l}\text { Gravel and boulders } \\
\text { Gravel and clay } \\
\text { Fine sand } \\
\text { Clay } \\
\text { Gine sand } \\
\text { Gravel and clay }\end{array}$ & $\begin{array}{r}18 \\
7 \\
5 \\
6 \\
23 \\
3 \\
22\end{array}$ & $\begin{array}{l}288 \\
295 \\
300 \\
306 \\
329 \\
332 \\
354\end{array}$ \\
\hline
\end{tabular}

\section{RANKIN}

\section{Population in 1940: 672.}

Source of information: W. J. Pollard, operator, Sept. 22, 1948.

Ownership: Municipal.

Source of supply: Two wells.

Well 1. About 100 yards northeast of Yates Hotel; drilled in 1939; depth, 170 feet; diameter, 8 inches; deep-well turbine pump ard 71/2-horsepower electric motor; yield, 50 gallons a minute.

Well 2. About 300 yards south of well 1; drilled in 1948 by N. C. House; depth, 166 feet; diameter, 7 inches; deep-well turbine pump and 5horsepower electric motor; yield, 60 gallons a minute.

Pumpage (estimated): 100,000 gallons a day.

Storage: Concrete ground storage reservoir, 50,000 gallons; e'evated tank, 50,000 gallons.

Number of customers: 270.

Treatment: None. 


\section{Analyses}

[Collected Sept. 22, 1948. pH, each well, is 7.2. Analyzed by J. R. Avrett and I. E. Weaver]

\begin{tabular}{|c|c|c|c|c|}
\hline & & ell 1 & & ell 2 \\
\hline & $\begin{array}{c}\text { Parts per } \\
\text { million }\end{array}$ & $\begin{array}{l}\text { Equivalents } \\
\text { per million }\end{array}$ & $\begin{array}{l}\text { Parts fer } \\
\text { million }\end{array}$ & $\begin{array}{l}\text { Equivalents } \\
\text { per million }\end{array}$ \\
\hline 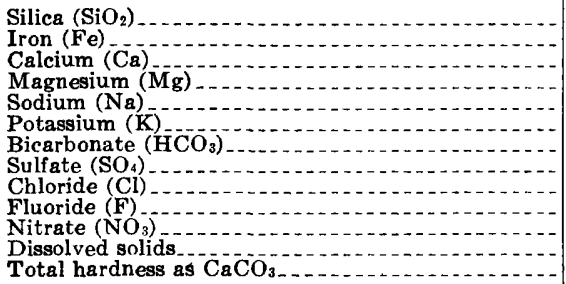 & $\begin{array}{l}8.2 \\
150 \\
110 \\
117 \\
276 \\
7.6 \\
312 \\
919 \\
165 \\
3.0 \\
15 \\
1,820 \\
856\end{array}$ & $\begin{array}{r}7.49 \\
9.62 \\
11.99 \\
.19 \\
5.11 \\
19.13 \\
4.65 \\
.16 \\
.24\end{array}$ & $\begin{array}{c}18 \\
150 \\
78 \\
142 \\
5.2 \\
336 \\
545 \\
108 \\
2.0 \\
12 \\
1,230 \\
695\end{array}$ & $\begin{array}{r}7.49 \\
6.41 \\
6.18 \\
5.13 \\
11.31 \\
3.05 \\
.11 \\
.19 \\
\end{array}$ \\
\hline
\end{tabular}

Driller's log, well 2

\begin{tabular}{|c|c|c|c|c|c|}
\hline & $\begin{array}{c}\text { Thickness } \\
\text { (feet) }\end{array}$ & $\begin{array}{l}\text { Depth } \\
\text { (feet) }\end{array}$ & & $\underset{\text { (feet) }}{\text { Thickness }}$ & $\begin{array}{l}\text { Depth } \\
\text { (feet) }\end{array}$ \\
\hline $\begin{array}{l}\text { Surface soil } \\
\text { Gravel and caliche. } \\
\text { No record } \\
\text { Yellow lime... } \\
\text { Sand and gravel (water) }\end{array}$ & $\begin{array}{r}8 \\
17 \\
33 \\
10 \\
\mathbf{3 4}\end{array}$ & $\begin{array}{r}8 \\
25 \\
58 \\
68 \\
102\end{array}$ & $\begin{array}{l}\text { White sandrock } \\
\text { Red rock. } \\
\text { White lime } \\
\text { Yellow sand (water) }\end{array}$ & $\begin{array}{r}16 \\
11 \\
2 \\
35\end{array}$ & $\begin{array}{l}118 \\
129 \\
131 \\
166\end{array}$ \\
\hline
\end{tabular}

\section{VAL VERDE COUNTY}

\section{DEL RIO}

Population in 1940: 13,343 .

Source of information: C. C. Brown, water superintendent, Nov. 1, 1945.

Ownership: Municipal.

Source of supply: San Felipe Springs in northeastern Del Rio, about half a mile north of U. S. Highway 90.

\section{Average pumpage, in gallons a day}

\begin{tabular}{|c|c|c|c|c|c|c|c|c|}
\hline & 1943 & 1944 & & 1943 & 1944 & & 1943 & 1944 \\
\hline $\begin{array}{l}\text { January } \\
\text { February } \\
\text { Mareh } \\
\text { April }\end{array}$ & $\begin{array}{l}1,000,000 \\
1,330,000 \\
1,526,000 \\
1,758,000\end{array}$ & $\begin{array}{l}1,196,000 \\
1,305,000 \\
1,340,000 \\
1,675,000\end{array}$ & $\begin{array}{l}\text { May_... } \\
\text { June..- } \\
\text { July_-- } \\
\text { August_- }\end{array}$ & $\begin{array}{l}2,050,000 \\
1,748,000 \\
2,416,000 \\
3,030,000\end{array}$ & $\begin{array}{l}1,865,000 \\
1,800,000 \\
2,267,000 \\
3,010,000\end{array}$ & $\begin{array}{l}\text { September - } \\
\text { October-- } \\
\text { November - } \\
\text { December.- }\end{array}$ & $\begin{array}{l}2,6 \mathrm{f} 6,000 \\
1,240,000 \\
1,5 ? 4,000 \\
1,1: 7,000\end{array}$ & $\begin{array}{r}1,543,000 \\
1,295,000 \\
1,331,000 \\
915,000\end{array}$ \\
\hline
\end{tabular}

Storage: Two concrete elevated reservoirs, 2,250,000 gallons each.

Number of customers: 3,100 .

Treatment: Chlorination and ammoniation.

\section{Analysis}

[Collected Nov. 1, 1945. $\mathrm{pH}$ is 6.8. Analyzed by C. B. Cibulka]

\begin{tabular}{|c|c|c|c|c|c|}
\hline & $\begin{array}{l}\text { Parts per } \\
\text { million }\end{array}$ & $\begin{array}{l}\text { Equivalents } \\
\text { per million }\end{array}$ & & $\begin{array}{l}\text { Parts per } \\
\text { million }\end{array}$ & $\begin{array}{l}\text { Equivalents } \\
\text { per million }\end{array}$ \\
\hline $\begin{array}{l}\text { Silica }\left(\mathrm{SiO}_{2}\right) \\
\text { Iron }(\mathrm{Fe}) \\
\text { Calcium }(\mathrm{Ca}) \\
\text { Magnesium }(\mathrm{Mg}) \\
\text { Sodium }(\mathrm{Na}) \\
\text { Potassium }(\mathrm{K}) \\
\text { Bicarbonate }\left(\mathrm{H} \mathrm{CO}_{3}\right)\end{array}$ & $\begin{array}{l}14 \\
58.05 \\
7.6 \\
27.6 \\
254.0\end{array}$ & $\begin{array}{r}2.894 \\
.625 \\
1.174 \\
.102 \\
4.163\end{array}$ & $\begin{array}{l}\text { Sulfate }\left(\mathrm{SO}_{4}\right) \\
\text { Chloride (CI) } \\
\text { Fluoride (F) } \\
\text { Nitrate (NO } \\
\text { Dissolved solids } \\
\text { Total hardness as } \mathrm{CaCO} \text { a }\end{array}$ & $\begin{array}{r}6.2 \\
13 \\
1.0 \\
5.8 \\
264 \\
172\end{array}$ & $\begin{array}{r}0.129 \\
.367 \\
.053 \\
.094 \\
-0 .-0 \\
\end{array}$ \\
\hline
\end{tabular}




\section{WARD COUNTY}

Population in 1940: 558.

\section{BARSTOW}

Source of information: M. W. Nichols, water superintendent, Oct. 9, 1948.

Ownership: Municipal.

Source of supply: Two wells.

Well 1. About $4 \frac{1}{2}$ miles east of Barstow; drilled in 1930 by James Miles; depth, 117 feet; diameter, 8 inches; deep-well turbine pump and $71 / 2$ horsepower electric motor; static water level, 95.72 feet below land surface Aug. 9, 1940; yield, 55 gallons a minute.

Well 2. About 30 feet south of well 1; drilled in July 1948 by C. C. and H. Drilling Co.; depth, 120 feet; diameter, 8 inches; deep-well turbine pump and 10-horsepower electric motor; yield, 72 gallons a minute.

Pumpage (estimated): 75,000 gallons a day.

Storage: Concrete ground reservoir, 50,000 gallons; elevated tank, 50,000 gallons.

Number of customers: 175.

Treatment: None.

Analysis, well 1

[Collected May 24, 1940. Analyzed by N. Talvite]

\begin{tabular}{|c|c|c|c|c|c|}
\hline & $\begin{array}{l}\text { Parts per } \\
\text { million }\end{array}$ & $\begin{array}{l}\text { Equivalents } \\
\text { per million }\end{array}$ & & $\begin{array}{l}\text { Parts per } \\
\text { million }\end{array}$ & $\begin{array}{l}\text { Equivalents } \\
\text { per million }\end{array}$ \\
\hline $\begin{array}{l}\text { Silica }\left(\mathrm{SiO}_{2}\right) \\
\text { Iron }(\mathrm{Fe}) \\
\text { Calcium }(\mathrm{Ca}) \\
\text { Magnesium }(\mathrm{Mg}) \\
\text { Sodium }(\mathrm{Na}) \\
\text { Potassium }(\mathrm{K}) \\
\text { Bicarbonate }\left(\mathrm{H} \mathrm{CO}_{3}\right)\end{array}$ & $\begin{array}{l}28 \\
149 \\
51 \\
76 \\
216 \\
6.2\end{array}$ & $\begin{array}{r}7.44 \\
4.19 \\
3.30 \\
.16 \\
3.64\end{array}$ & $\begin{array}{l}\text { Sulfate }\left(\mathrm{SO}_{4}\right) \\
\text { Chloride }(\mathrm{Cl}) \\
\text { Fluoride (F) } \\
\text { Nitrate (NO} \\
\text { Dissolved solids } \\
\text { Total hardness as } \mathrm{CaCO}_{8}\end{array}$ & $\begin{array}{c}428 \\
83 \\
1.2 \\
6.4 \\
935 \\
682\end{array}$ & $\begin{array}{r}8.91 \\
2.34 \\
.06 \\
.10 \\
\hdashline-. .-10\end{array}$ \\
\hline
\end{tabular}

\section{GRANDFALIS}

Population in 1940: 653.

Source of information: L. J. Adams, manager, Oct. 9, 1948.

Owner: L. C. Harrison Water Co.

Source of Supply: Two wells.

Well 1. About $4 \frac{1}{2}$ miles northwest of town; drilled in 1940 by Boyd Hopkins; depth, 95 feet; diameter $81 / 4$ inches; deep-well turbine pump and 100-horsepower natural gas engine; yield, 500 gallons a minute.

Well 2. South of well 1; drilled; depth, 95 feet; diameter, 8 inches; deepwell turbine pump and 75-horsepower electric motor; yield, 500 gallons a minute.

Pumpage (estimated) : 200,000 gallons a day.

Storage: Two steel surface reservoirs, 24,000 and 32,00 gallons, respectively.

Number of customers: 247.

Treatment: None. 
Analysis, well 1

[Collected May 15, 1940. Analyzed by N. Talvite]

\begin{tabular}{|c|c|c|c|c|c|}
\hline & $\begin{array}{l}\text { Parts per } \\
\text { million }\end{array}$ & $\begin{array}{l}\text { Equivalents } \\
\text { per million }\end{array}$ & & $\begin{array}{l}\text { Parts per } \\
\text { million }\end{array}$ & $\begin{array}{l}\text { Equivalents } \\
\text { per million }\end{array}$ \\
\hline $\begin{array}{l}\text { Silica }\left(\mathrm{SiO}_{2}\right) \\
\text { Iron }(\mathrm{Fe}) \\
\text { Calcium }(\mathrm{Ca}) \\
\text { Magnesium }(\mathrm{Mg}) \\
\text { Sodium }(\mathrm{Na}) \\
\text { Potassium }(\mathrm{K}) \\
\text { Bicarbonate }\left(\mathrm{H}^{2} \mathrm{CO}_{3}\right)\end{array}$ & $\begin{array}{l}18 \\
94 \\
40 \\
367 \\
9.11 \\
940\end{array}$ & $\begin{array}{r}4.69 \\
3.29 \\
15.96 \\
.25 \\
3.93\end{array}$ & $\begin{array}{l}\text { Sulfate }\left(\mathrm{SO}_{4}\right) \\
\text { Chloride }(\mathrm{Cl}) \\
\text { Fluoride (F) } \\
\text { Nitrate (NO}) \\
\text { Dissolved soilds } \\
\text { Total hardness as } \mathrm{CaCO}_{3}\end{array}$ & $\begin{array}{r}324 \\
463 \\
1.7 \\
1.0 \\
1,437 \\
399\end{array}$ & $\begin{array}{r}6.75 \\
13.06 \\
.09 \\
.02 \\
-.- \\
-1\end{array}$ \\
\hline
\end{tabular}

\section{MONAHANS}

Population in $1940: 3,944$.

Source of information: E. R. Blackmond, water superintendent, Sept. 29, 1948.

Ownership: Municipal.

Source of supply: Twenty wells.

Unit 1. Five wells seven blocks north of city hall; all wells about 130 feet deep and each equipped with deep-well turbine pump and 5-horsepower electric motor; combined yield of five wells, 300 gallons a minute.

Unit 2. Twelve wells northwest of city limits; drilled between 1942 and 1947; depths, about 130 feet; deep-well turbine pumps and electric motors; combined yield, 957 gallons a minute.

Unit 3. Well 40 feet south of Victory Avenue and 140 feet west of city limits; drilled January 1948; depth, 160 feet; diameter, 16 to 14 inches; deep-well turbine pump and 40-horsepower electric motor; static water level, 49 feet below land surface in March 1948; yield, 400 gallons a minute with drawdown of $\mathbf{5 2}$ feet after pumping 3 hours in March 1948.

Unit 4. Well about 1,100 feet south of Unit 3; drilled in January 1948; depth, 160 feet; diameter, 16 to 14 inches; deep-well turbine pump and 40-horsepower electric motor; yield, 350 gallons a minute with drawdown of 50 feet after pumping 6 hours in May 1948.

Unit 5. Well about 1,100 feet south of Unit 4; drilled in January 1948; depth, 160 feet; diameter, 16 to 14 inches; deep-well turbine pump and 25-horsepower electric motor; yield, 300 gallons a minute with drawdown of 50 feet after pumping 6 hours in August 1948.

Pumpage (estimated) : 1,000,000 gallons a day.

Storage: Two elevated tanks, 56,000 gallons and 500,000 gallons, respectively; two concrete ground storage reservoirs, 200,000 gallons each.

Number of customers: 1,825 .

Treatment: None.

Analysis, well 1, unit 1

[Collected Apr. 29, 1941. Analyzed by B. Irelan and N. L. Lewis]

\begin{tabular}{|c|c|c|c|c|c|}
\hline & $\begin{array}{l}\text { Parts per } \\
\text { million }\end{array}$ & $\begin{array}{l}\text { Equivalents } \\
\text { per million }\end{array}$ & & $\begin{array}{l}\text { Parts per } \\
\text { million }\end{array}$ & $\begin{array}{l}\text { Equivalents } \\
\text { per million }\end{array}$ \\
\hline $\begin{array}{l}\text { Silica }\left(\mathrm{SiO}_{2}\right) \\
\text { Calcium }(\mathrm{Ca}) \\
\text { Magnesium }(\mathrm{Mg}) \\
\text { Sodium and potassium } \\
\quad(\mathrm{Na}+\mathrm{K}) \text {. } \\
\text { Bicarbonate }\left(\mathrm{HCO}_{2}\right)\end{array}$ & $\begin{array}{c}17 \\
32 \\
6.7 \\
178 \\
265\end{array}$ & $\begin{array}{r}1.60 \\
7.76 \\
4.34\end{array}$ & $\begin{array}{l}\text { Sulfate }\left(\mathrm{SO}_{4}\right) \\
\text { Chloride }(\mathrm{Cl}) \\
\text { Fluoride (F) } \\
\text { Nitrate (NO}) \\
\text { Dissolved solids } \\
\text { Total hardness as } \mathrm{CaCO}_{3}\end{array}$ & $\begin{array}{c}149 \\
76 \\
3.7 \\
8.8 \\
602 \\
108\end{array}$ & 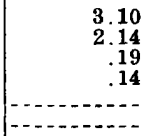 \\
\hline
\end{tabular}




\section{WHEELER COUNTY}

\section{SHAMROCK}

Population in 1940: 3,123.

Source of information: T. M. Dickey, water superintendent, Dec. 13, 1946.

Ownership: Municipal.

Source of supply: Bronco Springs and five wells 12 miles north of Shamrock.

Bronco Springs. In SE $1 / 4$ sec. 41 , blk. A8; water collected in galleries through tile drains; yield, about 150 gallons a minute.

Well 1. In NE1/4 sec. 2, Poitevent subdivision; drilled by Ke!ly Well Co. in 1928; depth, 50 feet; diameter, 18 inches; deep-well turbine pump and 71/2-horsepower electric motor; static water level, 15 feet below land surface in 1928 and 26.52 feet below land surface Dec. 13, 1946; original yield, 175 gallons a minute; pump broke suction afier pumping several hours in 1946.

Well 2. About 534 feet west of well 1; drilled by Kelly Well Co. in 1928; depth, 36 feet; diameter, 18 inches; deep-well turbine purp and 71/2horsepower electric motor; static water level, 8 feet below land surface in 1928 and 21.3 feet below land surface Dec. 14, 1946; original yield, 175 gallons a minute; pump broke suction after pumping 4 or 5 hours in 1946.

Well 3. About 638 feet north of well 2; drilled by Kelly Well Co. in 1928; depth, 48 feet; diameter, 18 inches; deep-well turbine pump and 15horsepower electric motor; static water level, 12 feet below land surface in 1928 and 31.0 feet below land surface Dec. 14, 1916; original yield, 250 gallons a minute; pump broke suction after pumping 4 or 5 hours in 1946.

Well 4. About 600 feet north of well 3; drilled by Kelly Well Co. in 1928; depth, 66 feet; diameter, 18 inches; deep-well turbine pump and 15horsepower electric motor; static water level, 13.8 feet below land surface in 1928 and 34.9 feet below land surface Dec. 14, 1946; original yield, 250 gallons a minute; yield in 1946, 200 gallons a minute; temperature, $62^{\circ} \mathrm{F}$.

Well 5. About 628 feet north of well 1; drilled by Kelly Well Co. in 1928; depth, 65 feet; diameter, 18 inches; deep-well turbine pump and 15horsepower electric motor; static water level, 25 feet below land surface in 1928 and 42.8 feet below land surface Dec. 14, 1916; original yield, 250 gallons a minute; yield somewhat less in 1946.

Pumpage: Average, 690,000 gallons a day in 1945.

Storage: Ground storage reservoir in well field, 50,000 gallons; ground storage reservoir $1 \frac{1}{2}$ miles north of city, 1,000,000 gallons; grcrund storage reservoir in city, 1,000,000 gallons; elevated tank, 75,000 gallons.

Number of customers : 1,129 .

Treatment: None. 


\section{Analyses}

[Collected Dec. 13, 1946. pH, Bronco Springs and well 4, each 7.4. Analyzed by C. B. Cibulka]

\begin{tabular}{|c|c|c|c|c|}
\hline & \multicolumn{2}{|c|}{ Bronco Springs } & \multicolumn{2}{|c|}{ Well 4} \\
\hline & $\begin{array}{c}\text { Parts per } \\
\text { million }\end{array}$ & $\begin{array}{c}\text { Equivalents } \\
\text { per million }\end{array}$ & $\begin{array}{l}\text { Parts per } \\
\text { million }\end{array}$ & $\begin{array}{l}\text { Equivalents } \\
\text { per million }\end{array}$ \\
\hline 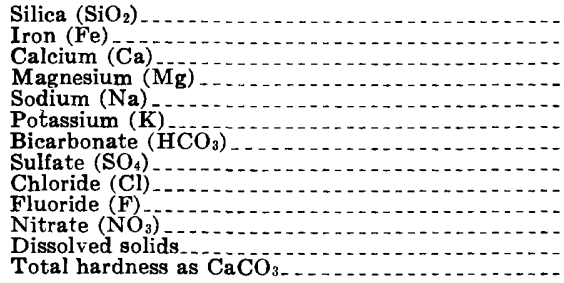 & $\begin{array}{l}19 \\
67.05 \\
5.1 \\
5.8 \\
2.3 \\
224 \\
5.1 \\
8.0 \\
2.6 \\
218 \\
188\end{array}$ & $\begin{array}{r}.344 \\
.419 \\
.254 \\
.059 \\
3.672 \\
.106 \\
.226 \\
.032 \\
.040 \\
-30\end{array}$ & $\begin{array}{c}18 \\
68 \\
6.04 \\
4.5 \\
7.2 \\
1.7 \\
202 \\
7.6 \\
6.0 \\
30 \\
36 \\
261 \\
188\end{array}$ & $\begin{array}{r}\mathbf{3} . \mathbf{3 9 4} \\
.370 \\
.315 \\
.043 \\
\mathbf{3 . 3 1 1} \\
.158 \\
.169 \\
.000 \\
.484 \\
\hdashline\end{array}$ \\
\hline
\end{tabular}

\section{WHEELER}

Population in 1940: 848.

Source of information: J. E. Risner, water superintendent, Dec. 16, 1946.

Ownership: Municipal.

Source of supply: Well $1 \frac{1}{2}$ miles west of town in SE1/4 SW $1 / 4$ sec. 13, blk. A4; drilled in 1926 by L. D. Lancaster and others; depth, about 100 feet; diameter, 15 to 8 inches, 40 feet of 8-inch screen, gravel-walled; de?p-well turbine pump and 15-horsepower electric motor; static water levol reported, 27 feet below land surface; yield reported, 200 gallons a minute with drawdown of 17 feet.

Pumpage (estimated) : Maximum 288,000; minimum 60,000; averrge, 150,000 gallons a day.

Storage: Steel ground tank at well, 50,000 gallons; elevated t־nk, 50,000 gallons.

Number of customers: 200.

Treatment: Occasional chlorination.

\section{Analysis}

[Collected Dec. 16, 1946. $\mathrm{pH}$ is 7.4. Analyzed by C. B. Cibulka]

\begin{tabular}{|c|c|c|c|c|c|}
\hline & $\begin{array}{l}\text { Parts per } \\
\text { million }\end{array}$ & $\begin{array}{l}\text { Equivalents } \\
\text { per million }\end{array}$ & & $\begin{array}{l}\text { Parts p or } \\
\text { millior }\end{array}$ & $\begin{array}{l}\text { Equivalents } \\
\text { per million }\end{array}$ \\
\hline $\begin{array}{l}\text { Silica }\left(\mathrm{SiO}_{2}\right) \\
\text { Iron }(\mathrm{Fe}) \\
\text { Calcium }(\mathrm{Ca}) \\
\text { Magnesium }(\mathrm{Mg}) \\
\text { Sodium }(\mathrm{Na}) \\
\text { Potassium }(\mathrm{K}) \\
\text { Bicarbonate }\left(\mathrm{HCO}_{3}\right)^{-}\end{array}$ & $\begin{array}{l}23 \\
83^{.08} \\
6.3 \\
24 \\
1.8 \\
294\end{array}$ & $\begin{array}{r}4.14 \\
.52 \\
1.06 \\
.05 \\
4.82\end{array}$ & $\begin{array}{l}\text { Sulfate }\left(\mathrm{SO}_{4}\right) \\
\text { Chloride }(\mathrm{Cl}) \\
\text { Fluoride (F) } \\
\left.\text { Nitrate (NO})_{8}\right) \\
\text { Dissolved solids. } \\
\text { Total hardness as } \mathrm{CaCO}_{3}\end{array}$ & $\begin{array}{l}14 \\
14 \\
15 \\
249 \\
233\end{array}$ & $\begin{array}{r}0.29 \\
.39 \\
.03 \\
.24 \\
-0 .- \\
\end{array}$ \\
\hline
\end{tabular}

\section{WINKLER COUNTY}

\section{KERMIT}

Population in 1940: 2,584.

Source of information: Fred W. Pearson, mayor, May 15, 1947.

Ownership: Municipal. 
Source of supply: Four wells.

Well 1 (Underwood). Drilled; depth, 700 feet; diameter, 121/2 inches; deep-well turbine pump and 25-horsepower electric motor; yield, 350 gallons a minute.

Well 2 (Walton). Drilled in 1935 by Crandell and Osmond; depth, 545 feet; diameter, $12 \frac{1}{2}$ inches; deep-well turbine pump and 25-horsepower electric motor.

Well 3 (Underwood). Drilled in 1946 by Permian Well Service; depth, 510 feet; diameter, $13 \%$ inches; deep-well turbine pump and 25-horsepower electric motor; pump set at 180 feet; static water level reported, 90 feet below land surface when drilled; yield, 360 gallons a minute.

Well 4 (Walton). Drilled in 1946 by Permian Well Service; depth, 471 feet; diameter, 20 to $13 \%$ inches; gravel-walled and gur-perforated; deep-well turbine pump and 25-horsepower electric motor; static water level reported, 95 feet below land surface when $d$ yilled; yield, 360 gallons a minute.

Average pumpage, in gallons a day

\begin{tabular}{c|c|c|c}
\hline 1943 & 1944 & 1945 & 1946 \\
\hline 195,000 & 262,000 & 335,000 & 590,000 \\
\hline
\end{tabular}

Storage: Two ground storage reservoirs, 300,000 gallons each; elevated tank, 150,000 gallons.

Number of customers: 1,172 .

Treatment: None.

\section{Analysis, well 4 (Walton)}

[Collected May 15, 1947. $\mathrm{pH}$ is 7.5. Analyzed by B. C. Dwyer]

\begin{tabular}{|c|c|c|c|c|c|}
\hline & $\begin{array}{l}\text { Parts per } \\
\text { million }\end{array}$ & $\begin{array}{l}\text { Equivalents } \\
\text { per million }\end{array}$ & & $\begin{array}{l}\text { Parts per } \\
\text { million }\end{array}$ & $\begin{array}{l}\text { Equivalents } \\
\text { per million }\end{array}$ \\
\hline $\begin{array}{l}\text { Silica }\left(\mathrm{SiO}_{2}\right) \\
\text { Iron }(\mathrm{Fe}) \\
\text { Calcium }(\overline{\mathrm{C}}) \\
\text { Magnesium }(\mathrm{Mg}) \\
\text { Sodium }(\mathrm{Na}) \\
\text { Potassium }(\mathrm{K}) \\
\text { Bicarbonate }\left(\mathrm{H} \overline{C O}_{3}\right)\end{array}$ & $\begin{array}{l}26 \\
40^{.02} \\
7.2 \\
25 \\
4.6 \\
146^{4} .6\end{array}$ & $\begin{array}{r}.997 \\
1.073 \\
2.118 \\
2.393\end{array}$ & $\begin{array}{l}\text { Sulfate }\left(\mathrm{SO}_{4}\right) \\
\text { Chloride }(\mathrm{Cl}) \\
\text { Fluoride (F) } \\
\left.\text { Nitrate (NO})_{3}\right) \\
\text { Dissolved solids } \\
\text { Total hardness as } \mathrm{CaCO}_{3}\end{array}$ & $\begin{array}{r}34 \\
19 \\
1.8 \\
3.0 \\
232 \\
129\end{array}$ & $\begin{array}{r}0.536 \\
.095 \\
.048 \\
-0 . \\
\end{array}$ \\
\hline
\end{tabular}

\section{Drillers' logs}

\begin{tabular}{|c|c|c|c|c|c|}
\hline & $\begin{array}{c}\text { Thickness } \\
\text { (feet) }\end{array}$ & $\begin{array}{l}\text { Depth } \\
\text { (feet) }\end{array}$ & & $\underset{\text { (feet) }}{\text { Thinkness }}$ & $\begin{array}{l}\text { Depth } \\
\text { (feet) }\end{array}$ \\
\hline \multicolumn{6}{|c|}{ Well 3 (Underwood) } \\
\hline $\begin{array}{l}\text { Caliche } \\
\text { Sand } \\
\text { Red beds. } \\
\text { Sand and gravel (water) } \\
\text { Red shale. } \\
\text { Red bed. } \\
\text { Gravel (water) } \\
\text { Red bed } \\
\text { Sand } \\
\text { Red bed. }\end{array}$ & $\begin{array}{r}44 \\
156 \\
10 \\
13 \\
3 \\
16 \\
6 \\
32 \\
5 \\
15\end{array}$ & $\begin{array}{r}44 \\
200 \\
210 \\
223 \\
226 \\
242 \\
248 \\
280 \\
285 \\
300\end{array}$ & $\begin{array}{l}\text { Red rock } \\
\text { Gravel a id sand (water) } \\
\text { Sand } \\
\text { Red bed } \\
\text { Sand } \\
\text { Red bed } \\
\text { Sand (water) } \\
\text { Red bed } \\
\text { Sand } \\
\text { Red bed }\end{array}$ & $\begin{array}{r}8 \\
7 \\
2 \\
13 \\
5 \\
81 \\
19 \\
21 \\
5 \\
40\end{array}$ & $\begin{array}{l}308 \\
315 \\
317 \\
330 \\
335 \\
416 \\
435 \\
456 \\
461 \\
501\end{array}$ \\
\hline
\end{tabular}




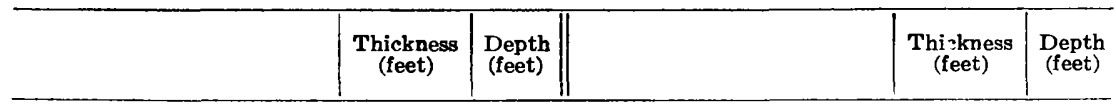

Well 4 (Walton)

\begin{tabular}{|c|c|c|c|c|c|}
\hline $\begin{array}{l}\text { Sand and caliche } \\
\text { Sand } \\
\text { Red bed } \\
\text { Sand and gravel (water) } \\
\text { Red bed } \\
\text { Sand and gravel (water) } \\
\text { Red bed } \\
\text { Sand and gravel (water) } \\
\text { Red bed } \\
\text { Sand } \\
\text { Red bed } \\
\text { Sand and gravel } \\
\text { Red bed. } \\
\text { Sand }\end{array}$ & $\begin{array}{r}24 \\
158 \\
13 \\
18 \\
38 \\
5 \\
24 \\
3 \\
37 \\
14 \\
4 \\
1 \\
9\end{array}$ & $\begin{array}{r}24 \\
182 \\
195 \\
213 \\
251 \\
256 \\
280 \\
283 \\
320 \\
334 \\
338 \\
\mathbf{3 3 9} \\
\mathbf{3 4 8} \\
\mathbf{3 5 0}\end{array}$ & $\begin{array}{l}\text { Red bed. } \\
\text { Sand bed } \\
\text { Red bed } \\
\text { Sand } \\
\text { Red bed. } \\
\text { Sand } \\
\text { Red bed } \\
\text { Sand } \\
\text { Red bed } \\
\text { Sand (water) } \\
\text { Red bed. } \\
\text { Sand } \\
\text { Red bed }\end{array}$ & $\begin{array}{r}8 \\
5 \\
5 \\
9 \\
\mathbf{3} \\
10 \\
13 \\
19 \\
\mathbf{3} \\
\mathbf{2 3} \\
\mathbf{8} \\
13 \\
\mathbf{2}\end{array}$ & $\begin{array}{l}358 \\
363 \\
368 \\
377 \\
380 \\
390 \\
403 \\
422 \\
425 \\
448 \\
456 \\
469 \\
471\end{array}$ \\
\hline
\end{tabular}

\section{YOAKUM COUNTY}

\section{PLAINS}

Population in 1940: 480.

Source of information: D. B. McGinty, owner, May 1945.

Owner: D. B. McGinty.

Source of supply: Well drilled in 1940 by W. A. Willis; depth, 128 feet; diameter, 12 inches; deep-well turbine pump and gasoline engine; static water level reported, 72 feet below land surface in 1940.

Pumpage: No record.

Storage: Elevated tank, about 50,000 gallons.

Number of customers: 65 .

Treatment: None.

\section{Analysis}

[Collected May 1945. pH is 7.7. Analyzed by J. H. Rowley]

\begin{tabular}{|c|c|c|c|c|c|}
\hline & $\begin{array}{c}\text { Parts per } \\
\text { million }\end{array}$ & $\begin{array}{l}\text { Equivalent } \\
\text { per million }\end{array}$ & & $\begin{array}{l}\text { Parts per } \\
\text { million }\end{array}$ & $\begin{array}{l}\text { Equivalents } \\
\text { per million }\end{array}$ \\
\hline 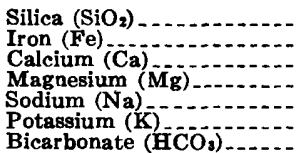 & $\begin{array}{l}42 \\
114 \\
111 \\
179 \\
34 \\
241\end{array}$ & $\begin{array}{r}\mathbf{5} .69 \\
\mathbf{9} .13 \\
7.78 \\
.87 \\
\mathbf{3 . 9 5}\end{array}$ & $\begin{array}{l}\text { Sulfate }\left(\mathrm{SO}_{4}\right) \\
\text { Chloride }(\mathrm{Cl}) \\
\text { Fluoride (F) } \\
\text { Nitrate (NO})_{3} \\
\text { Dissolved solids_. } \\
\text { Total hardness as } \mathrm{CaC} \mathrm{CO}_{3}\end{array}$ & $\begin{array}{r}779 \\
102 \\
5.5 \\
8.0 \\
1,490 \\
741\end{array}$ & $\begin{array}{r}16.22 \\
2.88 \\
.29 \\
.18 \\
\\
\\
\end{array}$ \\
\hline
\end{tabular}

\section{DENVER CTTY}

Population in 1940: 1,750 .

Source of information: Water superintendent, December 1944.

Ownership: Municipal.

Source of supply: Two wells.

Well 1. Drilled in 1939 by W. A. Willis; depth, 178 feet; ciameter, 8 inches; deep-well turbine pump and 5-horsepower electric motor; yield, 95 gallons a minute. 
Well 2. Drilled in 1940 by W. A. Willis; depth, 180 feet; diameter, 8 inches; deep-well turbine pump and 10-horsepower electric motor; yield, 150 gallons a minute.

Pumpage: Average, 48,300 gallons a day.

Storage: Two ground storage reservoirs and elevated tank, total capacity, 125,000 gallons.

Number of customers: 234.

Treatment: None.

Analysis, well 2

[Collected December 1944. pH is 7.8. Analyzed by W. W. Hastings'

\begin{tabular}{|c|c|c|c|c|c|}
\hline & $\begin{array}{c}\text { Parts per } \\
\text { million }\end{array}$ & $\begin{array}{l}\text { Equivalents } \\
\text { per million }\end{array}$ & & $\begin{array}{c}\text { Parts per } \\
\text { million }\end{array}$ & $\begin{array}{l}\text { Equivalents } \\
\text { per million }\end{array}$ \\
\hline $\begin{array}{l}\text { Silica }\left(\mathrm{SiO}_{2}\right) \\
\text { Iron }(\mathrm{Fe}) \\
\mathrm{Calcium}(\mathrm{Ca}) \\
\text { Magnesium }(\mathrm{Mg}) \\
\text { Sodium and potassium } \\
\quad(\mathrm{Na}+\mathrm{K}) \text {. } \\
\text { Bicarbonate }\left(\mathrm{HCO}_{2}\right)\end{array}$ & $\begin{array}{l}61 \\
62 \\
33 \\
44 \\
229\end{array}$ & $\begin{array}{r}3.09 \\
2.71 \\
1.91 \\
3.75\end{array}$ & $\begin{array}{l}\text { Sulfate }\left(\mathrm{SO}_{4}\right) \\
\text { Chloride }(\mathrm{Cl}) \\
\text { Fluoride (F) } \\
\left.\text { Nitrate (NO})_{3}\right) \\
\text { Dissolved solids. } \\
\text { Total hardness as } \mathrm{CaCO}_{3}\end{array}$ & $\begin{array}{c}110 \\
51 \\
2.7 \\
5.5 \\
559 \\
290\end{array}$ & $\begin{array}{r}2.29 \\
1.44 \\
.14 \\
.09\end{array}$ \\
\hline
\end{tabular}

\section{IITERATURE CITED}

American Public Health Association, 1946, Standard methods for the examination of water and sewage, 9 th ed.

Broadhurst, W. L., Sundstrom, R. W., and Rowley, J. H., 1950, Public water supplies in southern Texas: U. S. Geol. Survey Water-S'pply Paper 1070.

Collins, W. D., 1928, Notes on practical water analysis: U. S. Geol. Survey Water-Supply Paper 596-H.

Dean, H. T., 1938, Endemic fluorosis and its relation to dental caries: U. S. Public Health Repts., vol. 53.

Faucett, R. L., and Miller, H. C., 1946, Methemoglobinemia occrrring in infants fed milk diluted with well water of high nitrate content: Jour. Pediatrics, vol. 29.

Smith, H. V., Smith, M. C., and Foster, E. O., 1936, Mottled enamel in the Salt River Valley and the fluorine content of the water supplies: Univ. Arizona Agr. Exper. Sta. Tech. Bull. 61.

Sundstrom, R. W., Broadhurst, W. L., and Dwyer, B. C., 1949, Public water supplies in central and north-central Texas: U. S. Geol. Survey WaterSupply Paper 1069.

Sundstrom, R. W., Hastings, W. W., and Broadhurst, W. L., 1948, Public water supplies in eastern Texas: U. S. Geol. Survey Vater-Supply Paper 1047.

Wilcox, L. V., 1948, Explanation and interpretation of analyses of irrigation waters: U. S. Dept. Agr. Circ. 784. 


\section{INDEX}

A

Abernathy -
Acknowledgments
Adrian
Alpine
Amarillo
Amherst
Andrews
Anton
Aspermont
Balmorhea
Barnhart -
Barstow
Big Lake -
Big Spring
Booker -
Borger
Bronte
Brownfield -

Canadian

Canyon

Channing

Chemical character of the water.

Childress

Clarendon

Claude

Coahoma -.-._-

Colorado City

Crane -_- Con

Crosbyton

D

Dalhart

Del Rio

Denver City

Dickens

Dimmit

Dodsonvill

Dougherty

Dumas

e - - -

-.....--

Eldorado

\section{$\mathbf{E}$}

El Paso

Estelline

Extent of area
Page

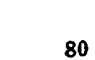

124-125

18-19

129-135

101

15-16

89-90

148-149

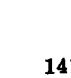

141

99

160

138-139

91-95

104

97-98

28-29

154-155

88-89

136-138

87-88

9-12

24-27

155

47-48

17

95

117-119

32-34

35

\section{(}

37-39

159

165-166

44-46

24

80

69

119-120

144-145

61-65

61-65

82-83

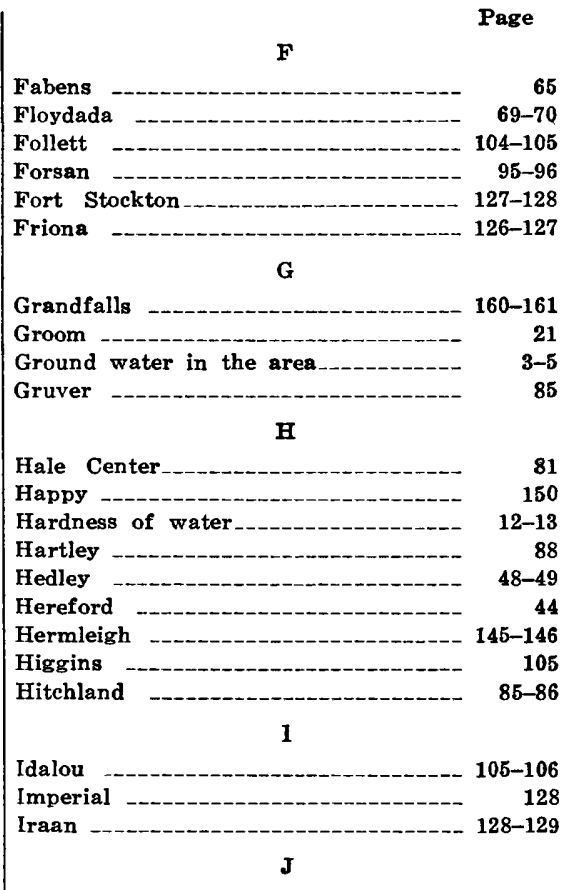

Jurassic rocks, Broad Pass area

Jayton _..._-_._. 101

$\mathbf{K}$

$163-165$

$\mathbf{L}$

$40-43$

77-78

90-91

166

102

70

36

$106-109$

M

McCamey -_-_- 157-158

McLean - 78-79

Marfa -_._- 135-136

Matador -.-_-_-_-_-_-_-_-_-_-_-_-_-_ 120-121

Memphis _._ 83-84

Mertzon - 


\section{INDEX}

\begin{tabular}{|c|c|c|c|}
\hline & Page & & Page \\
\hline Miami & 144 & Sanderson & $151-154$ \\
\hline Midland _-_-_- & $113-117$ & Santa Rita & 139 \\
\hline Monahans - & 161 & Seagraves & 71-73 \\
\hline Morse & 86 & Seminole & $73-74$ \\
\hline Morton & 28 & Shamrock & $162-163$ \\
\hline Muleshoe & $17-18$ & Sierra Blanca & $96-97$ \\
\hline $\mathbf{O}$ & & $\begin{array}{l}\text { Silverton } \\
\text { Skelleytown }\end{array}$ & $\begin{array}{r}20 \\
22-23\end{array}$ \\
\hline Odessa & $49-60$ & Slaton - & $109-110$ \\
\hline O'Donnell _- & $110-111$ & Snyder - & $146-147$ \\
\hline Olton & $102-103$ & Sonora & $149-150$ \\
\hline - n-m- & 34-35 & $\begin{array}{l}\text { South Plains } \\
\text { Spearman } \\
\text { Spur }\end{array}$ & $\begin{array}{r}71 \\
86-87 \\
46-47\end{array}$ \\
\hline aducah & $31-32$ & Stanton & $112-113$ \\
\hline Pampa - - - - & $79-80$ & Stinnett -- & 99 \\
\hline andle - & 21-22 & ford & 148 \\
\hline Pecos & $141-143$ & n & 103 \\
\hline Perryton - & -124 & Surface water in the area & \\
\hline Petersburg - & 81 & Nater & 123 \\
\hline Plains & 165 & & \\
\hline Plainview & 82 & 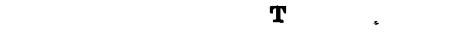 & \\
\hline 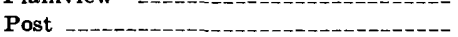 & 74-77 & Tahoka _.... & 111 \\
\hline idio - & 136 & Texline - & $39-40$ \\
\hline $\cos$ & $98-99$ & Texon - & $139-141$ \\
\hline $\mathbf{Q}$ & & $\begin{array}{l}\text { Tormillo - } \\
\text { Toyah }\end{array}$ & $\begin{array}{r}66 \\
143-144\end{array}$ \\
\hline Quitaque & $19-20$ & 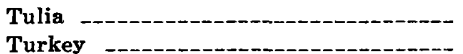 & $\begin{array}{r}151 \\
84\end{array}$ \\
\hline $\mathbf{R}$ & & $\mathbf{V}$ & \\
\hline 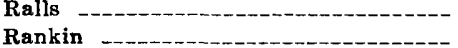 & $\begin{array}{r}36 \\
158-159\end{array}$ & Valentine - & 100 \\
\hline Roaring Springs & 121 & Van Horn & 37 \\
\hline Robert Lee & 29 & Vega - & 125 \\
\hline Roby - & 67 & W & \\
\hline Rocksprings _- & 61 & Vellington & $30-81$ \\
\hline Roseoe - - & 122 & $\begin{array}{l}\text { Wheeler } \\
\text { Whing }\end{array}$ & 163 \\
\hline Rotan - - & $67-68$ & $\begin{array}{l}\text { Wheeler } \\
\text { White Deer } \\
\text { Wilderado }\end{array}$ & $\begin{array}{r}103 \\
23 \\
125-126\end{array}$ \\
\hline ngelo & & $\mathbf{Y}$ & $66-67$ \\
\hline
\end{tabular}

